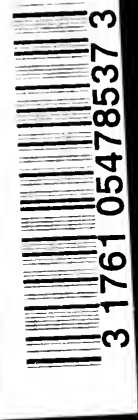


Digitized by the Internet Archive in 2007 with funding from Microsoft Corporation 

. 


\section{DISEASES OF THE SKIN}




\section{$1 \mu^{s * x}$ \\ Diseases of the Skin}

An Outline of the Principles and Practice of Dermatology

BY

SIR MALCOLM MORRIS, K.C.V.O.

Surgeon to the Skin Department of the Seamen's Hospital, Greenwich; Lecturer on Dermatology in the London School of Clinical Medicine; Consulting Surgeon to the Skin Department, St. Mary's Hospital

\section{FIFTH EDITION}

Revised by the Author, with the assistance of S. ERNEST DoRE, M.D. Cantab., M.R.C.P., Physician to the Skin Department of the Itampstead General Hospital and to the Skin Department of the Evelina Hospital for Sick Children; Assistant in the Skin Department of the Middlesex HLospital

WITH 10 COLOURED AND 67 BLACK-AND-WHITE PLATES

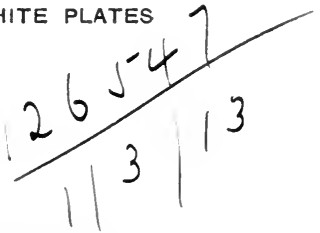

CASSELL AND COMPANY, LIMITED London, New York, Toronto and Melbourne 
First Edition 1893. Reprinted 1894 Second Edition 1898. Reprinted 1899 Third Edition 1903. Reprinted 1904 Fourth Edition 1908. Reprinted 1910 Fifth Edition 1911 


\section{PREFACE TO THE FIFTH EDITION}

Is the three years that have passed since the fourth edition of this Manual was published, advances have been made in so many branches of Dermatology, that the volume has had to undergo considerable revision, and a further enlargement to the extent of some seventy pages has been found unavoidable. An attempt has been made to estimate the true place of salvarsan in the treatment of syphilis, and the serviceability and limitations of the Wassermann test. The section on ringworm and other fungous diseases has been rewritten in the light of the researches of Sabouraud, Colcott Fox, Whitfield, and other workers. The conditions which offer scope to the newer methods of treatment, such as congelation, radium-therapy, serumtherapy, and vaccine-therapy, are considered. Some affections formerly included among neuro-dermatoses, or among new growths, have now been transferred to the ever-growing group of microbic diseases; and the influence of intestinal toxins in the production of cutaneous affections has been recognized. The diagnostic sections of the book have been strengthened, the descriptions of primary and secondary lesions in Chapter I. have been amplified, and space has been found for a new chapter on Affections of Mucous Membranes of Lips, Tongue, and Mouth. 
Some of the illustrations that appeared in earlier editions have been discarded, and many new plates have been included. These have been produced from photographs and other material kindly placed at my service by Mr. C. C. Choyce, Dr. J. A. Fordyce (New York), Dr. Wilfrid Fox, Mr. J. E. R. McDonagh, Dr. Ogle, Dr. Sabouraud (Paris), Dr. Stowers, Dr. Whitfield, and the Cairo School of Medicine. The Index has been extended, and it is still so arranged that the reader may see at a glance the main lines of treatment suitable for any given disease.

M. M.

8, Harley Street, W.

September, 1911. 


\section{CONTENTS}

CHAPTER

1. Pathology of the Skin .

Pathologieal Processes-Primary Lesions-Secondary Lesions.

2. Classification $\quad$ - . $\quad$. $\quad$. 20

3. Principles of Diagnosis * . . . . 24

Examination of the Patient-Chavacters of the Eruption-Diagnosis by Exelusion-Positive DiagnosisGeneral Eruptions-Local Eruptions.

4. Neuroses of the Skin : Classification . . $\quad 54$

5. Neuroses of the Skin (continued): Sensory Neuroses . . . . . . .

Hyperæsthesia-Meralgia Paræsthetica-Anæsthesia -Itching-Pruritus-Prurigo.

6. Neuroses of the Skin (continued)

Urticaria - Angio-Neurotic Edema - Hysterical Edema-Urtiearia Pigmentosa.

7. Neuroses of the Skin (continued)

Erythema Simplex-Erythema Fugax-Erythema ab Igne-Erythema Intertrigo-Infantile Erythema of Jaequet-Erythema Paratrimma-Erythema Scarlatiniforme-Erythema Infeetiosum-Rubeoloid Erythema-Erythema Uræmieum-Erythema SolareErythema Pernio-Frostbite-Erythema Keratodes - Erythema Multiforme-Erythema Perstans-Erythema Nodosum-Purpura Simplex-Purpura Hæmorrhagica-Purpura Rheumatica-Lupus Erythematosus-Flushing-Rosacea-Pellagra-Acrodynia.

8. Neuroses of the Skin (continued)

Dermatitis Herpetiformis - Herpes Gestationis Pompholyx-Pemphigus and Epidermolysis Bullosa -Irritative Herpes-Herpes Zoster. 
9. Neuroses of the Skin (concluded)

Sclerodermia - Morphœa - Morphœa Guttata Sclerodactylia - Ainhum - Lichen Planus - Lichen Nitidus-Lichen Annularis-Lichen Pilaris-Porokeratosis - Parakeratosis Variegata - Xantho-Erythrodermia Perstans-Pityriasis Rubra Pilaris-Congenital Ichthyosiform Erythrodermia-Erythrodermia Desquamativa-Anomalies of Pigmentation : Chloasma, Leucodermia, and HæmochromatosisRaynaud's Disease-Dermatitis Repens-Erythema Serpens-Diabetic Gangrene-"Hysterical" Gan. grene - Charcot's Bed-Sore - Trophic Ulcers Syringomyelia and Morvan's Disease-Glossy Skin -Atrophy of the Skin.

\section{Artificial Eruptions}

Eruptions due to Animal and Vegetable AgentsTrade Eruptions-Röntgen-Ray and Radium Dermatitis - Feigned Eruptions - Drug EruptionsVaccination Eruptions - Tuberculin EruptionsAntitoxin and Serum Eruptions.

\section{Eczema}

Definition-Symptoms-Modifying Influences: Distribution and Regional Peculiarities, Sex, AgeSpecial Forms: Seborrhœic Eczema, Sweat Eczema, Eczerna Folliculorum, "Nervous Eczema"-Summary of Subjective Symptoms-ComplicationsPathology-Etiology-Diagnosis-Prognosis.

\section{Eczema (concluded)}

Internal Remcdies-Local Treatment, including Radiotherapy - Diet - Clothing - Hydrotherapy Climate-Summary.

\section{Psoriasis and Parapsoriasis}

Psoriasis: Objective Symptoms-Subjective Symptoms-Clinical Course-Pathology-Etiology-Diagnosis-Prognosis-Treatment. Parapsoriasis.

\section{Pityriasis}

'Pityriasis Rubra and Ritter's Disease-Pityriasis Rosea. 
Scabies-Scabies of Animal Origin-Grain ItchBarley Itch-Pediculosis-Miscellaneous Parasites.

16. Parasitic Affections (continued): Vegetable Parasites

Ringworm : Tinea Tonsurans-Tinea Sycosis-Tinea Circinata-Ringworm of the Nails-Eczema Marginatum and Dhobie's Itch.

17. Parasitic Affections (concluded): Vegetable Parasites (concluded)

Tinea Imbricata-Tinea Versicolor-Other TineasErythrasma-Favus - Pinta - Mycetoma - Actinomycosis-Streptothrix Infection-SporotrichosisBlastomycetic Dermatitis-Labiomycosis.

18. Microbic Affections . . . . . .

Tuberculosis-Clinical Tests-Lichen ScrofulosusStrumous Ulcers-Tuberculous Ulcers-Verruca Necrogenica-Erythema Induratum ScrofulosorumHypodermic Sarcoids-Tuberculides-Acneiform Tuberculide-Acne Cachecticorum-Multiple Benign Sarcoid.

19. Microbic Affections (continued) : Lupus-Vúgaris Symptoms-Clinical Course-Secondary EffectsEtiology_Secondary Causes-Pathology-Diagnosis -Prognosis-Treatment.

20. Microbic Affections (continued): Syphilis .

Stages-Primary Lesion-Secondary Lesions-Tertiary Lesions-Hereditary Syphilis-Etiology-Diagnosis-Wassermann Test-Demonstration of the Spirochæte-Prognosis.

21. Microbic Affections (continued): Syphilis (concluded) .

Mercury : Oral Method-Intramuscular, Subcutaneous, and Intravenous Injection-Inunction-Mercurial Baths and Suppositories. Arsenic: Salvarsan - Superiority of Intravenous to Intramuscular or Subcutaneous Injection-Dosage-Results. Hectine.

22. Microbic Affections (continued): Leprosy-Yaws 
23. Microbic Affections (concluded)

Erysipelas-Cutaneous Diphtheria-Impetigo Contagiosa-Pemphigus Contagiosus-Bullous Impetigo of Infants-Dermatitis Gangrenosa Infantum-Impetigo Herpetiformis-Impetigo of Bockhart-Coccogenio Sycosis - Furunculi - Carbuncle - Dissection Wounds-Acne Vulgaris-Acne Varioliformis-Acne Keratosa and Acne Excoriata-Acnitis-GlandersRhinoscleroma - Malignant Pustule-Gonorrhœal Keratodermia-Ulcus Molle-Furunculus Orientalis -Elephantiasis Arabum-Granuloma Pyogenicum - Ulcerating Granuloma of the Pudenda.

\section{Affections of the Sebaceous and of the Sweat-} Glands

Seborrhoa-Seborrhœa Corporis-Milium-Comedones. Hyperidrosis-Granulosis Rubra Nasi-Bromidrosis - Chromidrosis - Uridrosis - Anidrosis Sudamina-Miliaria Papulosa.

25. Affections of the Mucous Membranes of the Lips, Tongue, Mouth, etc.

Perlèche-Cheilitis Exfoliativa-Cheilitis Glandularis -Fordyce's Disease-Ichthyosis Glossæ-Leucoplakia of the Tongue-Leucoplakia of the Penis, Vulva, and Vagina-Kraurosis Vulvæ-Grooved Tongue-Glossitis Areata Exfoliativa-Black Tongue.

26. Affections of the Hair and of the Nails .

Folliculitis Decalvans - Pseudopelade - Dermatitis Papillaris Capillitii-Hirsuties-Atrophic Changes in the Hair-Trichorrhexis Nodosa-MonilethrixCanities-Alopecia-Alopecia Areata-LepothrixTrichomycosis Capillitii-Piedra-Tinea Nodosa. Onychia and Paronychia-Ingrowing Toe-NailHypertrophy of the Nails-Egg-Shell Nail.

27. New Growths : Benign Neoplasms . . .

Cysts-Adenoma-Cheloid-Fibroma MolluscumDiffuse Fibroma-Von Recklinghausen's DiseaseHard Fibromas and Neuro-Fibromas-Myoma Cutis Myxoma-Telangiectasis-Nævus Vascularis-Nævus Anæmicus-Nævus Pigmentosus-Angio-Keratoma -Infective Angioma-Angio-Fibroma Contagiosum 
Tropicum-Lymphangioma-Xanthoma-Xanthoma of Balzer-Molluscum Contagiosum-Colloid Milium - Epithelioma Adenoides Cysticum-Keratosis Follicularis-Acanthosis Nigricans-Lentigo-Papilloma

-Keratosis Senilis-Verruca Peruviana-Clavus-Callus-Cornu Cutaneum.

28. New Growths (concluded): Malignant Neoplasms

Paget's Disease-Cancer en Cuirasse-Melanotic Cancer-Epithelioma-Rodent Uleer-Sareoma of the Skin-Idiopathic Multiple Pigmented Hæmorrhagic Sarcoma-Mycosis Fungoides-Leukæmia Cutis-Kaposi's Disease.

29. Malformations . . . . . . . 721

Ichthyosis - Tylosis - Sclerema Neonatorum and (Edema Neonatorum-Albinism.

Index 



\section{LIST OF PLATES}

Plate

Facing page

1. Urticaria Pigmentosa $\quad$ • . . . 88

2. Lupus ERythematosus (Colour) • • . . 122

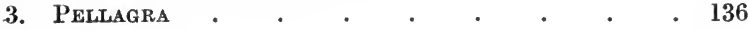

4. Dermatitis Herpetiformis . . . . 140

5. Microscopio Section of Vesicle in Dermatitis HeRPETIFORMIS . . . . . . . 141

6. Atrophied Skin and NaIls in Epidermolysis

BUllosa . . . . . . . 154

7. Bilateral Herpes in a Congenital Syphilutic - 176

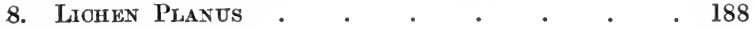

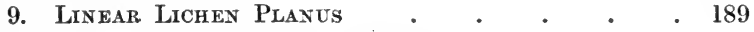

10. Hypertrophic Lichen Planus . . . . 192

11. Parakeratosis Variegata . . . . 202

12. Pityriasis Rubra Pilaris (Colour) . . . 204

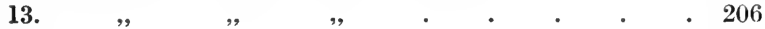

14. $\quad, \quad, \quad$. $\quad . \quad+\quad . \quad 207$

15. $, \quad, \quad$, $\quad$ - . . . . 208

16. LeUdodermia . . . . . . 209

17. Eruption due to Bromide of Potassium • 236

18. Generalized Vaccinia . . . . . 254

19. Mrcroscopic Section of Eczema Vesicle . . 284

20. Infectious Eczematord Dermatitis, following

Abscess of Breast . . . . . 285

21. AnNular Psorlasis . . . . . . 316

22. Microscopio Sfction of Psoriasis Lesions • $\quad 317$

23. Pityriasis Pubra (Colour) . . . . . 340

24. Animal Parasites . . . . . 352

25. Hairs in Microsporosis and 'Trichophytosis • 374

26. Hairs in Keriox and Favus . . . . 375 
Plate

Facing page

27. Cultures of Rrygworm Fungi . . . . 376

28. Cultures of Ringworm and Favus Fungi . . 377

29. Bullous Ringworm af Wrists . . . . . 394

30. Scrapings from Cases of Eczema Marginatum and

Ringworm of Toes . . . . . . 395

31. Favus and Tinea Fungr . . . . . 402

32. Author's Case of Favus . . . . . 403

33. Various Fuxgi axd Microörgaxisus (Colour) • 416

34. Actromycosis (Colour) . . . . . . 418

35. General Tuberculide $\quad$. $\quad . \quad$. $\quad . \quad 4442$

36. General Tuberculide, Showing Strumous Ulcers 443

37. Early Lupus Vulgaris . . . . . 446

38. Ulcerating Lupus Vulgaris . . . . 447

39. Lupus Vulgaris with Chronic OEdema . . 450

40. Microscopic Section of Nodule of Lupus VulGARIS $\quad . \quad . \quad . \quad . \quad . \quad . \quad . \quad 451$

41. Papulo-Maculo-Squamous Syphilide . . 476

42. Corymbose Syphilid . . . . . . . 477

43. Lenticular Syphilide (Colour) . . . . 478

44. Follicular Syphilide . . . . . . 480

45. Circinate Squamous Syphilide . . . . 481

46. Circinate Syphilide in a Negro. . . . 482

47. Leucodermic Syphilide in Neck (Colour) . . 484

48. Cutaneous Gumata of Foot; Nodular late SyPHILIS . . . . . . . 486

49. The Spirocheta Pallida . . . . 487

50. Nodular Leprosy with Lesions ox Toxgue . 518

51. Leprosy with Atrophic Skix Lesions axd MutiLATED FINGERS . . . . . . . 519

52. Nerve LeprosY . . . . . . . $\quad .520$

53. Nerve Leprosy with Muscular Atrophy . 521

54. Section of Pustule in Inpetigo Contagiosa • 546

55. Old Acne with Severe Scarring • . . 568

56. Elephantiasis of Foot, Legs, and Scrotum • 588

57. Elephantiasis of Mamia . . . . . . 590

58. Mrcroscopic Shotion of a Comedo . . 604

69. Alopecia Areata with Atrophied Nalls . 636 
Plate

60. Fibroma Molluscum . . . . . . 654

61. Von Recklinghausen's Disease . . . . . 655

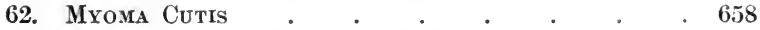

63. Telangiectasis with Ulceration (Colour) . . 660

64. Lymphangioma Circumscriptum Cutis . . . 668

65. Xanthoma Diabeticorum . . . . . 670

66. Microscopic Section of Moluuscum Contagrosum 676

67. Acanthosis Nigricans (Colour) . . . . $\quad 684$

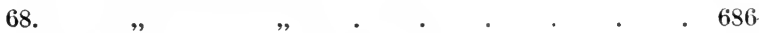

69. Linear and Unilateral Warts . . . . 687

70. Cancer en Cuirasse (Colour) . . . . 700

71. Microscopic Section of Epitheliona . . . 702

72. Early Rodent Ulcer . . . . . . 703

73. Rodent Ulcer-More Advanced Stage . . 704

74. Rodent Ulcer-Late Stage . . . 705

75. Microscopic Section of Rodent Ulcer . • 706

76. Mrcosis Fungordes . . . . . . 712

77. Tylosis in Father and Daughter . . . 726 



\section{DISEASES OF THE SKIN}

CHAPTER I

\section{PATHOLOGY OF THE SKIN}

\section{PATHOLOGICAL PROCESSES}

THE skin may be the seat of pathological processes similar in nature to those seen in other tissues and organs of the body, but modified to a greater or less degree by its position and anatomical structure. It may present congenital anomalies, the result of errors of development or intra-uterine disease, such as ichthyosis, nævi, moles, and albinism. Owing to its situation, it is particularly exposed to the injurious influences of heat and cold, which directly affect the circulation; of light, which in certain circumstances has an almost caustic action; of the friction or pressure of clothes or other substances in contact with the surface of the body; of the manifold sources of irritation furnished by fungi and other parasitic organisms, animal and vegetable; and of traumatism of various kinds. The skin may further be involved in processes which begin in the deeper tissues; it may be stretched and broken by the expansion of growths or the collection of fluid, or bound down by adhesions to the underlying parts.

Injuries to the skin have a special tendency to become complicated by infective processes, owing to the presence of pyogenic and other organisms on the surface of the healthy skin, in the sebaceous and other glands. 
Anomalies of secretion play a large part in the pathology of the skin. Retention of secretion, caused by mechanical obstruction or nervous influence, is frequently the starting-point of inflammatory processes. Excessive or diminished secretion is often dependent on abnormal states of the nerve centres or of the peripheral nerves. Profuse sweating may be the result of nerve exhaustion, or of the presence in the blood of toxic matters calling for elimination. An excessive secretion of sebum is often the starting-point of the inflammatory process in eczema seborrhoicum.

Iike all other tissues, the skin is liable to inflammation, and the process is essentially the same as in other organs. The classical signs of inflammation, as given by Celsus-redness, swelling, heat, and pain-are particularly manifest in the skin. A characteristic feature of inflammation of the skin, however, is that the disorder of sensation generally expresses itself in the form of $i t c h$ ing (see p. 64) rather than of pain. The general definition of inflammation given by Burdon-Sanderson-" the succession of changes which occurs in a living tissue when it is injured, provided that the injury is not of such a degree as at once to destroy its structure and vitality" -applies to the skin as to other tissues. The essential part of the process is increased diapedesis of white corpuscles, with escape of liquid exudation from capillaries and small veins, and accumulation of these bodies, causing obstruction in the lymphatics. The higher degrees of inflammation are marked by stasis in the capillaries, veins, and small arteries; if this condition persists it induces necrosis. If the necrosed part liquefies, the leucocytes which have left the channels of the affected vessels find their way in large numbers into the necrotic liquefied tissue and become pus cells, the result being the formation of an abscess. The process by which leucocytes are attracted or repelled 
by irritant materials, whether these be products of microörganisms or of any other kind, is termed "chemiotaxis," and the power which the leucocytes display of engulfing foreign bodies, such as bacteria, is termed "phagocytosis." According to investigations by Leishmann, Wright, and Douglas, this phagocytic activity depends upon the presence in the serum of certain substances which affect bacteria in such a way as to enable them to be taken up by the leucocytes. To these substances Wright gave the name of opsonins.

The first step in recovery from inflammation is the cessation of stasis followed by restoration of the blood circulation. Before stasis disappears, however, hæmoglobin, or red blood-corpuscles, frequently escape from a capillary into the surrounding tissue, with the result that pigmentation of a more or less permanent character is left behind. According to Virchow, the pigment is always derived from the blood, and is at first held in solution in the plasma which bathes the tissues. The pigment appears to be derived from certain special mesoblastic cells, to which Ehrmann gives the name of melanoblasts. The majority of observers agree that melanin, which can be distinguished from hæmatogenous pigments by morphological and chemical tests, has its origin in the blood, the pigment-bearing cells or chromatophores being variously regarded as connective-tissue cells (Unna, Kölliker), leucocytes (Schmidt), or protoplasmic processes from epithelial cells (Kromayer). Other writers (Kaposi, Delépine) hold that melanin is not a degeneration product of hæmoglobin, but a separation or secretion product of the protoplasm of pigmented cells. ${ }^{1}$

Slighter degrees of the inflammatory process, if long persistent, result in hyperplasia of the fixed connective-

${ }^{1}$ MacLeod, "Handbook of the Pathology of the Skin," p. 305. 
tissue cells and in the presence of plasma cells. The latter, which are variously regarded as pathologically altered connective-tissue cells (Unna), as mononuclear leucocytes or lymphocytes (Marschalko, Jadassohn), or as endothelial cells (Whitfield), ${ }^{\mathbf{1}}$ occur in the infective granulomas and other pathological conditions as well as in simple chronic inflammation.

All degrees of dermatitis may be set up by the application to the skin of irritants, such as mustard oil, in solutions of varying strength. The slightest irritation causes temporary hyperæmia, in which it would be impossible, on simple inspection, to say that exudation had taken place. The microscope, however, shows the process to be really inflammatory. By using progressively stronger irritants, papules, vesicles, blebs, and other lesions may be produced. On removing the irritant, recovery takes place with a greater or less amount of desquamation. Microscopic examination of an inflammatory papule shows that the cells of the rete are œedematous and proliferated (acanthosis). The cells of the horny layer are moist and imperfectly cornified, and retain their nuclei (parakeratosis). The corium is infiltrated with small round cells, which are most thickly clustered around the separate vascular areas. To these changes in the rete and corium are due the most marked appearances of the inflamed cutis, viz. the swelling, elongation, and flattening of the papillæ.

A further degree of irritation will transform the papule into a vesicle; the latter lesion is due to separation of the layers of the serrated cells of the rete and accumulation of fluid containing leucocytes in the clefts (spongy transformation). In the process of separation some of the rete cells are drawn out into fusiform or filiform figures, forming a meshwork in the vesicle. Involution of a vesicle may begin by absorption of the fluid without

${ }^{1}$ Brit. Journ. Derm., Jan. and Feb., 1904. 
breach of the superficial epidermic layer, or the vesicle may burst, leaving a red surface that secretes serous fluid, and is formed by the papillary layer of the corium, which is generally covered by the deepest layer of the epidermis; this is termed excoriation. In severer cases, not only the whole of the epidermis, but part of the corium, is destroyed; this is ubceration. If the irritant action be maintained, the contents of the vesicle are more and more charged with corpuscles, becoming opaque and afterwards puriform; thus the vesicle is transformed into a pustule.

Umbilication of vesicles or pustules takes place in several ways. Thus the fluid may not fully distend the cleft in which it lies, and the network of elongated rete cells may cause a dimpling (primary umbilication of Auspitz and Von Basch), or commencing absorption may cause a similar flaccidity of the sac; or again, a scab-covered umbilication is often seen after rupture. That pus can be absorbed without being discharged on a surface is proved by the frequent absorption of a collection of pus in the anterior chamber of the eye without perforation of the cornea. Desquamation in superficial dermatitis is analogous to excessive mucous secretion in catarrhal affections of mucous membranes. This is the course of events in a typical case of traumatic dermatitis; but an inflammatory process may be set up in the skin in various ways. Thus, the retention of secretion in a sebaceous gland may induce perifolliculitis, which the presence of micrococci may cause to become pustular. Slight injuries, such as those inflicted by the itch mite or by lice, may also become infected by pyogenic organisms. More intense infective processes are seen in the case of erysipelas.

Abnormal vascular or nervous conditions in the skin render it more vulnerable. The lower limbs show a marked proclivity to inflammation of all degrees of 
severity when they are the seat of varicose veins, or when they are paralysed owing to affections of peripheral nerves or of the spinal cord. Circulatory inadequacy may be due to abnormal conditions of the heart or lungs. There is a special vulnerability of the skin, as well as of the other tissues, which is associated with tuberculosis and ichthyosis, and also with diabetes. This vulnerability of tissue manifests itself in slowness of repair after injury, and in a marked tendency to become infected by pyogenic cocci and tubercle bacilli.

The influence of disordered nerve action in producing inflammation of the skin is displayed in such conditions as herpes and urticaria. Other examples of lesions dependent on nervous disorder are seen in acute bed-sore, perforating ulcer, glossy skin, etc., where severe lesions are directly traceable to inflammatory conditions of the peripheral nerve trunks or their origin in the spinal cord.

The results of inflammation vary according to the severity of the process and the structural peculiarities of the part affected. Pigmentation is a marked feature in syphilitic lesions, and in all lesions on the leg when the veins are varicose, and when there is therefore a tendency to disintegration of red blood-corpuscles.

Thickening of the epidermis is a frequent result of inflammation, and the increased rapidity of proliferation of epidermal cells leads, in eczema and certain other conditions, to the formation of visible scales and, when the nails are affected, to pitting or thickening.

Degeneration of the skin takes place naturally in old age, the corium becoming thinner, and the skin darker owing to increase of pigment. The elastic tissue is altered in its anatomical appearance and loses its function. A peculiar degeneration of the elastic tissue is associated with the disease known as "xanthoma of 
Balzer." Degeneration of morbid products takes place in xanthoma when the inflammatory cells become loaded with fat, and in the peculiar colloid degeneration of the skin which somewhat resembles xanthoma, but is due to changes in the walls of the blood-vessels.

The view that cancer and sarcoma are infective diseases is held by some pathologists; but the whole subject of the etiology of these conditions is still, in spite of the investigations of hosts of workers in many lands, shrouded in obscurity. Papillomatous growths (warts, horns, etc.) may result from constant irritation by such agents as strong lotions, but more commonly from prolonged irritation by microörganisms. From the epidermis and its glands other epithelial growths, such as adenoma and epithelioma (in what may be called the dermatological sense of the term), may arise. From the corium may develop such growths as fibroma, myxoma, myoma, etc., as well as those of malignant type, such as sarcoma and endothelioma.

Parasitic and microbic affections are common. Suppuration is usually the result of microbic infection, and parasites of various kinds are present in ringworm, favus, itch, etc. The list of such affections will no doubt be extended by further research.

Seats of pathological processes.-Some pathological processes are confined to a single structure of the skin; others affect a number of structures. The following summary of our knowledge of this aspect of cutaneous pathology we owe to Darier's gift of classification :-

Epidermis.-The epidermis as a whole is the seat of adenoma, epithelioma, keratoma, papilloma, and cysts. The stratum corneum is the structure affected in favus, microsporosis, trichophytosis, erythrasma, tinea versicolor, scabies, and superficial trauma. 
In the strata corneum, lucidum, granulosum, and upper rete are found the lesions of the hyperkeratoses, including ichthyosis, pityriasis rubra pilaris, keratosis pilaris, callosities and corns; of the parakeratoses, among them psoriasis, eczema seborrhoicum, dry seborrhœa, and pityriasis rubra; and of the sequelæ of burns.

In the strata corneum, granulosum, and upper rete are located the dyskeratoses, embracing psorospermosis, molluscum contagiosum, and Paget's disease.

In the strata lucidum, granulosum, and entire rete take place the acantholytic processes-herpes simplex and zoster, prurigo, dermatitis herpetiformis, impetigo, hydroa æstivale, varicella, and vaccinia.

In the lower rete are encountered the hyper-acanthotic processes - lichen planus, lichenification, acanthosis nigricans, verruca, and vegetations.

In the stratum germinativum are found the anomalies of pigmentation-chloasma, vitiligo, lentigo, melanodermia, and albinism.

Derma.-In the corium as a whole are located sarcoma, fibroma, neuroma, myxoma, xanthoma, and lipoma, cicatrices, and degenerative processes-senile, colloid, myxœdematous, and elastic.

The pars papillaris is chiefly the seat of the congestions, erythemas, and roseolas; of the cdemas, particularly urticaria; of the acute forms of dermatitiserysipelas, ecthyma, acne, infective folliculitis, etc.; and of the chronic types of dermatitis-lupus erythematosus, tuberculosis, leprosy, syphilis, mycosis fungoides, elephantiasis, and sclerodermia.

Hypoderm.-Beneath the derma occur the deep hæmorrhages of purpura, hydradenitis, and syphilitic, tuberculous, and leprous gummas. 


\section{MORBID ANATOMY OF THE SKIN}

Pathological changes in the skin are for the most part appreciable by the sight or the touch. Hence the gross anatomy of skin lesions constitutes the most important part of symptomatology, and must be firmly grasped by everyone who intends to hold himself responsible for the recognition of the infective fevers and of all diseases that affect the skin.

The complexity of the normal anatomy of the skin results in a corresponding complexity of morbid forms, or, as they are termed, lesions of the skin. These elementary lesions are primary when they result from a pathological process before or at its fullest evolution, and secondary when they result from the more or less complete subsidence of that process. Thus each vesicle in a case of herpes zoster is a primary lesion, and the scars which may remain in the place of the same vesicles are secondary lesions. It should be noted that identical lesions may be at one time primary, at another secondary:

\section{Primary Lesions}

Macule.-A macule is a discoloration or decolorization of the skin, having a definite outline, but no noticeable elevation or depression. Should a macule take on a slight degree of elevation, it may be classified as a maculo-papule. Macules may be caused (1) by the passage of blood, or of its colouring matter, into the skin, e.g. purpura; (2) by dilatation of the vessels of the skin, or the formation of new vessels, e.g. the capillary nævus; (3) by hyperæmia, either arterial or venous, e.g. erythema; (4) by changes in the pigmentation of the rete or of the corium-on the side of deficiency as in leucodermia, or on the side of excess as in chloasma-due to trophoneurosis, or to the administration of drugs. Macules of the second and third 
groups are temporarily effaced by pressure; those of the first and fourth groups are unaffected by pressure.

Macules may be congenital as in moles, or acquired as in the exanthemata; inflammatory as in the rose spots of typhoid, or non-inflammatory as in purpura; permanent as in leucodermia, or temporary as in drug rashes. They vary in size from a mere speck to, say, the palm of a man's hand. If the pigmentation be widely diffused, as in Addison's disease, it is termed a discoloration. Macules may be oval or of irregular shape, but they are usually more or less round; in colour they are red, brown, or yellow. Subjective symptoms, such as itching, are sometimes, but not often, present. Though generally a primary lesion, the macule may be secondary to excoriations, burns, blisters, and various kinds of eruptions. Macules caused by the passage of blood, or of the colouring matter of blood, into limited areas of skin are termed vibices when linear, and petechice when punctate. If of large size they are styled ecchymoses.

Papule.-A papule is a solid, circumscribed elevation of the skin, not larger than a pea. Papules are distinguishable from vesicles (p. 13) by their solidity, from nodules ( $p .11)$ by their size. But frequently they are transitional lesions, which may pass into vesicles, or pustules, or scales; may hypertrophy as into warts; may atrophy; or may break down into ulcers. If the transformation into vesicles or pustules be incomplete, the lesions are termed papulovesicles or papulo-pustules. Or if the lesions which originate as erythematous macules be only partly transformed into papules, they are styled maculopapules or erythemato-papules. Papules may occur in the derma as in urticaria papulosa, or in the epidermis as in verruca plana, or in both these structures as in lichen planus. Dermic papules may be 
œdematous or infiltrated; the former may be recognized by their pinkish colour and their momentary yielding to pressure; the latter by their redness, induration, and elasticity. Epidermic papules are distinguishable by their superficial elevation, their solidity, and their dryness; dermo-epidermic papules by their union of some of the characters of the other varieties.

Papules may be inflammatory as in eczema, or noninflammatory as in severe goose-skin, or when they are produced by excessive cornification round the mouths of hair-follicles, or by retained secretion as in acne. If inflammatory, they are usually attended by itching. In colour they may be coppery as in syphilis, violet or purplish as in lichen planus, pink or rose as in urticaria papulosa, bright red as in eczema, yellow as in xanthoma, almost black as in sarcoma, whitish as in milium, or simply skin-coloured as in prurigo. The limits of size are from that of a pin's head as in lichen scrofulosorum to that of a pea as in lichen planus. The papule in this latter affection, flattened, with an irregular base, presents the typical shape; but they may be cone-shaped as in pityriasis rubra pilaris, or rounded or oval as in prurigo; they may also be umbilicated. They may occur in patches as in lichen scrofulosorum, or be discrete as in prurigo; sometimes they arise in connection with the papillæ, the sweat-glands, or the sebaceous glands, or they may form round a hair-follicle as in eczema folliculorum.

Nodule.-The term nodule, or node, or tubercle, is generally interpreted to signify a solid elevation of the skin smaller than a tumour and larger than a papule. This, however, is but a rule-of-thumb definition: some lesions of tertiary syphilis and leprosy, although they may be smaller than a pea, are styled nodules instead of papules, while certain quite small neoplasms are classified as tumours rather than as nodules. The term 
tubercle, as a synonym for "nodule," should no longer be used now that it has acquired a specific meaning in connection with tuberculosis. From papules, nodules differ not only in size but also in their greater tendency to downward growth. They are sometimes flat, or conical, or of irregular shape, but most often they are rounded. As a rule, they are a dull or brownish red, but they may vary to pink on the one hand and to dark purple on the other. They may persist indefinitely, may degenerate and ulcerate and be followed by scars, or may be absorbed. The nodule is characteristically a cutaneous lesion, but subcutaneous nodules, presenting over the sheaths of tendons and the fascia covering bony prominences, around joints, and on the scalp, are one of the signs of acute rheumatism, while Heberden's nodes on the finger-joints are a sign of osteo-arthritis.

Wheal.-The wheal, which may be regarded as a special variety of the papule or of the nodule, is a flattish evanescent elevation of the skin, the effect of an œdema of the derma. Erythematous in character, it is the expression of an angio-neurotic excitation which dilates the capillaries and so permits an exudation of serum. The reaction assumes the form of a spasmodic contraction of the vessels, so that they do not take up the fluid immediately; when they return to their normal condition the lesion disappears, usually without leaving any trace of its presence. Wheals generally appear suddenly and last only a few hours, but may be followed by a fresh crop. They vary in size from a pin's head upwards. Though usually flat, or but slightly raised, the smaller ones may take the form of conical or acuminate papules, often with a tiny vesicle on the summit; while the larger ones, when not the effect of coalescence, are hemispherical. They may also be linear in shape, and may run together into roundish plaques. 
Generally pale in the centre, they may be a uniform rose-red, or there may be a whitish border, or they may be purplish from the presence of hæmoglobin. They are invariably attended by itching or burning. The only cutaneous affections which are characterized by wheals are the different forms of urticaria, including strophulus (urticaria papulosa), but they may also be set up by the bites of insects or by contact with the stinging-nettle.

Tumour.-A tumour is a solid elevation of the skin, usually larger than a nodule, seated in the derma and the subcutaneous tissue. As is pointed out under Nodule, tumours may be quite small. They vary greatly in character and in termination, according as to whether they are benign or malignant, ete.

Vesicle.-A vesicle is a circumscribed elevation of the epidermis, containing serous fluid, which may become sero-purulent or mixed with blood, and ranging in size from a pin's head to a small pea. Serous or sero-purulent elevations larger than a small pea are regarded as bullæ. Vesicles may originate as such, or may be transformed papules. The process of vesiculation may be either parenchymatous or interstitial. In parenchymatous vesiculation, e.g. varicella, the plasma accumulates within the Malpighian cells, the unicellular vesicles thus formed running into each other; in interstitial vesiculation, e.g. eczema, the plasma accumulates between the Malpighian cells, and distends them until they rupture, or, as in herpes, the process of "ballooning" is set up, the cells becoming rounded, losing their prickles and distinctive staining reactions, and undergoing turbid or fibrinous degeneration. At first the contents of vesicles consist almost always of pure plasma, either quite clear or very slightly tinged with yellow, though in rare instances the fluid is from the outset mixed with blood. Later the 
clear fluid becomes yellow. Usually vesicles are of short duration, (a) terminating by rupture and crusting over as in eczema, or (b) drying up and then crusting as generally in herpes, or (c) enlarging into blebs as often in dermatitis herpetiformis, or $(d)$ becoming pustules as in variola. In folds of the skin, on the lips, and on mucous membranes they rupture sooner than elsewhere and leave excoriations. Always the manifestation of a more or less inflammatory process, they generally give rise to itching and burning. The more usual shapes are rounded, conical, or acuminate; but vesicles may tend to the oblong as in scabies, or may be both oblong, and irregular as in dermatitis herpetiformis. Sometimes they are umbilicated as in smallpox; in dermatitis herpetiformis and other affections they may be flaccid instead of tense. Usually, as in herpes and eczema, they appear in a plentiful crop, which form groups or closely-set clusters; but in a few affections, e.g. chickenpox, they are few in number and discrete.

Bulla.-Bullæ, or blebs, are elevations of the epidermis larger than a pea, containing serous fluid which, as in vesicles, may be mixed with blood, or may be seropurulent. They bear, therefore, the same relation to vesicles that papules bear to nodules. They differ from vesicles, however, in mode of formation, as well as in size, for while vesicles are always the result of an inflammatory process (see above), with bullæ there is a cleavage of the epidermis. Bullæ, again, are unilocular, while vesicles, in the first stage at any rate, are often multilocular. They may originate as bullæ, or may result from the growth of a vesicle or the running together of several vesicles. Most frequently they are superficial, the serum accumulating between the mucous and the horny layers; but they may also be subepithelial and may elevate the entire epidermis. In colour they are clear or yellowish when serous, brownish or 
reddish when they contain blood which uniformly mixes with the serum, and pale or yellow, with red or brown streaks, if the blood be not evenly distributed. The typical shape is rounded or oval, but they may be irregular; and they may vary in size from a pea to a hen's egg, or larger. The surface is usually tense, but it may become flaccid, or may be so from the beginning. Terminating by rupture, by suppuration, or by absorption and desiccation, they form crusts which vary in colour and thickness according to the nature of the exudate. When the crusts fall off they generally leave a red or brownish stain, which, however, soon disappears. Ordinarily the only sensations which accompany bullæ are those of slight burning and tension.

Pustule.-A pustule differs from a vesicle or a bulla only in the nature of its contents: it is definable as an epidermal elevation, either unilocular or multilocular, containing a purulent liquid. It is always, like the vesicle, a product of inflammation; most frequently, indeed, it is a transformed vesicle, though it may also develop from a papule, or may be pustular from the beginning. Should the metamorphosis be incomplete, the lesion is styled a papulo-pustule or a vesico-pustule. Pustules may be situated in the epidermis, in the derma, or in a follicle. Accumulations of pus beneath the derma are classified as abscesses or gummas. In size, pustules vary from a pin's point to a split pea. Usually round or convex, they may also be acuminatc, or flat and irregular, while occasionally, as sometimes occurs in scabies, they may be oblong and tend to the linear form. In variola and the varioliform syphilide they may be flattened or concave, from commencing absorption, or because the fluid is insufficient to distend its cavity. Most frequently they are yellowish or greyish in colour, with a red areola; but if the pus be mixed with blood the yellow is tinged with red or brown. When a pustule 
is ruptured and its turbid contents are examined under a microscope they are found to consist of leucocytes and serum, with cocci. Usually developing rapidly, pustules terminate by desiccation or by rupture-more often accidental than spontaneous, and a yellow, brown, or blackish crust is formed, firm if it follow rupture, friable if it follow desiccation.

\section{SECONDARY LESIONS}

Lesions of this group are due to mechanical injuries, such as scratch-marks, or they form in the course of the involution of primary lesions. In the second category we may recognize four chief processes: desquamation, hypertrophy (persistent infiltration), scar formation (atrophic infiltration), and pigmentation.

Scale.-The scale, or squame, is a dry and usually laminated exfoliation of the epidermis, due either to inflammation as in psoriasis, to a previous acute hyperæmia as in erythematous eruptions, or to an abnormal dryness of the skin as in keratosis pilaris. The morbid process may take the form either of interference with the normal horny transformation or of an excessive formation of the epidermic cells. Scales may be large and thin as in pityriasis rubra, or small and branny as in tinea versicolor, and in the same affection there may be much variation in size in different regions. There may be several layers as in psoriasis, or but a single stratum as in scaly eczema. They may be present in immense profusion, as in pityriasis rubra, or in insignificant quantity as in tinea versicolor. Sometimes they are a dirty yellow as in some syphilides, or reddishbrown as in oily seborrhœe; but the usual colour is white or grey, either bright and silvery as in psoriasis, or dull and lustreless as in seborrhœea. Ordinarily they are dry and friable, but if a serous or sero-purulent discharge be present as in eczema, or an oily secretion 
as in seborrhœa, they may take the form of scaly crusts or crustaceous scales.

Scab.-The crust, or scab, consists of a dried-up mass of exudation-serum, pus, or blood, or a mixture of these fluids-on the surface of the skin, commingled, it may be, with epithelial débris, or fat, or fungous elements. Scabs form on matured vesicles, on bullæ, on pustules, and on wounds, ulcerations, erosions, and excoriations. When the exudation is thick, they are usually tough and adherent; when it is thin, or there is a considerable admixture of fungous elements, they are friable, and easily detached, as also are those resulting from the seborrhœic process. Fungous and seborrhœic scabs, however, e.g. the so-called crusts of favus, are scaly rather than crusty. In colour, scabs may be yellow as in impetigo contagiosa, red-brown as in ecthyma, or dark-brown or greenish or blackish as sometimes in syphilis. When they have been detached, the surface may be ulcerated as in rupia, or excoriated as in eczema, or if they have been long adherent, as in some cases of impetigo, it may be dry.

Pigmentation.-Excess or deficiency of the natural coloration of the skin may follow almost any primary lesion. It may be general, the result of irritation of the abdominal sympathetic, especially the solar plexus, or local, consequent upon the exudation or extravasation of the colouring matter of the blood. Local pigmentation may be due to the action of irritants, or to a condition of hyperæmia, or may be a sequela of skin eruptions.

Excoriation. - This lesion is usually superficial, due to traumatic or mechanical causes, by which the integument is denuded as far down as the rete. The most frequent cause of excoriation is the scratching provoked by itching affections. On such excoriations crusts composed of blood and exuded serum often form; 
they may be surrounded by an areola, and various secondary lesions may be caused by the constant scratching, such as papules and pustules, while the nearest lymphatic glands may undergo enlargement. In chronic cases there may be inflammation, infiltration, and, especially in the subjects of protracted pediculosis, pronounced and widely diffused pigmentation. Excoriation is not followed by scarring unless it extend to the corium, when small, whitish atrophic patches may appear.

Ulcer--An ulceration may be defined as a pathological destruction of the tissues of the skin, molecular or gangrenous, as opposed to a wound, which is due directly to traumatism. It may affect the cpidermis only, but in true ulceration the process involves the corium, and there may even be destruction of the subcutaneous tissues. When not due to trauma, ulcers most frequently occur in connection with syphilis, lupus, scrofula, leprosy, malignant disease, and varicose veins, or as boils or carbuncles. There is always some discharge, which may be serous, sanious, hæmorrhagic, or purulent. Scabbing usually takes place, but in some cases the discharge is constant and there is no formation of crust. In syphilis, and occasionally in ecthyma, the crust may consist of superimposed layers diminishing in size and resembling a limpet-shell (rupia). Most frequently ulcers are roundish, but they may be reniform, serpiginous, or quite irregular. The base may be soft and odematous, or infiltrated and hard; the edges may be sharp or rounded, sloping, everted, or undermined. Though varying greatly in their course, ulcers, unless malignant, tend to heal, leaving behind them a permanent cicatrix.

Fissure. - Rhagades or fissures are linear clefts in the epidermis only, or in both epidermis and derma. Most prone to arise in the natural lines and furrows, 
they are frequently encountered also on the joints, the palmar and plantar surfaces, the fingers and toes, around the anus, and at the angles of the mouth. They most often occur in such conditions as eczema, dermatitis, ichthyosis, and sclerodermia.

Scar-Cicatrices, or scars, are the result of the complete involution of an inflammatory infiltration sufficiently intense to destroy part of the corium, or they may follow the loss of tissue caused by injury. They consist of newly formed connective tissue containing blood and lymphatic vessels and nerves, but not usually supplied with hairs or glands. If there has been imperfect involution of inflammatory exudations, as in chronic eczema and elephantiasis, the condition of hypertrophic scar arises; or the scar tissue may depart still farther from the normal and develop into a distinctly fibrous growth-cheloid. Among the conditions which leave characteristic scars are smallpox, syphilis, lupus erythematosus, and lupus vulgaris. The fibrous, cord-like, cheloidal scars of burns are usually unmistakable. 


\section{CHAPTER II}

\section{LASSIF ICATION}

Classification is a good servant but a bad master, and the student must never allow himself to be beguiled into thinking that any system of pigeon-holing is an Ariadne's thread which will guide him safely through all the mazes of the pathology of the skin. There can be no finality in the classification of cutaneous affections till finality of knowledge of their causation, clinical phenomena, and pathological affinities has been reached. At present all attempts at classification must be provisional, shifting with the prevailing currents of scientific thought and liable to give way at any moment under the pressure of increasing knowledge. In these circumstances the best classification is not the most complete and most symmetrical, but that most likely to be practically useful for purposes of treatment, by grouping diseases according to their proved or probable etiological affinities.

The earliest attempt to classify diseases of the skin was made by Hieronymus Mercurialis in the first book on dermatology ever published. ${ }^{1}$ His classification was purely regional, skin affections being divided into those of the head and those of other parts. This simple arrangement was followed a century and a half later by Daniel Turner, ${ }^{2}$ and afterwards by Alibert (1806), who made two principal genera of cutaneous diseases, those of the head (which he called teignes), and those of

1 “De Morbis Cutaneis," 1572.

2 "A Treatise of Diseases Incident to the Skin," 1712. 
the body (which he called dartres). The former he subdivided into five, the latter into seven species, each with several varieties based on differences in the appearance of the lesion. Thus a scaly eruption on the trunk was a dartre squameuse, one with crusts a dartre crustacee, each being still further qualified according to shape, moisture, or dryness, etc. Affections too impartial in their attacks on the skin to be confined within the limits of a particular region were grouped in somewhat haphazard fashion as éphélides, syphilides, scrofulides, psorides, cancrö̈des, etc.

Scientific classification may be said to have begun with Plenck, ${ }^{1}$ who took as the basis of bis classification. the predominant objective feature of the disease, including, however, the results of the evolution of the process as well as the primary lesions. He grouped affections of the skin under fourteen heads as follows : (1) Macules, (2) Pustules, (3) Vesicles, (4) Bullæ, (5) Papules, (6) Crusts, (7) Scales, (8) Callosities, (9) Excrescences, (10) Ulcers, (11) Wounds, (12) Cutaneous Insects, (13) Diseases of the Nails, (14) Diseases of the Hair. Willan somewhat modified Plenck's classifica. tion, grouping skin lesions in the following "orders" : (1) Papules, (2) Scales, (3) Exanthemata, (4) Bullæ, (5) Pustules, (6) Vesicles, (7) Tubercles, (8) Macules. To these Willan's pupil, Bateman, added a ninth group, Dermal Excrescences. ${ }^{2}$ Passing over Joseph Frank's (1821) absurd classification of skin diseases into acute and chronic, we come to Erasmus Wilson, who, as an anatomist, naturally looked for a basis of classification in anatomy. He grouped cutaneous affections according to the structure in which they took their origin, making four divisions: (1) Diseases of the Derma, (2) Diseases of the Sudoriparous Glands, (3) Diseases of

1 " Doctrina de Morbis Cutaneis," Vienna, 1776.

2 " Practical Synopsis of Cutaneous Disease8," London, 1815. 
the Sebiparous Glands, and (4) Diseases of the Hair and Hair-Follicles. Meanwhile, the French school, of which Bazin may be taken as the representative, attempted to classify skin diseases according to certain constitutional states of which they were supposed to be an expression. To make such a scheme anything like complete, however, it was first necessary to create diatheses to account for a large number of affections, which were accordingly put down to the credit of sundry mythical dyscrasias, "herpetic," "dartrous," etc. In 1845, Hebra published a scheme of classification based on the more solid ground of pathology. He divided affections of the skin into twelve classes corresponding to the structural changes in the tissues of the body generally, which formed the foundation of Rokitansky's classification of the results of pathological processes. Thus, according to Hebra, a disease of the skin falls under one or other of the following heads: (1) Hyperæmias, (2) Anæmias, (3) Anomalies of Secretion of Glands, (4) Exudations, (5) Hæmorrhages, (6) Hypertrophies, (7) Atrophies, (8) Neoplasms, (9) Pseudoplasms, (10) Ulcerations, (11) Neuroses, (12) Diseases caused by Parasites.

From what has been said it will be seen that the classification of the English school was mainly objective, that of the French school diathetic, and that of the Vienna school anatomico-pathological, in character. A classification according to processes was attempted by Auspitz, and after him by Bronson, but, scientific though such a system undoubtedly is, in the existing state of our knowledge it is impossible to carry it out satisfactorily. At the present day Hebra's classification is generally adopted, with some slight modifications, by English writers.

In the present work no formal scheme of classification is propounded, but an attempt is made to group the diseases described in accordance with the tendency 
of modern pathological research-that is to say, etiologically. The lines followed are mainly those traced out by Unna in his arrangement of subjects in the Monatshefte für praktische Dermatologie. Thus the affections in the production of which disorder of the nervous system may reasonably be held to be the leading factor, form one class; the eruptions due to artificial irritation, external or internal, a second; and those caused by medicinal substances, a third. A large and composite group is made up of affections which, differing in every other respect, are linkud together by the fact that they are the results of the action of microorganisms or parasites. Diseases of which the etiology is at present obscure, or altogether unknown-such as eczema, psoriasis, pityriasis rubra, and new growthsare for the present necessarily left unclassified.

The progress of medical science lies almost entirely in the discovery of causes. As these become known, fresh groups of diseases will naturally be formed. The outline of a scheme here sketched must not be looked upon as a classification of skin diseases, but only as a provisional arrangement which has at least the advantage of bringing into strong relief the chief point to which treatment is to be directed. Thus, if it be known that an affection is of nervous origin, that fact of itself at orce supplies the leading indication for treatment; and if the lesions belong to the category of artificial eruptions or drug rashes, it follows naturally that in order to remove the effect we must suppress the cause. ${ }^{1}$

1 For a fuller account of the various schemes of classification of diseases of the skin that have been proposed, the reader is referred to an address delivered by the author as President of the Section of Dermatology at the annual meeting of the British Medical Association held at Montreal in the autumn of 1897 (Brit. Med.Journ., 1897, i. 697).! 


\section{CH.ATER III}

\section{PRINCIPLES OF DIAGNOSIS}

THE diagnosis of any case of shin disease implies an sdequate knowledge not only of the nature and erolution of the lesions by which it manifests itself, but of the process of which these are the result. When, in addition to this, the cause which is the origin of the pathological mechanism can be discorered, the diagnosis is complete. It is not enough to recognize that an eruption is papular, resicular, or pustular; as a rule, the indiridual lesion br itself is no more an index of the disease which produced it than a single brick is of the building of which it forms a part. Fach case must be studied in all its relations as a clinical entity, not as a mere illustration of a h.pothetical trpe. Facts must be observed with an open mind and a resolute endeavour to see things as they are, and not to be misled by names. The object of the present chapter is not to enumerate all the points which differentiate one affection from another, but to set forth the principles of a diagnostic method which may enable the observer, if not to decide at once what the particular disease before him is, at least to say with greater or less probability what it is not.

Examination of the patient.-The first thing necessary is to make a thorough examination of the patient. This should always be done in clear daylight; in the dusk, colour, which is always a most raluable guide in the diagnosis of skin affections, becomes invisible; and by artificial light it is so changed as to be mislead- 
ing. All the lesions should be seen, and the ideal plan is to have the patient completely stripped; in the case of females, however, we must generally be content with inspecting the affected parts piecemeal. On no account should the practitioner ever allow himself to be betrayed into giving an opinion on the nature of a skin lesion which he has not had an opportunity of seeing. The examination should in the first instance be purely objective; no reliance should be placed on statements made by the patient, but all possible information should be got from the study of the lesions themselves. When this has been done, the patient's deposition may be taken, but it is most important that no questions of a leading nature should be put, and statements as to the history and course of the lesions must always be carefully checked by the results of objective examination. The interrogatory should be particularly directed to the following points: What is the chief symptom complained of? How long have you had it? When, in what form, and where did the eruption first show itself ? Does it itch? Does it come and go, or is it constant? What are the general features in the development of the lesions-has there been "weeping," discharge of matter, etc.? In interpreting the patient's answers allowance must be made for inaccuracy of description and misuse of terms : thus even well-informed persons will include under the term "blister" not only" vesicles and blebs, but wheals. The nationality of a patient, or the fact of his having resided in the tropics or other regions where certain diseases-such as leprosy and malaria-are endemic, is often a most important link in the chain of evidence. MLreover, the occupation of the patient should always be noted. The other relevant points of the medical history, such as age and sex, should be ascertained in the ordinary way.

Characters of the eruption.-In studying an 
eruption, not only the shape, colour, and appearance of the lesions, but their place and mode of origin, their distribution, their arrangement in groups or otherwise, the pigmentation which they leave behind them, the presence or absence of induration in and around them, their individual and corporate life-history, the presence or absence of local rise of temperature or the other classical signs of inflammation, and the general symptoms, if any, by which their development is preceded, accompanied, or followed, must be taken into account.

Thus certain diseases almost invariably begin in particular parts, as, for example, psoriasis on the elbows and knees, and seborrhœic eczema on the scalp. In some affections, as in lichen, the elementary lesion remains unchanged and unmingled with other forms throughout; in others, as in erythema multiforme and dermatitis herpetiformis, it undergoes various transformations, and lesions of the most diverse type are present at the same time.

The lesions may be symmetrical in distribution or the reverse: they may be grouped or isolated and irregularly scattered about. Symmetry may be the effect of an irritant circulating in the blood-stream, and acting on the skin. The tissues at corresponding parts of the cutaneous surface have equal powers of resistance; hence symmetry is a characteristic of drug rashes, the eruptions of specific fevers, and generally of skin lesions due to constitutional disturbance.

On the other hand, lesions dependent on other than constitutional causes are often asymmetrical : exemplifications of this law are seen in herpes zoster, local diseases such as ringworm, tertiary syphilis, growths such as nævi, etc. Sometimes lesions follow the natural lines of cleavage in the skin; this may perhaps be explained by the fact that cutaneous blood-vessels and nerves run along these lines. In many cases the 
arrangement of lesions in a particular way may be accounted for by structural conditions : thus new patches of lupus frequently develop in the track of lymphatic vessels communicating with pre-existent foci, and the lesions of anæsthetic leprosy correspond with the direction and branching of a nerve trunk. In the majority of cases, however, it is impossible to account for the concentric rings and patches of irregular outline in which lesions tend to group themselves, unless these complex figures may be thought to represent some related conditions of the central nervous system, which has a common origin with the epidermis in the epiblast of the embryo.

The evolution of lesions is important in regard to diagnosis, as a knowledge of their mode of spreading and of the phases through which they pass enables us to recognize the identity of lesions differing widely in appearance. Many lesions, as in psoriasis, tinea tonsurans, etc., increase in size by peripheral extension. Some, while continuing to spread at the edge, undergo involution in the centre, as in erythema iris ; in others, again, as in tinea imbricata, extension takes place simultaneously in a centripetal as well as in a centrifugal direction, the area of healthy skin enclosed by the primary ring of eruption being gradually converted into a uniform patch. When neighbouring rings in their expansion meet each other, the parts in contact disappear, the remaining segments forming broken, curved, or wavy lines, or irregular festoon-like figures which sometimes, as in the so-called erythema gyratum, continue to advance at the edge independently.

Much of the history of the affection is sometimes written in the lesions themselves or in their results. For instance, yellowish scabs usually imply previous pustulation; the record of a discharge may often be seen in stiffened linen; every stain and scar bears its own 
witness to those who have eyes to read such signs. It is in the earlier stages of an affection that lesions are most likely to be seen in their typical character, unmodified by natural evolution or artificial changes. The edge of a patch must always be examined with particular attention, for it is there, when the process is active, that lesions can be seen in their original form. Hence the edge of a patch very often supplies the key to the nature of a disease which in the absence of such evidence it would be difficult, if not impossible, to identify with certainty. The apple-jelly nodules of lupus, the red, moist surface of eczema, the glistening papules of lichen planus, the yellow cups of favus, are generally to be found at the edge of areas of disease when elsewhere all typical lesions have been swallowed up in the secondary changes accompanying the evolution of the process.

The observer must carefully discriminate between the lesions which are the direct result of the morbid process and those which are the consequence of modifying influences, such as scratching (wheals, excoriations, blood-crusts, dermatitis), scarring, with atrophy or hypertrophy, thickening of the epidermis (lichenification, keratosis), secondary inoculation of cocci or other microörganisms, and local treatment, whether soothing, stimulating, caustic, or surgical. It must be borne in mind that two or more affections may coexist (for instance, scabies or psoriasis with syphilis), and in such cases of mixed disease it usually happens that one condition more or less completely overshadows the other: thus scabies may mask syphilis, and syphilis may more or less completely disguise lupus vulgaris.

In studying an eruption it is always well to compare corresponding parts together-arm with arm, leg with leg, ear with ear, and so forth. Concomitant lesions of mucous membranes and enlargement of lymphatic 
glands must be looked for, and all stains, scars, and other marks of past or present disease must be noted. Lastly, an estimate must be formed of the state of the patient's health, apart from his skin affection.

There are certain affections which can at once be diagnosed by the presence of lesions peculiar to themselves. Thus, burrows, from the distal end of which the itch mite can be extracted, are pathognomonic of scabies; nits on the hair and "hæmorrhagic spots," of pediculosis; broken hairs, of ringworm of the scalp; sulphur-yellow cups, of favus; apple-jelly nodules, of lupus vulgaris ; and flat, glistening, purplish papules, of lichen planus. In all these cases the changes incidental to the progress of the disease may so far modify the characteristic lesion as to make it difficult of recognition; but, whenever found, it is conclusive as to the nature of the disease.

\section{Diagnosis by Exclusion}

In cases of less obvious nature than those referred to above, the first step towards the identification of the disease is the elimination of conditions which are clearly "out of court." In the case of chronic processes, congenital malformations, such as xerodermia, must first be excluded. In the presence of an acute eruption the observer must guard himself against ridiculous, and possibly disastrous, error by considering the possibility of the eruption being the rash of an infectious fever. In practice it is comparatively seldom that such a question arises; the epidemic prevalence of the disease, the fact of exposure, and the presence of grave constitutional disorder generally leave little room for doubt as to the nature of a febrile exanthem. Now and again, however, the practitioner finds himself confronted with a case in which a diagnosis has to be made almost entirely on the evidence of the eruption itself; 
and this is not always an easy matter, even for the most experienced. A brief summary of the main features of the rashes of the principal infectious feversscarlet fever, measles, rötheln, enteric fever, typhus, smallpox, and chickenpox-will therefore not be out of place here. Erysipelas must also be included. The rashes occasionally seen in diphtheria, influenza, cholera, and cerebro-spinal meningitis do not concern us, as they are merely accidental phenomena, presenting no characteristic features, and are never likely to be a source of difficulty in diagnosis.

The rash of scarlatina shows itself on the first or second day, its appearance being heralded by general febrile disturbance of a more or less severe kind. It is erythematous in character, consisting at first of a multitude of tiny red points, which soon coalesce into a diffused redness of a tint like that of a boiled lobster. The redness disappears on pressure. In very severe cases the eruption presents a purple mottled appearance; it is purpuric in character, and is therefore not obliterated by pressure. It is usually bright red, but sometimes dusky ; sometimes it is general, in other cases scattered in patches. The rash usually comes out first on the chest, belly, neck, wrists, or back, and spreads to the limbs; it comes out in fresh crops on one part of the body, while fading on another. It generally disappears by the tenth or twelfth day. Desquamation always follows, and is directly proportionate in its abundance to the intensity of the rash. Sometimes the eruption is so faint and transient as to escape recognition. The skin affections most likely to be mistaken for the exanthem of scarlet fever are certain forms of erythema, and especially that described by French writers as desquamative scarlatiniform erythema; urticaria when the wheals have disappeared, leaving small red spots; belladonna or other medicinal rashes, derma- 
titis exfoliativa neonatorum and pityriasis rubra. Billet ${ }^{1}$ records a case in which an eruption of malarial origin was at first mistaken for scarlatina. In doubtful cases the chief guides must be the presence or absence of the characteristic strawberry tongue, sore throat, and fever. The strawberry tongue is quite different from the appearance presented by the tongue in smallpox. Between the tenth and the twentieth day the illness the occurrence of albuminuria may reveal the nature of the disease. The history of a previous attack is not absolutely conclusive against its being one of scarlet fever. Exposure to contagion must also be taken into account.

The rash of measles comes out on the fourth day, and almost always appears first on the face It consists of raised red spots or patches; the latter often run together, and have a marked tendency to assume a crescentic or circular outline. The rash spreads from the face to the body, and from the latter to the limbs. It usually fades on pressure, but in serious cases it is dusky, and even petechial ; there is usually considerable swelling of the skin of the face. Desquamation occasionally occurs. On the mucous membrane of the mouth, opposite the molar teeth, may usually be seen small red spots surmounted by tiny bluish-white specks -Koplik's spots. The eruption as a whole differs from that of smallpox in being macular instead of papular, and in never passing into a vesicular or a pustular stage. The characteristic symptoms of measles-fever, coryza, and cough-will usually prevent its being mistaken for a skin affection.

The rash of röthein sometimes resembles that of measles, sometimes that of scarlatina; occasionally it begins like measles and ends by resembling scarlet

1 Bull. et Mém. de la Soc. Méd. des Hôp. de Paris, April 11 th, 1902. 
-fever. The rash, however, does not, as a rule, tend to assume the crescentic shape so markedly as that of measles, nor has it the same preference for the face. It comes out on the second, third, or fourth day, sometimes on the first ; it may be accompanied by sore throat, but without the patches and ulceration on the tonsils characteristic of scarlet fever: Some enlargement of the posterior cervical glands is a constant sign and is of great diagnostic importance. The eruption disappears in three or four days. It is most likely to be confounded, apart from scarlet fever or measles, with copaiba rash: It is distinguishable from smallpox by the implication of cervical glands, which is never met with in that disease at an early stage.

The enteric fever rash is not as a rule conspicuous. It occurs chiefly on the abdomen and back, and consists of rose-red lenticular spots slightly raised and fading on pressure. They appear in successive crops, each crop lasting some four or five days. They are neither so hard nor so elevated as the papules of early smallpox, and the distribution is different, for the face, the arms, and legs almost always escape, their usual site being the trunk. The purpuric lesions sometimes present in typhoid may suggest toxæmic smallpox, but there is neither pronounced systemic disturbance nor severe pain, nor is there an erythematous rash. From the dermatologist's point of view, the main thing in connection with rose spots is not to mistake them for fleabites, or vice versa, an error which I have known to occur. The great point of distinction is that typhoid spots have not, as a rule, a central dark-red point of hæmorrhage. Flea-bites, moreover, are generally more numerous than rose spots. The two kinds of spots may, of course, co-exist.

The typhus rash appears from the fourth to the seventh day. The eruption consists of a general mottling 
with spots, usually red, slightly elevated, at first disappearing on pressure, but in a day or two ceasing to do so. They ultimately become bluish or brown in colour, distinct petechiæ or subcutaneous hæmorrhages becoming developed in the spots. The general appearance of the typhus rash is fairly well expressed by the term " mulberry rash." It first appears on the front of the trunk, sometimes on the arms and hands.

The smallpox eruption generally appears on the third, sometimes on the second, fourth, or fifth day. The true variolous eruption is occasionally preceded by a roseolar rash resembling that of scarlatina. Beginning as a mere fleck of the size of a pin's head, flush with the surface and impalpable, it swells in the course of a few hours into a hard pink papule, which can be felt embedded in the skin like a small shot, and is surrounded by an erythematous zone, the areola. In a few days the papule, growing larger, undergoes vacuolation, becomes grey and translucent, and is transformed into a loculated vesicle. The smaller vesicles are usually hemispherical, the larger ones flat-topped. Some twentyfour hours later the pustular stage is reached, the grey, translucent centre meanwhile becoming surrounded at the periphery of the crown by a white or yellow ring. The lesion attains maturity at the sixth day from its birth, becoming uniformly yellow, with a dome-shaped crown. As the pustule develops, the areola, which was biggest and brightest in the vesicular stage, begins to wane, and soon disappears. When the pustules dry up or rupture, scabs are formed, which on separation leave dark stains, scars, and "pits." The number and depth of the pits are usually proportionate to the severity of the attack.

In mild attacks the pustules remain discrete, in severer cases they are confluent. In bad cases hæmorrhage takes place into the skin, and into the interior 
of the pustules. The mucous membranes, especially that of the mouth, are not infrequently invaded; the extent to which they are involved depends rather upon their susceptibility than upon the severity of the attack. In modified smallpox the eruption may resemble that of the unmodified disease, the lesions, however, being less abundant and rarely confluent; or it may consist of merely scattered papules, which abort without vesication or pustulation.

Both the pustules and the vesicles of smallpox may be indented owing to the absorption of their contents, and the vesicles may be umbilicated in the stricter sense of the term. Inasmuch as the indentation of the pustule is not peculiar to smallpox on the one hand, and is often absent on the other, its value in diagnosis is easily overestimated. The same caution may be given with regard to indentation of the vesicle, which is not present in most of the cases of modified smallpox.

Ricketts, in his masterly monograph, 1 has shown that in the diagnosis of smallpox the distribution of the lesions is of greater import than their character, upon which the chief stress has hitherto been laid. Its significance consists chiefly in the eruption being neither localized nor elliptic, but generalized; in its being symmetrical and graded-graded, too, evenly in homogeneous areas; and in its preference for surfaces exposed to friction. The parts most liable to attack are the face and hands, which owe their susceptibility to their exposure to constant stimulation from wind and weather, from light and temperature, and from friction. Next in liability come the upper limbs, then the trunk, then the lower limbs.

The eruption of chickenpox bears considerable likeness to that of smallpox, and the two diseases are 1 "The Diagnosis of Smallpox." By T. F. Ricketts and J. B. Byles. London, 1908. 
frequently confused; but chickenpox is essentially vesicular, only occasionally becoming pustular. There are no hard shotty papules. The commonest situations are the face, the chest, the shoulders, the back, and the scalp. Slightly raised red spots generally precede the vesicles. A few vesicles form on the mucous membrane of the palate, mouth, or lips. The rash usually comes out within the first twenty-four hours. There is often scarcely any constitutional disturbance. Ricketts has admirably summarized the differences between chickenpox and smallpox. In chickenpox the vesicles are usually unilocular and never umbilicated; in smallpox they are usually multilocular and frequently umbilicated. In chickenpox the abdomen and chest are the seat of the eruption equally with the face, if not more so ; in smallpox the rash is most abundant on the face and least abundant on the chest and abdomen. In chickenpox the abdomen and the back receive equal attention; in smallpox the rash is much more abundant on the back than on the abdomen. In chickenpox the distribution as between shoulders and loins, chest and abdomen, is indifferent; in smallpox it favours the shoulders more than the loins, the chest more than the abdomen. In chickenpox the limbs usually escape, and when they are invaded the rash is centripetal; in smallpox the rash shows a predilection for the limbs and is distributed centrifugally. In chickenpox it shows no such preference for prominences and surfaces exposed to irritation as it displays in smallpox. Lastly, in chickenpox the lesions are superficial, have no infiltrated base, are frequently irregular in outline, or else oval or elongated, and are not, as a rule, homogeneous ; whilst in smallpox they are deep-seated, with an infiltrated base, and are generally circular and homogeneous.

In the great majority of cases, as already said, there is little real danger of a purely cutaneous affection 
being mistaken for the exanthem of an eruptive fever, or vice versa. It is only when the constitutional disorder is so slight as to escape observation that any diffculty as between a symptomatic and a purely cutaneous eruption can oceur. It is just these slight, ill-marked cases, however, that constitute a danger to the community, and if the practitioner has any doubt he will do well to isolate the patient at home for two or three days. A precipitate notification of the case as one of infectious disease, with removal of the patient to a fever or smallpox hospital, is not unlikely to lead to the supposed fever or some other infectious disease being contracted at the hospital.

Erysipelas is usually ushered in by considerable constitutional disturbance (rise of temperature, headache, and often vomiting). The eruption, which is erythematous in character, starts, in the majority of cases, from a fissure, abrasion, or wound; in other cases, from the margin of a natural orifice where skin and mucous membrane meet. In simple cutaneous erysipelas it may spread over the skin like fluid on blotting-paper, as a red rash with a well-defined edge, or at some part of the process there may be formation of vesicles or blebs. When the underlying connective tissue is involved there is swelling proportionate in amount io the depth to which the process extends. The eruption does not occur in patches, but there is a variety of the affection in which the inflammation moves from place to place, remaining only for a short time in each locality (erysipelas fugax).

Zymotic fevers and erysipelas having been eliminated, feigned eruptions must next be excluded. In such cases the lesions are always on a part of the body easily accessible to the patient, the front of the chest, the arms, and the thighs being the regions most frequently operated upon. Noreover, the lesions have not the characters 
of Nature's handiwork, nor do they conform to the type of any known disease. They generally give evidence of their artificial origin in the regularity of their outline and in the absence of any commencing elementary lesion likely to develop into the conditions present. The subjects are invariably persons of highly neurotic temperament, the large majority of them being young women.

The next group to be considered is the class of infective granulomata, particularly tuberculosis, syphilis, and leprosy. Tuberculous lesions, with the exception of lupus, are as a rule associated with the well-known signs of the scrofulous diathesis or with actual tuberculous disease in the lungs or elsewhere. There is no feature per se distinctive of a tuberculous lesion in the skin, except the apple-jelly nodule of lupus vulgaris. The diagnosis must therefore be made from concomitant circumstances.

Syphilitic lesions usually betray their nature in their appearance; but no disease is more likely to perplex the inexperienced, on account of the protean character of the lesions which it causes and the extraordinary closeness with which it often imitates those produced by other affections. There are certain general features more or less characteristic of syphilitic lesions which, taken singly, are inconclusive, but cumulatively have a force amounting almost to proof. These are, in the case of most secondary eruptions, symmetry of distribution, erratic localization, multiformity of lesion, absence of itching, and, to a lesser extent, peculiarity of colour and shape. With regard to localization, syphilis should always be suspected when lesions resembling those characteristic of other diseases are found in situations generally avoided by the latter. Thus a patch resembling psoriasis is possibly syphilitic if there are not and have not been any similar lesions in the situations most affected by psoriasis, especially the tips of the elbows 
and the fronts of the knees. Polymorphism is a character common to all secondary syphilitic lesions, except macular and erythematous syphilides. A livid colour like that of the lean of raw ham, tending with the lapse of time to become brown and coppery, is always suggestive of syphilis, but is by no means pathognomonic. The same may be said with regard to the shape of lesions. Both eruptions and ulcers due to syphilis have a tendency to assume a horseshoe outline ; this by itself, how ever, is not distinctive of syphilis. Squamous syphilides sometimes have indefinite objective characters, but their nature will be recognizable in the light of a clear history of a primary sore and subsequent signs of constitutional infection. It must be remembered that syphilis often co-exists with other skin affections: thus a squamous syphilide may be found grafted, as it were, on seborrhœa. There are also frequently to be found other co-existing evidences of the disease, such as remains of chancre, falling out of the hair, ulcerative grooves in the tonsils, mucous tubercles on the tongue and in the mouth, pains in the bones; or marks of its presence in the form of scars or enlarged glands in the suboccipital region, the groins, and other parts, of leucodermia in the neck, or of nodes on the shins, etc. In late tertiary syphilides the distinctive features are absence of symmetry, their marked tendency to spread serpiginously and to ulcerate. Furthermore, they are followed by scarring, and on the scalp by total destruction of hair. Whenever the diagnosis is in doubt the Wassermann test should be applied.

In leprosy the diagnosis usually presents little difficulty, except in an early stage. Anæsthesia in the lesions, or in some area of the cutaneous surface, is seldom absent, and its presence is conclusive. Leprous spots, again, seldom perspire. The patient's previous history in respect of residence in an affected area may 
afford confirmatory evidence. In doubtful cases search should be made for the lepra bacillus.

The other inoculable diseases to be excluded comprise those caused by (1) animal and (2) vegetable parasites, and (3) those due to various microorganisms. In the first of these subdivisions the affection of greatest practical importance is scabics. Here conclusive proof is afforded by the presence of the acarus. The burrows must be looked for in the webs between the fingers, and about the wrists. The fact that there are no lesions on the face in a given case is presumptive evidence that the disease is itch. The presence of nits on the hairy parts, or of the characteristic "hæmorrhagic spots," is conclusive of pediculosis. Among the vegetable parasitic diseases the most important are ringworm, favus, and tinea versicolor. Each of these affections has characteristic features, by which it can at once be identified. Thus in ringworm the broken hairs on the scalp, the circinate lesions on the body, and the presence of the special fungus, are conclusive. Favus is recognized by the sulphur-yellow cups and mousy smell; tinea versicolor by the characteristic fawn-coloured spots almost exclusively seen on covered parts of the body and in adults. In the third subdivision contagious impetigo is recognizable by the isolated scabs without inflammatory halo, looking as if they had been stuck on with gum (Tilbury Fox).

The skin diseases of nervous origin are recognizable either by the lesions being distributed in correspondence with the area of distribution of a particular root, as in herpes, or by their occurring in persons of markedly neurotic temperament, or as the result of a definite injury to the nervous system, or of a severe mental shock.

New growths on the skin may be confounded with 
nodular formations of tuberculous, syphilitic, or leprotic nature, with the swellings of erythema nodosum, or with abscesses and cysts. Erythema nodosum may be identified by its localization (legs and arms), the associated rheumatic symptoms, if present, and history, and the speedy subsidence of the swellings; collections of fluid by fluctuation or thrill.

\section{Posituve Diagnosis}

Having by this process of exclusion come to a decision-subject, of course, in many cases to revision in the light of fuller knowledge-as to what the affection is not, the next step is to form a judgment, or, rather, a working hypothesis, as to what it is. In the first place it must be noted whether the eruption is general or local; next the nature and distribution of the lesions must be observed in greater detail than has already been done.

General eruptions are, as a rule, more or less symmetrical. A diffuse red rash is seen in scarlet fever, measles, and the period of invasion in syphilis: such an eruption often accompanies the development of nodules in tubercular leprosy; it occurs in urticaria, erythema, eczema, pityriasis rubra, and follows the internal administration of various drugs - chloral, belladonna, copaiba, antipyrin, mercury, opium, nux vomica, quinine, tar, stramonium, sulphonal, and salicylic acid and the salicylates. The diagnosis must be made by the clinical history, the degree and character of the constitutional disturbance, and the nature of the associated symptoms. Thus, in syphilitic roseola there will be a history of infection, cnlargement of glands, ulcerated throat, mucous tubercle, etc., and if doubt still exists the Wassermann test can be applied, always remembering that it is positive in leprosy and in sleeping sickness, as well as in syphilis ; in tubercular leprosy 
there will be found more or less perceptible infiltration of the erythematous patches, usually accompanied by some functional disorder of the glands of the affected, skin and by abolition or exaltation of sensation. In the case of scarlet fever and measles the date of invasion is important, and the other points already indicated must be taken into account. Diffuse red rashes due to drugs have nothing characteristic about them, and can be diagnosed only by the exclusion of other possible causes combined with examination of the urine and such circumstantial evidence as can be gleaned from associated symptoms, the discovery of bottles, and so on. The more purely cutaneous affections, such as urticaria and eczema, will be recognized with little difficulty as the lesions develop into typical forms.

Local eruptions.-In the diagnosis of eruptions in particular regions we have, generally speaking, fewer side-lights from constitutional disturbance and clinical history to guide us. There are, however, certain features characterizing lesions in special situations which often furnish a clue to their nature. The following is a summary of these as they relate to eruptions of different types-erythematous, papular, vesicular, bullous, pustular, wheals, ulcers, and dry scaly lesions -when met with in a particular part, such as the scalp, the face, the hands (especially the palm), the genitals, the mucous membranes.

Scalp. - On the scalp the chief difficulty in diagnosis is with regard to pustular lesions and dry scaly eruptions. Of the pustular type the chief are contagious impetigo and pustular syphilides. The distinctive feature of the former is that the lesions are not surrounded by a zone of hyperæmia, but, as already said, look as though they were stuck on with gum; on the other hand, in the case of pustular syphilides, when the scab is picked off there is usually an ulcer underneath, in the older lesions. In 
pustular eczema, again, the course of the disease is different; there is, or has been, "weeping," especially behind the ears, and the lesions are not isolated like those of contagious impetigo. In lupus erythematosus there are often crusts which resemble scabs; they are not, however, formed by the drying-up of pustules, but by sebaceous matter; moreover, on picking off a portion of the crust its under surface will be seen bristling with prickle-like projections, corresponding to the dilated orifices of ducts which they have plugged.

A dry scaly eruption of the scalp is either seborrhœa, seborrhœic eczema, psoriasis, tinea tonsurans, favus, or a squamous syphilide, which again may be secondary or tertiary. The distinctive feature of seborrhœa is that there is no redness or sign of inflammation under the scales. In seborrhœic eczema, on the other hand, the surface beneath the scales is red, and each patch has an erythematous zone around its edge. Moreover, the scalp alone is seldom affected, and the disease spreads downwards to the face, the back, and the chest. Psoriasis, also, is present in other parts, especially on the elbows and knees, and has, as a rule, spread upwards to the scalp. In this situation it generally occurs in localized patches, and in typical cases the scales have a characteristic silvery-grey appearance. It may here be said, however, that little reliance can be placed on mere differences in the character of the scales in any of the conditions here referred to, when they occur on hairy parts. Ringworm and favus can always be recognized by the distinctive characteristics already mentioned, and, if there be any doubt, it is removed by the detection of the fungus with the microscope.

In the case of secondary squamous syphilides there is nothing characteristic in the appearance of the lesions, and the diagnosis can be made only by the history, the presence of more distinctive lesions or marks elsewhere, 
and the effect of specific treatment. In the case of tertiary squamous syphilides there is often no other concomitant lesion to guide one, but the characteristic serpiginous outline and the marked tendency to ulceration, followed by scarring, are in many cases sufficiently distinctive.

Face.-Red patches limited to the face, and especially affecting the cheeks and the nose-the so-ealled "flush area "-may be erysipelas, erythema, lupus erythematosus, rosacea, or lupus vulgaris. Erythema comes on suddenly; the patch has a well-defined edge, and the eruption is not accompanied by constitutional disturbance. Erysipelas, on the other hand, is accompanied by more or less severe febrile phenomena; the patch has a well-defined edge, which advances rapidly while the process is in the active stage; the affected skin is tense, often to such a degree as to cause great pain on movement. Both in erythema and in erysipelas, vesicles and bullæ may form on the inflamed surface. Lupus erythematosus is much slower in its course than either of the affections just named; the patch has often a characteristic outline like a butterfly with expanded wings ; there is almost invariably more or less atrophic scarring in the centre, and on detaching a portion of the crust, tags of sebaceous matter will be seen projecting from its under surface. Lupus vulgaris can in most cases be recognized by the characteristic apple-jelly nodules; if these are not at first visible, they can often be brought into view by stretching the skin, or by pressing the blood out of it with the finger. In rosacea there is no defined edge, the surface is knobby with papules and pustules, and is traversed by small varicose veins, and there is no scarring. Most of the conditions that have been mentioned may be more or less closely simulated by syphilis; there is always something wanting, however, which makes the imitation imperfect. Thus the absence of acute general symptoms differentiates a syphilitic 
lesion from erysipelas; the absence of sebaceous plugs from lupus erythematosus; the absence of apple-jelly nodules from lupus vulgaris ; and the absence of dilated veins on the affected surface from rosacea.

Ulcers on the face may be scrofulous, lupous, syphilitic, or malignant. Scrofulous ulcers are mostly seen in children of strumous aspect or in elderly people with marks of lesions dating from early life. They have no absolutely distinctive characters, but the edge is often undermined and the surrounding skin blue and of low vitality. In lupus, ulceration is extremely chronic; the edge of the sore is generally more or less rounded, and the process is very superficial, never extending to the bones. The sore is covered with greenish-black crusts, but around it may be recognized the apple-jelly nodules in different stages of development. Syphilitic ulceration, on the other hand, frequently attacks the bones of the face and is more rapid in its course. In many cases the ulcers, while healing in the centre, extend at the margins, and so assume a circinate or serpiginous form which is characteristic. They are most frequently met with in the late secondary and tertiary stages. Rodent ulcer usually occurs in persons beyond middle life, and often attacks the face about the outer edge of the orbit or the side of the nose. The ulcer is rounded in outline, has a firm raised " rolled" edge and a depressed centre with little appearance of granulation, and a scanty inoffensive discharge; the process in many cases is almost painless until the ulcer has reached its later stages and is actively destroying the surrounding tissues. In ordinary epithelioma, on the other hand, the edge is everted and very hard; the base of the ulcer is foul and roughened with granulations; the neighbouring glands are enlarged; pain is often very severe, and the whole process is more rapid and more aggressive than in rodent ulcer. 
Nodular lesions on the face may be due to tuberculosis, syphilis, or leprosy. The tuberculous (lupus) nodule has a characteristic gelatinous or apple-jelly appearance, which once seen cannot be mistaken for anything else. Nodular syphilides may be secondary or tertiary manifestations. In the former case they are generally solitary or very few in number ; they are coppery in colour, and are usually associated with other syphilitic lesions elsewhere. In the tertiary form they are frequently dotted thickly over the face, especially on the forehead and on the nose; they often coalesce, giving rise to a diffuse infiltration which is apt to break down into ulcers, at the edge of which younger nodules are visible. Gummata are painless and develop rapidly; when they break down a puriform fluid exudes and a cavity is left which, if the patient is left untreated or is out of health, may spread. There is no induration or turning-out of the edge, nor is there involvement of neighbouring glands. Leprotic nodules develop slowly; they are yellowish-brown in colour, and may attain the size of a hen's egg. They are at first hyperesthetic, but, when fully developed, usually anæsthetic. Their formation is in most cases associated with a presumption of leprosy from the co-existence of other signs of the disease, and from the fact of a patient having lived in a region where it is endemic.

Small tumours on the face may be molluscum contagiosum, milium, adenoma sebaceum, or xanthoma tuberosum. In molluscum contagiosum each of the growths has a central depression with a small opening out of which a substance like sebaceous matter can be squeezed. This substance consists of particles of new growth. Milium, on the other hand, has no external opening; but when it is pricked, exit is given to sebaceous matter. Adenoma sebaceum is usually congenital, 
and occurs with nævoid conditions. Xanthoma tuber. osum is of a yellowish pearly colour; when it is pricked nothing can be squeezed out, the growth being com. posed of connective tissue.

The vermilion of the lips may be the seat of chancre, which may take the form of a crater-like infiltrated ulcer, or may be flattened, and covered with a false membrane. In the secondary stage condylomas may be present in this situation. The red of the lips may also be involved in many cutaneous affections, the nature cf which will be more clearly indicated by the accompanying lesions on the integument. In some cases eczema is limited to the lips and immediately adjacent parts. Cheilitis exfoliativa, in which there is persistent and repeated desquamation of the red of the lips, is usually associated with slight seborrhœa of the scalp and with the neurotic temperament. Cheilitis glandularis, however, a chronic inflammation of the lower lip, with swelling of the mucous glands, may be associated neither with seborrhœa nor with the neurotic state. Fordyce's disease specially attacks the red of the lips and the oral mucous membrane, and takes the form of small whitish or yellowish milium-like bodies, accompanied possibly by slight burning or itching, with a feeling of stiffness in the affected surface. Perlèche usually starts at both angles of the lips as a whitening and maceration of the epithelium, and extends along the middle line, and also involves both the surrounding skin and the mucosa of the inside of the lips. Other lesions than the exuberant epithelium may also be present, such as vesicular erythema, crusts, and diphtheroid or impetiginous stomatitis.

Hands.-Eruptions met with on the hands are principally vesicular, bullous, or dry and scaly in character. Artificial dermatitis, arising from contact with irritating substances, such as lime, ete., must first be 
excluded. Vesicular lesions are present in eczema, pompholyx, and scabies. In eczema the lesions tend to run together, the disease spreads to other parts, and there is, or has been, "weeping." In pompholyx, on the other hand, there may be discharge, but there is no weeping ; the lesions do not tend to run together as in eczema, and there is no eruption in other parts. The affection runs a more or less regular course, and shows a marked tendency to recur. In scabies the lesions are isolated; the characteristic burrows and acari at once establish the nature of the affection. Lesions on the hands that may appear to be eczematous or dysidrotic may be really due to ringworm.

Dry scaly eruptions are mostly localized on the palm. Both hands, or only one, may be affected. In the former case the affection may be psoriasis, eczema, syphilis, lichen planus, xerodermia, or keratosis. It is impossible to diagnose the nature of the case from the dry scaly character of the eruption alone. Psoriasis is indicated by the presence of characteristic lesions elsewhere, notably on the elbows and knees, and perhaps on the scalp; or there may be a history of an eruption on these parts. In eczema there is a history of "weeping " in the part itself, or eczematous lesions are present in other situations. Lichen planus of the palm is also associated with similar lesions in other parts. If it is a secondary syphilitic lesion, there will be a history of infection and other signs of the disease. Xerodermia is nearly always congenital. Keratosis is also sometimes congenital, in which case it is to be regarded as a form of xerodermia; and the affection of the palms is generally associated with dryness and hardness of the skin in other parts. Keratosis may, however, be the result of a previous inflammatory process, such as dermatitis or eczema, or of arsenic taken internally; the history in such cases will give the clue to the nature of 
the affection. Scaly eruptions affecting one palm, if syphilitic, are tertiary. It is, as a rule, only by such side-lights as have been mentioned that the nature of a dry scaly eruption of the palm can be recognized. The eruption itself, however, often presents definite features which, even in the absence of collateral evidence, should at least suggest the nature of the process of which it is a part. In psoriasis, and also in lichen planus, the scales are usually massed in small, hard, circumscribed, corn-like patches; but in acute cases of the latter affection, the whole hand, both palm and back, may be uniformly affected with general thickening and œdema. In eczema there is not only scaling, but thickening, and often fissures. Syphilitic patches are irregular in shape, and often cracked on the surface; the scales are not piled up, but peel off; the lesions spread serpiginously. In xerodermia there is comparatively little scaling; the skin is dry and polished. In keratosis the thickening is very marked, especially round the circumference of the palm, the hollow of the hand being generally less affected.

Fingers.-Digital lesions may be present in a large number of cutaneous affections, and may be vesicular, bullous, papular, pustular, scaly, erythematous, or ulcerative. The vesicular affections which may attack the fingers include eczema, dermatitis herpetiformis, scabies, pompholyx, chilblains, and the irritation set up by a variety of substances handled in the course of occupation; the bullous, dermatitis herpetiformis, pemphigus, epidermolysis bullosa, scabies, leprosy, and syphilis ; the papular, eczema, lichen planus and lichen annularis, angio-keratoma, pityriasis rubra pilaris, and papular syphilide; the pustular, impetigo contagiosa, boils, scabies, eczema, and pustular syphilide ; the scaly, lichen planus, eczema, psoriasis, ichthyosis, syphilis, verruca necrogenica, and acanthosis nigricans; the 
erythematous, erythema, lupus erythematosus, eczema, urticaria, chilblains and frostbite; the ulcerative, disscetion wounds, chilblains and frostbite, X-ray ulcer, lupus vulgaris, leprosy, epithelioma, chancre and syphilitic ulcer, Raynaud's disease, diabetic gangrene, bed-sore, trophic ulcer, and sclerodermia. The most characteristic affections of the finger are chilblains and frostbite, pompholyx, eczema, Raynaud's disease, diabctic gangrene, dissection wounds, and post-mortem wart. The possibility of ringworm, mentioned above (p. 47), must not be lost sight of.

Nails.-Isesions of the nails may be due to psoriasis, eczema, lichen planus, syphilis, epidermolysis bullosa, favus, or ringworm. Most of these affections can be diagnosed only from the co-existence of characteristic lesions in other situations. In the case of ringworm and favus the fungus can be detected by examining scrapings of the affected nail with the microscope. The nails are also subject to trophic changes, due to acute illness or senile decay, or occurring without any apparent cause. These changes may take the form of leuconychia, or white nail ; of spoon-nail, in which the nail becomes thin and hollowed; of onychia, or inflammation of the nail, going on in some cases to paronychia, or whitlow ; of onychoschizia, or separation of the nail from its bed; of onychorrexis, or brittle nail ; of onychauxis, or hypertrophied nail, which may develop into onychogryphosis, in which the free end of the elongated nail becomes twisted; or of so-called egg-shell nail, in which the colour is precisely that of the inside of the shell of a hen's egg.

Feet.-Lesions on the feet, as on the hands, may be due to a great variety of cutaneous diseases. Those of the affections to which the feet are particularly liable - perforating ulcer, Raynaud's disease, diabetic gangrene, chilblains and frostbite-are too distinctive to be 
mistaken, especially when interpreted in the light of the history. The feet may be the site of lesions due to ringworm. They may also present lesions peculiar to tropical affections. Thus, a greatly swollen foot, in the tissues of which black or pink granular pustules are found, will suggest a diagnosis of mycetoma (Madura foot), a rare condition only found in the tropics.

Genital region. - A vesicular eruption about the genitals of either sex may be herpes, eczema, scabies, or ringworm. The first of these is characterized by tiny vesicles, grouped on an inflamed base; when suppuration occurs it may simulate a soft sore, but the discharge is not auto-inoculable. Eczema usually begins in vesicles which are arranged in groups; it is aggravated by chafing (as between the scrotum and the thigh), and shows an erythematous surface which may be moist or dry and scaly, but is always inflamed and angry; the itching is almost intolerable, and pustules and various other secondary lesions are produced by scratching. In scabies the lesions are scattered about, not grouped as in eczema; here again the typical appearances are generally more or less destroyed by scratching, but careful search will reveal burrows and acari on the penis or scrotum. Ringworm and other fungous diseases affecting the perineum and genitals (eczema marginatum, "dhobie's itch," and erythrasma) can be identified by the fungus. Papules among the pubic hairs, the tops generally scratched off, point to pediculosis pubis. Patches of steel-grey colour, about the size of the finger-nail, due to a pigment contained in the thorax of the parasite, will clench the diagnosis. If the neighbourhood of the genitals presents an almost uniformly deep-red surface, with abundant desquamation, the case must be diagnosed as one of psoriasis, although on the arms and legs the psoriatic lesions may be insignificant.

Ulcers of the genitals are chiefly venereal. The 
syphilitic or infecting sore is distinguished by its raised edge, indurated base, and the fact that it is usually single ; the non-infecting, or soft sore, by the irregularity of its shape, the absence of hardening, and the fact that it is usually multiple. Squamous and other secondary syphilides about the genitals are to be recognized by the absence of itching and by other symptoms of the disease.

An affection which is usually, though not always, venereal is ulcerating granuloma of the pudenda, met with in certain warm climates. It takes the form of a chronic vascular granuloma of the genitalia, tending to cause a deep formation of dense fibrous tissue, which does not caseate or suppurate.

In infants, as the result of irritation set up by napkinis, the region of the genitals may be the seat of the eruptions grouped under the designation of Jacquet's infantile erythema ; they may be erythematous, erythemato-vesicular, papular, or ulcerating, or all four forms may be present simultaneously. They are distinguishable from congenital syphilis by the marked preference they show for the convex surfaces of the buttocks, thighs, scrotum, or vulva, by the course they run, and by the absence of the familiar specific signs and symptoms. Should the whole napkin area be occupied by a uniform bright-red rash, covered for the most part with moist or greasy yellowish scales, and extending both upwards and downwards, whilst the scalp is the seat of a red, scaly, or crusty eruption, and numerous pinhead papules are found elsewhere, the case is one of seborrhœic eczema of infants, known also as seborrhœic dermatitis of infancy.

Mucous membranes.-The mucosæare the seat of lesions in such general infections as the acute exanthematous fevers, syphilis, lupus vulgaris and leprosy, in drug eruptions, and in many cutaneous affections, among 
them the erythemas, urticaria, pemphigus, dermatitis herpetiformis, epidermolysis bullosa, herpes febrilis and herpes zoster, lupus erythematosus, lichen planus, impetigo contagiosa, impetigo herpetiformis, ulcerating granuloma of the pudenda, sclerodermia, rhinoscleroma, acanthosis nigricans, and xanthoma. The mucous membrane lesions in these various conditions are described under each, and it would be a profitless task to discuss them in detail from a diagnostic point of view, since they are seldom distinctive enough to form a basis for diagnosis, the variety of colour which is so helpful in the diagnosis of cutaneous lesions being absent. In pemphigus the shreds of the broken blebs, and in herpes zoster the ragged fringes of the ulcer, partly covered with the remains of vesicles, may point to the nature of the disease; but even in these affections the lesions do not usually tell their own story, as those of the integument frequently do. It is necessary, therefore, to look for guidance to the lesions on the skin, when these are present, and also to the history of the case. But the fact that the mucous membrane is liable to be affected in so many cutaneous diseases suggests the importance, in all cases of such diseases, of carefully examining the mucosa of the mouth and nose, and of extending the examination to other mucous surfaces, should there be any sign pointing to their implication.

Certain mucous membranes, e.g. those of the lips, tongue, and mouth, may be affected apart from, or primarily to, the integument in such affections as perlèche, cheilitis glandularis, Fordyce's disease, and idiopathic leucoplakia. Whenever lesions limited to a mucous surface leave the diagnosis in doubt, the practitioner's first thought should be of syphilis, not forgetting, however, that such conditions as herpes of the prepuce or of the vagina, lupus of the mucosæ, and idiopathic leucoplakia, which are so apt to suggest 
a mistaken diagnosis of syphilis, are by no means infrequent. A useful aid in doubtful eases of lupus is to press with a glass the strip of skin which extends inwards from the surface of the nostrils, the characteristic apple-jelly nodules, if present, often becoming visible when the skin is thus blanched.

In concluding this rapid survey of the salient points which the observer should take as his guides in the diagnosis of skin affections, I wish once more to emphasize the fact that in the majority of instances they will only suffice to establish a prima-facie case as regards any particular disease. The object $I$ have had in view has not been to give a full account of all the features which differentiate one affection from another, but to put the student in the way of "reckoning up" a ease in a simple, rapid, and logical manner. By the process of exclusion which has been briefly, but I hope sufficiently, illustrated, the observer will, if he fail to identify the disease at once, at least be able to reduce the case before him to a group of affections having close affinities with each other, the study of which he ean then pursue in detail in the sections treating of them. The chapter is, in fact, intended to be an introduction to the right use of presumptive evidence, and of elues supplied by the disease itself to the identification of affections of the skin. It can hardly be necessary to repeat that a diagnosis of the kind here referred to must, as a rule, be regarded as merely provisional until it has been supplemented and confirmed by the results of a study of the ease in all its bearings. 


\section{CHAPTFR if}

\section{NEUROSES OF THE SKIN}

\section{Clasificatios of Tetro-Dermatoses}

THE diseases treated of in the following chapters (Chapters r. to IX.) are generalls described separately as different forms of infiammstion of the skin. An attempt is here made to group them together $b 5$ the bond of $a$ primary cause common to them all. Widely different from each other as herpes and leucodermia mar appear to be in erers other respect, the essential etiological factor-namely, disturbance of innerration-is the same in both. Errthems, pemphigus, herpes, and perhaps lichen, may be regarded as connecting links beiween simple raso-motor disturbance, as represented br urticaria, on the one band, and the results of grave structural lesions of the nerrous srstem, as displared in Raynaud's disease and diabetic gangrene, on the other. In studying this chapter the reader will do well to draw a sharp line of demarcation between the clinical and pathological facts herein set forth and the chain of theory by which it is sought to bind them together. The former rest on a sure foundation of observation and experience; the latter, lise all chains, is onlt as strong as its weakest link.

The ghin affections dependent on lesion or funcdional disorder of some part of the nerrous system roat provisionally be classified under the following heads:-

1. Pure sensory disturbances - anæsthesia, irperesthesia, parzsthesia, pruritus. 


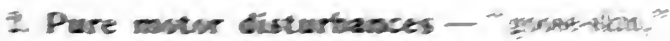

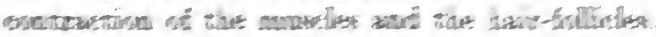

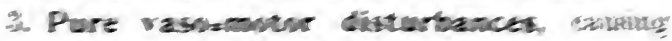

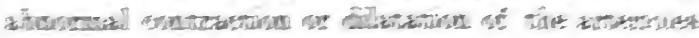

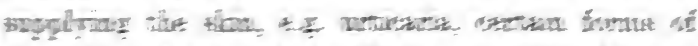

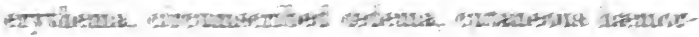
Titangate

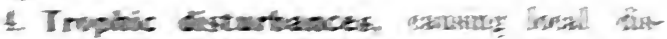

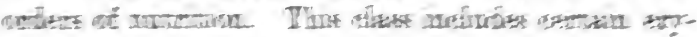

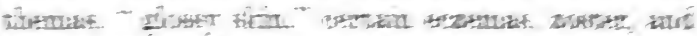

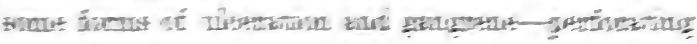

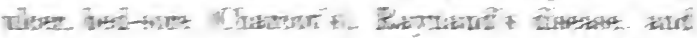

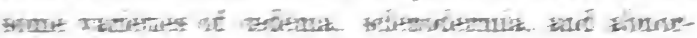

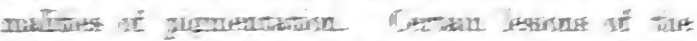

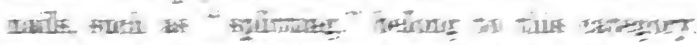

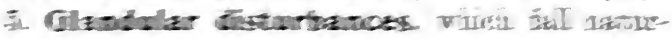

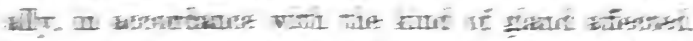

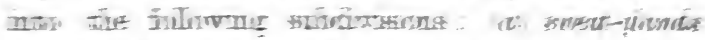

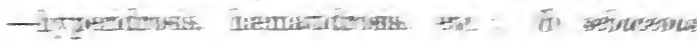

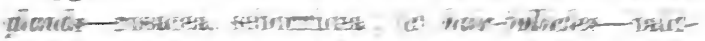

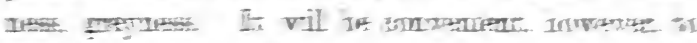

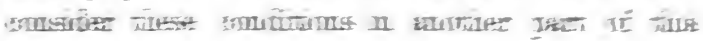
vinet

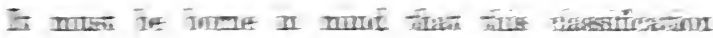

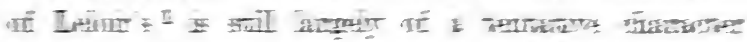

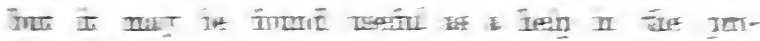

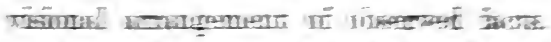

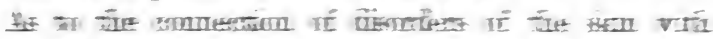

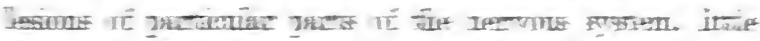

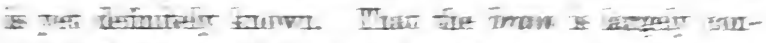

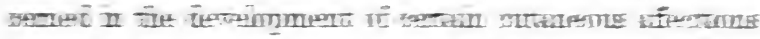

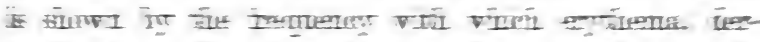

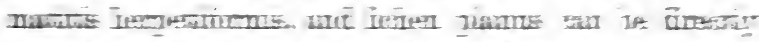

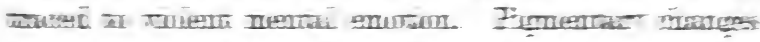

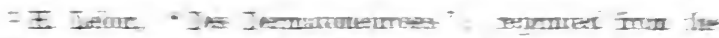

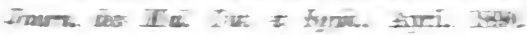


are also often the result of nervous shock-a fact illustrated by the whitening of the hair which sometimes takes place under the stress of sorrow or anxiety, or even suddenly under the influence of a great fear. The comparative frequency of leucodermia in the insane and in epileptics is probably attributable, at least in some measure, to abolition or suspension of cerebral control. Facts have been recorded which seem to indicate that severance of nervous communication with the brain may affect the distribution of an eruption. ${ }^{1}$ The brain acts on the skin through the medium of the sympathetic, and its influence in the production of cutaneous eruptions is measured by the degree to which it inhibits the vaso-motor centre. In the majority of cases no visible changes in the encephalon have been found in relation with lesions in the skin. Bourneville and Poirier have, however, reported a case in which partial discoloration of the skin was associated with a tumour in the left fronto-parietal lobe. ${ }^{2}$

Cutaneous eruptions are frequently associated with lesions of the spinal cord, the posterior columns of which play a leading part in the nutrition of the skin. Any abnormal condition which affects them is, therefore, not unlikely at some stage of the process to find an echo in the integument. This is especially the case in locomotor ataxy, in which skin lesions of the most varied kinds are of common occurrence. In the early stages erythema simplex and erythema nodosum, urticaria, papular eruptions, eczema, herpes zoster, pemphigus, pustules, ulcers and gangrene, have been met with; their appearance is usually coincident with exacerbation of the lightning pains, and, as a rule, their distribution is limited to the

${ }^{1}$ See Crocker, “Lesions of the Nervous System Etiologically Related to Cutaneous Disease," Brain, vol. vii. (1884-85), p 343.

2 Progrès Médical, 1879. 
course of the nerve along which the pain is felt. ${ }^{1}$ In the later stages of ataxy, perforating ulcer of the foot, shedding of the great toe-nail, leucodermia, petechiæ and ecchymoses, unilateral swelling, and odema have been observed. It is probable that sclerosis of the posterior columns is the particular condition most frequently associated with skin eruptions; but as, even in ataxy, such eruptions are not the rule but the exception, it would seem that something besides the lesion of the cord is required for their production. In some cases of acute disease characterized by bullous eruptions (Schwim. mer, Meyer), the most striking lesion in the cord was sclerosis of the columns of Goll. As to the relation of disease of the other divisions of the cord to affections of the skin, the pathological evidence is at present ambiguous or negative. ${ }^{2}$ In spinal meningitis herpetic and pemphigoid eruptions are not uncommon; and Erb says that herpes and bullæ are often associated with slow compression of the cord. In both cases the skin lesions are probably in direct relation with changes in the pos. terior columns or the issuing nerves. Skin eruptions may, however, occur in connection with disease in the cord-as in the case of acute ascending paralysis-where no visible lesions are to be found.

The influence of disease of the spinal cord on cutaneous eruptions is well demonstrated in cases of syringomyelia, and even more so in Morvan's disease.

Bärensprung has shown that herpes zoster is the direct effect of inflammation of the spinal ganglia corresponding to the nerves in the area of distribution on which the eruption occurs. In some cases, however,

1 Crocker, loc. cit., p. 350.

2 Schwimmer's cases are reported in his "Die neuropathischen Dermatonosen," a work in which the nervous origin of many skin lesions was first fully discussed and illustrated by striking cases. (Vienna, 1895.) 
herpes zoster seems to depend on a lesion of the posterior spinal roots, the cord and the ganglion being to all appearance healthy. Herpes frontalis has been found associated with inflammation of the Gasserian ganglion, or hæmorrhage into that body (Kaposi). In other cases herpes has seemed to be due to injury or neuritis of the trunk itself (Dubler); but in these cases it is obvious that the inflammation may easily have extended upwards to the spinal ganglion. The same may be said with regard to other cases in which herpes is a consequence of peripheral irritation. The predilection of arsenic for the peripheral nerves explains the cases of zoster, peripheral neuritis, etc., which have occurred in prolonged administration of the drug, as well as those reported in connection with the contamination of beer by this poison.

The skin lesions that have been observed to follow gunshot and other injuries to nerves are a very persistent variety of erythema resembling abscess and described by some writers as erythema nodosum, herpes, bullæ, ulceration-simple and perforating-eczema, "glossy skin" (Weir-Mitchell), defects of hairs and nails, pigmentary changes, chronic œdema, and a condition resembling ichthyosis. The eruption of bullæ on the fingers and toes which often accompanies the shooting pains in the early stage of anæsthetic leprosy may be grouped under this head, as they are caused by inflammation of the nerves of the limb.

In cases of skin eruption (pemphigus, for example) the cutaneous nerves in the neighbourhood of the affected part have sometimes been found to be in a condition of atrophic parenchymatous neuritis; but it is doubtful how far in such cases the peripheral lesion has been independent of central changes. It must be recollected that in many forms of so-called peripheral neuritis the norve changes are in reality degenerative, and secondary 
to influences acting on the cell in the cerebro-spinal axis, of which the axis cylinder process is only the remote peripheral prolongation. It seems to me at any rate probable that, as Crocker says, the cutaneous nerves do not give way until the central influence is weakened. The direct evidence as to the influence of lesions of the sympathetic in the production of skin eruptions is inconsiderable.

Eruptions, such as erythema of a transient kind, urticaria and rosacea, may also be caused by absorption of toxins from the stomach and intestines, or by reflex irritation from organs such as the uterus or the ovary.

Many of the eruptions associated with nervous lesions are modified by the fact that the skin, deprived of efficient trophic control, becomes an easy prey to bacteria of various kinds. ${ }^{1}$

To sum up, the action of the brain on the skin varies according as its control over the vaso-motor system is increased or diminished. In the cord, the fibres that regulate the nutrition of the skin are bound up with the sensory fibres, and consequently are in the posterior columns ; outside the cord they run through the posterior roots and spinal ganglia, with the sensory fibres, and lesions of one or more of these may be followed by eruptions on the skin. It must be borne in mind that precisely similar lesions in a nerve centre may, in different individuals, or in the same person at different times, produce widely different effects on the skin, and still more often may produce none at all. There are, as already sajd, other conditions which have a determining influence on the development of eruptions, but of which little is at present known.

Besides the various modes of influence of the nervous system upon the skin which have been referred to, cutaneous lesions may be indirectly of nervous origin when,

1 Galloway, Brit. Journ. Derm., vii. 304-8. 
owing to injury or to the condition of impaired nerve force conveniently designated by the term "neurasthenia," the innervation of the tissues is defective, and the skin and other parts are therefore more vulnerable than in the normal state.

Of skin lesions in connection with hysteria and other neurotic conditions there is not much to be said in the present state of knowledge. Among the forms of cutaneous affection which have been observed in connection with hysteria are erythema, urticaria, pemphigus, dermatitis, pigmentation, hyperidrosis, chromidrosis, and hæmatidrosis. ${ }^{1}$ There is nothing characteristic in the lesions. One point of difficulty in the subject is to eliminate the element of frand or unconscious deception in such eases. Van Harlingen ${ }^{2}$ holds that while in some cases the lesions are self-inflicted, the majority of cases are the result of a profound affection of the nervous system. Charcot has recorded cases of "hysterical œdema" which may ulcerate and simulate cancer; under the name "unilateral swelling of hysterical hemiplegia," a similar condition has been described by Weir-Mitehell; and Renaut has reported a "gangrenous urticaria" of purely neurotic origin. Even self-inflicted lesions are to be regarded as a manifestation of hysteria, and such cases are therefore properly included in the group of neuro-dermatoses.

It has already been stated that in the production of skin lesions the nerve centres operate mainly through the agency of the vaso-motor system. In all cutaneous eruptions of nervous origin the mechanism of their production is the same. The process is "angio-neurotic"

${ }^{1}$ A large number of cases of hysterical neuroses of the skin in which recovery took place are collected and critically analysed by Van Harlingen in the Amer. Journ. Med. Sci., July, 1897. See also Rasch, Derm. Centralbl., 1899, No. 11.

2 Journ. Cut. Dis., Sept., 1903. 
in character-that is to say, a disturbance propagated from the centre, or reflected from the periphery, sets up a corresponding disturbance in the vaso-motor centres in the spinal cord, with the result that the circulation at certain parts is thrown into disorder. The blush of shame and the pallor of fear illustrate the effect of mental emotion-i.e. disturbance of the higher cerebral centres-on the vaso-motor system, and through it on the skin. The rashes of fevers and the eruptions caused by certain drugs exemplify the action of the cerebrospinal centres on the integument; these centres are in the first place irritated by the poisonous material circulating in the blood, and this irritation reacts through the vascular system on the skin. The effect of peripheral irritation is illustrated by the consequences which in some persons follow contact with certain species of hairy caterpillars. Intense local hyperæmia, quickly followed by the development of a wheal, is the first result of the direct irritation of the sensory filaments. Soon, however, when the peripheral irritation has had time to make itself felt in the centres, an answering disturbance is excited in parts around the original seat of irritation, and this may reach such a pitch that scratching will at once bring out an abundant crop of similar lesions.

The character of the lesion produced by disordered innervation in any particular case is to some extent a question of the degree of vascular disturbance; but that other elements of a less simple nature are concerned in the process is proved by the fact that in varicella or pemphigus exudation may occur without precedent hyperæmia. 


\section{CHAPTER V \\ NEUROSES OF THE SKIN (Continued)}

\section{SENSORY NEUROSES}

THE sensibility of the skin may be exaggerated, disordered, or abolished without any visible lesion to account for the subjective phenomena. When itching is present, secondary lesions produced by scratching can nearly always be seen. In prurigo the papular eruption may precede the itching (p. 73).

\section{HYPER ESTHESIA}

Hyperæsthesia of the skin is met with in certain nervous affections; the excessive sensibility may be general, or may be limited to the area of distribution of a particular nerve. The increased keenness of the pain-sense is often accompanied by a greater or lesser degree of diminution of tactile sensibility. In hysteria the sensibility of the skin is often greatly exaggerated, a characteristic point being that the hyperæsthesia is very inconstant, both in position and in duration. This painful sensation is produced by light stroking rather than by firm pressure.

Actual neuralgic pain in the skin is not uncommon in locomotor ataxy, and sometimes it seems to be the result of cold. It is generally localized in hairy parts, and ruffling, or even touching, the hair sometimes causes much discomfort, of a character akin to the pain of so-called "muscular rheumatism." Spontaneous pain in the toes, followed by patchy red dis- 
coloration of the skin, and aggravated by warmth, was first described by Weir-Mitchell under the name of erythromelalgia. The pain was so severe in the case which formed the basis of his description that the patient submitted to amputation of one of his toes. H. Batty Shaw found arterial changes present in the parts removed by amputation from three cases of erythromelalgia, and F. W. Weber ${ }^{1}$ argues that there is no distinct boundary between this affection and Raynaud's disease.

\section{Meralgia Paresthetica}

In this condition, first described by Bernhardt and Roth, abnormal sensations, such as tingling, formication, darting or dull aching pains, are felt in the skin of the outer lower two-thirds of the thigh, over an area strictly corresponding to the distribution of the cutaneous filaments of the external cutaneous femoral nerve. The affection is of rare occurrence, only some thirty-four cases having been reported up to 1905 . In a typical case described by Professor White, of Boston, U.S.A., ${ }^{2}$ the morbid states with which it is said to be often associated -neuritis, tabes, rheumatism, gout, alcoholism-were absent, as also were all other disturbances, systemic or local, which might account for the neurosis. In some cases massage has given partial and temporary relief, but the affection appears to be beyond the control of remedies. I have seen three cases of this disorder; in one, the pains were increased by fatigue.

\section{Anfesthesia}

Loss of sensibility depends on various central and peripheral nerve lesions, and, as a rule, lies beyond the province of the dermatologist. It is a prominent symptom of non-tubercular leprosy, in which the absence

1 Brit. Journ Derm., 1904, xvi. 71.

2 Truns. Amer. Derm. Assoc., 1905, p. 44. 
of common sensibility is often associated with increased sensitiveness to cold. Owing to this, lepers frequently inflict on themselves severe burns by pressing their hands and feet against the bars of the grate. Anresthesia is sometimes a symptom of hysteria; in that case it is apt to shift about very suddenly from one part of the body to another.

\section{ITCHING}

This may be a substantive affection, associated with no visible lesions of the skin, except those due to scratching (see Pruritus), or may be a subjective symptom of various cutaneous diseases. These affections may be neuroses, such as hysteria and hypochondriasis, or general nutritive disorders that affect the nervous system secondarily, e.g. diabetes mellitus and gout. Or the irritation may be set up by definite cutaneous lesions or by the attacks of parasites. It may be described by the patient as a pricking, or tingling, or as a formication. Slight in seborrhœea, erythema, pemphigus, and pityriasis rubra pilaris, it is, in varying degrees, more severe in eczema, prurigo, and some cases of psoriasis, in dermatitis herpetiformis and dermatitis gestationis, in lichen urticatus, lichen planus, and lichenification, sometimes in pityriasis rosea, in some forms of tinea, in scabies and pediculosis, in flea-bites, mosquitobites, and bug-bites, in urticaria, in pompholyx and chilblain, and in prickly heat. It also varies greatly in degree in different cases of the same affection.

\section{Pruritus}

The term "pruritus," though often used synonymously with itching, should be reserved to denote itching without any visible cause to account for it. It is a true sensory neurosis, due to some functional disorder of the related nerves, independently of any source of irritation on the surface. The symptom may be so mild as 
hardly to interfere with the patient's comfort, or it may be so severe and persistent as to endanger his life from sleeplessness, or his reason from the nervous irritability which it causes. It is usually aggravated by errors of diet, by the warmth of the bed, and by mental excitement. The strongest will cannot keep the patient from seeking relief in scratching, and, as a matter of fact, the itching often ceases when excoriation has been produced.

Pruritus may be general or local. Of generalized pruritus, four varieties are described-pruritus universalis, pruritus hiemalis, pruritus senilis, and bath pruritus. In pruritus universalis the itching, though affecting the whole body, is not felt all over the surface of the skin at one and the same time; it is, fortunately, also subject to remissions. The causes are mostly constitutional-gout, rheumatism, jaundice and functional derangement of the liver, diabetes, Bright's disease, cancer of the stomach or liver, dyspepsia, uterine diseases, and pregnancy. Many sufferers from universal pruritus are the subjects of lithæmia or oxaluria. The affection often begins in cold weather, but it is by no means confined to the winter. Pruritus hiemalis, on the other hand, begins, in cold climates, between October and January, and ceases about April or May. The itching generally affects the extensor surfaces of the limbs, especially the thighs, but the whole surface of the skin may be involved. The itching is usually more severe when the patient is in bed, probably owing to the greater degree of warmth and the absence of preoccupation. During the day, when the patient's attention is otherwise engaged, he is but little troubled. In this form of pruritus, though the exciting cause seems to be cold, the patients are generally of gouty or rheumatic antecedents or inheritance. Many of them are of neurotic constitution, and are the subjects of hay fever. Others have a naturally dry and thick skin. 
Pruritus senilis is probably the expression of senile changes in the skin. It begins usually after the age of 65 , and is extremely persistent. A remarkable feature of this form of pruritus is that scratching leaves little or no mark (Brocq).

Bath pruritus most frequently affects the legs, from the hips downwards; but the forearms also may be involved, and the neurosis may take even wider range. An affection of adolescence and adult life, it is more frequently met with in males than in females.

Local pruritus affects the anus, the vulva, the scrotum, the nares, the palms of the hands, or the soles of the feet. In most cases some local cause of irritation will be found if carefully looked for. Thus, pruritus ani may be due to hæmorrhoids, to the presence of scybala in the rectum, to ascarides, to fissures or ulcers, or to discharges from the rectum; sometimes it appears to depend on dietetic errors. Pruritus of the vulva may be caused by ovarian, uterine, or vaginal disease, and especially by the presence of sugar in the urine. It is often also a climacteric symptom. In young children pruritus may be due to the presence of ascarides in the rectum. Pruritus of the scrotum, apart from eczema or intertrigo, is rare; when present, however, it is a most distressing affection. The point of maximum intensity of the itching is the raphë (Brocq). Pruritus narium is generally a trivial affection; those subject to it are usually of gouty strain. Pruritus palmarum et plantarum is rare. The sufferers are mostly gouty. In women it is sometimes associated with uterine disorders. Bottstein ${ }^{1}$ reports three cases of pruritus which he attributes to smoking. The affection is symmetrical, and is often extremely troublesome.

Diagnosis.-When pruritus is complained of, the first thing to be done is to exclude all possible sources of

1 Monats. $f$. prakt. Derm., Nov. 15, 1904, p. 577. 
parasitic irritation-lice, bugs, fleas, et hoc genus omne. Nothing in this matter must be taken for granted; lice and itch are sometimes found in the most unexpected quarters. The situation of the scratches must be noted. If they are found on the shoulders, especially in elderly people, the presence of pediculi should be suspected; if on the wrists and the interdigital spaces, the burrows of the Acarus scabiei must be carefully looked for. In all cases of local pruritus the parts must be examined for the conditions that have been mentioned as often producing it. The urine must be examined for sugar, and the constitutional state inquired into. It is a sound rule of practice, however, to fall back on general causes for pruritus only when minute investigation fails to reveal any local source of irritation.

Treatment.-The first indication is to discover and remove any local source of irritation. Linenmesh or silk underclothing should be substituted for flannel. In the itching about the anus, vulva, and urinary meatus, that makes life a misery to some patients, careful examination will often reveal a definite focus of irritation recognized by the sufferer as the point from which the trouble starts. There may be nothing to see at the spot indicated; or slight localized congestion or a tiny excoriation may be visible. In such cases the application of menthol or cocaine will generally relieve the itching for a time. When milder measures fail, the best plan is to destroy the focus of irritation. For many years I have been in the habit of destroying the point to which the source of irritation is referred by touching it with Paquelin's thermo-cautery. X-rays, high-frequency current, and radium have all been used with success in severe oldstanding cases of pruritus ani and in other forms of local pruritus; but such methods are not indicated in generalized pruritus. Whenever itching about the 
genitals, especially about the orifice of the urethra, is complained of by a person of either sex, the urine should be examined for sugar, and, should this be discovered, antidiabetic treatment must be begun. Irritation due to glycosuria may be relieved by the application of menthol, or the parts may be bathed with water as hot as can be borne, and after drying smeared with ichthyol ointment (10 per cent.). In other cases it may be found that the irritation is caused by ascarides, hæmorrhoids, or leucorrhœa. These various conditions must be treated with suitable remedies.

If no local cause can be discovered, general measures must be employed. The patient's diet must be carefully regulated, abstinence from coffee, tea, and sugar, in particular, being enjoined, and alcohol being abso. lutely forbidden. It will be well also if the patient can be induced to exclude shell-fish, pickles, and all highly seasoned, salted, or preserved food from his dietary; white meats, green vegetables, and light milk puddings should form his bill of fare, and he should drink nothing but aerated waters. If there be any evidence or reasonable suspicion of gout, a combination of calomel, guaiacum, and sulphurated antimony in the form of Plummer's pill given at bedtime is often of great service. The bowels should be carefully regulated, but purgation should be avoided. Such cases are likely to derive benefit from a course of sulphur waters-particularly those of Harrogate (Old Sulphur Well), Strathpeffer, Schinznach, Aix-les-Bains, and Luchon. In senile pruritus, indifferent waters, such as those of Bath, Buxton, or Gastein, are more likely to be serviceable.

Internal medication.-The drugs most generally useful are carbolic acid and cannabis indica. Carbolic acid may be given in pills composed of absolute phenol gr. ii, glycerine $m \frac{1}{4}$, powdered marshmallow gr. ii (to make one pill); or in perles of carbolic oil, each containing 
gr. i of carbolic acid. I often use this drug (gr. $\frac{1}{2}$ ) with valerianate of zinc (gr. iii.) in a capsule taken after meals; its benefit consists in its acting as an intestinal antiseptic. Cannabis indica is particularly recommended by Bulkley in senile pruritus; he begins with $m x$ of the tincture, usually increased by degrees to $\mathrm{m} x \mathrm{x}$ or even M $x x x$, three times a day. The drug should be given largely diluted, and its effect should be watched. Ichthyol is often an efficient remedy ; it may be given in the form of capsule, tabloid, or coated pill, gr. $v-x$ being an average dose. Aspirin also is an effective remedy. Another drug that is sometimes very useful is antipyrin, in doses of gr. $\mathrm{x}-\mathrm{xv}$.

External remedies.-When pruritus is general, Turkish baths often give great relief, owing to their diaphoretic action. Continuous emollient or alkaline baths are also most useful. The former may consist of bran 2 to $6 \mathrm{lb}$., potato starch $1 \mathrm{lb}$., or linseed $1 \mathrm{lb}$., in 30 gallons of water; the latter, of bicarbonate of soda zii to $\bar{\jmath} \mathrm{x}$, or carbonate of potash $\bar{\jmath}$ ii to $\bar{\jmath} \mathrm{vi}$, or borax $\xi \mathrm{iii}$, in the same quantity of water. I have kept a highly neurotic patient, affected with intense generalized itching, in a bran bath for several days almost continuously in comparative comfort. An excellent bath is made by mixing zii of sulphurated potash with 30 gallons of water. All these baths should be taken warm, and the skin may afterwards be rubbed with a lather of medicated soap or smeared with a soothing ointment.

Among the simplest and most generally available remedies is the application of plain hot water. A sponge dipped in this and partly squeezed out should frequently be firmly pressed on the itehing part at short intervals. This method is particularly useful in itching of the anus and scrotum. When other applications are employed, it is a good plan always to bathe the parts with hot water before putting on a fresh dressing. The use of a 
[CHAP.

cooling lotion or ointment gives more relief if preceded by the local use of hot water as described; indeed, sudden alternations of heat and cold are of themselves useful in relieving itching. Simple evaporating lotions hardly ever fail to afford temporary relief; they should be applied by means of pieces of linen or lint kept constantly wetted with the solution. A good evaporating lotion may be made by mixing ordinary vinegar with an equal quantity of water. A better application consists of equal parts of eau-de-Cologne or spiritus ammonia aromaticus and water. An excellent anti-pruritic lotion is liquor plumbi subacetatis 3 ii to $3 \mathrm{iv}$, distilled water to 亏 viii, or $3 \mathrm{i}$ of the solution of the subacetate in $亏$ ii of fresh milk. Among other useful lotions must be mentioned the following: Borax 3ii, glycerine $3 \mathrm{ss}$, water 1 quart; carbonate of potash 3ii, water 3 viii; bicarbonate of soda $3 \mathrm{i}$ or 3ii, glycerine 3iss, elder-flower water $3 \mathrm{vi}$.

Carbolic acid may be used in a watery solution (gr. ii-iv to zi) or in the form of a lotion composed of $3 \mathrm{i}$ of acid and 3 ii of pure glycerine, with water to $\xi$ viii, or as a liniment containing 1 part of carbolic acid in 19 of olive oil. Another useful lotion is acid. carbol. 3i, glycerin. pur. 3ii, sp. vini rect. 3iii, aq. camph. ろv. Compresses soaked in these lotions should be applied every hour or two. Carbolic acid may be combined with cocaine in an ointment or a lotion. A useful formula for the former is acid. carbol. $m \times x$, hydrochlorate of cocaine gr. $\mathrm{x}$, vaseline $\mathrm{z}$; and for the latter, acid. carbol. $3 \mathrm{ss}$,

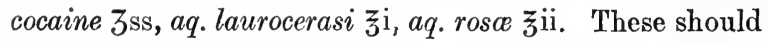
be applied several times a day. Carbolic acid may also be advantageously combined with mercury in an ointment, as follows: Hydrarg. perchl. gr. ii-v, acid. carbol. mxx, ol. oliva $3 \mathrm{i}$, benzoated oxide of zinc ointment $3 \mathrm{i}$. Brocq's carbolized pomade, consisting of gr. xv of carbolic acid, lard 3v, and lanolin 3x, is an excellent application. He recommends that after it has been applied the 
parts be well dusted with starch powder. Mercurial applications are extremely valuable. Among them is black wash, which may be used either alone or in a vehicle of mucilage of tragacanth, as follows: Lot. nigrae, liq. calcis, āā żiv, mucilag. tragacanth. 3i. Other excellent mercurial applications are hyd. perchlor. gr. v., sp. rosmar., sp. vin. rect., āā そ̇, emuls. amygdal. amar. そviii; and

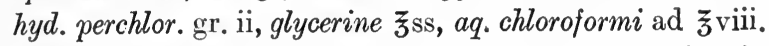
Citrine ointment freely diluted is often of service in pruritus senilis. Naphthol is useful in the form of a soap, or as an ointment prepared as follows : $\beta$-Naphthol gr. xx, lanolini 3ii, ung. simpl. zi.

Cocaine may be used not only in combination with carbolic acid, as mentioned above, or with almost any other substance, but alone. The most convenient form for general use is in an ointment with lano-vaseline or boric-acid ointment as a base. In pruritus ani a half-grain suppository of cocaine will usually give relief. Another valuable local application is menthol, which leaves the parts numb and cold for some time, to the great comfort of the patient. This may be applied either by rubbing the affected surface with the solid cone previously wetted with alcohol or water, or, better, in a solution of gr. $\mathrm{v}-\mathrm{x}$ in dilute alcohol zi. It may also conveniently be used in the form of soap. Menthol and eucalyptol soap is particularly useful. The refreshing coolness caused by menthol is, however, often replaced after a time by heat, tingling, and even slight pain, somewhat resembling the re-establishment of the circulation after partial frostbite. Chloroform may be employed in the form of an ointment containing $3 \mathrm{i}$ to $\bar{z}$ vi of lanolin, or as a lotion of $m_{x v}$ to $\overline{z i v}$ distilled water, and put into an eight-ounce bottle so that it can be thoroughly shaken up before use. Chloral may also be recommended; a solution of the drug in spirit or eau-de-Cologne may be sprayed on 
the affected part after it has been exposed for some time to hot steam and then dried. Equal parts of chloral and camphor rubbed up together make a good anti-pruritic application.

Salicylic acid can be applied diluted with glycerine or alcohol, or as an ointment containing gr. $\mathrm{x}-\mathrm{xv}$ of the acid, vaseline and carbonate of zinc $\bar{a} \bar{a} \overline{3}$, and cold cream ad ̋i.

The most convenient form of applying tar is the liquor picis carbonis, which may be used diluted with water or spirit to the proportion of 1 in 4 or weaker; or combined with solution of subacetate of lead, āā $3 \mathrm{i}-\mathrm{ii}$, rose-water ろviii. Lotio picis carbonis may also be used with calamine lotion as a vehicle (3ii of the former to $\overline{3}$ viii of the latter). Liquor rusci detergens, a solution of oleum rusci in spirit, can be used in the same way as lotio carbonis detergens. Tar may also be applied in the form of ointment, ol. rusci $3 \mathrm{i}$, camphorce gr. $\mathbf{x}$, adipis $\mathrm{z}$; or in pastes.

Nitrate of silver in solution (gr. $\mathrm{v}-\mathrm{xv}$ in $\mathrm{z}_{\mathrm{i}}$ of water, or spiritus ætheris nitrosi) often gives relief. Benzoin in the form of compound tincture painted on with a camel-hair brush, or a solution of benzoic acid $3 \mathrm{ii}$ in diluted alcohol そviii, applied by means of compresses, is useful as an application for very limited areas. Ichthyol may almost always be used with advantage. It is well to begin with a weak solution, such as 1 in 16 parts of water, and gradually increase the strength up to equal parts. The effect is often increased by the addition of a small quantity of precipitated sulphur. Ichthyol may also be applied in ointment, soap, or salvemull.

In conclusion, a word of warning in regard to the choice of a remedy to commence with may not be out of place. One should begin with simple soothing remedies, reserving stronger ones, should they be 
necessary, until the patient's tolerance has been cautiously tested. Spirituous solutions or sprays should never be applied when the skin is excoriated, as they cause considerable smarting and thus intensify the mischief.

\section{Prurigo}

This affection may be of the type which is named after Hebra, or it may be ordinary prurigo, the prurigo vulgaire of French writers.

Etiology.-The actual cause is unknown. Some authorities consider prurigo to be a neuro-dermatosis expressing itself through the vaso-motor apparatus in an inflammatory process; others regard it as a tropho-neurosis, the result of a chronic irritation of the trophic centre, and belonging to the group of dystrophies of the corium-the pruritus, on this hypothesis, being probably secondary to the papule. Brocq and Darier hold that the papule, like the subsequent roughening or lichenification, represents the special reaction of the skin to scratching provoked by the itching, which is the only primary symptom. This view I am unable to accept in its entirety. It may be that in some cases the pruritus is primary; but, with the elder Hebra, I am inclined to believe that more often the papular eruption is the characteristic lesion of the various forms of prurigo.

Prurigo of Hebra.-This type of prurigo generally begins in the first year of life, ${ }^{1}$ and, unless treated

${ }^{1}$ Vidal ("Considérations sur le Prurigo de Hebra," Ann. de Derm. et de Syph., Sept.-Oct., 1892) says that, like Besnier and the majority of French dermatologists, he has seen the affection begin between the ages of 10 and 15 , and even later. In one of his patients the first symptoms of the disease showed themselves at the age of 35 . In nine cases eited by Ehlers (Bull. de la Soc. Franc. de Derm. et de Syph., 1892) the affection commenced between the ages of 15 and 30 . In a case reported by Bernhardt (Arch. f. Derm. u. Syph., 1901, p. 173) it began at the age of 8 . 
in the very early stage, usually lasts until the patient's death, becoming better or worse, however, under the influence of season, the general state of health, etc. The characteristic lesion is an eruption of discrete, slightly raised papules, at first of the same colour as the skin, but afterwards, when subjected to irritation by scratching, becoming reddened and increasing in size. There is often a blood-crust at the top. The eruption is most abundant on the extensor surfaces of the limbs, but it also occurs on the chest (back and front), the lower part of the belly, the sacral region, and the buttocks. It is rarely seen on the flexor aspects of limbs, and occurs sparsely on the face. The itching is intense, and the changes produced by scratching are very marked. Besides these, other lesions often develop, which may resemble those of eczema (except that the flexor surfaces are generally spared) or of urticaria. Pustules and sores, often accompanied by considerable enlargement of the femoral and axillary glands, are not infrequent. In the rare and severe type of Hebra's prurigo which is styled prurigo ferox, to distinguish it from prurigo mitis, the ordinary form of the disease, the elementary lesions are more developed and more numerous, and the skin in certain parts, notably the legs and forearms, gives a sensation to the touch like coarse brown paper or a nutmeg-grater (Crocker). The lymphatic glands in the groin and in the axilla are enlarged, and may even suppurate. Poverty and insanitary conditions of life are predisposing causes, and males are more often affected than females.

Pathology.-Structurally, the papule is a localized hypertrophy of the prickle-cell layer. At first it is urticarial, there being odema of the derma, with proliferation about the vessel walls. The stratum corneum is thickened, the upper layers of the derma are infiltrated with cells, the arrector muscles are con- 
tracted, so that the follicles are thrown into a state of erection. Finally there is chronic hypertrophy of the prickly and horny layer, the papillæ are depressed, and the panniculus adiposus disappears.

Diagnosis. - This is made partly by a process of exclusion, partly by the sum of the clinical facts. Other itching conditions, such as scabies, pediculosis, etc., are excluded by the absence of the characteristic lesions. The positive characters are the origin of the affection in infancy, its persistence, the poor general health, the preference the papular eruption shows for the extensor surfaces of the limbs, and the immunity enjoyed by the bends of the joints. A pathognomonic feature is the nutmeg-grater-like feeling of the skin on the outer side of the legs and forearms. The glandular enlargement, which in the groin often attains a very large size, is another distinctive feature.

Treatment. - The disease can, as a rule, be cured only in the very earliest stage-that is to say, in childhood, before it has become inveterate. As already said, however, it is subject to spontaneous remissions, and it can always be greatly mitigated by treatment. This must be conducted on the lines laid down for pruritus. In addition to the internal and external remedies for itching already described (pp. 67-73), a liberal supply of nutritious food is always of the greatest importance, especially in the case of children. Of the various local applications, strong tar in lotion or ointment is the most generally useful. Systematic bathing and massage may also be recommended. Cod-liver oil and iron may be given in most cases with advantage.

Prurigo of the ordinary type.-This form of prurigo is at once less severe and less refractory to treatment than that described above. It may begin in childhood, but more often it does not appear until the patient is in the third decade. It usually develops on a neuro- 
pathic soil, and auto-intoxications of various kinds, alcoholism, uterine disorders, and other conditions which depress the health are frequently its antecedents. It may be either circumscribed or diffuse. The circumscribed form (the névrodermite circonscrite or prurit circonscrit of Brocq), more common in women than in men, begins as itching, which is almost always intermittent, there being crises separated by periods of remission. The skin loses its normal colour, and soon there is an eruption of papules grouped into one or more plaques, which may be, according to a distinction set up by Brocq, complete or incomplete. A complete plaque presents simultaneously three concentric zones, but much more frequently the plaque is incomplete. Any part of the cutaneous surface may be attacked, but the regions most often affected are the neck, the upper and inner surfaces of the thighs, the loins, the lower and outer aspect of the leg, the popliteal and axillary folds, the palmar and plantar surfaces, the scrotum in men, the labia majora in women. It is this circumscribed type of prurigo to which Vidal has given the name of lichen simplex chronicus. The diffuse form, like the circumscribed, begins with severe itching, which, either continuous or paroxysmal and intermittent, ushers in the papular eruption. Large portions of the cutaneous surface, including all the limbs, may be attacked, and even the whole body, and the affected area may be deeply pigmented. After a period varying between two or three weeks and several months, the pruritus may subside and the lesions heal, but there is great proneness to recurrence, and the relapses may be seasonal.

In both the circumseribed and the diffuse forms of prurigo, as in eczema and certain other conditions, there occurs the change which is known as lichenification or lichenization. The skin becomes thickened and rugose; its peculiar aspect is due to an 
exaggeration of the normal strix of the integument, so that it is quadrillated into a reticulum composed of square, lozenge-shaped, or polygonal meshes, with a surface which frequently, to borrow Darier's simile, resembles glossy and brilliant facets, as of a mosaic. In some cases there is a covering of fine scales. Skin which has become lichenified is, of course, less supple than the normal integument. Its colour may remain unchanged, but often it takes on a greyish or brownish hue. The margins of the patches are ill-defined.

Diagnosis.-The most important differentiating characters of common prurigo, whether circumscribed or diffuse, are the long duration, the acute itching, the character of the papules, and the lichenification. The last-named feature occurs also in other affections, but it is different from the lichenification of prurigo. Thus, in eczema and psoriasis the thickened and rugose skin has no glistening facets, the margins are clearly defined, and the colour is red. In lichen planus the plaques are made up of papules that have run together, and they are surrounded by characteristic papules.

Treatment.-The least unpromising methods of treatment are applications of the $X$-rays or of radium, or of static electricity. But when the itching ceases and the lesions clear up, there is marked liability to recurrence. The affected areas may be covered with the leucoplast of Unna, or some other adhesive dressing or plaster. Irritating preparations must be carefully avoided. Sedatives may be administered internally, all occasions of excitement must be avoided, the diet regulated, and the general health carefully attended to. 


\section{CHAPTER VI}

\section{NEUROSES OF THE SKIN (Continued)}

\section{URTICARIA}

Symptoms.-The characteristic lesion of urticaria is a wheal or raised patch of skin flattened on the surface, firm to the touch, and at first uniformly red in colour, but afterwards white and bloodless in the centre, with a bright-red border, which often has an areola of erythematous redness outside it. Sometimes, however, the patch remains red throughout. When the wheal subsides the centre becomes red and the border pale. When wheals are numerous their areolæ become confluent, so that the white centres stand out boldly on a red ground. Wheals vary in size from a threepenny piece or smaller to a florin or even a four-shilling piece. The lesion is seen in its most typical form in the wheal which is caused by the stinging nettle, whence the name "urticaria" (urtica, nettle) or nettle-rash.

Urticaria comes on quite suddenly, the appearance of the eruption being accompanied by intense itching and burning. Scratching gives some momentary relief, but is followed by the development of large numbers of fresh wheals, which spring up, so to speak, under the patient's fingers, or may arise at a distance. Sometimes the affection is purely local, as when it arises from insect bites, but in severe cases the skin eruption is usually associated with some systemic disturbance. In the common form of the affection, urticaria fugax, the individual wheals last only a few hours at 
most, and disappear, leaving no trace of their presence. Fresh crops, however, may continue to appear, and the attack may last for some days. In some cases the eruption comes out in successive crops day after day, for weeks or months or even years. ${ }^{1}$ To this form of urtiearia the term "chronic" is usually applied; but as there is no difference in respect of the severity of the local symptoms between it and the more common variety, urticaria fugax, it would be more logical to call it urticaria perstans. In certain cases not only the duration of the disease but that of the individual wheal is considerably prolonged. Cases of this kind have been reported in which wheals on the limbs, the back, and the belly, varying in size from a lentil to a haricot bean, persisted for three months. ${ }^{2}$ Hartmann ${ }^{3}$ describes seven cases of urticaria perstans in which the leading symptoms were itching and a papular eruption with excoriations, with no evidence that the lesions had begun as ordinary wheals.

Urticaria sometimes invades the mucous membranes of the eyelids, lips, mouth, tongue, pharynx, vulva, and possibly of the digestive canal and stomach (Pringle) and of the bronchi. Involvement of the bronchi probably affords an explanation of the frequent association of urticaria with asthma, the same causes determining an attack of both affections. The asthma may either accompany or alternate with the wheals on the skin. On the mucosæ named, œdematous swellings may occur (see Angio-Neurotic CEdema, p. 86), and should the glottis be attacked there may be difficulty of breathing, going on even to suffocation. A patient of mine, a medical man, has suffered periodically from urticaria of the buccal mucous membrane for the greater part of his

1 Dubreuilh, Gaz. des Hop., Oct. 22, 1892.

2 C. Boeck, Norsk Magazin for Laeg., 1888.

3 Arch. f. Derm. u. Syph., March, 1903. 
life, the swelling affecting the palate and the tongue. Wheals in the Eustachian tube and the middle ear have also been reported.

The wheals have no definite arrangement, and are never symmetrical. There may be only a few on some particular part of the body, or they may cover nearly the whole of its surface. A striking feature of urticaria when it has obtained a hold on the patient is that the slightest contact with the clothing or the least scratch will at once bring out a crop of wheals on any part of the skin; even when the rash is not present the patient can often write his name with his finger-nails on apparently healthy parts of his skin, especially on the back and chest (autographism, or urticaria factitia).

Several varieties of urticaria have been described, according to the size, configuration, and structural peculiarities of the characteristic lesions. Thus the wheals may be small and on their subsidence leave papules. Hence the name urticaria papulosa. It is to Colcott Fox that we owe the proof of the urticarial nature of these lesions and their identification with the lichen urticatus of Bateman and the lichen strophulus of Rayer and Biett. ${ }^{1}$ Urticaria papulosa, sometimes styled strophulus, or simple prurigo, or gum rash, is chiefly met with in children. The wheals are, as a rule, no larger than a lentil, and on the top of each is a tiny red point or inflammatory papule, which is usually covered with a darkish scab, the result of scratching. If the red papule is not at first visible, it can always be brought into view by pressure, when the colour fades from the circumference of the papule, leaving a minute red spot in the middle. The eruption affects all parts of the body, but shows a preference for the trunk. It is sometimes

1 Colcott Fox, "Urticaria of Infancy and Childhood," Brit. Journ. Derm., May, 1890. 
markedly vesicular in character, closely simulating varicella ${ }^{1}$ or scabies. Fresh crops of lesions come out at night, and cause such intense itching that sleep is impossible. I have seen some cases in which the general symptoms were very severe. The disease may last for several years, becoming milder or practically remitting in winter, and returning with the warmer weather, or vice versa. Urticaria papulosa may be looked upon as a connecting link between urticaria and prurigo. I agree with Colcott Fox in thinking that its transition into prurigo, if it ever takes place, is an extremely rare occurrence.

When ordinary urticaria attacks parts like the eyelids, scrotum, etc., where there is much loose connective tissue which offers comparatively little resistance to the diffusion of the infiltration, it is termed urticaria œdematosa. The œdema as a rule comes on suddenly, to the great alarm of the patient, especially when mucous membranes such as those of the tongue and throat are involved; but it seldom lasts longer than twenty-four hours. Alcoholism and neurotic inheritance seem to be predisposing causes.

Urticaria gigas, known also as the acute circumscribed oedema of Quincke (see also p. 86), is characterized by the rapid development on the cutaneous or the mucous surface of large patches of localized œdema, varying in size from a hazel-nut to an ordinary orange. They are hard to the touch, like the biceps muscle when strongly contracted. Itching is seldom complained of, but there is usually a sensation of tension and of heat. The swellings last a day or two, and subside as quickly as they came. There may be a slight feeling of malaise before the eruption, but often there are no prodromal symptoms. The affection may persist for several years, and the intervals between the attacks may be regular or

1 Colcott Fox, Brit. Journ. Derm., 1899, p. 157. G 
irregular. The malady is attended by no danger so long as the upper respiratory passages do not become the seat of the lesions. The affection is due to causes similar to those of ordinary urticaria, and requires the same treatment. Cases are sometimes met with which are intermediate between the two forms.

When effusion of blood takes place into the wheals the condition is termed urticaria hæmorrhagica or purpura urticans; when bullæ form on the surface, it is spoken of as urticaria bullosa. Urticaria pigmentosa is not really an urticaria, and is dealt with separately.

Etiology.-It has been suggested by Wolff-Eisner and Bruck that the urticarial condition may be explained by hypersusceptibility or anaphylaxis to foreign albuminoid bodies. The theory derives some support from Bruck's experiments, ${ }^{\mathbf{1}}$ and deserves consideration. In the present state of knowledge the causes of the affection may be provisionally classified as predisposing, external, and internal. Among predisposing causes are sexfemales being considerably more liable to the affection than males; age--infants, owing to the irritability of their skin, being particularly prone to nettlerash; the neurotic temperament; indigestion; gout; functional and organic disease of other organs, notably the uterus and ovaries, and of the nervous system. In infants urticaria is often associated with rickets and dilatation of the stomach. Malaria is so strong a predisposing cause that some writers make a special variety of the affection, under the name of " paludal urticaria." Urticaria is often associated with jaundice, rheumatism, purpura, and occasionally co-exists with albuminuria and glycosuria. Violent mental emotion may be sufficient of itself to bring on an attack.

Among external causes are local irritants - the stings of nettles, jelly-fish, or wasps ; the bites of insects-bugs,

${ }^{1}$ Arch. f. Derm. u. Syph., 1909, xcvi., 241. 
mosquitoes, etc.; contact with or proximity to hairy caterpillars; the direct application of cold to the skin, and especially sudden alternations of temperature. $^{1}$ S. B. Ward ${ }^{2}$ records a case-that of a woman of 47 -in which urticaria was caused simply by exposure to the sun's rays. Exposure to the heat of a fire or to X-rays had no such effect.

Among internal causes are certain articles of food which irritate the alimentary canal and reflexly the skin (through the pneumogastric nerve). Every variety of idiosyncrasy is displayed by patients in this respect; but to shell-fish, especially mussels, crabs, and lobsters, must be assigned the chief place among dietetic irritants. Among other substances which cause urticaria in certain individuals may be mentioned pork, almonds, strawberries, parsley, mushrooms, and oatmeal. Certain medicinal substances also give rise to it. These are dealt with in the chapter on "Artificial Eruptions" (see p. 231). Among the internal causes of the affection should also be mentioned the presence of hydatid cysts, and especially of their fluid contents, in the abdominal cavity; and worms. Chronic urticaria has in some cases been traced to the colon bacillus. In a case of Winkelried Williams's, ${ }^{3}$ urticaria gigas appeared to be due to absorption of toxins from a chronic otorrhœa. In a case of my own, severe angio-neurotic odema of the glottis was clearly traceable to injury to the brain.

Pathology.-Urticaria is held by Philippson, as the result of experiment, to be due to the action of irritants either directly on the walls of the blood-vessels or by circulating in the blood, and this conclusion finds support in the experiments of Török and Hari. ${ }^{4}$ Gilchrist,

1 Crocker, "Diseases of the Skin," 3rd edition, p. 126; Brit. Journ. Derm., Feb., 1902, p. 43.

« N.Y. Med. Journ., April 15, 1905, p. 742.

${ }^{3}$ Brit. Journ. Derm., 1907, ix. 12.

${ }^{4}$ Arch. f. Derm. u. Syph., April, 1908. 
who has made a study of the question, holds that a toxin is present in the blood-stream, and that when the wheal is produced some of the poison is set free, causing death of the cells, which is followed by acute inflammatory changes. His sections show an emigration of leucocytes and lymphocytes, fragmentation of polynuclear leucocytes and fixed connective-tissue cells, apparent increase in mast cells, and swelling of the cells of the sweat-glands, with a dispersal of fibrin throughout the derma. ${ }^{1}$ Another view is that the affection is a result of reflex vaso-motor disturbance. Stephen Mackenzie placed the nervous centre of the reflex mechanism in the dense plexus of fine nerve fibres in the superficial layer of the corium. The variations in the size and other characters of the wheal are due to the different depths to which the infiltration penetrates. In ordinary urticaria only the upper layer of the integument is affected, while in urticaria gigas the whole thickness of the skin is involved, and in the odematous variety infiltration takes place into the loose meshes of the subcutaneous areolar tissue.

Diagnosis.-Urticaria, as a rule, presents no difficulty in diagnosis, the sudden onset, the presence of wheals, and the fugitive nature of the eruption being the characteristics of the disease. In certain cases, however, in which the wheal is surmounted by vesicles or bullæ, urticaria may for a time simulate pemphigus, or the first stage of dermatitis herpetiformis; and if the constitutional symptoms are well marked, the rash may at first be mistaken for that of scarlet fever, or even for erysipelas. But the history and course of the eruption, and the almost invariable presence of typical lesions, soon reveal the true nature of the affection. Urticaria papulosa is frequently confounded with scabies, but the distribution of the lesions on the

${ }^{1}$ Trans. Sixth Internat. Derm. Congr., 1907, ii. 905. 
trunk and the absence of the characteristic burrows are sufficient to exclude that disease. For the diagnosis between urticaria papulosa and erythema papulatum, see p. 109.

Prognosis.-This is always favourable, although as has been said, in some rare cases the disease may run a more or less prolonged course. Its duration depends upon the cause. If this be an acute toxæmia from the ingestion of unsuitable food the symptoms may quickly disappear; if it be a chronic toxæmia, the affection may be much more persistent. Severe cases of doubtful origin are often very intractable.

Treatment.-The first thing to be done is to discover and, if possible, remove the cause (errors of diet, especially shell-fish, worms, parasites, or other source of reflex irritation). If the attack is distinctly traceable to indigestion, or to poisoning by mussels, etc., an emetic should be given if the symptoms are very severe; in milder cases a smart saline purge will cleanse the intestinal canal of the toxins which are the cause of the trouble. The patient should be kept on a bland, unirritating diet for a few days, if fever be present ; and, especially if the urticaria be of malarial origin, quinine in full doses may be given with advantage. If there be any reason to suspect a gouty element in the case, it must be dealt with on general principles, alkalis being particularly useful. Sir A. E. Wright, who calls urticaria "a serous hæmorrhage," recommends calcium chloride in doses of gr. $\mathrm{xx}$ three times a day. The itching may be allayed by means of any of the local applications recommended for the treatment of pruritus (pp. 69-73), simple evaporating lotions generally being sufficient for the purpose. Brocq advises that the patient's body-linen be impregnated with starch powder, and that he sleep in fine sheets sprinkled with the same material. It is most important to prevent chill. 
For this reason it is well, whenever the patient will submit to such a course, to keep him in bed. I have known patients derive benefit from exchanging a flannel for a linen nightdress. Excessive heat should also be avoided. The clothing should be light, and the underclothing especially should not be of such a nature as to cause irritation of the skin. The effects of scratching must be dealt with as already indicated.

In chronic cases the bowels must be carefully regulated, and any constitutional state that may appear to be associated with the skin affection should be treated on general principles. In those cases which, from examination of the blood, urine, or fæces, appear to be due to the colon bacillus, a vaccine prepared from the bacilli may be administered, and lavation of the colon employed. Salol also is very useful. All food of a stimulating character, and alcohol in any form, must be avoided. Should these general measures prove unavailing, an attempt may be made to act directly on the vaso-motor centres by means of sulphate of atropia, which may be given internally as a pill, containing gr. $\frac{1}{200}$ to gr. $\frac{1}{1} \frac{1}{0}$, with sugar of milk and glycerine of tragacanth. This pill should be given at night. The drug may also be administered by subcutaneous injection (gr. $\frac{1}{150}$, very cautiously increased). Administration of adrenalin, which contracts the capillaries, has been found serviceable. Ichthyol in gradually increasing doses is one of the best drugs at our disposal. Chronic urticaria which has resisted all medical treatment is often cured by the rest and freedom from worry given by a holiday. A sea voyage is sometimes efficacious when other means fail.

\section{Angio-Neurotic CEpema}

This condition, also styled acute circumscribed œdema, resembles urticaria œdematosa, but occurs independently of urticaria. 
Symptoms.-Circumscribed swellings of varying consistency develop in the loose tissue of the scrotum, penis, and eyelids; they'may also occur in the hands and feet, and on the forehead. The odematous swellings are isolated, well defined, more or less red in colour, smooth and glistening on the surface. They vary in circumference at the base from a five-shilling piece to the palm of a man's hand (Brocq). They are not the seat of pain or itching, but they sometimes give rise to a slight feeling of tension. The affection is sometimes associated with purpura, or with colic and gastro-intestinal disturbance. As a rule, the swellings last only a few hours, or at most a day or two. The affection may, however, persist a considerable time, as fresh swellings may continue to appear. The mucous membranes may be attacked, and, if the swellings develop in the pharynx or larynx, alarming symptoms may ensue.

Etiology.-The causes are the same as in urticaria (p. 82). The affection is sometimes hereditary, and Osler has shown that it is related to peliosis rheumatica and erythema nodosum.

Prognosis and treatment.-The attack generally subsides under treatment, but there is great liability to recurrence. Administration of adrenalin has been tried with good results, as also has the administration of pituitary gland. Care must be exercised as to food; the stools, the urine, and the blood must be examined, and if the colon bacillus is found to be present an autogenous vaccine should be employed, and lavation of the colon practised.

\section{Hysterical Cedema}

This is a form of œedema which, though noticed by Sydenham, was fully described only some twenty-five years ago by Charcot, and notably by Renaut. ${ }^{1}$ It is

${ }^{1}$ Médecine Moderne, Feb. 20, 1890. 
usually met with in hysterical subjects, but in cases reported by Strübing ${ }^{1}$ nervous symptoms were not an invariable feature, nor, where such symptoms were present, was he satisfied of any immediate causal connection between the hysteria and the state of the skin. The cedema is a hard swelling of a violet colour (cedème bleu des hystériques); it scarcely pits even under prolonged pressure. The local temperature is usually subnormal, and numbness and sometimes pain of greater or less severity are complained of. The swelling, which is, as a rule, associated with hysterical paralysis or contracture, is very persistent; but it is subject to extremely sudden variations under the influence of emotional disturbance or in connection with the menstrual function. If the œedema reaches a certain degree of intensity it may induce gangrene of the skin, followed by deep and widespreading ulceration, which may be mistaken for malignant disease. A remarkable case of an apparently miraculous cure of such a "cancer" is related by Charcot. ${ }^{2}$ The main part of the treatment must be directed to the restoration of the nervous system to a condition of healthy equilibrium.

\section{Urticaria Pigmentosa (Plate 1)}

This affection, as mentioned above, is not essentially an urticaria, though it is so styled because the macules or nodules by which it is characterized take on an urticarial character upon slight irritation.

Symptoms.-Urticaria pigmentosa usually begins very soon-often a few days-after birth, more than half the cases arising before the age of 6 months is attained; but Graham Little, ${ }^{3}$ in a careful study based

1 Arch. f. Derm. u. Syph., Feb., 1902, p. 171.

2 "La Foi qui Guérit."

'Brit. Journ. Derm., Oct., Nov., and Dec., 1905, and Jan., 1.906. 


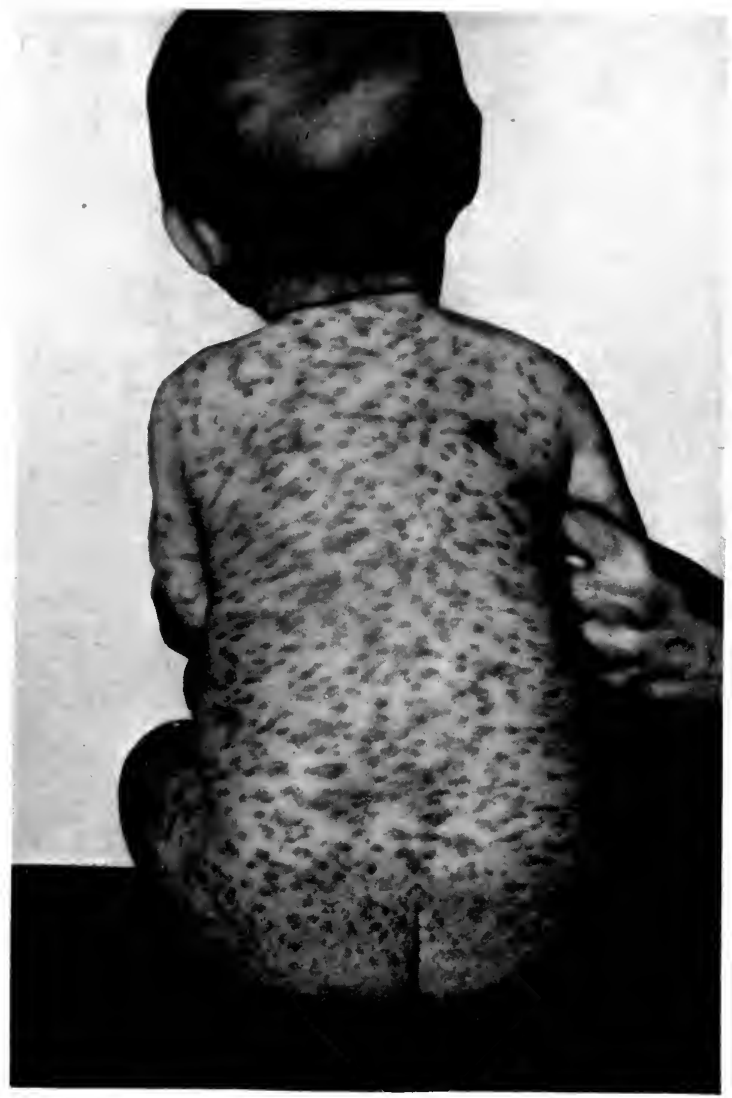

Plate 1.-URticaria pigmentosa. 

upon all the cases which he found recorded up to the end of 1905, shows that it occasionally appears after puberty. In a case of a woman whom I showed at the Dermatological Society of London the disease began at the age of 38 . The essential feature is the appearance of raised patches somewhat conical in shape and red or pink in colour; these afterwards become flattened on the top, and their hue deepens gradually to dark brown. In a case under the care of Mitchell Bruce and Galloway, which was carefully studied by $H$. R. G. J. Brongersma, superficial scarring was noted in the centre of the patches; this feature of the lesions of the disease had previously been reported by Hallopeau to the French Dermatological Society (1892). The individual lesions do not disappear like the wheals of ordinary urticaria, but persist, while others come out in successive crops. When the disease is fully developed the child is spotted with more or less prominent patches varying in size from a split pea to a sixpenny-piece, and in colour from bright red to dark brown, according to the age of the wheal. The parts usually affected are the front and sides of the chest, the back, the belly, and the limbs; the face is not always spared, nor is the buccal mucous membrane. The disease is usually distinctly symmetrical, in contrast with ordinary urticaria, a point illustrated in a remarkable manner in a case shown by me at the Clinical Society:

At varying intervals, especially in summer, the morbid process seems to be quickened into fresh activity. At such times the patches become intensely congested. Vesicles and bullæ may develop on their surface, and new lesions appear on parts of the skin previously healthy. These phenomena are accompanied by intolerable itching, and the scratching which is the result adds fuel to the fire. In some cases the raised red 
patches predominate; in others the flattened pigmented lesions. Usually the two forms, which, as already said, represent different stages of the same process, coexist in varying proportions.

Two collateral symptoms usually found associated with urticaria pigmentosa are, according to Graham Little, general enlargement of the glands, not proceeding, however, to suppuration, and a condition of the unaffected skin, styled by French writers dermographism, in which artificial wheals may easily be produced by scratching.

The natural tendency of the disease is to disappear as the patient grows older. Three well-defined stages can be recognized in the large majority of cases. There is a period of activity, during which successive crops of the eruption continue to appear. This lasts about a year, occasionally longer. Next follows a period, lasting from two to five years, during which the disease is more or less stationary. Lastly, there is a period of retrogression, during which the spots gradually fade away, though complete disappearance of the pigmentation is rare. This retrogressive period may last several years.

Pathology and etiology. - The special pathological feature of urticaria pigmentosa is that the local infiltration which gives rise to the distinctive lesions is largely made up of mast cells. These cells exist in such large numbers in the pigmented spots that sections especially stained for their recognition assume a reddish colour owing to the reaction given by the mast cell to granules. In the case of a child suffering from urticaria pigmentosa, Gilchrist showed by experiment that in the skin which appeared to be normal, as well as in the lesions, mast cells were present in considerable numbers. Brongersma is of opinion that the prime cause of the disease is not a congenital increase of the vaso-motor irritability of the skin, but "a con- 
genital increased tendency for the connective tissue to change into mast cells," the urticarial wheal being a secondary symptom. He thinks it possible that mast. cell granules which are seen lying free in the lymph spaces are degenerative products, and that, gaining access to the blood, they act as a toxin that is capable of producing the changes in the superficial circulation which give rise to the urticaria. Darier claims that Ranvier has demonstrated that the mast cells are not altered connective-tissue cells, but are derived from the blood.

Diagnosis.-The clinical diagnosis of the condition rests chiefly on the appearance and mode of evolution of the wheal-like patches and the persistence of their pigmentation.

Treatment. - Various kinds of treatment have been tried, without producing any appreciable modification of the morbid process. Belladonna internally, and atropine in hypodermic injections, have been recommended; but the clinical evidence at present available is insufficient to warrant a definitive judgment as to the efficacy of this method. The itching may be relieved by the measures that are found useful in ordinary urticaria. Apart from this, the principal indication is to build up the general health on as solid a foundation as possible. 


\section{CHAPTER VII}

\section{NEUROSES OF THE SKIN (Continued)}

\section{ERYTHEMA}

ERYThema strictly means nothing more than superficial redness, disappearing on pressure; that is to say, a local congestion of the skin. A good deal of confusion as to the nature of the affection has been caused by the fact that different stages of the same process have been described as distinct diseases, and a further element of perplexity has been imported into the subject by classifying the rashes of infectious diseases as varieties of erythema. Erythematous they doubtless are anatomically, but they have no independent existence as pathological processes, and it is illogical to consider them apart from the diseases of which they are manifestations. The eruption of an infectious fever is, in fact, the result of the irritation of certain specific poisonous matters circulating in the blood. The eruptions caused by certain drugs, which are often erythematous in appearance, are the result either of a toxic action of the chemical substance on the nerve centres, or of direct irritation of the peripheral ends of the nerves supplying the integument. These will be dealt with in some detail in Chapter $\mathrm{x}$. (Artificial Eruptions).

Types of erythema. - Erythema, as a substantive disease, shows itself under various forms, all of which may, however, be grouped under two heads, viz. (a) hypercemic, (b) inflammatory. In the 
former category the mechanism of the process consists in localized vascular disturbance, which gives rise to hyperæmia-at first active, but, if the cause persists, soon becoming passive owing to vaso-motor paralysis. The colour of the affected area of skin, which at the outset is bright scarlet, changes as the bloodstream becomes more sluggish to dull red, deepening as the tendency to stagnation increases to livid blue or purple. In correspondence with the variations in the blood-current, the skin at first feels hot both to the patient and to the observer; but the heat subsides as the congestion assumes a passive character, and often, especially in the extremities, the local temperature falls below the normal point. In erythema of the inflammatory type the retardation of the blood-current goes on to stasis, exudation of serum takes place, leucocytes escape into the tissues around the vessels, and sometimes subcutaneous hæmorrhages occur. In this way the various lesions-vesicles, blebs, œdema, and pigmentation-seen e.g. in erythema multiforme, are produced. If the inflammatory process is severe it gives rise to more serious lesions, such as local asphyxia, ulceration, sloughing, and even gangrene. Widely different as the hyperæmic and inflammatory forms of erythema are in their clinical aspects, pathologically no definite boundary line can be drawn between them.

Etiology.-Individual predisposition is a necessary condition of the development of erythema. This predisposition appears to be simply an exceptional instability of the vaso-motor system, rendering it unduly susceptible to irritation. The irritation may be direct, as by the action of cold or heat, acrid discharges, certain vegetable or chemical substances (Primula obconica, rhus, mustard, etc.), the bites or stings or mere contact of certain insects (fleas, bugs, hairy caterpillars), coarse flannel or dirty underclothing ; 
or indirect, i.e. reflected to the nerves of the skin from internal organs, more particularly the organs of digestion and the female genital apparatus. It is also sometimes a manifestation of the rheumatic or gouty poison. Frequently, too, it is the result of intestinal toxæmia. Thus, Galloway has shown that cutaneous manifestations of the erythematous type occur in diseases of the liver, especially in cases in which the portal blood passes into the general, and therefore the cutaneous, circulation without having been subjected to the purifying action which it is the function of that organ to perform. ${ }^{1}$ It is not always possible, however, to trace an attack of ery. thema to any distinct cause ; in such cases, no doubt, sources of irritation of one or other of the kinds just mentioned are present if only they could be found.

\section{Hyperæmic ERythema}

Of the hyperæmic type of erythema there are several varieties.

Erythema simplex is characterized by patches of redness, at first scarlet, afterwards pinkish in hue. These may come out on any part of the cutaneous surface, showing a preference, however, for the face and portions of the skin which are in contact with each other or exposed to the air. The affected parts feel hot to the hand, and the patient complains of a sensation of burning or itching; but there is seldom any fever or systemic disturbance. The redness gradually fades and finally disappears, leaving no discoloration behind. Slight desquamation often accompanies the subsidence of the eruption. The affection may last an indefinite time. The diagnosis, as a rule, presents no difficulty. Erysipelas may be excluded by the absence of serious constitutional disorder, by the mildness of the local symptoms, and especially by the fact that the reddened

${ }^{1}$ Brit. Med. Journ., March 21, 1908. 
area is not raised and is not bounded by a sharply defined edge. From urticaria, on the other hand, erythema simplex is differentiated by the absence of the characteristic wheals and by the comparatively persistent nature of the eruption.

A variety of erythema simplex which deserves special mention on account of its recurrent character shows itself in the form of congestive redness of the cheeks and nose. This recurs again and again, and may finally become permanent. (See Rosacea, p. 133.)

Erythema fugax is simply a more transient variety of erythema simplex. Patches of redness come out suddenly on the face or body, and disappear in a day or two. In children the eruption is usually the result of reflex irritation, as by teething, or disorder of the intestinal tract by unsuitable food, or worms. In adults it is sometimes associated with mental emotion. The redness may be either diffuse or scattered over the body in irregular patches of varying size. Under this head may be placed the fleeting rashes described by some authors under the designation of "roseola."

Erythema (or ephelis) ab igne, or livedo reticularis.-In this condition, as the result of long exposure to heat, the affected surfaces present a dark, reddish-brown, reticulated coloration. Under pressure the redness temporarily disappears, leaving a brownish stain. In a case shown by Adamson, ${ }^{\mathbf{1}}$ the patient was a cabinet-maker's assistant whose work necessitated his standing before a large fire, and the seat of the erythema was the anterior and lateral surfaces of the legs, from the middle of the thighs to the ankles. The condition has also been observed as a result of the application of hot compresses for abdominal disorders, or of the use of hot-water bottles for lumbago, etc. This form of erythema probably represents a venous stasis, the ${ }^{1}$ Brit. Journ. Derm., 1911, xxiii. 48. 
reticulated pattern depending upon the anatomical distribution of the vessels in the skin.

\section{INFLAMMATORY ERythema}

Under this heading may conveniently be grouped certain diseases which, differing from each other in some particulars, are all characterized by lesions of an inflammatory erythematous nature.

Erythema intertrigo, as the name implies, occurs in parts where two opposed surfaces of skin chafe each other (inner aspect of thighs, groins, axillæ, under pendulous breasts, at the lower part of the abdomen, etc.). Infants and fat persons are most liable to the affection; in the former the eruption is commonest on the parts which are chafed by the napkins. The affected surface is reddened and glazed; there is no exudation, but the epidermis is generally to some extent macerated by sweat. Intertrigo is differentiated from eczema by the absence of "weeping." In the case of young children it is sometimes difficult to distinguish intertrigo from the erythema of congenital syphilis. The eruption is very similar in both affections; but while in intertrigo the redness is usually limited to the parts covered by the napkin, in congenital syphilis it extends down the legs, often to the heels and soles of the feet. ${ }^{1}$ The chief point of distinction, however, is that if the affection is syphilitic other characteristic lesions are sure to be present.

Infantile erythema of Jacquet.-Erythema of the napkin area, named by Adamson ${ }^{2}$ after the observer who was the first to distinguish the condition from congenital syphilis, affects specially the convex surfaces of this region rather than the folds. It manifests itself

${ }^{1}$ Crocker, "Diseases of the Skin," 3rd edition, p. 77.

2 "On Napkin-Region Eruptions in Infants," Brit. Journ. Derm., 1909, xxi. 37. 
in (1) simply erythematous, (2) erythemato-vesicular, (3) erythemato-papular, and (4) ulcerating forms, which may develop consecutively or simultaneously. The commonest forms are the erythematous and the papular. In the simple erythemas the rash may be limited in mild cases to the genitalia, the inner sides of the thighs, and the perineum; in severer cases it may extend to the lumbar region, the lower abdomen, and the calves and heels. In the erythemato-vesicular form, on the convex surfaces towards the centre of the erythematous areas, small bright-red lesions appear, which may run into each other. The typical lesion is a vesicle, which may usually be found near the border of the reddened area. The erythemato-papular form is met with when the erosions just described have thrown up flattened granulations, which make the lesions look like flat, reddish papules. In this stage the heels and the lower abdomen may be involved. In the ulcerating form the erosions, instead of granulating, develop into ulcers with sharply defined borders, or running together into vermicular lesions. They are entirely confined to the convex surfaces, the folds always escaping. All four forms of the eruption are no doubt partly due to the irritation set up by moist or soiled napkins, but vaso-motor irregularities and gastro-intestinal toxæmia may also be exciting causes. Adamson has frequently observed that this erythema is associated with, or is followed by, urticaria papulosa, and he suggests that the two conditions may be etiologically related. The affection is often met with in children's clinics, and is of importance because, like erythema intertrigo, it is apt to be mistaken for congenital syphilis. Confusion with that disease may be avoided by noting the absence of other specific signs and symptoms and by attention to the appearance and distribution of the lesions, as here described, and the course they run. In seborrhœic dermatitis there are, as 
a rule, greasy yellow scales; the lesions are moist, and they will be present also in the axillæ and other flexures. In intertrigo they begin in the folds of the thighs, groins, etc., and the convex surfaces are only affected later.

Erythema paratrimma is a term sometimes used to denote the effect of long-continued pressure on a particular part of the skin, as from long continuance in a recumbent position. The mechanical effects of pressure are aggravated by the irritation of urine and fæces when the patient is not properly nursed, and by conditions which lower the vital power, particularly by lesions of the spinal oord, which interfere with the nutrition of the part. Erythema paratrimma, if not carefully attended to, is certain to end in bed-sore. This lesion may appear on the knuckles, induced by friction with the bed-clothes as the patient raises himself to the sitting posture.

Erythema scarlatiniforme is a febrile affection characterized by an eruption closely resembling that of scarlet fever, but not contagious. The onset is marked by shivering and systemic disturbance, which is accompanied or quickly followed by the appearance, on the trunk or elsewhere, of efflorescences, vivid red in colour and variable in size. These often run together so as to cover extensive areas of skin, and the whole surface of the body may be involved. The tongue is foul and has a more or less distinct "strawberry" appearance, and there is usually some reddening of the fauces, with soreness of the throat. In extreme cases the nails may be shed and the hair fall out. The fever speedily subsides, and before the eruption has begun to fade desquamation begins. The average duration of the affection is from two to six weeks, but in some cases it lasts much longer. Two distinct types of erythema scarlatiniforme can be recognized clinically - one running a more or less definite course and dis- 
appearing after a few weeks; the other severer and more prolonged. Relapse is not uncommon, a fresh crop of lesions coming out before the first has disappeared. Erythema scarlatiniforme shows a marked tendency to recur, sometimes every year, and sometimes at shorter intervals. Those subject to it can generally tell beforehand when an attack is impending. Various complications-pulmonary, cardiac, renal, etc. -have been described in association with erythema scarlatiniforme, ${ }^{1}$ but it appears more probable that such conditions, or the drugs employed to combat them, may have been the exciting cause of the skin affection.

The etiology of the disease is by no means clear. A certain idiosyncrasy on the part of the patient is required, and among the exciting causes one of the most potent appears to be exposure to a very high temperature. Crocker has seen it in connection with sewergas poisoning, and it has been found in association also with various toxæmias, general or intestinal, with digestive derangements, septic infection, prolapsed and enlarged ovary, and obscure changes of tissue or secretion about wounds. In a large number of the cases reported by French dermatologists-to whom we are chiefly indebted for the recognition of the disease-the use, internally or externally, of certain drugs, notably mercury, would seem to have played an important part in its causation (see Chapter x.); but the fact that erythema scarlatiniforme may occur when the possible influence of drugs or toxic agents of any kind can be absolutely excluded justifies us in placing it provisionally among the ery themas proper. Rheumatism, malaria, syphilis, gonorrhœa, albuminuria, and alcoholism have been indicated as possible causes of the affection, but in

1 Besnier and Doyon's French translation of Kaposi, 2nd edition, vol. i., p. 343. 
all these cases it is obvious that the real source of the mischief may be mercury, quinine, salicylate of soda, or some other drug.

Diagnosis.-.-Erythema scarlatiniforme derives its chief importance from its resemblance to scarlet fever. This is so close that the most experienced observer may be unable to give a definite opinion as to the nature of the rash during the first few days. The most striking point of distinction is the early commencement of desquamation in erythema scarlatiniforme, and the fact that it begins when the eruption is still in the florid stage-as early as the second day, if the patchcs are carefully examined with the lens, and at latest on the third or fourth day. Again, in scarlet fever the eruption does not last longer than ten days, whereas in erythema scarlatiniforme it may persist several weeks, and sometimes indefinitely. In the case of a person who has had previous attacks the history will often be helpful; but in all cases it will be safer to isolate the patient till the diagnosis is clear. It is probable that, in some at least of the cases in which recurrence of scarlet fever has been reported, the disease in one or other of the attacks has really been erythema scarlatiniforme. From pityriasis rubra, to which the affection under consideration bears considerable resemblance, it may be distinguished by the less general diffusion of the scaliness and by the repetition of the desquamative process.

Erythema infectiosum is an acute infectious disease which attacks children between the ages of 4 and 12 in the spring and early summer. After an incubation period of six to fourteen days, bright-red confluent patches, sometimes preceded by sore throat and lassitude, appear on the cheeks ; occasionally the extensor surfaces of the limbs may be involved. The redness disappears on pressure. There is some swelling, but no 
pyrexia. The affection may become epidemic, and hence is alternatively known as megalerythema epidemicum. ${ }^{1}$

Rubeoloid erythema-that is, an "ephemeral" eruption of measles-like character-has been described by Besnier; but he himself admits that when " abortive measles without catarrh, rubeola, and the unlimited series of modified roseolæ are eliminated, there remain very few true rubeoliform erythemas." I only mention it here, on the authority of that distinguished dermatologist, as affording a possible clue to errors of diagnosis which occasionally occur.

Erythema uræmicum, a rare condition described by Huet in 1870, and subsequently by Bruzelius, Lancaster, Lindley Scott, and others, appears as the immediate precursor of uræmic symptoms in interstitial and more larely in parenchymatous nephritis. It is first seen on the extensor surfaces of the hands and feet as discrete macules of a bright-red colour, which become papular or nodular, and in a few days become merged in a general dusky erythema affecting the whole body and face. Desquamation ensues in about a fortnight in the form of small branny flakes, or thin strips. Occasionally vesicles or blebs form. Death usually occurs within five or six weeks of the appearance of the rash. ${ }^{2}$

Erythema solare, or sunburn, appears to be an effect of the light rather than of the heat of the sun; the violet rays are thought by some to be the actual agents in its production. The electric light has been found to cause an erythema indistinguishable from sunburn (Charcot). The effect of other forms of energy

${ }^{1}$ See Ruhrah's article in Osler and McCrae's "System of Medicine."

2 For an account of this disease, see "Cutaneous Affections in Various Diseases, with special reference to certain AngioNeuroses," by S. E. Dore, Brit. Journ. Derm., Sept., 1906. 
related to light, shown in the various forms of erythema, and even more severe lesions, caused by the Röntgen rays, are now well known. A number of cases have been reported in which the $\mathrm{X}$-rays have produced a severe and circumscribed form of dermatitis. Actual burns of all degrees of severity, even to necrosis of the deeper tissues of the $\lim b$, have also been recorded.1 (See p. 229.)

Erythema pernio, or chilblain, is characterized by the development of small patches, dusky red or bluish in colour, and slightly raised. These generally form on the hands (edge and dorsum of fingers) and feet (heel and outer edge, especially on the little toe); but they may occur at any part distant from the heart where the local circulation is much exposed to the influence of cold air (nose, ears, cheeks). Subjectively, the symptoms are great tenderness of the affected parts, and itching, which becomes almost unbearable when they get warm. The subsidence of the inflammation is frequently followed by desquamation. If neglected, the skin often breaks, and ulcers of greater or less extent may form, particularly in under-fed or tuberculous children. Chilblain is more common in childhood and old age than in adult life. It has been suggested that the disease is of tuberculous origin $;^{2}$ but there is no cogent evidence of such a connection. Scrofulous children are undoubtedly more liable than others to chilblains ; but that is on account of the feeble circulation which is so pronounced a feature of the tuberculous diathesis. The disease is a result of local disorder of the circulation. The arterioles are at first contracted under the influence of cold; but this condition soon gives way

${ }^{1}$ See "Light and X-Ray Treatment of Skin Diseases," pp. 24-6.

${ }^{2}$ Cazin and Iscovesco, Congrès Intern. de Dermatol. et de Syphilis tenu à Paris en 1889; Comptes-Rendus, p. 511. 
to dilatation from vaso-motor paralysis, and the other phenomena of the inflammatory process follow in due course.

Frostbite.-In connection with chilblain, frostbite, which is pathologically a more advanced stage of the same process, may conveniently be considered. The first effect of the cold is to blanch the part (fingers, nose, or other extremity) by constriction of the vessels; dilatation follows, and the part becomes congested and swollen, assuming a peculiar violet colour. Some itching and pricking are usually complained of. In the milder cases the skin soon recovers its natural appearance; but sometimes the capillaries remain dilated, causing permanent erythema. In serious cases vesicles form. This is a sign of ominous import. The severer degrees of frostbite, in which gangrene of a part occurs, belong rather to the domain of general surgery than to that of dermatology.

Erythema keratodes.-Under this name Brooke ${ }^{1}$ has described a rare form of sharply circumscribed chronic erythema of the palms and soles, leading to overgrowth of the horny tissue, and accompanied by œdema and tenderness, which interfere considerably with movement. Besides the lesions on the palms and soles, more or less horny erythematous nodules are seen on the backs of the finger-joints. The affection begins with the development on the palms and soles of red patches, which speedily coalesce. The thickening of the epithelium quickly follows the first signs of inflammation. The surface of the skin is smooth and the furrows are well preserved. The progress of the affection is gradual; it responds readily to treatment, but tends to relapse. In this respect it differs from the affection described by Besnier ${ }^{2}$ under the name of

${ }^{1}$ Brit. Journ. Derm., 1891, p. 335.

2 "Intern. Atlas of Rare Skin Diseases," Pl. v., Fig. 1. 
keratodermia erythematosa symmetrica, which is continuous. Brooke thinks that the symmetry of the lesions, in conjunction with the synchronous implication of both hands and feet, indicates a central tropho-neurosis as the cause. Dubreuilh, however, who has recorded a similar case, points out that the symmetry of the lesions is no proof of such an origin, and that the rapid and complete cure of the affection by treatment, mainly local, does not accord with the hypothesis of a central neurotic origin.

Erythema multiforme is an inflammatory affection of the skin characterized by a polymorphous eruption, in which papular, vesicular, bullous, nodular, œdematous, and hæmorrhagic elements are mingled together, or succeed each other, so as to form a clinical picture that is kaleidoscopic in its infinite variety. As Besnier and Doyon truly say, "You may pass twenty years of your medical life in observing and collecting cases of erythema, and each year will bring you forms which you have never before seen. Not only does this variety defy all complete description, but categories ad infinitum would be needed if one wished to classify all the facts in methodical series. The authors who have attempted to do so have invariably failed, and have only succeeded in producing undigested and useless compilations." 1 All that can be done here is to indicate the salient points-the types of the different forms assumed by the process in the various stages of its evolution. To these typical forms distinctive names have been given, as erythema papulatum, erythema annulare, and so forth. These names serve a useful purpose as indicating the predominant character of the lesions in a particular case or at a given time. It must be clearly

1 Kaposi, "Maladies de la Pean," Besnier and Doyon's translation, 2nd edition, tome i., p. 364 (Paris, 1891). Translators' footnote. 
understood, however, that they denote, not different diseases, but phases of the same process.

The eruption, which is generally more or less sym. metrical in distribution, first shows itself, as a rule, on the back of the hand and the dorsum of the foot; it may, however, appear on any part of the body. In the progress of the disease the forearm and arm, the leg and the thigh, and the trunk and face may be invaded. In exceptional cases the mucous membrane of the mouth and pharynx and the conjunctiva may suffer. At the height of the attack the tongue is sometimes greatly swollen. The backs of the hands seldom escape ; otherwise the disease follows no rule, either as to the extent of surface over which it is distributed or as to the points which it selects for attack.

Erythema multiforme is often ushered in by rise of temperature, congestion of the pharynx, gastro-intestinal disturbance, and other signs of systemic disorder. Pain in or about one or more of the joints is perhaps the most constant of these premonitory symptoms. Any or all of them, however, may be absent, and the temperature may be below, instead of above, the normal standard. The eruption, as already said, is markedly polymorphous not only in the form but also in the nature of the lesions. As Jamieson points out, ${ }^{1}$ however, in their evolution a gradual rise from simple to more complex forms can usually be traced. Thus the commencement of the process is marked by the appearance of a crop of papules no larger than a pin's head, of a bright-red colour, which fades on pressure, and is hard and distinctly hot to the touch. (erythema papulatum). These papules quickly spread centrifugally so as to form small tubercles (erythema tuberculatum); or, if closely grouped together, they may coalesce and form raised patches of the size of a threepenny or sixpenny piece. 1 “Diseases of the Skin," p. 87. Edinburgh, 1888. 
Each patch presents a sharply defined border, and has around it an areola of congested skin; the centre is of a less vivid red than the edge, and its tint soon deepens to violet, then to purple. Bullæ occasionally develop on the patches, and after a time shrink and form scabs. The eruption may disappear in a few days, leaving behind it only a slight brownish discoloration. More commonly the centre of the patch undergoes absorption, while the edge continues to advance. In this way rings of varying circumference are formed, the centre of which is depressed and pale or bluish-red in colour, while the edge is raised and of a florid scarlet hue (erythema annulare). The rings, as they enlarge, come in contact with others. This leads to the disappearance of the eruption at the points where the edges meet, so that only segments of the pre-existing circles remain, either isolated or variously joined in the form of curves or wavy lines (erythema gyratum). Some of these may continue to spread as narrow raised bands with a sharply defined edge (erythema marginatum). As fresh crops of papules continue to come out from day to day, several or all of the phases that have been described may be present in one case at the same time. The multiformity of the lesions may be still further increased by the formation of vesicles and bullæ on the patches and on the centre and borders of the rings, by scabs, and by escape of the colouring matter of the blood or actual hæmorrhage beneath the epidermis. The average duration of the individual lesion in erythema multiforme is little more than a week, but the process as a whole usually lasts a month or six weeks; and as recurrence is very common and often takes place at short intervals, the duration of the disease may sometimes appear to be indefinitely prolonged.

Erythema iris. - This form of erythema multiforme requires separate mention, because its appearance is so 
characteristic as almost to entitle it to be classed as a distinct disease, and because, as a matter of fact, it often occurs independently of any of the other lesions that have been described. Erythema iris is met with under two typical forms. One of these begins as a small red spot. On this, in a few hours, a vesicle forms, and around the vesicle a zone of redness quickly develops. The central vesicle soon dries up, leaving a small scab, and a ring of secondary vesicles forms on the red zone encircling it. When the central scab separates, the skin underneath presents a blue, congested appearance, which takes some time to disappear. The process here described may be repeated several times, the concentric rings of vesicles and reddened skin producing an appearance not unlike a target. There may be only a single lesion of the kind, or there may be several scattered about the wrists, arms, and legs. On the fingers, owing probably to the anatomical peculiarities of the part, the target-like appearance is not so well marked. The other form is characterized by the development of a large central bulla surrounded by a ring of vesicles of considerable size; hence the misleading name of herpes iris is often applied to it. Another ring of vesicles may develop outside the first, and outside the second there is sometimes a third. The intervening circles of skin are of a purplish hue. In both these forms the process is essentially that of erythema multiforme, the vesication being only an accidental complication.

Although, as has been stated, erythema iris may occur independently of any other form of eruption, it is often associated with the more ordinary lesions of erythema multiforme. In my own experience, it is more apt to occur alone in cold weather, in policemen and other persons whose occupation involves a good deal of exposure. The condition runs a definite course, 
lasting from two to three weeks, and leaving behind it only a brown stain.

The subjective symptoms in erythema multiforme are not, as a rule, of any importance. The fever does not always subside with the appearance of the eruption, and in that case some degree of malaise may be complained of; but this seldom persists for more than a very few days. . There is not generally any itching or burning, and when such sensations are present they are never very severe. In children, pyrexia and the other constitutional symptoms are more marked than in adults, and the vesicles are apt to become transformed into pustules, followed by scarring.

Clinically there are two types of erythema multiforme-viz. the ordinary form, which runs a benign course and ends, after a longer or shorter series of relapses, in complete recovery; and a severe form, characterized by visceral manifestations of various kinds, gastro-intestinal crises, acute nephritis, hæmorrhage from the mucous surfaces, pericarditis, and endocarditis. This form usually ends in death.

Pathology.- The pathology of "idiopathic" erythema multiforme is summed up in the statement that the process is a toxic angio-neurosis. It differs from hyperæmic erythema only in the fact that exudation is a far more pronounced feature than it is in that form of erythema. In the severer type the skin lesions are secondary to septic and suppurative processes in the viscera. Thus they have been observed in cases of cystitis from stricture, of rectal chancre (Finger), of diphtheria, of cholera, etc. Whitfield ${ }^{1}$ records an anomalous case associated with cardiac and renal disease, in which the lips and mouth were enveloped in a sheet of vesicles precisely resembling those of confluent smallpox; while others were scattered about on the arms ${ }^{1}$ Brit. Journ. Derm., 1903, xix. 273. 
and legs. The lesions, formidable as they were, cleared up, leaving only slight pitting and in some instances no perceptible scar. Galloway and MacLeod ${ }^{1}$ describe a ease in which the lesions, on the face and the hands, formed a connecting link between a circinate erythema multiforme and acute lupus erythematosus. They conclude that certain cases of lupus erythematosus and certain types of erythema multiforme are so closely related as to form the ends of a chain in which all transitional stages may be met with, and that both are due to toxins of which the exact nature is still uncertain.

Etiology.-Though still obscure, the causation of erythema multiforme has gradually had more light shed on it. Probably many causes produce similar results in this disease, and many hold that toxic material circulating in the blood is the chief cause of the symptoms of erythema multiforme, while rheumatism is little concerned in its causation. ${ }^{2}$ It may also be due to drugs, such as iodide of potassium and copaiba, and to antitoxic serum. It occurs more frequently in spring and autumn than in the other seasons, and is commoner in females than in males.

Diagnosis.-This seldom presents any difficulty. The appearance of erythema iris is so characteristic as to make it impossible to mistake it for anything else, and the multiformity of the lesions in other cases is sufficient to differentiate the disease from other conditions. Occasionally urticaria of the papular variety bears some resemblance to erythema papulatum, but the

1 Brit. Journ. Derm., March, 1908, xxiv. 65.

${ }^{2}$ Cf. Veiel, Trans. Internat. Congress of Dermat., 1896; Mackenzie, ibid., 1896 ; Osler, "On the Visceral Complications of Erythema Exudativum Multiforme" (Amer. Journ. of the Medical Sciences, Dec., 1895); Finger, “Beitrag zur Aetiologie und pathologischen Anatomie des Erythema Multiforme" (II. Internationaler Dermatologischer Congress abgehalten in Wien im Jahre 1892). Wien, 1893, p. 754. 
latter can usually be identified by the absence of itching, by the longer persistence of the lesions, and by the fact that they leave stains. The subjects of urticaria, further, are usually children, and it is chiefly the covered parts of the body, and particularly the lower lumbar region, that are attacked. In the papular stage of eczema, as in urticaria, the itching is a very marked feature. When the rash of erythema multiforme is widely diffused, and even invades the face, it may suggest smallpox. The diffusion, however, is seldom so general as that of the variolous rash, nor are the parts attacked in the same order. For the diagnosis between erythema multiforme and herpes zoster, see p. 178.

Prognosis. - In the vast majority of cases the outlook is good as regards the particular attack; but recurrence is almost certain, and it is quite impossible to predict that the patient will remain free from the disease. If serious complications occur, the forecast must be based on them, not on the skin affection.

Erythema perstans.- Under this designation are grouped cases of erythema analogous to those for which Radcliffe Crocker and Campbell Williams have proposed the name erythema elevatum diutinum (p. 197). In one of two cases described by Wende ${ }^{1}$ the affection had persisted for four and a half years, and never during that period was the patient completely free from lesions. First appearing simultaneously on the arms and legs, they quickly spread over the abdomen, gradually developing from a red spot to a ring, a process attended with intermittent itching. The lesions, violet-red in colour, varied in size from that of a pea to three inches in diameter, and the larger ones were well defined. The smaller patches were elevated; the larger ones, made up of rings or segments of rings, formed gyrate or serpiginous figures. During one summer the body was ${ }_{1}$ Trans. Amer. Derm. Assoc., 1905, p. 141. 
almost free from lesions, but with the onset of cold weather the eruption grew worse. In Wende's other case also there was a fresh exacerbation when the patient was subjected to marked changes of temperature. Wende classes these two cases with others of longcontinued erythema that have been reported, some of them exhibiting simple chronic inflammatory patches, others diffused patches, papules, or nodules. Evolution of the lesions frequently begins in the centre and leaves annular or gyrate figures.

Etiology.-The causation of erythema perstans remains obscure, but the cases reported suggest as possible causes intestinal toxæmia, the gouty or rheumatic diathesis, and atmospheric changes. No age is exempt, nor is either sex, but men seem to be more liable to the affection than women.

Erythema nodosum is characterized by the formation of node-like swellings on the legs and feet, less frequently on the forearms, thighs, buttocks, and over the scapulæ, and in rare cases on the face. The distribution of the swellings is generally symmetrical: they come out in crops of two or three at a time, the first point of attack generally being the leg, along the tibia. Their appearance is preceded and accompanied by a greater or less degree of constitutional disturbance, one constant symptom being pain of a rheumatic character about the joints, especially of the lower limbs. The swellings are oval in shape, and lie with the long axis corresponding to that of the limb. They have no well-defined border, and vary in size from a walnut to a hen's egg. At first bright red in colour, they soon become bluish in the centre and purple at the circumference, and as they subside they exhibit the various changes of tint that are seen in a bruise. They are not, as a rule, painful, but are very tender on pressure. Firm and tense in the beginning, they soon soften and 
give a sensation somewhat resembling fluctuation to the finger, but they never suppurate. The individual swellings last about a fortnight; but as fresh ones come out in successive crops for two or three weeks, the duration of the affection averages from three to six weeks.

Etiology.-Erythema nodosum is very rare after the age of 20, and girls show a greater proclivity to it as compared with boys in the ratio of about two to one. It is more common in the spring and the autumn than at other seasons of the year. Exposure to cold, and especially, according to Crocker, to brine-laden winds, may be an exciting cause. Stephen Mackenzie ${ }^{\mathbf{1}}$ showed from an analysis of 108 cases that erythema nodosum is frequently associated with rheumatism. Even when there are no actual rheumatic lesions the patients often present the signs of the rheumatic diathesis. The affection sometimes occurs in tuberculous subjects, or is followed by tuberculosis. The condition is sometimes complicated by endocarditis or some other acute cardiac mischief.

Pathology.-The pathological process is that of hyperæmic erythema. Local vaso-motor disturbance is followed by inflammatory effusion of fluid and escape of white blood-corpuscles.

Diagnosis. - There is seldom any room for doubt as to the nature of the affection. The appearance of the lesions and their association with pains in the joints are characteristic. I have, however, known instances in which erythema nodosum on the face has been mistaken for tubercular leprosy. For the diagnosis from the early stages of nodular leprosy, see p. 532. From the nodules of syphilis the lesions of erythema nodosum are distinguished by the joint-pains and swellings and short duration, and by the absence of ulceration. These ${ }^{1}$ Clin. Soc. Trans., xix. 215. 
features also serve to differentiate erythema nodosum from erythema induratum scrofulosorum (Bazin's disease), in which, further, the nodules are violet instead of bright red, and undergo successive changes of tint. Mention should also be made of another variety of nodelike swelling which is of not infrequent occurrence in the legs of young women suffering from varicose veins ; these swellings are nodules due to capillary phlebitis. In neither of these affections, however, are there any concomitant rheumatic symptoms.

Prognosis.-In uncomplicated cases of erythema nodosum the prognosis is always favourable, the disease. tending to subside spontaneously after running its course. If any serious cardiac complication be present, the prognosis must be based on that, and not on the affection of the skin.

\section{Treatment of the erythemas.-For erythema} simplex no treatment is required beyond the removal of any obvious source of irritation. Itching may be relieved by the treatment described under the head of Pruritus (p. 67). In intertrigo the opposing surfaces should be separated by small pads of lint or cotton-wool placed above and below the diseased area, or by the interposition of a muslin bag filled with powder. Inasmuch as in the situations where intertrigo is apt to occur decomposition of the secretions is likely to take place, with the result of greatly intensifying the irritation, the parts should frequently be washed with a solution of boric acid (gr. $\mathrm{x}-\mathrm{xv}$ in distilled water $\jmath^{\mathrm{i}}$ ), then carefully dried, and finally thickly dusted over with some protective powder. In the case of infants the strictest cleanliness must be enjoined: napkins must be changed as soon as they are wet; other conditions keeping up irritation-such as diarrhœa or worms-must be treated by appropriate remedies. 
Similar methods should be followed in the infantile erythema of Jacquet.

In commencing erythema paratrimma (bed-sore) the pressure must, as far as possible, be neutralized by the use of air-cushions or circular pads, or by keeping the patient on a water-bed. The greatest attention must be paid to local cleanliness, and the nutrition of the affected area should be kept up by frequent washing with stimulating applications-such as a mixture of brandy or rectified spirit and white of egg, or camphorated spirit of wine. If, in spite of this treatment, a bedsore forms, it must be dealt with on general surgical principles.

In scarlatiniform erythema the cause must first, if possible, be removed; in other respects treatment must be symptomatic. It is most important, for obvious reasons, to avoid the use of drugs that have the property of causing rashes (see under Artificial Eruptions, p. 231); Besnier has even recorded fatal results from this cause. Locally cooling and soothing applications. (simple or boric-acid ointment, calamine liniment, dusting powders, etc.) are grateful to the patient and may do a certain amount of good. Payne ${ }^{1}$ found quinine in large doses (gr. $\mathrm{xx}-\mathrm{xxx}$ a day) and sodium salicylate very efficacious.

In the treatment of erythema pernio (chilblain) the principal indication is to stimulate the circulation in the affected region. For this purpose the parts should be kept warm ; and; unless the feet are disabled, brisk walking exercise should be taken. One of the best local remedies is iodine, applied in the form of the tincture. Friar's balsam and camphorated spirit are excellent remedies; so, too, is ichthyol. One point of great importance is to dry the part as thoroughly as possible after washing. If vigorous friction with a towel or piece ${ }^{1}$ Brit. Journ. Derm., May, 1894. 
of lint can be borne, it will be useful. Ulceration, should it occur, must be treated on general surgical principles. If the patient is anæmic, ferruginous tonics should be given; and if the heart's action is weak, it may with advantage be strengthened by the administration of digitalis. As regards prevention, it is important to keep the circulation active by warmth (woollen gloves for the hands, thick worsted stockings for the feet), and especially by vigorous exercise. The skin may also be hardened by the use of toilet vinegar in the water used for washing. Calcium chloride gr. $\mathrm{x}-\mathrm{xv}$, or the lactate gr. $x v$, may be given three times a day for two days, and repeated for the same time a few days later. Adrenalin may also be recommended.

In the milder cases of frostbite care should be taken not to warm the parts too quickly. Rubbing with snow is recommended, and this must be continued till the circulation begins to be restored. Ichthyol, owing to its influence on hyperæmia and circulatory anomalies generally, is of great service; it may be taken internally and used locally, a 10 per cent. ointment being rubbed into the affected part. Massage and galvanism are valuable adjuncts to the treatment.

Erythema keratodes, according to Brooke, yields readily to the internal administration of ichthyol (miii in capsules thrice daily), and the constant application of an ointment containing ichthyol and salicylic acid. Dubreuilh cured his case with iodide of potassium internally (given on the hypothesis that the affection was syphilitic), and the application of diachylon ointment to which 20 per cent. of salicylic acid had been added.

Erythema multiforme runs a definite course, and is seldom much influenced by treatment. The important thing, as in the treatment of urticaria (p. 85), is to give antiseptics, such as salol, in cases in which an intestinal toxæmia is suspected. In such cases a colon bacillus 
vaccine may be administered. In a case of severe relapsing bullous erythema multiforme T. C. Gilchrist reports marked improvement and final cure from the use of staphylococcus albus vaccine. ${ }^{1}$ The diet should be of the plainest and least stimulating character, and alcohol must be forbidden. Arsenic may be of service when the inflammatory symptoms are not intense; if they are, antimony should be given in the form of vinum antimoniale ( $\mathrm{miii}-\mathrm{v}$ in $\overline{3}$ of water). The internal administration of adrenalin has been found useful. The calamine lotion already mentioned is the best local application to relieve the pain and burning. In severe cases sedatives may be employed; they should be given at bed-time.

Like erythema multiforme, erythema perstans is obdurate to treatment. In one of the cases described by Wende (see p. 110), the only remedy which appeared to influence the eruption was chrysarobin ointment.

The chief indications in the treatment of erythema nodosum are rest and the neutralization of the effects of the rheumatic poison if there be evidence of its presence. Salicylate of soda in doses of gr. $\mathrm{x}-\mathrm{xv}$, according to age, should be given three times a day for this purpose. When the swelling and other local symptoms have subsided, an iron tonic is generally indicated. Rest in bed, with elevation of the affected limbs, in addition to the application of soothing or cooling lotions, is necessary. The swellings should never be opened, however distinctly they may fluctuate.

\section{Purpura}

Whether this is ever a substantive disease, or is only the result of pathological processes that may occur in a number of different morbid conditions, is a question that cannot be regarded as finally settled. The

${ }^{1}$ Trans. Amer. Derm. Assoc., 1909, p. 19. 
older view that purpura may be an idiopathic affection finds support in the discovery in the blood of purpuric patients, by Letzerich and Kolb, of a bacillus, cultures of which injected into animals caused characteristic hæmorrhages. In Letzerich's blood was found the same bacillus, and his illness, it is suggested, was the result of infection from his purpuric patients. ${ }^{1}$ The extravasation of blood into the cutis may take place either as a mechanical effect of over-dilatation or as the result of changes in the blood or in the vessels, or of impaired nerve control. The hæmorrhage, whatever the agency to which it is to be ascribed, gives rise to different appearances in the skin; hence various names have been given to purpuric lesions, according to their shape. Thus the extravasation may cause spots or puncta, lines or vibices, small patches or petechioe, or diffuse patches, bruises or ecchymoses. In all these forms the note of the lesion is that it cannot be obliterated by pressure with the finger, showing that the discoloration is due to effused blood, not to congestion. The lesion, so far as it concerns the dermatologist, is referred to in dealing with the various affections in which it occurs, but it may also be a symptom of certain toxic conditions, such as the exanthematous fevers, some drug eruptions, and scurvy. Török ${ }^{2}$ regards all true purpuras as due to infective, or toxic, or autotoxic agents, acting directly on the vascular walls and reaching their point of action by way of the blood-stream. Purpura sometimes occurs in connection with various visceral hæmorrhages-in the brain, lung, retina, and gastrointestinal canal, in tuberculosis, especially in the last stages of phthisis, and in sarcoma and lymphadenoma. Shattuck reports a case in which it was associated with lymphatic leukæmia.

${ }^{1}$ Wien. klin. Rundschau, May 14, 1905.

z Journ. des Mal. Cut. et Syph., April, 1903. 
Purpura simplex.-The lesions of purpura simplex are small bright- or dark-red petechiæ, which usually appear suddenly, as a rule on the lower extremities. The buccal membrane is occasionally involved. Sometimes the lesions assume the form of wheals, when the affection is styled purpura urticans; this condition, however, is usually urticarial in character rather than purpuric. The petechiæ come out in successive crops, and generally the distribution is symmetrical.

Treatment.-Except in the mildest cases, rest in bed should be prescribed, with a generous diet. Recovery will usually take place within ten days or a fortnight without the aid of drugs. If these should be required, iron (or arsenic) and chlorate of potassium may be ordered. The internal administration of adrenalin has been found serviceable.

Purpura hæmorrhagica.-This affection, known also as Werlhof's purpura, land scurvy, and morbus maculosus, is one of great gravity, which often has a fatal termination. Extensive ecchymotic areas are seen on the surface of the integument. There is bleeding from the gums and the mucous membranes of the mouth and throat, and there may also be hæmorrhage from the serous surfaces. The disease belongs to the province of the general physician rather than to that of the dermatologist.

\section{Purpura or peliosis rheumatica. - Schönlein's} disease, as this condition is also styled, is an acute affection, the symptoms of which are pains in the joints, with purpuric spots appearing in patches, especially in the neighbourhood of the joints in which the pain is most severe. It bears a general resemblance to some forms of erythema multiforme, but the articular pain is generally more pronounced, and the subepidermic hæmorrhages, instead of being occasional, are constant, and form the only lesion of the skin. The onset of the 
affection is sometimes marked by constitutional disturbance; swelling of the joints with pain comes on, and a day or two later the eruption appears, usually during the night. The spots always come out on the knees and ankles, and often on the elbows and wrists, but the trunk is seldom attacked. The pain in the joints frequently abates or ceases on the appearance of the eruption. The lesions consist of slightly raised papules or patches, bright red at first, but not fading on pressure. They soon change colour, becoming purplish and then black; they are, in fact, obviously hæmorrhages, and exhibit the usual discoloration of the skin caused by extra. vasated blood. The affection in the acute stage lasts only a few days, but recurrence may take place in two or three weeks; and this may be repeated, so that altogether the affection may last several weeks or even months.

Pathology and etiology.-The pathology of the disease is obscure, but the general trend of opinion is that rheumatism plays no part in its causation, and that the pains in the joints are caused by the effused blood. Stephen Mackenzie; however, held the belief that it is of rheumatic nature. ${ }^{1}$ The pathological process is carried a step beyond exudation of serum or effusion of hæmoglobin as in erythema multiforme, and actual hæmorrhage takes place. Why hæmorrhage should be a constant phenomenon is not clear, though it may be conjectured that it is due to some alteration in the constituents of the blood dependent on one of those infective, toxic, or autotoxic agents to which the purpuras are attributed by Török (see p. 117). Women are more often affected than men. The disease is most common between the ages of 20 and 30 , but is sometimes seen in children, in whom it is known as Henoch's purpura.

1 "On the Relationship of Purpura Rheumatica to Erythema Exudativum Multiforme," Brit. Journ. Derm., 1896, viii. 116. 
Diagnosis. - Peliosis rheumatica can hardly be mistaken for any other disease, the combination of pain in the joints with a purpuric eruption around them being almost absolutely distinctive. This form of purpura and purpura hæmorrhagica (p. 118) are distinguishable from smallpox in that the petechiæ are not preceded by papules, vesicles, or pustules, and from the purpuric variety of smallpox by the absence of severe backache and high fever. From typhus fever these graver forms of purpura differ by the presence of hæmorrhage from the mucous surface, and the absence of mottling. The disease which they most resemble is scurvy, in which, besides the ecchymoses and petechiæ, there may be bleeding from the mucosæ. Confusion between the two affections should be obviated by the pronounced anæmia with which scurvy begins, the sponginess of the jaws which is an almost invariable feature of that condition, and the history of deprivation of fresh food.

Prognosis.-In uncomplicated cases recovery is certain, but recurrence is almost as certain. When grave complications are present, they must of course be taken into account in forecasting the issue of the disease.

Treatment. - This may be summed up in the following recommendations : Rest in the horizontal position until the lesions have disappeared; the administration of quinine, iron, and other tonics; and a liberal diet.

\section{Lupus ERythematosus}

Lupus erythematosus - ulerythema centrifugum (Unna), or, as I should prefer to call it, erythema atrophicans-is an inflammatory process giving rise to cellular infiltration, ending in atrophy of the affected part of the skin.

Symptoms.-The disease begins with the appearance of "primary eruptive spots" (Kaposi), character- 
ized by a red, elevated hyperæmic and thickened border, with a central scar-like depression, which is either smooth, or covered with a dry, firmly adherent scab or thin papery greyish scales (Jamieson). These small red spots fade on pressure. The distribution of the lesions is frequently symmetrical. Saalfeld ${ }^{1}$ holds that in the majority of cases the starting-point of the disease is a more or less marked seborrhœa. When the disease attacks a part provided with sebaceous glands, the skin is usually covered with small adherent scales of sebum, which at the margin of the patch plug the dilated orifices of the glands, thus forming numerous comedones. In parts where the adherent scales become detached, these plugs are seen hanging from their under surface as thread-like tags. In some cases this sebaceous covering is absent, and then the erythematous character of the lesion is more evident. According to Unna, the plugs are the result of acanthosis (p. 4), and are not sebaceous or necessarily connected with the sebaceous glands; other authorities regard them as processes of cornification. The affected area is often surrounded by a zone of dilated blood-vessels. In its evolution the process conforms to one of two principal typesspreading either by the peripheral enlargement of single spots (lupus erythematosus discoides), or by the successive appearance of fresh crops of spots, which coalesce and form patches of considerable size (lupus erythematosus aggregatus or disseminatus). The former may also be distinguished as the "slow-spreading," the latter as the "eruptive," form of the disease.

Crocker 2 describes a "telangiectic" form, "in which there is no marked change of the surface except persistent circumscribed redness, which close inspection

1 Derm. Zeitsch., Bd. viii., Heft 3 (abstr. in Brit. Journ. Derm., 1901, p. 436).

2 "Diseases of the Skin," 3rd edition, p. 761. 
shows to be due to dilated vessels." This is commonly situated symmetrically on both cheeks, the affected area being very much of the size and shape of the red patsh which the clown paints on his face ; it is not very noticeable to the eye, though on pinching up the tissues marked thickening can be felt. Crocker also describes a nodular form of the disease, in which the lesions are exactly like those of erythema tuberculatum, and vary in size from a hemp-seed to half an inch in diameter.

The face is the part most commonly attacked by lupus erythematosus, especially by the discoid variety of the disease (Plate 2). The lesions usually appear symmetrically on both cheeks, where they form wide blotches, which spread inwards and meet in a narrow strip over the bridge of the nose, thus giving rise to the "butterfly" or "bat's-wing" appearance characteristic of the disease. On the other hand, in some cases the process has its starting-point on the nose, and extends thence outwards across the cheeks. It occasionally begins on the helix of the ear, the tip of the nose, the scalp, the hairy part of the face, or the margin of the lips; in rare cases it commences on the nape of the neck. Next in order of frequency to the head and neck as points of attack come the hands (Plate 2) and the feet; neither the flexor nor the extensor surfaces are spared. In some rare instances the trunk is invaded in several places. In one of my cases the disease was limited almost exclusively to the scalp, which was almost entirely denuded of hair; the only other lesion of the disease was a small red atrophic patch on the tip of the nose. Another interesting feature of this anomalous case was that the posterior cervical glands were enlarged on both sides.

The mucous membrane of the inner surfaces of the lips and cheeks, the hard and soft palate and the larynx, and the conjunctiva may also be attacked, usually by extension from the skin. When the lips are attacked 


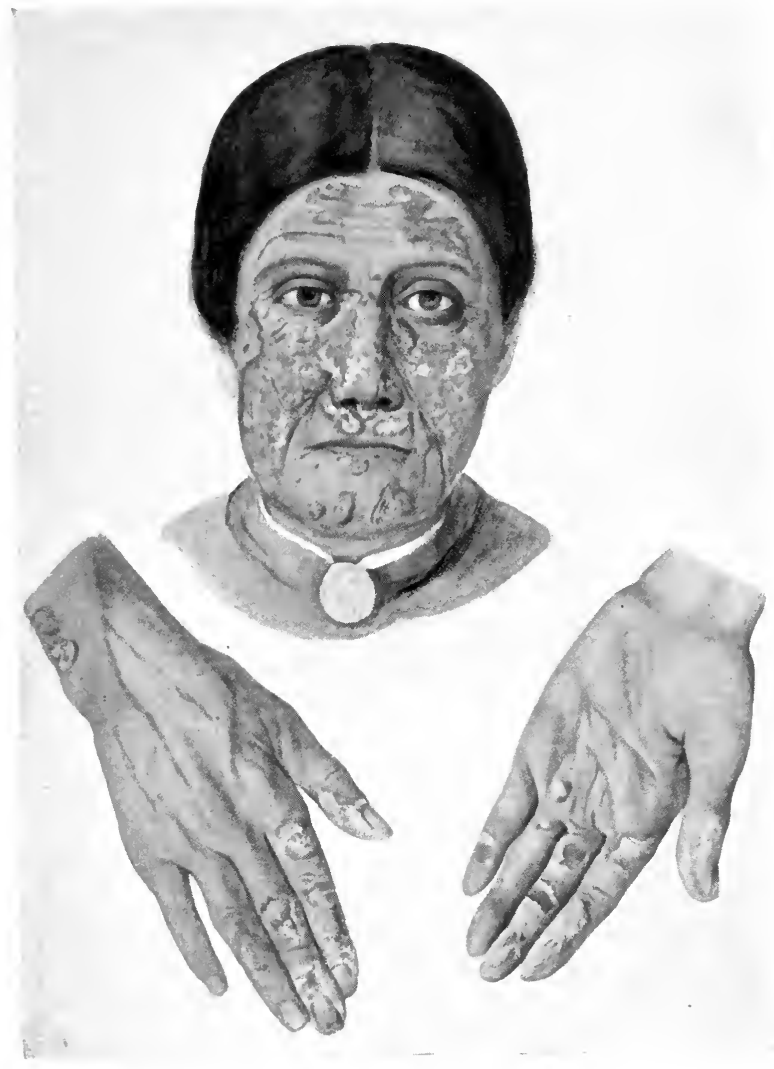

Plate 2.-Lupus eRYTHEMATOSUS. 

they become swollen and purplish-red, afterwards dry and scaly, and finally, according to Fordyce, the lesions show an atrophied greyish centre encircled by a hyperanæmic zone. On the buccal mucous membrane the patch is sometimes an abraded surface, varying in colour from a bright red to pale. Sometimes the lesions in the mouth, including those on the tongue, closely resemble leucoplakia, or lichen planus. In a case of mine, coincidentally with lesions on the skin of the face there was a crack extending through the whole substance of the lower lip, with thickening around. There was also a patch of leucoplakia on the left side of the mouth, which had been diagnosed as syphilitic; it had precisely the appearance of a patch of lichen planus. If the conjunctiva is attacked, there is congestion of the surface, sometimes with slight thickening. In a case recorded by Stelwagon, in which a patch extended from the skin to the lining membrane of the nose, there was considerable infiltration in that part.

Some authorities hold that lesions of the mucous membranes, especially of the mouth and nose, are comparatively frequent in lupus erythematosus. Of 56 consecutive cases examined by Dr. Thomas Smith, 16 (28 per cent.) had some affection of the mucous membrane. In 15 cases of Wilfred Warde's ${ }^{1}$ he found that in 9 there was associated rhinitis, and in 6 of those there was ozæna. In his experience atrophic changes in the tympanum and external auditory meatus are frequent concomitants of lupus erythematosus. In 8 of his 15 cases the lining of the auditory meatus and the drums presented well-marked atrophy. In several cases there were atrophic patches in the mouth. Only in 4 cases of the whole number did the mucous membrane appear to be healthy, and even in these he was not sure that the appearance was not delusive. I have seen atrophic

${ }^{1}$ Brit. Journ. Derm., Sept., Oct., Dec., 1902; May, 1903. 
scars on the lining of the meatus and the drum after the lupus erythematous lesions had disappeared from those situations. In one case of mine the patient had lupus erythematosus in the vagina coincidently with a "raspberry tongue."

Course and prognosis.-The disease usually runs a very slow course. The lesions continue to enlarge for ten, fifteen, or twenty years, when the process seems to have, as it were, spent itself, leaving, however, ineffaceable atrophic scars, and in hairy parts permanent baldness. In certain circumstances, especially when the disease is of the aggregate or disseminate type, the inflammatory process may be quickened into greater activity, so that it sometimes resembles severe persistent erysipelas. In such cases the change in the character of the inflammation is heralded and sometimes accompanied by fever and systemic disorder. Sequeira and Balean made repeated examinations of the urine in 27 cases, 10 of the disseminated and 17 of the discoid type, and found albumin in 7 . Five of these were of the disseminated variety, and the disease was in an active stage. In a fatal case in which they had the opportunity of making a post-mortem examination, parenchymatous nephritis was found. They are inclined to believe the albuminuria to be of toxic origin. ${ }^{1} \mathrm{Kaposi}$, Boeck, Jadassohn, Pernet, ${ }^{2}$ Leslie Roberts, and others have recorded cases of acute disseminated lupus erythematosus (aigu d'emblée) which had a fatal termination. One, described by Dr. F. Beetham and Professor Eurich, ${ }^{3}$ that of a girl of 15 , ended in death a month after coming under observation. The post-mortem threw not the faintest ray of light upon the case. There were

${ }^{1}$ Brit. Journ. Derm., Oct., 1902.

2 "Le Lupus erythémateux aigu d'emblée." Thèse de Paris, 1908.

${ }^{3}$ Brit. Med. Journ., Nov. 13, 1909, p. 140. 
no signs of tuberculosis, no bacteria or protozoa were found, and the only distinctly pathological condition discovered was a small calcified mesenteric gland. The most recent of such cases is one described by Leslie Roberts, ${ }^{1}$ who points out that in all of them the maximum eruption appears upon the head, chiefly the face, and next upon the hands, and that on the body the symptoms lose their distinctive characters and become extremely multiform in different cases. In a case of acute lupus erythematosus shown by the author and S. E. Dore the lesions on the hands were polymorphic, some of them, when the patient was first seen, resembling lichen planus, otluers psoriasis, and yet others erythema multiforme. ${ }^{2}$

A constitutional state may in certain cases tend to cure. Fordyce mentions a case of the disseminate type which disappeared during pregnancy, leaving only atrophic patches.

Pringle $^{3}$ has recorded a case in which multiple epithelioma developed on lupus erythematosus in a woman aged 36 . He refers to similar cases published, by Riessmeyer and J. Dyer in America, by Stopford Taylor in this country, and by Kreibich in Germany. A case has also been reported by E. Hollaender, ${ }^{4}$ who points cut that the conjunction of carcinoma with lupus erythematosus is much less malignant than with lupus vulgaris.

Pathology.-The pathological process is essentially inflammatory in nature. According to Veiel, the primary and essential feature of the disease is an accumulation of blood corpuscles in the dilated capillaries in the papillary layer and the corium, with cell infiltration

1 Brit. Journ. Derm., 1911, xxiii. 167.

2 Ibid., 1911, xxiii. 187. 3 Ibid., Jan., 1900.

+Derm. Zeitsch., Bd. vii. (abstr. in Brit. Journ. Derm., 1901, 1. 103). 
in the neighbourhood of the blood-vessels. More recent researches have proved that the inflammatory process begins in the blood-vessels of the superficial layers of the cutis.

Microscopic sections show heaping up of small cells which have escaped from the vessels by diapedesis. These cells are especially abundant around the hairfollicles and the sebaceous and sudoriparous glands. The small vessels become thickened, and proliferation of connective-tissue corpuscles and epithelium takes place. According to Schoonheid, ${ }^{\mathbf{1}}$ mast cells are present in the infiltration, but in inconstant numbers.

Granular and fatty degeneration and disintegration of the cellular elements occur, resulting in the formation of a thin scar-like cutis destitute of glands or hair-follicles, covered by an atrophied epidermic layer. In short, the process presents the usual characters of slow inflammation, the only feature that can be called characteristic being the peculiar cicatricial atrophy to which it leads. The scarring is only superficial.

Etiology.-The causation of lupus erythematosus is obscure. Sex appears to be a predisposing factor, twothirds or more of the subjects of the disease being women. Of 71 cases under the observation of J. H. Sequeira and H. Balean, only 11 were males, a proportion of 84.6 females and 15.4 per cent. males. Many of the females attacked are chlorotic, and a tuberculous inheritance or tendency is sometimes associated with the disease. Sequeira and Balean found that in 18 of their 71 cases there was evidence of tuberculous disease. ${ }^{2}$ Other authorities also have reported a frequent association of lupus erythematosus and tuberculosis. In the great majority of cases that have come under my own

1 Arch. f. Derm. u. Syph., Dec., 1900.

${ }^{2}$ J. H. Sequeira and H. Balean, Brit. Journ. Derm., Oct., 1902. 
observation, however, the patients have shown no sign whatever of constitutional disease; and of 43 cases in Neisser's clinic at Breslau between 1892 and 1901 there were evidences of tuberculosis in only $18 .^{1}$ The statistics, therefore, are discordant and confusing.

Lupus erythematosus seldom begins before 25 or after 45 , though in a case of Kaposi's the patient was a child of 3 . In 8 of the cases reported by Sequeira and Balean the disease began before the age of 16 , and 28 before the twenty-first year. Its starting-point is often a congestive seborrhœa of the nose, occurring either spontaneously or as a sequel of erysipelas, smallpox, scarlet fever, or measles. The immediate cause of the affection is some local disturbance of the circulation; this may be due in some cases to an external agency, such as cold or heat-a circumstance which helps to explain the marked preference shown by lupus erythematosus for exposed parts of the body, such as the face and hands. In a case shown by me and Wilfrid Fox to the Dermatological Section of the Royal Society of Medicine a slight frostbite of the ear which never completely healed passed into this condition. In the case of a nurse under my care the starting-point was a mosquito-bite. $^{2}$ I have since seen several cases which began in insect-bites. In a case reported by Whitehouse the application of a cantharides plaster is said to have been the exciting cause. ${ }^{3}$ In other cases the circulatory disturbance is doubtless due to nerve disorder. L. Perrin, of Marseilles, has recorded the case of a girl aged 18 who, after a violent mental shock at the time of the earthquakes of 1887, followed by temporary mania and suppression of menses, developed lupus erythematosus of the disseminate variety. Perrin thinks-and I am

1 Arch. f. Derm. u. Syph., Dec., 1901, p. 358.

2 Brit. Journ. Derm., Jan., 1896.

${ }^{3}$ Quoted by Sequeira and Balean, loc. cit. 
disposed to agree with him-that the nervous shock here paved the way for the onset of the disease. Sequeira and Balean think that the peculiar limitation of the areas affected affords strong support to the angio-neurotic theory of the disease. Galloway ${ }^{1}$ holds the cause to be a toxæmia, as in erythema multiforme, coupled with a tendency to easily produced paralysis of the vaso-motor mechanism, and suggests that there is evidence that tends to associate the affection with chronic nephritic toxæmia. He has also described a case in which it was probably connected with cirrhosis of the liver, that organ being no longer able to exert its cleansing action upon the blood. In the extensive chronic cases the affection certainly seems to be a toxæmia of some kind, the patients in such cases being invariably debilitated, with feeble circulation. The relation between certain cases of lupus erythematosus and certain types of erythema multiforme has been pointed out by Galloway and MacLeod, but in concluding that both are due to toxins in the circulation, they add that other cases of lupus erythematosus are probably ascribable to external causes. Leslie Roberts, in the paper already referred to, suggests that lupus erythematosus is due to a cytolytic toxin derived from the leucocytes in the lymphglands, and that the formation of the toxin is favoured by, though not absolutely dependent upon, the presence of the tubercle bacillus in the gland. The available evidence, in my opinion, does not warrant the assertion of any closer connection than this between lupus erythematosus and tuberculosis.

Diagnosis.-Lupus erythematosus may be distinguished from other varieties of erythema by the slowness and persistence of the process. The lesion itself, with its central atrophy, surrounded by a well-defined red border, studded with plugs, is sufficiently characteristic

${ }^{1}$ Brit. Journ. Derm., July, 1903. 
to enable it to be identified when it occurs on the face. On the hands, however, it often resembles chilblain so closely that the diagnosis must rest ehiefly on the fact that chilblain disappears in the summer, and in the winter usually yields readily to treatment.

In rosacea the lesion has no central cicatrix and no scab adhering to its surface. Ringworm, which occasionally simulates lupus erythematosus, runs a more rapid course, and its lesions present the characteristic fungus when examined microscopically. The points of distinction between lupus erythematosus and lupus vulgaris are of special interest and importance. They will be discussed under Lupus vulgaris (p. 458), but the chief points may be summarized here as follows: 1. In lupus erythematosus the primary lesions are minute red points; in lupus vulgaris, soft applejelly nodules. 2. Ulceration, which never oceurs in lupus erythematosus, is frequent in lupus vulgaris. 3. Lupus erythematosus never penetrates below the surface; lupus vulgaris often attacks the deeper parts (eartilage, etc.); hence the old division of lupus into exedens and non-exedens. 4. While lupus erythematosus always develops at or after puberty, lupus vulgaris almost invariably shows itself before that period.

Under the name of lupus vulgaire érythématoide, Leloir ${ }^{1}$ described a class of cases in which lupus erythematosus is closely simulated by lupus vulgaris. The lesion occurs as a patch of varying size, sometimes as two or three patches, beginning generally on one cheek. It is usually confined to one side, but in some cases attacks the nose and both cheeks symmetrically, so as to produce the elassic appearance of the "butterfly" or "bat's-wing." The appearance of the surface closely resembles that of true lupus erythematosus, but fre-

1 Journ. des Mal. Cutan. et Syph., May, 1891. 
salicylic acid plaster mull, will sometimes effect a cure. The procedure may be repeated as often as required. In suitable cases carbon-dioxide snow may be used with much benefit, but not for acutely erythematous lesions. The thermo-cautery lightly applied, followed by the application of iodoform, boric acid, or other antiseptic powder, also gives good results. The results of $X$-ray treatment are uncertain. In the follicular or sebaceous types, improvement, and frequently arrest of the morbid process, may be looked for in the majority of cases; but my experience is that it is difficult to secure com. plete removal of the affection, and relapses often occur. I have obtained better results with the high-frequency current and the Finsen light, the former in subacute, the latter in chronic cases. ${ }^{1}$ Some cases of a chronic; character are benefited by ionization with zinc or copper.

\section{Flushing}

This condition differs from blushing in that it is not invariably due to emotion, nor are the emotions those of shyness, shame, and modesty. A flush may arise simultaneously in all the parts in which it is felt ; it may start in the head and take a downward course ; it may begin in a lower region and ascend to the head; or it may travel both upwards and downwards. ${ }^{2}$ It is one form of expression of a nerve-storm, which may either be preceded or, as is more frequent, followed by a cold stage. It may be attended or succeeded by a great variety of general and nervous symptoms-nausea, vomiting, faintness, a feeling of suffocation, tinnitus, giddiness, numbness, tremors, palpitation, paresis. Among the physical conditions to which flushing is due are menstruation, normal and abnormal, the menopause,

1 For details of cases, see " Light and X-Ray Treatment of Skin Diseases" (1907), pp. 91-94.

${ }^{2}$ Harry Campbell, "Flushing and Blushing." 
pregnancy, lactation, defective circulation, chlorosis, dyspepsia, and general debility. It may also be caused by intemperance or by violent emotion, or may merge into an epileptic aura.

\section{Rosacea}

In its simplest form, rosacea is nothing more than temporary congestion of the face caused by reflex circulatory disturbance, set up by such causes as those just mentioned. The condition, however, gradually becomes chronic, and the skin in the middle third of the face becomes permanently reddened, the point of maximum intensity being in most cases the nose. Subsequently there is almost always considerable dilatation of the superficial vessels. After a time hypersecretion and retention of the sebaceous matter occur, followed in some instances by inflammation. The affected area is thus studded with pimples marking the obstructed ducts. This is the condition popularly known as " grog-blossoms" a designation as unscientific as it is uncharitable, for, though drink may be an aggravating circumstance, the affection is often seen in the most temperate persons. The disease sometimes passes into a further stage, the chronic inflammatory process giving rise to hypertrophic thicken. ing with lobulation of the skin of the nose, known as rhinophyma. This is particularly seen in habitual spirit-drinkers who are much exposed to the weather, cabmen furnishing a large proportion of victims. The hypertrophy occasionally takes the form of pendulous masses.

Rosacea is much more common in women than in men, owing, doubtless, to the periodical disturbances of the circulatory equilibrium to which they are subject. Women who have passed the "change of life" show less proclivity to the affection than men of the same age. Over-indulgence in alcohol, chronic dyspepsia 
feebleness of circulation, and exposure to sudden changes of temperature may all help to cause it, especially when two or more of these factors are combined. The use of cosmetics containing irritant substances may also play a part in its production.

Pathologically the condition is a vaso-motor neurosis called into action by reflex irritation, and followed by inflammation in and around the sebaceous glands with permanent dilatation of superficial blood-vessels, and occasionally by overgrowth of connective tissue around them.

Diagnosis.-The recognition of rosacea can hardly ever present difficulty. The conditions for which it might possibly be mistaken are lupus erythematosus, certain tertiary syphilides, and acne vulgaris. From lupus erythematosus it is distinguished by the absence of scaliness, by the border, which is not raised and shows no signs of active spreading, by the absence of atrophic scarring in the centre, and by its fluctuations dependent on digestive disorder and other causes. From tertiary syphilides it is distinguished by its symmetry, by its slow course, by the absence of any tendency to ulceration and of marks or history of previous lesions. The possibility of a mixture of diseases must, however, always be borne in mind. Rosacea is, as a rule, sharply differentiated from acne vulgaris by the age of the patient, the absence of comedones, and the redness of the affected surface. For the diagnosis of rhinophyma from rhinoscleroma, see p. 583.

Prognosis.-This is generally favourable as regards mitigation of the condition, and in the majority of cases a complete cure can be effected.

Treatment.-If the condition is found to be due to intestinal toxæmia, an autogenous vaccine and intestinal antiseptics may be given. Any functional disorder of the stomach, liver, bowels, ovaries, etc., should be cor- 
rected. The diet must be regulated, whatever causes flushing of the face being avoided. Abstinence from alcohol should be enjoined, and it would be well also if the patient could be induced to forgo coffee. Arsenic is seldom of use. After the removal of any obvious cause, the most trustworthy internal remedy is ichthyol, which often brings about a marked improvement after even a few days' administration. It regulates the bowels, prevents flatulence, helps the digestion, stops the reflex flushing, and steadies the circulation. I usually begin by ordering gr. $\mathrm{v}$ in capsules, tabloids, or pills, to be taken on an empty stomach early in the morning and late at night. In a few days the dose is increased to gr. viiss, and afterwards to gr. $\mathrm{x}$ and upwards, until the desired results are obtained. In addition to the internal administration of ichthyol, salol, or $\beta$-naphthol, local treatment on the lines laid down for acne vulgaris (p. 573) will be required if there be inflamed papules and pustules. The varicose venules may be destroyed by scarification, the superficial use of Paquelin's cautery, or, better still, by electrolysis. Hypertrophic excrescences should be pruned with the knife, and pendulous growths must be dealt with by ordinary surgical procedures. I have had good results with $X$-rays and with radium. Radium is effective also in rhinophyma, and the same may be said of carbon-dioxide snow.

\section{Pellagra (Plate 3)}

This toxic affection is endemic in Northern and Central Italy, in Spain, Roumania, and Egypt. Of late years it has been met with in the United States.

Symptoms.-Pellagra generally commences in the spring with malaise, pains in the joints, a burning sensation in the back, radiating through the limbs to the hands and feet, and gastro-intestinal disturbance. An early symptom is spastic paresis of the 
lower limbs. The skin affection consists of an erythematous eruption, chiefly affecting parts exposed to the sun. The skin is swollen and tense, and is the seat of burning or itching sensations; petechiæ are frequent, and bullæ also occur, which on rupturing leave indolent ulcers. In about a fortnight from the commencement of the attack the erythema subsides, and desquamation follows, leaving the underlying skin thickened and stained to the colour of café au lait or sepia. The symptoms usually subside towards the end of summer, only to reappear, however, in the following spring. The attacks thus recur regularly every year, the thickening and pigmentation being increased on each occasion in the first four or five years. Afterwards the integument undergoes atrophy, and becomes dry and wizened a 3 in old age. This is especially marked on the backs of the hands. The nails and hair show no change. When the patient has suffered from the disease for three or four years he becomes weak and wastes, his vision grows dim, swallowing is painful, colliquative diarrhœa sets in, symptoms of cerebro-spinal irritation increase, and he sinks into a typhoid condition, in which he passes away. Insanity is an extremely frequent complication, the mental disorder chiefly showing itself in the form of melancholia, with marked suicidal tendency. The disease lasts on the average five years; in mild cases patients may live ten or fifteen years.

Etiology.-Poverty, insufficient nourishment, and insanitary surroundings are predisposing causes. The immediate etiological factor has been held to be the prolonged use as food of decomposed or fermented maize, which has a toxic effect analogous to ergotism. De Giaxa thinks the disease may be caused by the use of even sound grain by imperfectly nourished individuals, auto-intoxication being caused by the formation of toxic substances in the intestines, owing to modifications in the 


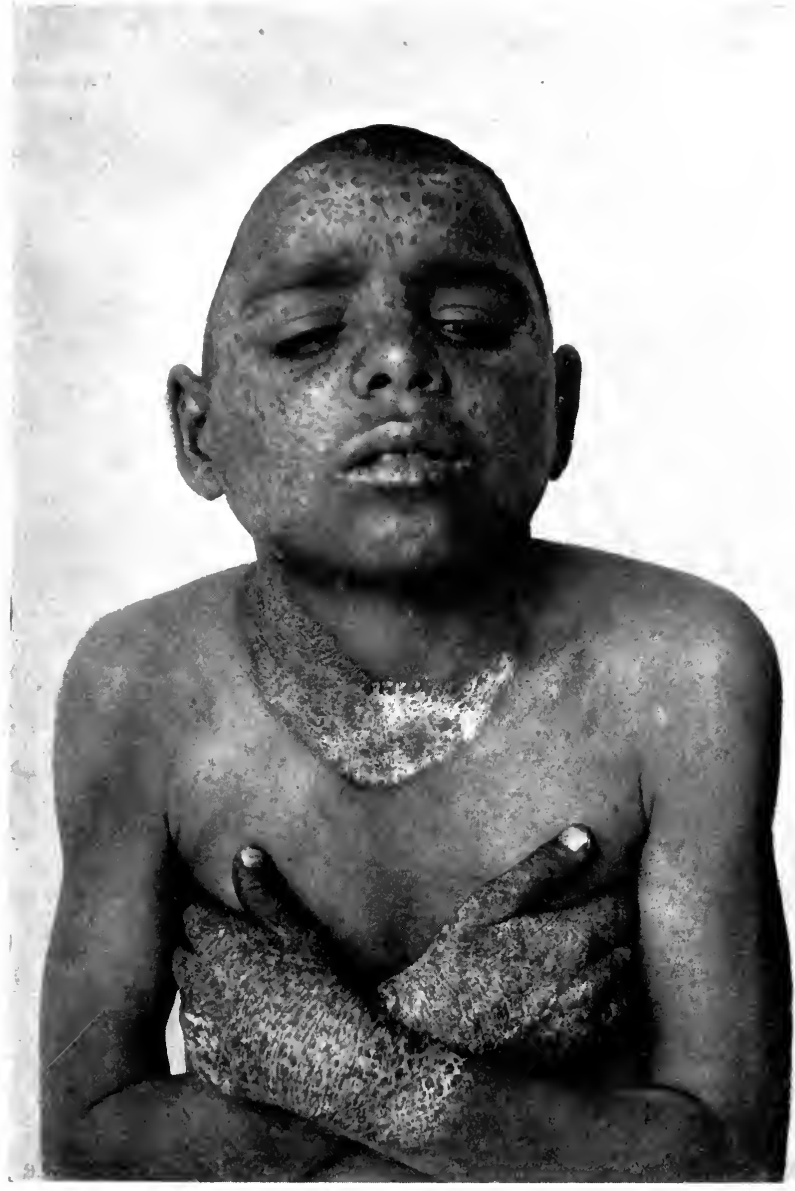

PLATE 3.-PELLAGRA.

(By permission, from the Photographic Allum of the School of Medicine, Carro.) 

substances of which the grain is composed. ${ }^{1}$ The maize theory in its various forms is subjected to close and damaging scrutiny in the last edition of Manson's "Tropical Diseases." The question is still unsettled. The disease is most common between the ages of $\mathbf{3 0}$ and 50 ; females are more often attacked than males, and children are less liable than adults.

Pathology.-The pathological process consists in a toxic effect on the vagus and sympathetic nerves, giving rise to hyperæmia and inflammatory processes in the membranes of the brain and cord, in the liver, spleen, kidneys, etc. ; to atrophy of the principal viscera and of the skin; and to fatty degeneration of various organs. In some cases there is actual wasting of the brain; in the cord the lateral column and the crossed pyramidal tract.are chiefly implicated. Both anatomically and clinically there is much resemblance between pellagra and general paralysis of the insane.

Diagnosis and prognosis.-The diagnosis can hardly ever be doubtful, the disease presenting features clearly differentiating it from other affections. The outlook is very gloomy, except in very slight cases.

Treatment.-The most important point is prophylaxis. When the disease is developed it is virtually incurable, and treatment must be symptomatic, opium, quinine, and calomel being used according to the indications. Arsenic is said by Lombroso to be the most efficient remedy. Attention must be paid to the hygienic surroundings of the patient.

\section{Acrodynia}

This affection is closely allied to pellagra. The disease so far has been observed chiefly in France, where it has several times occurred epidemically in the

1 "Contributo alle cognizioni sull' etiologia della pellagra," Annali dell' Istituto d' Igiene Sperimentale, vol. ii., fasc. 1, and vol. iii., fasc. 1. 


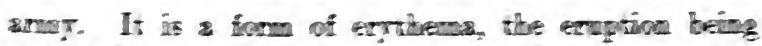

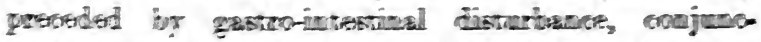

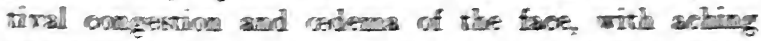

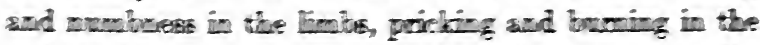

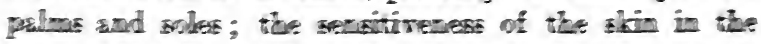

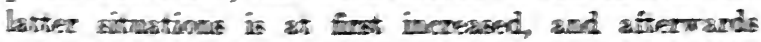
abolictied Tte ecrution, which consiste of erribems-

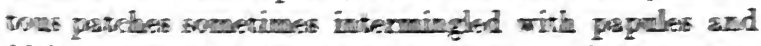

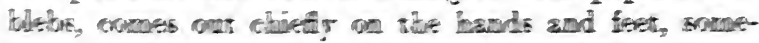
timer strexding over the limbe to the trunk. It is followed by exfoliarion of the epidermie, s bleckioh diaodoration being keft in the sfferted parte, expecially in wirm rupions, as hetween the thighs. In serebe cases wationged parexis of tbe limbe are sometimes oberred. The errytion is pot, as a rule, acoompanied by any tebrile phenowens, and the distase seastely ever peoves istal, except in elderly or weakly subjects, who sometimes exorumito to dischuces. Pexcover? gecorally tokes plaoe in a fev wek. The etiology of acrodyria is obsecure; it har then sorribed to some toric element in the food, bout of this no proof is fortborming. There are no postmortem changes that can be called characteristic of the sfiertion. 


\section{CHAPTER VIII}

\section{NEUROSES OF THE SKIN (Continued)}

\section{Dermatitis Herpetiformis (Duhring's Disease)}

AfFections of the skin, differing from each other more or less in certain particulars, but all characterized by pemphigoid eruptions, causing intense itching and burning, have been described under various names by different authors. Thus dermatologists are acquainted with the eczema pruriginosum and herpes circinatus bullosus of Erasmus Wilson, the hydroa vacciniforme of Bazin, the hydroa herpetiforme of Tilbury Fox, and the pemphigus pruriginosus of Hardy. Though each of these affections, as described by the author who named it, has features of its own, they are essentially nothing more than varieties of the extraordinarily polymorphous disease to which Duhring has given the name of "dermatitis herpetiformis." 1 The affection has been defined by Unna as " a chronic neurosis of the skin, associated with some yet unexplained blood-changes not markedly interfering with the general health.

Symptoms. - There is a more or less universal eruption, coupled with burning or itching sensations, and

${ }^{1}$ A summary of Professor Duhring's obserrations and researches on this affection will be found in his "Cutaneous Medicine, a Systematic Treatise on Diseases of the Skin," Part ii. (Philadelphia, 1598). The affection appears to have been first recognized and was clearly described by Tilbury Fox (see a posthumous article, with annotations by Colcott Fox, in Amer. Arch. Dermatology, 18s0), whose claims to priority in this matter have been overlooked both in America and on the Continent. 
regularly recurring for an indefinite period after intervals of complete or comparative immunity. The type is erythemato-bullous, which, however, may undergo considerable modification. (Plate 4.) Characteristic objective features are the multiformity and herpetiform grouping of the lesions. The most marked subjective symptom is intense itching and burning. This is sometimes relieved, though it is occasionally aggravated, by the appearance of the eruption, and in most cases it is subject to paroxysmal exacerbations. The symptoms are frequently of such severity as to rob the patient of sleep and keep him in a state of constant nervous excitement. When the erythema is spread over an extensive area, great pain and tension in the skin are complained of.

Almost any part of the cutaneous surface may be invaded, the limbs (both flexor and extensor aspects), the scalp, the face, and the trunk being all equally liable. In the majority of cases the limbs, especially the wrists and forearms, are the first points of attack. The lesions, as they subside, leave pigmented areas of greater or less extent, the pigmentation varying from dirty yellow to an almost coppery brown; the discoloration is often very persistent. The skin remains thickened and rough, and pitted and scarred here and there from the healing of excoriations underneath the scabs. The mucous membranes of the mouth, throat, nose, pharynx, and larynx, as well as the conjunctiva, may also be the seat of lesions. In a case of mine the vagina was severely attacked, as well as the mouth. Blebs in the mouth may precede those of the cutaneous surface. I have seen a case in which the tongue was first attacked, vesicles then appearing on the face and arms, and there being subsequently a more general distribution of the lesions.

In severe cases the disease is ushered in by fever and general constitutional disturbance, and there is often 


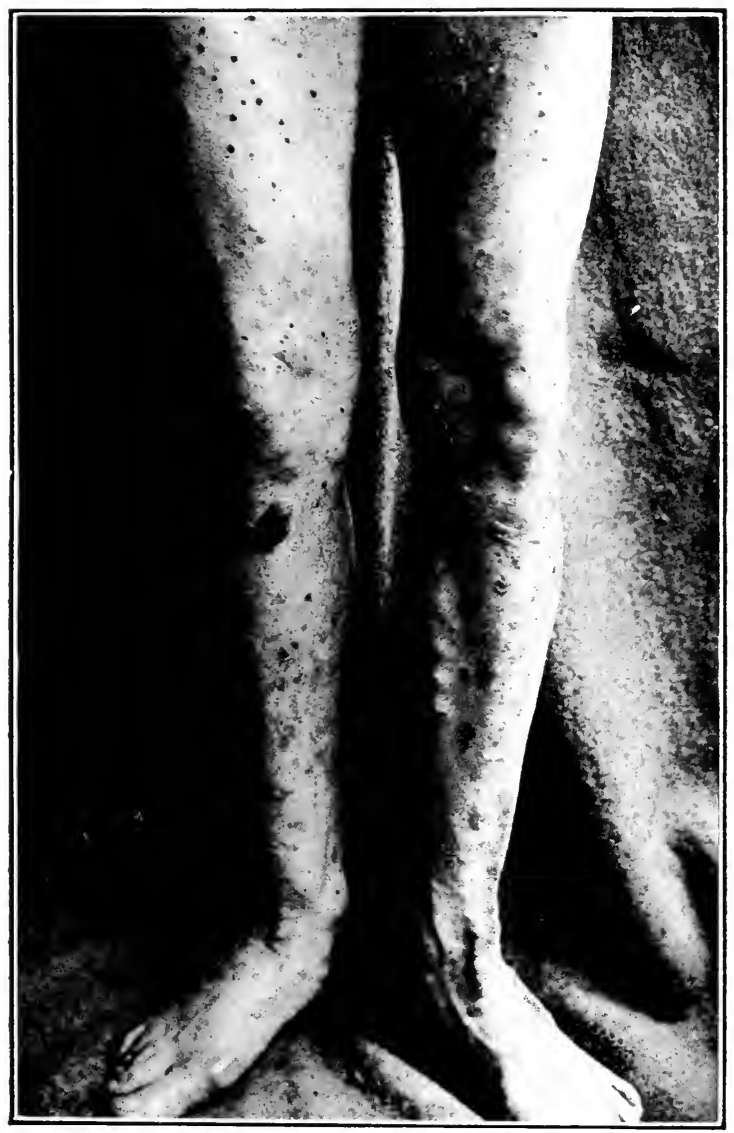

Plate 4.-DERMatitis herpetiformis. 
Dermatitis herpetiformis may be said to combine in itself the characteristics of several different varieties of skin affection, the herpetic, pemphigoid, and urticarial types on the whole predominating. The essential features of the process are: (1) The multiformity of the eruption-a multiformity showing itself not only in the appearance of crops of lesions of different types in different phases of the disease, but in the co-existence of several different types at the same time. (2) Disorders of sensation of varying intensity, but always present in greater or less degree-itching, burning, and pain; these paræsthesiæ may precede or accompany the eruptions, and may exist in the intervals between the successive crops. (3) The protracted course and constant tendency to exacerbation and recurrence. (4) The absence in most cases of any grave impairment of the general health, in spite of the physical suffering and mental anguish caused by the disease. In some cases, however, especially in the later stages, the attacks are accompanied by symptoms of septicæmia, and death has been known to occur. I have seen eight cases in which death occurred as the direct result of the disease. In two of these the fatal issue was due to heart failure, and in the others to exhaustion after prolonged attacks. Pringle 1 has also seen two cases in which the disease ended in death. In one of these the patient, who had suffered from the disease for seven years, died of peritonitis foilowing perforation of the ileum, which was the seat of numerous ulcers, others of the same kind being scattered about the cæcum. These were regarded as internal manifestations of the disease. Throughout the illness there had been indications of marked implication of the alimentary mucous membrane (dysphagia, vomiting, diarrhœa, and melæna).

The sexes appear to be equally liable to dermatitis

${ }^{1}$ Brit. Journ. Derm., 1899, p. 130. 
herpetiformis, and no age is exempt. Unna has described a variety of the affection which he considers peculiar to childhood, and which he therefore proposed to call hydroa puerorum. ${ }^{1}$ The following are, according to him, its distinguishing features: (1) It begins in the first years of life. (2) Continual relapses take place during childhood. (3) The attacks reach their maximum of intensity in the hot season. (4) Multiformity of lesion is not so marked a feature as in ordinary dermatitis herpetiformis, the eruption almost exclusively consisting of papular erythema, vesicles and bullæ. Conversely to what is the rule in adults, itching is a much less prominent symptom than pain. (6) The acuteness of the attacks is in itself a characteristic feature. (7) The general health is affected even before the appearance of the eruption.

(8) The attacks become progressively less severe as the period of puberty is approached. (9) The disease disappears or becomes extremely mild in adult age. (10) Boys are more liable to the disease than girls. The affection seems to be identical with that described by Bazin under the name of hydroa vacciniforme and by Hutchinson under that of hydroa astivale. Meynet and $\mathrm{Pehu}^{2}$ argue that there is no reason for making of the juvenile cases a separate group. John T. Bowen, of Boston, U.S.A., ${ }^{3}$ agrees with Unna that the cases described by him form a special subdivision of dermatitis herpetiformis, but himself reports fifteen cases of the adult type of the affection in children. In certain cases, he suggests, vaccination may be the exciting cause of the eruption.

Cases of dermatitis vegetans, in which lesions characteristic rather of dermatitis herpetiformis than

${ }^{1}$ Congrès Intern. de Derm. et de Syph. tenu à Paris en 1889 ; Comptes-Rendus, Paris, 1890, p. 185.

${ }^{2}$ Ann. de Derm. et de Syph., Dec., 1903, p. 893.

3 "Dermatitis Herpetiformis in Children," 1905. 
[CHAP.

of pemphigus vegetans were followed by the occurrence of vegetations, have been described by Jamieson, Hartzell, Hallopeau (under the name of pyodermite végétante), W. A. Pusey, Fordyce and Gottheil, and others. Fordyce and Gottheil ${ }^{1}$ remark that in its preference sites - the mouth, genitals, and lower extremities-dermatitis vegetans resembles pemphigus vegetans, but in its relatively benign course is in striking contrast with that affection. "If we assume," they add, "that the disease in its inception was a dermatitis herpetiformis, the late persistent and vegetating lesions could be explained by reason of a secondary pyogenic infection." Pusey ${ }^{2}$ also explains the vegetations by assuming a secondary infection, consequent upon a "weeping" dermatosis, not necessarily eczematous.

Etiology and pathology.-As to causation, all that can be said with certainty is that the neurotic disposition is a predisposing cause. In the great majority of cases the outbreak of the disease is preceded by a definite nervous shock or long-continued depressing influences. As in all other diseases, some predisposition is necessary before the exciting cause can produce its effect; in the case of dermatitis herpetiformis the susceptibility of the patient is probably determined simply by loss of nerve force. Of the sudden onset of the disease after extreme nervous shock Duhring relates a striking example. A strong, healthy man, aged 34, who had never before had any disease of the skin, narrowly escaped being buried alive in a quagmire. Three days later the eruption appeared in the form of small variously-shaped vesicles, and he continued subject to the disease in a well-marked form for at least four years.

Investigations by Leredde, Perrin, Darier, and

1 Trans. Amer. Derm. Assoc., 1906, p. 170.

2 Ibid., 1906, p. 160. 
others into the changes occurring in the blood of cases of dermatitis herpetiformis, pemphigus, and bullous leprosy have disclosed the fact that the eosinophile cells of the blood are usually, if not always, in great excess. Thus, instead of finding them present in the proportion of 1.4 per cent. of all leucocytes, as in normal blood, they are usually found increased to from 8 per cent. to 20 per cent., and cases have been reported with an even higher percentage $\left(69^{\circ} 6\right.$ per cent., rising to $77^{\circ} 2$ per cent., in a case of dermatitis herpetiformis reported by Bushnell and Winkelried Williams, ${ }^{1}$ and 60 per cent. in pemphigus). In a case of mine, in which the blood was examined by Whitfield, the eosinophilia rose from 4.9 per cent. in the blood at the commencement of an acute attack, to 12 per cent. of all leucocytes present when the eruption was at its height.

This discovery led to the examination of the fluid obtained from the bullæ and vesicles in various diseases, with the result that it was found that whereas in artificial blisters produced in the ordinary way the eosinophiles amount to about 8 per cent., in the bullæ of pemphigus and dermatitis herpetiformis the proportion is very much larger-from 15 to as much as 93 per cent.

Sections of the skin of the diseased area in cases of dermatitis herpetiformis also show a certain number of eosinophiles among the other leucocytes present in the inflammatory exudation. These cells may be stained by a mixture of methyl green, orange, and acid fuchsin; blister fluid or sections may be stained with eosin and afterwards with hæmatoxylin, and Leredde recommends first staining with Mayer's hæmatoxylin, afterwards with a mixture of 1 per cent. eosin in alcohol and 1 per cent. orange in water.

Comparatively little diagnostic significance can be attached to these cells, since it has been found ${ }^{1}$ Brit. Journ. Derm., 1906. 
that they occur in the blood of patients suffering from pemphigus, syphilis, leprosy, and erythema multiforme, and in the serum of the bullæ and vesicles in pemphigus, erythema multiforme, eczema, dysidrosis, and ecthyma. Brocq, ${ }^{1}$ however, holds that it is the presence of eosinophiles in large numbers both in the blood and in the vesicles that is characteristic of dermatitis herpetiformis.

Although dermatitis herpetiformis is probably a functional neurosis, it is possible that in some of the severer cases peripheral neuritis may be present, but no proof of this has yet been advanced. It has been suggested by Hallopeau and others that the neurosis may depend on the presence of a toxin in the blood, but of this there is as yet no conclusive evidence. In a collection of fourteen cases analysed by Engman, ${ }^{2}$ indican was present in marked excess in the urine, and the indicanuria was coincident with the eosinophilia. 'Adenocarcinoma of the thyroid has been found in some cases. Microscopical examinations by Gilchrist ${ }^{3}$ (see Plate 5) have shown that the disease is characterized in its earlier stages by a very acute inflammation of the papillary layer of the corium with formation of vesicles immediately beneath the epidermis and the migration of large numbers of polynuclear leucocytes; the epidermis is only passively engaged.

Diagnosis.-Dermatitis herpetiformis may be mistaken for any of the diseases whose characteristic lesion predominates at any given period of its course. The diagnosis must rest on the following points : (1) The multiformity of the lesions: and under this head must be counted the scars, pits, and pigmentary blotches left by

1 Ann. de Derm. et de Syph., t. ix., Oct. and Nov., 1898 (abstr. in Brit. Journ. Derm., 1899, p. 213).

2 Trans. of Amer. Derm. Assoc., 1905, p. 173.

3 Johns Hopkins Hosp. Repts., vol. i. 
previous attacks as well as the vesicles, blebs, etc., actually present. (2) The intensity of the itching, which, as already said, often vexes the patient when otherwise the disease appears to be quiescent. (3) The frequency of relapses; and (4) the general refractoriness of the affection to treatment of every kind. The practitioner must be guided by the aggregate of symptoms rather than by one or other feature which may happen to be predominant at a particular time. The diagnosis from herpes zoster will be found at p. 178 .

Treatment.-No measures appear to be of much avail in curing or even controlling dermatitis herpetiformis. All that can generally be done is to relieve pain and induce sleep by hypodermic injections of morphine or by opium internally, and soothe irritation by some of the means already described. No spirituous lotions should, however, be employed, as they cause smarting of the skin, which is always raw and tender. The rubbing in of weak sulphur ointment is the local measure which has so far given the best results; the inunction should be done with some degree of force, so as to rupture the vesicles and bullæ. This method should be employed at first over a limited area, so as to minimize the risk of setting up dermatitis. The application of almond or carbolic oil, or, better still, olive oil combined with lime water, to the whole surface sometimes gives relief. Salicylic acid is often useful as a local remedy. Schwim. mer obtained satisfactory results with thiol, a solution $\left(10^{\circ} 0\right.$ to $\left.30^{\circ}\right)$ ) of which was painted over the affected surface twice daily for two or three days, the skin being then carefully washed with pure water. Weak ichthyol ointment or solution is of value as a local application. Of internal remedies, arsenic is probably the most efficient, although in many cases it seems to have little or no effect. The dose of arsenic required is smaller than that generally used in pemphigus. In the early 
stages, when the inflammatory symptoms are very marked, antimony may be useful, but its use should be continued only for a short time. I have seen good effect from small doses of quinine. Iron, phosphorus, and nerve tonics may do good by maintaining the strength and bracing up the nervous system, especially in the later stages of the disease. I have seen good results in subduing nervous symptoms from the use of phenacetin-gr. $\mathrm{v}$ in the middle of the day, and gr. $x-x v$ in the evening. The mid-day dose may with advantage be combined with citrate of caffeine, gr. ii. Phenacetin has proved beneficial also in the hands of Pringle, who has, besides, seen diminution in the amount of itching from antipyrin. Warm bathing gives relief in some cases, but in others appears to aggravate the symptoms. The diet should be strictly regulated, all substances that have any tendency to disagree being carefully avoided, and liquids, such as coffee, generous wines, and spirits, which stimulate the heart and cause an increased flow of blood to the skin, being absolutely prohibited. Disturbing emotions of all kinds are likely to intensify the evil, and the patient should expose himself as little as possible to vicissitudes of temperature.

Herpes Gestationis (Hydroa Gravidarum, Hydroa Gestationis)

This affection occurs in association with pregnancy, and is characterized by multiformity of lesion and excessive itching.

Symptoms - The clinical features of herpes gestationis are practically identical with those of dermatitis herpetiformis, the only point of distinction being, according to Brocq, that among the lesions observed pustules are less frequent than in the latter affection. The symptoms come on during the last six months 
of gestation, sometimes a few days after delivery. The eruption, which is multiform in character, appears usually first on the limbs, especially the hands and arms; sometimes the umbilicus is the point first attacked. The subjective phenomena (itching, burning, etc.) are constant and very pronounced. Sometimes the eruption is accompanied by slight febrile disorder, but on the whole the affection has little effect on the health beyond causing a certain degree of fatigue. When the period of parturition is over, the disease, as a rule, disappears spontaneously; but it has a marked tendency to recur with each successive pregnancy, increasing each time in severity, and to merge into ordinary dermatitis herpetiformis. A curious fact pointed out by Brocq ${ }^{1}$ is that true dermatitis herpetiformis seems to disappear in women suffering from it if they become pregnant.

Treatment.-There is nothing to be added to what has been said concerning dermatitis herpetiformis, except that, in view of the patient's condition, internal remedies must be employed with caution.

\section{Pompholyx (Dysidrosis)}

This disease, sometimes known as cheiropompholyx, a term which does not fully cover the ground, is marked by an eruption consisting of vesicles symmetrically distributed on the extremities. The feet sometimes escape, but the hands are always attacked.

Symptoms.-The affection begins with sensations of burning and itching, quickly followed by the appearance of numerous tiny vesicles deeply embedded in the skin, and showing through the epidermis like boiled sago grains. Their advent is accompanied by increase of the itching. As they become more prominent on the surface they run together and form large irregular bullæ 2 "Traitement des Maladies de la Peau," p. 135. Paris, 1890. 
containing clear fluid. These show little tendency to burst, but become more and more distended for a time; and then, as the contents become opaque and thicken, they begin to shrink, and finally form crusts. When these are thrown off, the surface of the skin underneath is found smooth, red, and exquisitely tender. The itching sometimes ceases when the bullæ are fully developed, as if some irritant substance had been thereby eliminated from the skin. When the bullæ are pricked the liquid which issues is clear, and neutral or alkaline in reaction. The first tiny vesicles may usually ba seen grouped around the orifices of the sweat-ducts. The eruption comes out along the sides and palmar aspects of the fingers, and in the interdigital spaces. In severe cases the whole surtace of the hands may be involved. Sometimes an eczematoid eruption spreads up the arms from the hands, or may develop at distant parts, allying the disease with some form of eczema, with which many authorities consider it identical. Its pathological anatomy on the whole rather supports this view. The duration of the disease is about a fortnight, but recurrence is almost certain, and may occur at such short intervals as to make the disease all but continuous. Repeated attacks at the same parts leave the skin discoloured, harsh, thick, and dry, and some time elapses before this inconvenient covering, which deadens sensation and hinders the movements of the fingers, is shed.

Etiology and pathology. - The disease was named "dysidrosis" by Tilbury Fox, on the supposition that the process was primarily set up by retention of the sweat secretion. Crocker regarded the process as one of hyperidrosis rather than dysidrosis, and thought that excessive sweating is a predisposing condition. Later observers have shown that the disease is not intimately connected with the sweat-glands, and some regard it as a vesicular eczema modified by the anatomical peculiari- 
ties of the part. There can be little doubt that the affection is, in the first instance, a vaso-motor neurosis, and it is in harmony with the notion of its nervous origin that it is much more common in women than in men, and that its especial victims are young women of neurotic temperament or who have been exposed to worry or excitement. So strongly marked, indeed, is the neurotic character of the affection, that in many cases the slightest unpleasant emotion or mental agitation is sufficient to bring on an attack. Among the immediate causes of the disease, next to nervous shock, is temperature. The affection is more common in spring and summer than in the colder seasons, and hot weather has a marked effect in determining an attack or aggravating an already existing one. Artificial heat acts exactly in the same way, and exposure of the hands to the fire, as in cooking, often induces an attack in those subject to the complaint. ${ }^{1}$

Winkelried Williams has shown that the anatomical life-history of the pompholyx vesicle is as follows : (1) A mild inflammatory action in the papillary layer of the corium results in an exudation of serum, which finds its way between the rete cells and leads to their compression, degeneration, and destruction. Vesicles are thus formed which receive fresh fluid and so increase in size. (3) The vesicular contents dry up, fresh epithelium forms below, and the superficial together with the dried contents of the vesicles are thrown off. The anatomical characters of pompholyx thus closely resemble those of vesicular eczema.

Diagnosis.-The distinctive features of pompholyx are the limitation of the eruption to the extremities, and particularly to the hands; the prone-

1 Unna (" Histopathology," p. 179) has found a bacillus like the $B$. tuberculosis but stouter, in all sections, which he believes to be pathogenic. 
ness of the vesicles to run together and form bullæ which seldom rupture spontaneously; the tendency to recovery, followed by repeated recurrence, and the constant association of the disease with the summer season. The co-existence of all these points suffices to identify the affection. The absence of "weeping" differentiates pompholyx from eczema; the formation of bullæ by coalescence of vesicles, from pemphigus; and the size, situation, and duration of the vesicles, from sudamina. Before concluding that an affection of the hands or feet is a simple pompholyx, care should be taken to exclude ringworm. In many such cases the discovery of the Epidermophyton inguinale or some other ringworm fungus in scrapings of the lesions has revealed the true nature of the affection.

Prognosis.-This is always good as far as recovery from any given attack is concerned, but the great probability of recurrence must always be borne in mind.

Treatment.-The local lesions must be treated on the lines laid down for pruritus (p. 67). Constitutional tratment is almost always required, tonics in the form of iron and arsenic, separately or in combination, quinine, and strychnine, being especially indicated. Violent exercise, alcohol in excess, and anything tending to promote sweating, must be avoided. Dietetic errors must be corrected and digestive disturbance rectified. Change of scene and mental diversion are often important factors in the treatment.

\section{Pemphigus}

Pemphigus may be defined as a condition characterized by the eruption of bullæ on previously healthy skin. Fresh crops of bullæ come out, not only on the skin, but sometimes on one or other of the mucous membranes, either continuously or at varying intervals of time. The chief varieties of pemphigus are-(1) a 
type in which the bullæ follow throughout a definite line of evolution and finally disappear without causing any loss of substance in the epidermis. To this group, the characteristic feature of which is the formation of bullæ, is applied the name of pemphigus vulgaris.

A type in which the epidermis tends to become detached in large sheets, leaving the deeper layer exposed over an area which afterwards enlarges circumferentially. To this process, in which the essential phenomenon is exfoliation, the term pemphigus foliaceus is applied. Pemphigus vegetans, in which, following an eruption of bullæ, papillary excrescences sprout up, the epidermis strips off in large sheets, and the process ends in gangrene and death. This form, described by Neumann, ${ }^{1}$ presents features so peculiar as almost to entitle it to rank as a distinct disease. Its only relation with ordinary pemphigus lies in the fact that the eruption is at first bullous in character.

Before dealing with those types of the disease, cousideration may be given to an affection which should perhaps be grouped under the same general heading, namely epidermolysis bullosa. ${ }^{2}$ In this group the affection seems to be in nearly every case congenital, and to be associated not only with an insufficient resisting power in the skin, but with a general tendency to nondevelopment throughout the body. On the slightest injury to the cutis, superficial bullæ of varying size arise, frequently with blood-stained contents. The mucous membrane of the lips and mouth may be attacked. Three children in one family whom I watched almost from their birth all had lesions of the buccal mucous membrane. In one of them the intestinal tract also

1 Congrès Internat. de Dermatol. et de Syph. tenu à Paris en 1889 ; Comptes-Rendus, Paris, 1890, p. 81.

2 See article by Wallace Beatty, Brit. Journ. Derm., 1897, ix. 301. 
was affected, bleeding being caused by the ingestion of any food that was at all hard. In a typical case of mine of twenty-eight years' duration there were blebs under the tongue which the patient traced to the biting of hard substances. In the course of years both skin and nails atrophy, as in the case illustrated in Plate 6.

There is still some question whether the bullæ are invariably the result of trauma, or whether a tendency to pemphigus does not co-exist. By the repeated formation of these very superficial bullæ the skin gradually assumes a peculiar papery, atrophied appearance, which is characteristic. Bukovsky ${ }^{1}$ reports a case which he studied in Janovsky's clinic. He believes the bullæ to be due simply to loss of continuity of the epidermis and corium, caused by traumatism, and maintains that the susceptibility to injury is the result of a marked difference in the contractility of epidermis and corium. Near the scars in this case were miliumlike bodies, which were found to be retention-cysts of the sweat-ducts, of which the orifices had been blocked by the healing of the bullæ. Petrini de Galatz ${ }^{2}$ describes in detail three cases in which, though they were congenital, there was no heredity, nor was there traumatism. He maintains that the disease is a definite dermatosis, with dystrophy for an essential feature, and suggests that the cause may be found in some alteration of the peripheral nervous system at the site of the lesions. The epidermal cysts, he asserts, are due to the transformation of epidermic cells, in which the central cells of the mass break down and are absorbed. In a case which came under the notice of Winkelried Williams ${ }^{3}$ there was evidence

1 Arch. f. Derm. u. Syph., 1903, lxvii, 163.

${ }^{2}$ Ann. de Derm. et de Syph., Aug. and Sept., 1906 (abstr. in Brit. Journ. Derm., Sept., 1907, vol. xix.).

${ }^{3}$ Brit. Journ. Derm., 1907, xix. 12. 


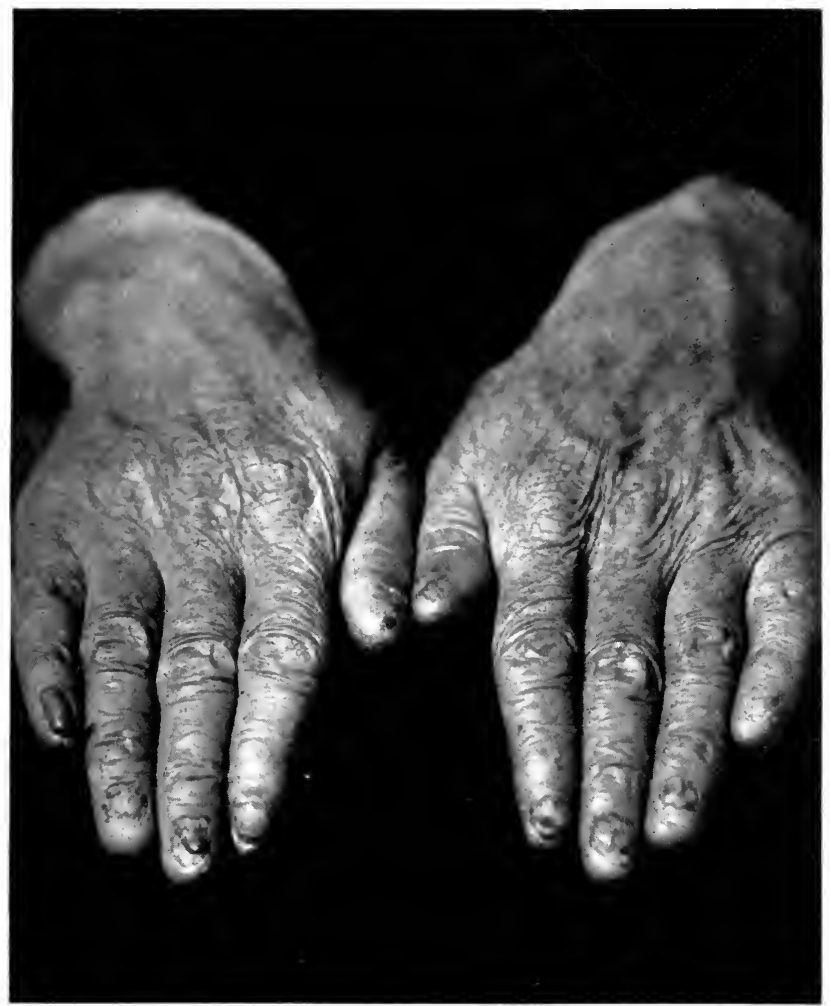

PLATE 6.- ATROPHIED SKIN AND NAILS IN EPIDERMOLYSIS BULLOSA. 
they stand out on the skin as hemispherical blebs, without any inflammatory areola around their base. They are scattered about irregularly, or arranged more or less symmetrically on the limbs, trunk, or lower part of the face. Sometimes they are set so close together as almost to deserve to be called " confluent," and in rare cases they actually do run together. Occasionally they are grouped around bullæ of older date so as to form circles, which, as they in turn gradually disappear, leave irregular wavy lines. The contents of the bullæ are at first clear and transparent, but they soon become opaque; the bullæ then dry up, forming brownish-yellow scabs. If the surface of skin covered by these scabs is extensive, they give rise to a disagreeable feeling of tension, and excoriation may be caused by their premature separation. When the scabs fall off naturally, the surface underneath is seen to be covered with newly-formed epidermis, which is at first purple in colour, but gradually turns brown and remains pigmented for some weeks. In some instances the ulcers under the scabs become covered with fibrinous exudation, and leave more or less scarring.

Pemphigus also sometimes attacks mucous membranes. I have seen cases in which the mouth and the conjunctiva were affected, the process being accompanied by " essential shrinking" of the latter. ${ }^{1}$

The life-history of each bulla extends only over a few days; but as successive crops of them come out, more frequent and abundant in proportion to the severity of the attack, the disease may last for several months. In certain cases hæmorrhage takes place into the interior of the bullæ, the contents of which are then pink, red, or blackish, according to the amount of blood

1 See a report of the case by the Author and Leslie Roberts, Brit. Journ. Derm., April, 1889. 
effused. In other cases the bullæ may end in sloughing and more or less extensive gangrene of the surrounding skin. These, however, are not varieties of the disease, but pathological accidents. Nikolsky's sign (p. 141) is present. Sometimes the general health is little, if at all, affected; but in persons of feeble constitution the discomfort of the lesions and the consequent insomnia cause depression, loss of strength, exhaustion, and even death. When the disease is on the decline the bullæ no longer come out in crops, but singly here and there; the fever ceases, sleep and appetite return, and the health is rapidly restored. There may be no recurrence after a first attack, but it more often happens that after some months, or even a year, the patient is again attacked, perhaps more than once. The disease may then definitively cease from troubling; or, on the other hand, it may get so firm a hold that it cannot be shaken off, attacks following each other at such short intervals as to make the affection practically continuous. In such cases the whole body may be invaded by the lesions, to the grievous detriment of the patient's health, and sooner or later to the destruction of his life ; or the process, though persistent, may be mild, the bullæ, though never altogether absent, being few and far between. These "sporadic" bullæ (if the term may be allowed) are apt to select parts where the circulation is sluggish (extremities, nose, etc.) for their appearance.

Though, as a rule, essentially chronic in its course, pemphigus is occasionally so acute in its manifestations as to warrant the term "malignant" which has been applied to such cases. The bullæ form in enormous numbers, crop following crop so closely that there is no remission of the process, which is accompanied by high fever and rapid wasting, and ends in death in two or three weeks or even a few days. This form of the 
disease is usually seen in young children, and must not be confounded with syphilitic pemphigus. Pernet and Bulloch have recorded ${ }^{1}$ a number of cases of acute pemphigus which followed wounds of the hands in butchers, and mostly ended fatally. A similar case has been observed by Wilfred Hadley and Bulloch. ${ }^{2}$ In all these cases diplococci were found by Bulloch in the fluid of the bullæ.

In pemphigus foliaceus, originally described by Cazenave, by whose name it is sometimes called, the bullæ are not rounded and tense like those of pemphigus vulgaris, but flattened and flaccid. They break easily, and the affected surface has a blistered appearance. The bullæ form yellowish crusts and, as the disease spreads, scales of considerable size are formed. These, as they become detached, leave red excoriated areas on which new layers of epidermis are formed, only to be quickly shed again or brushed away mechanically. Nikolsky's sign (p. 141) is present. After a period of months or years the whole cutaneous surface may be invaded; the skin readily ulcerates wherever it is subjected to any pressure, the face becomes disfigured by cicatricial contraction, causing ectropion, etc. The patient loses flesh, and as the disease advances the febrile symptoms and constitutional disorder become intensified; he cannot move or lie down without pain, and his condition is one of great misery. Pemphigus foliaceus generally ends in death. The affection may begin and run its whole course as an independent disease, or it may follow long-standing pemphigus vulgaris, when the eruption has become continuous and widely distributed, and cachexia has been induced.

In pemphigus foliaceus, as in pemphigus vulgaris, the mucous membranes of the mouth, pharynx, and

1 Brit. Journ. Derm., May and June, 1896.

${ }^{2}$ Lancet, May 6, 1899. 
larynx may become the seat of eruption. If bullæ form on the epiglottis there may be danger of suffocation. If the bullæ on the mucous membrane follow the same course as in pemphigus foliaceus of the skin, swallowing becomes impossible, the voice is lost, and the respiration may be embarrassed. In such circumstances the patient is in a condition of the gravest danger. The lesions of pemphigus may extend far into the lower air-passages, and in the last stage of pemphigus foliaceus the trachea and bronchi are often invaded. Kaposi holds that an eruption on the mucous surface may be the precursor of the cutaneous disease; and J. A. Fordyce 1 reports a case in which lesions in the mouth preceded the development of general pemphigus by several months. Ulcers resulting from pemphigus bullæ have been observed on the vagina, and Cheadle has reported a fatal case in which ulcers that apparently had the same origin were found in the intestine.

The initial lesions of pemphigus vegetans are bullæ of the size of lentils, which gradually distend the epidermis with the colourless exudation which they contain. Excoriation takes place, and in four or five days the centre of the denuded surface is occupied by a pale white protuberance which grows rapidly in height and width, so that in a short time warty or granulation-like excrescences are formed. These are at first bounded" by a circle of excoriation, later by bullæ, which form at the circumference. The surface of the patches is uneven, slightly raised, flesh-coloured, and discharges a thin, foul-smelling secretion. The discharge, as it dries, forms a thin crust, which can easily be stripped off, when an excrescence, partly covered with a thin stratum of epidermis, is seen. Sometimes the points first attacked are the labia majora and minora; next, the mouth and lips; then the skin, axillæ, hands, feet, inner parts of I.Y. Med. Journ., March 6, 1909. 
thighs, face (where the eruption joins that of the lips and mouth). In other cases the buccal and pharyngeal mucous membranes, or the neighbourhood of a nail, may be the starting-points. The mucous membrane becomes dry and fissured, and swallowing is so painful that the patient does not care to attempt it. On the skin the bullæ, instead of drying up into scabs, break down and form excoriations, upon which, in parts where the integument is folded on itself (armpits, junction of thighs with perineum), papillary excrescences sprout up. Fresh crops of bullæ continue to come out, and the epidermis strips off in large sheets, leaving the papillary layer exposed, as in a burn of the second degree. The diseased surface is dirty, wet, and warty. The secretion decomposes rapidly and is horribly offensive. Finally, superficial gangrene takes place, and the patient dies exhausted by his sufferings and by want of food, or of some intercurrent disease (e.g. nephritis, or œdema of the lungs), a few months after the first appearance of the eruption.

Pemphigus vegetans is rare. Neumann in 1889 had seen only 14 cases, and up to 1906 not more than 58 cases had been recorded. Crocker met with a typical example in 1887, which he considered at that time to be the only one observed in England, ${ }^{1}$ though he thought that some cases of "a rare pustulating disease of the skin and mucous membranes," allied to foot-and-mouth disease, reported by Hutchinson, might have been examples of a mild variety of pemphigus vegetans. Allan Jamieson ${ }^{2}$ describes a case in which Professor Welsh discovered, postmortem, in the spinal cord, and also, though less advanced, in the sympathetic ganglia and the cerebral

1 Trans. Roy. Med.-Chir. Soc., lxxii. (with bibliography up to date).

2 Brit. Journ. Derm., Aug., 1902. 
cortex, nerve-cell changes representing a primary degeneration. In a case reported by Hamburger and Rubel ${ }^{1}$ all the internal organs except the lungs were found to be healthy. Professor Winfield's analysis of the 58 cases recorded up to 1906 shows that the disease occurs most frequently between the thirty-fiftl and forty-fifth years, and that females are more liable to it than males-31 as against $25^{2}$ Dr. W. J. Rutherford has published an elaborate clinical description of a case which proved fatal in seventeen weeks. ${ }^{3}$

Pathology.-The characteristic bulla is the result of inflammatory exudation from the vessels of the papillary layer. Crocker states that in the case of a very large bulla which he examined, the fluid poured out had stretched the lower rete cells until they were separated from the corium ; and as the process continued, the lower layers were destroyed and the upper compressed until, at the centre, the roof was formed by the horny layer and about the upper two-thirds of the rete, with here and there a fragment of a sweat-duct or hair-follicle depending. At the border the lower stretched cells of the rete were still present. The fibres of the corium below the bulla were compressed, and there was free cellinfiltration of the upper layers.

The liquid contained in pemphigus bullæ has most of the characters of blood serum. Even when it is clear, leucocytes may be found in it; and when it becomes opaque, pus corpuscles and red blood-corpuscles abound in it. It is, as a rule, weakly alkaline in reaction. The eosinophile cells are usually present in great excess in the blood (see p. 145). The urine of patients suffering from pemphigus shows a diminution in the normal amount of urea. Among the complications of pemphigus

1 Johns Hopkins Hosp. Bull., April, 1903.

${ }^{2}$ Trans. Amer. Derm. Assoc., 1906, p. 199.

${ }^{3}$ Brit. Journ. Derm., 1910, xxii, 118. 
[CHAP.

are Bright's disease, pneumonia, tuberculosis, and ulceration of the intestinal follicles.

Etiology.-Of the causation of pemphigus vulgaris nothing is known with certainty. New-born babes and young children are more liable to it than adults, and debilitated subjects more than persons in good health. It is not clear that sex has any influence, statistics collected by different observers giving contradictory results. It is occasionally hereditary. Kaposi cites the case of a patient whose mother, sister, and maternal uncle had been sufferers; several of the man's own children were also subjects of the disease. I have treated three members of the same family for pemphigus vulgaris. A predisposing cause of the disease is possibly some instability or over-excitability of the nervous system. Changes in the peripheral nerve ends under the bullæ have been found in a few cases of pemphigus vulgaris by Dejérine and others, and Weir-Mitchell has shown that bullous eruptions sometimes follow injuries of the nerves, especially such as cause neuritis. In certain forms of nerve degeneration or irritation bullæ are apt to be induced along the course of the affected nerve trunks by heat, cold, or slight injury. The pemphigoid blebs which are a frequent accompaniment of leprosy are probably the result of direct irritation of the vaso-motor nerves by the leprotic infiltration. Bullous eruptions are also not uncommonly associated with sclerosis of the posterior columns of the cord. Possibly pemphigus vulgaris is, as Schwimmer suggested, a tropho-neurosis, but in the present state of knowledge no conclusive proof of this theory is obtainable. Some confirmation of the view just expressed as to the nervous origin of pemphigus is afforded by the fact that it is not infrequent in neurotic and hysterical subjects. According to Kaposi, in women the disease is occasionally associated with gestation, the eruption showing itself in the course of every preg- 
nancy and disappearing after delivery. In such cases, however, it is probable that the affection is not true pemphigus, but the same as that already described under the name of "herpes gestationis," and therefore of the nature of dermatitis herpetiformis.

It has been pointed out by Bunch ${ }^{1}$ that some cases of pemphigus do not fit in with the theory of the nervous origin of the disease, but suggest rather a toxic or infective origin. The toxins, he surmises, may be due to the metabolic products of bacteria, and may induce a functional or organic change in the peripheral or the central nervous system, which reacts on the skin by disturbed innervation of the area on which the bullæ arise.

Microörganisms have been found in the blood and in the urine of the patients by Paul Gibier, and in the contents of the bullæ, in the urine, and in the blood by Spillmann. Demme found, in the bullæ and in the blood, diplococci from which he succeeded in making pure cultures. Similar organisms have, as already said, been detected by Bulloch. Crocker found a few micrococci in recent bullæ, and under cultivation in peptonized gelatine minute bacilli developed. In a case of pemphigus vegetans reported by Winfield ${ }^{2}$ the Bacillus pyocyaneus was present in the bleb contents, in the blood, and (post-mortem) in blood from the right auricle. In a case of acute pemphigus vulgaris recorded by Bunch, ${ }^{3}$ in a girl of 7 , streptococci were found in film preparations and in pure culture, and the patient rapidly recovered under treatment with a streptococcic vaccine prepared from cultures of the bullæ. It is obvious that, in view of the numerous microörganisms of the most diverse kinds which are found on the epidermis

1 Brit. Journ. Derm., 1908, xx. 336.

2 Trans. Amer. Derm. Assoc., 1906, p. 199.

${ }^{3}$ Loc. cit. 
under normal conditions, all observations on the bac. teriology of skin lesions must be received with greater caution than those relating to any other part of the body.

Leredde ${ }^{1}$ considers that pemphigus foliaceus is to be regarded as essentially a blood disease, the cutaneous manifestations being secondary, while both the blood-changes and the skin lesions depend, in his opinion, on an affection of the bone marrow set up by toxic bodies of one kind or another. Cranston Low, in a paper which gives a complete bibliography up to date, ${ }^{2}$ suggests that the alimentary canal is a possible source of the origin of pemphigus foliaceus by the absorption of toxic substances. Thus he explains the presence of indican in the urine in one of the cases described by him, as well as the irregular temperature, the general wasting, and the occasional attacks of diarrhœa which are features of the disease. The changes in the blood and nervous system, he adds, may similarly be due to the action of intestinal toxins. The nervous theory of the origin of this disease has had advocates, but nervous symptoms are seldom present, and nerve lesions are either absent or variable.

Diagnosis.-The diagnosis of pemphigus vulgaris seldom presents any serious difficulty. The presence of the characteristic bullæ and of scabs and pigmented spots representing bullæ of earlier formation, and the absence of pustules, erythematous patches, and other lesions, taken together with the history of successive crops of exactly similar eruptions, are points which will in most cases suffice to identify the disease. Pemphigus may sometimes be confounded with bullous forms of

${ }^{1}$ Ann. de Derm. et de Syph., July, 1899, vol. x. (abstr. in Brit. Journ. Derm., 1899, p. 406).

${ }^{2}$ Brit. Journ. Derm., 1909, xxi. 101 and 135. See also a later paper, ibid., 1911, xxiii. 1. 
urticaria and erythema. In both these conditions, however, there are other lesions besides the bullæ; moreover, except in pemphigus, the bulla is more an adventitious than a primary lesion-implanted on a wheal (as in urticaria), or on a raised red plateau in a setting of vesicles (as in erythema multiforme), not rising out of healthy skin, which is the pathognomonic feature of pemphigus. From dermatitis herpetiformis, again, pemphigus is distinguished by the uniformity of the lesion. Pemphigus foliaceus may be mistaken for eczema rubrum and pityriasis rubra, and the diagnosis can sometimes be made only by taking into account the history of the case and by carefully watching its course. Thus in eczema the scales are not so large as in pemphigus foliaceus, nor is the disease often universal. In pityriasis rubra there are no bullæ, and the surface is dry. Moreover, the scales are smaller and thinner than in pemphigus foliaceus. In all forms of pemphigus, and especially in pemphigus vegetans, one of the first things to be done is to exclude syphilis. Neumann gives the following three points of distinction: (1) In pemphigus vegetans the excrescences are always surrounded by a zone of bullæ, while condylomata have an infiltrated border. (2) In pemphigus vegetans the surface is excoriated and warty; in condylomata it is even and smooth. (3) The sequence of events and concomitant circumstances in the two cases, condylomata being almost invariably the consequence of an acute process, and being accompanied and followed by other signs of syphilis; moreover, if left to themselves they finally tend to involution. In pemphigus foliaceus, on the other hand, the lesions continue to multiply, and the disease goes steadily from bad to worse.

Prognosis. - In pemphigus vulgaris the prognosis is, as a rule, favourable as to life, though recurrence is only too likely, and it is impossible to say 
how often this may take place. In acute cases there is nearly always a greater or less amount of danger, especially in young children or old people. The longer the disease lasts the less hopeful is the prospect. One element of danger in very chronic cases is that the process may pass into pemphigus foliaceus, which is always fatal, though life may be dragged on for years. As to pemphigus vegetans, Neumann says that in no disease is the prognosis so gloomy: "A small excoriation in the axilla, one or two bullæ on the mucous membrane of the lips, are often sufficient grounds for prognosticating death, irrevocably impending, in a few months." Crocker, however, held that early treatment before the skin is much involved offers some chance of recovery.

Treatment.-Should microörganisms be found in the bulla or in the excreta, an autogenous vaccine should be administered. In other cases chief reliance must be placed in the internal administration of arsenic, which is more of a specific in this than in any other skin affection. It must not, however, be looked on as an absolutely unfailing remedy. It should be given in the form of Fowler's solution, beginning with a dose of three drops, gradually increased to five, seven, eight, ten, and even larger doses, three times a day. When arsenic fails, quinine is often beneficial; in other cases opium is the most efficient internal remedy. Phosphorus, ichthyol, and belladonna are all occasionally usefui. The local lesions must be treated on general principles, the itching being relieved by one or other of the methods already described. If the bullæ are very large and tense they may be pricked with a sterilized needle, and afterwards dressed with boric-acid ointment or carron oil, or weak lead lotions or ointments; if the skin around them be much inflamed, cooling ointments will give relief. In pemphigus foliaceus little 
can be done. None of the many drugs that have been tried internally has yielded satisfactory results. Local treatment should be limited to the application of a bland ointment or paste to prevent evaporation and cover the denuded areas. A vaccine of a culture of staphylococci found in the blebs has been injected without conclusive results. Fever and other constitutional disturbances accompanying the skin affection must be treated on general principles. A leading indication in pemphigus foliaceus, as in all forms of pemphigus, is to support the strength by suitable food.

\section{HERPES}

Herpes may be taken as the type of a skin lesion of nervous origin, as its connection with certain abnormal conditions of the nerves supplying the affected area can be clearly established. The term "herpes," in strictness, denotes merely a particular lesion which may be an incidental phenomenon in a variety of diseases, or it may be the expression of a definite morbid state, or, as some (Erb, Landouzy, Brocq, Wasiliewski) believe, the exanthem of a specific fever.

The lesion itself is a cluster of transparent vesicles varying in size from a pin's head to a pea, and in number from two or three to twenty or more, seated on an erythematous patch, and surrounded by a narrow red zone. The eruption is almost always preceded by a feeling of heat and tension, sometimes itching, in the part about to be attacked. The life-history of the individual lesion comprises four stages: (1) A slightly red spot appears on the skin; (2) soon serous effusion takes place under the epidermis, and vesicles are formed ; (3) the vesicles become opaque-occasionally purulent-shrivel up, and form yellowish-brown crusts which, (4) after some days, become detached, usually leaving no scar, but a brownish stain, which slowly fades and disappears. These 
four phases in the evolution of the lesion are named by Brocq congestive, vesicating, desiccating, and macular. The whole process occupies from a week to a fortnight. On mucous membranes the lesion runs a somewhat different course. Owing to the macerating action of the secretions the vesicle is quickly reduced to a whitish pulp, which, when the eruption is extensive, gives the part the appearance of being covered with false membrane. When the sodden epithelium becomes detached, roundish excoriations are seen underneath. These may be scattered irregularly about, or, intersecting each other, may form largish ulcers with wavy borders. Healing, as a rule, takes place without scarring.

Two distinct types of herpes may be ricognized: one which I propose to call irritative or symptomatic herpes (herpes febrilis); the other a definite morbid process which manifests itself by a herpetic eruption following certain definite lines of distribution-herpes zoster, or zona, or shingles.

Irritative herpes chiefly affects the face and the genital organs-hence the herpes facialis, or labialis, and herpes progenitalis (or, as I prefer, with Besnier, to call it, genitalis) of authors; but it may appear on the neck or the buttocks, and H. G. Adamson ${ }^{1}$ has recorded four cases in children in which the fingers were attacked.

Symptoms. - In the face and the genital organs the process is essentially the same; the only difference between them is that the lesions, and also to some extent the symptoms, are modified by the anatomical relations and the functions of the parts affected. In the face the eruption most frequently comes out on the lips, especially the lower, and about the mouth; but any part of the face below the forehead may be invaded. Nor are the conjunctivæ and the mucous membrane of the mouth and throat exempt from attack. The lesions pass through

1 Brit. Journ. Derm., 1909, xxi. 323. 
the four stages of evolution which have already been described. The attack usually occurs in the course of some febrile disorder-catarrh of the respiratory passages, pneumonia, scarlet fever, typhoid fever, influenza, diphtheria, cerebro-spinal meningitis, malaria-and is generally ushered in by a sensation of chill, or even actual shivering. In scarlet fever and in diphtheria it is essentially a phenomenon of the acute stage. ${ }^{1}$ Herpes facialis used to be considered a sign of " crisis" in acute febrile diseases, and in the case of pneumonia in particular it was looked upon as of good augury for the favourable issue of the illness. It is now, however, regarded as a simple incident in the general morbid process without any special significance. It is, in short, merely symptomatic of feverishness with shivering. In some persons herpes is produced by local irritation; hence the frequency with which the upper lip is the seat of an eruption after an attack of nasal catarrh. In many persons herpes of the lip shows a marked tendency to recur.

In the genitals the favourite points of attack in men are the prepuce, especially its internal surface, the sulcus, the glans, and the meatus; and in women the labia and the cervix uteri. The symptoms are in proportion to the severity of the lesions. In men the eruption is usually discrete, and, with the exception of the burning and itching which it causes, but little inconvenience is felt by the patient. If neglected, however, and especially if irritated, as by frequent coitus or the application of caustics, the ulceration may spread, and the glands in the groin may become enlarged and painful. In women the eruption is apt to become confluent, and in some cases not only the vulva, but the perineum, the inside of the thighs, and the mons Veneris may be invaded. The labia majora and minora

1 J. D. Rolleston, Brit. Journ. Derm., 1910, xxii. 310. 
and the mucous lining of the vagina become immensely swollen, and covered with macerated epithelium, which, as it separates, leaves extensive excoriations. There is an offensive muco-purulent discharge, and the pain on movement is so great that the patient can hardly walk. The itching and burning are almost unbearable. Enlargement of the inguinal glands is a frequent complication.

Genital herpes is more common in men than in women. It is sometimes symptomatic, occurring in the course of some febrile disorder, such as pneumonia; but most commonly it appears to be the result of local irritation. Ravaut and Dané ${ }^{1}$ found that, in a number of cases in which it was associated with nervous symptoms, there was modification of the cerebro-spinal fluid. In men the eruption is sometimes preceded by a gonorrhœa or a venereal sore, and it is apt to recur at frequent intervals after sexual intercourse (especially, according to Brocq, after intercourse with different women), the passage of an instrument into the urethra, or other local irritation, or after fatigue, or over-eating. The tendency to recurrence may last for years, but sometimes, as pointed out by Berkeley Hill, ceases under the alterative influence of a severe intercurrent illness. In women, genital herpes is often brought on by the first attempts at sexual intercourse. It may also be the result of irritating discharges (leucorrhœa, gonorrhœa), or it may be related to the menstrual function.

Diagnosis.-On the face the affection may sometimes be mistaken for impetigo, but the acuteness of its course, its limited distribution, and the facts that it is not autoinoculable, and that it does not spare the lips, as impetigo usually does, will serve to distinguish it. In genital herpes the diagnosis presents no difficulty if the case is seen before the characteristic vesicular eruption has

${ }^{1}$ Ann. de Derm. et de Syph., June, 1904, p. 481. 
become obscured by the violence of the inflammatory process. If ulceration is extensive, and especially if there is much suppuration, it may be impossible at first to distinguish genital herpes from soft sores. The latter, however, are multiple, secrete little liquid, have a flattened and fouler base, and excavate more deeply. Time will also help to clear up the question, the lesions of herpes disappearing, as a rule, in a few days, while soft sores are much slower in healing. If positive proof be required, the test of auto-inoculation may be applied. From true chancre, genital herpes can usually be distinguished without difficulty by the absence of induration, the multiplicity, irregular form, and small size of the ulcers, the intense burning and itching which they cause, and the less considerable and more transitory gland-enlargement. It is not uncommon, however, according to Fournier, for a chancre to develop in the midst of a premonitory eruption of herpes. For the diagnosis of herpes from eczema, see Chap. xI.

Herpes zoster.-Zona, or shingles, is an affection characterized by the eruption of clusters of vesicles seated on an erythematous base, not along the course of one or more peripheral nerves, as used to be taught, but in the region of distribution of one or more of the posterior spinal roots of the skin. ${ }^{1}$

Symptoms. - The intercostal variety of herpes zoster, being by far the most common, may conveniently be taken as a type in describing the disease. The appearance of the eruption is usually preceded by pain of neuralgic character and tenderness over the area of distribution of the nerve or nerves corresponding to the part of the

${ }^{1} \mathrm{Head}$, as the result of careful investigation ("On Disturbances of Sensation, with especial reference to the Pain of Visceral Disease,"-Brain, Parts 1 and 2, 1893), found that the areas occupied by the eruption of herpes zoster corresponded with those which become tender in visceral disturbances. See also Head's article in Allbutt's "System of Medicine." 
surface about to be attacked. Sometimes there is also slight constitutional disturbance. The eruption invariably first appears at certain points, from which, in most ca ses, it spreads. However extensive the area involved may be, these points are always those where the affection is at its maximum intensity (Head). As a rule, though by no means without exception, the neuralgic pain ceases on the appearance of the eruption, but the lesions cause considerable smarting and tension, and there may be severe pain owing to neuritis of the implicated nerve. Children seldom suffer much pain; as a rule they complain more of itching. In old people, however, pain is often most persistent and severe. The eruption shows itself in the form of erythematous patches, which can be made to disappear on pressure. They are more or less oval in outline, with their long axis parallel to the underlying nerve. They come out in crops, beginning, as a rule, nearest the corresponding nerve centre, and are scattered at irregular intervals along the track of the nerve with which they are in relation, especially at the points where its twigs pierce the fascia, or are distributed in the skin. The number of lesions varies from two or three to twenty or thirty. The full development of the eruption generally occupies about a week. In a short time the surface of the red patches becomes studded with papules, which are quickly transformed into vesicles. These are grouped in clusters to the number of about ten, or even twenty, on each patch. The vesicles are sometimes discrete, sometimes confluent, forming irregular bullæ; but the edge of the erythematous patch on which they rest is always visible as a red zone around the base of each cluster. Most of the lesions go through the regular phases of evolution already described; but some of them may abort, while others, instead of drying up in the ordinary way, may burst and give issue to a fluid which by and by forms 
yellowish or brownish crusts. Occasionally hæmorrhage takes place into the vesicles, and in such cases little ulcers are apt to form under them. These may give rise to permanent scars, which are sometimes whiter than the surrounding skin, sometimes pigmented, or they may be white in the centre and pigmented at the circumference (Brocq). In some cases cheloid develops in the scars. It is well to make a point of warning patients as to the possibility of such lesions being left. In elderly or weakly subjects the lesions of herpes zoster sometimes assume a gangrenous character. Enlargement of the glands in the neighbourhood of the lesions is not uncommon.

The eruption is, in the great majority of cases, unilateral, the right side being far more often affected than the left. Sometimes it comes out on both sides, though at different levels. In certain rare cases, however, the lesions form a complete girdle round the body: hence zona. Occasionally, while remaining unilateral, the lesions may overstep the middle line in front for one or two inches. James Mackenzie ${ }^{\mathbf{1}}$ has shown that the terminal branches from neighbouring intercostal nerves frequently cross each other.

All the different phases of herpes zoster may be seen in the same patient at one time. The total duration of the disease till the separation of the scabs is from a fortnight to three weeks, but in severe cases it may be much longer. One attack appears to confer immunity; but this rule is not absolute, Kaposi having seen no fewer than eleven recurrences in one of the patients under his care.

Herpes zoster is most frequent on the trunk, but does not spare any part of the body, though it is extremely rare below the knee. A case in which herpes

1 "Herpes Zoster and the Limb Plexuses of Nerves," Journ. of Path. and Bacter., Feb., 1893, p. 332 et seq. 
zoster limited to the foot followed a twist of the ankle has been recorded. The process is everywhere the same, but on the head and limbs the lesions are distributed in more or less irregular lines, and have not the girdle character which is seen on the trunk. On the face the eruption follows the ramifications of the fifth nerve, especially the supra-orbital branch and the ophthalmic division. In the former the inner third of the frontal region is the favourite seat of the disease. The lesions extend upwards in vertical lines, or spread out fanwise from the supra-orbital foramen and extend on to the scalp. Sometimes the mouth is attacked, especially the tongue; but occasionally, as Head has shown, the lesions on the tongue occur independently of any eruption on the lips or the palate. The mucous membrane of the nose also may be involved, and cases have been reported in which the eruption is limited to the nasal and buccal mucosæ. In ophthalmic zoster, especially when the nasal branch is implicated, severe pain around the orbit and photophobia are prominent symptoms, and eye lesions (conjunctivitis, keratitis, iritis) are usually caused, which in rare cases lead to permanent mischief (posterior synechiæ, deformity of the pupil, and even amblyopia and atrophy of the papilla). This variety of herpes zoster is also frequently followed by indelible scars. Among other parts liable to be the seat of herpes zoster may be mentioned the nape of the neck and the occiput, and the skin supplied by the various branches of the superior cervical plexus. The eruption in this case spreads over the scalp along the branches of the occipital nerve. The arm, the thigh, the buttock, and the genitals are also liable to be attacked; in fact, it may be said that wherever there are cutaneous nerves, there herpes zoster may break out. The musculo-spiral and sciatic nerves are sometimes affected. Zoster is, however, rare on the 
forearms and legs, and all but unknown on the hands and feet.

Etiology and pathology. - The affection is common at all ages, and there does not seem to be any marked difference in the relative proclivity of the two sexes. Nearly all authorities are agreed that chill may be an exciting cause of zoster, and the epidemics of the disease that have been reported are probably to be explained by the influence of the weather. The cold probably causes neuritis, which in turn gives rise to zoster. Arsenic, which, according to Hutchinson, sometimes causes herpes zoster, no doubt acts in the same way. The association of the disease with croupous pneumonia, pleurisy, tuberculosis, cancerous and other tumours, syphilis, and various inflammatory lesions, may also be explained by the irritation to which the peripheral nerves, or their spinal roots or ganglia, are subjected when involved in such processes. In short, whatever causes neuritis-cold, injury, poison, or long-continued irritation-may also induce zona.

The lesion of the nerve may be in any part of its continuity, from its origin in the spinal cord to its peripheral end. Bärensprung ${ }^{1}$ first demonstrated that in most cases of herpes zoster there is interstitial neuritis of the posterior ganglion and of the trunk of the nerve issuing therefrom which is distributed to the affected area of the skin. In some cases the lesion is in the posterior spinal root between the cord and the ganglion, or in the posterior columns of the cord. Dubler found zoster associated with peripheral neuritis without any trace of central disease; and cases have been reported (Curschmann, Eisenlohr) in which the disease was apparently caused by multiple neuromas in the course of the

${ }^{1}$ Charité Annalen, Bd. ix. 2; Bd. x. 1; Bd. xi. 2. Danielssen seems to have been the first to observe (in 1857) that in a case of intercostal zoster the corresponding nerve was greatly congested. 
affected nerves without any central change. The lesion may be due to hæmorrhage as well as to inflammation. Herpes zoster sometimes occurs in association with locomotor ataxy. Willmott Evans ${ }^{1}$ holds that in no inconsiderable number of cases a meningitis is the startingpoint of the herpetic eruption. In his experience it is rare in connection with tuberculous meningitis, but more frequent in the non-tuberculous basal meningitis of children. It also occurs as a consequence of meningitis from extension of disease of the middle ear. The characteristics of herpes zoster resulting from meningitis are, according to Willmott Evans, a tendency for the eruption to be bilateral and to be more persistent than usual. The fact that meningitic herpes is so often bilateral may, he thinks, account for the belief, so widespread among the public, that when the disease encircles the body it is fatal. He points out that the herpes arising from meningitis always corresponds to the distribution of a nerve root and not to the distribution of a nerve, except when they are practically identical, as in the dorsal region of the cord.

Herpes may also be bilateral when it is of syphilitic origin, as in a case of Dr. Ogle's, illustrated in Plate 7 . The patient, a boy of 6 , was admitted to St. George's Hospital with paretic symptoms which were provisionally attributed to tubercular meningitis. Eleven days later the herpetic eruption came out on both sides at the same time. Congenital syphilis having been suspected, treatment by mercurial inunction had already been begun. The herpes improved rapidly, and at the end of ten days had practically disappeared, except at one point. The general condition also improved.

Wasiliewski ${ }^{2}$ rejects the theory of the nervous origin

1 Brit. Journ. Derm., 1900, p. 83.

2 " Herpes Zoster und dessen Einreihung unter die Infections. lsrankheiten." Jena, 1892. 


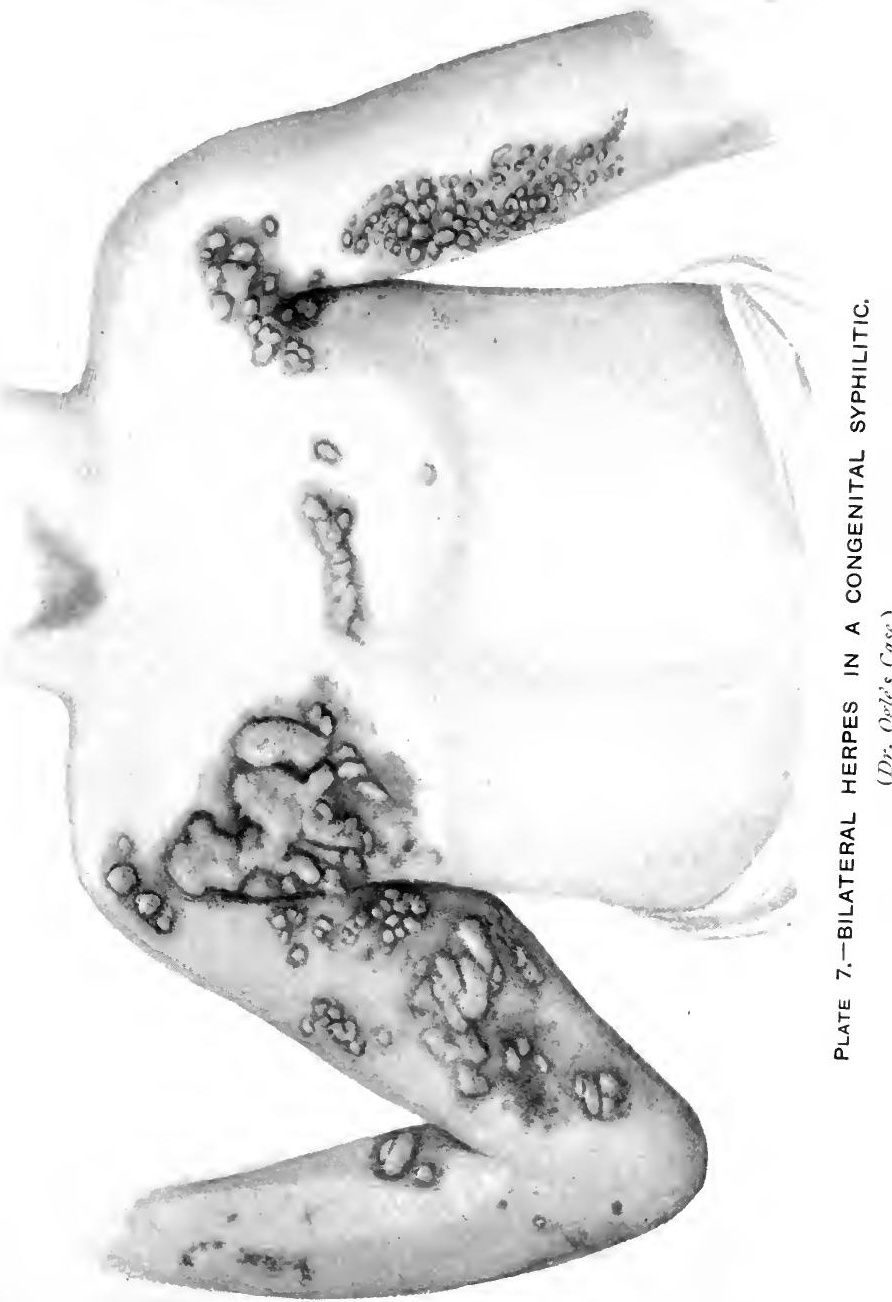



of herpes zoster, on the ground that the clinical phenomena correspond closely with those of infectious fevers. His view is based on 274 cases gathered by collective investigation by the Medical Society of Thüringen. Wasiliewski thinks the distribution of the eruption is better explained by the blood-stream than by nerve ramification. He points out that in some cases no nerve lesions can be found. Pfeiffer ${ }^{1}$ has attempted to prove that the distribution of the lesions in herpes zoster is determined by the arterial supply; but, as pointed out by J. Mackenzie, ${ }^{2}$ all the cases he gives show clearly the distribution of the eruption in regions supplied by definite spinal nerves.

The lesions of herpes zoster are produced by a peculiar process of epithelial degeneration, known as ballooning, which is also seen in the epithelium of the rete in such diseases as variola, varicella, etc. The cells become rounded, lose their prickles, a vacuole appears in its centre, gradually becoming larger, swelling the cell and causing both protoplasm and nucleus to lose their distinctive staining reactions and to degenerate. At the same time considerable leucocytic exudation takes place into the papillæ, and the leucocytes ultimately escape into the epithelium between its degenerated cells. According to Haight, of New York, the nervous filaments going to the affected parts are profoundly altered. They are swollen, and their neurilemma is full of small nucleated cells. The connective tissue around the nerves is infiltrated with leucocytes, and the nerve tubes themselves are abnormal in appearance.

Diagnosis.-Herpes zoster has to be distinguished from eczema, erythema multiforme, dermatitis herpetiformis, and irritative herpes (herpes facialis and

1 “Die Verbreitung des Herpes Zoster längs der Hautgebiete der Arterien." Jena, 1889.

${ }^{2}$ Loc. cit., p. 339. 
genitalis). From eczema it can, as a rule, easily be distinguished by the fact that the vesicles dry up and do not keep up a continuous "weeping," and, moreover, are distributed in the area of a particular nervous supply. From erythema multiforme, dermatitis herpetiformis, and irritative herpes, zoster is clearly distinguished by its unilateral character, by the distribution in one or more nervous territories, and by the neuralgic pain which precedes and sometimes accompanies it. The history is also an important diagnostic point, zoster being a disease which, as a rule, attacks a person only once. About the genitals it may not be easy to distinguish zoster from irritative herpes. The presence of pain of a neuralgic character is, however, a certain sign that it is the former we have to deal with. The neuralgic pain . may at first suggest pleurisy, but the course the affection runs points conclusively to its true nature.

Prognosis in herpes generally.-Both in irritative herpes and in zoster the prognosis is favourable. The disease runs a regular course, and tends to spontaneous recovery in from a fortnight to a month. If ulceration has been severe, and especially if gangrene has occurred, the lesion will take a considerable time to heal. Weakly people, particularly if advanced in years, may be exhausted by the severity of the process and by the pain which accompanies the eruption and may persist long after its disappearance. In a case of zoster of the ophthalmic division, death has been known to occur as the result of embolism of the ophthalmic vein (Brocq). Impairment of vision has also been known to follow this variety of zoster. Genital herpes may recur again and again if irritation is kept up; but patients suffering from zoster may be comforted with the assurance that it is practically certain that they will not be troubled by the disease again.

Treatment of herpes.-In irritative herpes 
the only treatment usually required is the application of soothing and anti-pruritic lotions or ointments, or protection of the affected surface by sprinkling with powder (oxide of zinc, stearate of zinc, starch, subnitrate of bismuth, talc, etc.), or by muslin bags. When the genitals are the seat of the eruption the parts must be kept scrupulously clean, and the surfaces should be kept apart with a piece of lint steeped in boric acid or calamine lotion. Black wash is a particularly useful application in genital herpes. If the patient be of gouty constitution, appropriate medication will be required.

In the treatment of herpes zoster the chief indication is the relief of pain, which is frequently acute. For this purpose menthol is often useful, but subcutaneous injections of morphine may sometimes be required. It is important to protect the lesions from friction and to keep the parts warm; they should be dusted with a protective powder, such as oxide of zinc and bismuth, with the addition of a small quantity of morphia if necessary, or painted with collodion; they should then be covered with a thick layer of cotton-wool. Internally, both iodide of potassium and antipyrin in doses of gr. $\mathrm{x}-\mathrm{xv}$ are useful in allaying the neuralgic pain, and tonics such as quinine, iron, and strychnine are generally beneficial. If the patient is in a low condition of health, cod-liver oil and feeding-up are indicated. If the pain is very severe, the application twice daily of ethyl chloride to the seat of the disease in the spine and chilling of the lesions are most valuable measures (Howard Morrow); or the continuous current may be applied along the course of the nerve. Division, stretching, or resection of the nerve has been known to relieve the pain in severe old-standing cases affecting the supra-orbital nerve. 


\section{CHAPTER IX \\ NEUROSES OF THE SKIN (Concluded)}

\section{Sclerodermia}

Sclerodermia is a disease characterized by hardening of the skin, either diffuse or circumscribed. The latter condition is usually known as morphœa ${ }^{\mathbf{1}}$ (p. 183).

Symptoms.-Diffuse sclerodermia is very rare. It occurs in two forms-as an infiltration and as an atrophy of the skin. In either case the affection often follows chill, and is sometimes ushered in by pains in the joints. A large part or the whole of the skin may be affected almost suddenly, or the disease may spread so slowly that it is some time before it is noticed. Some part of the upper half of the body is, as a rule, first attacked, and the limit of the disease is often indicated by a line of demarcation invisible to the eye but faintly perceptible to the touch. The distribution is always symmetrical. The affected skin becomes rigid, tense, and hard, like that of a frozen corpse, but withont the coldness, its temperature being only a degree or two below normal (Crocker). It does not pit, nor can it be pinched up ;

1 As has been shown by Colcott Fox, in an interesting paper entitled "Note on the History of Sclerodermia in England" (Brit. Journ. Derm., 1892, p. 101), what is now known as sclerodermia was described by Willan under the name of "ichthyosis cornea," by Addison under that of " true cheloid," by Wilson and others under that of "morphœa," and by Gibert under that of "lèpre vitilige." Much light has been thrown on the nature and pathology of the condition by Crocker, whose description of it has mainly been followed here. 
the joints which it covers are immoblized, as if swathed in a stiffened bandage; the features are drawn, and the face becomes fixed into an expressionless mask; the chest walls are so tightly bound that breathing is seriously hindered. Sometimes the mucous membranes (mouth, pharynx, larynx, vagina) are attacked. At first sight the skin often does not seem to be much altered in appearance, but it is whiter than normal, and, on looking closely at it, the natural lines are seen to be obliterated. Erythematous patches, with telangiectases and mottling from scattered pigmentation of varying hue, are often present. Sensation is usually unaltered. The skin is dry, owing to diminution or suppression of the sweat and sebaceous secretion, and itching is sometimes troublesome. The general health is often not appreciably affected, but the patients are extremely sensitive to cold.

In the atrophic form the shrinking of the skin is always preceded by an œdematous stage, in which pitting is produced with some difficulty, as if the finger were pressed into a bladder of lard (E. Wilson). After this has lasted some time, the skin shrinks and becomes ivory-white in colour. The distribution is symmetrical, as in the infiltrated form, but, as a rule, not so extensive, only the face and upper limbs being attacked in many cases. The skin is stretched tightly over the bones, pinching the features like those of a corpse, shrivelling the limbs, fixing the joints, and distorting the hands. The skin is so tightly drawn over the underlying parts that ulceration occurs on slight provocation.

In the infiltrated form the tendency is to gradual softening of the skin and recovery, with occasional relapses from taking cold or less obvious causes. The atrophic form is more chronic, the condition often persisting for years, and sometimes ending in death from exhaustion; the stiffening of the skin may, however, 
disappear, but the shrunken tissues never recover their normal state, and some deformity may be left. The affection runs a more acute course in children than in adults.

Etiology and pathology.-Of the causation of sclerodermia nothing is known, but nervous depression and privation are believed to be predisposing factors. It is not infrequently associated with acute rheumatism, and cardiac lesions are sometimes present. Meneau believes that the disease may be secondary to various other processes-cardiac œdema, thickening of varicose veins, scleroses due to traumatic lesions of nerves, ataxia, and elephantiasis. Sclerodermia and vitiligo may coexist in the same person, and a case has been reported by Haushalter and Spillman which, it was suggested, may be a connecting link between the two diseases. Tedeschi reports a case in which sclerodermia was associated with neuritis, the two affections, in his opinion, being interdependent and due to a common cause. The disease is much more frequent in the female sex than in the male. No age is exempt. It has been suggested by Gustav Singer and other authors that myxœdema, Graves's disease, and sclerodermia are loosely allied affections, all springing from the same cause, namely, a lesion of the thyroid body. Enlargement of the pituitary gland has been found in association with sclerodermia. 1 Henry H. Whitehouse ${ }^{2}$ calls attention to the fact that in five cases of diffuse sclerodermia three gave a strongly positive Wassermann reaction, one was faintly positive, and one was negative; the two latter had been under prolonged anti-syphilitic treatment. He suggests that, should these observations be confirmed, syphilis may prove to be one of the etiological features of the disease. The anatomical conditions are due to obstruction of the circulation-arterial, venous, and lymphatic

${ }^{1}$ Hektoen, Centralbl. f. allg. Path., viii. 17.

${ }_{2}^{2}$ Trans. Amer. Derm. Assoc., 1909, p. 77. 
-by narrowing of the vessels consequent on the pressure of layers of cells which surround them like a sheath ; in some cases further narrowing has been caused by concentric hypertrophy of the inner and middle coats of the vessels. How this accumulation of cells is caused is not known; it does not, however, appear to be the result of inflammation. The most probable cause of sclerodermia is defective innervation, the source of which must be situated high up, not improbably in the vasomotor centre (Crocker).

Prognosis.-From what has been said it will be gathered that the prognosis is much more favourable in the infiltrated than in the atrophic form.

Treatment.-The indications are to guard the patient against cold, to.improve nutrition by cod-liver oil, etc., and to stimulate the circulation in the affected parts by massage and galvanism. Arsenic is sometimes useful. Singer suggests thyroid feeding.

\section{MoRPHEA}

Morphœea, or circumscribed sclerodermia (p. 180), occurs in the form either of patches or of bands, the former being more common in adults and the latter in children.

Symptoms.-The patches, which are generally level with the surrounding skin, though sometimes slightly depressed, are irregular in outline and white or creamy in colour; the edges are streaked with small dilated vessels, making a pink or violet border. They occur most frequently on the limbs, especially the lower, on the trunk, especially on the breasts, and on the face; they are not, as a rule, symmetrical, and in their distribution they sometimes follow the course of a nerve distribution in the sense that herpes zoster does (p. 171). The affected skin is not adherent to the underlying tissues; on pinching it up it feels like parchment or 
stiff leather (Crocker). The patches may remain stationary for a long time, or they may gradually extend, small atrophic spots appearing in their neighbourhood, and in time coalescing with them. The condition causes no symptoms except itching and suppression of sweat secretion in the patches. It may last for years, fresh patches forming while some of the older ones disappear. Bands usually cause grooving of the skin, owing to their being adherent to the underlying structures; sometimes they form ridges on the surface. They often have the appearance of a cicatrix.

Telangiectases, patches of pigmentation and atrophic striæ, are frequently intermingled with the lesions of both forms of morphœa.

Etiology and pathology.-The affection is more common in females than in males. It may occur at any age after infancy. The neurotic temperament and nervous depression from any cause are predisposing factors. The determining cause sometimes appears to be local irritation, as by garters, the pressure or friction of clothing, stays, blows, etc. The pathology is essentially the same as that of diffuse sclerodermia-namely, local obstruction to the blood supply, probably dependent on defective innervation. Sequeira, ${ }^{1}$ who had two cases of frontonasal morphoa at the Skin Clinic of the London Hospital in two successive weeks, holds that morphœa is due not to an affection of the peripheral nerves, but probably to a ganglionic lesion, acting by vaso-motor or trophic influence. Cases of a mixed nature have been recorded, a primary diffuse sclerodermia or lupus erythematosus being followed by the development of typical morphœa patches. In one of Sequeira's cases a patch of lupus vulgaris developed on the tip of the nose ; the patient, a woman of 00 , had had a "silver streak" on her forehead as long as she could remember.

1 Brit. Journ. Derm., 1911, xxiii. 40. 
Diagnosis.-Morphœa is distinguished from leucodermia by the absence of hardness of the integument in the latter.

Prognosis and treatment.-As a rule, morphœa tends to spontaneous recovery, though the bands are more persistent than the patches. Local treatment, except gentle massage, generally does more harm than good. Brocq, however, has been successful with electrolysis. The improvement of the general tone of the circulation by massage is likely to assist the curative efforts of nature.

\section{Morphea Guttata (“White-Spot Disease ”)}

Under these names has been described a rare but fairly distinct clinical variety of morphœa occurring in girls and women. The parts chiefly attacked are the neck, the shoulders, the upper parts of the back and chest, and the breasts. The characteristic features of the affection, as set out by Drs. Montgomery and Ormsby, ${ }^{1}$ are, besides the location of the lesions, their dense whiteness, their sharpness of outline, making them look as though they were let into the healthy skin, their small size, and their tendency not to coalesce even when grouped closely together. Nor do they show the coloured border exhibited by morphœa. In one of the two cases observed by Montgomery and Ormsby some of the lesions, after a few years, were transformed into typical areas of morphœa, and in all the ten cases reviewed the last atrophic stage appeared to be identical with the same stage of morphœa and with macular atrophy of the skin, save that the scars were small and remained discrete.

\section{Sclerodactylia (Acrosclerodermia, Progressive ScLerodermia)}

Symptoms.-This form of sclerodermia usually begins in the fingers; but the nose or the auricles may 'Trans. Amer. Derm. Assoc., 1906, p. 88. 
be starting-points. At first it resembles Raynaud's disease, there being darting pains, with "dead fingers," or blueness of the extremities. In rare cases there is profuse sweating, and blebs may form. The fingers gradually waste; the skin atrophies, and is attached to the bones, and becomes greyish in colour. As the result of atrophy from the periphery a tapering digit is produced, "like an elongated radish," to use Sequeira's simile. The process slowly extends to the forearms. The nails atrophy and become claw-like. Callous ulceration or necrosis, with absorption of the bones, leads to spontaneous amputation. Occasionally the face is attacked; the features are fixed, mastication and swallowing become difficult, the tongue and the larynx become involved, and there may be extension to the trunk. The pigmentation which is invariably present may not be limited to the sclerosed areas.

Prognosis and treatment.-The disease runs a slow course, and death usually occurs from intercurrent disease. Little can be done in the way of treatment beyond attending to the complicating affections as they arise. ${ }^{1}$

\section{Ainhum}

This affection may be referred to here because, like sclerodactylia, it is characterized by the spontaneous amputation of a digit-in this instance usually the little toe, but sometimes the fourth, and very rarely the second, or the hallux. Confined to the dark races, it was first reported from the West Coast of Africa, but is now known to have a wide distribution in tropical countries. It usually begins as a furrow around the junction of the toe with the foot. The distal portion of the toe becomes swollen and odematous as the result of constriction, and in many cases an ulcer forms on

${ }^{1} \mathrm{James}$ H. Sequeira, “Diseases of the Skin." London, 1911. 
the inner side of the groove. Finally, after from two to many years, the toe falls off, or is knocked off. Eyles and Moreira suggest that the process originates in irritation to the skin caused by walking barefoot, setting up proliferation, endarteritis, and rarefying osteitis. The disease may be cut short by incision of the contracting band; in advanced cases, amputation is the only course.

\section{Lichen Planus}

The term "lichen" is often loosely used to designate a number of diseases which have nothing in common but the fact that at some time or another the eruption has been papular in character. Thus lichen simplex and lichen agrius are really varieties or phases of eczema. Lichen strophulosus is a form of miliaria occurring in infants. Lichen tropicus, or prickly heat, is also a form of miliaria; and lichen urticatus has already been described as a form of urticaria affecting children. Accepting Hebra's restriction of "lichen" to conditions characterized by papules of typical form, which persist as such throughout their whole course, I recognise only one form of lichen, i.e. lichen ruber planus or, more shortly, lichen planus. The affection termed by Kaposi "lichen scrofulosorum " is described among tuberculous diseases (p. 433).

Lichen planus was first described by Erasmus Wilson, and is still accepted by leading dermatologists as the type of the group of affections designated by the name of "lichen." The condition described by Hebra under the name of lichen ruber is identical with Wilson's lichen planus, as from personal observation of the cases on which both these distinguished men based their descriptions I am able to testify. Kaposi describes two forms of lichen ruber, namely, lichen ruber planus and lichen ruber acuminatus. In my opinion, however, 
these names represent two distinct diseases, the latter being the same as pityriasis rubra pilaris, or Devergie's disease, under which names it is described (see p. 204). The view that lichen ruber acuminatus is identical with pityriasis rubra pilaris receives strong confirmation from the similarity of the process in the two conditions, as shown by the histological researches of Lukasiewicz ${ }^{1}$ and Max Joseph. ${ }^{2}$

Symptoms.-Lichen planus is ordinarily characterized by an eruption of small, irregularly shaped papules (Plate 8), flat on the top and sometimes "umbilicated." As to how this umbilication is produced there has been much speculation. The most probable theory seems to me to be that propounded by Radcliffe-Crocker and accepted by Fordyce: the thickened, horny layer sinks into the rete in the shape of a funnel, and the elimination of the "funnel" by exfoliation produces the depression. An admirable plate accompanying Fordyce's remarks on this subject ${ }^{3}$ shows that it is in the area where hyperkeratosis is most marked that the rete is depressed. The papules, of a violet or lilac tint, have a little scale in the centre which at first sight, especially if looked at sideways, makes them appear as if they were vesicating. At first the papules are irregularly scattered about, but they soon group themselves in lines, curves, or rings (annular lichen planus). If one of the lesions becomes annular the others do so also; but Whitfield ${ }^{4}$ has observied that on the glans penis they frequently assume this form without the rest of the eruption undergoing the same evolution. The favourite situations are the flexor surface of the wrists, the popliteal space, and the limbs. They do not, however,

1 Arch. f. Derm. u. Syph., Bd. xxxiv., 1896, p. 163 et seq.

2 Ibid., Bd. xxxviii., Jan., 1897.

3 Trans. Amer. Derm. Assoc., 1909, p. 57.

'Brit. Journ. Derm., 1910, xxii. 130. 


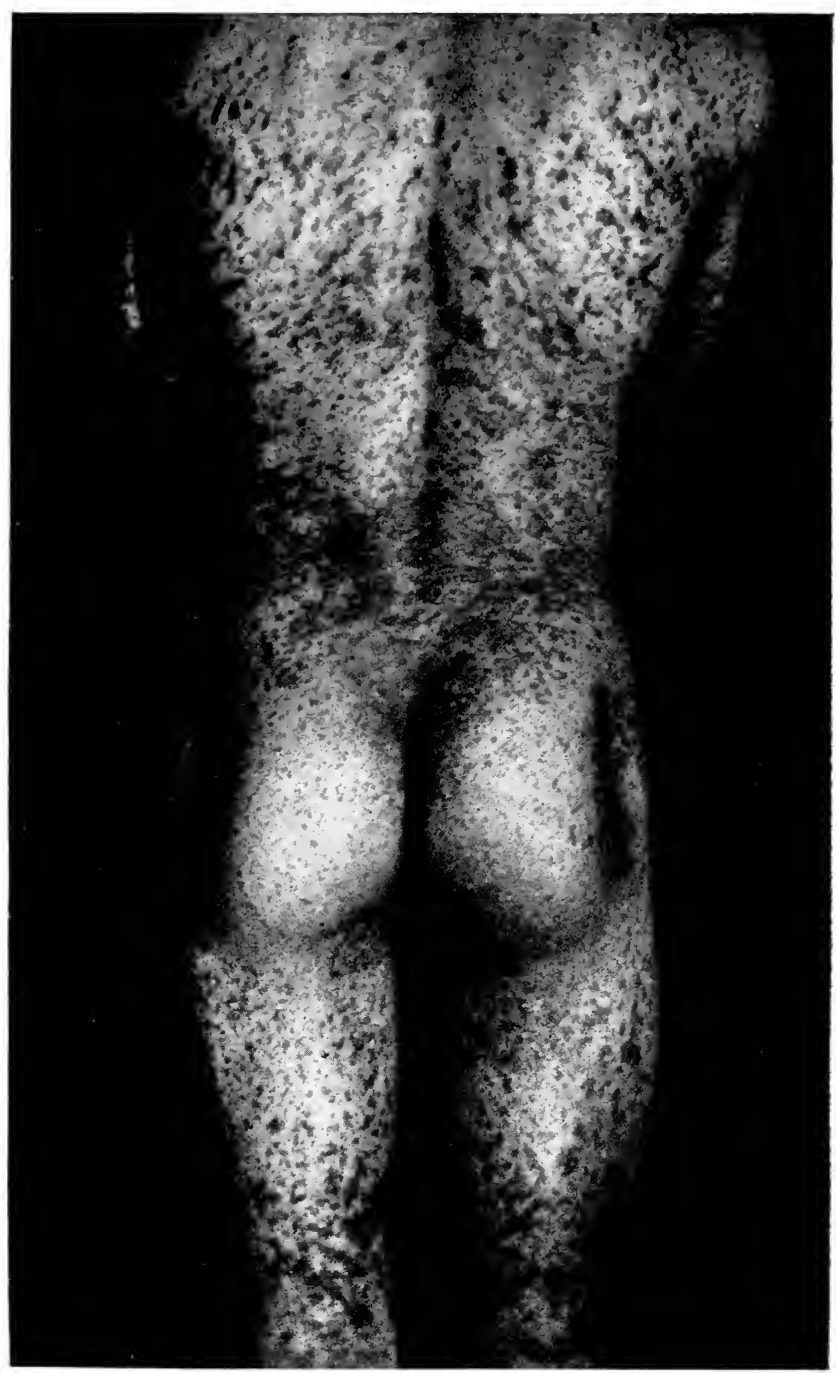

Plate 8.- lichen planus. 
spare the trunk of the body, and they are seen on the mucous membrane of the lips and tongue; in some cases umbilication has been observed in lesions of the mucosa. In the palms of the hands the papules feel like small corns. On the trunk they generally lie very close together, like the pieces of a mosaic; the older papules in the middle become flattened and usually of a sepia colour, whilst a new crop springs up around them, producing something of the effect of a dark stone set in pearls (Kaposi). In acute cases, however, the lesions are red, and if the affected papillæ are replaced by sclerotic tissue (lichen atrophique, p. 191) they may be ivorywhite. Occasionally the lesions of lichen planus follow the distribution of a nerve, and appear in long streaks (Plate 9). Galloway ${ }^{1}$ has reported a case in which the eruption corresponded to the distribution of the small sciatic nerve, and Stephen Mackenzie has observed it around the body like zoster. In a case described by. Gunsett $^{2}$ the rows of lesions corresponded with the distribution of the subcutaneous veins of the thighs, and for the most part were disposed like the beads in a rosary. This necklace-like arrangement. of the papules is the lichen ruber moniliformis of Kaposi, but it occurs too seldom to deserve recognition as a distinct variety oi lichen planus. A case of unusual distribution has been shown by Sequeira, ${ }^{3}$ the eruption being almost limited to the middle line of the back between the shoulders and spreading out below in the loin. Except for a few spots on the left thigh, the fronts of the forearms and legs and the thighs were quite free from lesions.

In course of time large areas of skin may be invaded, and the integument then has a uniform dark-red colour; it is distinctly thickened, and feels rough to the touch.

${ }^{1}$ Brit. Journ. Derm., 1896, viii. 436.

${ }^{2}$ Arch. f. Derm. u. Syph., May, 1902, p. 179.

${ }^{3}$ Brit. Journ. Derm., 1911, xxiii. 119. 
At this stage the disease has more or less the appearance of psoriasis, but without the general scaliness characteristic of that affection. In the adult, pustules are never mingled with the papules, but in some cases vesicles, and more rarely bullæ, appear. Whitfield ${ }^{1}$ has collected the reports of 17 cases, in addition to one of his own, in which bullæ were present. In at least 9 of them the bullæe occurred before arsenic was administered, so that they could not be attributed to the drug. In 8 cases they did, and in 3 they did not, correspond exactly, with the papular lesions. Roná has reported a case, thought to be unique, in which the bullæ appeared before the papules. In children vesicles are more frequently seen than in adults.

The mucous membranes are not seldom attacked. Heuss found them involved in 40 per cent. of the cases, Gautier in about two-thirds, Herxheimer in 93 cases out of 127 ; Dubreuilh holds that the mucous membrane alone is more often attacked than the skin alone. The mucosa most often involved is that of the cheek; next comes that of the tongue; lesions may also appear on the lips, gums, uvula, and palate, the nose, the epiglottis, the larynx, the urethra, the anal mucosa, etc. Gastric symptoms may be accounted for by involvement of the mucosæ of the stomach and intestines. The eruption shows itself in the form of Chinese-white patches. It has the appearance of a streak of milk on the mucous membrane, and it is often confounded with the mucous patches of syphilis. When the red of the lips is affected, it looks as though it had been tattooed with some white substance (Brooke). On the tongue the lesions elosely resemble leucoplakia, and in one of my cases the buccal lesions had been attributed to that condition. The true nature of the disease was disclosed by the lichen papules on the arms, wrists, abdomen, and legs. The eruption in

1 Brit. Journ. Derm., 1902, p. 161. 
the mouth may precede any cutaneous lesions, or it may appear when those have cleared up. It may, indeed, be the only sign of the disease; but the diagnosis, in my opinion, can never be conclusive in the absence of lesions on the skin. The mucous membrane of the genitals is seldom attacked, but occasionally the milk-white patches of lichen planus are seen on the inside of the labia.

The acute form of the disease, described on p. 192, and attended by pronounced nervous symptoms, such as great depression of spirits, loss of sleep, restlessness, and irritability, usually lasts for a few weeks only, though it may persist for years. In the more ordinary chronic form the papules disappear after a few weeks, leaving in their place stains varying in hue from light brown to black. Later, these stains lose their pigmentation and become white and atrophic, like scars. As one crop of papules disappears, others come out in different places. The disease sometimes remains limited to particular parts of the body for many months, but it may in course of time invade nearly the whole surface of the skin. In some cases-especially on the legs and in persons with varicose veins-lichen planus assumes a hypertrophic form, the patches being raised so as to form plateaux of considerable extent (lichen hypertrophicus) (Plate 10). It may also assume an atrophic formlichen planus atrophicus or sclerosus, the lichen atrophique of Hallopeau. Two such cases, in which the patches were demarcated by a raised bluish-red margin surrounding atrophic skin, have been reported by Zarubin. Other cases are described by Reiss and Wechselmann. Montgomery and Ormsby ${ }^{1}$ report a case of "lichen planus et atrophicus," which they hold to be entitled to recognition as a distinct type of lichen planus. In rare cases lichen planus occurs in association with glycosuria.

1 Trans, Amer. Derm. Assoc., 1906, p. 99. 
The affection varies very greatly in severity in different persons. In the later stages, when the lesions extend over a considerable portion of the body, the skin becomes very tender, and great pain is experienced when the parts are pressed. Occasionally old-standing lesions take on a warty character (lichen verrucosus). ${ }^{1}$ Emery and Humbert ${ }^{2}$ describe a case in which the warty lesions were present on the scalp as well as on the internal surface of both knees.

The acute variety of lichen planus, referred to above, is characterized by the rapidity of its onset and the intense severity of the lesions, the extremities being swollen and tense, and the blue or purple appearance being very marked. I have seen several examples. In a very severe case under my care the patient was a man aged 31 , otherwise healthy. Within a few weeks the whole body was covered with the eruption, the hands and feet being most severely affected, swollen, blue, œdematous, and subsequently desquamating in large masses as in scarlet fever. On the body, though the eruption was so extensive, the characteristic appearance of the individual papules was not lost.

Lukasiewicz has recorded a case in which lichen planus and acuminatus occurred in the same patient, a man aged 26. The lesions characteristic of the former affection predominated on the face, neck, trunk, scrotum, and penis ; those of the latter on the extremities. The man was in hospital nine months, and Lukasiewicz had the opportunity of observing on the trunk, during a period of exacerbation, distinct transitional forms of planus and acuminatus.

Pathology.-Crocker, whose careful examinations have been, in the main, confirmed by more recent

1 See paper (with illustrations) by Fordyce, Journ. Cut. and Gen.-Urin. Dis., 1897, xv. 49.

${ }^{2}$ Ann. de Derm. et de Syph., Jan., 1904, p. 41. 


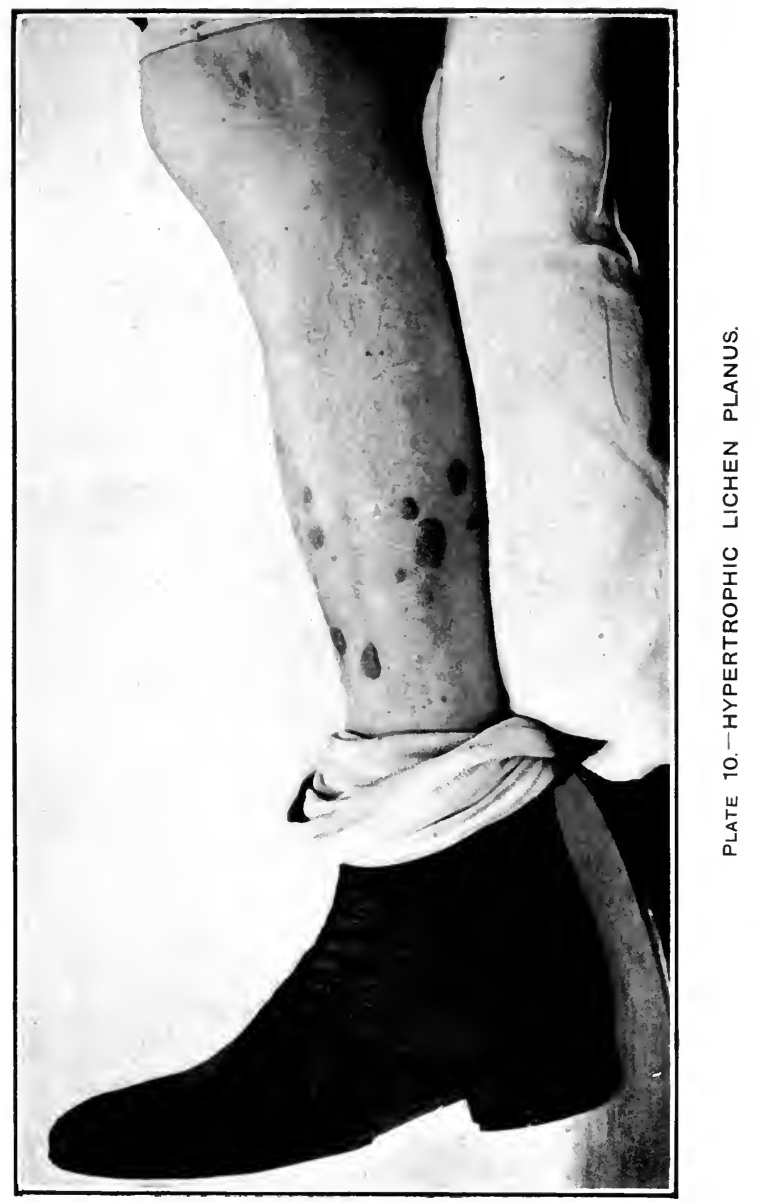



investigators, has shown that the process is inflammatory, the starting point being generally a sweat-duct in the upper part of the corium. The inflammation results in thickening of the rete, with enlargement of the papillæ, the papillary vessels being dilated, and downgrowth of the interpapillary processes taking place. It is possible that the process is angio-neurotic, but so far this has not been proved.

Etiology.-The causation of lichen planus is obscure. The process, as already said, is essentially inflammatory in character; but, on the other hand, it is, in my experience, not infrequently the result of a violent nervous shock or emotional disturbance. One of the worst cases I have seen was that of a lady whose husband died suddenly in a railway carriage while travelling with her from the South. Besides the shock of this event, she was subjected to much worry and anxiety by the necessity of going through, without assistance, the vexatious formalities insisted on by officials in such circumstances. She bore up well, however, till after the funeral, when she was suddenly seized with a severe attack of lichen planus, in which the subjective symptoms were of such intensity as almost to upset her reason. In other cases the neurotic element is very strongly marked, and I think it not improbable that this may be a leading factor in the causation of the disease. I have therefore included lichen planus among neuro-dermatoses provisionally, but it must be understood that the evidence of its nervous origin is so far entirely clinical. It occurs in persons otherwise perfectly healthy. It is neither contagious nor hereditary. The majority of patients are between 20 and 50 years of age, and the disease is very rare at each extreme of life. In some cases there is evidence of auto-intoxication from the intestines.

Diagnosis. - Lichen planus has to be distinguished from psoriasis punctata, papular eczema, and 
papular syphilitic lesions. From psoriasis it is differentiated by the fact that the papules remain unaltered instead of spreading out into scaly patches; from eczema, by the fact that usually no vesicles are formed; and from syphilis, by the dryness of the papules. In all doubtful cases the characteristic primary papules of lichen planus must be looked for. Generalized lichen planus is sometimes difficult to distinguish from generalized psoriasis. The points of distinction are that in the former there is less scaliness and more thickening, and characteristic papules can usually be found at the margin of the patches. For the diagnosis of lichen planus from verruca plana juvenilis, see p. 689 ; from pityriasis rubra pilaris, p. 207.

Treatment.-Lichen planus must be treated on the general lines already laid down for the treatment of skin affections of nervous origin. Arsenic is particularly valuable if given in large doses and continued for a long time. Kaposi, following Hebra, looks upon this drug as a specific. In the case of children he gives it in the form of Fowler's solution, beginning with two drops daily and increasing the dose by very slow degrees ; in adults he gives it in the form of hypodermic injections of Fowler's solution or of Asiatic pills. The treatment is begun by the administration of 3 pills a day, increasing every four or five days by 1 pill, until a daily total of 8 to 10 pills is reached. As a rule no improvement is perceptible before a period of six to eight weeks has elapsed, in which time the patient will have taken from 200 to 500 pills. The patient continues taking 8 or 10 pills daily till the disease has almost entirely disappeared, when the quantity is gradually reduced to 6 pills daily. This number the patient continues to take for three or four months after the final disappearance of the eruption. I agree with Besnier, however, who, while admitting that arsenic often gives satisfactory 
results in lichen, says that in some cases it fails, while in others recovery takes place without it. However free from danger the method may be in experienced hands, the use of arsenic in such heroic doses is hardly to be recommended as a routine practice. In a case under the care of Pringle, ${ }^{\mathbf{1}}$ remarkable subsidence of the inflammatory lesions occurred during the administration of antipyrin in 10-grain doses thrice daily, which appeared to arrest all itching. In generalized lichen planus I have found the internal use of biniodide of mercury most useful from its intestinal antiseptic action; I usually give it according to the following formula : Liq. hydrarg. perchlor. そi, potass. iodid. gr. xl, decoct. sarsa co. ऊviii.-M. : two tablespoonfuls three times a day. C. J. White has given injections of the salicyl-arsenate of mercury (énésol), with excellent results. Locally, the remedies indicated in lichen are those recommended for itching. Unna cured a series of cases in three weeks, without any internal treatment whatever, by means of frictions twice a day with an ointment composed of corrosive sublimate grm. i., carbolic acid grm. xx, and grm. 500 of simple ointment, the patient afterwards being wrapped up in linen cloths and put to bed. Pyrogallic acid (5 to 10 per cent.) rubbed on the affected parts is useful in old-standing patches. Mercurial plasters are beneficial when the lesions are confluent, but if the surface thus treated is extensive, it is necessary to be on the watch for symptoms of mercurialism. In old atrophic patches the cautery may be required. In a case under my care, hypertrophic masses which microscopically presented all the appearance of commencing epithelioma were left in the labium and had to be removed surgically. Cases of hypertrophic lichen planus which were intractable to the ordinary forms of treatment have 1 Brit. Journ. Derm., 1901, p. 12. 
cleared up under the influence of the $X$-rays ; and radiotherapy is also serviceable in relieving the itching of large patches in which there is no hypertrophy.

\section{LICHEN Nitidus}

Under this designation Pinkus has described a condition somewhat resembling lichen planus, but in which the exanthem gives rise to no symptoms and is so little prominent that it may be readily overlooked. In the nine cases reported by him, and in thirteen since reported by Arndt, ${ }^{1}$ the patients were men suffering from venereal disease, and the genitals were the seat of the eruption, consisting of shiny, slightly raised papules, the colour of the normal skin. In only one case, reported by Josef Kyrle and J. E. R. McDonagh, ${ }^{2}$ was the patient a female, and in this case the eruption was generalized. R. L. Sutton ${ }^{3}$ has also reported a case in which the axillæ, groins, forearms, wrists, and thumbs were affected. These observers found in the lesions epithelioid cells and giant cells of the Langerhans type, and they sum up the disease as being brought about by a tubercular toxin and characterized by the formation of a granuloma unattended by the customary inflammatory changes. Confusion with lichen planus may be avoided by noting that the papules in lichen nitidus present a transparent appearance, undergo no change of colour, and do not tend to increase in size. Moreover, in the centre of the lesions there is a horny depression.

\section{Lichen AnNularis}

Under this name Galloway ${ }^{4}$ has described an affection the distinctive characters of which are summed

${ }^{1}$ Derm. Zeitschr., Bd. xvi., Hefte 9, 10.

2 Brit. Journ. Derm., 1909, xxi. 340.

3 Journ. Out. Dis., Nov., 1910, p. 597.

4 Brit. Journ. Derm., June, 1899. 
up by him in the following definition: "A chronic inflammatory disease of the upper layers of the cutis, associated with increase in the overlying epithelium, commencing as a nodule, spreading peripherally and healing in the centre, without suppuration or any rapid form of degeneration." The disease especially affects the dorsal surface of the phalanges in the neighbourhood of the joints. In the nature and distribution of the inflammatory infiltration it closely resembles lichen planus, while in its progress it simulates certain other conditions whose toxæmic origin is better defined. Although there are wide clinical differences between the two diseases, Galloway considers that the histological characters of the affection bring it within the strictest definition of the term "lichen," while the ringed arrangement of the lesions is distinctive. Lichen annularis has certain analogies with a condition described by Crocker under the name of erythema elevatum diutinum ${ }^{1}$ and with lupus erythematosus. From a study of a number of cases recorded under various names by Hutchinson, Dubreuilh, and others, Galloway concludes that there are certain chronic inflammatory conditions of the skin which produce lesions resembling in many points those of lichen planus, and caused by poisonous substances circulating in the blood, the nature of which is yet undetermined. These lesions present differences in the amount of congestion, in the appearance of the lesions, and in their duration. They agree, however, in their chief clinical features and in the histological changes which they produce. They do not suppurate. It is possible that gout and rheumatism are factors in their causation.

In January, 1902, Radcliffe-Crocker ${ }^{2}$ described six cases of a nodular ringed eruption which he termed

1 Radcliffe-Crocker and Campbell Williams, Brit. Journ. Derm., 1894, pp. 1, 3, 335.

${ }^{2}$ Brit. Journ. Derm., 1902, p. 1. 
granuloma annulare. Graham Little ${ }^{1}$ also had six cases under his observation, and gives a review of all the cases published up to 1908 . He believes in the close relation and possible future identification of granuloma annulare with lichen annularis. The eruption usually appears on the hands and feet, or on the neck; but in young children it may occur on the buttocks and thighs, as in the case of a male infant of 16 months shown by Haldin Davis. ${ }^{2}$

\section{Lichen Pilaris or Spinulosus}

Radcliffe-Crocker and Colcott Fox were the first (in 1883) to call attention to the affection to which this name has been given. Occurring chiefly in children, it is marked by the projection of filiform spines from pilosebaceous follicles, of which the mouths are elevated into small acuminate papules, pinkish or pale in colour, disposed in patches on the limbs and trunk. There is almost invariably no itching or other subjective symptom, nor is there any appreciable interference with the general health.

The reported cases, English and Continental, are carefully reviewed by Adamson, ${ }^{3}$ who, finding neither clinically nor histologically any evidence of pronounced inflammatory action, deprecates the application of the term "lichen," and suggests that Unna's name, keratosis follicularis spinulosa, is a more appropriate designation. While allowing that at first the lesions are slightly inflammatory, he holds that the essential part of the process is a hyperkeratosis of the follicular wall, due, perhaps, to toxic rather than to parasitic action, or mechanical irritation. In adults the typical spiny lesions are generally, if not always, associated with lichen planus, and attended with pruritus. In a case

1 Brit. Journ. Derm., July, Aug., Sept., Oct., 1908.

2 Ibid., 1910, xxii. 90. ${ }^{3}$ Ibid., Feb. and March, 1905. 
shown by Adamson in 1906 the lesions began as simple spines without inflammatory papules, confirming his view that the affection is primarily a follicular hyperkeratosis.

\section{Porokeratosis (Mibelli)}

By this name Mibelli ${ }^{1}$ las designated an affection presenting a certain resemblance to lichen annularis. It is characterized by patehes of irregular shape and size, surrounded by a horny linear edge. Galloway, ${ }^{2}$ who showed a case at the Dermatological Society of London, pointed out that the lesions closely resembled those seen in the examples reported by Mibelli, Gilchrist, and others, being generally circular in outline, with irregularly advancing and receding margins. "Its periphery is marked by a raised border of loose, horny epithelium, the summit of which has given way and allows a crack to appear in the greater part of its course. Within this horny outline numerous small conical elevations of the horny layer are obvious, while the greater part of the enclosed area of skin is smooth, and shows the normal furrows of the skin with very slight alteration." There seemed to be no appreciable thickening of the cutis. The disease, in Galloway's opinion, is of a totally different nature from lichen annularis, in which there is marked infiltration of the eutis, with much less disturbance of the horny layer than in porokeratosis. Mibelli regards as an eminently characteristic feature of porokeratosis the depressed furrow of the lesion. So far, however, very little has been found on histological examination to differentiate the one disease from the other. Galloway thinks it probable that some of the cases described by foreign observers under the name of porokeratosis have really been examples of lichen

${ }^{1}$ Monats. f. prakt. Derm., xvii., 1893, and Ann. de Derm. et de Syph., June, 1905, p. 503.

2 Brit. Journ. Derm., 1901, p. 262. 
annularis. Mantoux ${ }^{1}$ describes a papillomatous form of porokeratosis, the localization of the tumours round the sweat orifices being his reason for terming the affection a porokeratosis. A similar case has been reported by Besnier, and another by Hallopeau.

\section{Parakeratosis Variegata}

The term "parakeratoses" was suggested by Unna ${ }^{2}$ to denote superficial inflammatory processes affecting the epidermis and characterized clinically by scaliness. Under this general head he grouped scaly forms of seborrhœa, pityriasis, psoriasis, and other conditions in which scales are a marked objective feature. The addition of the qualification "variegata" was meant by Unna to designate a form of dermatitis, chronic in course, with spontaneous remissions, attacking the hand and extending to the extremities, marked by flat papules, often surmounted by scales, the eruption as a whole producing a "variegated" appearance owing to the enclosure of areas of healthy skin in a meshwork of disease. There is no disturbance of the general health, nor are there subjective symptoms, except a trifing amount of itching. In the cases on which Unna's original description was founded the histological examination showed that the papillary and subpapillary layers were alone affected, presenting dilated vessels, œdema, cellular infiltration, and a few leucocytes. Interepithelial and intra-epithelial œdema and thickening of the prickle layers were present, but there was no increase in mitoses. The stratum granulosum was present and the horny layer slightly thickened.

At the meeting of the British Medical Association held in Edinburgh in 1898, Jamieson ${ }^{3}$ presented three

1 Ann. de Derm. et de Syph., Jan., 1903, p. 15.

2 Monats. f. prakt. Derm., Bd. x., 1890.

${ }^{3}$ Rrit. Journ. Derm., Sept., 1898. 
cases for diagnosis, which were regarded by several of those present as examples of parakeratosis variegata. In one case the essential lesions were papules "which, clinically and microscopically, were identical with those of lichen planus"; in another "the lesions were indeterminately lichenoid"; while the third case was considered by Jamieson to form a connecting link between the other two. Jamieson classified the cases as anomalous forms of lichen planus; Radcliffe-Crocker, and the author, suggested the possibility of their being instances of a premycotic condition; while Unna identified them as excellent examples of parakeratosis variegata. Boeck stated that he had seen several cases of similar nature, and had described them under the name "dermatitis variegata." It may be mentioned that Crocker bas suggested the name "lichen variegatus" for the disease. In one of Jamieson's cases tumours like those of mycosis fungoides appeared subsequently on the face and body. Colcott Fox and J. M. H. MacLeod have published a most instructive report of a case under their observation, and have collected and analysed all the records of similar cases published up to that time. ${ }^{1}$ The conclusions at which they arrived are summarized by themselves as follows: "Parakeratosis variegata attacks chiefly the male sex ; the patients are usually adults, and are generally in robust health when attacked. The clinical appearances and histological changes suggest vaso-motor disturbance as the etiological factor; this is associated with œedema and infiltration of cells in the corium and secondary changes in the epidermis. The initial lesion is a macule or maculo-papule of small size, flat on the surface and covered with a fine adherent scale which may be scratched off without causing bleeding. By the coalescence of the lesions a peculiar retiform arrangement results, in

${ }^{1}$ Brit. Journ. Derm., Sept., 1901. 
which areas of normal skin are enclosed, and which, combined with differences in the colour of the lesions in the more dependent parts of the body, produces a marbled or variegated appearance, which is one of the most pronounced characteristics of the dermatitis. It affects the skin almost: universally, except, as a rule, that of the face, scalp, palms, and soles. It is subject to remissions and exacerbations, but is peculiarly chronic in its course. Marked subjective symptoms are singularly absent. It is strangely resistant to local treatment. It consists histologically of a superficial inflammation affecting the subepidermal layer, with dilatation of vessels, œdema, and infiltration of cells; and an œdematous condition of the epidermis, with more or less defect in the process of cornification. It may be regarded as balonging to a group of superficial inflammations of the corium, with secondary changes in the epidermis, which we have provisionally entitled 'resistant maculo-papular scaly erythrodermias,' which might include, besides this variety, érythrodermie pity. riasique en plaques disseminées (Brocq), pityriasis lichenoides chronica (Juliusberg), dermatitis psoriasiformis nodularis (Jadassohn), and the lichenoid psoriasiform exanthem (Neisser)."

In 1908, W. T. Corlett and Oscar T. Schultz ${ }^{1}$ described two cases which were clinically identical with that described by Colcott Fox and MacLeod. Recently the author and S. E. Dore reported a case which was quite typical, except that the eruption was not universally distributed on the trunk, as it usually is. It presented its most striking appearance on the right upper arm (Plate 11), where there were irregular-shaped patches of a bright-red to crimson colour, extending longitudinally or obliquely along the main axis of the limb, with intervening areas of healthy skin. It was difficult to make out

${ }^{1}$ Trans. Amer. Derm. Assoc., 1908, p. 51. 


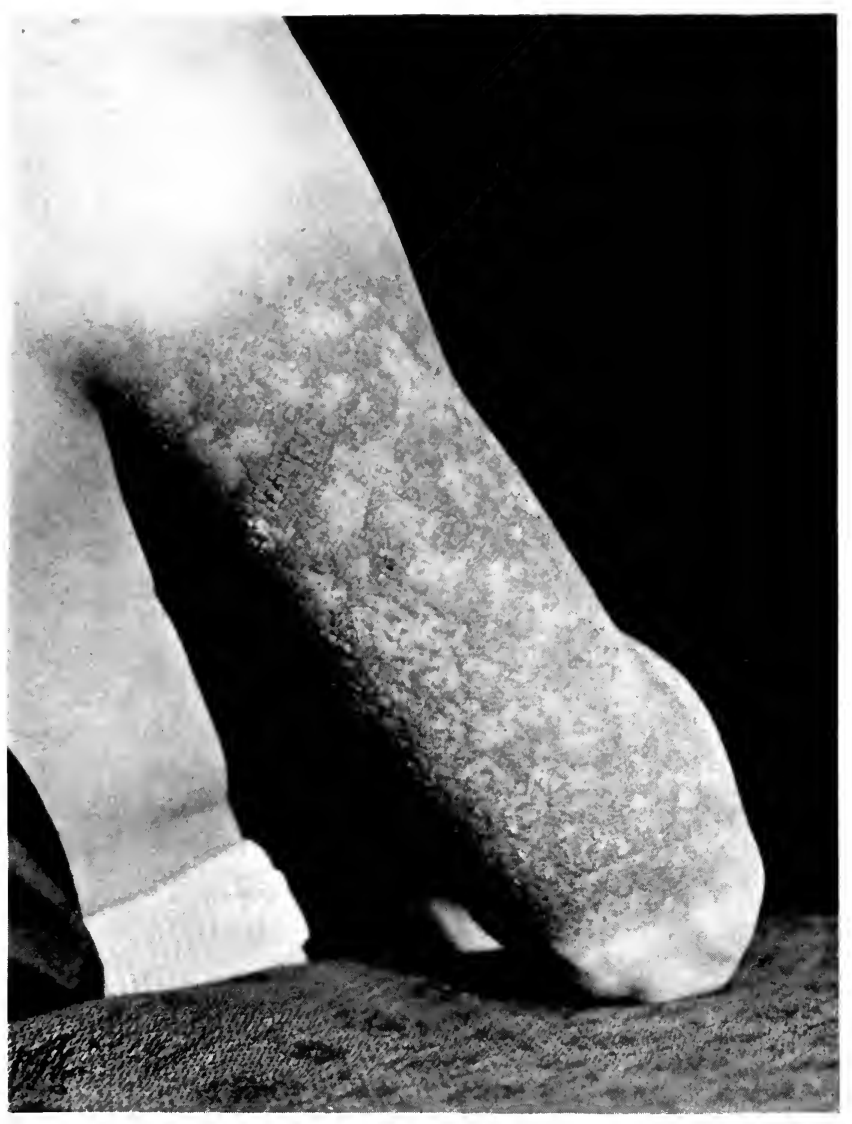

PLATE 11.-PARAKERATOSIS VARIEGATA. 

the elementary lesions, but the patches had a quadrillated appearance, and seemed to be made up of shiny, flat-topped papuies, resembling those of lichen planus, but lacking their definite outline. There was no difficulty in referring this case to Brocq's lichenoid parapsoriasis, the second of his types of parapsoriasis (p. 336). ${ }^{1}$

In a case observed by Professor Anthony ${ }^{2}$ the retiform arrangement of the papules was absent, while there was pronounced pigmentation (light-brown, chestnutbrown, and bluish plaques), with some atrophy. $\mathrm{He}$ maintains, however, that the case was one of parakeratosis variegata, and points out that since the original case (Unna's) was published, in 1890, a number of cases have been reported in which there was no mesh-like disposition of the lesions.

The prognosis of parakeratosis variegata is bad, the tendency of the disease being gradually to spread: Treatment can effect little beyond the removal of the scaliness.

\section{Xantho-Erythrodermia Persisans}

Pernet suggests this name for an affection of which ten eases were deseribed by Radeliffe-Crocker, ${ }^{3}$ who regarded it as having many analogies with that group of Broeq's which includes his érythrodermie pityriasique en plaques disseminées, but as being separated from it by differences sufficiently marked to entitle it to be regarded, provisionally, as a dermatosis sui generis. This affection, like parakeratosis variegata, is now referred to the group of conditions styled parapsoriasis, and eomprising three types-(1) parapsoriasis guttata, (2) lichenoid parapsoriasis, and (3) parapsoriasis en plaques. They are eonsidered elsewhere (p. 336).

1 For further details, see Brit. Journ. Derm., 1910, xxii. 249.

2 Trans. Amer. Derm. Assoc., 1906, p. 79.

${ }^{3}$ Brit. Journ. Derm., April, 1905, p. 119. 


\section{Pityriasis Rubra Pilaris (Devergie), or Lichen Ruber Acuminatus (Kaposi)}

This affection (Plates 12, 13, 14, 15) is an anomaly of cornification primarily affecting the hair-follicles, at the orifices of which characteristic papules form, and secondarily leading to inflammatory changes of the dermic structures. There has been a good deal of discussion as to the relation of the affection to lichen ruber acuminatus. Kaposi thinks the two conditions identical, and I agree with him. What may fairly be called a test case was shown at Budapest to some members of the Congress of Dermatology held at Vienna in 1892. The patient was exhibited as an illustrative example of lichen ruber acuminatus, and the affection was unhesitatingly pronounced to be pityriasis rubra pilaris by the French dermatologists present. Neumann, however, maintained that lichen ruber acuminatus and pityriasis rubra pilaris are two distinct affections. ${ }^{1}$

Neisser ${ }^{2}$ holds that there is a disease, differing from both lichen planus and pityriasis rubra pilaris, for which the name of lichen ruber acuminatus may conveniently be retained. Two cases were shown to illustrate this view, one being pityriasis rubra pilaris, the other lichen ruber acuminatus. The main points in the differential diagnosis are, according to Neisser, the following: Lichen ruber acuminatus affects the general health very seriously, is benefited by arsenic to a very marked extent, and shows usually more distinctly papule formation and less hyperkeratosis. Under the microscope the papules of this disease are seen to be situated round a hair-follicle, and to consist almost wholly of an infiltration of small cells in the corium. In pityriasis rubra pilaris, on the other hand, the disease causes

1 Arch. f. Derm. u. Syph., 1892, Heft 1.

2 Trans. Fourth German Congress of Dermatology. 


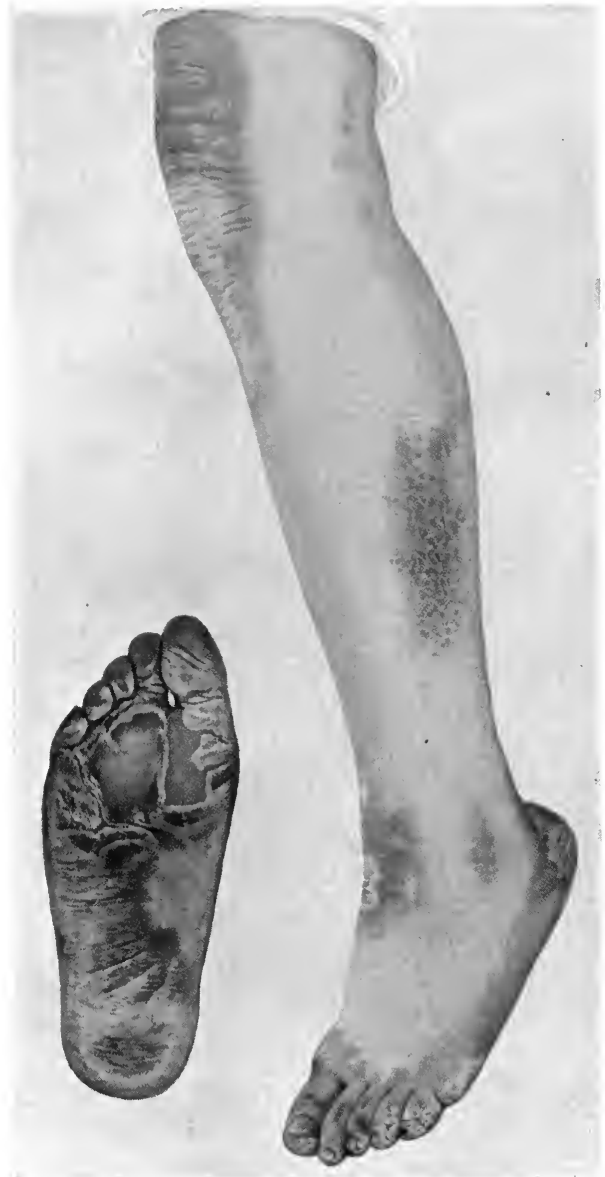

PLATE 12.-PITYRIASIS RUBRA PILARIS. 

scarcely any alteration of the general health, is essentially chronic in nature, is quite uninfluenced by the administration of arsenic, and shows less marked papule formation but great hyperkeratosis. Under the microscope there was seen to be very little infiltration of the corium and a marked increase of the epidermis. Neisser admits that Kaposi described Devergie's disease under the name of lichen ruber acuminatus, but thinks that this disease was also included in the description. On the other hand, he considers that the French school have fallen into the same error, and described two diseases under the one name of pityriasis rubra pilaris. Lastly, Neisser allows that the initial lesion of lichen ruber acuminatus may also show hyperkeratosis, but even then the state of health and the action of arsenic make a great difference. This appears to coincide with Unna's lichen neuroticus. ${ }^{1}$

Symptoms.-Pityriasis rubra pilaris usually comes on, so to speak, in disguise. Sometimes it appears in the form of scaly patches resembling psoriasis on the palms and soles, sometimes as a dry eruption, covered with eczematous-looking crusts, on the scalp and face. Soon, however, the characteristic papules become visible a the orifices of the hair-follicles. These papules are small, red, hard, dry, harsh to the touch, and more or less conical in shape, each having a single atrophied hair in the centre surrounded by a kind of horny sheath which penetrates into the follicle. The projection of these tiny, cone-shaped papules is sufficient to roughen the surface of the integument, so that it feels like the skin of a newly-plucked fowl (Besnier). The papules are distributed on the limbs, especially where the hair is most abundant-that is to say, on the backs of the fingers (particularly the first and second phalanges), on the outer aspect of the forearms, on the outside of the

1 " Histopathology," p. 303. 
thighs, and on the buttocks. They are also, though less frequently, seen about the elbows and knees. On the trunk they chiefly affect the waist and the lower part of the belly. They are at first discrete, but as they increase in number they tend to become confluent, and thus form patches. In these patches the distinguishing characters of the individual papules are lost in a pale yellowish-red surface, covered with papery scales, or with small adherent ones resembling mica, which, when situated in the positions most affected by psoriasis, may closely simulate the lesions of that disease. At the edge of the patches the characteristic conical papules are always to be seen.

The three marked objective features of pityriasis rubra pilaris are: (1) the "goose-skin" appearance and grater-like feeling caused by the conical papules at the orifices of the hair-follicles; (2) the desquamation; (3) the redness of the surface. The natural folds of the affected parts of the skin are always exaggerated. The eruption often spreads over a large part of the body, and in some cases becomes universal. The lesions present certain differences of appearance, according to their situation. On the face they are often of a seborrhœic type, a red base being covered with adherent crusts; sometimes they have the characters of pityriasis rubra. They are always dry, and there is usually considerable tension of the skin, which may give rise to ectropion of the lower eyelid. On the scalp they are generally of seborrhœic type; the hair is often matted together by firm crusts. The nails become soft, greyish in colour, and marked with longitudinal yellow stripes. On the hands, however extensive the eruption may be, small blackish cones can always be seen around the hair-follicles.

Beyond a trifling amount of itching, which, moreover, is by no means a constant feature, there are no 


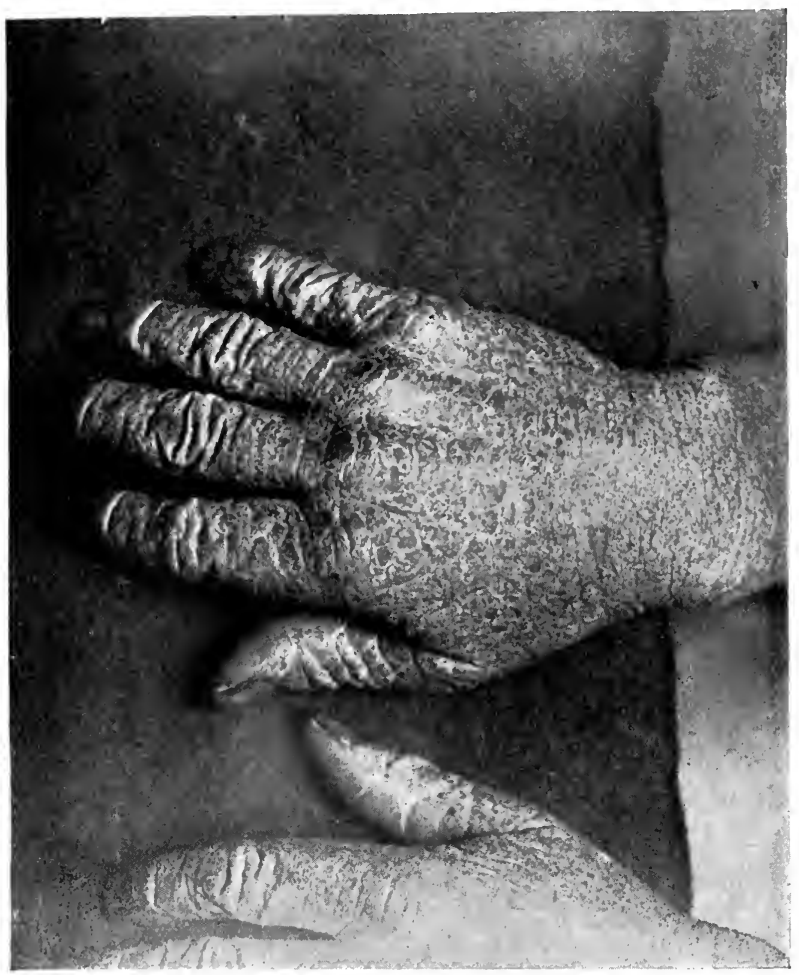

Plate 13.-PITYRIASIS RUBRA PILARIS.

(Galloziny's Case.) 


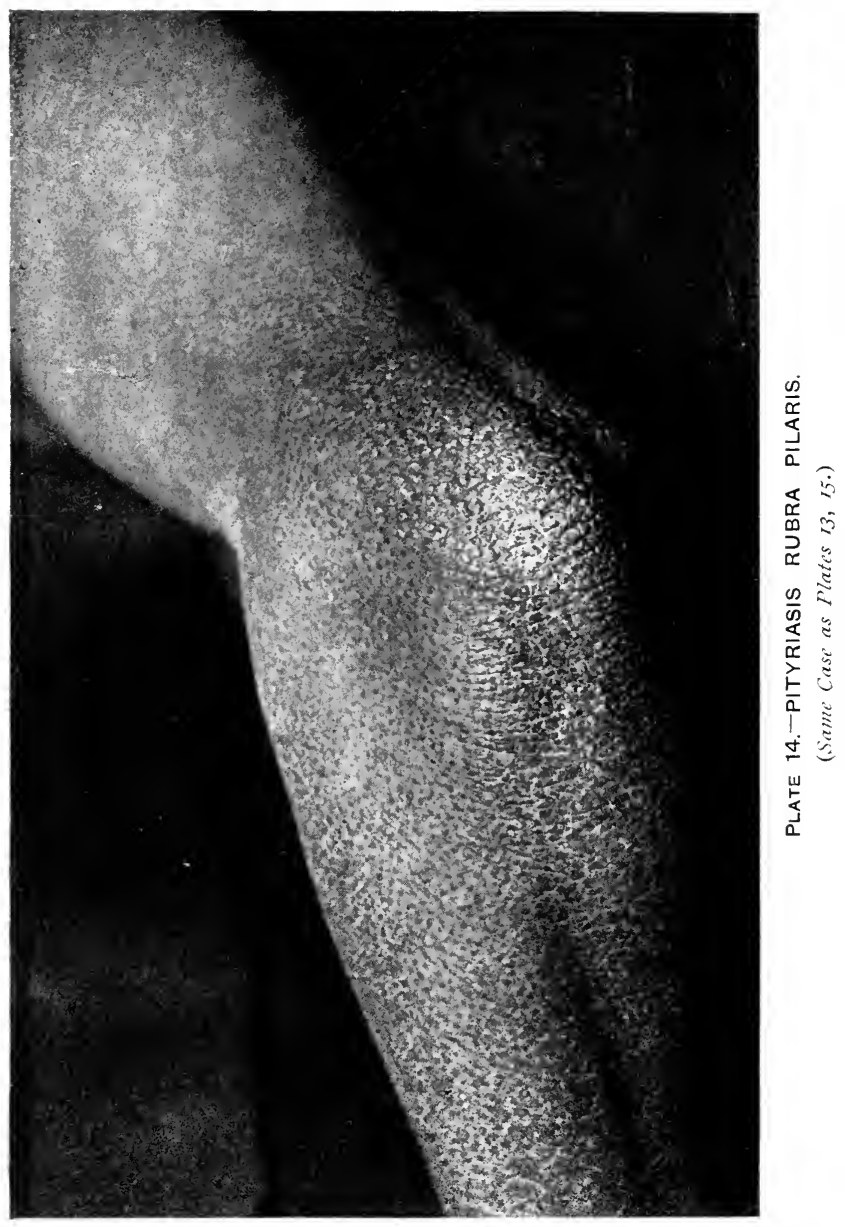


subjective symptoms in pityriasis rubra pilaris. The general health is seldom affected. The onset of the disease may be preceded by great irritation in the parts about to be attacked. Its course is slow, and subject to sudden remissions and exacerbations without obvious cause. Even when the affection appears to be completely cured, relapse may occur at any time.

Pathology.-Jacquet has shown ${ }^{1}$ that the conical papule, which is the essential lesion of the disease, is caused by exaggerated cornification of the epithelial wall of the infundibulum of the hair-follicle. The plugging of the follicle is followed by inflammatory lesions in the dermic structures.

Diagnosis.-This is almost always easy. The characteristic conical papule, with its single hair plugging the mouth of a follicle, is conclusive as to the nature of the disease. The best place to look for the lesions is on the backs of the fingers : they can be picked off, little pits being left which give the skin a cribriform appearance. The absence of any attendant disorder of the general health distinguishes the affection from other forms of exfoliative dermatitis, in which also there is more pronounced scaliness, with greater redness, while there is seldom any appreciable thickening of the integument. From lichen planus it is differentiated $(a)$ by the absence of itching; $(b)$ by the absence of impairment of nutrition; and $(c)$ by its resistance to the therapeutic action of arsenic. In lichen planus, further, the papules are flattened, and frequently umbilicated. When the lesions of pityriasis rubra pilaris appear in the situations most common to psoriasis-the tips of the elbow, the fronts of the knees, and the extensor surfaces of the limbs-and the patches are covered with mica-like scales, they may suggest that affection; but at the edge of each pateh the characteristic conical papule, with its

${ }^{1}$ Quoted by Brocq, op. cit., p: 644. 
single hair plugging the mouth of a follicle, will always be found.

Treatment of pityriasis rubra pilaris is unsatisfactory. Arsenic appears to be contra-indicated; but Brocq, while admitting that the drug cannot be relied on, recommends arseniate of soda in gradually increasing doses. ${ }^{1}$ Heidingsfeld discountenances the injection of arseniate of soda, and reports three cases which yielded to the administration of intestinal aseptics, such as $\beta$-naphthol and guaiacol carbonate, or carbolic acid, with the hypodermic injection of arsenic in the form of atoxyl or cacodylic acid, the external application of tar, and attention to diet. ${ }^{2}$ Sudorifics are clearly indicated by the dryness of the skin; for this purpose pilocarpin or jaborandi is likely to prove useful, or, as suggested by Brocq, violent exercise may be indulged in, of course with due regard to the special circumstances of each case. Locally, oil of cade may be applied. Brocq speaks well of pyrogallic acid. If inflammation runs high, soothing applications are required. Sebaceous concretions on the face or scalp should be removed in the usual way.

\section{Congenital IChthyosiform Erythrodermia}

Brocq has given this name to an affection closely resembling pityriasis rubra pilaris; he has seen two or three cases of it, and others are recorded by various writers under different names. ${ }^{3}$ A case has recently been shown by Adamson ${ }^{4}$ in which there was marked horny thickening of the palms and soles. The objective symptoms are generalized redness, less accentuated on the face, thorax, and abdomen, and exaggerated on the neck,

${ }^{1}$ Op. cit., p. 644 .

2 Journ. Cut. Dis., Aug., 1906.

3 Ann. de Derm. ot de Syph., Jan., 1902 (abstr. in Brit. Journ. Derm., (oct., 1902).

${ }^{+}$Brit. Journ. Derm., 1910, xxii. 163. 


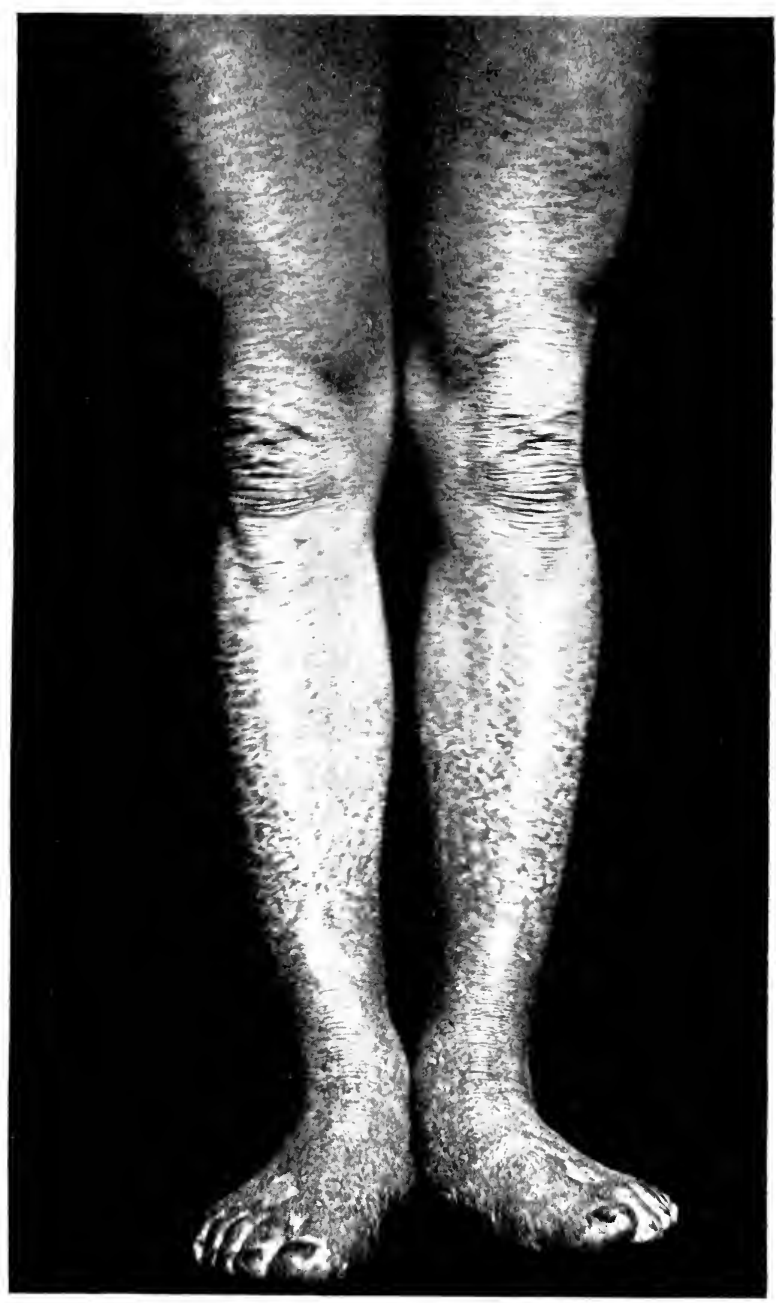

Plate 15.-PITYRIASIS RUBRA PILARIS.

(Same' Case as l'late's 13, 1\%.) 


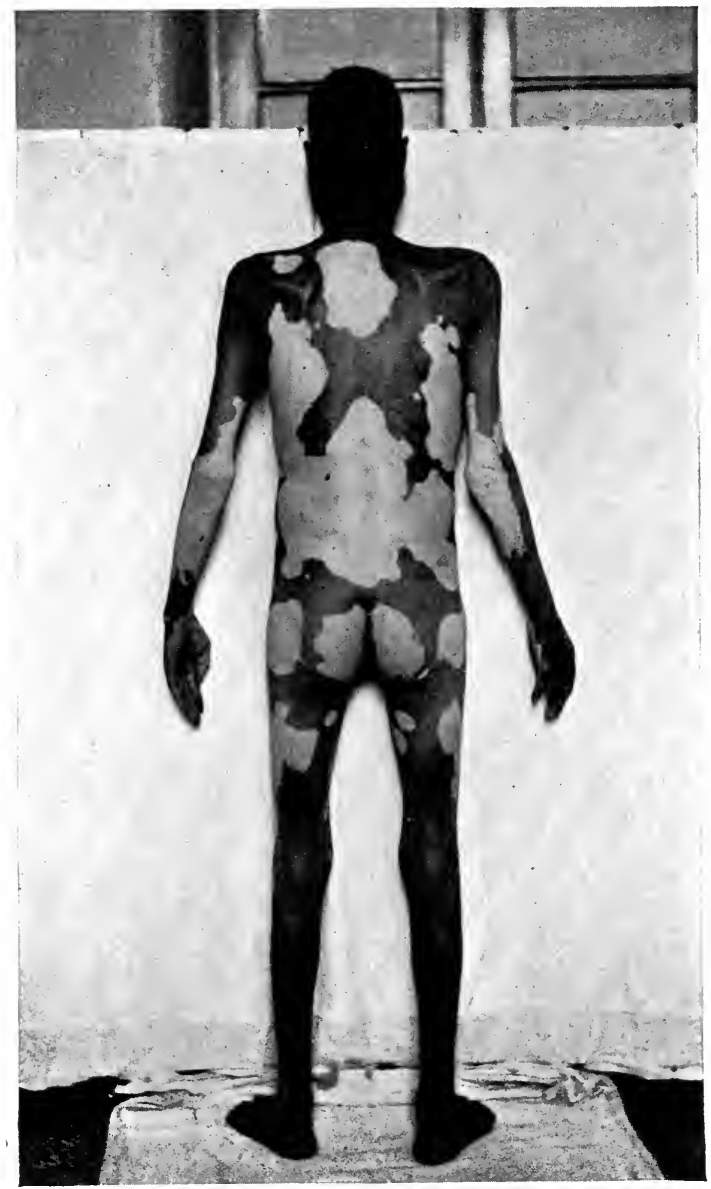

Plate 16.-LEUCOdeRMia.

(By permission, from the Photographic Album of the School of Medicine, Cairo.) 
the folds of the joints, and the limbs. The papillo of the skin are much enlarged, and this is accompanied by a pronounced general hyperkeratosis. The scalp is always markedly seborrhœic. In all cases the affection was either congenital or began in earliest infancy. There was no particular disturbance of the general health, and the affection lasted for many years. In certain cases it seemed to become mitigated as age advanced, but in no case has a cure been observed. C. Rasch 1 suggests that the affection should be styled ichthyosis rubra. The features which are held to differentiate it from pityriasis rubra pilaris are that it is congenital, that it runs a different course and lasts longer, and that it is not accompanied by appreciable impairment of the health.

\section{Erythiroderma Desquamativa}

Under this designation Carl Leiner ${ }^{2}$ describes a peculiar universal dermatitis of breast-fed children, of which he has seen forty-three cases. The scalp is usually covered with fatty greyish-white scales; the skin is intensely red and shiny. The lesions extend to the face, and, finally, the whole cutaneous surface is involved; in some cases there is a parakeratosis. In about one out of every three cases there is a fatal termination. Leiner claims that the disease is an idiopathic erythrodermia, of which the distinctive features are the fatty constitution of the scales at the outset, the absence of an atrophic stage, and its occurrence in children at the breast, to whom it is almost peculiar. It presents some resemblance to Ritter's disease (p. 343).

\section{Anomalies of Pigmentation}

These may occur as the result of inhibition of the regulating influence of the nervous system, as by menta

${ }^{1}$ Derm. Z $Z$ sitschr., viii. 669.

2.Arch. f. Derm. u. Syph., Jan. and Feb., 1908, vol. 1xxxix. 
shock or long-continued depressing conditions, or by reflex disturbance. 'Thus, as is well known, the hair may grow rapidly grey under the stress of fear or sorrow; and Paget mentions the case of a lady subject to nervous headache who always found in the morning, after an attack, that some patches of her hair were white, as if powdered with starch. The change was effected in a night, and in a few days the hairs gradually regained their dark-brownish colour. ${ }^{1}$ The commonest errors of pigmentation are those met with in chloasma, whether idiopathic or symptomatic. Idiopathic chloasma is generally due to some kind of external irritation, such as that set up by vesicants, but in some cases the cause cannot be traced. Symptomatic chloasma is the result of abnormal conditions of the uterus, or of other abdominal viscera, or of cachexia, or it may be an accompaniment or a sequela of cutaneous eruptions. Its most frequent form is that of chlcasma uterinum. The refiex nature of this affection is shown by the fact that it is not always associated with pregnancy, but may occur in connection with any form of uterine irritation. The smooth, yellowish-brown patches most commonly appear on the forehead, cheek and nipples, but almost the whole face may be involved, and also the trunk and limbs. The diagnosis of chloasma is not usually difficult, though the particular cause of the pigmentation can only, of course, be deduced from the general symptoms. Chloasma may be differentiated from tinea versicolor and some other fungous diseases by the fact that the colour cannot be scraped off, nor are the patches scaly, but quite smooth. It differs from chromidrosis in that the colour does not disappear if washed with ether or chloroform. The pigmentary syphilide is seldom met with except on the neck. For the diagnosis between leucodermia and chloasma, see p. 213.

1 "Surgical Pathology," p. 31, 3rd edit. London, 1870. 
The general bronzing of the skin observed in Addison's disease is due to irritation of the abdominal sympathetic, particularly the solar plexus. Pigmentation is also a . symptom of Graves's disease, ${ }^{1}$ as well as of abdominal tuberculosis, and of other disorders of the abdominal viscera. Pigmentary abnormalities also occur in the cachexia of malaria, cancer, nodular leprosy and secondary syphilis. In malaria it is a yellowish-brown to black tint ; in cancer, a sallow hue; in nodular leprosy, a fawn colour early in the disease and a general bronzing at a later stage; in secondary syphilis, an earthy tint, affecting the face. The rare condition designated by Virchow ochronosis-a blackening of the cartilages, sclerotics, and skin-has been traced in some cases to alkaptonuria, in others to the prolonged absorption of carbolic acid. ${ }^{2}$ The pigmentary changes in the macular form of leprosy and in leucodermia are tropho-neurotic in their nature. The former will be described under the heading of Leprosy (Chap. XIII.), but a brief account must be given here of the latter, as well as of hæmochromatosis.

Leucodermia, or vitiligo (Plate 16), is somewhat rare in Great Britain, and in Europe generally; but it is common in the tropics, and especially in the dark races.

Symptoms.-The characteristic feature of the disease is the formation, in different parts of the body, of white patches, surrounded by a pigmented border. The appearance is as if the pigment had receded from the affected area and heaped itself up at its circumference. The patches are at first small, and more or less rounded in shape. As they spread, however, their outline becomes irregular, but the border always remains convex. The

1 Brit. Journ. Derm., Oct., 1900 (Dore).

${ }^{2}$ See Lancet, Jan. 21, 1904 (Osler), and July 11, 1908 (A. E. Garrod). 
pigmented zone surrounding them merges insensibly into the healthy skin around it. The white patches may be few or many in number, and they may be scattered about irregularly, giving the surface of the integument a maplike appearance, or distributed with some approach to symmetry, especially on the limbs. The neck is a common situation; but the face, the scalp, and the trunk, as well as the limbs, may be the seat of the affection. The disease is very slow in its course, and in some cases after a time it becomes stationary. In other cases, again, it spreads over the whole body, taking, however, many years to do so. The affected skin is smooth and supple, and shows no sign of scaliness; the physiological functions of the skin are intact, and sensation is unaltered. Sometimes slight itching may precede the formation of a patch. The hairs in the affected areas participate in the loss of pigment, and turn white. Both sexes are equally liable to the disease. Between the ages of 10 and 30 is the time of life when it generally commences.

Etiology.-There can be little doubt that leucodermia is a disease of neurotic origin, and Leloir in some cases found changes in the nerves supplying the whitened patches of skin. It also not infrequently follows violent mental emotion, or prolonged depression from illness or anxiety. It sometimes occurs in connection with tabes and with Graves's disease. Extreme heat or cold appears to have some influence as an exciting cause. By some authors leucodermia is supposed to be usually, if not invariably, associated with syphilis, but this view, which is shared by Pierre Marie, is combated by Thibierge, ${ }^{1}$ who cites three cases in which leucodermia developed from one to three years before the contraction of syphilis. Leucodermia is, however, met with in women in connection with pigmentary syphilides (see p. 481). It

1 Ann. de Derm. et de Syph., Feb., 1905, p. 128. 
is occasionally associated with alopecia areata, with lichen planus, with prurigo, and with selerodermia.

Diagnosis.-Leucodermia can be distinguished from macular leprosy by the absence of ansesthesia in the white patches, from sclerodermia by the absence of the parchment-like stiffness and thickening of the skin characteristic of that condition, and from the atrophic patches of $X$-ray burns by the absence of telangiectases. When in leucodermia the white areas have spread over the greater part of the body and are taken for the normal colour, the condition may have to be diagnosed from chloasma. But in chloasma the border is convex, not concave, and the history is not the same. From morphoea leucodermia may be diagnosed by the absence of a pink or violet border to the lesions and the thickened edge, nor is there any intermingling of atrophic striæ. With partial albinism it could only be confused by ignoring the history.

Prognosis and treatment.-The prognosis is by no means favourable, so far as restoration of the pigment is concerned. The process, as already said, sometimes comes spontaneously to a standstill. There is little to be done in the way of treatment. The lost natural colour cannot be restored, though the surrounding increase of pigment may be modified by the application of a weak solution of corrosive sublimate or peroxide of hydrogen.

Hæmochromatosis.-This anomaly of pigmentation may be mentioned here, although it appears to be directly due to diseases of the alimentary tract and liver. In a case described by Galloway ${ }^{1}$ the patient was pigmented from head to foot, the colour in the flexures of the body, where it was most distinct, being of a deep grey-blue slate tint. A collection of free, clear fluid, containing a few flakes of lymph, was evacuated from the abdomen by operation, and the patient, who

${ }^{1}$ Brit. Med. Journ., March 21, 1908. 
was emaciated to an extreme degree, made an uninterrupted recovery, though the skin did not quite regain its normal colour. This condition may be closely simulated by argyria (p. 251), and it is not always easy to obtain the true history where nitrate of silver has been administered. In a case of argyria shown by F. Parkes Weber and R. H. Norman, ${ }^{\mathbf{1}}$ it was not until a late stage in the exploration of the history that the patient produced an old prescription for a pill which contained argenti nitras, and which she had taken at intervals for two years.

\section{Raynaud's Disease}

Symptoms.-Symmetrical gangrene of the extremities (including in that term the tip of the nose and the ears), is a disorder of the peripheral circulation, and has three well-marked stages: first, spasm of arterioles, with pallor and loss of sensibility in the affected parts (local syncope, "dead fingers") ; secondly, stagnation of the venous circulation, with consequent cyanosis of the parts; thirdly, superficial gangrenethe skin becoming black, the epidermis becoming covered with eschars, and being raised here and there into bullæ, which dry up or burst and leave persistent ulcers. A line of demarcation is formed, and in severe cases separation of the affected part takes place. Carl Beck ${ }^{2}$ has reported two cases in which not only the soft tissues but also the bones were affected. The gangrenous process is at first accompanied by sharp pain, formication, and itching. In slighter cases, after the sloughing of the superficial tissues is complete, healing takes place, the fingers, however, remaining thinned, and covered with small white depressed cicatrices of considerable toughness. The process may be arrested in any of the three stages above described:

${ }_{1}$ Brit. Journ. Derm., 1910, xxii. 136.

${ }^{2}$ Amer. Journ. Med. Sci., Nov., 1901. 
Raynaud's disease is almost invariably symmetrical, but the process may be mild on one side and severe on the other. In a case under my own care it was asymmetrical. The order of frequency with which different parts are attacked is as follows: Fingers, toes, heels, nose, and ears. Any part of the body, however-limbs, trunk, or face-may be assailed.

Etiology.-Females are more liable than males. No age is exempt, but children are more often attacked than adults. Persons in whom the circulation is weak, and especially those who are subject to "deadness" of the fingers or to chilblains, are especially prone to Raynaud's disease. The most favourable predisposing condition for its development is the combination of a sluggish circulation with an unstable nervous system. Both in Raynaud's disease and in chilblains there is an undue susceptibility to the affection, apart from exposure to cold; in chilblains, however, reaction follows early, while in Raynaud's disease necrosis occurs before reaction can take place. Malaria, gout, and diabetes are believed to have a predisposing influence. The most frequent exciting causes are cold and an attack of acute disease (scarlet fever, measles, diphtheria).

Prognosis.-This depends on the severity and extent of the process and the constitutional state of the patient. Death is rare; but, on the other hand, the disease is always likely to recur, and permanent changes in the parts or mutilation may occur.

Treatment.-The most efficient treatment is galvanism. The constant current should be applied by immersing the affected extremity in a large basin of salt water, one pole being placed in the water while the other is applied to the limb. If this treatment be employed at a sufficiently early stage, the progress of the disease will often be cut short. The high-frequency current also is sometimes beneficial. Massage is very 
useful, and the internal administration of ichthyol, arsenic, or quinine may sometimes prove of service. The diet should include foods which are rich in hydrocarbons. When gangrene has taken place, the treatment must be conducted on ordinary surgical principles.

\section{Dermatitis Repens.}

Under this title Crocker has described a form of spreading dermatitis occasionally following injuries. It commences almost exclusively in the upper extremities, and is probably neuritic in character. I have seen a case in which the disease began on the sole of the foot, due to injury from a tack. The condition might sometimes be mistaken for eczema, but the oozing surface entirely denuded, and the sharply defined undermined spreading edge, are quite different from anything seen in that affection. The disease, though primarily the result of peripheral neuritis, is probably kept up and aggravated by secondary parasitic irritation. This view is confirmed by the beneficial effect of the local application of anti-parasitic remedies. The affection is sometimes persistent and difficult to cure.

\section{Erythema Serpens (Erysipeloid)}

Morrant Baker ${ }^{1}$ gave this name to an affection which has since been described independently by Rosenbach under the designation of erysipeloid. ${ }^{2}$ It appears to be due to inoculation with poisonous matter, and is seen most frequently in cooks, butchers, and persons who handle game, skins, etc. It shows itself as an erythematous eruption which starts from the seat of a small injury, doubtless the point of inoculation, generally on the fingers, especially about the knuckles. It spreads centrifugally, clearing up in the centre as the edge

'St. Bartholomew's Hosp. Repts., vol. ix., 1873.

${ }^{2}$ Verhandlungen d. deut. Gesellschaft f. Chir., April, 1887. 
advances; the circle soon breaks up, and when first seen the lesions generally present themselves as blotches of pink erythematous rash. There is no suppuration, and no involvement of the lymphatics of the arm. The subjective symptoms consist of local tingling, burning or shooting pains; there may also be some nervous excitement. The duration is from a fortnight to six weeks, the average being three weeks. Rosenbach believes that the affection is due to a microörganism, supposed to be a cladothrix, which exists in decomposing animal matter; but Gilchrist, ${ }^{1}$ in 329 cases at Baltimore, could find no such organism. Treatment consists in the application of a salicylic-acid (25 per cent.) or ichthyol (25-40 per cent.) ointment.

\section{Diabetic Gangrene}

In diabetes localized inflammation, ending in gangrene, is not infrequently observed in the foot, especially in one or other of the toes. It is not always the distal end that is attacked. The lesion sometimes affects a circumscribed area on the sole, the ball of the toes, or the dorsum. The part becomes inflamed, bullæ are formed, and more or less extensive sloughing takes place. The process, as a rule, affects only one side. Kaposi has described a case of what he calls "bulloserpiginous diabetic gangrene," in which the left leg was the seat of an eruption of disseminated bullæe on an inflamed base, with subsequent formation of eschars. From the affected part, as from a centre, the process extended serpiginously; the lesions took several months to cicatrize, and death occurred only after the process had invaded the tibio-tarsal joint. Gangrene of the penis, etc., has also been observed in association with diabetes, and I have met with cases in which the fingers have been affected.

1 Journ. Cut. Dis., 1904. 


\section{"Hysterical" Gangrene}

So-called "spontaneous" gangrene of the skin has occasionally been seen in young women, mostly in those presenting unmistakable signs of hysteria and anæmia. The patient suddenly feels a sensation of burning on some part of the skin, usually the chest or the arms. On examination, a raised and somewhat red spot, varying in size from a slilling to a crown piece, is seen in the place where the sensation was localized. In a few hours the skin becomes bluish-black or greenish-brown in colour, and a leathery eschar is formed resembling that produced by the application of sulphuric acid. This separates in due course, and its place is taken by a hypertrophic cicatrix. The same process is repeated in other parts at intervals of a few days or weeks, and this may go on for months or even years, and then finally stop. This description is taken almost verbatim from Kaposi, ${ }^{1}$ who expresses no suspicion of the genuineness of the phenomena. To me, however, the facts, as given by him, and those of similar cases of what is sometimes termed dermatitis factitia, are strongly suggestive of imposture. As the result of a study of five cases S. Roná concludes that the lesions of "hysterical gangrene" are artificially produced by some form of caustic." Max Joseph has recorded a case of multiple neurotic gangrene of the skin ${ }^{3}$ and Brandweiner another, in which the patient, a woman of 31 , had been an hysteric from the age of 13, and had several times been treated in asylums. Injection of serum from the patient's own blood caused gangrene, but no such result followed control experiments performed upon her. ${ }^{4}$ Pernet, availing himself of the

\footnotetext{
${ }^{1}$ Op. cit., p. 489.

${ }^{2}$ Arch. f. Derm. u. Syph., June, 1905, p. 257.

${ }^{3}$ Ibid., June, 1895.

4 Monats. f. prakt. Derm., Sept. 1, 1904, p. 241.
} 
theory of dissociated personalities, has suggested that in some cases the lesions may be inflicted while the patient is in the state of altered personality, and that no knowledge of what she has done is present to her normal consciousness. A moiety, or some smaller fraction, of the patient may therefore be truthful!

\section{Charco't's Bed-SORE}

A form of localized gangrene of the skin has been described by Charcot under the name of "acute bedsore." Its characteristic feature is the suddenness of its development. It is generally associated with transverse myelitis, but sometimes with abscess of the brain, and is in that case situated on the side of the body opposite to that of the cerebral lesion.

\section{Trophic Ulcers}

Trophic ulcers are the result of direct injury to nerves, or in some cases of reflex irritation. They generally spread serpiginously, and are preceded and accompanied by pain of neuralgic character referred to the area of distribution of a particular nerve. The ulcers often form under vesicles or bullæ, and leave indelible depressed or cheloid scars (Brocq). In some cases the process takes on a gangrenous character.

Perforating ulcer of the foot is a special form of trophic ulceration seen on the foot, but occasionally also on the hand. It is the result of pressure or injury in an extremity in which, owing to peripheral or central lesion, the proper nervous supply is interfered with. It occurs in locomotor ataxy and in syphilis, leprosy, etc., as well as in cases of injury to the nerve. The most common situation of the ulcer is at the point of greatest pressure, such as the under-aspect of the metatarsophalangeal joint of the big or little toe, or the ball of the great toe. It is more a sinus than an ulcer, and is 
usually painless. The process is generally very chronic, and if the pressure from walking is continued, the thickened epidermis forms a kind of natural corn-shield around the opening. The only lesion with which perforating ulcer can be confused is a suppurating corn, from which it is differentiated by the absence or slight degree of pain, and by the inefficacy of simple surgical treatment.

T. J. P. Hartigan ${ }^{1}$ reported a case of trophic ulcer in the flexure of the right big toe, extending down to the bone, and of three years' duration, which, treated by cataphoresis, with copper ions, healed up within a month "as completely as one can expect such tissue to heal."

\section{Syringomyelia and Morvan's Disease}

In syringomyelia the skin becomes the seat of various lesions, such as "glossiness," hyperkeratinization, excessive secretion of sweat, and whitlows, leading to necrosis of the phalanges. There is nothing characteristic about the skin lesions, which are tropho-neurotic in origin. The disease itself belongs to the domain of neurology.

Morvan's disease is characterized by paroxysmal attacks of neuralgic pain, ushering in various disorders of sensation and the development of bullæ, followed by ulcers and fissures, on the palmar surface of the hands and fingers. Usually one or more whitlows form, and necrosis of the phalanges takes place. A peculiar deformity of the hand, exactly resembling the main en griffe of anæsthetic leprosy, is produced. The disease appears to be connected with lesions of the cord. It was first described by Dr. Morvan, of Lannilis, in Brittany, in an admirable series of articles. ${ }^{2}$ This form of disease seems to be fairly common in certain rural parts of

1 Brit. Journ. Derm., 1908, xx. 369.

${ }^{2}$ Gaz. Hebdomadaire, 1883, No. 35 et seq. 
Brittany, and the hypothesis was put forth by Zambaco Pasha of Constantinople, and supported by others, that the cases of Morvan's disease were examples of leprosy attenuated by descent in an ancient population. Repeated pathological observation failed to give support to this hypothesis, and it has now been conclusively proved that Morvan's disease is a special form of syringomyelia, in which trophic skin lesions are prominent. ${ }^{1}$

\section{GLOSSY SkIN}

As the result of injury to the trunk of a nerve supplying a particular part of the integument, a peculiar change is often observed which is known as "glossy skin." The first account of this condition was given by Paget many years ago. After injury to the brachial plexus, he noticed that the fingers assumed "a smooth, glossy, tapering appearance, almost void of wrinkles, and hairless, pink, or ruddy, or blotched as if with permanent chilblains, and associated with this condition of the skin was distressing local pain." A fuller account of the condition was given by Weir-Mitchell, Morehouse, and Keen from their vast experience of nerve injuries during the American Civil War. They compare the appearance of the affected skin to that of a highly polished scar. The skin easily becomes inflamed, excoriated, and fissured. Characteristic changes in the nails are also observed. They are curved both in the longitudinal and in the transverse direction, and the cutis beneath their free ends is sometimes thickened. The condition, in short, is one of atrophy with degeneration of the skin, rendering it more vulnerable to injurious influences of all kinds owing to impaired nutrition. This is dependent on neuritis of the trunks from which it derives its nervous supply, and the effect is the same

1 Joffroy and Uchard, Arch. de Med. Expérimentale, 1890-95. Galloway, Brit. Journ. Derm., 1895, vii. 304. 
whether the nerve lesion is the result of injury or of disease. This "glossy" skin is observed in nontuberculous leprosy, gout, rheumatism, etc., as well as after traumatism.

I'the condition tends to disappear as the nervous influence is restored either by subsidence of the neuritis or by the establishment of a collateral supply.

\section{Atrophy of the Skin}

Localized atrophy of the skin may either be primary, or may be secondary to a great variety of conditions, which are thus enumerated by Sequeira ${ }^{1}$ : (1) Injury, traumatism, wounds, burns, scalds, the application of caustics, X-rays, and radium; (2) xerodermia pigmentosum and epidermolysis bullosa; (3) certain acute specific fevers-variola, vaccinia, varicella; (4) bacterial infections; acne vulgaris and other suppurative lesions of the follicles; ulcers due to syphilis, tuberculosis, leprosy, etc.; (5) favus of the scalp; (6) nervous diseases-herpes zoster, glossy skin, syringomyelia, nerve leprosy; (7) interstitial affections without ulceration -lupus vulgaris, lupus erythematosus, lichen planus atrophicus; (8) hydroa vacciniformis, and occasionally pemphigus and dermatitis herpetiformis; (9) stretehing of the skin as in strix atrophice; (10) senile degeneration.

Primary atrophodermia, which is sometimes the result of a tropho-neurosis, may take the form of linear streaks or striæ (atrophodermia striatum), or less commonly of maculæ (atrophodermia maculatum, the anetodermia erythematosa of Jadassohn), or the two forms may be combined (atrophodermia striatum et maculatum). A good example of linear atrophy has been recorded by OhmannDumesnil. $^{2}$ A little girl, who had been severely burnt

1 "Diseases of the Skin," by James H. Sequeira. 1911.

${ }^{2}$ Brit. Journ. Derm., 1890, p. 246. 
on the wrist, some years afterwards presented atrophic rectilinear areas about $\frac{3}{4}$ inch in width, and varying from $\frac{3}{4}$ to 2 inches in length, on the front of the arm and forearm, apparently following or lying directly over the brachial and radial nerves. The areas were five in number; they were distinctly depressed, and the colour was paler than that of the normal skin, but warmth made them redder than the healthy integument. On pinching up the affected skin it was felt to be thinner than in other parts. At first many of the striæ may be red, and somewhat raised, but after a time the redness disappears and formation of scars takes place. Strix may also be the result of injury during growth, pregnancy, and other conditions in which the skin is subjected to stretching. Many cases of patellar striæ following typhoid fever have been recorded, and Köbner ${ }^{1}$ concludes, from the histological and clinical evidence taken together, that in such cases the striæ are due to tension of the skin produced by rapid growth of the long bones and in other ways. In a case of mine, that of a girl of 15, there were linear striæ on the thighs, abdomen, and breasts, the result of overgrowth following scarlet fever. They have also been described in phthisis, lichen scrofulosorum, and colitis. Bunch ${ }^{2}$ has given an account of cases recorded up to 1905, and reported two observed by himself. One of these followed typhoid fever, and the other occurred in a case of pleurisy and endocarditis of long standing. The histological appearances pointed to the conclusion that rupture of the elastic fibres of the corium had taken place, due probably to stretching or tension rather than to inflammation.

Macular atrophy is usually associated with tuberculosis, and Heuss and others have expressed the opinion

1 Münch. med. Woch., May, 1904, p. 928.

${ }^{2}$ Brit. Journ. Derm., 1905, p. I. 
that there is connection between the toxins of tubercle and this dermatosis. A remarkable case, in which the patient had nephritis, but had no history of tuberculosis, has been reported by Adamson.1 There were oval, whitish to bluish atrophic macules, about the size of a finger-nail, over the whole trunk, on the arms to the elbows, and on the thigh. The macules followed the lines of cleavage, radiating from the axillæ, and sloping downwards and forwards at the sides of the chest. The openings of the follicles were larger and more conspicuous over the macules than in the surrounding skin. There were no signs of erythema, infiltration, or pigmentation. On the lower part of the abdomen the patches were distended into dome-shaped elevations by the œedema present in that part. Sections made by McDonagl showed a marked cell-infiltration immediately around the blood-vessels and a total loss of elastic tissue in the lesions.

A peculiar form of atrophy of the skin has been described by Herxheimer and Hartmann under the name acrodermatitis chronica atrophicans. These writers have published ${ }^{2}$ a study of twelve cases, and at the time their paper appeared the total number of cases recorded was twenty-seven. The affection begins with inflammation and infiltration, and, as in lupus erythematosus, these symptoms are succeeded by atrophy. Its etiology is not understood, and it runs an obstinately chronic course. It is differentiated from so-called idiopathic atrophy in that the atrophy is preceded by an inflammatory stage.

Senile atrophy of the skin.-This form of atrophodermia manifests itself in a parchment-like thinning and yellowish or reddish pigmentation of the skin, which loses its natural elasticity, and sometimes becomes dry

1 Brit. Journ. Derm., 1910, xxii. 351.

2 Arch. t. Derm. u. Syph., July and Aug., 1902. 
and ichthyotic. Frequently there are telangiectases and keratomas, which may undergo malignant transformation. In some cases the skin, instead of wasting, becomes thickened and wrinkled, and soft to the touch. Pruritus is frequently present. Allied to senile atrophy is the condition which Unna styles seaman's skin, in which the unprotected parts of the integument of those whose occupations expose them to extremes of cold and heat, undergo a degeneration which not infrequently leads on to epithelioma. Tropical skin is a form of this atrophodermia. It is not uncommon to see cases in which, as the result of residence in the tropics, the skin has shown atrophy, pigmentation, warts, and epitheliomas. 


\section{CHAPTER $\mathrm{X}$}

\section{ARTIFICIAL ERUPTIONS}

Artificial eruptions include all skin lesions produced by the external or internal action of some substance foreign to the economy. They form naturally two great groups-(1) eruptions caused by the direct contact of irritant substances with the skin (dermatitis venenata); (2) eruptions following the ingestion of substances that have a toxic effect on the system, manifesting itself by the production of certain lesions on the skin (toxic dermatitis).

\section{EXTERNAL AGENTS}

These may be of animal, vegetable, or inorganic nature. Among the animal irritants affecting the skin are $(a)$ parasites (lice, fleas, etc.) ; (b) jelly-fish, gnats, wasps, mosquitoes, etc.; (c) irritating discharges from the body itself (in coryza, gonorrhœa, and diabetes). Vegetable irritants include substances that come accidentally, or in the way of occupation, in contact with the human skin (Rhus venenata and toxicodendron, Primula obconica, thapsia, the common orange, eucalyptus leaves, arnica, etc.). Among other substances giving rise to skin eruptions by direct contact may be mentioned blood, mustard, ammonia, sugar, soap, and paraffin. The lesions caused by parasites, whether of animal or of vegetable nature, are described in Chapters Xv.-XvII.

The influence of these various agents on the skin shows the greatest diversity as regards the nature and 
severity of the lesions. As a general rule, it may be stated that the effect is proportionate to the length of time during which the contact is prolonged. The lesions may simulate almost any disease of the skin. The erythematous type predominates, but frequently the eruption takes the form of urticaria or lichenoid eczema. The severity of the process varies from a simple patch of erythematous redness, readily disappearing under pressure, to violent inflammation of the skin, presenting all the outward characters of the formation of eschars and ending in widespread ulceration and gangrene. Between these limits every degree of the inflammatory process -papules, vesicles, blebs, wheals, and pustules-may be seen. The erythema is always followed by more or less desquamation; the vesicles, blebs, and pustules by crusts and scabs. As the result of prolonged irritation the skin sometimes becomes thick, harsh, and wrinkled, while it is at the same time the seat of a chronic eruption characterized by papules and excoriated vesicles and resembling lichenoid eczema (Brocq).

As typical examples of the effect produced by certain vegetable irritants on the skin, mustard and rhus may be taken. The former produces redness and vesication; in some cases the process may run on to an actual dermatitis of erysipelatoid character, and even ulceration may be produced. The lesions may persist for several weeks. There are three varieties of rhus, all of which have strongly irritant properties, but only certain persons are susceptible to their action. Those in whom the idiosyncrasy is very pronounced may be affected even by the volatile emanations from the plant. The eruption is usually eczematous in character; the hands, arms, and face may be enormously swollen. Distant parts may share in the general eruptive disorder. There is always intense itching. Erysipelatoid inflammation of the skin and dermatitis exfoliativa are 
not infrequently observed. Rhus vernix is much em. ployed in Japan, and to it the so-called "lacquer poisoning" is due. Touching furniture that has been varnished with this substance, or even sleeping in a room where some of the furniture has been so treated, often suffices to induce an attack in those predisposed thereto. The effect on the skin is violent dermatitis, with much swelling of the eyelids and face generally, and with more or less severe headache, dizziness, and constitutional disturbance. Dermatitis also occurs from handling Primula obconica and teak. In the Scilly Islands, those employed in gathering and packing the various kinds of daffodils grown there are liable to what is known as the "lily rash," which may become chronic, or may generalize, and may be so severe as seriously to affect the health.

Trade eruptions.-Among eruptions caused by the contact of irritant substances, many are of the nature of diseases of occupation. Thus persons who often handle paraffin, petroleum, tar, bichromate of potash, sugar, salt, lime, sulphur, croton oil, etc., are all subject to eruptions of varying character and severity directly due to their occupation. The same is true of bakers, paperhangers, dyers, tanners, chemists, workers in the silver and electro-plating trades, washerwomen, etc. A case of antimonial and lead poisoning has been recorded by Leslie Roberts. The lesions in each case may assume any of the forms that have been mentioned, but in the majority the affection more or less closely simulates eczema; and in patients predisposed to affections of the skin the trade eruption not seldom develops into true eczema if the irritation causing it is sufficiently prolonged.

Röntgen-ray dermatitis.-A form of artificial dermatitis was sometimes set up by prolonged exposure to the Röntgen rays, until means had been devised by 
Holtzknecht, Sabouraud and Noiré, and others, for measuring the doses. X-ray dermatitis may be considered as $(a)$ an acute inflammation resulting from a single over-exposure or several exposures which are excessive in the aggregate, or $(b)$ as a chronic dermatitis affecting $\mathrm{X}$-ray operators or those who are constantly exposed to the rays over prolonged periods. Acute X-ray dermatitis occurs in three or four degrees of severity, which have been compared to those of ordinary burns. The first is erythema, with swelling and desquamation. In the second, there is erythema with vesicle and bulla formation. In the third, ulceration occurs, with destruction of the epidermis, corium, and, to a greater or less extent, of the subcutaneous tissue. The Röntgen ulcer is indolent and may be many weeks in healing; it is covered with a whitish or yellowish adherent slough, which has been compared to a diphtheritic membrane. Necrosis may ensue, the necrosed tissues persisting for several months or even a year or more. X-ray lesions are accompanied by severe pain, and leave deep vascular scars. The inflammation is apt to recur periodically. It is often sharply limited, stopping abruptly where the skin is covered by the clothing. Chronic X-ray dermatitis begins with erythema, pigmentation, atrophy, and the formation of telangiectases on the exposed partsusually the backs of the hands. The skin becomes thin, roughened, and fissured. In course of time local hyperkeratosis occurs, and the small warty growths thus formed may eventually become epitheliomatous. Dystrophy of the nails is also a common feature; these become pitted, striated, and brittle, and may be exfoliated. The condition described above occurs after many months or years, in those who have not taken means to protect the skin from the rays. Short of operative procedures which may become necessary, many applications have been suggested, but without much effect in 
alleviating the pain or subduing the process. Radium may, however, be of service. To avoid the cumulative effects of the X-rays, there should be an interval of from fifteen to twenty days between one large dose and the next.

Radium dermatitis.-Workers with radium may be affected with a dermatitis similar to that set up by the $\mathrm{X}$-rays. If there is over-exposure, an ulcer may occur.

Feigned eruptions.-Artificial eruptions are sometimes produced by the patients themselves, either to excite sympathy or to escape work. The subjects are mostly hysterical girls, beggars, prisoners, malingerers, or lunatics. Some of these impostors become by practice artists of sufficient skill to deceive the unwary. The substances used by them are chiefly croton oil, nitric acid, carbolic acid, essence of turpentine, iodine, mustard, thapsia, cantharides, and urine. The points which should give rise to suspicion are the situation of the lesion (breast, limbs, or other easily accessible part, the left side being for obvious reasons much more often chosen as the seat of operation than the right); the total absence of eruption in other situations; the anomalous outline of the lesions, which may be angular, and may resemble nothing seen in disease; the want of symmetry, or less frequently the too perfect symmetry, at once suggesting the work of art rather than of nature. Circumstantial evidence of fraud is also frequently supplied by the smell of the agent with which the lesions have been produced.

The affections most, often simulated are erythema, ulcerations, and chromidrosis (caused by blacklead, etc.). Colcott Fox and Sangster have reported cases in which sores were produced by perseveringly rubbing a spot with the ends of the fingers moistened with saliva. ${ }^{1}$

${ }^{1}$ Lancet, Dec. 30, 1882. 
In the French army, thapsia juice is in great favour with malingerers, on account of the erysipelas-like inflammation of the skin which can be induced by means of it. Patients of this kind will often inflict a good deal of pain on themselves, and will snip out pieces of skin with scissors, burn themselves with lighted matches, etc., with a fortitude worthy of a better cause.

\section{INTERNAL AGENTS}

Among eruptions caused by internal agents are included all those produced by substances swallowed either as food or as medicine. In the former case the agent is generally a particular article of diet in regard to which the patient exhibits an idiosyncrasy. The eruption which in many persons follows the eating of shell-fish, especially mussels, may be taken as the type of this skin affection $a b$ ingestis. The process has already been described under Urticaria (p. 83), and need not be further referred to here.

\section{Drug ERuptions}

Drug eruptions, properly speaking, include those caused by the external as well as the internal use of medicinal substances, inasmuch as a drug applied to, and producing lesions in, the skin may also be absorbed into the circulation, so that it is difficult to separate the one effect from the other. In this province, as Brocq well says, individual susceptibility is the most important factor; it is that which determines the appearance of the eruption and the form which it assumes. The eruptions caused by drugs present a variety of type that defies all classification : they may be erythematous, urticarial, papular, vesicular, bullous, and even cancerous-at least indirectly. A particular patient generally reacts in the same way to the same drug. The lesions are seldom multiform at a given 
time, though almost every variety may be exhibited in the course of an eruption at different stages.

Pathology.-Various theories have been advanced as to the mode in which drugs produce eruptions. According to Farquharson, when from any cause there is diminished activity of the kidneys, which are the natural channels by which most medicinal substances are eliminated, the skin vicariously assumes the functions of these organs, and the drug, in working its way outwards through the cutaneous glands, irritates the skin and produces lesions of various kinds. This theory would imply that before an eruption can be produced the drug must have accumulated to a greater or less amount within the body. This, however, is not by any means the rule, for the smallest dose of a drug will produce an eruption in some persons, while in other cases very large doses may be taken for a long time continuously without producing any effect whatever on the skin. But in the case of the halogens it is probable that the eruptions which they produce are due to the excretion of the drug by the cutaneous glands. Another theory is that certain drugs have an elective affinity for certain anatomical elements, and that in this way some medicinal substances naturally gravitate, as it were, to the cutaneous glands. In proof of this is adduced the fact that traces of the drug are often found in the lesions which it has produced. This, however, is probably nothing more than an accident; it is certain that the most careful tests frequently fail to reveal any trace of the drug in the cutaneous lesions, while it is readily found in the urine.

Behrend has propounded the view that drug eruptions, with the exception of those caused by the bromides and iodides and the erythemas produced by belladonna, hyoscyamus, stramonium, and possibly arsenic, are due to the presence in the blood of some foreign material 
generated by the action of the drug; this material he thinks is probably of chemical nature. It is a sufficient refutation of this theory, that drug eruptions are often confined to particular parts of the cutaneous surface, whereas, if they were due to an alteration in the blood, one would expect to see them wherever that fluid circulates. My own view is that-at least in the majority of cases-the mechanism of drug eruptions is the same as that of the erythematous, vesicular, bullous, and pustular affections which they simulate-that is to say, the process is angio-neurotic in character. It has already been explained that the simple mechanism of vaso-motor paralysis, followed by the phenomena of congestion and inflammation in varying degrees, is sufficient to account for the production of an ascending series of lesions, ranging from simple erythema up to gangrene; and inasmuch as all these various lesions are simulated by drug eruptions, there appears to be no reason to look farther for an explanation of their mode of action. In short, it may be stated that drug eruptions arise in response to irritation of nerve-endings, as when medicinal substances are applied externally to the skin, or to irritation of nerve-centres (vaso-motor), as when drugs are taken internally. In some cases, as Engman and Mook say of iodine and bromine eruptions, ${ }^{1}$ it may be that lesions are prone to appear on the sites of previous inflammation, and that trauma, pressure, and quick changes of temperature may precipitate an eruption in tissues charged with the drug.

Morrow has pointed out that a large proportion of the medicinal agents which determine eruptive disturbance act specifically upon the nervous system. From this point of view, the individual predisposition or idiosyncrasy, which is a necessary underlying condition for the production of drug eruptions, is, as has already

${ }_{1}$ Trans. Amer. Derm. Assoc., 1906, p. 152. 
been said in a previous chapter, nothing but abnormal excitability or instability of the nervous system. This may possibly be combined in the class of cases under consideration with undue susceptibility of the skin to irritation. The skin, being the organ of tactile sensation, is in the most intimate connection with the nervous system. So close, indeed, in some persons is the sympathy between the nerve centres and the skin, that the latter is, as it were, a mirror on which every passing shade of nervous impression or mental emotion is reflected. It is not, therefore, to be wondered at that it should often respond sympathetically to nervous disturbance produced by central or peripheral irritation. In the case of drugs which excite or irritate the nervous system, it may be laid down as a general rule that the greater the nervous disturbance, the more severe will be its manifestations on the skin. ${ }^{1}$

Diagnosis.-Drug eruptions following the external application of irritating substances are usually limited to the part with which the agent has been in contact ; moreover, in some cases the lesions themselves present certain definite characters by which they can be recognised. These will be referred to in connection with the several agents. The rashes produced by drugs taken internally often simulate those of the specific fevers, or of certain toxæmic conditions, so closely that, if rise of temperature and constitutional disturbance happen to be associated with them, it is almost impossible to distinguish them. Thus, copaiba eruption resembles that of measles or smallpox, and those of belladonna and quinine that of scarlet fever. An important point is the sudden occurrence of an

${ }^{1}$ For a lucid discussion of the mode of action of drugs in producing skin lesions the reader is referred to the valuable papers by H. G. Brooke on "Behrend's Division of Drug Rashes into Specific and Dynamic Groups" (Brit. Journ. Derm., Oct., 1890), and to Colcott Fox's "Contribution to the Study of Drug Eruptions" (ibid., Nov., 1890). 
eruption during the administration of a drug ; and if, on discontinuing the use of that drug, the eruption vanishes, it may safely be concluded that the two stood to each other in the relation of cause and effect. On the other hand, a drug eruption sometimes lasts for a considerable time after the drug is stopped. Another diagnostic point is the presence of the drug in the urine, the saliva, or the sweat. This, as a rule, holds good only when the drug has been taken in large quantities or for a long period of time. In the case of certain substancessuch as turpentine and other essential oils - their presence in the urine is often obvious to the sense of smell; in the case of the balsamic preparations the drug reveals itself by the smell of the patient's breath. Others, again - such as arsenic and nitrate of silver-produce a characteristic discoloration of the skin which is suffcient of itself to indicate the cause. As a general rule, it may be said that in the case of eruptions appearing suddenly, or presenting features different from those seen in idiopathic skin affections, the practitioner should always make careful inquiry as to what medicines the patient has been taking. It is impossible, within the limits of the present volume, to deal exhaustively with all the varied lesions that may follow the use of drugs; and, after all, there are only two drugs that cause eruptions of a sufficiently definite character to be pathognomonic; these are bromine and iodine and their compounds. The skin lesions to which these substances are apt to give rise will therefore be considered in some detail, and a brief summary of the principal effects on the skin that may be produced by some of the drugs in everyday use-such as arsenic, copaiba, belladonna, mercury, opium, and quinine-will be given. The effects of other drugs on the skin are indicated in a tabular summary (see p. 250).

Bromide eruptions.-Characteristic eruptions are 
caused by the use of bromine or its compounds-bromides of potassium, ammonium, sodium, etc.

Symptoms.-The primary lesions may be papules, vesicles, wheals, bullæ, or erythematous patches, but by far the most common and characteristic lesion is a papulopustular eruption (bromide acne) which is said to occur in about 75 per cent. of all patients treated with bromide of potassium. Bromide acne presents a considerable resemblance to acne vulgaris. Unlike the latter, however, the bromide lesion does not confine itself to parts rich in sebaceous glands, and the papulo-pustules always develop without the antecedent existence of comedones (Morrow). Bromide acne shows a marked preference for hairy parts of the skin. The papules, as a rule, precede the pustules, and they are seen about the forehead and nose and the backs of the shoulders, especially in persons whose skin is thick and greasy. They commence as small hyperæmic patches on an indurated base. Most of them are pierced by a hair. They may undergo no change for weeks, or they may quickly become transformed into pustules of a yellowish-white colour. Sooner or later the contents escape and a hard nodule or pigmented spot remains. They often give rise to small rounded cicatrices. This pustular eruption generally persists as long as the administration of the drug is continued, and the number of lesions increases as the dose is augmented (Veiel). On discontinuing the drug, the eruption, as a rule, disappears in from one to three weeks. In women and in children taking bromides, and in infants nursed by mothers who are taking them, the predominant type of lesion caused by the drug is the " confluent acne" described by Cholmeley. This at first resembles varicella, the vesicles, however, running together instead of drying up, and forming clusters, which continue to enlarge, and finally suppurate. In course of time, in this way, flattened elevations are 


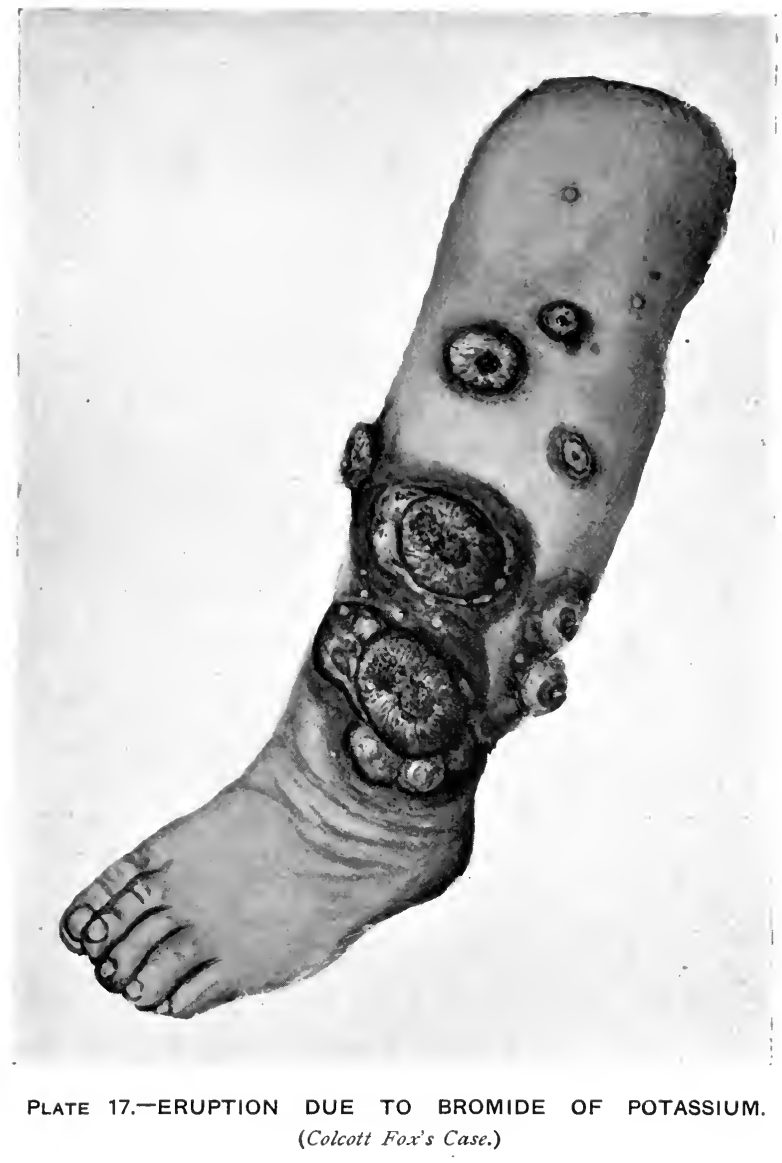



formed, covered with thick light-brown crusts and surrounded by a zone of redness. There is a tendency in these lesions to papillary hypertrophy, sometimes to such an extent as to simulate condylomas. The legs are the chief seat of the eruption. (Plate 17.)

Furuncular and anthracoid forms of bromide eruption are not uncommon. The boils, which are mostly of small size, are commonly seen in the situations generally affected by ordinary furuncles (forehead, neck, hairy parts of face), while the anthracoid swellings are usually found on the face and limbs, seldom on the trunk. The swellings are red in colour and well defined. The tops are dotted with numerous yellow points which give them something of the appearance of a carbuncle. After a time a scab is formed, and involution takes place rapidly if the drug is discontinued. If it is pushed, however, ulceration is pretty sure to take place. Sometimes the bromide eruption assumes an ulcerative character almost from the first. Large, irregular ulcerated patches form symmetrically on the legs. The granulomatous tumours arising in such cases may be mistaken for certain other forms of tumour. ${ }^{1}$ The drug may be continued, to allay the discomfort from eruptions of which it is actually the cause. The ulcerated surface is firm, and is composed of large raised masses often papillomatous in appearance. Warty growths on the face have been described as a result of bromide medication (Veiel). Though bullous elements are sometimes associated with other lesions due to bromide, true bullæ without more or less solid base and with fluid contents are rare (Colcott Fox). The appearance of bromide eruptions is not, as a rule, accompanied by fever or constitutional disorder. They not uncommonly develop on scar tissue. They often begin in the neighbourhood of the sebaceous glands

1 Cf. Jacquet, "The St. Louis Atlas of Skin Diseases" (part vii., 1897). Galloway, Brit. Journ. Derm., ii. 156. 
and hair-follicles, but are not by any means confined to these situations. Idiosyncrasy plays a comparatively subordinate part in the production of bromide eruptions. So constant, indeed, is their occurrence, given the necessary conditions of dose and persistence of administration, that the changes in the skin may with propriety be classed among the exaggerated physiological effects of the drug. Idiosyncrasy does, however, come into play in some cases when very small doses are followed by the development on the skin of some of the lesions that have been described.

Pathology. - The theory of Engman and Mook ${ }^{1}$ as to the modus operandi by which bromine (like iodine) produces its effects is that in certain conditions the. drug circulating in the body tissues acts as a toxin, causing at points of local disturbance-the sites of previous irritation or of injury-all the symptoms of an inflammation. Their suggestion is that it plays the part of a toxin when there is a disturbance of the normal equilibrium between the drug contained in the serum and the tissues. My own view is that the drug probably produces its effect through the nervous system.

Diagnosis.-The acneiform bromide-eruption lesions are easily distinguished from those of acne vulgaris by the absence of comedones, and by their occurrence at any period of life and on any part of the body. The anthracoid swellings are differentiated from carbuncle by the absence of a red border and of brawny induration around. Oliver S. Ormsby ${ }^{2}$ has described three cases of bromide eruption which were mistaken for blastomycosis. In the latter affection the papulo-pustular lesions are deeper, and less irregular in shape and size, nor is the earlier stage so actively inflammatory. In many cases the smell of bromine in the breath and its presence in
${ }^{1}$ Loc. cit.
${ }_{2}$ Journ. Cut. Dis., Oct., 1909. 
the urine at once point to the true origin of the skin lesions.

Iodide eruptions. Symptoms.-Eruptions produced by the action of iodine or its salts (iodide of potassium, iodide of ammonium, iodide of sodium, etc.) are erythematous, papular, urticarial, vesicular, and sometimes bullous in type. The erythematous form is the most frequent among the earlier manifestations of the influence of the drug on the skin. The redness may be scattered about in small or large patches, or pretty generally diffused, the favourite situations being the chest, the face, and the forearms. At a later period papules and wheals may develop on the erythematous ground, and on these wheals large capillary vessels are frequently seen. Vesicles may also develop on the erythematous patches. These are usually discrete, and are sometimes associated with wheals, around which a ring of clear vesicles may form. The bullous type of eruption is comparatively rare. The bullæ are sometimes mingled with vesicles and pustules. They may be as large as a pigeon's egg, and if two or three coalesce, as they sometimes do, enormous blisters may be formed.

The iodide eruptions often commence as hard papules which have the shot-like feel characteristic of the earliest stage of smallpox pustules. As the papules become transformed into vesicles they frequently show a tendency to umbilication. They are for the most part surrounded by an erythematous areola, and the skin about them is generally more or less infiltrated. The papulopustular form is the most common and the most characteristic eruption caused by the iodides. The face, the upper part of the chest, the backs of the shoulders, and the arms are the parts in which it chiefly shows itself. This form also begins as shot-like papules, which become pustular either at the summit or throughout their whole 
depth as they develop. These pustules dry up and form crusts, which leave a scar on becoming detached. In other cases the papules develop into vesicles and even bullæ, or they may become transformed into red, hard nodules deeply implanted in the tissues and disappearing very slowly. From these elementary lesions various more complex forms of eruption-ecthymatous, condylomatoid, molluscoid, etc.-may arise. Among the other forms of eruption caused by iodides there is one of carbuncular type resembling the "confluent acne" already described as a frequent effect of the bromides. The little boil-like nodules are violaceous in colour, with a depressed centre covered with a scab and studded at the circumference with numerous sebaceous-looking pustules. When these lesions disappear they leave a brownish scar. A purpuric eruption sometimes appears on the legs as the result of treatment with iodides (Fournier). The petechiæ almost always come out within a very few days of the beginning of treatment. Stephen Mackenzie reported a fatal case of iodide purpura in a child caused by a single dose of two and a half grains. A nodular form of iodide eruption has been described. Hard, red, painful nodules, varying in size from a nut to an egg, come out on the face, neck, buttocks, thighs, and calves. The eruption closely resembles erythema nodosum. As a rule, the effect of iodides on the skin is restricted to one type of lesion in any given case, but sometimes the eruption is polymorphous. Iodide eruptions are often associated with renal and cardiac inadequacy, and, though usually of little practical importance, may occasionally assume a grave character and react unfavourably or even dangerously on the patient's general condition. The eruption generally shows itself within a week of the commencement of administration of the drug, but the interval varies according to dosage and to individual 
susceptibility. After it has subsided one small dose may suffice to bring it out again in a very few hours. According to some observers, the salts of iodine vary somewhat in their power of producing skin eruptions, the iodide of ammonium being the most and iodide of sodium the least active in this direction.

Diagnosis. - In the early stages the papular form of iodide eruption may simulate smallpox, and the resemblance is increased by the umbilication which occurs when the papules develop into vesicles. The absence of severe constitutional symptoms, however, and the rapid disappearance of the eruption on discontinuing the drug, will quickly clear up any doubt that may exist. In some cases iodide eruptions may simulate acne or varicella, but here again the coincidence of the skin lesions with the administration of the drug, their aggravation by increase of the dose, and their disappearance on suspending the treatment, will prevent any misapprehension as to their nature. Rupial and other forms of iodide eruption may be mistaken for syphilitic lesions, and, in the words of Morrow, "iodide of potassium may be continued, possibly in increasing doses, for the very condition which it has caused."

Iodide and bromide eruptions compared.-The essential lesion in both these eruptions is a dermatitis showing a tendency to localization about the sebaceous glands. In each the eruption may take the form of papules, pustules, vesicles, bullæ, nodules, and almost every variety of combination of these elementary lesions. These often coalesce, and large swellings with crusts, warty excrescences, and ulcers may result. The bromide eruptions are, as a rule, slower in their development, and less painful than those caused by the iodides. Moreover, the latter are usually smaller than the former, and confluence is less frequently observed. In the case of both bromide and iodide eruptions the parts 
chiefly affected are the face and limbs, especially around hair-follicles.

Iodoform.-The use of iodoform in surgical dressings sometimes causes irritation of the skin. This is in the majority of cases accompanied by greater or less constitutional disturbance. The rash is generally erythematous in character, papules, vesicles, and even bullæ not infrequently developing on the inflamed surface. Sometimes the eruption rather approximates to the eczematous type. Purpuric lesions have in rare cases been observed in connection with the application of iodoform. In cases where idiosyncrasy in relation to the drug is pronounced, intense itching, with rise of temperature and swelling of the hands, arms, and face, may be caused by simple contact, as in dressing a wound with an iodoform bandage (Morrow).

Orthoform.-Orthoform, when applied to ulcerated surfaces, may also give rise to eruptions. Dubreuilh ${ }^{1}$ groups them under two heads : erythematous eruptions, with or without complicating vesiculation or pustulation; and gangrene. In the latter case the appearance is said to resemble that of lupus vulgaris treated by pyrogallic acid.

Arsenic.-Arsenic, when applied to the skin, acts as an irritant, causing dermatitis; used in a concentrated form, and for a long period, it is a caustic. The irritant effects are usually seen after the use of the drug in lotions for the complexion, in dusting-powders for children, and in various industrial products-notably artificial flowers, green wall-papers, certain aniline dyes (in stockings, under-vests, etc.).

Symptoms. - The lesion is at first erythematous in character, and on this vesicles and pustules often develop; and sometimes, more especially about the scrotum and pudenda, small, shallow, clean-cut ulcers

${ }^{2}$ La Presse Méd., No. 40, 1901. 
may result. When given internally, arsenic may cause exacerbation of acute inflammatory disorders of the skin. When no previous cutaneous affection exists, the internal administration of the drug may cause dermatitis, with papular, vesicular, urticarial, petechial, and pustular lesions; boils and carbuncles and multiple ulcerations are also sometimes observed. A general scarlatiniform eruption, with inflammation of the conjunctiva and mucous membrane of the respiratory passages (leading in the nose not infrequently to ulceration and perforation of the septum), has sometimes been observed. A common effect of arsenic on the skin is the production of a peculiar greyish or brownish discoloration, with desquamation in various parts. The prolonged administration of the drug also sometimes gives rise to general thickening of the epidermis on the palms and soles, 1 or in some cases to the formation of small corns; if the drug is persevered with, these corns may assume an epitheliomatous character (Hutchinson, Brocq, Hartzell, and others). Aldrich suggests that arsenical poisoning is one cause of white transverse lines on the nails. In the case which drew his attention to the point, the white lines, judging from the growth of the nail, corresponded to the time when the patient had taken arsenic with suicidal intent, and in other cases also he succeeded in obtaining confirmatory evidence. ${ }^{2}$ It is well known that arsenic, like other drugs of the metallic group, has been given

${ }^{1}$ For a remarkable example of this effect of arsenic, see " A Case of Keratosis of the Palms and Soles," by Pringle, in the Brit. Journ. Derm., 1891, p. 390. Another striking case, in which the keratosis was followed by cancer, is described by Schamberg (Trans. Amer. Derm. Assoc, 1906, p. 144). It is possible that some of the cases in which keratosis of the palms and soles has seemed to follow lichen planus (see Brooke, Brit. Journ. Derm., 1891, p. 19) may have been of arsenical origin.

2 Amer. Journ. Med. Sci., April, 1904, p. 702. 
for a long time in large doses, as in chorea, and that in such cases it sometimes produces peripheral neuritis; this fact probably explains the occurrence of zoster and other forms of herpes in association with treatment by arsenic.

On the basis of facts observed in the epidemic of arsenical beer poisoning which occurred in the north of Fngland and midland counties in 1900, Brooke and Leslie Roberts ${ }^{1}$ conclude that arsenic and the other members of the same group must be distinguished from all other medicaments by the fact that their action, whether therapeutic, pharmacological, or toxicological, is entirely dynamic, and consists essentially in altering the ratio to the tissues of one of the most active normal constituents of the body, namely, oxygen.

Chloral.-Chloral hydrate acts as an irritant when applied to the skin, and Ritter thinks it superior in some ways to cantharides as a vesicant. When given internally it occasionally causes a diffuse erythematous eruption on the skin; this generally begins on the face, and may spread to the neck and chest, and may also affect the extremities. An erysipelatous-looking flushing of the head and face is one of the commonest forms of chloral rash. On other parts of the body the eruption sometimes occurs in patches or scattered dusky-red spots, giving the skin a mottled appearance (Morrow). The rash comes out, as a rule, within ten days of the commencement of administration, is unattended with constitutional disturbance, and quickly fades. The taking of food or the drinking of tea, and especially of alcohol, has a marked effect in intensifying and extending the eruption; and even when chloral is no longer being taken, the rash may for some days come out after each meal. In some cases it is distinctly scarlatiniform in character, and may spread over the ${ }_{1}$ Brit. Journ. Derm., April, 1901. 
entire surface of the skin. This condition is often accompanied by fever and is followed by desquamation. Papular, urticarial, vesicular, and petechial eruptions have also been described as occurring in connection with the taking of chloral. The mucous membranes may be affected as well as the skin. The chloral rash bears a close resemblance to that produced by copaiba, belladonna, and quinine. The characteristic odour of copaiba is, however, absent, while the throat is not affected as in the case of belladonna; the absence of mydriasis, which is so characteristic an effect of the latter drug, is another point of distinction. From quinine eruption the skin lesions caused by chloral can be distinguished by the marked effect which a full meal or alcohol almost always has on the latter. From measles and scarlatina chloral eruption is differentiated by the absence of coryza and sore throat respectively.

Copaiba and cubebs.-These drugs cause eruptions on the skin that vary in character, the erythematous and papular forms, however, predominating. The lesions are generally seen around the wrists, ankles, and knees; often on the hands and feet, breast, and abdomen; sometimes they spread over the whole body. The most characteristic effect of copaiba on the skin is the so-called "balsamic erythema," which consists of small discrete erythematous papules, apparently seated at the follicles, and sometimes agminated into patches. These patches may also become confluent. Vesicular, urticarial, bullous, and petechial forms also occur, and the eruption may simulate erythema multiforme. The copaiba rash might possibly, from its appearance, be mistaken for an erythematous syphilide. The charasteristic violet-like odour of the drug will, in the majority of cases, prevent such an error; but it must be remembered that the balsamic eruption and erythematous syphilide occasionally co-exist. I have known a copaiba 
eruption to be mistaken for smallpox. The chief differentiating features, besides the odour of the drug, are the history, the heterogeneity of the lesions, and the absence of systemic disturbance, especially lumbar pain.

Belladonna.-The rash caused by the use (external or internal) of belladonna or atropine is generally erythematous in type, and of a dark-red or copper colour; it is, as a rule, diffuse, and closely resembles the exanthem of scarlet fever. The face, neck, and trunk are the usual seats of the eruption, and a stinging or pricking sensation in the affected skin is commonly complained of. The rash quickly disappears, and is not followed by desquamation. Dilatation of the pupil assists in making a diagnosis. Children with fine skin are particularly subject to eruptions from the use of belladonna. In ophthalmic practice the use of atropine is sometimes followed by severe dermatitis resembling erysipelas.

Chlorine.-An acneiform eruption caused by contact with chlorine has been noted by Herxheimer. Bettmann $^{1}$ has reported two cases in vigorous men who had been at work cleaning out the place for the manufacture of hydrochloric acid in a chemical factory. The disease was very obstinate.

Formalin.-The use of formalin occasionally produces eruptions on the skin. In a case under the care of Dr. Lewis G. Glover, of Hampstead, ${ }^{2}$ a young lady was attacked by violent urticaria, practically covering the whole body, after using a hair lotion containing formalin in bay rum. The face was swollen so that the features were scarcely recognizable, and great wheals were observed on the trunk and extremities. A large amount of erythema was also present, and the dis-

${ }^{1}$ Deut. med. Woch., July 4, 1901.

${ }^{2}$ Brit. Journ. Derm., 1901, p. 154. 
comfort was so great that sleep was impossible. No local dermatitis on the scalp was produced. An eczematoid eruption on the fingers is sometimes caused by handling solutions of the substance in the preparation of museum specimens. ${ }^{\prime}$ After putting cotton-wool soaked in a weak solution of formalin into a hollow tooth, Fisher noted an urticarial eruption on his own body.

Mercury.-The irritation of the skin caused by the external use of mercury varies, according to the strength of the application and the length of time contact is prolonged, from slight erythema to severe dermatitis, which may run on to ulceration and sloughing. The most common lesion is erythematous redness with the formation of vesicles, especially around the hair-follicles; these vesicles often develop into pustules. Such eruptions have been very common since corrosive sublimate became fashionable among surgeons as an antiseptic. Certain widely advertised preparations for the removal of pimples and blotches from the face contain corrosive sublimate, and serious effects, local and general, have been known to follow the use of them. When taken internally, mercury may produce almost any kind of skin lesion, and the effect of the drug may simulate urticaria, herpes, impetigo, or furuncle; sometimes it produces extensive ulceration. In the majority of cases, however, the eruption is erythematous or scarlatiniform in type; desquamation sometimes follows. In certain cases violent eruptions, resembling pityriasis rubra, may be produced. The skin lesions caused by the internal use of mercury are not infrequently, however, polymorphic. The eruption is often preceded by itching and dryness of the skin, and in severe cases it is ushered in by constitutional disorder. The symptoms often come on quite

1 T. Fisher, Brit. Journ. Derm.; 1901, p. 306. 
suddenly, not seldom after a single dose of the drug; but Thimm ${ }^{1}$ reports a case in which a bullous eruption appeared so late as eighteen days after the last of twelve mercurial inunctions. The malaise generally ceases on the appearance of the eruption.

The visceral and other symptoms of mercurialism (stomatitis, etc.) are frequent accompaniments of the skin eruption. The average duration of the latter is from one to three weeks, but the condition may persist for six months or more. The diagnosis is not always easy; measles and the other exanthematous fevers have to be excluded, and all other possible sources of drug eruption have also to be eliminated. When the affection is severe the prognosis is often serious. A case of malignant mercurial dermatitis ending in death has been recorded by Mari. ${ }^{2}$

Opium.-The intolerable itching sometimes caused by opium was known to Dioscorides and other ancient writers, who speak of it as pruritus opii. The eruption caused by it is mostly scarlatiniform in character; sometimes it is morbilliform, consisting of small discrete spots, bright or dusky red in colour. The face, neck, and flexor surfaces are the usual seats of the eruption, the appearance of which is generally preceded by local heat and itching. The rash may involve the whole cutaneous surface, making the patient "as red as a lobster." Desquamation is the rule. The rash quickly disappears on discontinuing the drug; but in those susceptible in this way to the influence of opium an eruption is almost certain to follow the administration of it in any form: Similar effects often result from the internal or subcutaneous administration of morphia. The latter is also apt to cause local inflammation and

1 Derm. Zeitschr., Bd. lx., Hft. 6, Dec., 1902.

${ }^{2}$ Giorn. Ital. delle Mal. Vener. e della Pelle, fasc. ii., 1896 ; Brit. Journ. Derm., 1897, ix. 118. 
abscesses in the skin, unless proper antiseptic precautions are employed; these conditions may lead to the formation of very obstinate ulcers.

Quinine.-Quinine, and all preparations of cinchona, may give rise to skin eruptions. Externally applied, it is not an irritant to the healthy skin, but workers in quinine factories are subject to eruptions which are no doubt due to absorption of the drug. These lesions are mostly eczematous in character, and generally come on suddenly; the parts chiefly affected are the hands and forearms, thighs, and genitals. Lichenoid and urticarial eruptions have been seen to follow the application of ointments or solutions containing sulphate of quinine. The hypodermic use of the drug is sometimes followed by widespread erythema, abscesses at the sites of injection, and ulceration. When given internally it causes skin lesions of the most various types. The erythematous form predominates; but macules, papules, vesicles, bullæ, pustules, wheals, and petechiæ are not uncommon. On analysing sixty cases of quinine eruption, published during a period of ten years, Morrow ${ }^{1}$ found that in thirty-eight the general character of the eruption was erythematous ("scarlatinal," "measly," etc.); in twelve it was urticarial, with " œdema," " puffiness of the face," etc.; in a few cases it was papular and vesicular or petechial. Bullous and gangrenous forms of quinine eruption have also been described. In diagnosis, the chief source of possible confusion is the close resemblance of the rash to that of scarlatina in many cases-a likeness which is made all the greater by the fact that the quinine eruption may affect the mucous membrane of the throat as well as the skin. Usually, however, the quinine eruption is not accompanied by fever; but sometimes there is considerable constitutional disturbance. The subsidence of the eruption on ${ }^{1}$ N.Y. Med. Journ., March, 1880. 
discontinuing the drug and the presence of the latter in the urine are the points of distinction.

Salicylic acid, salicylate of soda.-Externally applied, salicylic acid is more irritating than carbolic acid, even a 2 per cent. solution causing the appearance of irritable vesicles in the neighbourhood of wounds (Callender). The internal use both of salicylic acid and of salicylate of soda sometimes gives rise to erythematous, urticarial, vesicular, pemphigoid, and petechial lesions on the skin. The erythematous lesions resemble those caused by antipyrin, chloral, etc., and their appearance is generally accompanied by some febrile disturbance. Sometimes the rash closely resembles that of scarlet fever, and, as it is occasionally accompanied by sore throat and systemic disturbance, it may be difficult to distinguish the one condition from the other. In other cases the rash is morbilliform.

Other drugs. - A summary of the eruptions caused by other drugs in common use follows :-

\begin{tabular}{c|c}
\hline Aconite & $\begin{array}{c}\text { Externally applied: Redness, itching vesicles, } \\
\text { erysipelatoid inflammation. } \\
\text { Internally administered : Vesicular eruption with } \\
\text { formication and itching; sometimes } \\
\text { pustules and blebs. }\end{array}$ \\
\hline Antifebrin & $\begin{array}{c}\text { Internally administered : Slate-coloured cyanosis. } \\
\text { Antimony }\end{array}$ \\
\hline Anternally applied: Varioloid eruption ; some- \\
times ecthymatous ulcers and exten- \\
sive destruction of tissue. \\
Internally administered : Vesiculo-pustular and \\
urticarial eruptions ; sometimes vario- \\
loid eruption like that produced by \\
external application.
\end{tabular}




\begin{tabular}{|c|c|}
\hline $\begin{array}{l}\text { Argenti } \\
\text { nitras }\end{array}$ & $\begin{array}{l}\text { Internally administered: Peculiar bluish-grey or } \\
\text { greyish-black discoloration of skin, } \\
\text { somewhat resembling Addison's disease, } \\
\text { especially on face and flexor aspects } \\
\text { of limbs (argyria). Erythematous and } \\
\text { papular eruption with pruritus. }\end{array}$ \\
\hline Arnica & $\begin{array}{l}\text { Externally applied : Erythemato-vesicular erup- } \\
\text { tion resembling that caused by "poison } \\
\text { oak"; often eczematous; sometimes } \\
\text { erysipelatous dermatitis. } \\
\text { Internally administered: Erythema with formica- } \\
\text { tion ; diaphoresis. }\end{array}$ \\
\hline $\begin{array}{l}\text { Balsam of } \\
\text { Peru }\end{array}$ & $\begin{array}{l}\text { Externally applied: Frythematous, eczematous, } \\
\text { and urticarial eruptions. }\end{array}$ \\
\hline Boric acid & $\begin{array}{l}\text { Externally applied: Erythematous rash on face, } \\
\text { trunk, and extremities (after washing } \\
\text { out pleura); acute dermatitis of scrotum } \\
\text { and thighs after injection in to bladder ; } \\
\text { impetigo after long use of borax. Some- } \\
\text { times burning and charring of skin. }\end{array}$ \\
\hline $\begin{array}{l}\text { Borate of } \\
\text { sodium }\end{array}$ & $\begin{array}{l}\text { Internally administered: Eruption like psoriasis } \\
\text { (Gowers); also eruption like pityriasis } \\
\text { rubra. }\end{array}$ \\
\hline Cade oil & $\begin{array}{l}\text { Externally applied: Erythematous eruption, } \\
\text { which may spread over large area ; ery- } \\
\text { sipelatoid dermatitis; papular eruption, } \\
\text { like "tar acne" on hairy parts (sycosis } \\
\text { cadique of Bazin). }\end{array}$ \\
\hline $\begin{array}{l}\text { Cannabis } \\
\text { indica }\end{array}$ & $\begin{array}{l}\text { Internally administered: Papulo-vesicular erup- } \\
\text { tion on scalp, face, neck, trunk, and } \\
\text { limbs. Only one case on record (Hyde). } \\
\text { In poisoning by the drug, œdema of } \\
\text { face. }\end{array}$ \\
\hline Cantharides & $\begin{array}{l}\text { Externally applied : Vesicant; skin around } \\
\text { blister may become covered with vesicles, } \\
\text { which are often confluent; eruption } \\
\text { sometimes assumes eczematous charac- } \\
\text { ter, and extends over whole body. In } \\
\text { persons of feeble constitution ulceration } \\
\text { and gangrene may follow application. }\end{array}$ \\
\hline Carbolic acid & $\begin{array}{l}\text { Externally applied: Erythema up to complete } \\
\text { destruction of tissues, according to } \\
\text { strength of preparation. Rash often } \\
\text { accompanied by toxic effects (headache, } \\
\text { vomiting, oliguria, and dark urine). }\end{array}$ \\
\hline
\end{tabular}


Chrysarobin

Chrysophanic acid

Croton oil

Ergot

Iron

Lead (acetate and carbonate)

Nux romica Strychnia

Phenacetin

Pix liquida (tar)
Externally applied: Hyperæmia with prunejuice discoloration of skin ; erythematous, papular, pustular, and furuncular eruption. Erysipelas-like swelling of head and face. Exfoliative dermatitis.

Externally applied : Erythematous, papular, vesicular, pustular eruptions. Sometimes secondary eruptions appear on distant parts (from absorption?).

Hypodermically given: Painful black swelling at site of puncture, phlegmonous inflammation round it.

Internally administered: Vesicular, pustular, petechial, furuncular lesions, sphacelus, gangrene of extremities (ergotism).

Internally administered: Acneiform eruption on face, breast, and neck. Iodide of iron causes erythematous, papular, urticarial, eczematous lesions (probably chiefly from iodine contained in it).

Externally applied: Blackish or brownish discoloration.

Internally administered: Erythematous rash; petechiæ.

Internally administered: Pruritus and formication. Miliary or scarlatiniform eruption.

Internally administered: Great heat and erythematous eruption on face.

Externally applied: Erythematous, papular, vesicular, pustular eruptions. Tar acne, consisting of small, hard, red nodules, distinguishable from ordinary acne by black tarry points in centre of each papule; they persist long after the application, and may require three or four weeks for their complete involution. Erythema papulatum and violent dermatitis may follow the application of a pitch-plaster.

Internally administered: Copious red rash with fever, nausea, etc.; erythematous, rubeoloid, urticarial lesions. 
Podophyllin

Stramonium



Sulphonal

$\underline{ }$

Sulphur

Terebene

Turpentine
Externally applied (in workmen who pulverize it): Irritant, especially on scrotum and genitals.

Internally administered: Eruption like that produced by belladonna, but less vivid in colour; numbers of small brilliant petechiæ on face (Meigs); erysipelatoid inflammation.

Internally administered: Diffuse scarlatiniform eruption with intense itching; generalized macular erythema.

Externally applied: Redness, papules, painful vesicles (often confluent); artificial eczema. Papular and vesicular eruption common in those taking sulphur thermal baths.

Internally administered: Dark discoloration of skin; eczematous eruption, boils, carbuncles.

Externally applied: Turpentine causes extensive redness, vesicles, and inflammatory lesions. Very persistent and intractable.

Internally administered: Terebene may cause a bright-red papular rash. Turpentine may cause erythema of wine-red hue on face and upper part of trunk ; profuse papulo-vesicular eruption; sometimes eruption becomes eczematous in character.

Eruptions are occasionally caused by bitter almond, calcium sulphide, capsicum, chinolin, conium, hyoscyamus, ipecacuanha, cod-liver oil, castor oil, phosphorus, santonin, tannin, and veratrum viride; but these are of such rare occurrence that they are of little practical importance, ${ }^{\mathbf{1}}$ and it is not necessary, therefore, to enumerate symptoms.

1 For full information on drug eruptions the reader is referred to Prince Morrow's work on the subject, edited for the Sydenham Society by Colcott Fox, who has enriched the text with copious notes which add greatly to the value of the book. 


\section{INOCULATION RASHES}

This seems to be the most appropriate place for a brief description of certain eruptions that follow vaccination and other inoculations performed for a therapeutic purpose. At present our experience of skin eruptions due to this cause-apart from vaccination -is very scanty; but as the current of therapeutical opinion has been setting in the direction of injections of organic liquids (tuberculin, thyroid extract, antitoxic serums of various kinds, vaccines of microörganisms, etc.), it will probably before long be considerably extended.

Vaccination eruptions. - From the etiological standpoint I have suggested a division of vaccination eruptions into groups, which has been slightly modified and extended by others, as follows:-

Group I.-Eruptions which are due to vaccine virus.

Group II.-Eruptions which are due to mixed inoculation-that is to say, to vaccine together with an additional virus.

Group III.-The sequelæ of vaccination.

Table of Vaccination Eruptions

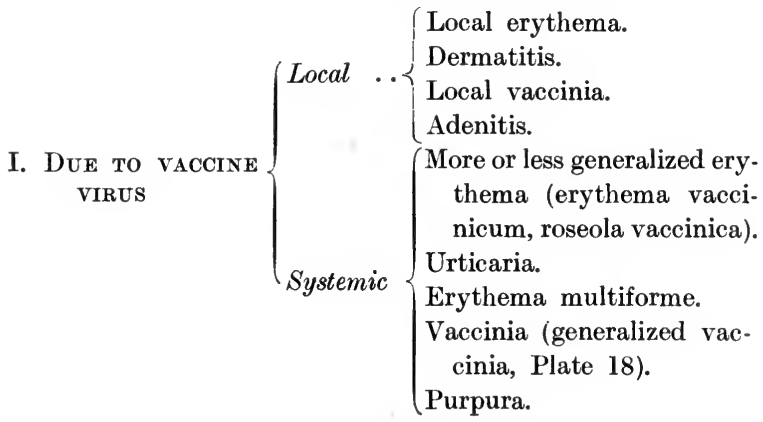




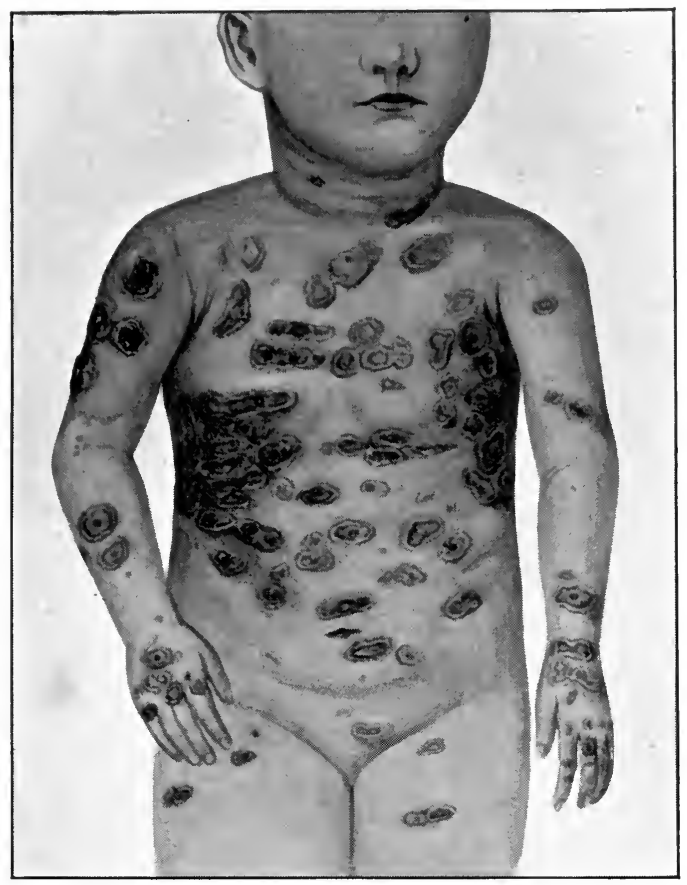

Plate 18.-Generalized Vaccinia. 

Table of Vaccination Eruptions (continued)

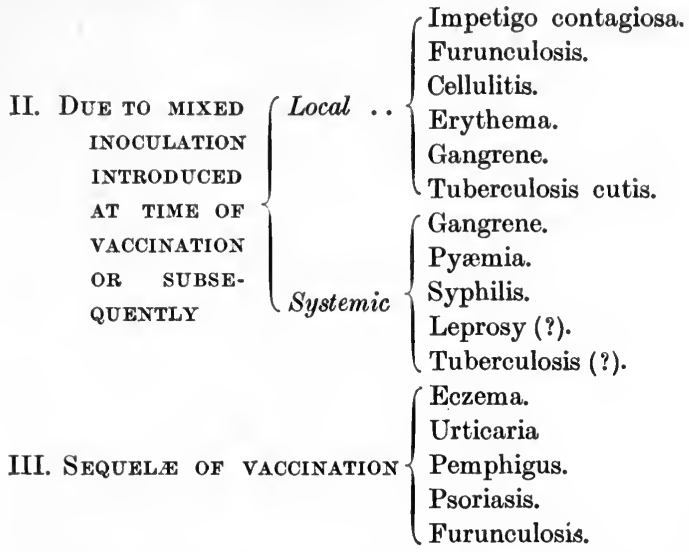

The eruptions belonging to Group I., depending, as they do for the most part, on idiosyncrasy, are practically unavoidable. On the other hand, those in Group II. are preventable by using only pure vaccine lymph with the strictest antiseptic precautions.

The eruptions belonging to Group II. may be local lesions, or manifestations of constitutional disease. To the former category belongs contagious impetigo, which can be inoculated with the vaccine virus, become developed in the vesicles, and spread by autoinoculation to all parts of the skin. Another local manifestation is a dernatitis or erythema, which starts from the areola and spreads over a limited area, passing imperceptibly into healthy skin. This is often spoken of as true erysipelas, but as it never extends to other parts of the skin, it is in reality only a local dermatitis.

A peculiar "raspberry excrescence" has been described by American writers as sometimes appearing from three to seven days after vaccination. It begins 
as a red elevation at the site of inoculation; but, instead of advancing to the vesicular stage, it remains hard, dense, bright red in colour, and nodular in form, looking not unlike a small nævus. It is very persistent, is not followed by a scar, and does not confer immunity. ${ }^{1}$

As regards constitutional disease, Hutchinson has proved that syphilis may be transmitted by vaccination. That leprosy may be transmitted by vaccination is inherently probable from the fact that the disease is inoculable. That it has actually been so transmitted there is extremely little decisive evidence to show. I know of only two published cases of the kind which will bear examination; both of these were recorded by Daubler. ${ }^{2}$ I know of no evidence that general tuberculosis has ever been transmitted by vaccination. Graham Little ${ }^{3}$ has reported four cases observed by himself and three by Colcott Fox, which seem to show that the transmission of lupus is at least a possible accident of vaccination. Fox, however, does not think that any conclusion of scientific value can be drawn from these cases. The vaccination sores may, he suggests, have been inoculated secondarily, or a previous tuberculous centre may have existed and an embolus found its way to the scar.

True erysipelas occasionally occurs in connection with vaccination. It is distinguished from the local dermatitis above referred to by its characteristic margin, swelling and tension of the skin, high fever and general constitutional disturbance, and by the rapidity with which it spreads over the limbs and the body. Gangrene

1 Welch and Schamberg, Morrow and others. See also Colcott Fox, "The Complications of Vaccination," Brit. Med. Journ., July 5, 1902.

2 "Ueber Lepra und deren Contagiosität," Monats. f. prakt. Derm., Feb. 1, 1889, p. 123.

${ }^{3}$ Brit. Journ. Derm., March, 1901. 
has in rare cases attacked the vaccine vesicles, causing extensive sloughing, and in one instance a general vaccinia is said to have become gangrenous (Hutchinson). Pyamia is extremely rare; it is caused by the introduction of pyogenic organisms into the wound.

The sequelæ of vaccination (Group III.) are eczematous, urticarial, pemphigoid, and psoriatic eruptions, and boils. I showed at the Dermatological Society of London a middle-aged man in whom vaccination was apparently the starting-point of eruptions having the characters both of psoriasis and of lichen planus. ${ }^{1}$

An eruption which mimics vaccine lesions is described by Colcott Fox ${ }^{2}$ under the name vacciniform ecthyma of infants. The resemblance to the vaccination eruption is so close that Jacquet has suggested the possibility of the lesions being an accidental localization of abnormal and deformed vaccine lesions. The age at which the affection develops, the sites of predilection-the neighbourhood of the genital organs and anus-and the morphology have sometimes led to a diagnosis of congenital syphilis. Colcott Fox suggests on both clinical and bacteriological grounds that the affection may be of streptococcic origin. It readily yields to local antiseptic measures.

Tuberculin eruptions.-The injection of Old Tuberculin (T.O.A.) may sometimes give rise to a diffuse scarlatiniform or morbilliform eruption. The lesions are generally situated about the hair-follicles, but small erythematous patches are sometimes scattered about the trunk. The eruption, as a rule, recurs after each

${ }^{1}$ For further information on vaccinal eruptions the reader is referred to a paper on the subject read by the Author at the meeting of the British Medical Association in 1890, and to the discussion which followed it (Brit. Med. Journ., Nov. 29, 1890).

2 Brit. Journ. Derm., 1907, xix. 191. 
injection. It is in some instances followed by slight desquamation. The New Tuberculin (T.R.) sometimes produces similar rashes.

Antitoxin and serum eruptions. - The use of serums and antitoxins may set up a condition of anaphylaxis, or hypersusceptibility, which manifests itself, as in the anaphylaxis due to food or bacterial proteins, in the form of eruptions. ${ }^{1}$ Barth reports a case in which injections of tetanus antitoxin (Tizzoni and Cattani) as a remedy for tetanus caused an urticarial eruption which lasted thirty-six hours. Erythematous rashes also frequently follow the injection of diphtheria and streptococcus antitoxins and various therapeutic serums. It is estimated that, on an average, one in three of the patients injected with diphtheria antitoxin develops a rash. As a rule, it is erythematous or urticarial, occasionally scarlatiniform or morbilliform; in very rare cases purpuric hæmorrhages into the skin have been observed. The rash affects the body generally, but especially the extensor surfaces of the limbs. It comes out usually on the eighth day, but it may appear immediately, or as late as the thirty-first day. It lasts from forty-eight hours to five or six days, and sometimes there are relapses. Frequently there is some desquamation. Rawlings ${ }^{2}$ has recorded a case of severe poisoning by antidiphtheria serum, in which the febrile condition was accompanied by a blotchy erythema around the site of infection, which rapidly spread to the trunk. It was markedly circinate in places. There was also a good deal of urticaria, to which the boy had previously been liable. The eruption was accompanied by intense

1 See “Serum Disease," by G. H. Weaver, Arch. Intern. Med., June 15, 1909. A review of the literature of Anaphylaxis, by J. G. Anderson and M. J. Rosenau, appears in the same number.

${ }^{2}$ St. Bartholomew's Hosp. Repts., Dec., 1898. 
itching, and by severe pains in the limbs, without swelling of joints.

\section{Treatment of drug and trade eruptions. - In} the majority of cases of drug eruptions the only treatment required is to discontinue the use of the drug that causes the skin lesions; cessante causâ cessat effectus. In some cases, however, the artificial eruption may be so severe in itself or may be attended with complications of such a nature that both general and local treatment are required. The chief indication in most cases after discontinuance of the drug is to stimulate the renal function so as to promote elimination by that channel. Diuretics should therefore be freely used, and drugs such as iodide of potassium, the use of which sometimes cannot be inte-. rupted without disadvantage to the patient, should be given copiously diluted with Vichy, soda or barley water, milk, etc. A saline purge is also generally useful. Bromide eruptions should be treated with arsenic internally (miii to $m v$ of Fowler's solution thrice daily), and by the application of lead lotion. If the drug must be persevered with (as in the case of epilepsy), a drop or two of Fowler's solution added to each dose of the bromide will often prevent the skin affection. Crocker suggests salol (gr. v thrice daily) as an intestinal antiseptic. The same lines of treatment should be followed in the case of iodide eruptions. The local treatment must be conducted on general principles. It should be borne in mind that stimulants often appear to increase the tendency to drug eruptions and to aggravate them when already existent. This is particularly the case with chloral.

Persons whose occupations bring them constantly into contact with irritant substances must be advised to change their trade; but this, of course, is in many cases out of the question, and the only measure of precaution 
that can be recommended is scrupulous cleanliness and care to avoid touching any unexposed part of the skin with hands or articles of clothing impregnated with the offending substance.

Complications of vaccination.-These must be treated on the general principles applicable to skin eruptions, and by the local remedies suitable to the special lesions produced. The possibility of the inoculation of constitutional disease can now be guarded against with practical certainty by the use of glycerinated calf lymph. In regard to other complications, I endorse the following recommendations ${ }^{1}$ made by the Lancet Special Commission :-

"We are strongly of opinion that many of the bad results obtained in vaccination are due to the presence of one or other of the following conditions: Imperfect sterilization of the skin and want of protection against the invasion of the weakened and abraded tissues by extraneous organisms. We have found that, given a good lymph, the application of a plentiful supply of soap and water, a razor to take off hairs and surface epithelium, ether to remove fatty and sebaceous matter, alcohol to wash away the ether, superficial vaccination (by sterilized instruments), and protection of the vaccinated surface throughout the whole course of the local manifestation of vaccinia, most excellent results may be obtained. The best means of protecting the local lesion is the application of several folds of sterile lint containing no chemical disinfectant; that is held in position by strips of adhesive plaster ; a layer of boric lint may then be placed outside this dressing, and the whole may be changed as soon as the slightest evidence of moistening by serum appears in the boric lint."

${ }^{1}$ Lancet, April 28, 1900, and June 27, 1902. 


\section{CHAPTER XI}

\section{ECZEMA : DEFINITION, SYMPTOMS, PATHOLOGY, ETIOLOGY, DIAGNOSIS, PROGNOSIS}

Definition.-In no subject within the province of dermatology has the loose use of a term given rise to greater confusion than in the description of the various affections of the skin which have, at one time or another, been grouped under the head of "eczema." Willan and Bateman restricted the name to a process in which vesicles were an essential lesion. The meaning of the term was afterwards expanded, especially by French observers, so as to include nearly all the skin lesions which were supposed to stand in relation to a constitutional dyscrasia, such as gout, rheumatism, or "herpetism," that pathological phantom which is held accountable for such varied disturbances. On the other hand, Hebra, and after him the Vienna school, went to the opposite extreme, contending that eczema is a purely local disease, which can be excited artificially by the use of external irritants ; that, in fact, the process is simply superficial inflammation of the skin dependent on some external cause. Hebra, it is true, admitted that constitutional conditions might predispose to the affection, but so strongly did he maintain the determining cause to be a local irritation, that he included itch in his definition of eczema, on the ground that it is an inflammatory process caused by a local irritant-that is to say, the Acarus scabiei.

It is clear, then, that in order to avoid confusion 
it is necessary in the first place to define the sense in which the term eczema is to be employed, and in the second to adhere strictly to the meaning thus attached to it. In the present work the term is used as connoting a catarrhal inflammation of the skin, originating without visible external irritation, and characterized in some stage of its evolytion by serous exudation. By "catarrhal" all that is here meant is that an essential feature in the process is an exudation of serum, either on the surface of the inflamed skin, or into its deeper parts where the horny layer prevents the fluid from escaping. Although eczema is essentially a catarrhal disorder, and the idea of moisture is an integral part of our conception of the disease, it does not follow that external discharge must always and in all cases be present; all that is implied is that exudation, either on the surface or into the deeper layers of the skin, is, has been, or will be a prominent feature of any given case.

The definition of eczema here given excludes all forms of inflammation of the skin caused by chemica] or mechanical irritants. The artificial dermatitis set up by such agents is identical anatomically with the eczematous process, and gives rise to lesions indistinguishable from those of eczema, but it is not eczema. The source of irritation is visible, and can be applied or withdrawn at will; the lesions are distributed over what may be called the area of exposure, and their severity is mostly proportionate to the strength of the irritant or the length of time during which it is applied. Moreover, artificial eczema runs a definite course, and the process is always under the patient's own controlto this extent at least, that he can at any time interrupt the action of the irritant, when, as a rule, the eruption will at once begin to subside. All the phenomena can be reproduced on any part of the cutaneous surface to which the irritant is applied; and although, owing to 
structural differences, the skin of different individuals varies greatly in vulnerability, and the patient's state of health may have some influence on the severity or duration of the process, the constitution has nothing to do with the development of the disease.

Eczema, on the other hand, arises to all appearance spontaneously - that is to say, not in response to any visible cause of irritation; its distribution has no relation to exposure to the action of external irritants; it is not confined to one particular spot, nor even to one region of the body, but may affect all in succession or simultaneously. Lastly, it does not run a definite course, but may smoulder on for long periods of time, breaking out into active conflagration at irregular intervals without any assignable cause. It may be added that, so far from being under the control of the patient, it too often defies all the efforts of the practitioner. It is evident, therefore, that there is something more in eczema than inflammation of the skin due to a local and transient cause; there is an unknown quantity beyond this-a pathological $x$, which may be either some invisible source of irritation or some constitutional peculiarity, or a combination of both these factors.

Most of the recent writers follow Erasmus Wilson in describing a number of different forms of eczemaerythematous, vesicular, papular, pustular, squamous, etc. All these, however, are but different stages of the same process, and there is no advantage in considering them separately, although the terms are sometimes of use in practice to indicate the predominant type of lesion in a given case or at a particular time. Eczema is essentially a polymorphous affection, and there is no particular lesion which can be regarded as distinctive of the disease. Tilbury Fox, following Willan, held that in all cases the initial lesion is a vesicle, though 
this may not infrequently be so small or so evanescent as to escape observation. Colcott Fox adheres to the same opinion. Though the vesicular stage is not a necessary phase in the evolution of eczema, it may be admitted that the vesicle is the most constant of all the primary lesions by which the disease manifests itself:

As no two cases of eczema are exactly alike, and as even in the same case there may be the utmost diversity not only in the lesions but in the symptoms which they produce in different parts and at different times, it is impossible to give a complete clinical picture of the disease in all its varied aspects as met with in practice. All that can be attempted here is an outline sketch, the details of which must be filled in by each practitioner for himself as his knowledge grows by experience. It will add to the clearness of the following description if it be premised that by the terms "acute" and "chronic" I do not mean to indicate suddenness of onset or slowness of course, but only greater or less intensity of the inflammatory process at a given time.

Symptoms.-An attack of eczemais generally ushered in by sensations of itching and burning in some parts of the cutaneous surface. Soon the skin at these spots becomes the seat of an erythematous blush, on which numerous tiny vesicles speedily form; the affected part presents the classical signs of inflammation-swelling, heat, redness, and tension-the itching, as a rule, becoming more troublesome as the lesions develop. The vesicles grow larger and often run together, but they soon burst or are broken by the patient's fingers in scratching, and give issue to a clear fluid which stiffens linen. The discharge does not at once dry up, as is the case in other vesicular eruptions, but continues to exude, more fluid being poured out as vesicles of more recent formation in their turn break and add their 
contents to the general ooze. In mild cases the inflammation begins to subside in a few days; the redness fades and the "weeping" gradually ceases, scales or crusts being formed, under which the abraded surface heals. As a rule, however, the process continues, fresh crops of vesicles starting up around the edge of the older patches, and new centres of disease being formed as the eruption breaks out in distant parts. In this way eczema may in time spread over nearly the whole of the body.

Sometimes papules are the predominant lesion, and the affection in such a case may simulate lichen planus. The papule of eczema, however, is rounder and more conical than that of lichen planus, and with the help of a lens a minute vesicle can sometimes be seen on the top of it. A characteristic appearance in this socalled papular eczema is that, owing to the rupture of the vesicles by scratching, the papules are covered by a tiny dome of blood-crust. The course and symptoms of the affection are as already described, except that the itching is usually more pronounced.

In other cases, again, erythematous lesions may predominate, especially on the face. The affected surface is red but not shiny; it is dry, and sometimes covered with small scales. These appearances may gradually fade away or may linger on, the process being now almost quiescent and again starting suddenly into activity for a time. The epidermis is apt to crack, and serous discharge oozes through the broken integument. This is especially likely to occur on surfaces of skin which rub against each other, forming an eczematous variety of intertrigo.

Eczema varies considerably in intensity at different times. As a rule, the onset is more or less acute, the affection gradually passing into a more chronic stage as it tends to recovery. Both acute and chronic forms 
may, however, co-exist-that is to say, while the process is intense at one point it may be quiescent at another, and every intermediate stage may be exhibited in other parts. Sometimes the affection begins in a trivial chronic lesion. 'Thus a red scaly patch that may have existed on the leg for years may suddenly wake "u into activity, causing intense irritation and exhibiting all the phenomena of acute eczema. Again, in cases in which an old-standing eczema has subsided, leaving only a small patch apparently dying out, this may at some subsequent time form a focus for a fresh development of the disease, from which it may spread over nearly the whole body.

The worst forms of eczema are ordinarily accom. panied by some constitutional disturbance, not amounting to fever, in the earlier stages; and the same thing occurs at each fresh exacerbation of the process. The general health, however, is seldom appreeiably affected, except when the itching is so intense as to make sleep impossible; but the attacks rarely follow each other so closely as to leave no intervals during which the patient ean make up arrears of rest. So slight is the effect of eczema on the system that in the most intense form of the generalized disease, when the discharge is so profuse as to glue the hair to the pillow and the linen to the body, and when the itching is maddening and almost continuous, fresh outbreaks occurring every few hours, there may be no rise of temperature, the tongue may be quite clean, and every function in perfect working order; in short, with the exception of nervous excitement, there may be absolutely no disorder of the general health. 'T'he itching and heat are often out of all proportion to the visible ehanges in the skin, and these symptoms are usually intensified to an extreme degree at night, especially in the small hours. I have often seen strong men literally reduced to tears by the 
irritation and discomfort which they experienced when there was nothing particular to see in the skin. Even persons of the strongest will are umable to control them. solves, and sernteh as if by traring their skin they could root out the cause of the irritation. They will tell you that they feol a kind of mavage sutisfuction in tearing their skin till the hlood comes, mol, as $n$ matter of fact, the pain of the severe axcoriation easesed by their nuils soems for a time to subdue the intolorulde itching. A state of mental enlm follows the nerve storm enused by the irritation, and the putiont is able to sleop. In severe cases mental oxcitement is often very pronounced. especinlly in persons of neurotic tempernment.

In the great majority of ouses of cezema the following stages are more or less direotly reconnizable: (I) An initial erythema, the affected surface presenting the usual sigus of inflammation, and generally soon becoming studded with vericles; (2) exulation of a clear serous fluid, which stiftons linen, the surface heing rod and "weeping," and often excorinted by meruteling ; crustation, the discharge " metting" into greyish-yollow crusts of varying thickness, which, as they heeome detuehed, are suceeded by others as lomg as tho ooving continues; (1) a dry slage, during which no further formution of crusts takes place, and the surfuce is covered with a thin, red, glistening epidermis, dotted with smull points of a decperered tint: (5) lastly, desquamation, the new opidermis being shed in scules, which gruduslly become smaller snd thimer till mothing remuins to mark the site of the lesions but a bownish stain. All these stages aro esurlly present, nt, ones in $x$ given case, and this, combined with the modilientions of the lesions in diflerent cirenonstances about to be described, together with the arevidental complientions produed by seratehing, and hy inoculation of micro. coeci (pustules, boils), gives aczems the multiformity 
of aspect which has been mentioned as one of its most striking characteristics. The process always begins with more or less violent inflammation- in other words, there is in all cases an "acute" initial stage, though sometimes this is so brief in duration that the disease might easily be thought to have been of the "chronic" type from the first. It may run through all the various phases that have been described, or it may abort at any stage, without in either case leaving permanent changes in the part attacked. On the other hand, it may be indefinitely prolonged, though in an almost dormant state, leading to thickening and other results of slow, persistent inflammation. Even in the oldest of such patches, however, the disease may start into activity at any time and without any visible provocation. Eczema may, in fact, as regards the vicissitudes and the varying degrees of intensity of the process, be compared with inflammation of a joint. First there is the period of onset, the heat, pain, and tension in the joint having their analogues in the heat, swelling, and itching of the skin; next comes effusion into the joint, corresponding to the "weeping" stage of eczema; lastly, absorption of fluid in the one case and drying up of the discharge in the other, followed by more or less complete restoration of the status quo ante. Again, there is in the joint, as in the skin, the liability to sudden exacerbation of the inflammatory process even after long quiescence, and the tendency to structural changes after long persistence or frequently repeated attacks.

The eczematoid lesions which precede, usually for a long time, the onset of mycosis fungoides are really the prodromal eruption of that affection.

Modifying influences.-While the eczematous process is always essentially the same, its manifestations in individual cases are more or less modified by special conditions of structure or situation in the affected parts 
of the skin and by the age and sex of the patient. The various factors will be considered separately.

\section{Distribution and regional peculiarities. - There} is no part of the skin which may not be attacked by eczema, but there are certain regions for which it exhibits a more or less marked predilection and in which it usually begins. These are the flexor surfaces of joints-the bends of the elbows, the backs of the knees, and the groins; other favourite situations are the groove behind the ears, the scalp, the palms and the soles, the breasts in women, the lumbar region, and the back at the level of the lower angles of the scapulæ. On the limbs, eczema sometimes gives rise to considerable infiltration and induration; hence deep, painful cracks are apt to be formed on the flexor surfaces when the inflamed skin is subject to frequent movement. The eruption is generally symmetrical.

On the fronts of the legs and arms, and occasionally on the flexor surfaces of joints, the disease assumes a peculiar form, which, from the uniform redness of the part attacked, has been dignified with a special name -eczema rubrum. The affected area is of a bright-red colour and glistens with moisture, beads of exuded fluid standing on the surface like dewdrops-hence the term " madidans," sometimes used to denote this form of eczema. The discharge quickly dries, forming extremely thin scabs like flaky piecrust or goldbeater's skin ; these, when torn off, reveal a wet, raw, tender surface beneath. Sometimes, especially in parts where the skin is more or less tightly stretched, as on the front of the leg and the forearm, the exudation cannot force its way to the surface, and the skin is dry, but very tense and red. When the inflammation is of a slight degree of intensity the patches are often covered with scales, which are easily detached, exposing a dull-red surface which is not raw nor tender. As a rule, no constitutional 
disturbance accompanies eczema rubrum, unless a very large area of skin be involved, when the condition approximates to pityriasis rubra.

On the scalp eczema is generally of the seborrhœic type. Another form is, however, met with which seems to be unconnected with seborrhøa. The scalp is red and covered with crusts, but the hair does not fall out. In children, and also in adults, the affection is sometimes associated with pediculi, and in such cases pustules are almost sure to be produced by inoculation.

About the nostrils eczema often accompanies coryza of an irritating character, complicated at times by painful boils. The disease may attack the nasal fossæ, where it may cause considerable oedema. Eczema in that situation sometimes leads to catarrh of the nasopharynx, and so to catarrh of the middle ear (Gruber). The upper lip may suffer in consequence of the nasal discharge trickling over it. The special features are great swelling and redness of the part of the lip lying below the nostrils, with painful papules about the orifices of the hair-follicles, and almost unbearable itching; crusts form, and a good deal of thickening of the lip, causing deformity and even obstruction of the nostrils, may be left. A particularly painful form of eczema may attack both upper and lower lips, which swell and discharge, and sometimes become so stiffened under a carapace of crusts that the patient can hardly move his lips without cracking the integument.

The ear is a favourite point of attack for eczema, which often lingers there when it has disappeared from other parts, and invades neighbouring regions from it as from a centre, when kindled into fresh activity. Sometimes the whole external ear is involved, the disease occasionally even spreading along the meatus to the membrana tympani ; in other cases the lesions are confined to the groove behind the ear. 
On the face eczema is usually of the seborrhœic form, and is, as a rule, the result of the extension of the process from the scalp. The conjunctiva is sometimes attacked.

Eczema of the chin is often confounded with sycosis, from which, however, it is to be distinguished by the absence of indurated nodules and cicatricial alopecia (Brocq).

On the wrists the dorsal surface is the usual seat of the disease, the irritation being kept up by the chafing of the cuffs. On the feet the spaces between the toes most frequently suffer. On the palms and soles the most common effect of eczema is great thickening of the epidermis, which impairs the flexibility of the parts and leads to the formation of cracks (eczema rimosum), making the use of the feet and hands so difficult and painful as to disable the patient for active life. The nails are discoloured and undergo degenerative changes. 'The first sign of the affection is usually pitting, which gives them an appearance somewhat resembling the rind of an orange. They become thin, split transversely and longitudinally, and exfoliate; in old-standing cases they sometimes become thickened to the extent of deformity.

Eczema may attack the nipple, especially in nursing mothers; but this part may also be the seat of the affection in unmarried women, and even in men. It begins in seborrhœa of the nipple and the areola, and presents the ordinary characters of seborrhœic eczema. Cracked nipple is a frequent result. The affection is generally symmetrical. It is not to be regarded as the first stage of Paget's disease; it is innocent in character, though often extremely obstinate.

On the genitals eczema is chiefly of the erythematous form, and it is naturally worst where two surfaces of skin rub against each other. 'J'he irritation is 
excessive, and the temptation to scratch more difficult to withstand than in almost any other situation. The scrotum and penis sometimes become greatly swollen, and the disease may spread over the perineum, round the anus, into the fold between the nates, and over the gluteal region; not infrequently it invades the whole of what may be termed the "bathing-drawers area." In such cases the patient cannot sit down or walk without the crusts and the inflamed skin beneath them giving way somewhere. In the female the state of things is even worse. The process is generally stirred up to a violent degree of intensity by the chafing of the parts ; the swelling may be enormous, and almost every variety of lesion that can be produced by acute inflam. mation aggravated by scratching and urine-foul crusts and scabs, fissures, and disgustingly offensive discharge -may be present, while walking is so painful as to be almost impossible, and the itching is so distressing that life becomes a burden.

Eczema of the anus is often associated with piles or worms; the skin is thickened, and painful fissures are frequently present. The itching is in most cases intense, and the harassing character of the affection gives an anxious and haggard expression to the sufferer's countenance.

The umbilicus is sometimes the seat of an obstinate eczema, usually seborrhœic in form. The lesions are circular in outline, and do not, as a rule, extend far beyond the edges of the umbilicus.

Sex.-Although eczema spares neither sex, males are perhaps, on the whole, more liable to be attacked than females. In childhood, Crocker's statistics show a preponderance of boys to girls of five to three. ${ }^{1}$ In middle age, when the burden of life is heaviest, the greater proclivity of the male sex is still more marked. 1 "Diseases of the Skin," 3rd edition, p. 157. 
Bulkley ${ }^{1}$ gives an analysis of 5,000 cases of eczema under his own observation, which shows that in the period from thirty to fifty years of age the number of male patients in his private practice was about double that of female. Hebra's estimate, that the proportion of females to males among the subjects of eczema in his clinic was as two to one, is probably to be explained by the greater opportunities women have of attending as out-patients at a hospital. There are, however, two periods of life at which women are more liable to eczema than men, namely, between the ages of ten and twenty, when menstruation is becoming established, and again at the menopause. ${ }^{2}$ In old age the influence of sex appears to be lost in the degenerative tendencies com. mon to both.

Age.-In children eczema is mastly of the seborrhœic form, and in a large proportion of cases it begins in the earliest years of life: As a rule, the starting-point is the head or face. The focus from which the disease starts is generally a patch of dried sebaceous matter. Such a patch, dirty brown in colour and consisting of greasy material, is often seen soon after birth. From the head or face the disease spreads downwards, generally in the middle line of the body (front and back), but not sparing the limbs. Vesicles show a much greater tendency to become pustular than in adults, forming on the head moist yellowish crusts, which glue the hair together, while from underneath them frequently wells up a sickly-smelling sero-purulent discharge. On the face the crusts often have a dark-green, or tbrownish tint, and cover the face, leaving the mouth, eyes, and nose free, like a mask with an, opening cut in the centre

1 "On the Relation of Eczema to Disturbances of the Nervous System." Reprinted from the Medical News, Jan. 31 and Feb. 7, 1891.

${ }^{2}$ Bohn, Deutsch. Arch. f. Klin. Med., Oct., 1886. 
(Unna). On the trunk, where the exudation is usually less abundant, thin scales are more common than crusts.

Itching is sometimes very troublesome, especially where cleanliness is neglected and the lesions caused by the disease are aggravated by pediculi. The lymphatic glands are frequently enlarged, and subcutaneous abscesses, particularly in the suboccipital region, are a not uncommon complication. In babies at the breast the natural folds and creases of the skin-nates, thighs, neck, etc.- are often the seat of eczematous lesions which are frequently overlooked, mothers and nurses not separating the parts properly for fear of making the child cry. Kaposi ${ }^{1}$ says that in these cases the dermatitis sometimes assumes a very intense character, rapidly becoming gangrenous or diphtheritic, a cure taking place in the most favourable cases with loss of substance and cicatrices, or death ensuing in a few days from convulsions and collapse. I can only say that no case of this kind has ever come under my observation.

According to Brocq, ${ }^{2}$ the rapid disappearance of an eczematous eruption in a young child may be followed by pulmonary congestions of the most dangerous kind.

Unna $^{3}$ recognizes three absolutely distinct types of eczema of the face in infants-nervous, tuberculous, and seborrhoic. The first occurs during dentition. It is symmetrical in distribution, and usually affects the middle of the cheeks, then the forehead, and almost at the same time the radial side of the backs of both hands and wrists. The itching is intense, and the healthier the child is the worse the symptom seems to

1 "Maladies de la Peau," t. i., p. 658. French translation; Paris, 1891.

2 "Traitement des Maladies de la Peau," p. 169. Paris. 1890.

${ }^{3}$ Journ. Cut. and Gen.-Urin. Dis., Dec., 1887. 
be. On the appearance of a few teeth the eczema dies away, probably to come out again a few days later. The tuberculous form is localized in the neighbourhood of the eyes, nose, mouth, or ears, and is often associated with scrofulous rhinitis and otorrhœa, and swelling of lymphatic glands. There is little or no itching. I shall return to this point in the section $\mathrm{cn}$ the etiology of eczema. The seborrhœic form is described at p. 277.

At puberty eczema may occur in the seborrhœic form just referred to. Beginning on the scalp, it may spread to the face and other parts, apparently by local infection. It also occurs in connection with the peculiar dryness of the skin known as xerodermia. In early life this condition is scarcely noticeable, but towards puberty the skin becomes dry and harsh, and on it eczema may develop. Another form of eczema which appears at puberty alternates with neurotic conditions, more especially with asthma and commencing osteoarthritis.

In middle life eczema presents little peculiarity either in the nature of the lesions or in their distribution. "Weeping" and scaly forms are, however, far more common than the pustular lesions that predominate in infantile eczema. It is at the middle term of life, moreover, that the influence of constitutional conditions, such as gout or rheumatism, is most likely to make itself felt. These conditions do this not so much by exercising any direct effect on the eczematous process as by modifying the general health in a way favourable to the continuance of the skin affection. The affection often comes on very acutely after a chill. According to Brocq, it is especially in middle life that alternations between eczematous lesions on the skin and "visceral manifestations" of greater or less gravity (pulmonary, renal, intestinal, cardiac, cerebral, etc.) 
are most likely to show themselves. When all the lesions have disappeared, there often remains an intensely irritable state of the whole cutaneous surface. The patient lives in constant dread of a new attack, and this, combined with the itching, may bring him to the verge of insanity. In women at the change of life eczema shows a marked tendency to relapse in particular regions. According to Jamieson, more than threefourths of the cases occur on the scalp and ears. The extremities may also suffer to some extent, but the trunk generally escapes.

Elderly persons are particularly apt to suffer from a form of eczema which is really an expression of enfeebled vitality or the result of degenerative changes in the skin. The disease is generally chronic in character, with short acute exacerbations. The irritation is often very great, making sleep impossible. The favourite situation of the disease in such cases is the lower part of the leg, where it is frequently associated with varicose veins and ulcers. The irritation of the skin may, as pointed out by Kaposi, set up reflex irritation in the intestine, preventing the proper digestion of food. The irregularity of the bowels reacts in turn on the skin, and thus a vicious circle is established. The patients are reduced to so miserable a condition that they are sometimes driven to end their sufferings by suicide. In milder forms the skin is only slightly roughened and red, the surface being covered with a thin film of scales; in severe cases there is often great thickening of the skin, accompanied by distressing itching. When the skin is very dry and atrophic, as it usually is in persons of advanced age, it is apt to crack along the lines of cleavage, causing great pain on movement. In old men eczema not infrequently spreads from an old, almost forgotten patch, commonly on the leg, involving wide areas and developing fresh 
centres in distant parts, till nearly the whole surface of the body may be invaded. The erythematous form already mentioned, which attacks the face and neck, is common in elderly people.

Special forms of eczema.-The general phenomena of the eczematous process having been described, certain variations in the clinical aspect and course of the affection, dependent on differences in its mode of origin, remain to be considered. By the terms of the definition of eczema given at the beginning of this chapter, all forms of inflammation of the skin due to definite chemical or mechanical irritation are excluded. But, even in the restricted sense in which the term is here used, eczema is still rather a pathological formula expressing the results of several forms of morbid action than a distinct disease.

The nature of eczema is one of the vexed questions of dermatology, and a full discussion of the question would be out of place in an elementary text-book. Such a discussion is the less necessary since for all practical purposes it is sufficient to recognize two kinds of eczema, or, to speak more precisely, two great groups of eczematous eruptions-those which come out on previously healthy skin, and those for which the way has been prepared by some pre-existisig local disorder of the secreting apparatus of the skin.

Of the latter category there are three special forms, according as the source of the mischief is in the sebaceous glands (seborrhœa), the sweat-glands (hyperidrosis, anidrosis), or the hair-follicles (folliculitis).

1. Seborrhœic eczema, for our knowledge of which we are indebted to Unna, ${ }^{1}$ begins, as a rule, in seborrhœa

${ }^{1}$ Journ. Cut. and Gen.-Urin. Diseases, Dec., 1887, p. 449 et seq. 'The paper was a communication to the Dermatological Section of the Ninth International Medical Congress, held at Washington. 
of the scalp, which in some cases has existed since birth; in rare instances the starting-point may be the margin of the eyelid, or a part like the axilla, the bend of the elbow, or the cruro-scrotal fold, where sweatglands are abundant. In connection with this point it should be noted that, according to Unna, what is usually called "seborrhœa" is often a fatty hypersecretion poured out not from the sebaceous but from the sudoriparous glands, and should be regarded as hydrosis oleosa. The affection begins as a latent catarrh ; it first manifests itself by the agglutination of epidermic scales, which are thrown off in large lamellæ. That there is a faulty distribution of the fat in the skin is shown by the fact that the hair becomes abnormally dry from closing up of the hair-follicles, while the epidermis and exfoliating scales are abnormally fatty. The scales may simply increase in quantity, or they may become massed into fatty crusts between the hairs, which are thus crushed out, leaving a bald patch on the top of the head (corona seborrhoica). In other cases the catarrhal phenomena are more pronounced; the skin is red and swollen and "weeps" profusely; the fatty scales either are not formed or are washed away by the discharge; the rete may be laid bare. Unna calls these respectively the scaly, the crusty, and the moist forms of what is generally termed "chronic eczema of the head." The sternal region may also be the seat of a primary seborrhœic eczema, which is almost always of the "crusty" form; the patches are usually made $u_{i}$ of segments of circles, and present different shadings of colour, from yellow in the centre to bright red (after removal of the scales) at the outer edge.

Eczema seborrhoicum spreads slowly in a peripheral direction; a patch may remain almost stationary for years. Beginning, as already said, on the head, it extends over the scalp, thence to the ears, the forehead 
and cheek, the neck, and down the front of the ehest and the back, especially in the interscapular furrow, into the axillæ and the bends of the elbows and on the hands, into the groin and the cruro-scrotal fold, over the genitals, behind the knees, and between the toes.

Seborrhœic eczema is nothing more than the eczematous process going through the various phases of its evolution in a skin that has long been the seat of seborrhœa. The latter prepares the ground for the eczema. The discharge itself may possibly have an irritant action on the skin, but the real irritant-the efficient cause of the lesions-is, there is every reason to believe, of infective nature. This affords an explanation of the suppurative processes which often complicate seborrhœic eczema. Much discussion has taken place regarding the microörganisms, especially the "bottle bacillus" of Unna (Plate 33, Fig. 9) and the microbacillus of Sabouraud, which are associated with this form of eczema. The question is still undecided whether these organisms are actually pathogenic in seborrhœic eczema. Sabouraud is convinced that the Staphylococcus epidermidis albus is at least a contributory if not a causal factor in the production of eczema of this type.

A special form of the affection is that which is usually designated seborrhoic eczema of infants, though Adamson, ${ }^{1}$ who was the first to lay stress upon its special incidence on the napkin region, prefers to style it seborrhoeic dermatitis of infancy, holding that it is not a true eczema. The entire region is occupied by a red rash of uniform brightness, with sharply defined margins. The prominences may be smooth and shiny, but the rest of the area is covered with yellowish scales, either moist or greasy. The eruption is not necessarily limited to the napkin area, but may spread downwards to the

${ }^{1}$ Brit. Journ. Derm., 1909, xxi. 37. 
thighs and calves, and upwards to the umbihicus ; elsewhere there may be smaller patches and an abundance of tiny red scaly papules. The flexure of the elbow, the axilla, the side of the neck, the naso-labial fissure, and the bend of the knee are frequently attacked...The scalp is always the seat of a red scaly or crusty eruption. The affection may be recognized by the sharply defined margins, the distribution as described above, the patches and minute scaly papules, and by the readiness with which it responds to mild local anti-parasitic remedies, such as sulphur ointment, gr. $\mathrm{x}$ ad $3 \mathrm{i}$.

2. Sweat eczema. - Excessive secretion of sweat, without any alteration in the character of that fluid, may also prepare the way for eczema by so modifying the condition of the skin as to make it prone to become the seat of the eczematous process as already defined. The most common situations for the development of this form of eczema are the parts where two opposed surfaces of skin rub against each other-between the nates, between the scrotum and the thigh, in the axilla, between the toes, in the deep folds under an overhanging breast, and in the hypogastric region under a prominent abdomen. The sweat in such parts is apt to undergo decomposition, and this fluid, mixed with shreds of macerated epithelium and "fluft" from the underclothing, forms a substance highly irritating to the skin. It must be understood, however, that hyperidrosis plus friction can only produce a dermatitis similar to that caused by other chemical and mechanical irritants ; for the production of eczema-i.e. of a train of lesions which may persist after removal of the conditions that engendered them, and which may be followed by the development of -similar lesions in other parts that have not been exposed to the same irritation -a tertium quid is required. This factor, which dermatologists of the older school assumed to be gout or 
some equally convenient dyscrasia, will in all probability be shown to be the action of microörganisms. Sweat eczema is almost always, in the first instance at least, an intertrigo, but is distinguishable from the erythematous form of that affection by the "weeping" of the opposed surfaces and the resulting crusts. It is not necessary, however, for the development of the eruption that there should be chafing; the eczema, which is one of the signs of the "crisis" of the coldwater cure, is due to the profuse sweating that is the principal effect of that method of treatment.

3. Eczema folliculorum, which was first described as a special form of the disease by the author, begins in inflammation of the hair-follicles. Each inflamed follicle stands out on the skin as an angry-looking red papule; the capillaries around are congested, and soon the skin is involved in the process. In this way red patches dotted with inflamed follicles are formed, which tend to spread by the extension of the inflammation from follicle to follicle. As a patch spreads at the edge it usually undergoes resolution in the centre, desquamation takes place, and the redness fades into a yellowish stain. The itching is often most intense. The patches are generally multiple and are scattered about the body, especially on the extensor surfaces of the arms and legs. The predilection of eczema folliculorum for the extensor surfaces of the limbs is a distinctive feature as regards distribution, other forms of eczema showing a preference for the flexures of joints. The affection is obstinate, and recurrence is almost the rule. It is closely allied to sycosis, and there can be little doubt that it is of microbic origin.

"Nervous eczema." - Apart from the special forms of eczema that have been described, there is a large class of cases in which the disease springs up de novo in skin that has not been the seat of seborrhœa or other 
preparatory process. This class, in the absence of any definite objective characteristic, I propose to designate "nervous eczema," though, as will be explained farther on, I include under that term many eczemas in which the nervous system is not the only, or the chief, etiological factor in operation. That eczema may be of purely nervous origin appears to be admitted by Unna himself, inasmuch as he expressly states that one of his three types of infantile eczema is caused by reflex irritation during dentition, and disappears when the tooth has cut its way through the gum. Elliot ${ }^{1}$ has applied the name of "reflex neurotic eczema" to what he considers to be a definite type of the disease which he has seen in babies and young children. Barham ${ }^{2}$ has described a "neurotic eczema" presenting objective features sufficient to distinguish it from other forms of the disease. These are: (1) Grouping of the lesions in circumscribed patches sharply separated from adjoining lesions; (2) symmetry of the eruption as a whole; (3) preference for the extensor surfaces of the extremities; (4) absence of peripheral spreading or contraction of the separate patches. My own experience leads me to the conclusion that when eczema arises in apparently normal skin it is always nervous in origin, though the parasitic element often comes into play as a secondary factor. I cannot say, how ever, that I have observed any peculiarities of appearance or distribution whereby a purely neurotic eczema could be distinguished from other forms of the disease.

Summary of subjective symptoms.-The objective phenomena of eczema have been described in the preceding pages, and incidental mention has been made of the subjective symptoms characterizing the different forms of the disease. It may not be amiss,

1 Internat. Med. Magazine, Oct., 1892.

${ }^{2}$ Med. Record, July 9, 1892, and Med. News, March 25, 1893. 
however, here to pass the latter rapidly in review for purposes of comparison. The only ones that need concern us here are itching and pain. These symptoms, particularly the former, vary greatly in intensity according to the temperament of the patient or the structure and condition of his skin. The lesions which in a person of "lymphatic" temperament cause only slight annoyance, may in a neurotic or gouty subject give rise to nerve storms of such intensity as to banish him from society and almost wreck his reason. Nor is the intensity of the itching proportionate to the severity and extent of the lesions: it is often worse when there is little or nothing to see, e.g. in the erythematous eczema of the scalp common in old people. In such cases the exudation imprisoned beneath the horny layer probably presses on or irritates the terminal filaments of the sensory nerves of the skin, and the relief given by free searification of the parts with the finger-nails seems to afford some confirmation of this view. It not infrequently happens that, owing to disturbance of innervation, itching persists long after every trace of lesion has disappeared. How profound an impression eczema may leave on the nervous apparatus of the skin is shown by the fact that in some cases in which the disease has lasted a long time the skin appears to be so much under its dominion that the slightest accidental irritation is sufficient to bring on an attack. Pain is not often severe, except when inflammation runs high and causes great heat and tension of the skin; the pain generally subsides as soon as the effusion finds its way to the surface. In the neighbourhood of parts - as the mouth, genitals, anus-which cannot be kept at rest, the skin becomes thickened and tender, and the cracks caused by movement are so painful as to interfere with the performance of natural functions. The only other subjective 
symptoms caused by eczema are an exaggerated sensitiveness to cold and a feeling of lassitude or disinclination for work (Jamieson).

Complications.--Locally, the eczematous process is often complicated by inflammation of the related lymphatic vessels and glands. As the result of scratching, micrococci may be inoculated, and when these penetrate from the superficial to the deeper layers of the skin they cause the development of painful boils. Of internal complications, the most common is dyspepsia. Gout is also a frequent concomitant. Both these conditions have been supposed to stand in a causal relation to eczema, but to me they appear to be nothing more than accidental complications. The case is somewhat different as regards asthma. That affection is so often associated with eczema that, when a patient suffering from certain types of the latter affection comes before me, I am in the habit of asking if he is subject to asthma. It will be seen later that $I$ regard these two. affections as frequently dependent on a common cause.

Pathology.-Eczema is essentially a catarrhal inflammation of the skin, and the appearances found are those characteristic of that process, being more or less marked in proportion to its severity. Plate 19 shows well the microscopical appearances presented by a vesicle. Colomiatti, as already said, found changes indicative of neuritis in the nerves supplied to the affected parts of the skin, and in one case ${ }^{1}$-that of a patient suffering from acute universal eczema, who died of pneumonia - " the upper cervical ganglia of the sympathetic, as also the cœliac ganglia, were visibly hyperæmic to the naked eye, and on microscopic section the changes were still more evident." No conclusion can, however, be drawn from a single case, and it is obvious

1 Maracei : Giornale Ital. d. Malattie Vener. e d. Pelle, 1878. 


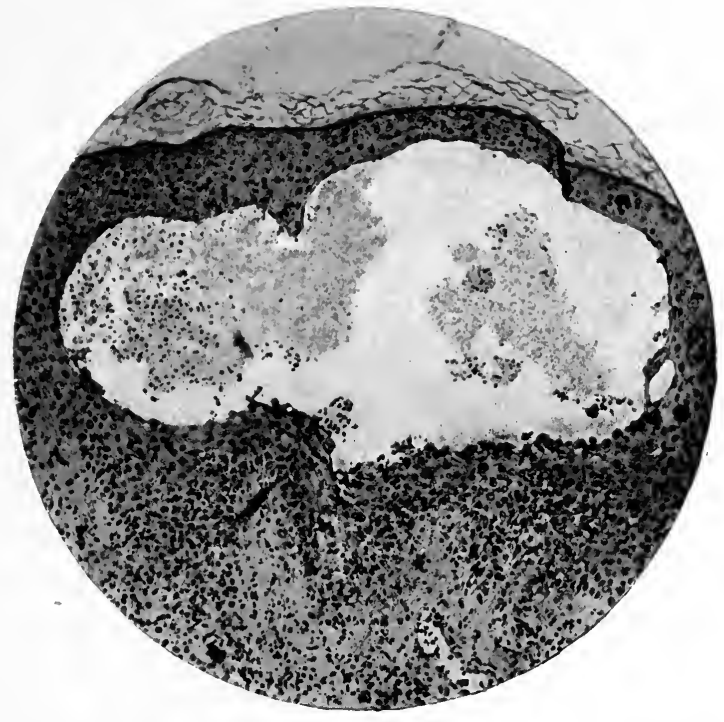

Plate 19.-MicRoscopic section of eczema Vesicle. (Gilchrist.) 


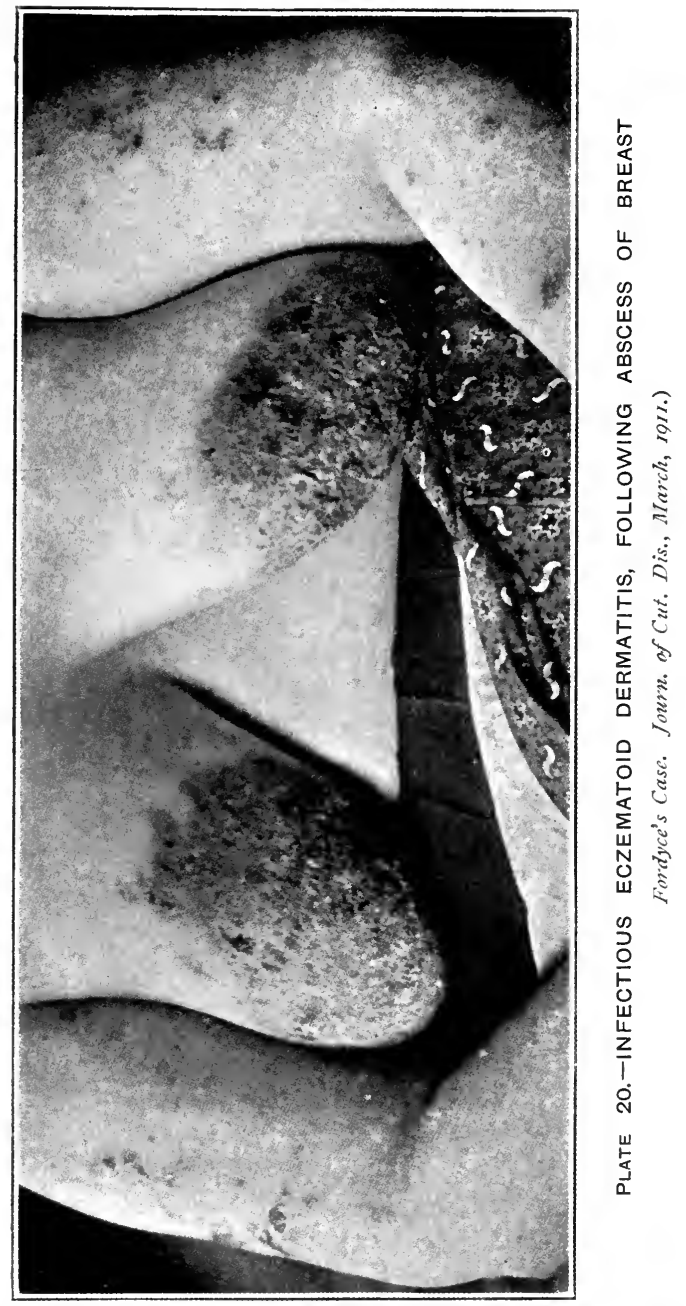


that the changes in the sympathetic ganglia here described may have been connected with the inflammation of the lung rather than with the eczema. In scaly eczema the desquamation is caused by parakeratosis, the cells of the horny layer preserving their nuclei. In chronic cases a further change takes place, a hyperplasia of the prickle-cell layer, with increase of the interpapillary processes. In rare cases there may be a hypertropliy so great as to simulate elephantiasis. In some other cases a persistent warty condition may be induced.

Etiology.-The causation of eczema has not yet been definitively established by scientific evidence, but it is clear that for its production two conditions at least are necessary: first, a special irritability of the skin, possibly inherited ; secondly, an exciting influence which brings this irritability into action. The abnormal vulnerability of the skin may depend on certain peculiarities of structure, or it may be the result of a pre-existing morbid condition; or, again, it may be connected with some underlying constitutional state. The exciting influences may act on the skin directly by setting up irritation and so causing the development of the lesions, or indirectly through the nervous system. In many cases both these modes of attack are combined. Lastly, the eczematous process, when set in motion by the causes that have been referred to, may be intensified and kept up indefinitely by secondary causes, such as the patient's state of health, his exposure to sources of additional irritation, etc.

As regards peculiarity of tissue, fair-haired persons appear to be somewhat more liable to eczema than those of darker complexion (Jamieson). A thin, dry, anæmic skin, with deficiency of subcutaneous fat, affords a very favourable soil for the development of the process. The disease is not infrequently associated with 
xerodermia, a congenital anomaly characterized by abnormal dryness of the epidermis - in fact, a mild form of ichthyosis. Such anomalies are often inherited, and the tendency to eczema may be transmitted with them; in this sense only is eczema hereditary. 'On the other hand, skins in which the sudoriparous glands are overactive are especially liable to "sweat eczema." But the condition of all others which makes the skin most vulnerable to attack is seborrhœa. I do not go to the length of saving with Unna, "Treat the seborrhœa of children, and you will not later have eczema in adults," but I am convinced that if there were no seborrhœa there would be much less eczema.

In the same way the ground may be prepared for eczema by artificial dermatitis. As has already been explained, I do not look upon the eruptions caused by chemical or mechanical irritants as coming within the category of eczema; undoubtedly, however, such lesions may be the starting-points of the disease. Thus it is by no means uncommon to see artificial dermatitis on a bricklayer's hands followed by the development of patches of true eczema on parts of the skin that have never been in contact with lime; and the eczema may persist and reproduce itself in different spots when the eczematoid lesions in which it took origin have disappeared. It is clear, therefore, that in such cases some other agency besides the original cause of irritation has come into play; to the lime there has been superadded an irritant of a different kind, the action of which is not temporary and localized, but continnous and self-multiplying. There can be little doubt that this additional irritant, which transforms a simple seborrhœa or dermatitis into an eczema, is the action of microörganisms. As has already been pointed out, the skin has an abundant and varied microbic flora of its own; under normal conditions these organisms do 
no harm, but it is easy to understand how the lesions produced by previous disease may make the integument more vulnerable to their attacks.

Unna ${ }^{1}$ formerly taught that in acute eczema the fluid in the vesicles contains a specific microörganism, which, from its tendency to form mulberry-like masses, he called "morococcus." By inoculation of cultures of this parasite he believed that he had produced eczema. He stated that he found the same microörganism in the scales in chronic cases. Seborrhœic eczema is believed by Leredde ${ }^{2}$ to be the result of a mixed infection due to the association of microbes, such as that of fatty seborrhœa, pityriasis capitis, etc., with Unna's morococcus. As a result of re-investigation of the question, however, Unna has made a public recantation of the "morococcus" doctrine. ${ }^{3}$ At the fourth International Congress, held in Paris in 1900, he summed up the conclusions to which he had been led by his more recent researches in the following propositions: 1. The uncertainty which exists concerning the pathogenic agent of eczema is due in great measure to the absence of precise knowledge as to the various forms of cocci, microorganisms which, presenting the closest similarity in appearance, possess widely different pathogenic properties. 2. In eczema numerous microörganisms are present, and among them are several which, when reinoculated, reproduce the disease, which is therefore contagious, and in certain circumstances may become epidemic.

James Galloway and J. W. H. Eyre, in a communication presented to the same Congress, reported the results of bacteriological examinations made by

1 "On the Nature and Treatment of Eczema," Brit. Journ. of Derm., 1890, p. 231 et seq.; see also Leredde, "L'Eczćma, maladie parasitaire," Paris, 1898.

2 O.p. cit., p. 6.

${ }^{3}$ Monats. f. prakt., Derm., Bd. xxi., Nr. 5. 
them in several cases of acute papulo-vesicular eczema. In early and uncomplicated lesions they found cocci producing whitish cultures, all of them examples of the type Staphylococcus pyogenes albus, and possessing to a greater or less extent the pathogenic powers of that organism. They expressed the opinion that in all probability there are many factors at work in the production of any attack of eczema, and, although they do not think this organism is the cause of the disease, they cannot help considering that this white coccus, and other cocci, such as the Staphylococcus pyogenes aureus and the Streptococcus pyogenes, which are so often present, especially in the later stages of the disease, must have very important influences on the development of the malady. The local infectivity and chronicity of eczema, the ease with which purulent manifestations occur, should be, in all probability, ascribed to the presence of such bacteria. Sabouraud, in a communication to the annual meeting of the British Medical Association held at Cheltenham in 1901, expressed the opinion that the staphylococcus is the cause of pustular lesions of the skin in eczema and other conditions. Neisser has summed up his belief in the formula, "No eczema without micrococci." Bender, Bockhart, and Gerlach,1 from an elaborate series of inoculation experiments performed upon themselves, preceded by artificially produced irritation of the skin with injury to the epithelium, conclude (1) that while inoculation of virulent cultures of Staphylococcus pyogenes aureus and albus in agar on the irritated human skin may produce impetigo staphylogenes, furuncle, and abscess, it never produces eczema; (2) that while isolated living and virulent staphylococci of the varieties specified, free from toxins, produce impetigo staphylogenes when inoculated on

- Monats. f. prakt. Derm., Bd. xxxiii., Nr. 4, Aug. 15, 1901 (abstr. in Brit. Journ. Derm.). 
the skin, they never cause eczema; (3) that filtered bouillon cultures of these staphylococci can produce on the human skin, with or without previous irritation, and with or without disinfection, typical acute papular or vesicular eczema when applied by means of moist, warm bandages for a period of from twenty to forty-eight hours; (4) that it is the staphylococcus toxin, and not the staphylococci themselves, which produces eczema. In a later number of the same journal (Nov. 1, 1901) Bockhart boldly says, "Staphylococci are the originators of eczema"-by means, that is, of their toxins.

On the other hand, Reibich examined bacteriologically the two- to four-days-old vesicles of forty-one cases of eczema, and found them for the most part sterile. Later in the course of the disease he found staphylococci and streptococci, but was unable to produce eczema experimentally by inoculation with these. Veillon ${ }^{1}$ also examined the fresh vesicles of ordinary eczema, and found them, almost without exception, sterile. Secondary infection with staphylococci and streptococci is common, but Veillon was unable to produce an eczematous eruption with those which he isolated. The serum of a horse immunized against the staphylococci, isolated, exhibited no influence on the course of the disease in the human subject. The accuracy of the observations of Reibich and Veillon is confirmed by Wilfrid Warde, ${ }^{2}$ who repeatedly found the clear eczema vesicle to be amicrobic. Fritz Veiel ${ }^{3}$ remarks that although a number of observers claim to have produced eczema by inoculation of staphylococci, no one has produced in this way a genuine chronic eczema, but he agrees with Neisser that staphylococci play an

1 Ann. de Derm. et de Syph., No. 6, 1900.

2 Brit. Journ. Derm., Oct., 1903, p. 349.

3 Münch. med. Woch., Jan. 6, 1904, p. 13. 
important part among the agencies that determine eczema and the course it runs.

The microbic theory must therefore for the present be dismissed as not absolutely proven, though it is impossible to believe that microörganisms known to possess definite pathogenic properties can be present in such numbers, as they have been proved to be by competent observers, without having a considerable effect on the character and severity of the disease, and there can be little doubt that many so-called pustular eczemas are from the first staphylococcic or streptococcic infections. The success of treatment based on the parasitic theory is a strong argument in favour of its truth in regard to a large proportion of cases. On the other hand, we know that the organisms referred to are harmless to a healthy skin. The ground must therefore be prepared for their action. Neisser holds that this is done by external irritants. But how is the ground prepared when there are no irritants? The distinguished Breslau professor gets over this difficulty by giving the chief place among "irritants" to soap and water. Thus, in his view, except among the "great unwashed," the human skin is always at the mercy of the golden-yellow staphylococcus. Galloway and Eyre include among predisposing factors (1) certain organic lesions, especially such as produce circulatory stasis in the skin and consequent œdema and malnutrition of both cutis and epidermis; (2) the seborrhœic state, which permits the free growth of vegetable parasites, and especially of certain bacteria; (3) certain conditions of imperfect metabolism, which predispose to the onset, or at any rate the recurrence, of eczema ; 'of these the most common are those associated with improper digestion and assimilation of food. Want of exercise, the impure atmosphere in cities, etc., aggravate this condition and increase the risk of recurrent attacks. 
In a valuable and suggestive study of the possible influence of anaphylaxis in skin reactions, ${ }^{1} \mathrm{~J}$. A. Fordyce points out that, assuming a state of hypersusceptibility which may either be present at birth or acquired from some change in the blood serum due to various affections, it is easy to understand how irritants of the most varied kind may evoke a dermatitis. Thus a purulent discharge may produce an eczema by the chemical product of pus organisms, and when the outbreaks occur at a distance from the primary focus it is possible that the explanation is to be found in changes induced in the blood serum by an extensive surface infection. He cites several cases of infectious eczematoid dermatitis following ecthyma, furunculosis, abscess of the breast, etc., among them the case illustrated in Plate 20.

Galloway and Eyre, in the paper referred to above, make no mention of a factor to which I attach great importance, that is, nervous shock and prolonged mental depression. I have so often seen the disease develop on skin previously quite healthy that I cannot doubt that the derangement of the nervous mechanism brought about by the influence of the mind on the body has been reflected on the integument. Bulkley gives some striking examples of eczema following worry, mental strain, and nervous shock. More than one case traceable to the "Black Friday" financial panic in Wall Street came under his notice. According to Radouan, the siege of Paris by the Germans and the brief " reign of terror" of the Commune in 1871 left their impress on the skins of many persons in the form of eczema. ${ }^{2}$ I have myself known the disease in its acutest form follow a fright.

1 "Infectious Eczematoid Dermatitis," Journ. Cut. Dis., March, 1911.

2 On the nervous origin of eczema, see Kromayer, "Allgemeine Derm." Berlin, 1896. 
The nervous depression caused by chill manifests itself in some persons as catarrh of the skin-that is, eczema-just as in others it shows itself as catarrh of the respiratory membrane, and in others again as catarrh of the intestine. Thus a man may go to business in the morning on the top of an omnibus, being at the time to all appearance in perfect health; he may feel that he has "taken a chill," and begin to shiver and complain of general malaise; on reaching home in the evening, however, he may find that, instead of a catarrh of his mucous membrane, he has developed a well-marked eczema. In the same way motoring and exposure to the sun may provoke an attack of eczema. Reflex nervous irritation from the uterus, the stomach, the intestine, etc., often seems to be an exciting cause of eczema. In some women menstruation and pregnancy are generally accompanied by an attack of eczema, and the disease is also not uncommonly one of the indications of the "change of life." Eczema is sometimes a result of intestinal toxæmia, or it may be due to the presence of worms in the intestinal canal. The origin of eczematous eruptions beginning on the cheeks, eyelids, etc., has been traced to disturbances of vision, and the skin lesions have ceased to appear when the eye affection has been cured. ${ }^{1}$

Unna, as we have alreday seen (p. 274), has described a special type of eczema on the face in infants, occurring in connection with dentition. Arthur J. Hall, in an elaborate analysis of 60 cases of infantile eczema under his care between the years 1897 and $1903,{ }^{2}$ maintains that neither dentition nor vaccination can be regarded as the cause in most cases, nor does the evidence support the theory which finds the cause in digestive disturbance or malassimilation.

1 Juler, Lancet, 1884.

${ }^{2}$ Brit. Journ. Derm., May, June, July, Aug., 1905. 
Holding the microbic theory also to be not proven, he attributes infantile eczema to such external irritants as chill to the skin, imperfect drying, soaps, irritating dust in the atmosphere, the sweat of the mother's skin, and the secondary action of microorganisms. His chief reason for tracing the affection to some or all of these irritants is the fact that in 95 per cent. of his cases the rash first appeared in what was practically the only exposed part of the infant, the head or face. Reviewing a further series of 40 cases in January, 1908, he holds that it supports his earlier conclusions. Of the 100 cases, there were 96 in which the head or face was the part first affected. He suggests broadly that eczema, whether in infants or in adults, is a form of reaction of the neuro-cutaneous apparatus to the external irritation. For my part, I am inclined to think that in the majority of infantile cases the production of the skin affection is rather to be explained by the seborrhøa which is apt to be set up by the abnormally large amount of blood supplied to the head in infants for the building up of bone and brain. The same influence is more or less actively at work in all growing children; hence the frequency with which seborrhoea is met with at that time of life.

Eczematous eruptions may also be produced by reflex irritation of peripheral origin, as in the case of burns, etc.; or they may be a consequence of changes in the nerves resulting from injury or disease, or they may be connected with functional neurosis. Colomiatti ${ }^{1}$ found structural changes in the cutaneous nerves in several cases of eczema, mostly of the papulosquamous type; and that these changes were in direct relation to the process in the skin he held to be proved by the fact that in cases in which the skin lesions were

${ }^{1}$ Giorn. Ital. $d$. Malattie Vener. e d. Pelle, 1879. 
wholly or partly cured the nerves also had in great measure recovered their normal appearance. These observations were afterwards confirmed by Leloir. ${ }^{1}$ Of the relationship between eczema and certain forms of functional neurosis there cannot be a better illustration than the fact that it is frequently associated with asthma; so close, indeed, is the connection between the two affections, that asthma is believed by some to be -at least in certain cases-simply eczema of the bronchial tubes. It is probable that both the eczema and the asthma are the response by the skin and respiratory mucous membrane respectively to some central or peripheral irritation to which both alike are exposed. Cases have been reported by Charcot, Vulpian, and others in which eczema occurred in association with disease of the brain or spinal cord, but there is not yet sufficient evidence to show whether the skin affection in these cases was the result of the nerve disease or an accidental coincidence. Anything, however, which interferes with the proper nutrition of the skin lessens its power of resistance, and in this way disease of the central nervous system may be regarded as a predisposing cause of eczema. The affection is said by some observers to be frequent among the insane. ${ }^{2}$

The exact mode in which eczema is induced by nerve disorder is still somewhat obscure. Such evidence as is available is almost entirely clinical. It is certain that under the influence of nerve shock and nerve exhaustion (neurasthenia), eczema may arise de novo in a previously healthy skin. In such circumstances the trophic influence of the nervous system on the skin is, to a greater or less extent, impaired; and, according to Leloir and Bulkley, eczema may be the result. In other words, eczema is, in the opinion of these der-

1 Ann. de Derm. et de Syph., 1890.

2 Fèvre and Nicol, quoted by Bulkley, loc. cit. 
matologists, simply a tropho-neurosis. It is not quite certain, however, that something more is not required for the development of a process so complex in its manifestations as eczema. There is something to be said for the view that the inhibition of trophic influence does nothing more than prepare the way for eczema by reducing the skin to a condition in which it is unable successfully to resist microbic action.

As regards reflex irritation, the case is somewhat different. It has already been shown that vaso-motor disturbance alone is sufficient to produce all the essential lesions of the eczematous process. Even here, however, microörganisms must often intervene, or there would be no pustules. While, therefore, not prepared to maintain, with Unna, that eczema is always microbic, I am still less disposed to accept the view of Leloir and Bulkley that it is never anything more than a neurosis. That in the majority of cases eczema is parasitic is proved by the effect of anti-parasitic treatment; that there are many cases in which the affection is of nervous origin is shown by the fact that it may be cured by remedies which act on the nervous system. Moreover, in many parasitic cases the neurotic element may be so pronounced as to furnish the leading indication for treatment.

Apart from the condition of the nervous system, I attach little importance to the constitution of the patient as an etiological factor in regard to eczema. The tendency to that affection is sometimes found associated with rheumatism, and sufferers from gout are prone to eczema as they are to other forms of catarrh. There is not, however, any form of skin lesion known to me which can properly be called "gouty eczema"; in other words, there is no special type of eczema that can be recognised objectively as of gouty origin. Brocq describes an eczéma érysipélatoïde 
récidivant des arthritiques, ${ }^{1}$ characterized by the rapid occurrence of inflammatory attacks of great intensity, almost always affecting the head and face, sometimes the hands, genitals, etc.; the skin is swollen and red as in erysipelas, and constitutional disturbance is more or less severe. Although gout is so common in Great Britain, I am not familiar with a type of skin affection answering to this description. In Germany, where gout is comparatively rare, eczema is just as common as it is in England. But, while denying that gout is of itself sufficient to produce eczema, I am willing to admit that the gouty diathesis or any other constitutional state characterized by a tendency to sudden vaso-motor disturbance may aggravate the skin affection to such an extent as to require to be taken into account in treatment.

There is no connection between eczema and rickets, nor has malnutrition any direct influence in its production. The disease is just as common in the wellnourished children of well-to-do people as in those of the poor, and breast-fed infants are no more exempt from it than those brought up by hand. Nor has scrofula anything to do with the production of eczema, except in as far as proclivity to catarrh is one of the notes of the scrofulous diathesis. It is true that many children suffering from eczema are the subjects of scrofula, but, on the other hand, there are far more eczematous children than scrofula can account for. It is almost unnecessary to say that, although scrofula cannot produce eczema, it may have a powerful modifying influence on the lesions.

Eczema is not as a rule contagious, but when caused or complicated by microörganisms it is autoinoculable; thus it reproduces itself in distant foci, while individual patches continue to spread at the edge.

${ }^{1}$ Op. cit., pp. 154-5. 
Sometimes it appears to be inoculable from one patient to another. Thus the arms of nurses who carry babies suffering from eczema of the nates may become irritated, and eczema may be induced by scratching.

To sum up : eczema in a large proportion of cases is of microbic origin, but the organisms cannot produce the lesions unless they find a suitable soil in which to proliferate. In some cases the skin is made suitable for this purpose by seborrhœa or other pre-existing morbid condition; in others by diminished resistance owing to loss of nerve control. In another class of cases the disease may possibly be altogether of nervous origin. When once started it spreads from one or two centres by auto-inoculation if microbic, by reflex irritation if neurotic.

Diagnosis.-In a certain proportion of cases of eczema the diagnosis presents no difficulty, the appearance of the lesions, and particularly the "weeping," being sufficient for the identification of the disease. Sometimes, however, the nature of the affection may be obscured by the very multiformity which is one of its characteristic features. In such cases one must have recourse to a process of exclusion. No reliance must be placed on subjective symptoms, as they are so variable that they can serve only as an index of the patient's temperament and of what may be called the temperament of his skin. All discharge, crusts, or accumulations of scales should first be removed, and a careful examination should be made of every affected spot. However multiform the lesions may be, one seldom fails, if an adequate search be made, to discover somewhere or other a patch which can be recognized as eczematous. This at once dissipates any doubt as to the nature of the disease. Secondary syphilis and erythema multiforme are the two conditions which, in 
the multiformity of their lesions, most resemble eczema. If the lesions are syphilitic there will be other signs of the disease, while erythema multiforme can be identified either by the presence of some typical lesion, such as so-called herpes or erythema iris, or by the preponderance of red raised patches without scales, and especially without any trace of " weeping." Erysipelas can be excluded by the absence of constitutional symptoms and of the characteristic brawny induration and ridged border.

Of parasitic diseases, the one which most closely resembles eczema is scabies; the lesions are so similar that, when the characteristic burrows are not visible nor the itch-mite discoverable, a mistake might easily be made. The lesions of itch are, however, isolated, not grouped into patches; instead of being small, acuminate, or circular, they tend to be linear, and they lack the spreading edge characteristic of eczema. There are, moreover, differences in the distribution of the two affections-scabies being scattered irregularly and showing a marked predilection for the hands, especially in the interdigital spaces, the wrists, the inner side of the thigh, the abdomen, the pubes, and the axilla; while eczema is nearly always more or less symmetrical, and mostly affects the head, the trunk, and the flexures of joints. Sycosis of the chin sometimes simulates eczema of that region so closely that it is almost impossible to distinguish the one from the other, except by the fact that sycosis shows no tendency to spread beyond the area covered by hair. Ringworm of the scalp can be identified by the broken hairs which can always be found on careful search, and by the presence of the fungus. Tinea circinata, if it occurs as a scaly patch on the trunk, can be recognized with the help of the microscope. Both tinea circinata and tinea cruris (eczema marginatum), when the hands and feet are 
attacked, may so closely simulate eczema that nothing but the discovery of the fungus can settle the diagnosis (see pp. 394 and 398). Favus of the scalp is distinguishable by its cup-shaped and sulphur-yellow crusts, their mousy smell, and the presence of the fungus. In old cases of favus, too, there is scarring.

From herpes in general, eczema is distinguished by the characteristic "weeping," by the multitude of minute vesicles, which run together without forming separate groups, by the slower course the affection runs, and by the usual supervention of inflammatory thickening; and from zoster in particular by the distribution, which does not follow any particular nerve area. Eczema papulatum often resembles lichen planus; in the latter affection, however, the papules are usually flat or umbilicated, with a glistening surface, are irregular in outline, and dark-red or violaceous in colour; and neither discharge nor crust formation is ever observed. Nor are the papules transitional, and while they leave behind them brownish or blackish stains, those of eczema are often transformed into vesicles and seldom produce discoloration. As already mentioned, the lens will often reveal, on the top of each papule in eczema papulatum, a minute vesicle-a phenomenon which is never observed in lichen planus. In the latter affection, too, characteristic discrete papules can usually be found at the margin of the patches.

Certain forms of dry seborrhœic eczema are frequently very difficult to distinguish from psoriasis. Attention to the following points of difference will help to a correct conclusion. In the first place, psoriasis is always dry; moreover, it has a typical distribution, and usually spreads from the elbows and knees. Eczema, on the other hand, in the majority of cases, spreads downwards from the head. Further, patches of psoriasis have a sharply defined border, and are not 
so stationary as those of eczema. In the former the scales are silvery-white, in the latter yellowish, with a distinctive fatty and crumbling character which is absent in psoriasis. Lastly, in psoriasis there is no history of previous seborrhoa. The point of diagnosis from a dry seborrhwic dermatitis so frequently emphasized-namely, that on removing the scales of psoriasis, the red or even bleeding tips of congested papillæ may be noticed-is of some value, but may be quite misleading. The absence of weeping is in itself sufficient to differentiate eczema from pompholyx, even in cases in which eczematous lesions assume the sagograin appearance characteristic of that affection. In all cases of vesicular or bullous lesions on the hands and feet a microscopic examination of the scales should be made with a view to the possible presence of ringworm. Certain cases of sudden and acute eczema may mimic smallpox, but the eczematous lesions are more superficial, and smaller, and seldom widely diffused, and the underlying skin is œedematous and infiltrated. Eczema of the nipple may be distinguished from Paget's disease by the absence of the parchment-like induration and retraction of the nipple which are characteristic features of the latter condition. For the diagnosis of eczema from pityriasis rubra, see p. 346 ; from miliaria rubra, see p. 614 ; from impetigo contagiosa, p. 550 ; from mycosis fungoides, p. 716.

Prognosis.-Eczema can nearly always be cured by a proper course of treatment perseveringly pursued. In many cases, however, the condition is extremely obstinate, and recurrence is the rule rather than the exception. When the neurotic element is strongly pronounced the prospect of cure is much less favourable than in cases of seborrhwic origin. The age and general health of the patient must be taken into account in forming a prognosis. 


\section{CHAPTER XII}

\section{ECZEMA : TREATMENT}

WITH regard to treatment, the question that meets us on the threshold is whether eczema should be treated at all. There is a popular notion that the affection is a kind of safety-valve which it is dangerous to close; nor is this idea confined to the laity. So experienced a practitioner as Brocq warns us against interfering too actively with eczema in elderly persons or in gouty, rheumatic, emphysematous, or asthmatical subjects, or sufferers from chronic bronchitis, melancholia, Bright's disease, dyspepsia, etc. A similar view is strongly held with regard to eczema in children by Gaucher. ${ }^{1}$ $\mathrm{He}$ appears to regard eczema as a provision of nature for the elimination of "toxic principles" resulting from constitutional and often hereditary disorders of nutrition. By shutting up this outlet these toxic principles are made to accumulate in the internal organs, "with consequences more or less rapid and more or less serious, according to the seat of the metastasis."

Holding, as I do, that in a large proportion of cases eczema is of microbic origin, and that the constitution, when involved at all, plays but a secondary part in the process, I am utterly opposed to the laissez-aller principle in dealing with the disease. The caution in treating eczema which is so emphatically enjoined on us is based on the assumption that we have a power of controlling

${ }^{1}$ Congrès Intern. de Derm. et de Syph. tenu à Paris en 1889 Comptes-Rendus, p. 538 et seq. 
the process which we are very far from possessing. Even if the "abrupt soothing down of the cutaneous phenomena" deprecated by Brocq were as dangerous as he believes, there need be no hesitation in treating them, since it is quite exceptional for the disease to be " abruptly soothed down" by any means at our disposal. My view, therefore, is that the practitioner should endeavour to cure eczema whenever he meets with it; the only caution necessary is that he should accurately adapt his remedies not only to the process but to the patient.

The first step towards successful treatment is to determine whether the disease is of microbic or of neurotic origin-that is to say, whether it is to be dealt with by local or by general remedies. As already said, these two etiological elements are often combined, and in that case it is important to ascertain which of them predominates in a given case.

Internal remedies.--In dealing with eczema the beginning of therapeutic wisdom is to clear one's mind of the notion that arsenic or any other drug is a specific. One must learn not to look upon it as a fixed law that internal remedies are to be given in every case. As a general rule, indeed, the less drugging the better. But if internal remedies have to be employed, they should be given only for a definite purpose and in accordance with definite indications. Random polypharmacy is often hurtful to the patient and an obstacle to scientific progress. How can we expect to gain any accurate knowledge of the action of medicines if they are used by the half-dozen at a time, like charges of small shot fired at the disease?

For the subduing the inflammation in acute generalized eczema there is, in my experience, nothing equal to antimony. Small doses of the vinum antimoniale quickly relieve the arterial tension and thus 
reduce the local inflammation. If the patient's constitution is sound, I generally begin by giving $m x$ to $m$ xiii of the wine, repeating the dose in an hour, and, if necessary, again two hours later. The interval between the administrations is gradually increased, while the amount is diminished till a dose of $m$ vi is reached. This should be given three times in the twenty-four hours as long as the acute symptoms last. When there is no great arterial tension, and when depression is a prominent symptom, antimony should not be given. In babies and young children small doses of calomel at bedtime occasionally are of great service. In all acute inflammatory conditions of the skin, iron only adds fuel to the flame by increasing the activity of blood formation. Arsenic also is contra-indicated in such circumstances. Stimulants must be forbidden, the diet should be of the simplest kind, and the bowels must be carefully regulated. The clothing should be light, and it is particularly important that the patient when in bed should not be covered with heavy blankets, as the symptoms are always intensified at night. Complete rest, both of mind and body, should as far as possible be secured. If the area of skin involved be very extensive, the patient should be kept in bed. When nervous symptoms are pronounced, appropriate sedatives must be administered. In the front rank of these is opium, which, if need be, should be given during the day as well as at night; sometimes it may be necessary to keep the sufferer almost continuously under the influence of opium or morphia. In such eases the constipating effect of the drug should be counteracted by giving a mild aperient, such as Carlsbad salts, Friedrichshall, or other saline purgative, in the morning: If opium disagrees, veronal, trional, chloral, sulphonal, or phenacetin may be substituted for it. If prostration be a marked feature of the case, it will be well to 
commence treatment by giving quinine; this remedy may often with great advantage be combined with opium. In chronic neurotic cases arsenic sometimes does good, but my experience is that this drug can never be relied upon in eczema. Strychnine, and especially phosphorus, are more frequently of use in such cases, and ergot may occasionally prove serviceable, probably by its action on the vaso-motor apparatus. In women at the climacteric period, and in hysterical subjects, such remedies as musk, valerian, etc., should be used; and in all cases, if any definite source of peripheral irritation can be discovered, it should, if possible, be removed. When the discharge is very profuse, quinine may usefully be combined with belladonna. When the disease is very rebellious, fresh exacerbations occurring every few days, Crocker has found counterirritation (by means of blistering fluid, mustard-leaf, etc.), applied over the vaso-motor centres of the part, very useful. In all cases the patient's general health must be attended to, complications like dyspepsia, etc., being dealt with as the occasion arises, and constitutional conditions such as rheumatism, gout, diabetes, renal disease, rickets, and scrofula being treated in accordance with the general principles of practice.

Having observed in cases of acne vulgaris treated with vaccine a clearing up of concomitant eczematous patches, T. C. Gilchrist used a staphylococcal vaccine in cases of eczema, with rapid and quite marked beneficial effects in a number of instances. This mode of treatment deserves, as he claims, further trial. ${ }^{2}$ In persistent acute cases in young children $I$ have found that thyroid extract in small doses yields good results.

Local treatment.-Although internal medication may be a useful adjuvant in the treatment of eczema, 1 "Diseases of the Skin," p. 182, 3rd edition, 1903.

2 Trans. Amer. Derm. Assoc., 1909, p. 18. 
the practitioner who, from a mistaken belief in the constitutional nature of the disease, trusts entirely thereto will find that he is leaning on a broken reed. Eczema, being in a large proportion of cases of microbic origin, requires appropriate local remedies, and in seborrhœic cases, when the patient's general health is sound, no other treatment may be required. It must, however, be understood that for local treatment to be successful two conditions must be fulfilled. First, the strength of the application employed must be judiciously tempered to the intensity of the process which it is intended to combat; secondly, the lesions must be kept continuously under the influence of the remedy. The mere perfunctory application, morning and evening, of a solution or an ointment can have little or no effect in checking the disease. The guiding principle in local treatment must be to destroy the irritant while soothing the inflammatory reaction set up by its presence.

A necessary preliminary to local treatment is the removal of all crusts and scales that prevent the free access of the remedy to the seat of disease. They can be softened by means of oil applied on strips of lint, or weak solutions of bicarbonate of soda. Crusts are readily loosened by keeping the parts covered for a few days with thin indiarubber; this method is especially useful on the head and limbs. When the crusts have been got rid of, the next, step is to attack the disease directly. In the local treatment of eczema three objects have to be kept in view. First, the removal of any source of irritation; secondly, the protection of the inflamed surface from the air and from possible invasion by fresh microbes ; thirdly, the relief of itching. As it is of the utmost importance not to aggravate the inflammatory process, an unirritating parasiticidal agent should be employed in the first instance; the strength of the 
application should be very moderate to begin with, and may be gradually increased as the symptoms subside. When there is much discharge a warm solution of boric acid is particularly useful for the washing of the affected part. The lotion should be dabbed on with wool or linen. A towel should not be used, but the discharging area may be dried by means of muslin bags containing starch, with a small quantity of powdered boric acid. Sometimes the parts are so sensitive that the patient cannot bear this application; in that case a calamine lotion should be applied. Or talc or starch with a little powdered boric acid may be dredged over the oozing surface; this procedure is, however, attended with the disadvantage that the powder becomes caked on the part, making it stiff and painful to move. It should be removed with the aid of weak carbolic oil.

During the acute stage the parts should never be washed with water, and even when the violence of the inflammation has subsided, washing should not be frequent, and friction with towels should be carefully avoided. Hard water should on no account be used; only rain-water or water that has been boiled should be allowed to come in contact with the eczematous skin. It is better not to use soap of any kind, but if any must be employed, one of the superfatted medicated class introduced by Unna should be selected.

For the protection of the inflamed surface from the air, and for the relief of irritation, greasy applications in the form of "creams" are most useful. These should be as emollient as possible. The following formula may serve as an example :-

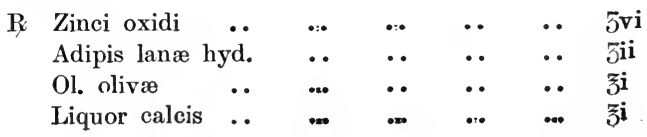


Some ointments have a tendency to heat the skin, while others impart to it a feeling of coolness. Unna attributes the latter property to the fact that in these "creams" a certain proportion of water is combined with the fatty base; this facilitates evaporation. A useful cooling salve consists of the following ingredients :-

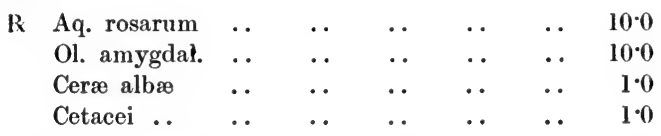

This cold cream forms a good base for various compound ointments, and may be made the excipient for different antiseptic agents. For the continuous application of parasiticidal agents, pastes, salve muslins, sticks, plaster muslins, and varnishes may be employed. A useful paste may be formed by mixing equal parts of starch and zinc ointment; to this any antiseptic that may be desired can be added. The following is the formula of Lassar's paste, which is valuable both by itself and as a basis for other drugs :-

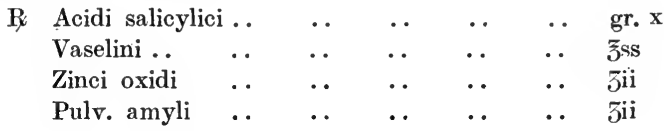

Resorcin, ichthyol, tar, etc., can be added to this paste. Sticks, as suggested by Brooke of Manchester, may also be the vehicles of antiseptic agents. The base of the stick is cocoa butter, and in this way boric acid, salicylic acid, ichthyol, oxide of mercury, resorcin, sulphur, etc., may be kept in contact with diseased surfaces. Both the pastes and sticks may be flesh-tinted with Armenian bole, so that they can be used on the face or hands without exciting notice.

Salve muslins were introduced by Unna, and form a 
very convenient means of keeping remedial agents in continuous contact with the parts on which it is desired to act. These consist of muslin spread with a consistent layer of benzoated lard and wax; vaseline or lanolin may, if desired, be substituted for the lard. These salve muslins may be the vehicles of carbolic acid, sulphur, resorcin, etc.; pieces of the salve muslin of the size required may be cut off and accurately fitted to the part to be treated. The salve muslins may be obtained spread on both sides. These preparations form the most convenient means of treating eczematous lesions in which the discharge is no longer profuse. The salve muslins are of use in the earlier, acute stages of eczema; plaster muslins are best adapted for chronic patches left behind when the acute stage is past. These plaster muslins may also be the vehicles for every kind of local remedy. Another equally convenient method of keeping remedies in contact with eczematous lesions is the glycerine jelly or varnish employed by Pick and modified by Unna. The advantage of these varnishes is that they can be applied to any part of the body, so as to form a tight-fitting and at the same time pliable covering, which can be easily removed and readily reapplied. Allan Jamieson envelops the raw, denuded, "weeping" surface with a starch jelly, with which is combined a proportion of boric acid.

In all cases, as has already been said, it is advisable to commence local treatment with very mild applications. One must feel one's way, so to speak, as it is impossible to know beforehand whether a particular remedy may not cause irritation. The best application in seborrhœic and all other parasitic forms of eczema is sulphur. At first a small quantity of sulphur, combined with a soothing application, such as zinc ointment, should be used. The proportion of gr. $\mathrm{x}$ of precipitated sulphur to $\mathrm{i}$ of zinc ointment is quite strong enough to 
begin with; the amount of sulphur should be gradually increased if the application is well borne. The ointment should be spread on strips of thin linen, which must be laid evenly on the part and fixed with a bandage. If the face is the part to be treated, it should be covered with a mask. Resorcin may be employed in the same way. Both that drug and sulphur have this special advantage, that they not only destroy the microorganisms on the surface, but cause rapid exfoliation of the horny layer. Ichthyol is useful in acute forms of eczema for its sedative as well as its microbicidal properties. In a large number of cases a solution of ichthyol in water ( 1 in 16 to begin with) painted over the inflamed area, or in an ointment, will allay irritation, cause contraction of the cutaneous bloodvessels, and lessen the discharge. In seborrhœic eczema ichthyol is best applied in the form of the varnish recommended by Unna, the composition of which is as follows : Ichthyol 40 parts, sturch 40 parts, albumin 1 to $1 \frac{1}{2}$ parts, water to 100 parts; or the albumin may be omitted and the proportion of the other ingredients modified as follows: Ichthyol 25 parts, carbolic acid $2 \frac{1}{2}$ parts, starch 50 parts, water $22 \frac{1}{2}$ parts.

Patches of chronic eczema may be the result of the acute form or the remains of seborrhœic affection. In the latter case they should be treated with stronger applications of sulphur and other antiseptics spread on linen, or, better still, in the form of the plaster muslins already referred to. Chronic eczematous patches of nonseborrhœic origin are often the seat of violent itching ; this can generally be relieved by applications of carbolic acid with a sponge or on a piece of rag. The following is a useful formula :-

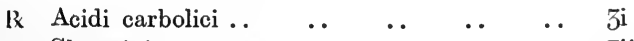

$\begin{array}{lllllll}\text { Glycerini } & \ldots & \ldots & \ldots & \ldots & \ldots & 3 \text { ii }\end{array}$

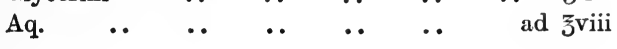


A lotion of $t a r$, in the form of liquor picis carbonis, (3ii to $\zeta$ viii of water), and a weak solution of nitrate of silver in $s p$. ceth. nit. (gr. $\mathrm{v}-\mathrm{xx}$ to $\mathrm{z}^{\mathrm{i}}$ ), are also useful for the same purpose. For the resolution of the patches a plaster muslin of yellow oxide of mercury, with or without resorcin, is a serviceable application. For the same purpose tar can be used in the form of ointment either alone or in combination with mercury. The following may be given as examples:-

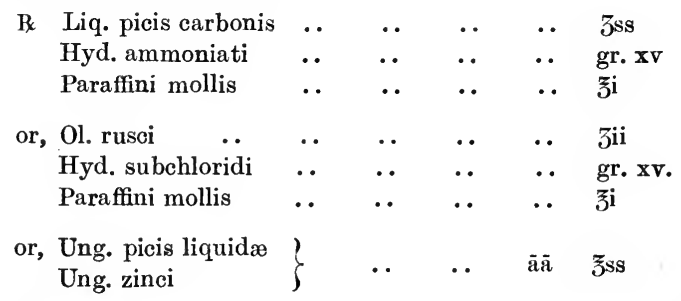

Salicylic acid in a plaster or as an ointment is of service in chronic eczema of palms and soles.

One of the best remedies for thickened chronic patches is chrysarobin; but the patient must be warned that the application sometimes causes redness and pain, and stains linen and clothes. It is best applied in the form of an ointment (the ung. chrysarobini B.P. being the most suitable), or in the form of a plaster muslin. For varicose eczema of the legs Martin's bandage or elastic stockings should be worn.

Certain modifications of local treatment are necessary according to the part that is the seat of disease. Thus, between opposing surfaces, as between the scrotum and the thigh, or beneath the breasts in stout women, there should be placed long narrow bags made of thin cambric or muslin, and partially filled with starch powder, powdered boric acid, or a mixture of powdered talc (87 parts), powdered starch (10 parts), and salicylic acid (3 parts); the 
parts are thus dried and kept in an antiseptic state. In seborrhœic eczema of the scalp and other hairy parts the hair should be cut short, and after softening and removal of the crusts, very weak sulphur ointment spread on strips of lint should be applied and fixed in position with a cap or bandage. About the ears, and on the vulva, in both of which situations the swelling is often very great, astringent and cooling lotions, such as lactate of lead and calamine lotion, give great relief: On the face, as already said, the local applications should, in the case of children, be kept in position by a mask. When extensive areas of skin are involved, as on the arms or legs, swathing the parts in strips of linen soaked in calamine lotion, or a warm saturated solution of boric acid, generally relieves the irritation; but when the inflammatory process begins to subside, stronger antiseptics must be kept continuously applied in one or other of the ways that have been mentioned.

Treatment requires to be adapted to the peculiar features of the disease manifested at certain periods of age. In infancy, when it generally starts from a focus of seborrhœa on the scalp, this should be treated by the gentle use of soap and water. Over-scrubbing should be avoided, and the soap should be superfatted. If there is any tendency to irritability of the scalp, the child should not wear a cap in the house, and its head should not be too warmly covered out of doors. A child's hands should never be tied to prevent scratching. The best local application is a very weak sulphur ointment-precipitated sulphur gr. $\mathrm{v}$ to $3 \mathrm{i}$ of benzoated lard. Xerodermia (which is sometimes associated with eczema) should be treated by soaking the dry hard parts with a mixture of 1 part of glycerine to 5 of water. For eczema at the menopause there is, in my experience, no remedy like ichthyol given internally in doses of gr. iiss after each meal at first, and gradually increased 
up to gr. $x$ or more. If in the eczema of old age, when the irritation is severe, opium is employed, it must be remembered that this drug has the property of causing severe itching, and the dose must be small. It should be combined with mild aperients if it causes constipation.

Good results have been reported from the treatment of eczema by radiotherapy, both in cases of the vesicular type and in rebellious cases of chronic dry eczema. In several cases of the latter variety under the care of the author and S. E. Dore, distinct benefit followed the application of $\mathrm{X}$-rays, though of course relapses may occur.

The methods of general and local treatment that have been described have often to be supplemented by other measures, such as diet, clothing, hydrotherapy, and climate.

Diet.-It is necessary, in the first place, to get rid of the superstition as to this matter which is so strongly implanted in the mind, not only of the public, but also of a section of the medical profession, especially those of the older school. The excessive "lowering" diet on which so much stress is still laid by some is not only unnecessary, but positively contra-indicated, except when the inflammation is extremely intense.

In microbic cases dietetic treatment is comparatively useless, and a recognition of this truth will save patients a good deal of needless privation. I might quote, in proof of what has just been said, numerous cases in which patients have been most carefully dieted for long periods without their eczema being in the slightest degree benefited; whereas on removing all restrictions of diet, and treating the affection by local remedies, a cure has speedily followed. Beer and other stimulants should be forbidden, especially in acute forms 
of eczema. There is no need to cut off tea, unless it be definitely contra-indicated by flatulence, palpitation, gastric acidity, or insomnia. Sugar may be allowed, except in the case of patients of gouty constitution, or when contra-indicated by glycosuria.

Clothing.-As already said, this should be as light as is consistent with proper protection from cold. Too much clothing increases the activity of the sweatglands, and thereby makes the skin moist, and to some extent predisposes it to eczema. Only silk, fine linen, or soft wool should be worn next the skin.

Hydrotherapy has little direct effect on eczema, though by its alterative action on the system it may indirectly modify the affection of the skin. Sulphur waters - notably those of Harrogate, Strathpeffer, Luchon, Aix-les-Bains, and Schinznach-often have a markedly beneficial effect in cases of obstinate eczema, especially in those who live too well and suffer from constipation. "Indifferent" waters, like those of Bath and Buxton, are often useful. Bromo-iodide waters are of use only. in very chronic conditions. The arsenical waters of La Bourboule and Levico are also of service in similar circumstances. Aperient waters, like those of Carlsbad and Marienbad, are indicated in the case of stout or gouty patients on account of their constitutional effect. Weyner ${ }^{1}$ recommends tar baths in chronic eczema, the crusts becoming detached and the infiltrated skin softened, so that the tar is enabled to reach the diseased surface.

Sea-bathing should never be indulged in while eruptions are present on the skin. I have, however, known patients subject to periodical outbreaks of eczema lose their proclivity to the disease as the result of a course of sea-bathing.

Climate.-All that need be said is that, the disease

${ }^{1}$ Balneol. Centralzeit., Dec. 2, 1901. 
being catarrhal, climates favourable to the production of catarrh of any kind should as far as possible be avoided.

The eczematous process in the skin has been compared to inflammation of a joint. The same analogy holds good with regard to the treatment of these two conditions respectively. The first indication in dealing with an inflamed joint is to keep it at rest; the next, to subdue the intensity of the process and bring about resolution or quiescence; lastly, the products of inflammation must, if possible, be got rid of, so that the joint may recover its natural suppleness. In eczema the same objects have to be aimed at, with the further indication that the microörganisms which find the lesions a favourable ground for their multiplication have to be destroyed or rendered inert.

Summary.-The fundamental principles by which one should be guided in the treatment of eczema are to soothe when the inflammatory process is acute, to stimulate when it is chronic, and in either case to keep the parts under the continuous influence of antiseptics and microbicides of a strength carefully regulated in accordance with the intensity of the disease and the tolerance of the patient's skin.

A word of caution may be added as to danger of over-treating eczema. When the disease is quiescent or in active retrogression, a masterly inactivity will be found the best policy. In all cases the greatest vigilance must be exercised in the adaptation of the strength of the remedies to the disease. I have seen many cases in which the condition has been aggravated by the injudicious use of baths and of stimulating treatment. 


\section{CHAPTER XIII}

\section{PSORIASIS AND PARAPSORIASIS}

\section{PSORIASIS}

Psoriasis is an affection of the skin characterized by flat, dry patches of varying extent, covered with white, silver-grey, or asbestos-like scales. There is no exudation, and consequently there are no crusts; the degree of scaliness varies from a thin film to a dense, heapedup mass. On removing the scales-which are, as a rule, tolerably adherent-a smooth, shining hyperæmic surface is exposed, dotted here and there with deep-red spots. This surface, the base of the lesion, though red, is not raw, and the tint varies from bright red in recent patches to a duller tint in those of older formation. The bright-red spots, which can always be seen with the help of a lens, are the tops of the hyperæmic papillæ; these bleed very readily on being touched. The typical lesion, or what may be termed the pathological unit, of psoriasis is a scaly patch, rounded or irregular in shape, with a sharply defined border standing out slightly but distinctly on the surface of the skin, and a hyperæmic base underlying the covering of scales. When the disease is spreading the patch is surrounded by a narrow zone of redness, but this is wanting when the process is inactive. The scales are of a dirty-white colour on the surface, but those underneath the uppermost layers have the appearance of frosted silver.

Objective symptoms.-The eruption first shows itself in the form of papules of the size of a pin's 
head; these are at first red, but they soon become white as the scales form. Besnier states that he has seen cases in which the appearance of scales was the first appreciable change. Whether scaliness precedes hyperæmia or vice versa is a point of no clinical importance. It is certain that even when no scales are visible on the red ground of the initial lesion, they can be brought into view by gently scratching the surface. The papules spread in a centrifugal direction and form patches, which are usually somewhat round or oval in outline when small, becoming more irregular as they get larger. After attaining a certain size they may remain stationary for a long time, and then gradually disappear; or they may continue to spread, and, becoming confluent, cover large areas of skin (Plate 21). The differences of aspect presented by the lesions at various stages in the evolution of the process have been dignified by distinctive names. Thus, the initial white scaly specks are sometimes spoken of as psoriasis punctata ; when the lesions are somewhat larger, so as to resemble splashes of mortar or drops of wax, the appearance is indicated by the name of psoriasis guttata; when still larger, and rounded like coins, we have pso= riasis nummularis, and so on. Other names sometimes used in describing the lesions of psoriasis are intended to denote not so much the shape as some other prominent characteristic. Thus, when the disease has involved extensive surfaces, the skin often presents a peculiarly harsh, dry, thickened, and cracked appearance ; this is sometimes called psoriasis inveterata. In other cases the scales may be heaped up into cone-shaped masses, arranged in layers forming concentric rings, and resembling rupial scabs; to this condition the term psoriasis rupioides has been applied by $\mathrm{M}^{\prime} \mathrm{Call}$ Anderson.

Sooner or later the patches undergo involution. They first begin to fade in the centre, leaving rings 


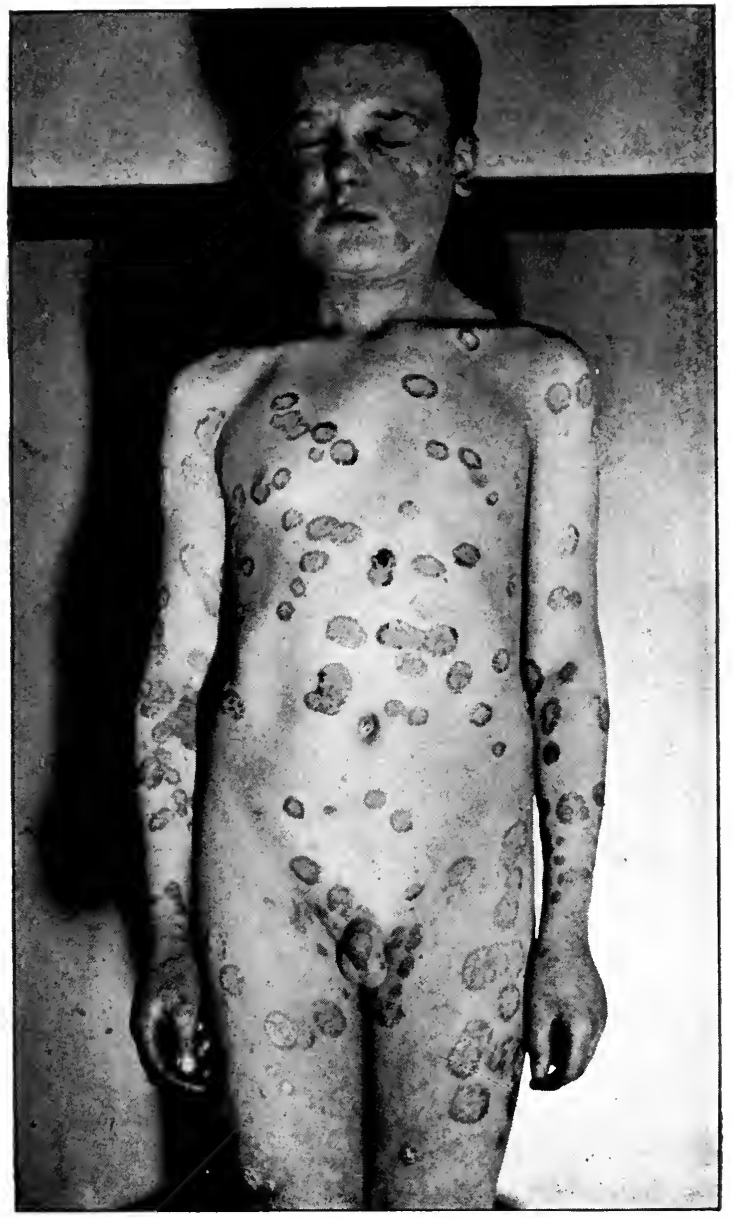

PLATE 21.-ANNULAR PSORIASIS. 


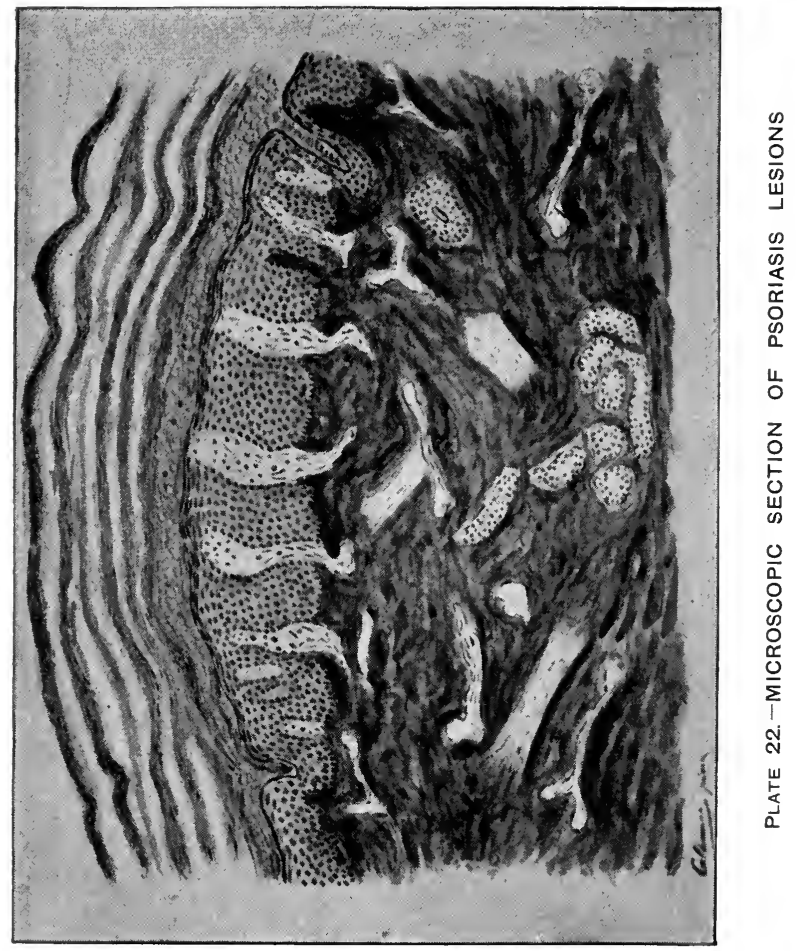


with a gradually narrowing border; and as the border itself in turn disappears at different points, segments of varying length remain, which, with similar relics of other patches, form wavy lines, festoons, and sometimes tracery of the most fantastic pattern. On the trunk it not infrequently happens that as involution goes on at the centre, the patch continues to spread at the edge; the spectacle is thus presented of a circle steadily increasing in diameter, the eircumference being formed by a border which, while constantly advaneing, never gains in width. To this condition the term "lepra" was formerly applied; but, apart from the possible confusion with the more formidable disease designated by that name, there is no advantage in using a special term to denote what is merely an accidental and evanescent appearance.

The lesions of psoriasis disappear completely, leaving. behind only some redness, which soon dies away. In cases in which the process has persisted for a long time, a deep-brown stain often remains; pigmentation is particularly likely to occur when arsenic has been freely used in the treatment. In rare instances superficial atrophy may mark the site of the patches.

Psoriasis is nearly always symmetrical in its distribution. It particularly affects parts where the skin is thick and frequently stretched, and where it is exposed to friction by the clothes, etc. Its favourite and almost invariable starting-points are the tips of the elbows and the fronts of the knees; it shows a special predilection for the extensor surfaces of the limbs. Next to these comes the hairy scalp; then the trunk, especially over the lumbar region. The face is rarely attacked, except in young people and in inveterate cases; the palms of the hands and soles of the feet still more seldom. As regards the proportion of cases in which these various parts are severally or jointly invaded, some idea may be 
gained from the following statistics given by Nielsen $:^{1}$ In a series of 862 cases of psoriasis the trunk, the extremities, and the head were all affected in 489 ; the trunk and extremities in 197; the extremities alone in 113 ; the extremities and the head in 53 ; the head alone in 5; the trunk and the head in 4 ; the trunk alone in 1. These figures may be taken as representing the area of territory invaded by the disease when it is of sufficient severity to induce the patient to seek medical advice; doubtless, however, there are very many cases that never come under treatment, and in a considerable proportion of these the disease is probably confined to the limbs and the scalp.

The lesions of psoriasis are more or less modified in appearance by the anatomical structure or other peculiarities of the regions which are the seat of them. On the scalp they are generally met with as scurfy patches, somewhat yellow or even brown in colour, the red ground of the hyperæmic base showing here and there, especially about the margin of the hair; it often extends on to the forehead, which it seems to bind with a narrow circlet. Sometimes the scales are piled on the scalp in thick, firm masses like dry mortar, in which the hair is embedded. The disease does not usually interfere with the growth of the hair; it is only in cases of exceptional severity that it causes baldness. Psoriasis seldom attacks the hairy parts of the face.

On the scrotum the skin is often red, swollen, indurated, and fissured, a thin secretion sometimes oozing from the cracks. Psoriasis is said to have been observed on the mucous membrane of the genital passages, and Fordyce has seen it on the lower lip; but manifestations of the disease on mucous membranes are, at any rate,

1 "Klinische und aetiologische Untersuchungen über Psoriasis," Sonder-Abdruck aus Monats. f. prakt. Derm., Bd, xv., Nos, 7 and 8. 
excessively rare. On the palms and soles the scales are usually heaped up into thick masses, which preserve the characteristic sharply defined border; the lesions in these situations show not a little disposition to crack. On the nails the disease is sometimes situated in the matrix, in which case the nail becomes dull and transversely furrowed. As the scales are more and more heaped up underneath it, the nail splits and is pushed out of its bed. In other cases psoriasis of the nails shows itself as a discoloration about the free border; this gradually extends downwards to the root, the nail becomes thickened, but there is no soreness of the matrix. Other modifications of the appearances characteristic of psoriasis may be produced by causes of various kinds. Thus, previous treatment may have removed the scaliness, or the same result, together with other lesions, may be produced by scratching. It is to be noted also that when the process is very acute in character the scales are often shed very rapidly, and there may be nothing to be seen but a red, inflamed surface.

Subjective symptoms.-These are seldom very pronounced. Itching is neither so constant nor so prominent as it is in eczema; indeed, in many cases there is little or no irritation. No rule can, however, be laid down on this point, as patients vary greatly in their sensitiveness to itching; all that can be said definitely is that in the acute forms of psoriasis itching is generally more marked than in cases of the ordinary type. In the more chronic forms, especially in patches about the elbows and knees, the itching is so often entirely absent that the disease may exist for a long time without the patient paying any attention to it. There is seldom any pain in chronic cases, except when the skin is fissured; this is especially apt to occur over the "bathing-drawers area," where the skin sometimes 
cracks every time the patient sits down. In very acute cases the skin may be the seat of pain from tension with heat and tenderness. In a certain proportion of cases of psoriasis there are, according to some French writers -notably Bourdillon ${ }^{1}$ and Besnier ${ }^{2}$-pains about the joints resembling those of osteo-arthritis. Besnier gave the proportion of cases in which this complication occurred in his experience as 5 per cent. In the first edition of this book it was stated that the author had not seen any clinical evidence of a relationship between psoriasis and osteo-arthritis. Further experience makes it necessary for him to correct that statement. In the last few years he has seen several cases in which joint-affection existed concurrently with psoriasis. Instances of the like association have been reported by Duckworth and Stephen Mackenzie. That the association is not common, however, is sufficiently proved by the experience of Brooke, who states that in some hundreds of cases of psoriasis he has hardly met with a single one in which joint-disease existed, although he has been on the look out for it.

Clinical course.-The course of the disease is essentially chronic, but it is subject to sudden exacerbations, during which it spreads over large areas. These exacerbations sometimes appear to be due to the influence of a particular diet, change of climate, or mental shock; in other cases they cannot be attributed to any definite cause. It is impossible to predict when or in what circumstances such an outburst may occur; some patients are attacked regularly once or twice a year, others at longer or shorter intervals. The disease, if left to itself, may last for months or even years, with intermissions of variable duration, during which it may entirely disappear. More

1 "Psoriasis et Arthropathies." Thèse de Paris, 1888.

2 French translation of Kaposi's "Maladies de la Peau," 2nd edition, vol. i., p. 553 et seq. 
frequently, however, patches remain on the elbows and knees in a state of inactivity until a fresh exacerbation occurs. Although a very large part of the cutaneous surface may be attacked, psoriasis is never absolutely universal. However completely the affection may disappear, recurrence is merely a question of time. Cheloid, warts, and even carcinoma may develop on the site of the lesions, or the disease may become transformed into pitvriasis rubra. The affection has generally little or no effect on the general health; Hebra considered that a certain standard of health is necessary for its development, and in fact patients have generally a robust appearance, their complexion being particularly clear and ruddy. In further confirmation of this it is to be remarked that if the general health be in any degree impaired the psoriasis tends proportionately to subside. Thus, during a severe attack of fever the patches often. fade, breaking out again, however, as convalescence is established. During pregnancy the disease usually disappears, but may recur after the birth of the child.

Psoriasis in its clinical manifestations follows certain types, which may be briefly summarized as follows: There is a simple, uncomplicated psoriasis with occasional outbursts of activity seen in young persons, without any sign of impaired health; there is an acute, rapidly spreading form, associated with headache, asthma, and other nervous symptoms; there is the form which occurs in connection with osteo-arthritis or acute gout, associated with severe constitutional disturbance; there is a psoriasis which tends to become transformed into pityriasis rubra. Lastly, there is a simple but a typical form which resembles seborrhœa.

Pathology.-There has been much debate as to the pathology of psoriasis, some considering the process to be essentially inflammatory, others a hyperplasia of the rete, others again a parakeratosis or anomaly of cornifi- 
cation. A contribution to our knowledge of the nature of psoriasis has been made by W. J. Munro,1 whose researches were carried out in Sabouraud's laboratory. As the result of the examination of 1,500 sections of psoriasis furnished by six biopsies of nascent lesions in different patients before any treatment had been employed, and of the study of many hundreds of psoriatic scales, recent and old, he came to the conclusion that the primary lesion is a tiny "dry " abscess at the surface of the horny layer. Immediately after the first stage two phenomena supervene-first, hypertrophy of the epidermic horny layer; secondly, the formation around the primary lesion of a number of similar ones. Munro defines the squama of psoriasis as a multitude of little dried abscesses included between sheets of exfoliated horny epidermis. He failed to find any microörganism in the lesions. He denies that psoriasis is " a vice of formation of the horny epidermis," and he holds that the hyperkeratosis is secondary to the primary lesion. Verrotti ${ }^{2}$ does not regard as the initial lesion the miliary abscesses described by Munro and Sabouraud. Among the other histological changes noted in psoriasis is the congestion of the vessels in the papillæ; the cells of the rete Malpighii undergo rapid multiplication and are at the same time much swollen (Plate 22). The stratum granulosum never forms; no granules of kerato-hyalin are formed. The swollen cells of the rete pass on, therefore, into an imperfectly cornified epithelium, constituting the distinctive scale of the disease. Blood cells frequently exude from the swollen papillary vessels, and become mingled with the rapidly dividing cells and overlying desquamating masses of cells. It has been shown by Robinson of New York and by Crocker that overgrowth downwards of the

${ }^{1}$ Brit. Journ. Derm., 1900, p. 63 et seq.

${ }^{2}$ Ann. de Derm. et de Syph., Aug. and Sept., 1902. 
interpapillary cones of the rete takes place, with œodema and exudation of leucocytes in the papillary layer. The epidermic horny layer and the papillary layer are immensely hypertrophied; the stratum lucidum disappears; no eleidin is to be seen, and the cornification of the epidermic cells either takes place not at all, or very imperfectly. According to Audry, ${ }^{1}$ the absence of eleïdin, together with the defective cornification of the epidermic cells, is the essential pathological feature of psoriasis. The characteristic silvery appearance of the scales is due to the entrance of air within and between them. The overgrowth downwards that has been referred to gives rise to an appearance which, on microscopic examination, is found somewhat to resemble that of epithelioma ; and in fact, in rare cases, the lesions of psoriasis undergo a malignant transformation.

Etiology.-Reluctantly I am compelled to record my agreement with Brooke's admission that "notwithstanding researches in every direction-social, statistical, chemical, microscopical, and bacteriological-the cause of psoriasis is still unknown." The disease is not common in early infancy, and it rarely begins after the age of 50 ; it is, on the whole, more frequent in youth than in later adult life. Statistics seem to show that men are somewhat more subject to it than women. Neither rank in life nor occupation has any influence in the production of the disease; but race and climate must be credited with a certain influence in connection with it. It is frequent among Jews, and rare among negroes. It is relatively common in northern latitudes, and very rare in tropical climes.

Psoriasis is to a certain extent hereditary, and Brocq ${ }^{2}$ says that it is not uncommon to see gout or some form of

1 Ann. de Derm. et de Syph., No. 4, April, 1893.

2 Op. cit., p. 702. 
neurosis replaced by psoriasis in a member of a family subject to one of the former complaints. Some dermatologists hold that gout is an important factor in a certain proportion of cases, but there appears to me to be no evidence to support this hypothesis, although gout is undoubtedly sometimes associated with psoriasis. Season seems to have a certain influence, not so much in causing the disease as in increasing its intensity: thus the subjects of psoriasis are, as a rule, more liable to exacerbations in the spring and the autumn than at other seasons ; recent cases, however, are often worse in the winter than at any other time. Polotebnoff has endeavoured to show that psoriasis is a neurosis of the skin; from an analysis of 67 cases he affirms that headache or other nervous disorder is present either in the patient himself or in other members of his family in a large majority of cases. ${ }^{1}$ It is certain that psoriasis often follows nervous shock, mental emotion, or depressing influences, such as insufficient nourishment, and in women childbirth or suckling. The neuropathic theory, however, rests on a very slight foundation of facts, and I am disposed to agree with Verrotti that the nervous disturbance does no more than determine the locality of the eruption.

Psoriasis also sometimes follows an abrasion or other injury of the skin, or even the irritation caused by the contact of clothes; in a case reported by Max Joseph ${ }^{2}$ it appeared to be due to exposure to sunlight; it is occasionally one of the sequelæ of vaccination, and it has been known to follow an attack of erysipelas or scarlet fever. There can be no doubt, however, that a special predisposition is required for the development of the disease, and the causes that have been enumerated. are probably only the immediate

${ }^{1}$ Ergänzungsheft d. Monats. f. prakt. Derm., 1891.

2 Derm. Centralbl., Sept., 1906, p. 358. 
determining factors which set the process in motion at a given time. Lang of Vienna attempted to show that psoriasis is a parasitic disease caused by a specific organism (Lepocolla repens), but this supposed fungus has since been demonstrated by Ries to be an artificial product. Destot, however, succeeded in producing the lesions of psoriasis on his own person by experimental inoculation with scales from a recent case of the disease; and at the International Medical Congress held at Copenhagen in 1884 Unna reported a case in which the disease appeared to have been communicated to three children by a nurse. A similar case came under the observation of Nielsen. Other cases in which there is prima-facie evidence of the transmission of psoriasis from one patient to another have been recorded by $M^{\prime}$ Call Anderson, Aubert, Augagneur, and others. Verrotti concludes that psoriasis is the result of an acid auto-intoxication, and that the activity of the psoriatic process is determined by the acidity of the blood and by the integrity of the renal functions. The skin, he holds, acts, in psoriasis, as a channel of elimination, supplementing or replacing the inadequate renal functions. In opposition to writers who have recorded observations tending to show that in psoriasis there is a diminution of the nitrogenous coefficient in the urine, Brocq and Ayrignac, as the result of experiments in which careful account was taken of the diet of the patients, maintain that there is no urinary modification which can be regarded as typical of psoriasis.

In an able review of the etiological theories that have been advanced, Pollitzer ${ }^{1}$ concludes (1) that while rheumatism, gout, neurosis, and heredity are not direct factors in the production of psoriasis, it can neither be denied nor affirmed that they may have some bearing on the obscure conditions of the system which render

1 Trans. Amer. Derm. Assoc., 1909, p. 122. 
-it more or less susceptible to this disease; (2) that psoriasis is "most probably" due to an external microbic infective agent; (3) that it is one member of a group of parakeratoses to which seborrhœa corporis and, in part, eczema seborrhoicum belong. Schamberg ${ }^{1}$ holds, with good reason, I think, that although the mode of growth and configuration of the patches, the occasional instances of apparent communication of the disease, and the results of Destot's inoculations are arguments in favour of the parasitic theory which are entitled to consideration, the general behaviour of the disease, the common experience of observers as to absence of contagion, and the almost universal failure of inoculation experiments constitute strong evidence against the theory.

Diagnosis.-In well-marked cases the diagnosis presents no difficulty. The patches with sharply defined border, covered with imbricated silvery scales; the bright-red points on a hyperæmic surface, bleeding easily when touched, which are exposed by removal of the scales; the absence of exudation at any period; the symmetrical distribution of the lesions and their predilection for the extensor surfaces of limbs, together with the unimpaired health and robust appearance of the patient, make up a clinical picture which can hardly be misinterpreted. In doubtful cases the fact of the eruption having first appeared on the elbows and knees is almost conclusive; but if this element in the history be wanting, it is sometimes impossible to be sure of the nature of the disease. The affections that are most likely to be confounded with psoriasis are eczema, lichen planus, lupus erythematosus, pityriasis rubra, and syphilis in the secondary and tertiary stages.

Eczema is distinguished from psoriasis (a) by its distribution-it prefers the flexures of joints; (b) by

1 Trans. Amer. Derm. Assoc., 1909, p. 139. 
the initial lesion-it generally begins in vesicles grouped on an inflamed base; $(c)$ by the serous discharge which is its characteristic feature; $(d)$ by the ill-defined margin of the patches, diseased fading insensibly into healthy skin; $(e)$ by the crusts, which are as characteristic of eczema as scales are of psoriasis. Less distinctive, but still important, features of eczema as compared with psoriasis are itching, which is at once more violent and more constant, and the muddy complexion, which often forms a marked contrast to the ruddy cheek of the subject of psoriasis. On the scalp, seborrhœic eczema almost always covers the whole surface, and often spreads on to the face and behind the ears to the neck; psoriasis, on the other hand, generally occurs in patches and ends abruptly at, or very slightly beyond, the margin of the hair. Another point of difference is that while seborrhœic eczema, as a rule, spreads downwards from the head, psoriasis almost invariably spreads upwards from its favourite situations. In certain very chronic forms of eczema, when there are only a few scattered patches with no history of an eruption on the elbows or knees, or of "weeping," the diagnosis may be all but impossible. Even in such cases, however, the intensity of the redness, if the affected surface be at the same time dry, may be some guide. It may be well to recall here that by gentle scratching the characteristic scales of psoriasis can often be brought into view when previously invisible.

Lichen planus is not likely to be confounded with psoriasis, except in the papular stage, when it sometimes presents an appearance resembling that described as psoriasis guttata. The former is, however, distinguished from the latter $(a)$ by its preference for the flexor aspects of the wrists and knees; $(b)$ by its characteristic shining smooth papules and the absence of scales; $(c)$ by the bluish-red tint of its ground as 
contrasted with the bright red of psoriasis; $(d)$ by its mode of extension, a lichen patch being formed by the aggregation of many papules, while psoriasis spreads at the edge. In doubtful cases careful search should be made over the whole body for the typical lesions of either disease; a single characteristic patch will settle the question.

Lupus erythematosus usually affects the cheeks, a part generally spared by psoriasis. In the former, moreover, scales are not abundant; the edge of the patch is more raised than in psoriasis; and the plugs in the orifices of the sebaceous ducts form a very distinctive feature. Further, in lupus erythematosus there may be scarring in the patch and atrophy of the ears.

Pityriasis rubra is differentiated from psoriasis (a) by its rapid development; (b) by the fact that it is almost always universal, psoriasis hardly ever being so ; (c) by its growth by peripheral extension instead of by the accretion of new papules; $(d)$ by its thin, waferlike scales, through which the reddened skin shows distinctly. For the diagnosis of psoriasis from pityriasis rosea, see p. 350 .

As regards syphilides, the only trustworthy means of distinguishing them from psoriasis lies in the his. tory, in the simultaneous presence of other lesions of skin, glands, or mucous membrane, and in concomitant cachexia. Syphilis has been justly called the "great imitator," and there is perhaps no dermatologist who has not been led by it into errors of diagnosis ; this can be avoided only by basing one's judgment on a comprehensive view of all the details of each case, or by applying the Wassermann test.

In the group of ill-defined affections styled by Brocq parapsoriasis (p. 336), although the desquamation presents a general resemblance to that of psoriasis, there 
is very little itching, or none at all; the lesions are pinkish in colour, are sluggish in evolution, are distributed in well-defined patches, and are markedly irresponsive to treatment.

Prognosis.-The prospect is favourable as far as any particular attack is concerned. By appropriate treatment the lesions can almost always be made to disappear for a time. Recurrence, however, after a longer or shorter period of complete or comparative freedom from the manifestations of the disease, is the general rule.

Treatment. Hygienic.-Attention must be paid to the clothing so as to avoid irritation of the skin, interference with perspiration, and chilling of the surface. Bulkley $\mathbf{1}$ has repeatedly seen such chilling followed by an outburst of the disease in a previously healthy person, and also by returns of the eruption. A warm and equable climate has often a most beneficial effect on psoriasis. Schutz has reported two very severe cases of generalized psoriasis in which spontaneous cure always took place on the patients exchanging their ordinary place of residence for a higher altitude, and Duhring cites a case in which a physician who had suffered from a severe form of inveterate psoriasis for many years found that it largely disappeared during a fortnight's voyage undertaken to put himself under special treatment in Europe. I have known several similar cases. Sea-bathing is often markedly beneficial, doubtless from its tonic action on the general system. Some cases that have proved refractory to other treatment quickly get well after a course of sea-bathing.

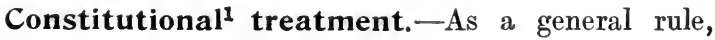

1 "Clinical Study and Analysis of One Thousand Cases of Psoriasis." Reprinted from the Maryland Medical Journal, Sept. 19, 1891, p. 14. 
no internal medication should be employed, except in response to a definite indication. Of internal remedies, arsenic is on the whole the most effective in the majority of cases; it is not, however, well borne by all persons, nor by the same person at different times. It is contra-indicated when hyperæmia is very marked; in such cases it only does harm, intensifying the process, aggravating the itching, and actually causing extension of the disease. In chronic cases, however, arsenic is often undoubtedly of the greatest service. It may be given in the form of Fowler's solution, freely diluted, beginning with a dose of miii-iv thrice daily after meals, gradually increased up to $m_{x}$ if no signs of intolerance manifest themselves. Kaposi gave arsenic in the form of Asiatic pills, each containing $\frac{1}{12}$ gr. of arsenious acid. He began with one pill thrice daily, gradually increasing the number to ten or twelve in the day, and continuing the administration, if necessary, for several months. If, after 500 or 600 pills had been taken, no decided improvement was observable, he considered that the drug had failed. It need hardly be added that during the administration of arsenic the effect should be carefully watched; gastric or intestinal irritation should be subdued by opium. As already said, when arsenic has been freely given, and especially if its administration has been long continued, deep-brown pigmentation is left, not only at the site of the patches, but over a more or less extensive area of skin. A peculiar thickening of the epidermis of the soles and palms is another occasional result of the prolonged use of arsenic. Hutchinson and others have described the development of corn-like projections, which may occasionally become the seat of malignant disease, as resulting from the same cause in very exceptional cases. Arsenic is of no value for the prevention of recurrence.

In acute forms of psoriasis, and in cases in which the 
subjective symptoms are very pronounced, I find antimony most useful. I give it in the form of vinum antimoniale, $\mathrm{mv}$ to $\mathrm{mx}$, thrice daily. Phosphorus is also useful under similar conditions. Radcliffe-Crocker found salicylate of sodium of great value, especially in the period of active development and in hyperæmic cases ; it is useful in all forms, except perhaps in old chronic patches. If the drug causes dyspepsia, of course it must not be continued. If symptoms of nervous disorder be present, nerve sedatives, such as bromide of potassium, bromide of sodium, or hydrobromate of quinine, may be useful; these and similar drugs are recommended as part of the regular treatment of psoriasis by those who hold that the disease is a neurosis. Crocker found salicin very valuable in acute and subacute cases of psoriasis ${ }^{2}$ he began with gr. $x v$ a day. In chronic cases he found the drug of little use. Of the treatment of psoriasis by large doses of iodide of potassium, as recommended by Haslund, I cannot speak from personal observation. Biniodide of mercury, in small doses given over a long period, is often of use in old-standing, recalcitrant cases. Unna has found naphthalan to be of the greatest service in cases that have been irritated by unseasonable treatment. Feeding with extract of thyroid gland, which was, on its first introduction, vaunted as almost a specific, has not answered the expectations which were formed of it; it sometimes does good, but more often does harm. Even when it is useful, the beneficial effects are not permanent. My own experience in this matter is confirmed by that of many other dermatologists. In a case of extensive psoriasis reported by Galloway the lesions disappeared under inoculation with vaccine prepared from the

${ }^{2}$ See report of a discussion on the treatment of psoriasis at the annual meeting of the British Medical Association, 1902, Brit. Med. Journ., Oct. 25, 1902. 
Staphylococcus aureus grown from them, but some months later there was recurrence, and under inoculation the patient grew steadily worse, and it was necessary to fall back upon ordinary treatment (soap baths, salicylic acid, and chrysarobin inunctions), in response to which the eruption cleared up until only slight traces remained. 1

In cases of psoriasis associated with osteo-arthritis, arsenic is useful. It may be given in Fowler's solution in gradually increasing doses. In cases of this nature I have seen good results follow the use of an exclusively meat diet washed down by copious draughts of hot water. As to the mode of action of this treatment, I can only suggest that the simplicity of the diet promotes the functional efficiency of the digestive apparatus, while the large quantity of water increases the action of the kidneys and bowels, and so brings about a complete elimination of waste products.

Passing next to the local treatment, the first thing to be done is to remove all scales, so that remedies may be applied directly to the affected part. For this purpose the free application of hot water and soft soap, more or less prolonged immersion in tepid water or an alkaline bath, or inunction with oil or vaseline, will be necessary, in order to soften and loosen the scales. Each patch must be dealt with separately, and the process of clearing the surface must be thoroughly carried out. A useful practical rule is that small patches should be dealt with by strong applications, while large areas require careful handling. A solution of salicylic acid in spirit, of the strength of 6 per cent., well rubbed in, will be found effectual in removing the scales in old patches. When the scales have been entirely got rid of, the next step is to attack the seat of disease with antimicrobic remedies. Although, as already stated,

${ }_{1}$ Brit. Journ. Derm., April and Dec., 1907. 
the theory that psoriasis is of microbic origin rests on a very slender foundation, it is a clinical fact that substances which have the property of checking the development of microbes are more useful than any other applica. tions. The pa icular remedy required in any given case must be judged of by the degree of intensity of the process. As a general rule, it may be laid down that, as in eczema, soothing applications are indicated in acute and stimulating remedies in chronic forms. If hyperæmia is very marked, the surface should be covered with strips of linen steeped in calamine lotion, or smeared with olive oil or cold cream. Alkaline baths (bicarbonate of soda $\xi \mathrm{v}$-vi in 30 gallons of water at a temperature of about $100^{\circ} \mathrm{F}$.) often give great relief. In less acute conditions mildly stimulating remedies should be employed. Mercurial ointments of moderate strength, the best being ung. hydrarg. ammon., will be found serviceable, but they should be applied only to a limited surface at a time. Tar may be applied in the form of cade oil or oleum rusci or creoline ointment, or liquor picis carbonis ( $m \times x \mathrm{x}$ to $\overline{\mathrm{i}}$ of water). Anthrasol, a colourless tar ( $3 \mathrm{i}$ to $\xi_{\mathrm{i}}$ of ung. petrolei), is at once unobjectionable and efficient. Resorcin (gr. $\mathrm{x}-\mathrm{xx}$ to $弓 \mathrm{i}$ of $\operatorname{lard}$ ) also is a very useful application.

The most rapidly acting and most efficient of all local applications, however, is chrysarobin. It may be used in the form of ointment (gr. $x v-3 i$ to $3 \mathrm{i}$ ). Unfortunately, there are several disadvantages attending the use of this substance which considerably limit its practical usefulness. If employed without proper precautions, it dyes the skin, the hair, and the nails bright yellow; it discolours linen in the same way, and the stain is not removed by washing, but is changed to purplish brown. A more serious drawback is the irritating effect of the drug on the healthy skin adjoining 
the diseased area; it may set up erythema of an acutely inflammatory form, with itching, swelling, etc. For this reason it is chiefly suitable for the limbs and parts of the trunk away from the neck and genitals; it must never be used on the face or head. In some cases chrysarobin may in this way determine the transformation of psoriasis into pityriasis rubra. The drawbacks attending the use of this powerful agent may be avoided by combining it with traumaticin, as suggested by Auspitz. This is made by dissolving $3 \mathrm{i}$ of pure guttapercha in $3 \mathrm{x}$ of chloroform; to this $3 \mathrm{i}$ of chrysarobin is added. This preparation is, after the removal of the scales, painted over the affected surface, where it forms a thin varnish, which should be renewed every two or three days. Chrysarobin should never be used in cases in which marked hyperæmia is present. It may here be pointed out that Walter G. Smith ${ }^{\mathbf{1}}$ has shown by experiment that chrysophanic acid is not an efficient substitute for chrysarobin in the treatment of psoriasis. In the case described by Max Joseph (p. 324) the lesions healed under application of Dreuw's ointment (acid. salicyl. 10, olei rusci, chrysarobini, āā 20, sapon. virid., vaselin flavi, āā 25), combined with the administration of arsenic internally. Pyrogallic acid, used in the form of an ointment (gr. $\mathrm{x}-\mathrm{xxx}$ to $\mathrm{z}_{\mathrm{i}}$ ), is also often useful, but it must be applied only to a limited area at a time, as toxic effects may be produced by its absorption. Eugallol, a derivative of pyrogallic acid, is preferable, but must be used cautiously, in strengths varying between gr. $x$ and gr. $x x x$ to the ounce. Allan Jamieson speaks highly of pyraloxin, which is pyrogallic acid modified by exposure to a current of air in presence of ammonia vapour. Achille Brenda ${ }^{2}$ records two cases in which patients suffering from psoriasis

1 Brit. Journ. Derm., vol. viii., July, 1896.

${ }^{2}$ Giorn. Ital. d. Mal. Ven. e d. Pelle, 1897, iii. 
had succeeded in causing the disappearance of patches by the application of caustics. The agent used in one case was nitric acid, in the other a solution of corrosive sublimate in alcohol $(1 \cdot 5)$. Brenda treated cases of his own with nitrate of silver and acetic acid applied on cotton-wool. The treatment seemed to be successful, but in one or two cases the application of nitrate of silver was followed by a cheloid condition of the scar. The same author reports a case in which massage of the skin carried out every day for a month cured a case which had resisted other remedies. There is a consensus of opinion that radiotherapy causes rapid removal of the lesions, but it appears to be no more successful than the application of ointments in preventing recurrence. The rays should not be applied to the scrotum because of their possible effects upon the testicles. Radium and the high-frequency current are beneficial in the same sense as the $\mathrm{X}$-rays.

Sulphur baths are often beneficial in chronic cases. The first, and perhaps the chief, effect produced is the softening and removal of the scales. One must then judge by the amount of hyperæmia present whether weak or strong local applications are required; if the latter are thought to be indicated, they must at first be used well diluted. The action of sulphur baths on psoriasis is no doubt mainly mechanical by removing the scales, but it is possible also that some further therapeutic effect is produced by the microbicidal action of the sulphur. The sulphur waters of Harrogate, Strathpeffer, Schinznach, Luchon, Aix-les-Bains, and Aachen are especially indicated in cases of chronic psoriasis. The waters of La Bourboule and Levico are also sometimes beneficial, both applied externally and taken internally, owing to the arsenic which they contain. Even the "indifferent" waters of Bath and Buxtor. often succeed when others have failed. It is important 
to bear in mind that in chronic cases the patient must be urged to persevere in the treatment, no matter what drug is used.

In the type of psoriasis in which there is a tendency to the development of pityriasis rubra, chrysarobin and all other local stimulating applications should be at once discontinued. The patient should, if necessary, be kept in bed, and the affected parts should be covered with carbolic oil or a weak lead preparation.

\section{Parapsoriasis}

Under this provisional name Brocq has grouped a number of conditions which have a superficial resemblance to psoriasis, but represent a more serious pathological process and are markedly persistent and refractory to treatment. Upwards of 40 cases have been reported.

Symptoms.-The eruption begins as small red papules, which, at first round, become flattened and less vivid in colour; they are usually indifferent in distribution and covered with thick, adherent epidermal scales, the removal of which discloses a bright-red, moist surface. The scalp is usually exempt, but in a case described by Rille, ${ }^{1}$ and in another reported by $\mathrm{A}$. Ravogli, ${ }^{2}$ this region was, badly affected. The palms of the hands, too, are usually spared, but in Ravogli's case the nails both of the fingers and toes were affected. Three types of parapsoriasis have been distinguished. In the first, parapsoriasis guttata (parapsoriasis en gouttes), the eruption consists of scattered pink or brownish-red patches, but slightly infiltrated, and covered with dry, adherent scabs. In the second type, lichenoid parapsoriasis, the eruption is of a more papular and less scaly character, with rather more infiltration; some-

1 Verhandl. d. Congr.f. inn. Med., xxi. 568.

2 Trans. Amer. Derm. Assoc., 1909, p. 70. 
times the lesions are depressed in the centre, they have a tendency to form semicircles, and they may be either grouped or scattered. In the third type, parapsoriasis en plaques, the lesions take the form of round or oval, well-defined, yellowish-red patches; there is little or no scaliness, nor is there infiltration. The first and second types correspond generally with the condition which has been designated parakeratosis variegata (p. 200); the third with that which Brocq at first styled érythrodermie pityriasique en plaques disseminées and with Radcliffe-Crocker's xantho-erythrodermia perstans (p. 203).

Pathology.-In psoriasis the pathological process is progressive, leading to epidermal hyperplasia, whereas in parapsoriasis, according to W. T. Corlett and Oscar T. Schultz, ${ }^{1}$ who have given an exhaustive account of the histology of three cases under their observation, the process is regressive, leading to intense cedema with resulting degeneration of epidermal cells and disturbance of cornification. These observers believe, further, that there is involvement of the deeper vessels of the corium, which is the cause of all the changes in the papillæ and in the epidermis. This is a point which calls for further investigation. Histologically, there are many resemblances between the various forms of the affection-marked cedema of the epidermis and of the papillæ, absence of the stratum lucidum, pronounced dilatation of the papillary capillaries and some infiltration by small round cells. In certain cases of the third type the stratum corneum contained no nuclei, and the stratum granulosum was more or less incomplete. In some cases of the other types the stratum corneum has been found nucleated. Other cases of these types appear to occupy an intermediate position so far as this point is concerned.

1 Trans. Amer. Derm. Assoc., 1908, p. 51. 
Etiology - In none of the cases reported has a definite cause been traced. There appears to be no ground for the theory that the disease is parasitic. No parasitic elements have been discovered, the affection is not contagious, it is rare, and it is unaffected by anti-parasitic remedies. Colcott Fox, MacLeod, and others have suggested that it is a vaso-motor neurosis, the destruction of the epidermic cells and the consequent parakeratosis being the result of dilatation of the blood-vessels, the œdematous condition of the tissues, and the small-cell infiltration. In two cases of Brocq's the condition followed in the train of emotional disturbance. It has been associated in some cases with syphilis and with tuberculosis, but there is no evidence that the connection is etiological, and the results of treatment lend no support to that suggestion.

Diagnosis.-The signs which differentiate parapsoriasis from psoriasis have already been mentioned (p. 328). It is not likely to be confused with any other condition.

Treatment.-I have known some improvement to follow the use of arsenic. Darier reports that injections of this drug are occasionally useful, and he has had a similar experience with injections of mercury. Local treatment should be always gentle. I have found a weak resorcin or salicylic-acid ointment useful. The constitutional symptoms require careful attention according to their nature. 


\section{CHAPTER XIV}

\section{PITYRIASIS}

THE term "pityriasis" indicates not a disease, but a symptom. It has been used to denote a variety of conditions, differing widely in their origin, course, and termination, but having one objective feature in common, namely, branny desquamation. The scales are small, easily detached, and not heaped up in layers, as is the case in psoriasis. Pityriasis simplex, whether on the head, the face, or the trunk, is now recognized to be identical with dry seborrhœa (p. 592); pityriasis versicolor is a parasitic disease, also called tinea versicolor (p. 403); pityriasis rubra, pityriasis rubra pilaris, and pityriasis rosea are inflammatory processes of which more or less abundant exfoliation of the epithelium is an essential element. Pityriasis rubra and pityriasis rubra pilaris constitute a class for which the general appellation of "exfoliative dermatitis" would be appropriate. In this category should probably be placed those cases in which the epidermis is periodically "cast," wholly or in part, like a serpent's slough. The most remarkable instance is that reported by Sligh. ${ }^{1}$ The patient, a man aged 36 , is said to have been taken ill with almost unfailing regularity every year since infancy. He complains of "bone-ache, weakness, nervousness, and inability to eat" ; his temperature rises $\left(101^{\circ} \mathrm{F}\right.$.), and he vomits. "Within a few days he has shed his skin from the entire surface of his body, including the finger- and

1 Internat. Med. Mag., June, 1893. 
toe-nails. The new skin is as soft and tender as a newborn babe's," but rapidly becomes sound, and in four or five days the man can resume work.

The affection already referred to under the name of erythema scarlatiniforme (p. 98) is a form of exfoliative dermatitis; clinically, however, it is more closely allied to simple inflammation of the skin than to the more formidable disease that is about to be described.

\section{Pityriasis Rubra (Dermatitis Exfoliativa)}

Pityriasis rubra (Plate 23) is an inflammatory affection of the skin marked by universal redness of the surface without infiltration or thickening, but accompanied by profuse desquamation. This varies in its character in different parts of the body; for example, it is branny on the head, on the trunk it consists of larger flakes, while from the hands and feet the epithelium is shed in huge scales. The disease used to be considered an affection sui generis till Buchanan Baxter ${ }^{1}$ showed that, while it may occur as an independent disease, it often follows other skin affections.

Symptoms. - The onset of pityriasis rubra is usually more or less sudden, and is accompanied by some malaise, though not of a very marked character. The eruption is symmetrical in distribution, and may select any part of the skin for its point of attack; most frequently, however, it begins on the limbs and chest. Red patches appear, which spread rapidly at the edge and coalesce with other patches so as to involve the whole surface of the skin literally from head to foot. The affected skin is uniformly bright scarlet in hue, but quickly becomes covered with thin wafer-like scales which overlap each other like slates on a roof, but are never fused together into crusts. There is very seldom any discharge on

${ }^{1}$ Brit. Med. Journ., 1879. 


$$
1
$$



e surface of the skin, and when exudation does take ace the fluid is thin and watery, like sweat (of which, inzed, it chiefly consists), and does not stiffen linen. The ales are easily detached, and when they separate the in underneath is seen to be intensely red. Although ere may be considerable tension, there are usually fissures. As a rule, itching is absent; this, howrer, depends on the temperament of the patient, and some cases itching is very pronounced. Over the hole surface of the skin an unpleasant feeling of stiffess, heat, and tenderness is often experienced. In my xperience a peculiar feature of the disease is that, in ite of the great redness and desquamation, there is no ickening of the skin; on the contrary, there is slight at distinct thinning of the integument, as can be felt a pinching it between the fingers. The disease usually reads with great rapidity, the whole body being inaded in a few days; sometimes, however, it remains onfined to certain regions, and never becomes uniersal.

Pityriasis rubra may develop as an entirely indeendent affection, or, as already said, it may be a quel of some other disease of the skin ; in other words, xfoliative dermatitis may be either primary or secondry. ${ }^{1}$ In the primary form the eruption first appears s a vivid red blush, which spreads so rapidly that it ecomes universal in a few hours. There is no infiltraon or thickening of the skin. Desquamation is most bundant, the whole skin seeming to be shed in some ases. The affection is extremely rare; I have seen nly three or four cases. The secondary form may start

${ }^{1}$ In a series of 21 cases published by Stephen Mackenzie Brit. Journ. Derm., July, 1889), 11 were primary and 10 condary in origin. My own experience, however, is that the tter form is far more frequent than the former. See also alloway, Brit. Journ. Derm., 1898, p. 448. 
from erythema multiforme, especially when the lesionssuch as erythema iris-are definite and characteristic. Gradually one sees the erythematous elements subside or disappear, their places being taken by patches of redness, which spread over the whole body and assume the aspect of pityriasis rubra, as described above. Again, one meets with cases presenting all the characters of typical eczema, with large discharging surfaces and other distinctive lesions, in which a sudden change comes over the face of the disease, the whole skin becoming crimson sometimes in a single night, and all the eczematous appearances fading away, or being swallowed up in pityriasis rubra. Psoriasis, again, with its characteristic lesions in typical positions, may suddenly lose all its distinctive features and become transformed into pityriasis rubra. Lichen planus and dermatitis herpetiformis may undergo a precisely similar metamorphosis. Examples of all these transformations have come under my own notice. The event is probably more frequent after psoriasis than any other affection. Sometimes the transformation is attended with considerable constitutional disturb. ance, but this is by no means invariable. However it may begin, pityriasis rubra varies in duration and intensity, sometimes not affecting the general health to any appreciable extent, and passing away in a few days or weeks; sometimes lasting for years, and leading to death from exhaustion or some intercurrent disease. In the cases in which recovery takes place, relapse is frequent. On the other hand, cases in which the disease has lasted for many years may take a sudden turn for the better and end in recovery; the patient is, however, always liable to subsequent attacks. In primary pityriasis rubra the health is not, as a rule, affected so early as in the secondary form of the affection. The disease is rare in children, but it is much more severe 
and fatal in them than in adults. The mental faculties are sometimes disordered in case of pityriasis rubra.

A special form of the disease affecting new-born infants has been described by Ritter of Prague, ${ }^{1}$ Kaposi, and other observers, and is variously known as Ritter's disease, as dermatitis exfoliativa neonatorum (or infantum), and as keratolysis neonatorum; but I have never myself seen a case answering to their descriptions, nor, so far as I know, has any such case been reported in England. The affection begins within the first fortnight-seldom beyond the first month-of infancy. The lesions are those of pityriasis rubra, sometimes with the flaccid bullæ of pemphigus foliaceus, with crusts and small fissures about the corners of the mouth, the openings of the nostrils, the commissures of the eyelids, and the anus. There is no constitutional disturbance, but in one-half of the cases the child dies of marasmus.

What appears to be a contagious form of pityriasis rubra has been described by Savill, ${ }^{2} 163$ cases having occurred in the Paddington Poor-Law Infirmary between July and October, 1891. The eruption appeared in the form of a thickly set papular rash, with general congestion and thickening of the skin. Vesicles occasionally formed. There were independent patches in different parts of the body, and in some cases the whole skin became crimson, inflamed, and painful. A sickening odour was perceptible. The epidermis was soon shed in small dry scales, or in large sheets, from the hands and feet. There was great constitutional disturbance, and 30 of the sufferers died from increasing weakness and coma. The disease ran a more or less definite course lasting from seven to eight weeks. The affection was clearly contagious, though its epidemic prevalence is

1 Vierteljahr. f. Derm. u. Syph., 1879, Heft 1.

${ }^{2}$ Brit. Journ. Derm., Feb. and March. 1892. 
difficult to account for. Similar outbreaks on a smaller scale have been recorded.

Pathology.-The pathological process is one of inflammation of the skin, at first superficial, later extending through the whole depth of the integument. The changes found on microscopic examination are simply those characteristic of chronic inflammation, varying in degree according to the length of time the process has lasted. They are present in all the layers of the integument. According to Petrini de Galatz, ${ }^{1}$ the essential lesion is in the papillæ. Besides hyperplasia of cells, there is in the earlier stages of the process a proliferation of round cells in the interior of the papillæ, around the vessels and in their walls. A similar proliferation is seen along the vessels of the cutis. This leads to sclerosis of the papillæ, and especially of their vessels, and in time to sclerosis and obliteration of the whole vascular apparatus of the skin, with granular and fatty degeneration of the neighbouring tissues. The glands atrophy, the sebaceous glands apparently becoming transformed into fat. The redness of the skin is due to the stasis of the blood in the vessels, and the ceaseless shedding of the cuticle is an indication of the degree to which the nutrition of the skin is impaired.

Etiology.-The causation of pityriasis rubra is very obscure. The primary form often follows a chill, but in many cases no cause can be discovered. The male sex shows a somewhat greater proclivity than the female, and the disease is more common in middle life than at any other period, though no age is exempt. In the secondary form the cause of the transformation is unknown. In some cases it appears to follow an injury to the skin. Thus, I have known pityriasis rubra develop suddenly after a burn, and spread over the whole body in a

${ }^{1}$ Congrès Internat. de Derm. et de Syph. tenu à Paris en 1889 ; Comptes-Rendus, Paris, 1890, p. 48. 
single night. In other eases it seems to be the result of the remedies used in the treatment of the pre-existing skin affection. In a healthy girl under my own care for chronic eczema, treatment with chrysarobin was followed by diffuse redness of the skin, which gradually assumed all the characters of pityriasis rubra. The disease has also been known to follow the use of mercury and other drugs. Crocker maintained that there was a close relationship between rheumatism (especially the acute form) and gout and pityriasis rubra, such an association having existed in 11 out of 18 cases which he had the opportunity of observing. Jadassohn has found tuberculosis (enlargement of the superficial lymphatic glands, and occasionally tubercle of the internal organs) associated with pityriasis rubra in a certain proportion of cases. Even if it be admitted, however, that rheumatism and tuberculosis may be predisposing causes, we are still in the dark as to the factors which determine the onset of the disease. It is possible that the absorption of poisonous products from the previously existing skin lesions might explain the development of secondary exfoliative dermatitis, but I am more inclined to believe that it will be found to be a result of parasitic invasion. Such evidence as we have on this point, however, is scanty and doubtful. Risien Russell ${ }^{1}$ found a diplococcus in the serum and the blood and also in the skin in some of Savill's cases. Petrini de Galatz, however, failed to find microörganisms either in the scales or in the blood. ${ }^{2}$

Diagnosis.-Pityriasis rubra is distinguished from other affections of the skin by (1) the vivid redness of the eruption, (2) its rapidity of diffusion, (3) its universality. (4) the constant and profuse desqua. - mation, and the characteristic papery scales and sheets,

${ }^{1}$ Brit. Journ. Derm., A pril, 1892.

${ }^{2}$ Loc. cet., p. 51. 
of epidermis, (5) the frequent absence of itching, and (6) its tendency to cause serious impairment of health and even death. From psoriasis it is distinguished by its rapid spread and the involvement of the whole area of the skin. Lichen planus is seldom universal, and does not spread so rapidly; moreover, it begins in characteristic papules. From eczema, pityriasis rubra is differentiated by the absence of exudation and crusts and the frequent absence of itching. From pemphigus foliaceus, which it sometimes resembles in other respects, it can be discriminated by the absence of the loose bullæ and foul-smelling discharge characteristic of that affection. Moreover, the general symptoms are more severe in pemphigus foliaceus than in pityriasis rubra, and the disease is less amenable to treatment.

Prognosis.-This depends chiefly on the extent to which the internal organs, especially the kidneys, are diseased. The presence of albuminuria adds much to the gravity of the outlook. In a considerable proportion of cases pityriasis rubra proves fatal, especially in children; and, however mild the symptoms may be, it is never safe, even if the patient appears to be on the way to recovery, to predict a favourable termination, as at any moment a turn for the worse may occur. Even after complete recovery, relapse may take place. In my own experience a fatal result has occurred much more frequently in cases occurring secondarily to a pre-existent skin affection.

Treatment. General.-The first thing to be done, if the disease be consecutive to some other affection, is to discontinue the use of chrysarobin or whatever other drug may seem to be the determining cause of the attack. Every effort must be used to keep the patient's health up to the highest standard. Over-drugging is likely to do great harm. The digestion and bowels must, of course, be carefully attended to ; 
nervous excitement must as far as possible be subdued by appropriate remedies. If the symptoms are acute, antimony, administered in the manner already described, will be found useful. In cases of a chronic type arsenic may do good, but it should never be given if the inflammation is at all intense. When the patient suffers from sleeplessness, and the urine is normal, the administration of opium is often of great use. Stimulants should be forbidden, unless definitely indicated by weakness of the heart's action. The strength must be maintained by nutritious food, and the least appearance of wasting should be the signal for cod-liver oil. Sufferers from pityriasis rubra are always unduly sensitive to cold, and, indeed, a chill is very likely to aggravate the symptoms during the course of the disease, or to bring on a relapse during convalescence or after recovery. It is of the greatest importance, therefore, that exposure to cold be most carefully avoided. In severe cases the patient should be kept in bed; even in apparently slight cases he should stay indoors.

Local treatment. - The obvious indications are to soothe irritation, disinfect the skin, and keep the affected parts warm. Tepid bran or alkaline baths are usually comforting. In cases in which the kidneys are sound, I have seen great benefit from the use of prolonged mucilaginous or demulcent baths. Patients are often able to sleep in these baths when they cannot do so in their beds. Tarry preparations are especially useful. The liquor picis carbonis freely diluted with water, or very weak creoline, or oil of cade ointment, may be applied. Carbolized oil (1 in 20) is often beneficial, but in using antiseptic applications care must be taken lest irritation be caused. Stephen Mackenzie got the best results from watery as distinguished from oily applications. His favourite application was a lotion of glycerine of subacetate of lead $\mathrm{i}$, glycerine 
§i, water to a pint. The patient is swathed from head to foot in a suit of lint and a mask soaked in this lotion, and is kept between blankets during the treatment, which is continued till all redness has disappeared. Then greasy applications, such as simple vaseline, are substituted for the lotion. Mercurial preparations should not be employed, as they are likely to aggravate the disease. For protective purposes the skin may be freely dusted with starch and oxide of zinc powder, and then covered with cotton-wool, or it may be wrapped in bandages steeped in calamine liniment.

With infants special precautions must be taken against cold. The skin should be smeared with fatty substances and covered with cotton-wool till the epidermis has been reproduced. Special attention should also be paid to the nourishment of the patient. ${ }^{1}$

\section{Pityriasis Rosea}

Pityriasis rosea, for the differentiation of which as a substantive disease we are indebted to Gibert, - is an inflammatory affection, the essential lesions of which are pink patches or circles, very slightly raised, and thinly covered with small scales.

Symptoms.-The eruption appears first as a single patch, which is situated on the trunk, the neck, or the arm. ${ }^{3}$ It is oval or circular in shape. Its edges are bright red in colour, somewhat raised, and covered with fine adherent scales; the centre is of a duller redbrownish tint, and slightly depressed. The patch spreads at the edge, fading in the centre as it does so.

1 For an instructive discussion of the whole subject of exfoliative dermatitis, in which Walter G. Smith, Stephen Mackenzie, Radeliffe Crocker, F. J. Payne, Colcott Fox, J. J. Pringle, Galloway, and others took part, see Brit. Journ. Derm., Dec., 1898, p. 437 et seq.

2 "Traité Pratique des Maladies de la Peau et de la Syphilis," 1860.

${ }^{3}$ Brocq, op. cit., p. 625. 
In a week or so this herald patch is followed by the appearance of a number of small bright-red spots, which soon grow into patches. These are of two types: one small, irregular in size, with an indistinet border and a scaly wrinkled surface; the other larger, rounded in outline, with a well-defined border like the herald pateh, and standing out among the other patches like medallions (Brocq). Lesions of the former variety are sometimes termed maculate, while those of the latter are known as circinate. Both forms usually coexist, the circinate lesions being scattered among the others, which are more numerous. As the circinate patches spread at the edge the centre undergoes involution, and rings, red and scaly at the circumference and fawn-coloured in the middle, are formed. In course of time the circle is broken by partial disappearance of the border, and segments remain, which, meeting similar relics of other patches, form wavy lines partly enclosing fawn-coloured areas. As the eruption fades at one spot it comes out at another, and at a given moment all stages of the process may coexist. Itching is not generally troublesome, but, owing to individual differences in the irritability of the skin, this rule is subject to numerous exceptions. The eruption usually first shows itself on the abdomen, but it may begin on the chest, the face, or the arm. It spreads rapidly, so as often to cover the trunk, the face, and the limbs in two or three weeks. It is generally thickest on the buttocks and abdomen, and it seldom extends below the elbow or the knee. I have, however, seen it in a corn-like form on both palms in a xerodermic patient. Occasionally it is universal. The appearance of the eruption is sometimes preceded or accompanied by constitutional disturbance, which is not, however, severe. The process terminates in spontaneous resolution within a period varying from a fortnight to two months. 
Etiology.-Pityriasis rosea may occur at any age, but is most common in the young, being generally seen in persons between 15 and 35 years of age. According to Brocq, ${ }^{1}$ it is more common in the female than in the male sex. It is said by some to be more common in spring than at any other season; of 56 cases observed by Moingeard, 53 occurred in the period between April and June. Jacquet says that it shows a preference for the subjects of dilated stomach, and Brocq believes it to be relatively common in those suffering from syphilis, especially in the early stage. It has been thought to be due to a fungus, the Microsporon anomoon (Vidal); but the very existence of such an organism lacks confirmation, and in any case its presence on the epidermis would be no proof that it had any causal connection with the disease. As the result of his investigation of pityriasis rosea, all that Sabouraud can say is that it is a polymorphous erythema due to some internal cause of unknown nature. ${ }^{2}$

Diagnosis.-As a rule, diagnosis is easy, owing to the well-marked objective features of the affection. The characteristic single patch which I have called the " herald," the pale-red tint, the slight scaliness, the want of elevation of the patches, the mingling of maculate and circinate varieties of lesion, and their spontaneous involution, make up a distinct clinical entity which can hardly be mistaken for anything else. From psoriasis, pityriasis rosea is differentiated by $(a)$ its more rapid onset; $(b)$ its slight scaliness; $(c)$ the absence of the characteristic hyperæmic spots on the red surface underneath the scales; $(d)$ its showing no preference for the situations most liable to be attacked by psoriasis. From maculo-papular syphilides it can be distinguished by

1 "La Pratique Dermatologique," vol. iii., p. 903. Paris, 1902.

${ }^{2}$ Rev. Prat. des Mal. Cut., Syph. et Vénér., June 1, 1902. 
the lighter colour, the absence of infiltration, the immunity usually enjoyed by the palms of the hands, the absence of a history of infection and of other concomitant signs of venereal disease. From seborrhoca corporis it is differentiated by $(a)$ the absence of the characteristic initial papules; $(b)$ its distribution, seborrhœa corporis affecting almost exclusively the middle of the chest and back, and avoiding the limbs; $(c)$ the dryness of the scales; $(d)$ the fact that it disappears spontaneously in a few weeks, while seborrhœa corporis, if untreated, will last for years; $(e)$ the absence of the bottle bacillus on staining. From tinea circinata, pityriasis rosea is distinguishable $(a)$ by the large number and wide distribution of the lesions, and $(b)$ by the absence of the fungus which is the cause of the former.

Prognosis.-This is always favourable, spontaneous resolution, as already stated, taking place in a few weeks.

Treatment.-All that is required is to soothe any irritation that may exist. For this purpose a weak lotion of liquor picis carbonis, or any of the anti-pruritic remedies already mentioned, may be employed. If the eruption be very. extensive, a tepid bran or alkaline bath will be useful. Allan Jamieson recommends that the patient be soaked daily for half an hour in a bath to which two or three teaspoonfuls of Condy's fluid have been added, and that then vaseline with salicylic acid be applied freely to the skin. Montgomery of San Francisco speaks well of this treatment. ${ }^{1}$ No internal medication is necessary.

1 Trans. Amer. Derm. Assoc., 1905, p. 196. 


\section{CHAPTER XV}

\section{PARASITIC AFFECTIONS}

\section{ANIMAL PARASITES}

A formidable list of the animal parasites that infest the human skin is given by Gebor $;^{\mathbf{1}}$ of these only the more common need be mentioned here. He divides the parasites into three classes: (1) Those (called by him "stationary") whose habitat is almost exclusively the human skin-including the Acarus (or Sarcoptes) scabiei hominis, or itch-mite; the pediculus or common louse in its three varieties, $(a)$ head, $(b)$ body (or, more properly, clothes), and (c) pubic or crab-louse; Pulex irritans, or the common flea; Demodex or Acarus folliculorum hominis. (2) Temporary or occasional parasites, which may be present either $(a)$ in a sexually mature or $(b)$ in a larval condition. Among the former may be mentioned Acanthia lectularia, or the bed-bug; Dermanyssus avium, or the bird-mite; Tabanidæ, or houseflies ; among the latter, Cestodes, such as Cysticercus cellulosa and the echinococcus or bladder-worm; Trematodes, such as Fasciola hepatica, or the liver-fluke ; Nematodes, such as Filaria medinensis, Filaria sanguinis hominis, Oxyuris vermicularis; and various flies (Muscidæ), such as Musca domestica, M. cadaverina, $M$. vomitaria, etc. (3) Accidental parasites, of which perhaps the most familiar is Leptus autumnalis, or the harvest-bug.

1 Ziemssen's " Handbook of Skin Diseases." 


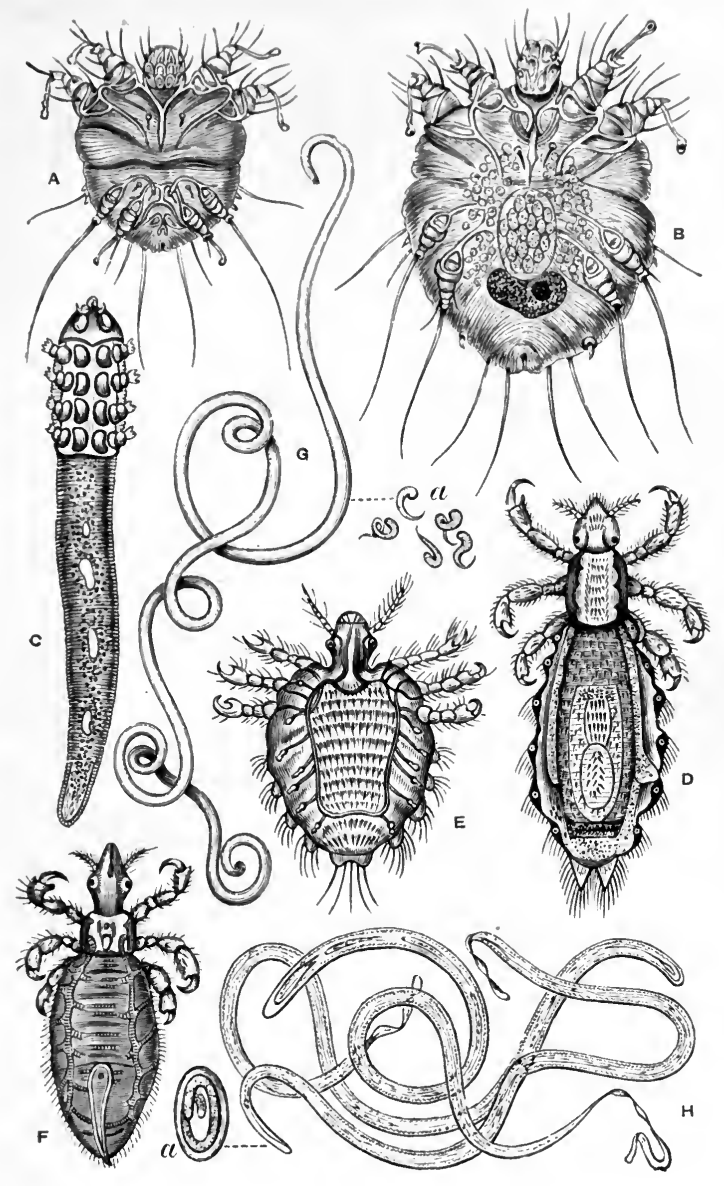

Plate 24.-ANIMAL PARASITES.

A, Acarus scabiei (male), $\times 100$ (Kaposi). B, Acarus scabiei (female), $\times 100$. C, Demidex folliculorum, $\times 250$ (Kuichenmeister). D, Pediculus corporis (female), $\times 50$ (Kiichenmeister). E, Peiiculus pubis, $\times 35$ (Küchenmeister). F, Pediculus capitis (male), × 35 (Kiichenmeister). G, Dracunculus medinensis, half natural size, with larvæ $(a), \times 30$ (Cobbold). H, Filaria sanguinis hominis, $\times 250$ (Lewis); with ovum $(a)$ (Cobbold). 



\section{Scabies}

Symptoms.-After a period of latency, which is usually about ten days, but may vary between two days and a month, a vesicle is seen at the point where the female acarus, the exciting cause of this affection, first enters the epidermis. The average length of the burrow is from $\frac{1}{8}$ inch to $\frac{1}{2}$ inch, but it may be a good deal longer. The burrow is the characteristic lesion of scabies, and its most common situations are the parts where the skin is least thick, namely, the webs between the fingers and toes (especially in infants), the fronts of the wrists, the ankles, the elbows, inside the umbilicus, the penis and other parts of the genitals, the breasts in women; occasionally, though rarely, except in very uncleanly people, they may be seen in other parts, but the head and face are never attacked except in children in arms, where, for obvious reasons, these parts are much exposed to contagion. The burrows can generally be found without difficulty in persons who are not too particular in their ablutions, the rough line marking the track being blackened by excrement which the acarus leaves behind her, and by dirt; in other cases the little vesicle at the entrance will indicate their position. In cleanly people they are often by no means easy to find, the line marking their course being ill defined. There are also certain periods in the disease when burrows are not present, namely, at the very beginning, when the acarus has only just penetrated the epidermis and has had no time to burrow, and later, when the burrows have been laid open and destroyed by scratching or by treatment.

The most marked subjective symptom is itching, which is usually extremely troublesome, especially at night. As in other conditions, however, it varies in degree according to the temperament of the patient, $\mathbf{x}$ 
some persons being the subjects of itch for weeks or months without being conscious of any particular irritation of the skin, others being driven almost frantic by it from the first. Irritation is not infrequently felt in places distant from the seat of the disease : thus, having once inoculated myself experimentally on the arm, I felt little or no itching at the site of inoculation, but after a time became aware of intense itching at the back of the shoulder. This reflex irritation may give rise to a sympathetic eruption in distant parts, as is observed in urticaria; and when the characteristic burrows are not readily discoverable, this may be very misleading. When the burrows have been destroyed, the itching and the other symptoms usually subside; sometimes, however, the lesions may persist for a long time, and in persons with an exceptionally irritable skin may be the starting-point of eczema and other troubles.

The secondary lesions are the results of inflammatory reaction, intensified by scratching and complicated by inoculation with cocci. The eruption is first vesicular; later, pustules and sometimes bullæ are formed. The distinctive feature of the lesions is that they are not grouped as in eczema, which they otherwise often resemble, but are isolated and irregularly scattered about. The marks of scratching are seen in all parts of the body which can easily be got at by the patient's fingers. In men they are chiefly found on the front of the body from the nipple to the knees; posteriorly they are almost exclusively on the buttocks. In women and children they are also visible on the lower part of the back. The eruption is usually most marked in parts subjected to friction, and over the ischial tuberosities in those whose occupation makes it necessary for them to sit long on hard seats. The eruption of scabies, therefore, presents a very pronounced multiformity of aspect. Burrows, vesicles, bullæ, pustules, 
are mingled in the most irregular manner with the marks of scratching and the results of secondary inoculations in the form of ecthymatous or impetiginous eruptions in various stages of development, and of destruction by scratching (ruptured vesicles and bullæ, pustules laid open and discharging or covered with scabs, hæmorrhagic points, etc.). The secondary lesions are sometimes so severe as to disguise the real nature of the affection. These severe cases are rare in England, but frequent in Norway and some other places.

Pathology,-The pathology of scabies is that of dermatitis, with the usual secondary lesions caused by scratching and inoculation with inflammatory products.

Etiology.-The Acarus (or Sarcoptes) scabiei hominis belongs to the tracheal order of the Arachnida. The female acarus (Plate 24, B) is the exciting agent in the initiation of the inflammatory process. After impregnation she measures from $\frac{1}{8}$ to $\frac{1}{4} \mathrm{~mm}$., and is then just visible to the naked eye as a whitish opaque spot. She has eight conical legs, the anterior ones furnished with suckers, the posterior ones with bristles. She penetrates the horny layer head first and wriggles through the mucous layer, leaving the ova behind her in the deeper epidermic structure as she progresses. She dwells at the blind end of the burrow for about two months, depositing altogether some fifty eggs. When they are hatched, after about a week's incubation, they find their way out of the burrow. The male acarus (Plate 24, A), about half the size of the impregnated female, and discoverable only with difficulty, does not dwell within the burrow. As a rule, it is only by close and prolonged contact between healthy and infected subjects that scabies can be conveyed, though indirect contagion, by means of underclothing or bedclothes, is possible. Want of cleanliness appears to be the only predisposing cause. Diagnosis.-In a well-marked case of scabies the 
diagnosis is easy, the characteristic burrows between the fingers and on the wrists being conclusive. As already said, the mouth of the burrow is usually marked by a vesicle, and in searching for the parasite the farther end of the passage away from the vesicle must be sought for. The following is the method of procedure most likely to be successful. A pin is laid on the surface of the epidermis, not point downward, but on the flat; it should then be pushed into the epidermis, at the end of the burrow away from the vesicle, with a rotatory movement, great care being taken not to draw blood. If the acarus is alive it will cling to the end of the pin, where it can be seen as a minute pearly object. It can then be mounted in glycerine and examined microscopically. When no burrows are to be seen, either because they have not yet been formed, or because they have been opened up by scratching, the diagnosis must chiefly rest on the distribution, and especially on the irregularity of the lesions. A pustular eruption on the hands should always excite suspicion; the distribution of the marks of scratching is a further guide to the nature of the affection, and any history of a similar affection in the same house is an important link in the evidence. The superficiality of the vesicles, and the heterogeneity of the secondary lesions, distinguish scabies from smallpox, and the distribution also is different, for, except in children, the face is not attacked. For the diagnosis from eczema, see p. 296.

Prognosis.-The prospect is always good as regards recovery, if proper treatment is submitted to; but, as already said, scabies may in certain persons be the starting-point of some other affection, of the skin. In nervous subjects, too, scabies may be followed by marasmus as a result of intense itching and insomnia.

Treatment.- The points to be aimed at are (1) the breaking up of the burrows; (2) the destruction of 
the parasites; (3) the relief of the subjective symptoms; and (4) the prevention or cure of secondary lesions caused by cocci, etc. The quickness of the cure depends on the thoroughness of the treatment. The patient should be stripped, and the affected parts soaked with hot water and vigorously scrubbed with soft soap; this will remove the superficial layers of the epidermis, and lay open the burrows. The next step is the application of parasiticidal agents in the form of a thickish ointment, or, better still, a paste, which should be thoroughly rubbed in and plastered over the affected parts, so as to fill every nook and eranny of the burrows. The usual application is simple sulphur ointment (3ss to §i) ; the sulphur ointment of the Pharmacopœia is unnecessarily strong, and should always be diluted. The application should be renewed every few hours for two or three days, the patient meanwhile wearing old undergarments. The treatment should be brought to a elose with a eleansing bath. An essential point is the disinfection of the patient's clothes by boiling or fumigation with sulphur.

At the St. Louis Hospital, in Paris, the favourite remedy is an ointment consisting of potass. carbonat. $3 \mathrm{i}$, sulph. sublim. 3ii, in an ounce and a half of lard. The treatment-la frotte-begins with rubbing the whole body vigorously with soft soap for twenty to thirty minutes and continuing the friction in a warm bath for a further period of an hour; the ointment is next thoroughly rubbed in, the patient resumes his clothes without washing off the ointment, and is usually cured. Another very effeetive ointment, recommended for cases in which there is much inflammation of the skin, is composed of sulph. sublimat., olei cadini, āā $3 \mathrm{ii}$, cret. preparat. Ziiss, saponis viridis and adipis āā $\bar{\jmath}$. If this application produces too severe a reaction, an ointment may be used consisting of styracis liquida, sulph. 
sublimat., āä 3i, adipis purificat. そi. A still milder ointment, which has little odour, and is white, is naphthol 孔ss, cret. preparat. $3 \mathrm{iii}$, saponis virid. 3 iss, adipis purific. 3iii. Max Joseph uses Kaposi's ointment, consisting of $\beta$-naphthol, cretae albœ, āā 10, saponis viridis 50, axungice porci 100 . In cases in which this fails he employs Hebra's modification of Wilkinson's ointment : Florum sulphuris, olei fagi, saponis viridis, $\bar{a} \bar{a}$ 40, axungice porci, pulv. cretae albce, āa 80 . When time is a matter of vital importance, the first and second indications in the treatment of scabies may be fulfilled by one remedy-namely, the application of Vlemingkx's lotion, which consists of quicklime 3ii, sulphur $3 \mathrm{iv}$, and water $\xi \mathrm{xx}$. The ingredients should be boiled in an iron vessel, and stirred with a wooden spatula to perfect union. The quicklime causes exfoliation of the epidermis, and gives the sulphur free access to the burrows. Medicated soapssuch as the sulphur precipitate soap, 10 per cent., prepared according to Buzzi's directions ${ }^{1-}$ are also useful. Balsam of Peru is now frequently used instead of sulphur, and is especially suitable for pregnant women and for cases in which there is exceptional sensitiveness of the skin or a predisposition to eczema. It is as rapidly fatal to the itch-mite as sulphur. It is painted over the whole surface of the body and allowed to remain on overnight, or longer if necessary. Peruol, an extract prepared from the balsam, is without the offensive smell of the latter drug; Juliusberg uses a 25 per cent. solution in olive oil. For infants and persons with a delicate skin, stavesacre, or weak balsam of Peru ointment, is very useful.

A word of caution may be added as to the way in which the mechanical and parasiticidal applications are made. Vigour must not be pushed to the length of violence, nor is it necessary to stir up acute inflammation

1 Monats. $f$. prakt. Derm., 1891, Ergänzungsheft 2. 
of the skin in order to kill the parasites. On the other hand, the mere smearing on of a little sulphur ointment is of no use. For the relief of the subjective symptoms, soothing applications, such as calamine lotion, alkaline baths, etc., and antipruritic remedies, such as carbolic or menthol soap, or any of the remedies recommended for pruriginous conditions, should be employed. The patient should be warned that itching sensations may continue for some time after the disease is cured. Secondary inflammatory or suppurative lesions should be treated with antiseptic applications, such as boricacid lotion or liquor picis carbonis.

In some cases the secondary lesions in scabies are of such a degree of severity that the application of the ordinary parasiticidal substances is out of the question. In these cases endeavour must first be made to subdue the inflammatory symptoms, and then one must cautiously feel his way towards the radical treatment of the disease by the graduated use of parasiticides, the effect of which should be carefully watched.

\section{Scabies of Animal Origin}

Scabies in which the parasite is derived from certain of the lower animals is sometimes met with. The most frequent of these severer forms of the disease is that known as Norwegian itch, which has been met with in lepers by Danielssen and Boeck, and in Germany and elsewhere by other dermatologists. Darier holds that it is due to a special variety of the parasite. The primary lesions are succeeded by large, thick scabs, and the whole body may be thus affected. Rarer forms of scabies of animal origin, derived, not improbably, from domestic and tame animals and from birds, are characterized by a diffuse miliary or multiform pruriginous eruption; as a rule, they readily yield to treatment. In cases of equine itch, reported by Besnier and Mégnin, 
and by Darier, the eruption assumed the form of a generalized pityriasis.

\section{Grain Itch}

This affection was first observed in Philadelphia, in 1901, when it appeared as an epidemic ; it was denominated grain itch (acaro-dermatitis urticarioides) by J. F. Schamberg, from whom the following description is derived.

Symptoms.-There is usually an eruption of wheals; many of them are topped by a vesicle, of which the contents soon become purulent. Instead of wheals, however, there may be slightly elevated erythematourticarial or papulo-urticarial lesions. As a rule, the eruption is abundant; the favourite sites are the neck, chest, abdomen, and back; the hands and feet nearly always escape. There is usually severe itching, which at night becomes almost intolerable.

Etiology.-The affection has almost invariably been traced to the use or handling of new straw mattresses. In the siftings of the straw was found a minute acarus, resembling or identical with the Pediculoides ventricosus.

Treatment.-Schamberg recommends the use of an ointment consisting of $\beta$-naphthol gr. xxx, sulphur pracip. gr. xl, adipis benzoat. $z$ i. This both destroys the mites and relieves the cutaneous symptoms.

\section{BARLey ITCh}

Cases of "barley-itch" have been described by W. Kenneth Wills ${ }^{1}$ as occurring in a number of grain porters who had unloaded a cargo of barley from West Africa. On the chest and abdomen, less abundantly on the neck, face, arms, forearms, shoulders, and sparely on the back and legs, a profuse rose-coloured papular 1 Brit. Journ. Derm., Aug., 1909. 
eruption of an urticarial nature appeared. When undenuded papules were compressed, a minute droplet of serum exuded, and under the lens a tiny black dot was detected in several of the papules; these dots were found to be lance-pointed hairs with a fractured proximal end and an air-containing medulla. Wills's conclusion was that the eruption was caused by vegetable hairs in the barley. Acari were discovered, but in so macerated a state that they could not be identified.

\section{Pediculosis}

The insects which are the exciting cause of phthiriasis, or pediculosis, are of three different species, which attack respectively the head, the body, and the pubic region.

Symptoms.-All three species of parasites cause similar lesions, modified by peculiarities of situation. The primary lesion is a wound inflicted by the parasite in feeding; possibly also a minute quantity of some poisonous secretion is inoculated at the same time. The process of feeding is effected by the insertion, into the opening of a sweat-duct, of a tube through which the blood is sucked up. When the louse has satisfied its appetite it extracts the sucker, and the blood welling up in the duct forms a minute red speck on the surface. This hæmorrhagic speck, which can be seen but cannot be felt, is, as was first pointed out by Tilbury Fox, the characteristic lesion of pediculosis, and its presence is conclusive evidence of the nature of the affection. There are no other lesions on the skin beyond such as are caused by scratching-erythematous red lines parallel to each other and marking the track of the finger-nails, hæmorrhages, excoriations, wheals, and impetiginous pustules. When the top of a congested papilla has been scratched off, a tiny blood-crust is often left; this is common in all conditions that are accompanied by scratching, and is distinguished from the hæmorrhagic 
speck characteristic of pediculosis by the fact that it can be felt as well as seen. If persistent, the lesions may result in the production of a peculiar leathery thickening of the skin with pigmentation-the so-called "vagabond's skin." This phenomenon is sometimes found in the buccal mucous membrane, a circumstance which shows, as Darier ${ }^{1}$ points out, that it cannot be due solely to scratching. Among the rarer symptoms of pediculosis may be mentioned pyrexia, which is believed by Jamieson ${ }^{2}$ to arise reflexly from cutaneous irritation; Payne, ${ }^{3}$ however, looks upon it as the result of a kind of poisoning.

In pediculosis of the head the occipital region, where the hair is thickest, is chiefly affected, but the itching is felt all over the scalp. In the healthy the scratching only causes excoriation, but in ill-nourished children a suppurative process is pretty sure to supervene from inoculation with pus cocci. Sometimes the occipital and other neighbouring glands become enlarged and inflamed, and abscesses may form. In very dirty persons a peculiar condition known as plica polonica is produced by the matting together of the hair with pus, nits, scales and scabs, and miscellaneous filth.

In pediculosis of the body the patient-generally an elderly person in low condition who disregards cleanliness-complains of irritation, especially about the shoulders, on the back, and on the extensor surfaces of the limbs, but not on the hands or feet. When the clothing

1 “"Précis de Dermatologie." Paris, 1909.

${ }^{2}$ Brit. Journ. Derm., vol. i., 1888-89, p. 321 et seq. A case is cited in which a healthy lad, aged 19, was on two distinct occasions admitted into the Edinburgh Royal Infirmary with a very high temperature $\left(106.2^{\circ}\right.$ on one occasion, $106.4^{\circ}$ on the other), which immediately fell to normal when he was freed by a bath and a change of linen from the innumerable pediculi with which he was infested.

Ibid., 1890, p. 209. 
is removed there is generally little or nothing to be seen beyond the results of scratching-namely, long lines torn by the finger-nails, with here and there wheals, but as a rule no vesicles or other definite lesions. On examination with a lens, the characteristic hæmorrhagic specks can be made out. No pediculi will be found on the skin, but on searching the clothes, particularly the folds of the under-linen, they will usually be discovered, unless, as often happens, the patient has taken the precaution to change his clothes before presenting himself for inspection. A favourite hunting-ground of the body-louse is the shirt-collar on its internal aspect. So partial is the parasite to this part that signs of severe scratching about the back of the neck and the shoulders in an elderly person of doubtful cleanliness are almost conclusive evidence of the presence of lice. It is in tramps and other persons infested with body-lice that the "vagabond's skin" already mentioned is most frequently seen.

In pubic pediculosis the only subjective symptom is itching. Papules (the tops of which are generally scratched off) are the usual lesions, but sometimes more or less severe eczematous inflammation is induced. A characteristic lesion produced by crab-lice, according to Mourson and Duguet, is a peculiar steelgrey pigmentation which appears in spots about the size of the finger-nail (macula ccrulea). The colour of these blue spots corresponds with that of a pigment contained in the thorax of the parasite, and is thought to be inserted by it through its sucker into the epidermic tissues. The stains fade when the pediculi have been destroyed.

Etiology.-The lice which are the cause of pediculosis belong to the order Aptera, of the family Pediculus. The pyriform head of the insect is provided with mandibles which grasp the skin, and a membranous 
sucker which penetrates the orifice of a sweat-duct; the thorax is furnished with six legs, ending in a mobile hook. The parasites differ according to whether they infest the head, the body, or the pubic hairs. The body louse $(\boldsymbol{P}$. corporis) is the longest, the crab-louse $(P . p u b i s)$ the widest, the head-louse ( $P$. capitis) being midway between the other two in both dimensions. The headlouse (Plate 24, F) has a triangular head, and varies in colour according to that of the skin which it feeds on, being grey with black margins on the European, yellowish-brown on the Chinaman, white on the Eskimo, and black on the negro. It especially frequents the hair of the heads of neglected children of both sexes, and of uncleanly women; it is sometimes found in the beards of men. The females are larger and more numerous than the males; each one lays from 50 to 60 eggs, so that multiplication is very rapid. The body-louse (Plate 24, D), besides being longer than the head-louse, has a more oval head and more developed legs, and is more active; it is dirty-white in colour, with black margins. Its habitat is the clothing, especially the underclothing; it infests adults and elderly persons more than children. Its fecundity is such that two impregnated females are capable of producing in two months a progeny of eighteen thousand. The crab-louse (Plate 24, E), which presents some resembance to the crab, is broader and flatter than either of the others; it is yellowish brown in colour, and has a rounded head wth five prominent antennæ; the female lays from 10 to 15 eggs, which hatch out in a week, the young being sexually mature in a fortnight. Its favourite dwelling-place is among the pubic hairs, but it may find its way to the abdomen, the thorax, the axillæ, and occasionally to the eyelashes, whiskers, and beard. It may be derived from the clothing of infected persons, but is more usually communicated in sexual intercourse. Pediculi deposit 
their ova on the hairs, one ovum or nit being usually attached to a single hair ; occasionally there are several. They are attached to the side of the hair by a glutinous material which binds them so firmly that they can be separated only by dissolving it with acetic acid, although they can be slipped along the hair (see below).

Diagnosis.-When itching of the scalp is complained of, and especially if impetigo contagiosa, which is of streptococcic origin, be present, and there are enlarged glands in the neck, the occipital region should be carefully explored for nits. Impetigo contagiosa alone, however, is not enough to found a diagnosis of lice upon, there being many other conditions with which that affection is associated. If the melanodermia has extended to the mouth, pediculosis of the body has to be distinguished from Addison's disease, of which buccal pigmentation is a symptom. This may be done by attention to the distribution, which is different in the two conditions, and by remembering that itching is not a feature of Addison's disease. Confusion between scabies and pediculosis of the body may be avoided by noting the absence, in the latter condition, of lesions on the hands and wrists. For the diagnosis from prurigo, see p. 75 .

Treatment.-The aim must be to destroy the parasites and induce healing of the secondary lesions by means of antiseptic remedies. In pediculosis of the head, if the patient be a child, the hair should be cut shor $t$ and white precipitate ointment applied. In women the hair need not be sacrificed; the lice can be killed by thoroughly smearing the scalp with the same preparation. The nits may be run down the hair nearly to the free end, and the hair then cut just above. Or the hair may be thoroughly wetted with acetic acid, which dissolves the glutinous material fixing the ovum to the hair, and then carefully combed out. The process 
should be repeated as often as may be necessary. A mixture of ether $\zeta \mathrm{i}$ and oleate of mercury (5 per cent.) $z^{\mathrm{i}}$ is an effective application for the destruction of pediculi and their ova; or the hair may be soaked with petroleum, turpentine, or oil of sassafras. The crusts should then be detached by softening them with carbolized oil or carbolic-acid lotion, and the impetigo contagiosa treated with weak mercurial or strong boric-acid lotions.

In pediculosis of the body the parasites must be killed by thorough disinfection of the clothes which are their habitat. For this purpose the most effectual method is baking in a disinfecting oven at a temperature of $212^{\circ} \mathrm{F}$. or more. The patient himself may with advantage take alkaline or ordinary hot baths, and the free use of some medicated antiseptic soap will be a most useful adjunct.

The treatment of pubic pediculosis must run on the same lines as that recommended for head-lice, but the pubic hair should not be cut. White precipitate ointment is an excellent remedy. Oleate of mercury (5 per cent.) $3 \mathrm{vi}$, cether. sulph. 3ii, kills the pediculi and destroys the nits. After the parasiticidal remedy has done its work some calamine or other soothing lotion should be applied. If the eyelashes are involved, the parasites should be picked off one by one with forceps.

\section{Miscellaneous Parasites}

The flea (Pulex irritans) makes a characteristic lesion, consisting of a small red spot with a central point of darker hue. Older spots become petechial, and sometimes in patients suffering from fever may be mistaken for the exanthem of typhoid or measles, or for purpura. The marks on the linen and the presence of recent spots will enable the observer to come to a correct conclusion.

The bug (Acanthia lectularia) produces a wheal with a whitish centre and a central punctum resembling that 
made by the flea. Great irritation and hyperæmia are usually caused by bugs, which excite artificial congestion by injecting an irritant substance so as to increase the supply of blood available for sucking. The irritation may be removed by the application of linen soaked in eau de Cologne, toilet vinegar, lead lotion, or strong ammonia, "eau de luce" being the most effective. Saturated solutions of soda or boric acid applied hot give great relief if the bites are extensive.

The harvest-bug (Leptus autumnalis) is active in July and August amongst those who work in the fields. It produces bright-red papules and wheals, generally on the ankles and legs, but often on other parts of the body. The itching is very troublesome, and scratching may cause secondary lesions of the usual kind. Treatment consists in the application of parasiticides such as naphthol or weak mercurial ointment.

The bites and stings of gnats, mosquitoes, and similar pests raise wheals, often with a vesicle in the centre, and usually accompanied by excessive itching. The remedies recommended for bug bites will be equally useful for these.

The chigoe, or jigger, a sand-flea (Dermatophilus penetrans), not unlike the common flea (Pulex irritans), is found in tropical countries, and is very prevalent on the East Coast of Africa among the coolies, by whom it has been introduced into India. The animal bores into the skin, and there gives rise to suppuration and ulceration-dermatophiliasis. The best treatment is to extract it with a needle.

In tropical America the ver macaque, the larva of the Dermatobia cyaniventris, attacks various parts of the body, both of men and of animals, and causes severe pains, especially when it is in motion. By the larva of Cordylobia anthropophaga Grünberg, Tambu-fly disease is set up in tropical Africa, and similar larvæ are 
encountered in this and other tropical regions. This parasite, the ver du cayor, burrows into the skin of man and beast, and causes an inflamed swelling not unlike a boil, from which it emerges in six or seven days. Both in dermatobiasis and in cordylobiasis, treatment consists in extracting the parasite with forceps and dressing the wound antiseptically.

The guinea=worm, Dracunculus (or Filaria) medinensis (Plate 24, G) is a parasite which in tropical countries is believed to gain admission to the body through the medium of water by drinking. I have seen but one case. The patient was a lady who had recently returned from India, the only symptom being one large bulla on the instep. I was able to prove the diagnosis and effect a cure by opening the bulla and winding out the worm on a match, a process which took twelve days. Emly, a French naval surgeon, has introduced a more expeditious method of extracting the worm by injecting it, if it protrudes, with a solution of perchloride of mercury; and so destroying it, extraction being, as a rule, easily effected after an interval of twenty-four hours. In the absence of protrusion he injects the solution as near the coiled-up parasite as possible; when the worm is thus killed, it may be cut down upon and extracted, or left to be absorbed. The parasite has been more frequently observed in England of late years. 1

Craw-craw, or nodular dermatitis, is a disease that occurs on the West Coast of Africa and in other tropical regions; it appears to be caused by a filarial organism, not improbably Microfilaria perstans. The lesions are papular, or papulo-pustular. Treatment consists in the persevering application of a 2 per cent.

1 For a full account of this and similar worms and the symptoms produced by them, see Manson's "Tropical Diseases," 4th edit. 
salicylic spirit lotion, followed by the use of a 5 to 10 per cent. $\beta$-naphthol ointment.

The veld sore of South Africa, from which so many of the troops suffered during the Boer War and after their return home, has resemblances to craw-craw ; but the most constant bacteriological feature of this affection is a diplococcus which Bishop Harman, who inoculated himself with it, considers to be a special organism (Micrococcus vesicans), and not simply an attenuated form of the Staphylococcus pyogenes aureus. The sore may follow insect bites or exposure to the sun, but is more often a sequela of abrasions. It mostly affects the extensor surface of the upper limbs, from elbow to digits. First there is a small blister or group of blisters, which break and lead to the formation of an ulcer that may remain open for months and is liable to exude pus as the result of secondary pyogenic infection. When the sore heals there is little destruction of skin. Treatment consists in removing the superficial epidermis for a considerable distance beyond the sore, rubbing the ulcersurface with lint soaked in a 1 in 1,000 solution of perchloride of mercury, and dressing it with the same solution.

Echinococcus hydatid, embryos of the Fasciola hepatica, and ova of Bilharzia hoomatobia (Schistosomum hamatobium) have also been found in rare instances in the human skin, and Cysticercus celluloso is sometimes present in the subcutaneous tissue. Other parasites which attack the skin are the gadfly or botfly (oestrus), and the wood-tick. In the former case treatment consists in incision and washing out the cavity with lotio acidi carbolici, 1 in 40 ; in the latter case the parasite may be killed with turpentine or paraffin. Eruptions are sometimes caused by infection of the skin by larvæ of certain members of the Arachnida, and by dipterous larvæ. 


\section{CHAPTER XVI}

\section{PARASITIC AFFECTIONS}

\section{VEGETABLE PARASITES}

THis group includes ringworm and all the other affections of the skin in which the process is set up by the growth of a fungus in the epidermis. I deal first with the ordinary forms of ringworm, which of late years have received so much attention from Sabouraud and other investigators as to require a chapter to themselves. In the next chapter various other tineas, with erythrasma and favus, will be considered, and, after them, a number of other fungous affections-pinta, mycetoma, actinomycosis, streptothrix infection, sporotrichosis, blastomycetic dermatitis, and labiomycosis.

\section{RINGWORM}

This affection may attack the hair, the skin, or the nails, and rarely the mucous membrane. On the skin the process is everywhere essentially the same, consisting in the inflammatory reaction excited by the growth of the fungus, and, usually, more or less suppuration. The appearance and evolution of the lesions are, however, so much modified by the structural peculiarities of the parts on which they are situated that clinically two distinct varieties are recognized, according as the disease affects hairy or hairless parts. Ringworm of the hairy parts is naturally subdivided into ringworm of the scalp (tinea tonsurans) and ringworm of the beard (tinea barbce, tinea sycosis). A rare form of ringworm, tinea palpebralis, which attacks the eyebrow, belongs, 
strictly speaking, to this category, but is generally classed with the next group, viz., ringworm of the hairless parts. This comprises ringworm of the body (tinea circinata), ringworm of the nails (onychomycosis), and ringworm of the mucous membrane (mouth, vulva). In addition to these, there is a speeial form of ringworm, occurring mostly in tropical climates, which attacks the inguinal, perineal, and gluteal regions; this generally goes by the name of cezema marginatum, but would be more appropriately designated tinea marginata.

Etiology.-In 1842-44, having in 1841 proved the cryptogamic nature of favus, Grüby, of Paris, published a series of papers reporting his discovery of three different cryptogamic fungi associated with as many different forms of ringworm. Four years later, Malmstein, of Stockholm, deseribed a parasite which he had independently discovered in ringworm, and designated it a trichophyton $(\theta \rho i \xi=$ hair, $\phi v \tau o ́ v=$ fungus), and afterwards Hardy coined the term trichophytie (trichophytosis) for the affection of which it was the cause. Of Grüby's three ringworm parasites, two were trichophytons and one was a microsporon. But his clinical descriptions were so vague that his distinctions were lost sight of, and for nearly half a century it was believed that in ringworm there was but one parasite concerned, causing a single diseasetrichophytosis-which manifested itself in different forms according to the soil on which the fungus grew. In 1891, Neebe and Furthmann isolated four ringworm parasites which yielded as many different cultures, though to what species they belonged is not known. In 1892, Sabouraud began the brilliant researches which, supplemented and confirmed by the work of other dermatologists and mycologists, British as well as Continental, have resulted in the general recognition of two different families of ringworm fungi, the 
Microspors and the Trichophyts, each of them com prising a number of different species.

Of the microsporons associated with ordinary ringworm, deven species have been identified; of the triohophytons, over thirty. The details of these parasites will be found in Sabourauds monumental work on ringworm. ${ }^{1}$ In these pages I must contine myself to these of clinical importance. These aro eight in number, four microsporons and as many trichophytons.

The four mierosporons are M. Audouini. M. felineum, $M$. cowis. and $M$. toriom. The type species is $M$. Andenimi. the special ringworm parasite of the child, for it sesreely ever attacks adults. It is the cause of 90 per cent. of juvenile ringworm in London. In a series of 126 consecutive cases of ringworm which I investigated 2 this fungus accounted for 92 per cent.; in more than $f(0)$ consecutive cases reported by coleott Fox and Blaxall ${ }^{3}$ the percentage was so to 90 ; in 17 consecutive cases of Adamson's it was as high as 97. This parasite is also the cause of much of the jurenile ringworm of Paris, and it is prevalent in the north-west of Europe generally, though met with most frequently of all in Fingland. South of the Loire it becomes rare, and it is seldom found in Italy or in Spain, in Denmark or in Sweden, in Germany or in Austria. The second and third clinically important microsporons, $M$. felineum and $M$. canis, closely related species, are responsible for an appreciable percentage of humsn ringworms- the one in Fngland, the other 1 "Les Teignes." Poris, 1940.

" "Ringworm in the Light of Revent kesewrch." London. 1893.

3 Brit. Jowrm. Derm.. July, 1s9ti

- Pasini has, however. recently shown that microsporosis is endemise in the provinee of Como. Grorn. Ital. d. Vab. Ven. e d. Pelbe, xlix, fass: 3. 
in France. The fourth species, M.tardum, is occasionally met, with in France.

The four trichophytons of clinical importance are $T$. crateriforme, $T$. acuminalum, $T$. suljureum, and $T$. violaceurn. In a series of some hundreds of scalp trichophytoses in London, Colcott Fox found $T$. crateriforme in 38 per cent. of the cases, $\boldsymbol{T}$. acuminatum in 26 per cent., $T$. sulfureum in 21 per cent., and $T$. violaceurn in 15 per cent. Of 100 cases at the St. Isouis Hospital, Paris, 50 were due to $T$. crateriforme, 30 to $T$. acurninuturn, and 15 to $T$. violaceum, while $T$. sulfureurn was not met with in a single instance. In 211 more recent Paris cases recorded in Sabouraud's work, $T$. crateriforme was present in 112, $T$. acuminatum in 47 , and $T$. violaceum in 35 , all the other species of trichophytons accounting for only 17 cases between them.

Differences between microsporons and trichophytons. - The ringworm which is caused by microsporons is styled small-spored tinea, or microsporosis, that which is due to trichophytons is known as largespored tinea, or trichophytosis. But the so-called spores of the parasites, i.e. the short elements, are not true organs of fructification, but, equally with the longer elements, are rather in the nature of mycelium. It should be understood, further, that among both microsporons and trichophytons the so-called spores vary greatly in size, according to the species. Those of the trichophytons may be as small as $3 \mu$, those of the microsporons as large as $4 \mu$, the limits of the one being 3-8 $\mu$, those of the other $2-4 \mu$. In my own series of cases Galloway found that the mean of ten measurements of detached spores of microsporons was $3.6 \mu$, the extremes being $2 \mu$ and $4 \mu$; and that the transverse diameter of the interwoven mycelium was $2.5-4.5 \mu$, giving an average of about $4 \mu$. The 
mean of as many measurements of trichophytic spores was $4.8 \mu$, the extremes being $5 \mu$ and $6 \mu$; the average diameter of the mycelium was about $5 \mu$, varying from $3 \mu$ to $6 \mu$. Certain species of trichophytons, indeed, now form a small-spored group (microīdes), and others a large-spored group (megaspores), the majority of the species of trichophytons constituting a middle group.

In a clinical sense, therefore, the microsporons and the trichophytons are differentiated not so much by the size of the spores as by shape and arrangement and mode of growth. Those of microsporons tend to be round or ovoid; those of trichophytons to be square with rounded angles, or oblong with sharper angles. In the microsporons (Plate 25, Fig. 1), the spores observe no definite arrangement, but are dotted about irregularly, and all the individual elements are discrete; in the trichophytons (Plate 25, Fig. 2) they are arranged in regular chains. In the microsporons the mycelium is irregularly jointed, curved and branching; in the trichophytons it is short and regularly jointed. In microsporosis the fungus lies around the hair, whether of the scalp or of the body, as a greyish sheath, visible macroscopically, which eats away the hair, frays the edges, works its way into the shaft, and grows downwards towards the root; presently the hair breaks off some way above the follicular orifice, the parasitic sheath undergoes disintegration, and a patch of ashcoloured scales is found on the epidermis. In trichophytosis the fungus first attacks the root and grows upwards, and the hair breaks off short. In both alike, however, the attack upon the hair is preceded by a lesion in the epidermis.

Endothrix and endo=ectothrix trichophytons. -In trichophytosis the hair is attacked in various ways; and these differences have been made the basis 


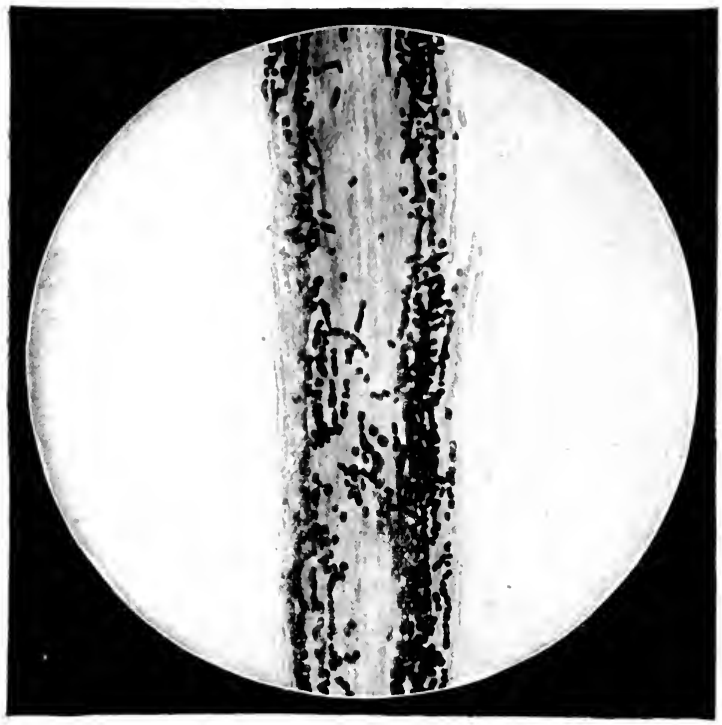

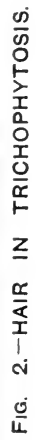

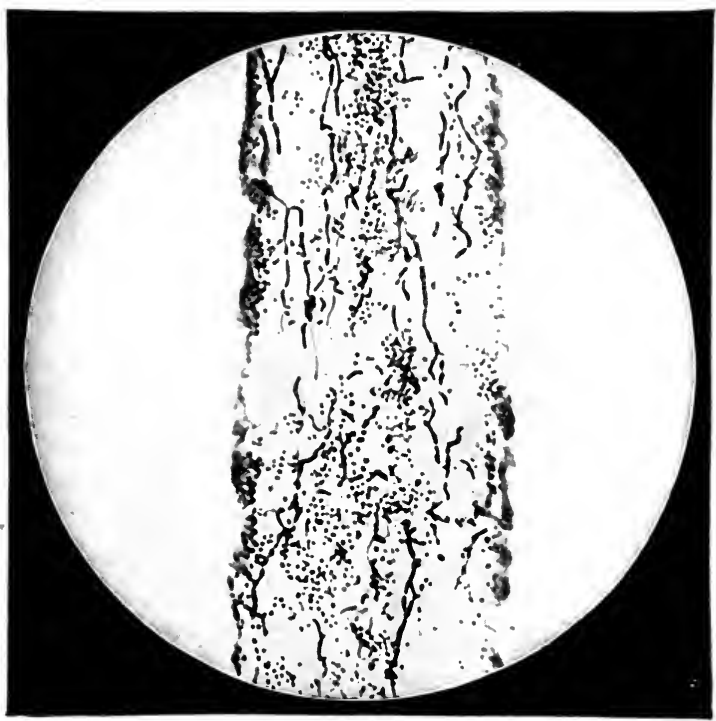

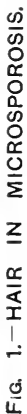




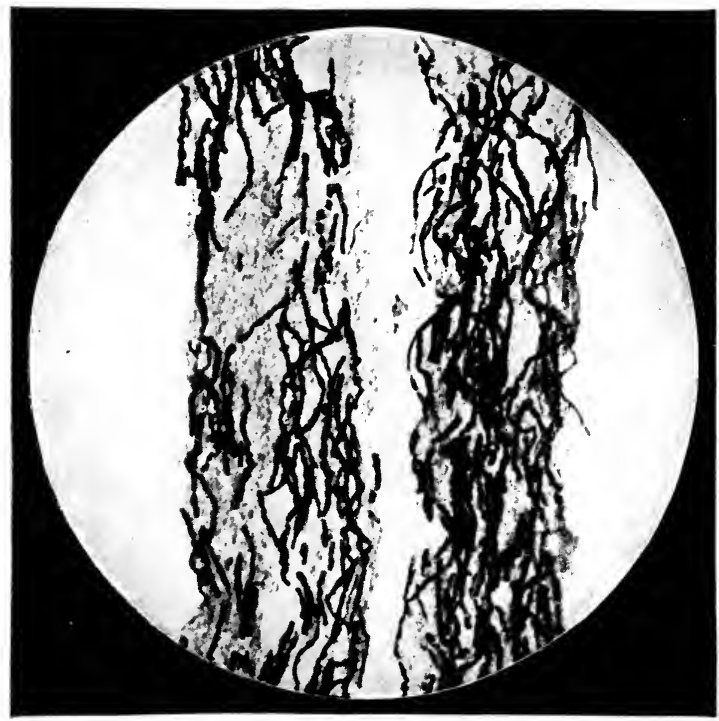

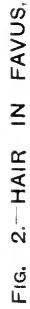

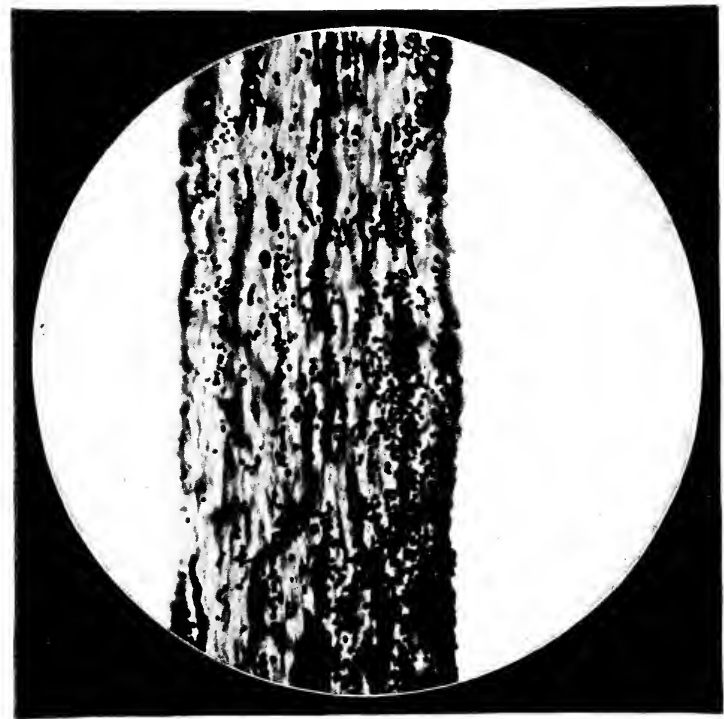

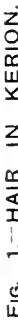


of a classification of the species into groups-the endothrix and the endo-ectothrix. Endothrix trichophytons make their way into the hair between the cells of the cuticle and develop exclusively within the hair-structure. Endo-ectothrix trichophytons not only develop within the hair, but continue to proliferate in the follicle outside. The name which Sabouraud proposed for this group, ectothrix, has led to much confusion, for it may suggest that the parasite does not find its way into the substance of the hair but occupies the surface only. The name should, therefore, be avoided in favour of endo-ectothrix, though, since it is the surface of the hair that is first attacked, ecto-endothrix might be still better.

The endothrix group is the cause of the great majority of cases of scalp trichophytosis both in London and in Paris; and some of the species may also attack the hairless skin, the beard, and the nails. But the endoectothrix group is responsible for a large majority of ringworms of the hairless skin. It also accounts for nearly all the cases of adult ringworm in all sites, for most of the specially inflammatory cases, and for a small minority of juvenile scalp ringworms. All four of the species which are most important in a clinical sense (p. 373) belong to the endothrix group.

The endothrix group is subdivided by Sabouraud into the true endothrix and the neo-endothrix, the prefix neo signifying not a later discovery of the parasite, but a prolongation of the invasion of the hair-of the early stage of the attack (stade de jeunesse). This stage, in an ordinary endothrix infection, is so transient that it may easily be missed; in a neo-endothrix infection it is so prolonged that the observer may mistake the case for one of tinea due to an endoectothrix.

The endo-ectothrix group is similarly subdivided 
into two-(1) those with small spores (microïdes), and (2) those with large spores (megaspores). In the microides the spores are not only small but form a sheath outside the hair, like that of the microsporons, but distinguishable from it by the chain-formation, which is never present in microsporosis. They are all pyogenic, and are responsible for many kerions (see p. 383). It is not unlikely that in the cases in which kerion has been reported in connection with microsporosis, the parasite was really one of these small-spored trichophytons rather than a microsporon.

Cultures of microsporons. - The species of microsporons are divided by Sabouraud into two cultural groups-(1) those of the M. Audouini type (Plate 27, Fig. 1), and those of animal origin. The one group gives a small (petite) or medium (moyenne), or, as he also terms it, a slow (lente) culture; the other a large, rapid (vivace) culture. In the latter case the preparation is crowded with multilocular segments heaped together in masses, and on a glucose or glycerine medium a white, downy pleomorphism, quite different from the mother culture, is exhibited. Microsporons of human origin, on the other hand, never produce a pleomorphic form.

Cultures of trichophytons. - There are three main types of endothrix cultures-(1) the crateriform, or sub-crateriform (resembling a volcano-crater), white, cream, or primrose-coloured (Plate 27, Fig. 2); (2) the acuminate (like a mountain-peak), grey or yellowish in colour (Plate 27, Fig. 3); (3) the violet. The cultures of the two neo-endothrix species are cerebriform in character. The endo-ectothrix cultures are - (1) the gypseum, large, white, plastery; (2) the niveum, large, white, downy (Plate 28, Fig. 1); (3) the large velvety; (4) the faviform. The gypseum and niveum cultures are those yielded by the small-spored endo-ecto- 

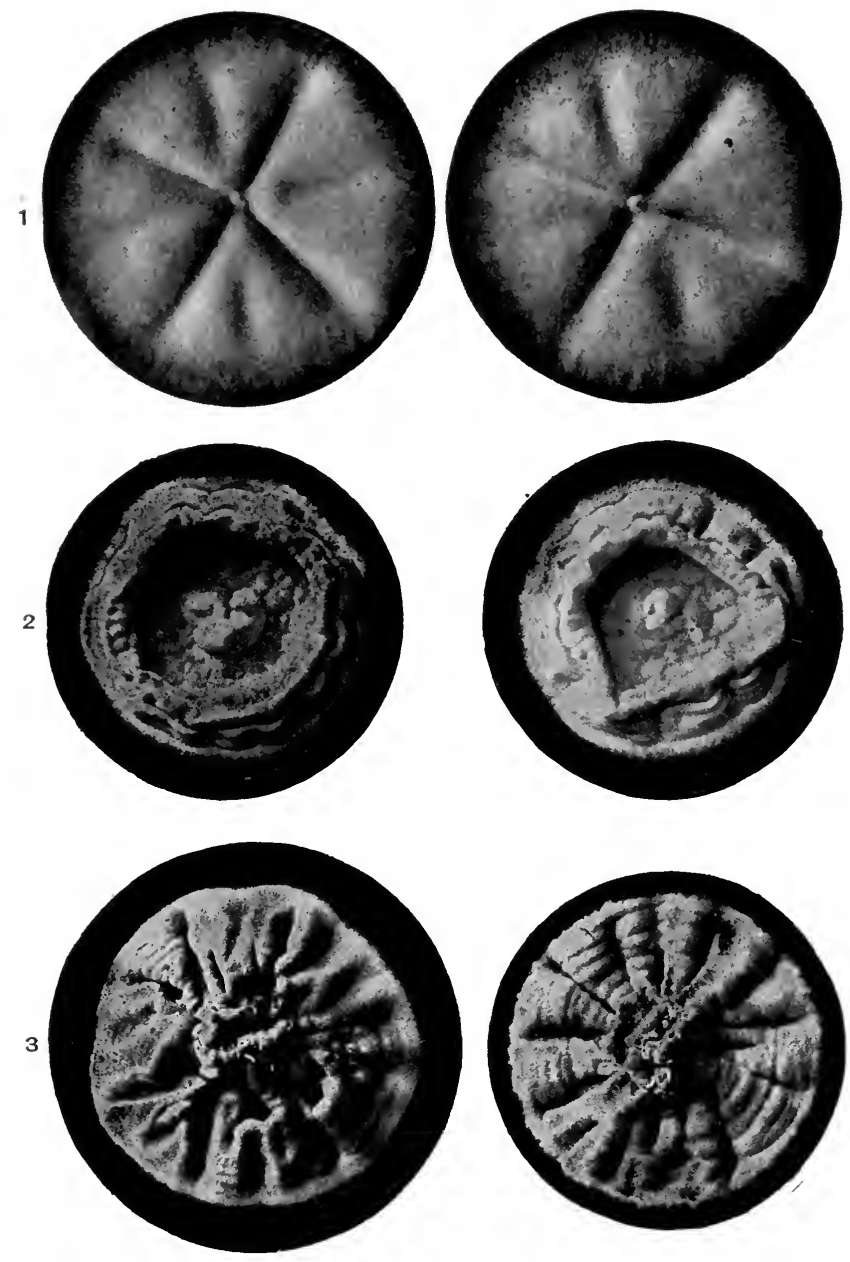

PLATE 27 -CULTURES OF RINGWORM FUNGI ON MALTOSE PROOF MEDIUM.

FIG. 1.-MICROSPORON AUDOUINI (2O DAYS).

FIG. 2.-TRICHOPHYTON CRATERIFORME (35 DAYS).

FIG. 3.-TRICHOPHYTON ACUMINATUM (35 DAYS).

(Sabourauls "Les Teignes.") 

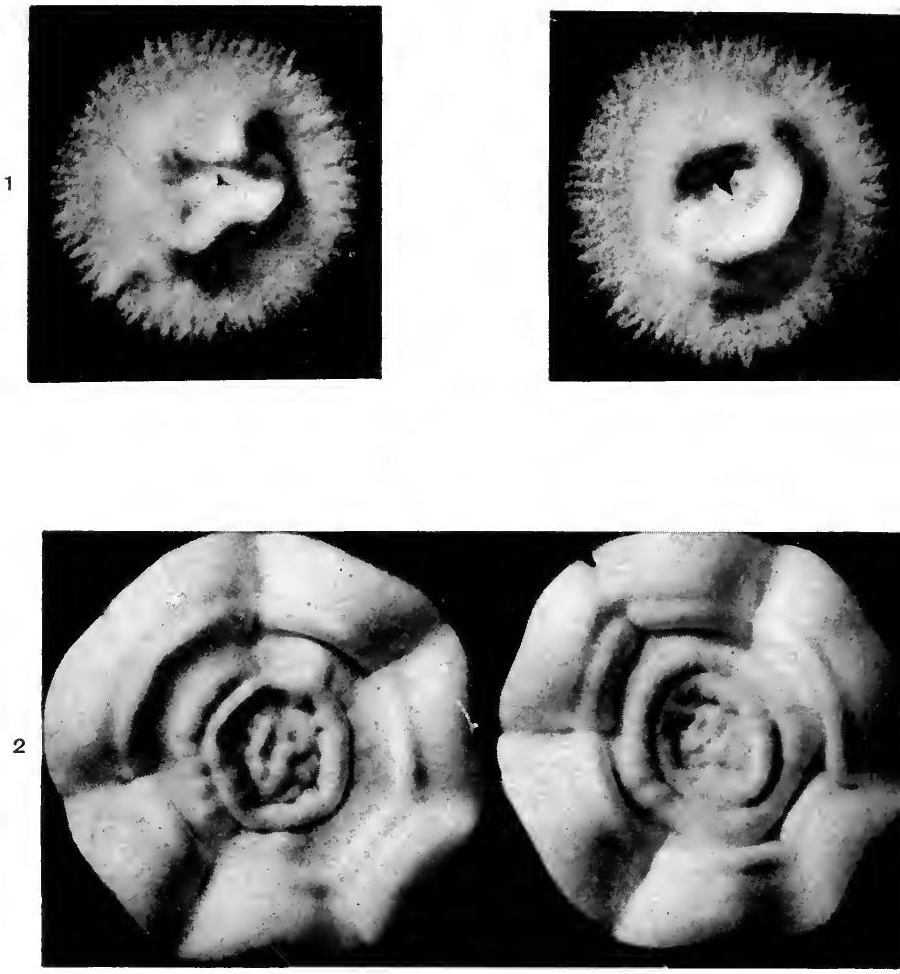
Plate 28.-CUltures of RINGWORM AND FAVUS FUnGI ON MALTOSE PROOF MEDIUM.

Fig. 1.-NIVEUM CULTƯRE (25 DAYS).

FIG. 2.-ACHORION SCHÖNLEINII (PLEOMORPHIC), 32 DAYS.

(Sabouraud's "Les Teignes.") 
thrices; the large velvety and the faviform by the large-spored endo-ectothrices. There is, further, a special type of culture, yellow and powdery, yielded by a single species which causes in man the eczema marginatum of Hebra, and which, although ranked as a trichophyton, is known among fungi as Epidermophyton inguinale.

Culture media.-The best culture material for ringworm parasites, as for dermatophytes in general, is Sabouraud's proof medium. Pure non-distilled water, 3 litres, is poured into a 5-litre flask, and finely divided agar 54 grm., Chassaing's granulated peptone 30 grm., and maltose (maltose brut de Chanut, obtainable from Cogit, 36 Boulevard St. Michel, Paris) 120 grm., are immediately added. The neck of the flask is stopped with wool, and the vessel is gradually heated in the autoclave to a temperature of $120^{\circ} \mathrm{C}$., and then cooled to $100^{\circ}$. Next, the contents are rapidly filtered through Chardin filter-paper into tubes or other vessels, which in their turn are stopped with wool and gradually heated in an autoclave to a temperature of $120^{\circ}$, when the gas is extinguished and the vessels left to cool spontaneously. For the maltose may be substituted glucose (glucose massée de Chanut). On the proof medium many ringworm parasites appear after a time as tufts of pleomorphic white down, representing a degeneration of the mother-culture, to which subcultures never return. This phenomenon may be avoided by using a medium which contains no glycerine or glucose.

Mycology of dermatophytes. - Colcott Fox was the first to insist (in 1896) upon the mycological relationship between the microsporons and the trichophytons. These, with a third family of dermatophytes, the achorions-the parasites of favus-are allocated by Matruchot and Dassonville to the Gymnoasceæ, of the order Ascomycetes. But the classification of dermato- 
phytes, as of fungi generally, is as yet only provisional. As to the origin of dermatophytes, they are probably, like most parasitic fungi, capable of a saprophytic existence, for they will grow on almost anything, and the most vigorous cultures are those from decayed vegetable matter. On favourable artificial media they present, even in primary cultures, close resemblances to the moulds which flourish on dead organic matters, and they develop true organs of fructification, as they never do in their parasitic life.

Infection.-At least a dozen trichophytons and four of the microsporons are common to man and to animals with which he comes in contact, and there is a large body of evidence that many cases of human ringworm are of animal origin. So long ago as 1871 Tilbury Fox exhibited seven cases of trichophytosis in man in which the contagion was derived from a pony, and Bunch has published a conclusive series of cases, both microsporic and trichophytic, in which the infection was conveyed to human beings by the bird, cat, and other animals. Ringworm which is derived from animals is, as a rule, more inflammatory than that of human origin. The affection may be communicated by direct contagion from one human being to another, or from an animal to a human being, or by indirect contagion through the medium of infected headgear, brushes, etc. Age is an important etiological factor in ringworm of the scalp, the affection being almost peculiar to childhood. In body ringworm there appears to be no age limit. The two sexes are about equally liable to the disease.

Epitome of etiology.-The complicated etiology of ringworm may be summarized in the following propositions :-

1. Tinea tonsurans is mostly due to Microsporon Audouini and to endothrix trichophytons, but the 
scalp, and especially the juvenile scalp, may be attacked by a great many of the fungi of both families.

2. The beard is attacked by trichophytons only.

3. The hairless skin is seldom infected by microsporons.

4. The nails are rarely involved, and almost always by $T$. acuminatum and $T$. violaceum.

5. Adults are scarcely ever attacked except by endo-ectothrix trichophytons, and this group is also responsible for much of the ringworm of the hairless skin.

6. The species of greatest clinical importance areamong the microsporons, M. Audouini, M. felineum, $M$. canis, and $M$. tardum; among the trichophytons, $T$. crateriforme, $T$. acuminatum, $T$. sulfureum, and T. violaceum.

Pathology.-The pathology of ringworm represents the results of the growth of the fungus in the epidermis-namely, destruction of the hair, and an inflammatory process set up by the irritation of the fungus and followed, in some cases, by suppuration.

That acute trichophytosis is a purely localized affection can no longer be maintained after the investigations of Calderone, Truff, Plato, Pautrier and Lutembocher, Bloch and Massini, Amberg, and others. If patients suffering not from mild but from severe trichophytosis are injected subcutaneously with trichophytin - a culture filtrate of trichophytons-there is not only a local reaction at the point of injection, but also a general reaction in the form of heightened temperature, malaise, headache, muscular pains, etc. In a series of 131 cases in which Amberg ${ }^{1}$ vaccinated inmates of a tuberculosis ward and others with trichophytin, he obtained positive reactions in 9 who had a history of trichophytosis, though in most cases they had been

${ }^{1}$ Samuel Amberg, Journ. Exper. Med., 1910, xii. 435. 
free from symptoms for many years. In 4 cases there was a positive reaction although there was no history of trichophytosis; two of these subjects were medical students, one of whom made special inquiries to satisfy himself that he had never suffered from any skin sffection. Four other cases in which there was a remote history of ringworm gave a negative reaction; but there was no evidence that the patients had suffered from acute trichophytosis; moreover, the time that had elapsed between infection and vaccination might have been sufficiently long to permit the disappearance of the antibodies. In all the other cases which remained under observation-cases, that is, in which there was no history of trichophytosis - the reaction was negative. The use of trichophytin is of no service in distinguishing between different trichophyton affections, for filtrates prepared from different species of trichophytons, derived from clinically different cases, act alike. Nor is a positive reaction of much practical significance, since it may be obtained many years after the subject has ceased to suffer from trichophytosis. It is the negative reaction, therefore, which possesses the greater diagnostic value. Amberg comments upon the analogies between the cutaneous trichophyton reaction and the cutaneous tuberculin reaction (von Pirquet's). In both the fact that a positive reaction may be obtained long after the disease has ceased to be active shows that the infection has left the organism in a state of altered reactivity - the state of hypersensibility which is designated allergy or anaphylaxis.

\section{Tinea Tonsurans}

Symptoms.-The incubation period of ringworm of the scalp, though variable within considerable limits, may for practical purposes be reckoned as under a fortnight. The affection is seldom, if ever, seen in its 
first beginning. Some localized scurfiness or loss of hair is discovered accidentally, or in consequence of the child scratching at the affected place. The initial lesion is often a small red papule, which develops about the orifice of a hair-follicle; sometimes it is nothing more than a minute scaly spot. The papule spreads peripherally, becomes scaly on the surface, and in a short time grows into a patch round or oval in outline, and slightly raised beyond the level of the surrounding skin. Similar patches are formed in the same way from other centres of infection. The patches vary in size from a threepenny-piece to a florin, but they are often as large as a five-shilling-piece, and sometimes they are several inches in diameter, equalling in area a clerical tonsure (hence the name tonsurans). Generally there are one or two small satellite spots in the neighbourhood of a patch. The patch, as a rule, stands out against the healthy skin more or less sharply by difference of colour as well as by scaliness. The hue varies from a dirty grey or slaty blue to reddish brown; in fair subjects it is generally yellowish.

In microsporosis of the scalp the typical patch is round, but it may be oval or irregular in shape, and the running together of neighbouring patches may give rise to areas of thickened desquamating integument with winding contours. The typical patch has a sharply defined margin, but sometimes, around what may be called a central clearance, there is an undergrowth of diseased hairs spreading out more and more luxuriantly towards the belt of healthy hair which marks the limit of the disease. The typical patch is often girt about by a narrow zone of erythematous redness; very rarely the edge of the ring is marked out by tiny vesicles. The patch is studded with dry, withered stumps of broken hairs, which stand out on its surface like the stubble on a mown field. The hair-stumps may be seen 
to have lost their natural gloss; they are thickened, and have a whitened, frosted appearance, produced by the parasitic sheath (see p. 374) which surrounds them. This sheath extends almost to the bulb, and, to use Sabouraud's simile, it makes the hair look like a sticky rod rolled in sand. Each stump sticks out of what may be called a miniature mole-hill or cone-like elevation thrown up around the hair by the massing of epithelial débris, caused by the burrowing of the fungus in the follicle. These tiny projections of the surface produce an appearance like "goose-skin." The individual hairs lose their elasticity, and are twisted and crumpled so as to have the appearance of cornstalks beaten down by wind and rain. They are also loosened, so that they can be pulled out without pain. The thickening of the hair is due to infiltration with fungus. Under the invasion of the parasite it becomes so brittle that it is broken to pieces by the epilating forceps, however gently handled, and is crushed with the greatest ease between the slide and the cover-glass.

An anomalous form of microsporosis of the scalp is bald ringworm (Liveing), or tinea decalvans (Tilbury Fox). The hair falls out in places, leaving a smooth bare spot of greater or less extent. This may occur in a spot to all appearance previously unaffected; more frequently it occurs in an ordinary patch of ringworm. Other patches generally become bald in like fashion, and the scalp presents an appearance resembling that of alopecia areata.

In a typical case of trichophytosis of the scalp the head is scurfy, and there are small patches of broken hairs, which may run together and form large patches. Examination with a lens discloses dark spots on the surface of the scaly patches among the remaining healthy hairs. From these dark spots trichophytosis of the scalp has been termed "black-dot" ringworm 
(Aldersmith). Sometimes the whole scalp is thus affected: the condition is known as "disseminated ringworm" (Aldersmith). The dots are pigmented, coiled-up hair-stumps, which are prevented from growing by the accumulation of scales at the orifice of the follicle. When the scales are removed the stumps begin to grow again.

In endothrix trichophytosis of the scalp-and most scalp trichophytosis is of this type-only some of the hairs in a patch are affected, so that in children with long hair the affection may be easily overlooked, or may be regarded as a slight pityriasis. In many cases the affected hairs are more or less pigmented, the result, it has been suggested, of the breaking-up of keratin. About the neck and face, and elsewhere, lenticular rosy macules often appear, and they are apt to recur.

The most severe form assumed by trichophytosis of the scalp is kerion (Plate 26, Fig. 1). The skin is raised into a dome-like elevation, which may be of considerable extent; the surface is angry-looking, smooth, and moist, and is thickly dotted with small holes, from some of which there projects a loose stump of hair, while others are filled with a plug of muco-purulent matter, and others, again, are empty and gaping. The holes are dilated follicles, and when a large proportion of them are plugged in the manner just described the appearance is very like that of a carbuncle. The swelling is tender and feels boggy, but does not distinctly fluctuate. Incision gives issue to little or no pus. The suppurative process is, in fact, localized in the follicles, at the bottom of each of which there is a little abscess. The pus loosens the hairs, and they are finally thrown off, the way being thus opened for the escape of a thick, viscid pus. Sloughing never occurs, but in rare cases a subcutaneous abscess may form. After the swelling disappears the site of it 
remains for some time red and bare, and it may be long before a new growth of hair takes place. In some rare cases the necrotic process is so intense as to destroy the roots of the hairs, and thus cause permanent baldness over the affected area.

The only subjective symptom in uncomplicated ringworm of the scalp, whether microsporic or trichophytic, is itching, and this is often absent. Even in strumous and ill-nourished children the affection causes no disturbance of the general health. The course is sometimes very rapid, especially in very young children. Dark hair is a less easy prey to the fungus than fair hair, and coarse hair resists more than fine hair. A patch of considerable size often takes several weeks, it may be months, to form. By continued spreading and confluence of patches, the whole scalp may in time be laid waste, its surface being covered by a thick layer of dry epidermic scales. As a rule, apart from kerion, the only inflammatory reaction in the scalp is the desquamation, though in young children red rings occasionally appear. In rare cases, however, inflammatory complications, vesicular, eczematoid, or impetiginous, arise. The commonest of these complications is impetigo, characterized by the appearance on the scalp here and there of isolated pustules, which on drying form scabs. If the impetiginous process is not speedily stopped, it is apt to spread over the whole scalp.

In an ordinary case of ringworm, when the fungus has worked its way to the bottom of the follicle there is little or no further reaction, and a dry, scurfy condition of the affected surface results. The disease then enters on an excessively tedious phase. In consequence of the thickening around the neck of the follicle, which is the result of the inflammatory process set up by the irritation of the parasite or by excessive treatment, the 
sac is converted into what may be called a bottle with a narrow neck; thus the fungus is imprisoned in the follicle, and remedial agents are prevented from gaining access to it. Disseminated ringworm is usually extremely obstinate, mainly, perhaps, because it is easily overlooked. I have known a boy suffering from this form of the disease to be a source of contagion in a school for many terms without suspicion attaching to him.

Diagnosis.-Microsporosis of the scalp may be distinguished from trichophytosis of the same region by the size, and yet more by the shape and arrangement of the spores, and the way in which the hair is attacked (see under Etiology) and broken off. In trichophytosis, again, on the surface of the scaly patches, dark dots are to be detected among the remaining healthy hairs. The scabs, too, are scantier, or may be absent altogether, nor is the outline of the lesions so rounded or so well defined. Tinea tonsurans, in either form, is not usually difficult to distinguish from other scalp affections. The clinical picture-the broken hairs, the black dots, the prominent follicles, the slight scaliness, the baldness in varying degrees, of the involved area-is, indeed, remarkably distinctive. From pityriasis of the scalp and from psoriasis of the hairy skin it is differentiated by the broken hairs, for in both those affections the hairs fall out unbroken. Psoriasis, further, exhibits a greater degree of scaliness; it is seldom that the scalp alone is affected, nor is it usual for the hair to fall out, though sometimes it does. The billiard-ball smoothness of the patches in alopecia areata is not present in bald ringworm, and the short hairs found at the edge of the patches, instead of being bent, as in tinea, resemble $\Omega$ note of exclamation, minus the point ('), while those that remain will be found free from fungus. In inflammatory cases of tinea a condition not unlike impetigo 
or eczema may be set up, but the characteristic broken stumps and the limited area involved, together with the history, will still point to the true nature of the disease. Moreover, the lesions in tinea, in these inflammatory cases, are sharply defined, and the pustules are always situated round the hairs. Seborrhœa differs from tinea tonsurans in the greasiness of the scales, the absence of bare patches, and the diffusion of the condition over the whole scalp. For the diagnosis from favus, see p. 413.

Prognosis.-However long the disease may last, it usually ends in cure-at puberty, if not before. I have, however, seen a few cases in which the disease has lasted from childhood to beyond the age of 25 . Permanent baldness sometimes results from the injurious application of irritants, such as croton oil, and small bare spots are sometimes left owing to destruction of hair-roots by kerion. Bald spots are also occasionally left in cases in which there has been neither artificial irritation nor suppuration. Kerion naturally tends to the cure of ringworm, the diseased hairs being cast off.

Apart from the nature of the soil, age greatly mitigates the disease. Other things being equal, ringworm of the scalp in a child of 15 is usually much milder than in a child of 10 . The constitutional state appears to have no influence either on the severity or on the duration of the affection. Some of the most persistent cases that have come under my notice have been in perfectly healthy children.

The question of immunity is not altogether determined. Children often have what appear to be second attacks of ringworm, but such cases are probably for the most part instances of relapse rather than recurrence.

Treatment.-This consists in the destruction of the fungus and the removal of the diseased hairs. These objects are attained (1) by epilation, effected by X-rays 
or otherwise; (2) by measures directed to the removal of the superficial parts of the epidermis, so that free access may be gained to the fungus; (3) by the application of parasiticidal agents, which may act $(a)$ directly on the fungus and $(b)$ on the tissues, making the soil unfavourable to its growth.

By the use of $X$-rays the treatment of tinea tonsurans has been revolutionized. By this means cases that took many months to cure can be cured in a few weeks. The efficacy of radiotherapy consists simply in the mechanical removal of the fungus-which is not killed by the rays-with the diseased hairs. It must, of course, be applied with judgment and caution, so as to avoid the risk of setting up a dermatitis. But the supposed danger of arresting the development of the child's brain is quite baseless. Of all the thousands of children whose scalps have been irradiated, there has not been a single case of ascertained injury to the brain.1 Sabouraud even cites a case in which a child whose anterior fontanelle was still open was $\mathrm{X}$-rayed with impunity. But it is assumed that the rays would not be applied to the scalps of infants before the fontanelle had closed.

Epilation may be brought about by a single exposure or by a series of exposures. The difficulty of estimating the exact amount of rays required to produce epilation is to a great extent overcome by employing SabouraudNoiré pastilles of platino-cyanide of barium, which change to a standard colour after exposure to the rays for a certain length of time. I do not, however, find it advisable to rely upon this method alone; the milliamperemeter, amperage in the primary circuit, spark-gap, appearance of the tube and anode, time of exposure, etc., should all be carefully watched and

1 See "The Present Position of X-Ray Treatment of Ringworm," by S. E. Dore (Lancet, 1911, i. 437). 
kept as constant as possible, so as to avoid any fallacies due to the pastille. Occasionally impetigo may occur owing to lowering of the vitality of the part as the result of the raying, and to consequent infection with staphylococcus. In cases in which complete epilation is necessary the Kienböck-Adamson method ${ }^{1}$ may be recommended. Five equidistant points are taken, each of which is irradiated in turn. The rays are so directed that where overlapping occurs their incidence is only just sufficient to produce epilation. Cylindrical or lead-foil localizers are not therefore necessary, an even radiation is secured, and the time occupied in raying the whole scalp is reduced from $3 \frac{1}{2}-4$ hours to about $1 \frac{1}{2}$ hours.

If the older methods of treatment be employed, the first thing to be done is to get a clear field of action by epilation. As many of the diseased hairs as possible should be picked out individually with forceps. Not only should all hair that is visibly affected be removed, but also a ring of sound hair around the seat of disease, in order to prevent its spreading. If done carefully, epilation causes but little pain. In quite young children epilation should be, if possible, avoided, but in all patients the hair should be kept clipped to a length of about half an inch, so that the whole scalp may be easily inspected. Commencing spots, and diseased areas generally, may be painted with an aniline pencil, as advised by Colcott Fox; and a head-covering of linen or other cool and inexpensive material should be worn day and night. As an unirritating parasiticide, which also helps to disclose diseased areas that may have escaped detection, Sabouraud recommends that tincture of iodine diluted with 3 volumes of 90 per cent. alcohol or eau-de-Cologne be applied to the healthy surface of

1 "X-Ray Application for Cure of Ringworm of Scalp," by H. G. Adamson (Lancet, May 15, 1909). 
the scalp. Other unirritating parasiticides are carbolized glycerine and vaseline. In a recent case the application of strong iodine or blistering fluid may, as in ringworm of the body, be the means of removing a large quantity of the fungus; but this should not be done too frequently lest thickening should ensue from the inflammation.

Next, a free way should be opened up into the interior of the follicles by clearing away obstructing fat and epithelial débris from their orifices. For this purpose the parts should be washed with spirit and ether lotion, which will dissolve fatty substances and dehydrate the tissues. The application of mild antiseptic washes is advisable. A good lotion for the purpose is salicylic acid dissolved in chloroform or ether (gr. v. to $\mathrm{xx}$ ad $3 \mathrm{j}$ ) ; this dissolves the fat, dehydrates the tissues, loosens the hairs, and directly attacks the fungus. By the use of salicylic acid in this form, if applied sufficiently early, before the fungus has had time to reach the deeper part of the follicle, a rapid cure may be effected. It is an essential condition of success that no fatty substances be used.

Such remedies, however, penetrate but a short distance into the epidermis. In a chronic case more powerful remedies are required, in order to set up a curative dermatitis. In such circumstances chrysarobin is the most effective agent. Unna has pointed out that the scalp does not react strongly to the drug. Duhring also speaks very highly of chrysarobin. He applies it in the form of an ointment containing from gr. $\mathrm{xv}$ to 3ii of chrysarobin to the ounce, the strength in common use being $3 \mathrm{i}$ to the ounce. A small quantity of the ointment is well rubbed in with a bit of cloth or a mop. Hodara uses a solution of equal parts of glycerine and chloroform, containing 5 to 10 per cent. of chrysarobin. Sulphur is very useful in the case of young children; 
it may be combined with mercury, salicylic acid, etc., in an ointment with a lanolin base. A formula that may be recommended is-Sulphuris $3 \mathrm{i}$, acidi carbolici $3 \mathrm{ss}$, lanolini c. oleo $z^{\mathrm{i}}$. Mercury, in the form of the perchloride, the biniodide, the oleate, the nitrate, or the red oxide, may be used in lotions, ointment, or plaster, but it should never be employed for very young children or for persons with a sensitive skin, and in strength it must always be adapted to the tolerance of the patient and its effects carefully watched. A good formula for the perchloride is-Hydrarg. perchlor. gr. vi, glycerini ziss, acidi acetici dil. ziiss. An explanation of the enormous number of remedies found useful in chronic ringworm is to be found in the fact that whatever excites inflammation is so far beneficial. This is the mode of action of Coster's paste, strong sulphur and mercurial ointments, oleate of copper, carbolic acid, etc., all of which are useful.

Lastly, in some cases a more destructive inflammation, producing a condition resembling kerion, is required, the object being to excite an inflammatory process and perhaps local necrosis, whereby the fungus, as well as the affected tissues, shall be involved in the destruction. Aldersmith uses croton oil for the purpose, and this substance is strongly recommended also by Sabouraud and by Colcott Fox, the latter of whom considers it to be the most effective of all drugs. An ointment containing $3 \mathrm{i}$ of croton oil to $\overline{3}$ of base is rubbed in daily, or as often as is necessary to excite the required degree of inflammation, and the application is continued until all the affected stumps have fallen or been epilated. Pus is regularly cleansed away and crust formation prevented. Needling with croton oil may be used for refractory stumps. The croton-oil treatment demands judgmentand care, and should not be undertaken by the inexperienced. Besnier went so far as to say 
that ringworm could be cured only by inflammation, as there was, in his opinion, no remedy that could destroy the fungus. Vidal's treatment is based on the fact that the fungus, being aerobic, can be destroyed by deprivation of air. The hair having been cut close, the head is rubbed with essence of turpentine and the affected parts are painted with tincture of iodine. The head is then smeared with vaseline, either pure or containing boric acid or iodine (1 per cent.), and covered with a caoutchouc cap or a guttapercha leaf which must be kept closely applied to the scalp with a bandage. The dressing is renewed morning and night, and the parts are washed with soap and water and carefully dried.

In schools special precautions are required to prevent the spread of ringworm. When a case is discovered the patient should at once be isolated, and a careful examination of each child should be made day by day.

\section{Tinea Sycosis, or Tinea Barbae}

Ringworm of the chin and other hairy parts of the face is a follicular inflammation set up mainly by endo-ectothrix trichophytons derived from the horse, the calf, etc., but caused in some instances by endothrices that yield the acuminate and violet cultures.

Symptoms.-The initial lesion is a red scaly spot, which soon enlarges; sometimes it undergoes involution in the centre, and forms a ring; in other cases it retains the character of a patch, with a defined margin and scaly surface. As similar lesions develop, they often become confluent. Pustules, each of which is traversed by a hair, form both on the surface of the patches and on the intervening skin. The eruption is accompanied by considerable itching. A more severe form of the affection, which corresponds to kerion of the scalp, is sometimes met with; 
the inflammatory process is more intense, and spreads rapidly; there is brawny infiltration of the skin of the chin and sides of the face, the surface of which is thrown up here and there into irregular lumps, and is thickly studded with hair-pierced pustules. The hair is loosened, but as a rule not damaged, except in very obstinate cases. The suppurative process may, however, be suffciently severe to destroy the follicles, leaving permanent scars, on which no hair can grow. The affection may persist indefinitely.

Tinea sycosis is communicated by contact with infected persons-especially children-or animals. The shaving-brushes and other instruments used by barbers are very often the medium of conveying the disease. This form of ringworm is naturally almost confined to the male sex, and it is most common in young adults.

Pathology.-The affection is a folliculitis and perifolliculitis, generally running on to suppuration. The process begins in the interior of the follicle, and spreads outwards, loosening the hair-shaft from the wall of the follicle.

Diagnosis.-The diagnosis between tinea sycosis and coccogenic sycosis will be found at p. 559. Eczematous folliculitis is distinguished from tinea sycosis by the fact that serous discharge is, or has been, a feature of the process; moreover, there is little or no loosening of the hairs, and the affection is not confined to the hairy parts, nor are the patches ring-like in form. The ring-formation is absent also in seborrhoea, nor is the hair involved. In severe cases of tinea sycosis the single tumour-like formation may be mistaken for a carbuncle; but the inflammation, and the swelling and pain, are never so marked as in carbuncle. Beard ringworm should not be confused with circinate syphilide, for in the latter condition the border of the lesion is darker in colour and more infiltrated, and there is 
either atrophy or pigmentation, or both. Whenever the diagnosis is in doubt the microscope will determine it by revealing the presence or the absence of the trichophyte.

Prognosis.-The prospect is good as regards ultimate cure, if the patient will persevere with suitable treatment.

Treatment.-This should be conducted on the same general principles as that of ringworm of the scalp. $X$-rays should be used when possible, but they require great care; if there is much suppuration, raying must be deferred until this has ceased. In cases in which radiotherapy is impracticable, epilation with forceps should be carried out piecemeal. This will give exit to the pus; incision is never required. Parasiticides must be applied, their nature and strength being carefully adapted to the condition of the affected parts and the susceptibility of the patient's skin. Chrysarobin, in the form of an ointment (gr. $x$ to $3 \mathrm{ss}$ of the drug to $z_{i}$ of lanolin or lard), is the most effective application. Sulphur or oleate of copper is useful in the milder forms of the affection. The case must be kept under observation for a long time after apparent cure.

For the prevention of the disease it would be well to follow the example of certain legislative authorities in Germany, France, and in some of the United States of America, which have made it compulsory on all barbers and hairdressers to disinfect their instruments thoroughly every time they are used.

\section{Tinea Circinata}

Ringworm of the hairless skin is almost always caused by trichophytons, but very occasionally, as Darier has pointed out, it is due to microsporons, usually $M$. lanosum.

Symptoms.-After an indefinite period of incubation 
-the length of which depends on the thickness of the epidermis-the affection begins as a small red spot, slightly raised, and having a well-defined border. This spot gradually spreads at the edge, its surface meanwhile becoming more or less scaly. As it extends peripherally the redness disappears in the centre, leaving a slightly discoloured branny area, which forms the inside of a red ring. The circle gradually enlarges like the fairy rings of the mushroom, without any widening of its edge, and it may expand so as to enclose a considerable area. There may be only one such ring, but more frequently there are several, and in that case adjoining ones may run together into festooned patterns. There is generally no tendency to symmetrical arrangement or grouping of the rings; occasionally, however, two or more rings are placed concentrically. The lesions are often situated on the face, neck, hands, wrists (Plate 29), or other exposed surface. In rare cases the buccal mucous membrane may be involved by extension from the skin. Whitfield has shown, first in 1908, and again and more fully in 1911, that in the hands and feet ringworm, both ordinary tinea circinata and tinea cruris (eczema marginatum), may simulate acute eczema of various types, vesicular, vesico-bullous, vesico-pustular, etc. ${ }^{1}$ Not infrequently involution does not take place in the centre as the edge advances, and the lesions take the form of patches instead of rings. These patches have a clearly defined border, but they are not always circular or oval in outline. The process is usually accompanied by inflammation, the intensity of which varies according to the idiosyncrasy of the skin. The ring or patch often becomes the seat of papular or vesicular eruption, and pustules may develop. Occasionally the neighbouring lymphatic glands are slightly enlarged.

1 Lancet, 1908, ii. 237. Brit. Journ. Derm., 1911, xxiii. 35. 


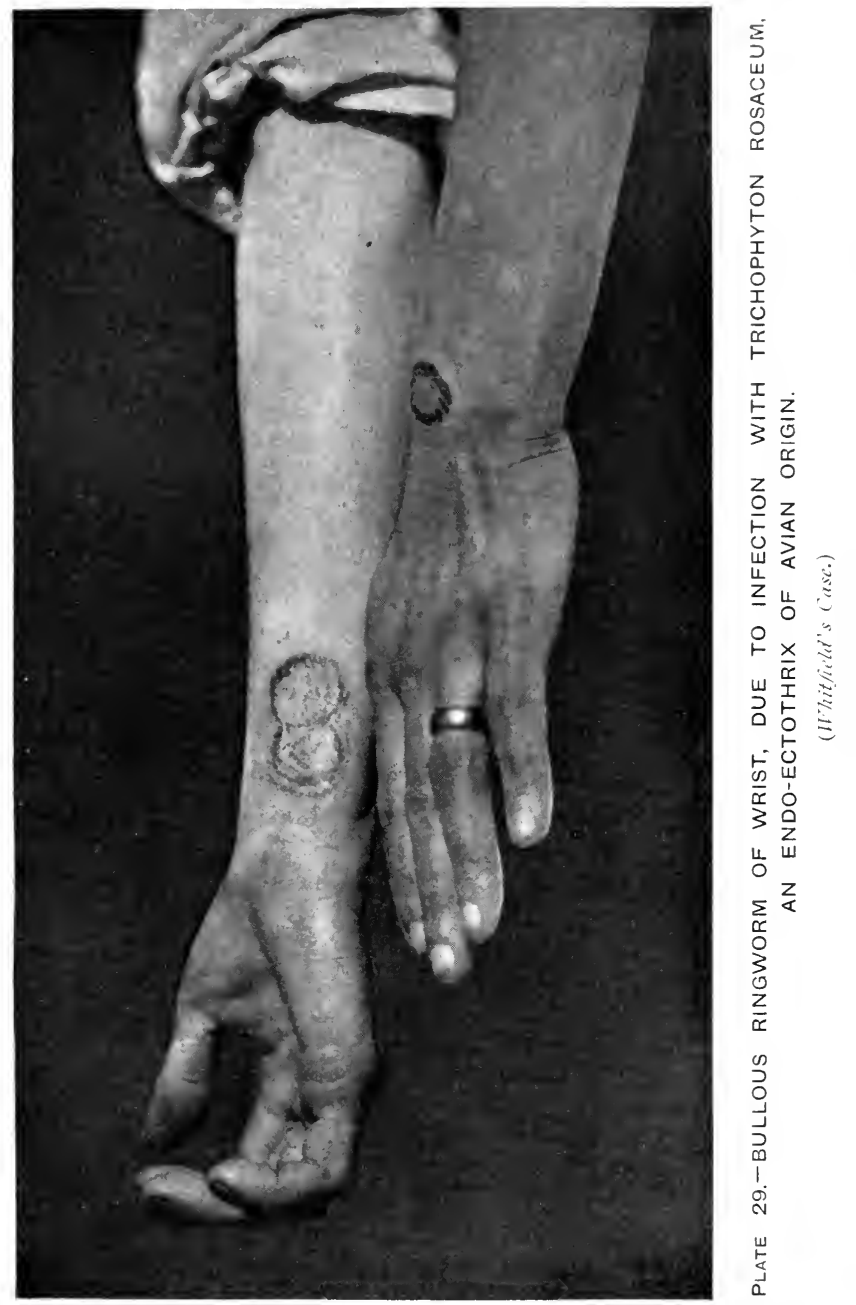


Diagnosis.-The recognition of tinea circinata seldom presents much difficulty, the lesions usually having a characteristic appearance. The presence of the fungus is conclusive, but it must be admitted that its discovery is not always easy even to an expert. In psoriasis the skin is affected in ring-like areas, but in all other respects the clinical features of the two affections are different. In the hands and feet tinea circinata may so closely imitate acute eczema (p. 394) that the true nature of the affection can only be determined by discovery of the fungus. In eczema seborrhoicum the scales are greasy, and frequently there are projections into the glandular openings. The points of distinction from the circinate syphilide are similar to those in tinea sycosis (p. 392). For the diagnosis from pityriasis rosea, see p. 351 .

Prognosis.-This is always favourable, there being no difficulty in bringing parasiticides to close quarters with the fungus.

Treatment.-The most effectual mode of procedure is the mechanical removal of the superficial layers of the epidermis by the application of iodine, liquor epispasticus, or some other blistering fluid. In this way the whole of the fungus may be destroyed at once. If some be left in the deeper layers of the rete, below the limit of the action of the blister, the applica. tion of a parasiticidal remedy will speedily destroy it. The most effective agent for the purpose is chrysarobin, which may be applied as an ointment composed of gr. $\mathrm{xx}$ of the drug to zi of lanolin, or in the form of Unna's ung. chrysarob. co., which consists of 5 parts of chrysarobin, 2 parts of salicylic acid, 5 parts of ichthyol, and 100 parts of unguentum simplex. Whitfield speaks highly of salicylic and benzoic acid ointment, 3 per cent. of the former and 5 per cent. of the latter in 1 part of soft paraffin with 3 parts of cocoa-nut oil. It must be used 
with discretion, for while it produces no immediate irritation, it may, if rubbed into tender parts too vigorously, give rise to a good deal of dermatitis a few days later. Other useful applications are ointments composed of oleate of copper or oleate of mercury 10 per cent.; or sublimed sulphur gr. iii, acid carbol. $\mathrm{m}_{\mathrm{xx}}$, lanoline 3ii, ol. oliv. 3ii ; either of these should be rubbed in thrice daily; For young children a milder application, such as hydr. ammon. gr. iii, lanoline or lard そi, should be used.

\section{Ringworm of the Nails}

The nails may be attacked by trichophytons, either in association with ringworm on some other part of the body or independently, especially in nurses who have to look after children suffering from the disease.

Symptoms.-The first sign is the appearance of greyish stains under the free border or the lateral borders of the nail, and at the root. Inflammation of the matrix is set up, and the nail becomes thickened, lustreless, uneven, and brittle. Exfoliation takes place, and under the free border is seen a mass of disintegrated nail structure, in which the fungus may be discovered.

Diagnosis. - Ringworm of the nails has to be distinguished from the onychomycosis of favus. In the latter affection the stains under the borders of the nails are yellow rather than greyish, and the mycelial elements are shorter and more irregular. From affections of the nails due to constitutional disorders, such as gout and rheumatism, or to such inflammatory affections as eczema and psoriasis, ringworm of the nails can be distinguished by the presence of the parasitic elements.

Prognosis.-Although the lesions may clear up spontaneously, they may persist for five years or longer, in spite of treatment. 
Treatment. - The affected nails should be thoroughly scraped, and chrysarobin or some other parasiticide applied. The treatment used by Harrison, of Bristol, for ringworm of the scalp is particularly useful for the disease as it affects the nails. He uses two solutions, No. 1 composed of liquor potassa and distilled water, $\bar{a} \bar{a} \bar{\zeta} \mathrm{ss}$, and iodide of potassium $3 \mathrm{ss}$; and No. 2 consisting of hydrarg. perchlor. gr. iv, spir. vini rect. and distilled water $\bar{a} \bar{a} \bar{\zeta}$ ss. The nail having been scraped, No. 1 is applied on lint under oiled skin for fifteen minutes; then No. 2 is immediately applied in the same way, and kept on for twenty-four hours. The nail is now again scraped, and the applications are repeated as often as may be necessary. H. Fournier recommends the removal of the whole of the affected parts by scraping, scratching, or avulsion, and by the action of various local remedies such as creosote, acetic acid, benzine, corrosive sublimate (2 per cent. in alcohol or chloroform), mercurial plaster, resorcin, or tincture of iodine. The two last named, combined with previous maceration of the nail by means of indiarubber coverings, are those which Fournier found most successful. Norman Walker's method, which Cranston Low ${ }^{1}$ has successfully employed, consists in soaking the nails under lint and finger-stall with ordinary Fehling's solution for a day or two, removing the softened nail, which easily comes out, and dressing continuously with copper sulphate of a strength of gr. $\mathrm{x}$ to an ounce of water.

\section{Eczema Marginatum (Tinea Cruris)}

This form of ringworm, which specially attacks the pubic, anal, perineal, and axillary regions and other parts where folds of the skin are in contact, was named by Hebra, whose description of it has become classical. But it is not essentially an eczema, and tinea marginata

${ }^{1}$ Edin. Med. Journ., Feb., 1911. 
would be a more appropriate designation. It is more common in the tropics than in Europe, and is at times epidemic.

Symptoms.-The characteristic features of the lesions are their broad, well-defined margin, scaly, and for the most part rough with papules. The mucous membrane of the vulva may be involved, as well as the skin of the regions enumerated above. The process often assumes an eczematoid character, though not a true eczema. Men are more often attacked than women, and young more often than middle-aged or elderly men.

In connection with eczema marginatum attention may be drawn to some anomalous cases of trichophytic infection. Whitfield, in a paper already referred to (p. 394), has reported three such cases in which the clinical features of the eruption were not characteristic of the disease. In the first case the skin and nails of the toes of a woman who had lived in China were affected by a condition resembling eczema; in the second case a man and his wife and maid suffered from peeling and itching of the soles of both feet, and the wife and maid also contracted the disease slightly in the palm from washing his stockings. In the third case, that of a young woman, there was a vesico-bullous eruption on the dorsal surface of the right forefinger and right hypothenar eminence, similar to the acute eczema of the hands often seen during great heat. Microscopic examination revealed trichophytic mycelium in all the cases, as it did in a fourth, in which the toes had been the seat of an eruption for nine years (Plate 30, Fig. 2 ). Sabouraud ${ }^{1}$ afterwards described a case of trichophytic infection, due to the fungus of eczema marginatum, the Epidermophyton inguinale, in which the patient, after being cured, returned with eczematiform or dysidrotic vesicles on the fingers and toes. The

1 Ann. de Derm. et de Syph., No. 6, June, 1910, p. 289. 
patient gave a history of having communicated the disease to someone else, and the scales were therefore examined, and showed the characteristic mycelium of eczema marginatum. In four later cases of intertrigo or intertriginous eczema of the toes, Sabouraud has found parasitic cultures showing the characters of the Epidermophyton inguinale.

Such cases as these of Whitfield and Sabouraud point to the necessity, in chronic eczema between the toes and fingers, especially in those who have lived in the tropics, of making a careful microscopical examination to determine whether the affection is parasitic. I have seen many cases, in Army officers and in schoolboys, of lesions on the toes which had all the appearance of eczema, but which were really manifestations of eczema marginatum. In one case the disease had lasted nine years, and was cured in a few weeks when the correct diagnosis was made.

Etiology.-The parasite of eczema marginatum is the Epidermophyton inguinale, which Sabouraud includes among the trichophytons. But in habits it is unlike any other known species of trichophyton, nor can it be traced to any source outside man. Alone among the trichophytons it never attacks the hair, but remains in the stratum corneum. It is remarkable for the rapidity with which, under culture, it develops degenerate forms. The disease may be communicated in sexual intercourse, and in the contact of family life.

Diagnosis.-From eczema seborrhoicum this affection can be distinguished by the ring-like formation of the early stage, the broad, elevated margin of the lesions, and their gradual spread. If any doubt is left, the parasite should be sought with the microscope.

Treatment.-Chrysarobin ointment (3ss of the drug to $3 \mathrm{i}$. of lanoline) may be applied, or a 5 per cent. naphthol ointment, or a 1 per cent. solution of naphthol 
in alcohol. Jamieson advises the free application, several times a day, of a freshly prepared solution of sulphurous acid; Sabouraud, vigorous friction with a hard pencil moistened with tincture of iodine $10 \mathrm{grm}$., and alcohol (90 per cent.) 100 grm., the treatment to be continued from ten to fifteen days.

Eczema marginatum is frequently known as dhobie's itch, but this expression has a much wider designation, being colloquially used in the East for all epiphytic skin diseases, from the notion that they are contracted from clothes that have been to the dhobie (washerman), but particularly for diseases that specially affect the inguinal region and the axillæ. Castellani holds that dhobie's itch, which he treats as synonymous with eczema marginatum, may be produced by three different species of epidermophyton-Ep. inguinale, mentioned above; Ep. Perneti, described by Pernet; and Ep. rubrum, so called from the deep-red pigmentation of its cultures in glucose, Sabouraud's medium, and mannite agar. Manson's view is that many cases of dhobie's itch are produced by Microsporon minutissimum and $M$. furfur. 


\section{CHAPTER XVII}

\section{PARASITIC AFFECTIONS}

\section{VEGETABLE PARASITES (Concluded)}

\section{Tinea Imbricata}

THis form of tinea, known also as Tokelau ringworm, is distinguished by a concentric arrangement of closely set rings of scaly epidermis. Formerly peculiar to certain Eastern oceanic tropical climates, it has more recently been encountered as far westwards as Burma and as far northwards as the coast of China, as well as in many of the islands of the South Pacific and in the interior of Brazil. I have no personal knowledge of tinea imbricata, and the following account is mainly derived from Manson, who named the disease and discovered the fungus.

Symptoms.-The lesions may at first be confined to one or two spots, but they soon spread over the greater part of the body, usually, however, sparing the hairy regions, including the scalp. The concentric rings of scales which form the characteristic lesion, about $\frac{1}{8}$ inch apart, spread at the edge both centrifugally and centripetally. The scales, resembling pieces of tissue paper, are firmly adherent at the opposite edge, and the free border of each is towards the centre of the circle or system of circles to which it belongs. When they separate, parallel lines, more or less concentrically arranged, are left; these represent the fungus as it proliferates and advances under the young epidermis. Nearly the whole of the cutaneous surface is sometimes 
involved, but the disease has no effect upon the general health. The only subjective symptom is itching, which is usually intense.

Etiology.-The parasite (Plate 31, Fig. 3), regarded by Sabouraud as a trichophyton resembling species of animal origin met with in Europe, was designated Endodermophyton concentricum by Blanchard; it is known synonymously as Trichophyton Mansoni (Castellani). Tribondeau, on the ground of aspergillus-like fructifications which he describes, maintains that it is a lepidophyton $\left(\lambda_{\varepsilon \pi i s}=\right.$ scale), and his results have been confirmed by Wehmer. Castellani and Chalmers, on the other hand, have never observed such fructifications in the fungus. Castellani has succeeded in cultivating both the Endodermophyton concentricum and an allied species, E. indicum, and in both instances has produced the disease experimentally by inoculation of the cultures. ${ }^{1}$

The incubation period lasts on an average nine days. Neither sex is exempt, and children are particularly liable.

Diagnosis.-The imbricated scales and concentric rings are so characteristic that there is little possibility of the affection being confused with any other. From tinea circinata it is at once distinguishable by the centripetal spread of the process, the abundance of the fungous elements, the absence of pronounced inflammation or congestion of the rings, their concentric disposition and the large size of the scales. It differs from ichthyosis in the concentric arrangement of the scales, their peripheral attachment, and the presence of the fungus.

Prognosis.-There is no tendency to spontaneous recovery. The disease is refractory to treatment, and after a few months relapses frequently occur.

Treatment.-Parasiticides such as strong linimentum 1 Journ. of Trop. Med., 1911, xiv. 81. 


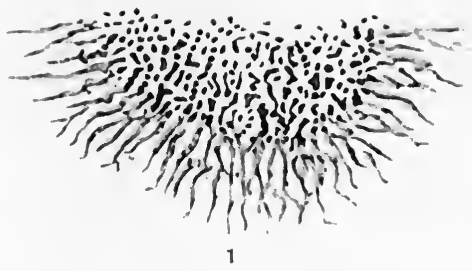

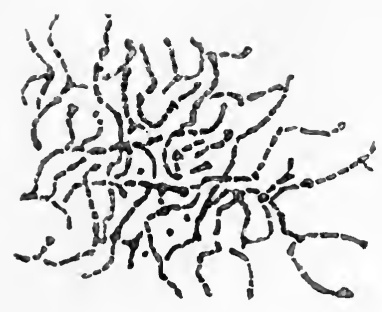

2

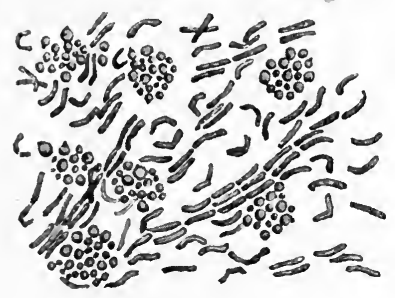

4

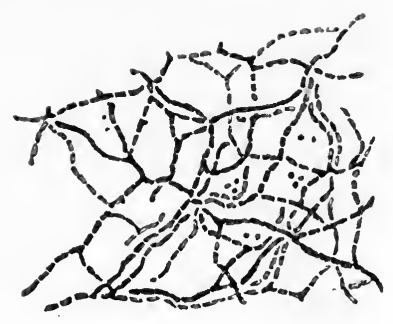

3

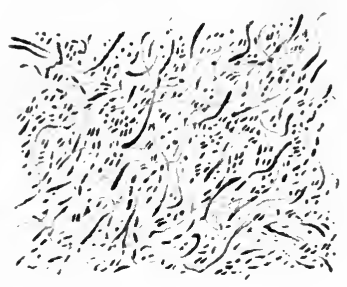

5

Fig. 1.-SCUTULUM OF FAVUS, SHOWING FUNGUS.

FIG. 2,-FUNGUS OF TINEA CIRCINATA.

FIG. 3.-FUNGUS OF TINEA IMBRICATA.

FIG. 4. MICROSPORON FURFUR.

FIG. 5.-MICROSPORON MINUTISSIMUM 


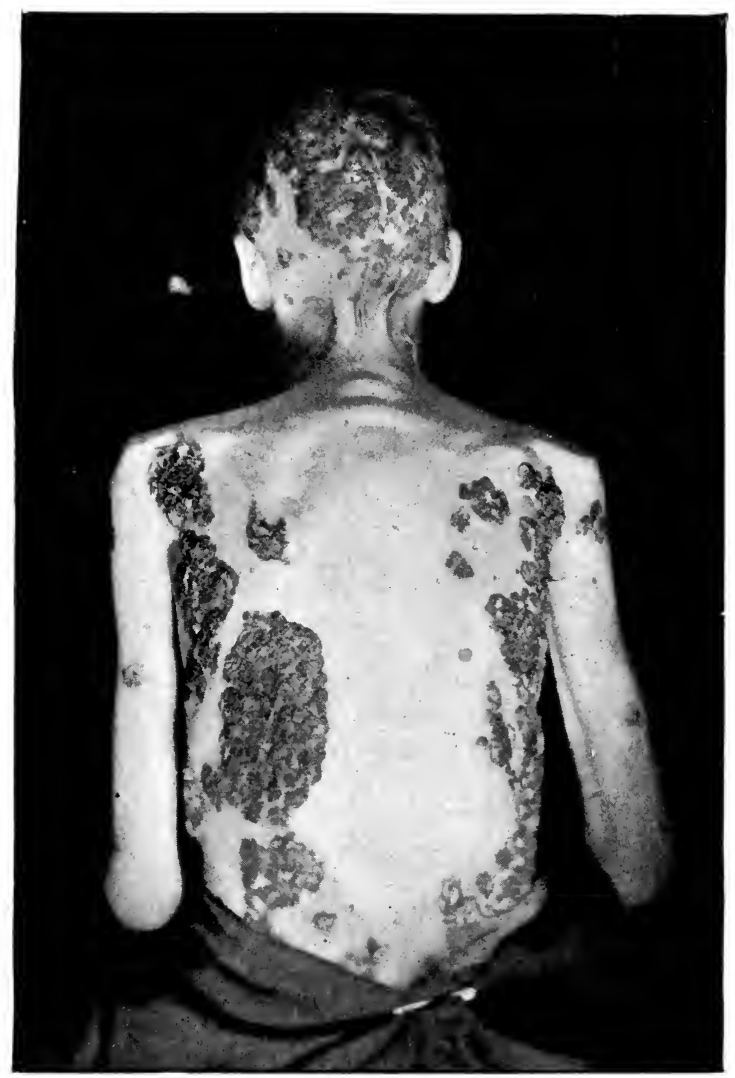

Plate 32.-Authior's case of favus.

(Brit. Joum. Dernu., ISgr.) 
iodi, or resorcin 3 ii dissolved in tincture of benzoin $\overline{3} \mathrm{i}$, or chrysarobin ointment (gr. $\mathrm{xx}$ to $\overline{\mathrm{z}}$ ), should be applied, or bruised Cassia alata leaves rubbed in. Chrysarobin ointment is now in general use among the natives of Tahiti as a prophylactic. The clothes, etc., should be disinfected or destroyed.

\section{Tinea Versicolor (Pityriasis Versicolor)}

This affection of the horny layer of the epidermis was styled pityriasis versicolor by Willan, by whom it was transferred from the group of pigmentary stains to that of desquamative diseases of the skin. The great variation to which the colour of the lesions is liable, not only in different patients, but in different regions in the same patient, is indicated by the specific name of the affection.

Symptoms.-The lesions of tinea versicolor are roundish, slightly raised, scaly patches, with a welldefined border; they are sometimes discrete and irregularly scattered about, but more often they are fused together so as to form large irregular areas. The trunk is generally the only part affected, and the front more than the back; occasionally the upper parts of the limbs are invaded. I have also seen the patches on the face, and similar cases have been reported by Besnier, Payne, and Dubreuilh. The characteristic feature of the lesions is the peculiar brownish discoloration which they present. The shade varies from "fawn" to "liver"; in persons who have lived in hot climates it is sometimes black, while in coloured races it is grey or white. The discoloration is quite superficial, affecting only the uppermost layers of the epidermis, so that it can in great measure be scraped away with the finger-nail. The patches, as a rule, spread very slowly. The only subjective symptom caused by the affection is itching, which is not generally very pronounced. 
In persons who perspire freely, however, the lesions may be the seat of slight inflammation, and even of an eczematoid process. In such cases there may be intense itching.

Etiology.-The parasite of tinea versicolor is the Microsporon furfur, of which cultures are grown with great difficulty. If a scale be removed from a patch and treated with potash, the parasite will be found in abundance. The spores are rounded, with a double contour, and $3-5 \mu$ in diameter; as a rule, they are grouped together in masses, like bunches of currants, between which can be seen short threads of mycelium, 3-4 $\mu$ in diameter, double-contoured, flexuous and occasionally $U$-shaped, some of them continuous, others formed of several cylindrical segments, divided by transverse septa. (Plate 31, Fig. 4.)

The disease is contagious, but the fungus requires a particularly favourable soil and prolonged contact before it can take root. Tinea versicolor has been produced by experimental inoculation both in men and in animals (Köbner). Rarely met with in young children, it occurs chiefly in early adult life, and men are rather more liable to attack than women. Profuse sweating prepares the soil to some extent for the fungus, and for this reason phthisical subjects are especially liable to attack. Neither good health nor scrupulous cleanliness, however, is an absolute protection.

Diagnosis. - Tinea versicolor is distinguishable from pityriasis rosea and from eczema seborrhoicum by the usual absence of inflammatory reaction, and from the former affection also by the fact that the limbs are not affected equally with the trunk. The lesions are occasionally mistaken for the pigmentary patches sometimes present in leprosy, and for secondary syphilides, but from these and from the other cutaneous 
manifestations mentioned they may be discriminated by the ease with which the scales can be removed by a stroke of the finger-nail, and by the fungous elements to be found in the scales.

Prognosis.-Untreated cases usually end in spontaneous cure, although they may persist for lengthened periods. They quickly yield to suitable remedies.

Treatment.-This consists in thorough washing with soft soap and warm water, afterwards rubbing the part with a flesh brush, to get rid of the natural oiliness of the skin. The part should then be treated with iodine, which effects a rapid cure, and also brings into view small and ill-defined spots. If the smell of iodine is objectionable, a solution of hyposulphite of soda-3ii to §-or sulphurous acid, diluted to one-fourth with water, may be used. For regions where the skin is delicate, such as the groins or the axillæ, naphthol (1 in 30), sulphur (1 in 20), or salicylic acid ointment may be used.

\section{OTHer Tineas}

Tinea flava, still regarded by some authors as a tropical form of tinea versicolor, is considered by Castellani and Chalmers ${ }^{1}$ to be a distinct affection, due to a fungus which they name the Malassezia tropica, resembling but not identical with the parasite of tinea versicolor. The roundish or oval spores have a diameter of 3.50 to $4.50 \mu$, and a double contour. Except in long-standing cases the fungus is present in the lesions in abundance, and mycelium and spores are often present together in clusters. The affection, which is not attended by itching, runs a very chronic course, and the lesions, beginning as tiny spots, may cover the whole of the face and the chest. The yellow colour from which this tinea is named is of various shades, from deep orange to very light canary.

1 Castellani and Chalmers, "Manual of Tropical Medicine." London, 1910. 
In some cases the healthy skin surrounding the patches appears to be intersected by yellowish, ribbon-like lines. Occasionally the affection co-exists with tinea nigra, due to Foxia (or Microsporon) Mansoni, and manifesting itself in dull, lustreless patches which are much darker than the healthy native skin. Tinea nigra, except on the palms of the hands, readily yields to resorcin ointment, following the application of a salicylic-alcoholic lotion ( 2 per cent.). Tinea flava is much less amenable to treatment. Castellani and Chalmers recommend the daily application of turpentine, followed by inunction with a naphthol or epicarin ointment (2 to 5 per cent.), or a salicylic-resorcin ointment (resorcin 3i, acid. salicyl. gr. $x$, vaseline $z i$ ) ; the treatment must be maintained for several months.

Yet other tineas which Castellani and Chalmers ${ }^{1}$ recognize are tinea albigena, tinea alba, tinea Sabouraudi, tinea nigro-circinata-all of them due to trichophytons-and tinea intersecta. The parasite of tinea albigena is Trichophyton albiscicans; the palms of the hands and the soles of the feet are specially affected, but the nails and the forearms and legs may be involved. Following the rupture of bullæ a diffuse keratosis develops until the palms and soles may be double their usual thickness; this in turn is succeeded by a process of decolorization. Tinea alba, caused by T. Macfadyeni, particularly attacks the arms and legs, but any region of the body, including the face, may be affected; the lesions consist of white, oval, roundish or irregular patches, the margins slightly elevated and sometimes dotted with papules. Tinea Sabouraudi, due to Trichophyton Blanchardi or $T$. Sabouraudi, which cannot be grown on Sabouraud's media, begins as erythematous patches; these become segmentary rather than circinate, and there is troublesome pruritus. In chronic cases there is thickening 1 op. cit. 
of the skin, with lichenification. In tinea nigrocircinata, set up by $T$. Ceylonese, the lesions, appearing mostly on the neck and scrotum, consist of black rings with thick elevated margins which may be pinkish in the upper portion, or covered with a dark crust. Tinea intersecta is due to the Endodermophyton Castellani, of which cultures have not yet been obtained. The affection is named from the fact that when the oval or roundish patches, usually situated on the limbs and the back and front of the chest, shrivel, the brown surface is intersected by cracks which show a white line. As the cracks deepen the epidermis splits into scales which leave white patches.

The treatment of the various affections mentioned in the preceding paragraph consists in the application of such drugs as chrysarobin and sulphur.

\section{Erythrasma}

Though seldom met with in the United Kingdom, this affection of the horny layer is said to be as frequent generally as tinea versicolor, to which it offers some resemblances. The characteristic lesions are brownish or yellowish-red, slightly scaly patches, of which the favourite situation is the genito-crural region.

Symptoms.-The lesions, when they appear in the usual sites, the genito-crural region, may be either unilateral or bilateral. Exceptionally they attack the axillæ, and, in fat persons, the abdominal and submammary folds, and those of the large joints. The colour of the patches varies from time to time, and may be dark-red, tawny yellow, or brownish with a red tint. The flat and scaly surface becomes moist and greasy during perspiration.

Etiology - The parasite of erythrasma is the Microsporon minutissimum, which was discovered by Burchardt in 1859. The spores and threads of mycelium 
are arranged almost the same as those of $M$. furfur (Plate 31, Fig. 5). The spores may be dispersed among the filaments, or massed together, or disposed in little chains of which the elements are only $0.6 \mu$ in diameter. The filaments, of the same diameter as the spores, are long and flexuous, and seldom ramified, and are so profuse and so twined together as to form here and there a network over the epidermic cells. De Michele's claim to have grown a culture with which he communicated the disease to a human subject by inoculation is disputed by Ducrey and Reale, though provisionally accepted by Bodin. Of the morphology of the parasite nothing is known. Like tinea versicolor, erythrasma has but a low degree of contagiousness. Children are not susceptible, and women are less liable than men.

Diagnosis.-Erythrasma is seldom difficult of identification. Exceptionally, it may be confused with one type of eczema marginatum, but it may be discriminated from that affection by its slow evolution and its low degree of contagiousness, and by the absence of inflammation, which also distinguishes it from eczema seborrhoicum and from pityriasis rosea. Any doubt may be cleared up by examination of a preparation under a microscope of sufficiently high power.

Prognosis and treatment.-Erythrasma quickly responds to suitable remedies, but relapse is frequent, and it is necessary to continue the course after apparent cure. Treatment is on the same lines as for tinea versicolor. Darier advises that the affected parts be painted with a solution of permanganate of potash, 1 in 1,000 , or that sulphur, cade, or naphthol ointment be applied. The delicacy of the skin at the sites of election makes it necessary that tincture of iodine, if used at all, should be well diluted. 


\section{Favus}

This affection is so rare in England that the replies to an inquiry on this subject addressed by me to the eleven metropolitan hospital schools a few years ago showed that only thirteen cases had been under treatment at those institutions during the previous year. It is less rare in Scotland, and is comparatively frequent in some parts of France-where it is a not uncommon cause of the rejection of conscripts for military service-in Russia and Poland, in Holland and in Italy. It was almost unknown in New York and other parts of the United States till it was imported by immigrants from Europe.

Symptoms.-The disease shows a marked preference for the scalp, but no part of the skin is exempt, and even mucous membranes are liable to be attacked. On the scalp it first appears as a tiny sulphur-yellow disk or scutulum, depressed in the centre like a cup and pierced by a hair. This is the characteristic lesion of favus. The little disks increase in size and become crusted over, the scutula being sometimes swallowed up in a large rugged scab. About the edge of the scab, however, the little disks can still be seen. The lesion generally takes several months to reach its full development, when the scab and scutula come away, leaving a glistening atrophic pit destitute of hair. The hair in the favus crusts is dry and dusty-looking, and is easily pulled out unbroken ; sometimes it is split longitudinally. No new hair is formed. In severe cases several lesions run together, forming raised crusted patches of irregular outline, which may be of considerable extent. Between the crusts there are often irregular pale, bluishpink scars. The lesions are usually present in various stages of development at the same time, scutula, large crusted excrescences, and scars being intermingled. The 
disease is not infrequently complicated by pediculosis, and there may be more or less suppuration. Itching is present in the majority of cases, but the most characteristic symptom, apart from the lesions, is a musty, mouse-like odour which is given off by the patient.

On hairless parts the lesions present the same general appearance. In a case which came under my observation the whole scalp was covered with large patches of favus crusts (Plate 32): A great portion of the back was occupied with similar masses; there were also crusts on the cheeks. The nails of both hands and both feet, particularly those of the first finger of each hand and the great toes, were thickened, uneven, and lustreless ; in some of them no trace of true horny substance remained, its place being occupied all over the matrix and nail-bed by an irregular, lumpy, dirtyyellowish crust. The disease began when the patient was 23 years of age, and persisted for fourteen years. She died of acute phthisis, which lasted nearly three months, and during that time the favus spread over the body with great rapidity. Kaposi has reported a case in which a patient suffering from universal favus died with symptoms of severe gastro-intestinal irritation, which was found after death to be due to the presence of the favus fungus in the stomach and intestine.

Etiology.-The vegetable nature of the dry pustules of favus was demonstrated by Schönlein, in 1839, but it was reserved for Grüby, not long before he discovered that ringworm was caused by a cryptogamic parasite, to prove, in 1841, that the fungus of favus, which he had independently discovered, was the cause of the disease. In 1842 he announced that he had succeeded in inoculating the skin, both of man and of certain lower animals, with the parasite of favus. In 1845, Remak separated the fungus from the genus Oïdium, to which it had been assigned, created for it the genus Achorion, 
and designated it the Achorion Schönleinii, in honour of his master. Later there appeared to be reason for concluding that more than one species of parasite was concerned in the causation of human favus, but the unity of the human fungus has been maintained by many weighty authorities, including Sabouraud. Bodin, while allowing that human favus can be produced by parasites of animal origin, maintains that such cases are exceedingly rare, and that $A$. Schönleinii is not a genus but a single species, the difference in the cultures being explained by the media employed, or by pleomorphism, and bearing no relation to the clinical variations which are met with. In Sabouraud's experience not one of his two hundred cases of scalp favus was due to any parasite other than A. Schönleinii. The unity of human favus is now generally accepted, and there can be little doubt that $A$. Schönleinii is responsible for quite 99 per cent. of the cases of favus in man. Four animal species of the parasite are recognized-A. Quinckeanum (mouse favus), A. gallina, Oospora canina, and A. gypseum. The spores of $A$. Schönleinii vary greatly both in shape and in size; some are round, others oval, others of irregular form. The segments of mycelium range between $4 \mu$ and $15 \mu$ in length, and from $3 \mu$ to $7 \mu$ in breadth. Both spores and mycelium are composed of granular protoplasm and a membranous substance. (Plate 31, Fig. 1.)

That favus can be communicated from the lower animals to man, and vice versa, is indisputable, but such transmissions are exceedingly rare. ${ }^{1}$ No classification of the favus fungi can be regarded as more than provisional, but $A$. Schönleinii is assigned by Matruchot

1 Adamson, having met with three cases of mouse favus in human beings, doubts the extreme rarity of this form of favus in the human subject. Brit. Journ. Derm., 1911, xxiii. 49. 
and Dassonville, on analogical grounds, to the Gymnoasceæ, like the trichophytons and microsporons of ringworm. The morphological resemblances between it and them are intimate and striking, marked as are the clinical differences. Bodin holds the saprophytic origin of the achorion to be possible but exceptional.

Favus is transmissible by direct contact, or through the medium of clothes, head-gear, etc. It is less contagious than trichophytosis. The chief predisposing cause is lack of cleanliness; and the skins of persons in feeble health, especially those affected with phthisis, appear to offer to the fungus a favourable soil. Favus is a rural rather than an urban disease; according to Darier, nearly all the cases met with in Paris are imported from the provinces.

Pathology.-The disease represents the reaction of the tissues to the irritation caused by the growth of the fungus. The spores generally find their way into the hair-follicles, where they grow round the hair. The fungus grows on the epidermis, the density of the growth causing pressure on the parts below, thus crushing out the vitality of the hair and giving rise to atrophic scarring. The characteristic cup shape is attributed by Unna to growth proceeding more vigorously at the sides than at the centre. The material of the lesion is easily broken up into a greyish dust; when this is done it is seen under the microscope to consist of abundant spores and of short tubes of mycelium. At the base of the cup are slender tubes of mycelium; above these are larger, sporulated tubes; on the surface are short particles of mycelium and spores; in some cases the whole lesion is covered with horny shreds of epidermis. The mycelium may penetrate bene th the disk into the stratum mucosum, and may even reach the derma, a phenomenon which is never observed in trichophytosis, and which, as Darier points out, explains the inflam- 
matory reaction in favus and the subsequent cicatrization. Favic hairs, though discoloured and lustreless, do not break off, as in ringworm. In a favic nail the ungual cells are separated by irregular filaments, which vary greatly in thickness, or by spores.

Diagnosis.-The identification of favus presents no difficulty in well-marked cases, the cup-shaped, sulphurcoloured scabs and mousy odour being characteristic. When, however, the initial lesions have coalesced into dense crusts, the affection may resemble psoriasis of the scalp ; the scales, however, are less pearly, and scutula or sulphur-yellow scabs can often be seen about the edges; the lustreless hair and atrophic scarring are also distinctive features. Favus can be distinguished from eczema and from seborrhoe by the fact that it is not diffuse, as the lesions in those conditions are, but is always surrounded by a well-defined margin. It is sometimes very difficult to distinguish from ringworm, and in some cases the diagnosis can be made only with the help of the microscope, or by culture of the parasite. All the lesions should be minutely examined with a lens for remains of the yellow disks of favus or the broken hairs of ringworm. It is sometimes a good plan to leave the disease to itself for a little time, so as to watch the development of fresh foci, when characteristic elements will be recognizable.

Prognosis.-The outlook as to cure is good, but the disease is sometimes extremely refractory to treatment. As in the case of ringworm, it is much more easily dealt with on hairless parts than on the scalp. In rare cases it comes to a standstill ispontaneously, but ordinarily, if left untreated, it is essentially chronic, and may persist for fifteen or twenty years, or even longer.

Treatment.-This must be conducted on the same general lines as that of ringworm. The crusts must be removed by thorough soaking with carbolized oil; the 
head should then be washed with soft soap. Epilation by X-rays, applied according to the Kienböck-Adamson method (p. 388), should be practised, and finally parasiticides of the same kind as those used for the destruction of the ringworm fungus (pp. 388-91) should be vigorously rubbed in. If the nails are affected, avulsion may be required so as to allow free access of the parasiticidal agent. Zinsser speaks highly of the application of heat by means of Leiter's tubes. The appearance of fresh disks must be carefully watched for ; when found, they should be at once dealt with as before. After apparent cure, the patient must be kept under observation for some time, owing to the tendency to relapse.

\section{Pinta}

Pinta, carate, or "spotted sickness," is an affection endemic in, and almost confined to, the tropical regions of America, though a few cases have been reported from Egypt by Madden and others, from the Gold Coast, and from the Philippine Islands. It is characterized by a peculiar discoloration of the skin, with continuous desquamation.

Symptoms.-Four forms of the affection are described-grey, blue, red, and white, but violet and yellow forms have also been reported; apparently they are all varieties of the same process. In the greyalso called the black-variety, spots of a leaden hue appear on the face, the tint deepening almost to black as they spread. The spots are irregular in shape, slightly scaly, and do not disappear on pressure ; the discoloration cannot be rubbed off. The whole face may be blackened, making the patient look like a negro, but usually there are patches of normal or less discoloured skin. Patches of discoloration also appear on the limbs, especially in the parts rich in pigment and most exposed to the sun, such as the external 
surfaces of the arms and legs, the dorsum of the foot, the back of the hand, the extensor aspects of the joints, etc. The trunk may also be the seat of similar lesions, but the whole of the skin is never invaded. Sometimes there is considerable itching, and then desquamation is more active. After a time the affected surfaces become harsh and rough, and the skin appears to be thickened and more vascular than normal. In this stage the patients often give off a penetrating, musklike odour. There is no sensory or other functional alteration in the skin.

The blue variety also affects the face and the limbs. The spots, which are more irregular in outline than in the grey variety, are of a bluish tint, sometimes of a leaden-grey shade, sometimes violet, sometimes dark indigo blue. The discoloration in some cases occurs in numerous small patches, giving the patient a "spotted" appearance ; in others it is diffused so generally over the body that the prevailing colour of the skin is blue.

In the red variety, which attacks by preference fair persons with a delicate skin, the distribution of the lesions is the same as in the two already described, but the patches of discoloration are smaller. The affected parts are blood-red, or sometimes of the colour of beetroot. The skin is rough and vascular, and is often marked with fissures, which bleed easily. Itching is intense; the skin is dry and hyperæsthetic. This variety is the most contagious. It is often associated in the same person with the two previously described.

The white variety is the terminal stage common to all the others. The spots of discoloration begin to fade in the centre, and gradually die away to a perfectly white tint, especially in parts where the skin is thin, as on the extensor surfaces of joints. In rare cases the spots are yellowish from the first, and soon pass into 
the white stage without ever having been red, blue, or grey. In such cases the disease is limited to certain regions, such as the roots of the hair, the parts about the eyes, and the hands and feet.

There is some doubt whether the disease was imported into America from Africa by the negroes, or whether it is indigenous. At the present day it is so generally prevalent among negroes that it has been said that none of them escape it. ${ }^{1}$

Etiology.-The disease is caused by fungi belonging to several genera-Penicillium, Aspergillus, Monilia, and Montoyella; these fungi are easily cultivated Sabouraud's maltose agar (p. 377). The physicians of Colombia are almost unanimous in looking upon the affection as not directly contagious. In the regions where it is endemic there is a general belief that the fungus is conveyed by mosquitoes. A tropical climate; dirt, pre-existing inflammation of the skin, and the use of water containing a large amount of mineral salts are predisposing factors. Both sexes are equally liable to attack, and no age, except early infancy, is exempt. The affection is rare among well-to-do people.

Diagnosis.-The disease may be mistaken for macular leprosy, but there is no anæsthesia, and the spots do not fade and reappear as in that affection. From leucodermia it is differentiated by the variety of the pigmentation, the itching, and the roughness of the skin. From tinea versicolor it is distinguished by the coloration, and by the distribution of the patches, which are mostly situated on parts of the skin exposed to the light, whereas the Microsporon furfur affects covered regions such as the chest and abdomen.

${ }^{1}$ In the official document already referred to the following words occur: "Puede decirse que todo negro es caratoso o lo será." ("It may be said that every negro suffers or will suffer from spotted sickness.") 

PLATE 33.

FIG. 1.-ACTINOMYCES.

FIG. 2.-FUNGUS OF MADURA FOOT.

FIG. 3.-TUBERCLE BACILLUS (LUNG).

FIG. 4.-LEPRA BACILLUS FROM SKIN.

FIG. 5.-ANTHRAX BACILLUS.

FIG. 6.-TUBERCLE BACILLUS IN GIANT CELLS OF LUPUS.

FIG. 7.-STAPHYLOCOCCUS IN PUS.

FIG. 8.-STREPTOCOCCUS IN ERYSIPELAS.

FIG. 9.-BOTTLE BACILLUS IN ECZEMA.

FIG. 10.-FILARIA SANGUINIS HOMINIS. 

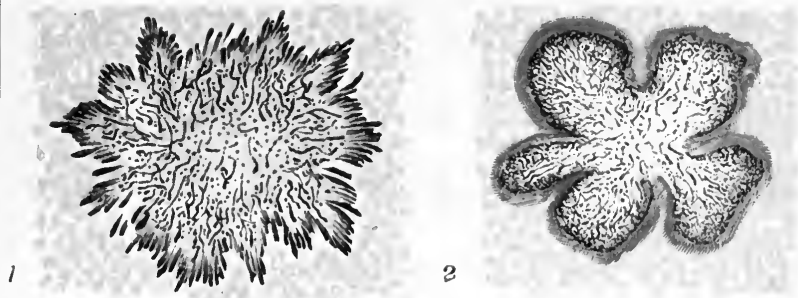

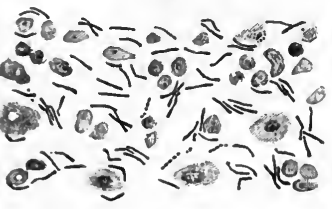
4 बำ

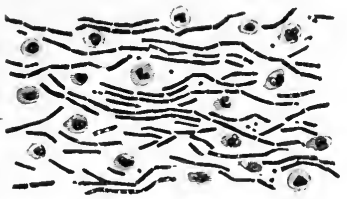

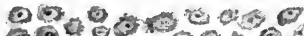
\% a - 615040

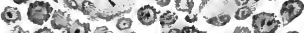

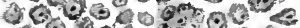

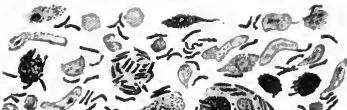

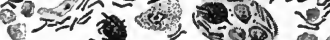
a) 2

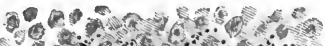

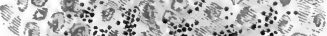
$1.5 \%$ or

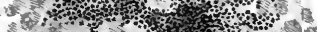

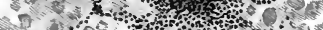

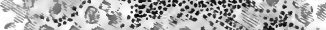
7 कोलीका
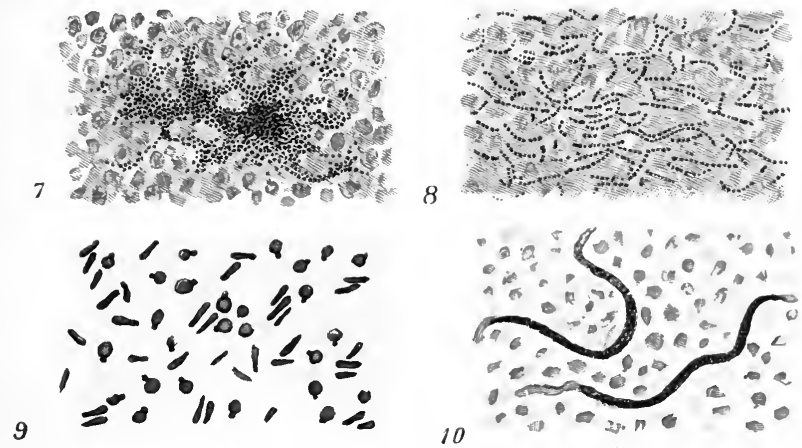

Prognosis.-The disease tends to become chronic, and is very refractory to remedies.

Treatment.-In early cases tincture of iodine may be tried. In more advanced cases chrysarobin yields the best results, but it must be used with caution, and not applied to the face. For facial lesions resorcin, or resorcin-sulphur, or salicylic-acid ointment may be used. In Colombia the favourite remedy is mercury nitrate ointment. With regard to prevention, close contact with patients suffering from the disease should be avoided; and in regions where it is endemic the local practitioners recommend that mosquito stings should be at once treated with an antiseptic application, such as carbolized oil, boric-acid ointment, etc.

\section{Mycetoma}

Mycetoma (Madura foot; fungus foot of India) is endemic in some parts of India, especially in Madura. It occurs in two chief varieties, the black and the pink, or, as Vandyke Carter preferred to call them, " melanoid" and "ochroid." The pink form is the more common of the two.

Symptoms, pathology, etiology.-The distinctive feature of the black variety of mycetoma is the presence in the affected tissues of black granular particles resembling gunpowder in the earlier stages, and in later stages of black or dark-brown truffle-like masses. The latter exhibit a faint pink mould in the earlier stages of development, and at a more advanced period characteristic pale-red, ovoid bodies resembling fish-roe. The pink mould is also visible in the pink variety of mycetoma. The disease as a rule affects the foot or the leg, sometimes the hand; in rare cases the shoulders and the scrotum. On the foot it begins with slight swelling and redness or local induration. In an advanced stage of the disease the foot is greatly swollen, the swollen surface being 2 B 
dotted with little nodules, in each of which is the opening of a sinus; from this comes a thin sero-purulent discharge containing rounded granules. Similar granules are visible on the little tumour around the mouth of the sinus. The pathology is simply disintegration of the foot by the fungus. Nine different varieties of mycetoma, caused by ten different parasites, are recognized, three belonging to the melanoid group and six to the ochroid. Three of the parasites are Discomyces and two of them Aspergilli, the others belonging, according to Castellani, to the Indiella, Madurella, and Oospora genera. (Plate 33, Fig. 2.) They are believed to effect entrance through abrasions or wounds caused by thorns, splinters, sharp stones, etc.

Treatment.-In some forms of the affection potassium iodide has been employed with benefit, but amputation is usually necessary. In the absence of treatment, the disease progresses slowly to a fatal termination. ${ }^{1}$

\section{Actinomycosis}

Actinomycosis (Plate 34) is a parasitic disease which chiefly affects the bones and the viscera, and only in rare cases the skin, but a case of primary actinomycosis of the skin has been reported by Wilhelm Dreyfus, ${ }^{2}$ another by A. Sichard, ${ }^{3}$ and another by D. E Allworthy. ${ }^{4}$ In Sichard's case infection resulted from the skin of the left index finger being cut by a spike of corn, and there was rapid extension of ulceration to the deep parts, the muscles and aponeuroses being destroyed and the periosteal and bony tissue affected.

${ }^{1}$ For further information as to Madura foot, see Manson's “Tropical Diseases," 4th edition. London, 1907.

2 Münch. med. Woch., Dec. 29, 1903, p. 2291.

${ }^{3}$ La Presse Med., Aug. 15, 1903.

4 Report of Belfast meeting of the Brit. Med. Assoc., Brit. Journ. Derm., 1909, xxi. 395. 


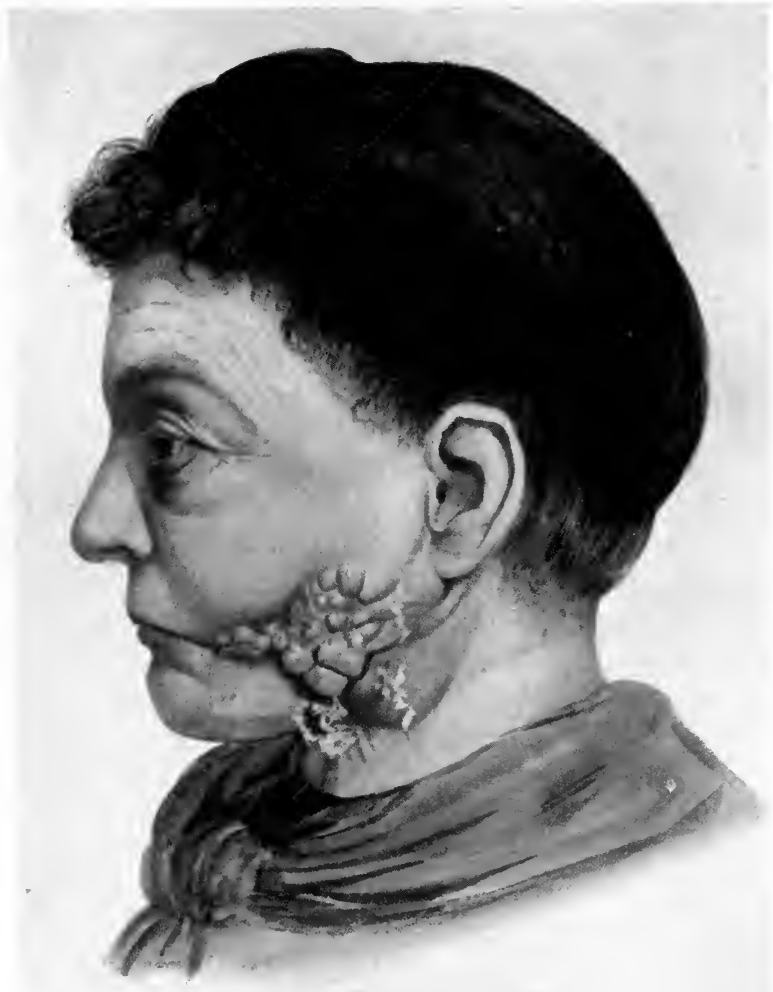

Plate 34.-ACTINOMYCOSIS. 

Deep-seated suppurating tumours are produced in bone or others of the deeper structures, and as these enlarge they gradually approach the surface, the skin over them presenting the usual appearance characteristic of abscess. The process is very chronic, and there is comparatively little pain. In course of time the skin breaks and sero-sanious or purulent fluid, containing peculiar sulphur-yellow granules, is discharged. If these granules are examined microscopically, the actinomyces, the ray-like fungus causing the disease, will be found (Plate 33, Fig. 1).

Etiology.-The ray fungus is believed to be derived from corn or hay. It may be conveyed to man by the sucking of straws, and especially the picking of carious teeth therewith, or by contagion from cattle or horses themselves suffering from the disease, or, in very exceptional cases, from man to man ; in Allworthy's case the medium of contagion was uncooked oaten meal which the patient was in the habit of eating. Males are, from their greater exposure to infection, more liable to the disease than females.

Diagnosis and prognosis.-The diagnosis will be made clinically by a process of exclusion. A tumour, especially if situated in the skin near the jaws, which presents neither the characters nor the symptoms of a malignant growth, a syphilitic gumma, a glanderous abscess, or lupus, should suggest the idea of actinomycosis, and a positive conclusion will be reached by puncturing and examining the contents for the actinomyces. The prognosis depends largely on the situation of the lesions. If these can be thoroughly removed the disease can be cured; otherwise it usually ends in death.

Treatment.-Actinonycosis can sometimes be cured by the internal administration of iodide of potassium alone. The earlier this is begun the surer and speedier 
is the effect. Beginning with gr. $\mathrm{x}$ or $\mathrm{xv}$ three times a day, it should be steadily pushed to gr. $\mathrm{xx}, \mathrm{xxx}, \mathrm{xI}$, or even much larger doses if necessary. Iodide of potassium ( 1 in 100) may at the same time be injected into the sinuses and fissures. Surgical treatment is, however, generally required. This consists in the completest possible removal or destruction of the diseased tissues. ${ }^{1}$ In a case in which iodide of potassium was not well borne, Zeisler, of Chicago, ${ }^{2}$ found that the affection was cured, at least temporarily, by $X$-rays combined with the internal administration of sulphate of copper.

\section{STREPTOThRIX INFECTION}

Foulerton $^{3}$ has described a case which he regards as an example of an infection by a streptothrix other than the ray fungus, while clinically it presented nearly all the features of ordinary actinomycosis, as just described.

The patient was a woman of 46 , who had suffered from an abscess in the left axilla, which had been opened surgically, a sinus running inwards and upwards for nearly five inches being left. After a time a red, painful nodule appeared above the left clavicle; this rapidly increased in size, and the skin over it soon broke. When seen there was ill-defined induration round about, and there were a few secondary swellings near the original one. The skin over the nodule was of a deep-reddish, and in some places purplish, colour; it was dotted here and there with yellow spots

1 See a report of a case of actinomycosis (Plate 34) involving the skin, by the Author (Lancet, June 6, 1896), to which a bibliography up to that date is appended. Poncet, of Lyons, has more recently published a comprehensive monograph on the whole subject.

Trans. Amer. Derm. Assoc., 1906, p. 104.

3 Brit. Journ. Derm., Nov., 1899, p. 417. 
varying in size from a pin's head or somewhat larger to a small pea. The spots marked the position of small abscesses, each of which contained a little thick yellowish pus. In places where these abscesses had broken, small punched-out ulcers remained, from which a thin, blood-stained, purulent discharge exuded. There were physical signs of lung disease. No treatment had any effect, the skin lesion gradually extending over the back of the left shoulder. The clinical evidence pointed to a primary lesion of the left lung, followed by extension of the infection through the chest wall to the skin. After a stay of four months in the hospital, the patient left, and at the date of the report nothing more had been heard of her. Pure cultures of a streptothrix fungus were obtained from the pus of a freshly opened abscess. Although, owing to the difficulty in obtaining free growth of the fungus, its natural history when growing on artificial media and its pathogenicity in animals were not fully worked out, the evidence was, in Foulerton's opinion, quite sufficient to show that it did not correspond with any of the previously described streptothriceæ. More recently, Hayo Bruns, of Strasburg, has published a case which clinically was thought to be one of actinomycosis, but from which he isolated a streptothrix fungus corresponding in some respects with that described by Foulerton.

A list of these streptothriceæ will be found in Foulerton's article. He makes the general statement that the anatomical results of an infection by any one of these usually appear as nodules of granulomatous tissure.

\section{SpOrotrichosis}

Several cases of this disease ${ }^{1}$ have been described by

1 H. G. Adamson gives a good résumé of the subject, with literature, Brit. Journ. Derm., Sept., 1908, p. 296. 
American and French observers, and more recently by Castellani and Chalmers in the tropics, and in 1910 the first case detected in Germany was reported by Arndt, ${ }^{1}$ who divides the affection into "regionally limited" and "disseminated" forms, corresponding with the "simple" and "multiple" forms of various other writers. In 1911, Adamson exhibited a case to the Dermatological Section of the Royal Society of Medicine. ${ }^{2}$

Symptoms.-Sporotrichosis begins with the development of subcutaneous nodules, which in two or three months soften to form abscesses with fistulous openings, discharging a greyish-yellow homogeneous pus. In the "simple" cases reported by Schenk, ${ }^{3}$ and by Hektoen and Perkins ${ }^{4}$ in America, the primary lesion occurred on the index finger and was followed by a train of abscesses along the line of the lymphatics; in the "multiple" cases of de Beurmann and Gougerot, ${ }^{5}$ Gaucher and Monier-Vinard, Cranston Low, and others, the abscesses were widely distributed over the borly. In some of the cases lesions were present in the mucous membranes, but the viscera do not appear to have been involved.

Etiology.-Three forms of the fungus have been identified: Sporotrichium Beurmanni in Europe and Brazil, S. Schenki in North America, and S. indicum in Ceylon. The ovoid spores are $2-6 \mu$ in length, and $1-4 \mu$ in breadth; the mycelial elements are very slender. Cultures are readily obtained, the best medium being Sabouraud's glucose agar (p. 377). Colonies appear

${ }^{1}$ Derm. Zeitschr., 1910, Hft. 1 and 3 (abstr. in Brit. Journ. Derm., 1910, xxii. 363).

2 Brit. Journ. Derm., June, 1911, p. 182.

${ }^{3}$ Johns Hopkins Hosp. Bull., 1898, p. 286.

${ }^{4}$ Journ. of Exper. Med., 1900, p. 77.

${ }^{5}$ Ann. de Derm., 1906, pp. 837, 914, 993. 
on the fourth to the tenth day as small white acuminate points $1 \mathrm{~mm}$. in diameter, surrounded by a white, finely rayed areola, and become brown in colour and convoluted as they increase in size. The fungus has been grown upon caterpillars, flies, larvæ, etc., and upon vegetable substances such as lettuce leaves. It has also been successfully inoculated into animals.

Diagnosis.-The lesions may resemble those of either syphilis or tuberculosis, or they may closely simulate those of glanders. There are also points of histological resemblance between sporotrichosis on the one hand, and blastomycosis and certain forms of deepseated ringworm on the other hand. The only satisfactory diagnosis, therefore, is that furnished by a culture, or, if this should be negative, by testing the power of the patient's serum to agglutinate the spores of the fungus.

Treatment.-The lesions have been found refractory to ordinary surgical measures, but rapidly disappear under the administration of iodides in large doses. The suppurative nodules are punctured and a 1 per cent. iodine solution is injected; dressings of iodine lotions are also used. The treatment must be prosecuted vigorously.

\section{Blastomycetic Dermatitis, or Cutaneous BLASTOMYCOSIS}

At the meeting of the American Dermatological Association in 1894, T. Caspar Gilchrist demonstrated organisms in a case of supposed scrofulodermia under the care of Duhring. Gilchrist suggested that these organisms were parasitic fungi having an etiological relation to the lesions in connection with which they were found. Two years later the same observer published ${ }^{1}$ a case of "blastomycetic dermatitis" in man,

1 "Johns Hopkins Studies in Dermatology," 1896. 
in which he classified the fungus as one of the yeast family. In conjunction with W. R. Stokes, Gilchrist published in 1898 a report of a case of "pseudo-lupus vulgaris" caused by a blastomyces, in which the results of experimental researches on animals were given. The elements of the organism were described as spherical and unicellular, with a double-contoured enveloping membrane, exhibiting budding forms in various stages and never enclosed in cells. A careful study of the skin disease, caused by this blastomyces has been made by Nevins Hyde, L. Hektoen, and A. D. Bevan, ${ }^{1}$ who collected all the cases recorded up to the date of their article, and compared and analysed the facts and phenomena, but other cases have since been reported by Gilchrist, Meneau, and others, and by 1904 over forty cases were on record. The disease is also met with in the tropics.

Blastomycosis in its wider sense, as an affection of the internal organs and structures as well as of the skin, forms the subject of a monograph by Dr. Buschke in the Bibliotheca Medica.

Symptoms.-As far as the symptomatology can be gathered from the cases on record, the affection begins as a maculo-papule of reddish hue, which afterwards suppurates, usually as the result of accidental infection. The papules gradually enlarge into nodules, and the skin over the area involved by the growth becomes raw and ulcerated; the ulcers spread widely, and sometimes extend deeply, destroying parts like the ala nasi and the lip. The ulcers leave scars with raised ulcerating edge, and small ulcers are scattered irregularly over the scar area. The parts affected are the ear, forehead, cheek, brow, nose, scrotum, thigh, leg, and the dorsum of the fingers, hand, and wrist. The parts first attacked are chiefly the dorsum of the hand and the front of the leg, a cir-

${ }^{1}$ Brit. Journ. Derm., July, 1899. 
cumstance which suggests that these regions infect each other. In the seven cases collected by Nevins Hyde, Hektoen, and Bevan, five of the patients were men, all of them at or near middle age; in some of them there was a family history of tuberculosis: Meneau ${ }^{1}$ attempts a division of cutaneous blastomycosis into two groups, the parasite in one being a yeast, in the other a mould.

Diagnozis.-Blastomycetic dermatitis closely resembles tuberculosis of the skin, esperially that form described by Riehl and Paltauf under the title "tuberculosis verrucosa cutis" (see p. 440). The vegetating forms of syphilis sometimes slightly resemble the symptoms of blastomycetic dermatitis. The nature of the disease is recognized by the presence or absence of the blastomyces. The features which are common to both protozoan and blastomycetic affections are-epidermal hypertrophy; the occurrence of multiple abscesses in both epidermis and corium ; a similar distribution of lesions ; and, principally, the general features of both scrofulodermia and tuberculosis of the skin. In a case reported by Sequeira, ${ }^{2}$ and shown at the Dermatological Society of London in 1902, the organism found in the lesions was much smaller than that found in the American cases. It was impossible to cultivate it, owing perhaps to the fact that the lesions examined were already infected with cocci. Sequeira suggests that the organism may have been a different species of blastomyces from those described by Gilchrist, Montgomery, and other American workers.

An interesting case of systemic blastomycosis with multiple cutaneous and subcutaneous lesions is reported by Oliver S. Ormsby and H. H. Miller, of Chicago. The cutaneous manifestations began two months after

1 Ann. de Derm. et de Syph., June, 1902, p. 578.

${ }^{2}$ Brit. Journ. Derm., April, 1903, p. 121. 
an affection of the lungs, and were obviously of internal origin, the infection reaching the skin through the circulation. The subcutaneous abscesses and (after death) various tissues and internal organs yielded pure blastomycetic cultures, and though the early implication of the lung and other symptoms, the microscopic appearance of the internal organs, and the family history suggested tuberculosis, it was proved by various tests that that affection was absent.

Prognosis.-As far as can be judged from the slender basis of statistics at present existent, the prognosis in blastomycetic dermatosis is fairly favourable. Usually, however, the disease runs a chronic course lasting from five to ten years.

Treatment.-The internal administration of iodide of potassium has been successful in causing the disappearance of patches (Bevan, Shepherd, Sequeira). Shepherd gave the drug in doses of gr. xx thrice daily. After a month of continued treatment, great improvement resulted. A further course of the same treatment effected a cure. Sequeira gave 5- and then 10-grain doses three times a day, but there was no marked improvement until the quantity had been increased to 105 grains a day, when the size of the lesions diminished, and many of the smaller ones cleared up altogether. After further improvement the patient insisted upon leaving hospital, and escaped observation. Meneau reports that cases that fell into his first group-those in which the parasite is a yeast-are more amenable to iodide treatment than those forming the second group, though the disease is more virulent and its course more rapid.

\section{LABIOMYCosis}

Under the name of labiomycosis, Willmott Evans describes an affection in children, resembling perlèche (p. 616), but, he holds, distinct from it and from eczema, 
both clinically and etiologically. It attacks the labial skin rather than the mucous membrane; and in the case of which he gives details the child had formed the habit of licking her lips. Among the epithelial scales scraped from the affected surface he found mycelium in such large quantity as to satisfy him that the organism was the cause of the lesions, although attempts at cultivation were " not very satisfactory." In twenty other cases he found the same organism present, but in many others, less well marked, there was but scanty mycelium. The weakest antiseptic ointments sufficed to remove the disease in a few days. 


\section{CHAPTER XVIII \\ MICROBIC AFFECTIONS}

\section{TUBERCULOSIS}

Scrofula and tubercle.-Before studying the effects of tuberculous infection of the skin, it will be well, for the sake of clearness, to define terms and to indicate the relation in which scrofula stands to tubercle. The progress of pathology has now definitively assigned to tubercle so much that used to be thought to belong to scrofula that there is some danger of the latter being swept away altogether. The reason for the confusion on this subject that still exists to a certain extent is that the term "scrofula" has been used not only as indicating a particular constitutional state, but also as connoting a variety of diseased conditions. Scrofula is not a disease, but a special pathological predisposition; it is a state of soil in which bacilliespecially tubercle bacilli-readily flourish. In view of the strong affinity of the tubercle bacillus for the strumous diathesis, scrofula might almost be defined as potential tuberculosis. It is not, however, for tubercle alone that scrofula prepares the way, but for many other diseases. The condition, in fact, is one of abnormal vulnerability to slight injuries. Lesions in a scrofulous subject are apt to take 'on a character of chronic inflammation of a peculiar type, of which a tendency to suppuration and the formation of unhealthy sores are the most marked features. Mucous membranes become the seat of catarrh on very slight irritation, and

- lymphatic glands readily become enlarged. The want 
of power of resistance in scrofulous subjects is seen in the fact that they suffer more severely than other persons from syphilis and gonorrhœa; and in them scarlet fever, measles, etc., are more likely than usual to run a fatal course. Such persons are also generally considered to be more liable to acute periostitis and necrosis of bone than healthy people. Their tissues are especially vulnerable not only to traumatic influences, but to the action of pathogenic microörganisms of all kinds, especially, as already said, to the bacillus of tubercle. To sum up, scrofula is merely a special vulnerability of tissue, making it abnormally sensitive to injurious influences of all kinds. Tubercle, on the other hand, is a new growth, presenting peculiar anatomical characteristics, and giving rise to definite lesions, which, though varying in appearance according to the situation in which they occur, and other circumstances, are the result of a process that is essentially the same in them all. To put the relation of scrofula to tubercle into the briefest form, it may be said that scrofula is the soil, the bacillus the seed, and tuberculosis the harvest.

The tubercle and its bacillus.-The anatomical element of tubercle is a nodule consisting of a rounded mass of epithelioid cells, containing in its centre one or more large multinucleated cells with branching processes-the so-called giant cells. These used to be thought characteristic of tubercle, but they are now known to occur in other conditions. Tuberculosis was first shown by Villemin to be an infective process, and in 1882 the specific microörganism causing the lesions was demonstrated by Koch. The tubercle bacillus (Plate 33, Figs. 3 and 6 ) is a rod-like organism, about one-third of the diameter of a red blood-corpuscle in length, and slightly curved longitudinally. It has no independent power of movement.

The bacillus appears to have a special affinity for the 
giant cell, which is, so to speak, its ordinary dwellingplace. In slowly growing tubercle very few bacilli are present, sometimes only one in each giant cell; hence it is often extremely difficult to discover them. Koch demonstrated the bacillary nature of tuberculosis by finding the microörganisms with the microscope, and by cultivating them to many generations outside the body; inoculations of these cultures in animals gave rise to genuine tuberculous disease, and from the affected tissues the microörganism was recovered. Tuberculosis, therefore, is a form of chronic infective inflammation caused by the irritant action of the specific microörganism and its chemical products. The disease spreads by infection of the neighbouring parts, and the virus may be carried to distant regions by wandering cells which enter the lymph-stream or by transport of the bacilli by the lymph- or blood-current. Fatty degeneration occurs in consequence of the gradual cutting off of the blood supply from the areas of infection. After this it may dry up and, becoming encapsuled in a fibrous envelope, may remain unchanged for an indefinite time; or it may soften, break down, and suppurate, and in this way be eliminated; or it may calcify, and at a later period become encapsuled. The particular change which the tubercle undergoes depends on its situation. Calcification is almost unknown in the skin.

The infective power of the tubercle bacillus is not great; diminished resistance in the tissues to which it may gain access is a necessary condition of its taking root and reproducing itself. The situation of the disease is often determined by some previous injury. Insufficient and unsuitable nourishment, exposure, and other unfavourable conditions of life, especially deprivation of light and fresh air, and insanitary surroundings of any kind, have a marked influence in preparing the soil for the multiplication of the bacillus. 
Clinical tests for tuberculosis. - The Old Tuberculin (Tuberculin Original Alt) is used diagnostically in several different ways. (1) It may be injected hypodermically; (2) it may be inoculated into the skin instead of subcutaneously-von Pirquet's method; (3) it may be applied in the form of an ointment-Moro's test; (4) it may be instilled into the conjunctival sacCalmette's ophthalmic reaction.

1. Subcutaneous injection.-The patient's temperature having been carefully observed for two days to make sure that the daily excursion is within moderate limits, an injection of 0.1 c.c. if the patient is in fair general health, or of 0.01 c.c. if in delicate health, is given beneath the skin of the back between the scapulæ. If reaction takes place, it will occur in about twelve hours. Local reaction, in the form of swelling and erythema, shows that the cutaneous lesion is tuberculous ; general reaction (i.e. rise of temperature of $1^{\circ} \mathrm{F}$.) indicates that there is a tuberculous focus. Should there be a very slight reaction, e.g. a rise of half a degree in the temperature, the dose may be repeated on the third day. In the absence of reaction, a dose of double the quantity may be given. In the former case there is often a pronounced rise of temperature, a phenomenon which Koch held to be very characteristic of tuberculosis. Should the small doses elicit no reaction, they may be increased to 0.5 and even to 1.0 c.c., and the final dose may be given twice to make quite sure that the reaction is negative. There is much difference of opinion and practice as to dosage, for the nature neither of tuberculin nor of the reaction it excites is properly understood, and these tests are essentially empirical. Bosanquet and Eyre ${ }^{1}$ are of opinion that the dose should never exceed 1 c.c. A temperature of $100^{\circ} \mathrm{F}$. is a contra-indication to the use of tuberculin.

1 "Serums, Vaccines, and Toxines." 2nd edition, 1909. 
2. Von Pirquet's, or the cutaneous test.-The skin having been surgically cleaned, one drop of a freshly prepared solution of Old Tuberculin is placed upon it, and it is then scarified as in vaccination, a control lesion being produced by scarifying through a drop of sterile saline solution at another point. A positive reaction, which may occur within about twenty-four hours, but is occasionally delayed, consists in an inflammatory lesion at the point where the tuberculin was inoculated, varying from a slight redness to an indurated papule, or a vesicle or group of vesicles, which a few days later may undergo desquamation. The value of the test is disputed; at most it only indicates that there is a tuberculous focus at some part of the body.

3. Moro's, or the percutaneous test.-A lanolin ointment containing 10 per cent. of Old Tuberculin is rubbed into the chest or abdomen over an area about 3 inches square. The reaction, if it occurs, follows in from 24 to 48 hours, and takes the form of an eruption of small red papules at the site of inunction.

4. Calmette's, or the ophthalmic reaction.-One drop of freshly prepared 0.5 solution of Old Tuberculin is instilled into the conjunctiva. If the patient be tuberculous the eye will become red and the lids will swell; the caruncle also will be red and swollen, and the inflammatory reaction may be even more marked. As soon as the reaction has begun. a boric-acid lotion should be applied. Any disease of the eye is a definite contra-indication to this test, and even in the absence of any such disease there is, in rare instances, severe conjunctivitis. A few cases of resulting blindness are on record.

The remainder of the present chapter will be devoted to a consideration of the various forms of tuberculosis of the skin, except lupus vulgaris, which is considered in a separate chapter. 


\section{SCROFUlOdermia}

Under this heading the following conditions are included, viz. (1) lichen scrofulosorum, and (2) strumous ulcers.

\section{Lichen Scrofulosus (Lichen Scrofulosorum).}

Symptoms. - This disease, improperly called "lichen," is characterized by a papular eruption, the elements of which are seldom larger than a pin's head, and are flattened and very slightly resistant. They are red in colour, the tint varying from light pink to violet. They are at first arranged in groups, forming patches of varying size. At the summit of each papule is a little scale, or, more rarely, a small pustule. In addition to the grouped papules, there are others arranged in ares of circles, which are chiefly seen about the orifices of the sebaceous glands. The eruption is attended with very slight itching. It may last for months without undergoing any visible change, and finally disappears completely by a process of very gradual exfoliation of the epidermis. The seat of the eruption is generally the trunk (back and lower part of abdomen). At first it consists of isolated groups of papules, but in course of time other groups form near them and the affection becomes generalized. In this state the whole skin is of a dirty reddish-brown colour, and is covered with thin scales which are easily detached. The course of the disease is extremely slow.

Etiology.-In ninety cases out of a hundred, according to Kaposi, the patients are the subjects of enlarged submaxillary, cervical, and axillary glands. In a few of the cases other evidences of tuberculous disease are present in the form of necrosis of bone or scrofulous ulceration of the skin. A certain proportion of the patients either suffer from phthisis or have a phthisical family history. The disease, according to Kaposi, is never $2 \mathrm{c}$ 
seen in perfectly healthy persons. It is not common after the age of 20, and sex appears to have little influence in engendering a tendency to it. Tubercle bacilli have been discovered in the lesions by Jacobi and Wolff, but many other practised observers-among them Neisser, Hallopeau, Darier, and Klingmüllerhave failed to find them. Klingmüller, ${ }^{1}$ while believing lichen scrofulosorum to be tubercular, holds it to be due to the action not of the bacilli themselves but of their toxins. Lesseliers ${ }^{2}$ accepts the theory of Jadassohn that in cases of apparent reaction to tuberculin there is pre-existent tuberculosis, which becomes active in response to the injection. Experimental inoculations in animals have almost invariably given negative results.

Pathology.-The process beginning in the hairfollicles and neighbouring sebaceous glands, each papule is situated close to the orifice of a follicle. The papule is formed by infiltration of the papillæ, and the central scale, or small pustule, on the top of the papule is constituted by the heaping up of hypertrophied epidermis or exudation at the orifice of the follicle.

Diagnosis. - The disease can be identified by the homogeneity of the papules, by their arrangement in groups, by their being situated chiefly on the trunk, by their painlessness, by their not projecting much from the surface of the skin, and by the absence of itching. These features, taken in combination with the youth of the patient, are sufficient in most cases to identify the disease. It sometimes closely resembles papular eczema ; but in that complaint itching is usually very troublesome, the papules are bright red and not limited to the trunk, and often vesicles or papulo-vesicles are present as well as papules. From lichenoid syphilides, lichen scrofulosorum is differentiated chiefly by the absence of any other

1 Arch. f. Derm. u. Syph., March, 1904, p. 167.

${ }^{2}$ Ann. de Derm. et de Syph., Nov., 1906, p. 897. 
sign or history of syphilitic infection. Moreover, in the former the papules are not generally arranged in groups, but mostly in circles, and they usually affect the bends of joints. They are also very hard, and have a shiny aspect. Lichen spinulosus is distinguished by the less inflammatory nature of the lesions, the situation on the limbs rather than on the trunk, and the absence of associated tuberculosis. ${ }^{1}$ Xerodermia (keratosis pilaris) differs from lichen scrofulosorum in that the papules do not form groups or patehes, and are generally found on the limbs, most frequently the thighs; they consist of projecting hair-follicles, which feel to the hand like a nutmeg-grater.

Treatment.-Lichen scrofulosorum can usually be cured, and even if left to itself is not likely to cause any particular inconvenience. It must be treated locally by soothing and mildly antiseptic applications, such as calamine lotion or boric-acid ointment, and constitutionally by measures appropriate to the state of health.

Eruptions of pustular and pemphigoid character, associated or not associated with lichen scrofulosorum, are occasionally met with.

\section{Strumous Ulcers}

Ulcers thus designated arise on the skin in different ways: (1) by extension of the inflammatory process from caseating lymphatic glands to the skin covering them; (2) by the formation of a nodule or circumscribed induration under the skin, which becomes involved in the process ; (3) by extension from bone which is the seat of tuberculous osteomyelitis.

Symptoms.-When a gland is the starting-point of the

1 Adamson, "Skin Affections in Childhood," 1907, p. 208; also " Lichen Pilaris seu Spinulosus," Brit. Journ. Derm., March, 1905, p. 77 . 
process the skin over it becomes red and infiltrated, and often adheres to the gland; after a time the skin breaks, sinuses form, and the tuberculous process becomes complicated by more or less profuse suppuration, owing to the entrance of pyococci. When nodules develop under the skin independently of glands, they give rise to what Erichsen calls "subcutaneous scrofulous abscess." The skin over the nodules is raised, and at first dusky purple in hue ; then, as the underlying growth softens, it breaks, giving exit to a thin curdy discharge, and an ulcer is formed bordered by dark-bluish thin undermined skin, the vitality of which is too feeble to allow of any attempt at repair. The edge is sometimes sharp-cut, but more often ragged; the floor is grey and irregular, the granulations are flabby and covered with unhealthy pus. These ulcers generally spread slowly but steadily, and in this way large indolent sores may be formed which are sometimes covered with heaped-up crusts simulating rupia. Such ulcers are common on the face and on the hands (where the process may extend to the bones, constituting one form of strumous dactylitis), and they are not infrequently seen on the feet and on the buttocks. In a patient under my care the elbows and knees were the seat of the affection. Healing seldom takes place spontaneously. These ulcers are, as a rule, seen in young people who have the notes of the scrofulous constitution plainly written on them in their physiognomy, or in the marks of similar lesions on the neck, the nose, the eye, or elsewhere. Flat ulcers, with clean-cut edges (as if the skin had been punched out) which tend to spread slowly, are sometimes seen in old people who bear scars of strumous sores with which they were afflicted in early life. These senile strumous ulcers occasionally assume the character of rodent ulcer or epithelial cancer.

Diagnosis. - The only conditions that are ever likely 
to be mistaken for strumous ulcers are syphilis and lupus. The syphilitic ulcer is met with in adults, and has not the characteristic undermined border; moreover, the process is generally much more active, and concomitant symptoms or marks usually indicate the nature of the disease. The absence of infiltration and of "applejelly" nodules will serve to distinguish scrofulous ulcers from lupus. The two conditions may, however, coexist, and Leloir believes that in the same way syphilis may be mixed with scrofulodermia in the same subject.

Treatment.-This must be conducted on ordinary surgical principles. Abscesses must be opened and their walls scraped; caseous glands must be removed, and ulcers cleansed and stimulated. The unhealthy undermined skin at the edge of the ulcers must be trimmed away, the floor thoroughly scraped, and antiseptic dressings applied. In cases of infection of the skin secondary to lymphatic infection, New .Tuberculin (Tuberculin Rückstand), in minimal doses, has been tried with favourable results in association with local treatment. X-rays are sometimes useful. The patient's constitution must at the same time be strengthened by plenty of good food, cod-liver oil, iron and other tonics, according to the indications, and especially by sea-air and a wholesome environment.

\section{Tuberculous Ulcers (Tuberculosis Cutis Orificialis)}

Symptoms.-Primary tuberculosis may occur on the face, on the breast, and elsewhere in the form of ulcers with an infiltrated, ragged, and undermined edge, and a slightly indurated floor covered with yellowish tubercles, moistened with a thin and scanty secretion. The surface is often more or less thickly crusted over. They are sometimes indolent, but usually they cause considerable pain. 
Occasionally the ulcers are the result of the breaking down of small tuberculous nodes. The lesion may be the precursor of tuberculous disease of the lung or intestine. Köbner has reported a case in which a tuberculous ulcer of the chin preceded the development of laryngeal phthisis. More commonly, however, such ulcers are secondary to pulmonary or intestinal tuberculosis. They are generally situated at the junction of skin and mucous membrane-about the corner of the mouth and margin of the nose in cases of lung disease, and at the anus, vulva, and glans when the intestine is the seat of the primary lesion. In the former case the ulceration may spread to the mucous membrane of the tongue, cheeks, soft palate and nose, and in the latter to the urethra and bladder. When the mucous membrane is the seat of these ulcers, yellow miliary tubercles can generally be seen in their vicinity. There may be one or several ulcers. They show no tendency to heal, but slowly spread by infection of the contiguous parts, sometimes attaining a considerable size. Occasionally they run together, forming serpiginous sores that cover a large surface. In a patient under my care, who died of phthisis at the age of 42 , numerous small ulcers coalesced and formed a large ulcerated surface which nearly surrounded the left ear.

Etiology.-Tuberculous ulcers of the skin are the result of direct inoculation with tuberculous matter. This often occurs in patients suffering from tuberculosis; hence their relative frequency in situations where bacilli in the fæces or sputa can readily find their way into any abrasion of the surface that may exist. I have seen such ulcers begin in a patch of eczema. Infection may also be conveyed from one patient to another. This is a not uncommon consequence of ritual circumcision, when the wound is sucked by an operator who is the 
subject of tuberculosis. ${ }^{1}$ The virus is also sometimes conveyed by tattooing.

Diagnosis.-Recognition of tuberculous ulcers is usually easy, owing to the presence of other signs of tuberculosis. When the ulcer is primary its surface should be scraped, and the shreds of tissue thus obtained examined for bacilli. In Köbner's case, above referred to, the lesion was judged to be syphilitic by several practitioners, and it was only the failure of treatment based on this view and the subsequent invasion of the larynx by tubercle that revealed the nature of the disease.

Treatment will be considered in the next section (p. 440).

\section{Verruca Necrogenica (Post-mortem Wart)}

This form of skin tuberculosis is seen on the hands of medical men, mortuary porters, butchers, cooks, and other persons who are in the habit of handling dead tissue containing living tubercle bacilli.

Symptoms. - The condition is characterized by the formation of red, indurated, wart-like growths, chiefly on the knuckles and in the interdigital folds, but occasionally on other parts of the hands, and on the arms. The lesion usually begins as a flat papule, which by and by becomes pustular. The pustule dries up and forms a scab, which in time falls off, leaving exposed a surface made irregular by prominent papillæ. These gradually become larger and harder, till they form a warty mass, which may spread slowly at the edge for an indefinite time. Hutchinson cites a case in which the growth continued to enlarge slowly for forty years. Sometimes spontaneous involu-

2 See Bernhardt, quoted by Graham Little (Brit. Journ. Derm, March, 1901). This method of stopping bleeding is no longer practised in Britain. 
tion takes place, and the warts disappear, leaving a scar.

The condition appears to be identical with that described by Riehl and Paltauf ${ }^{1}$ under the name of tuberculosis verrucosa cutis. This is a local tuberculosis of the skin, the affected tissues showing the changes characteristic of tubercle, together with the specific bacillus, which is present in larger numbers than is the case in lupus. The condition known as lupus verrucosus, and seen chiefly on the hands and feet, is also a form of local tuberculosis of the skin, having the same characters as post-mortem wart. Primary cutaneous inoculation of tuberculosis on the extremities in patients who have to attend to those suffering from tuberculosis frequently takes the form of verruca necrogenica.

Treatment.-The diseased tissue in all these conditions should be removed with salicylic acid, applied by means of Unna's plaster mull or Brooke's ointment. If the lesions are spreading actively they should be thoroughly destroyed with caustics, such as nitric acid, or the electric cautery, or treated by $X$-rays.

\section{Erythema Induratum Scrofulosorum}

The affection thus designated was first described by Bazin (hence it is sometimes called Bazin's disease), and has been exhaustively studied by Colcott Fox. ${ }^{2}$

Clinical features.-The special lesions are chronic, inflammatory, and deep-seated nodules, which develop chiefly on the legs, but also in other parts, and often closely resemble syphilitic nodular gummata. The lesions, which are painless; are at first subcutaneous, and can only be felt, not seen. They affect the back rather than the front part of the leg; the skin over them occasionally presents a violet-tinted discoloration. They

1 Viertelj. f. Derm. u. Syph., 1886, Hft. 1, p. 16.

a Brit. Journ. Derm., Aug., 1893. 
are generally discrete, but sometimes become fused together so as to form a solid mass of infiltration. They are apt to break down into irregular ulcers. The large majority of patients are young girls, and the disease is particularly common in washerwomen and other women whose occupation involves much standing. When ulceration occurs the affection is generally taken to be syphilitic, but in typical cases no evidence of syphilis is present, and anti-syphilitic treatment does harm rather than good. In many cases the patients present clear signs of scrofula, but sometimes they seem, save for the local affection, to be perfectly healthy. Numerous lesions resembling lichen scrofulosorum and erythema induratum scrofulosorum have recently been described and discussed under such names as " folliclis" and " acnitis" (see p. 443), and their relation to tuberculosis suspected. That one form of this disease is tuberculous was proved by Colcott Fox, who excised a deepseated nodule and submitted it to examination. Typical giant cells were found, though not in great abundance. The result of an experimental inoculation in a guineapig made by Eyre was that the animal died of tuberculosis. Hartung and Alexander also, ${ }^{1}$ in discussing a series of five cases treated in the General Hospital at Breslau, regard the affection as tuberculous, of hæmatogenous origin, and interpret erythema induratum and folliclis as variants of the same pathological process. In a case of MacLeod's a positive ophthalmotuberculin reaction was obtained. ${ }^{2}$ But Whitfield has reported two cases in which there was no sign whatever of tuberculosis, and in the light of these and other cases he concludes that there are "two well-defined types of the disease, one tuberculous and the other nontuberculous, the latter occurring usually, though not

${ }^{1}$ Arch. f. Derm. u. Syph., Sept., 1904, p. 384.

2 Brit. Journ. Derm., Jan., 1908, p. 16. 
invariably, in older patients than the former, running a more rapid course, showing less tendency to ulcerate and causing much more pain." 1 The earlier of the two cases was shown to the Dermatological Society of London, and the diagnosis of erythema induratum accepted.

Diagnosis.-From gummata the nodules of Bazin's disease differ in running a less rapid course, in being less painful and inflammatory and not suppurating, as well as in being more numerous and in attacking both legs. For the diagnosis between erythema induratum scrofulosorum and erythema nodosum, see p. 113.

Treatment.-This consists in rest in the horizontal position, compression by bandaging, and cod-liver oil internally.

\section{Hypodermic SARCoIdS}

A condition not improbably related to erythema induratum has been described by Darier and Roussy, the former of whom considers that the two affections are simply varieties of one species. It occurs chiefly in women between the ages of 30 and 40 . The lesions, chronic indolent neoplasms in the hypoderm, varying in size from a pea to a nut, are sometimes grouped into nodular patches. The preference site is the costal regions, but they may appear anywhere.

\section{TuBerculides}

Under this name a somewhat motley group, presenting a great variety in appearance, but having certain characters in common, has been provisionally brought together. In the words of Colcott Fox, who presented a masterly report on these eruptions to the Fourth International Congress of Dermatology, "the essential lesion is a small, extremely indolent granuloma, tending

${ }^{1}$ See also a paper by Whitfield, "On Multiple Inflammatory Nodules of the Hypoderm," Brit. Journ. Derm., 1909, xxi. 1. 


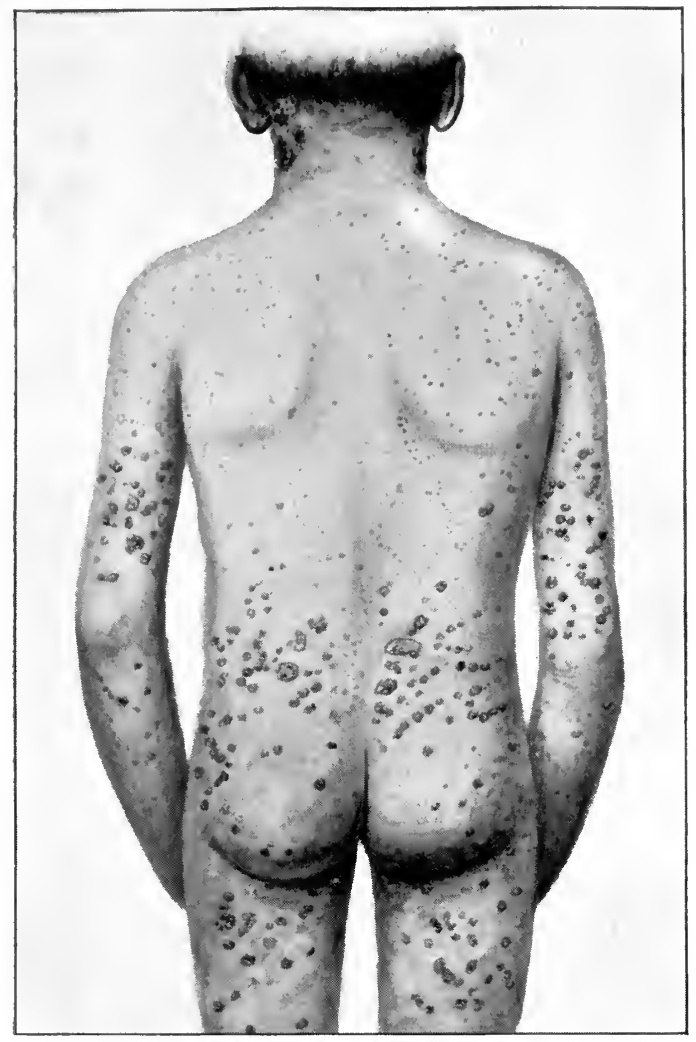

Plate 35.-general tuberculide. (Same' Case as in P'late 36.) 


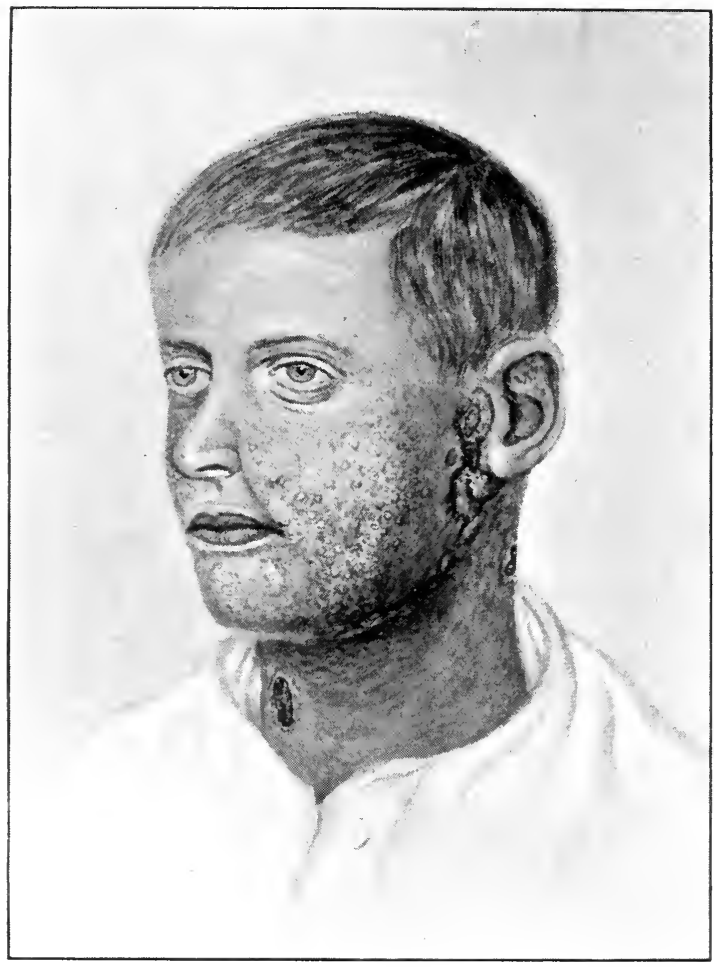

PLATE 36.-GENERAL TUBERCULIDE, SHOWING STRUMOUS ULCERS.

(Same Case as in Plate 35.) 
to undergo central softening and death, and thus leaving scars." According to difference in the size, character, grouping, and behaviour of the lesions, a bewildering complexity of affections, with a corresponding complexity of nomenclature, has been described by various observers. Among the names by which the lesions have been known are-lupus-psoriasis scrofulosa, folliculitis exulcerans, folliculitis scrofulosorum, hydradenitis destruens suppurativa, spiradenitis disseminata suppurativa, acnitis, acne telangeiectodes, impetigo varioliformis, acne varioliformis, and folliclis.

The evidence of the tuberculous nature of these varied eruptions is not by any means complete. They are often, though by no means invariably, associated with tuberculous disease in the lungs and lymph-glands or with strumous ulcers (Plates 35 and 36). The microscopical evidence so far obtained is inconclusive, while the bacteriological evidence is absolutely negative. Experimental inoculations have for the most part been unsuccessful. It has been suggested by Hallopeau and others that these tuberculides may be the result, not of the inoculation of tubercle, but of the circulation in the blood of toxins produced in tuberculous foci within the body. In view of the eruptions produced by other kinds of toxins and by certain drugs, the possibility of such an origin cannot be denied. But at present it is a theory resting on no solid proof. Colcott Fox points out that if these tuberculides are due to the implantation of tubercle bacilli, starting from some distant focus and coming by way of the blood-stream, the organisms must be of little virulence and are probably easily killed. This would, he suggests, explain why the pathological changes are often indecisive, and why inoculations fail. Pautrier, the author of a large work on the tuberculides, ${ }^{1}$

1 "Les Tuberculoses cutanées atypiques (Tuberculides)." Thèse de Paris, 1903. 
thinks that possibly the tubercle bacillus arrives in the skin either moribund or actually dead. In Fox's experience tuberculides are frequent sequelæ of measles, which, as is well known, is often the determining factor in the development of phthisis.

Articles on acne varioliformis and acnitis will be found in a later chapter (see pp. 575, 577). The reader who desires full information upon the tuberculides is recommended to consult Fox's paper, which is not only a summary of the whole subject, but a storehouse of references bearing thereon.

\section{Acneiform Tuberculide (Acne Scrofulosorum)}

An affection described under these names presents sufficiently distinctive features to be worthy of special mention. ${ }^{1}$ J. M. H. MacLeod and Oliver Ormsby of Chicago have made an exhaustive study of two cases. The following are the essential details. Case 1: The patient was a baby with tuberculous history and evidences of general tuberculosis-dactylitis, acneiform tuberculides on the arms, hips, etc. Histological examination revealed typical tuberculous cells; there was also endophlebitis in the veins of the hypoderm. Case 2: A woman, aged 25, with acneiform tuberculides on the legs. Typical tuberculous architecture was seen in the sections, with periphlebitis and endophlebitis. MacLeod and Ormsby conclude that acneiform tuberculides begin in an affection of the hypoderm, the cause of which is the tubercle bacillus. The process $\mathrm{r}$ sults in a deep-seated necrosis, definitely tuberculous in character, which is the consequence of the invasion of the tubercle bacillus and its toxins. Török describes under the name of dermatitis nodularis necrotica a case which is clinically identical with acneiform tuberculide. For treatment, see next section.

${ }^{1}$ Brit. Journ. Derm., 1902. 


\section{Acne Cachecticorum}

The eruption known by this name, occurring in patients of the same type as sufferers from acne scrofulosorum, consists of papules and pustules purplish-red in colour, not unlike syphilides. In some cases there are also hæmorrhagic lesions. Its favourite sites are the face, chest, and back, and lower limbs. It is very persistent, and may not clear up until the general health of the patient improves. This is the immediate object of treatment, both in acne cachecticorum and in acne scrofulosorum. The lesions in both conditions are benefited by the application of red oxide of mercury ointment, or other stimulant antiseptic preparation.

\section{Multiple Benign Sarcoid (Miliary Benign Lupoid)}

The condition which has received from Boeck these designations' must also be noticed. Often accompanying visceral tuberculosis, it presents resemblances both to lupus vulgaris and to sarcoma, but is distinguishable from both by histological examination, as well as by the course it runs. From lupus it is also differentiated by its negative reaction to experimental inoculation. It manifests itself by nodules, at first rose-coloured, afterwards livid, then brownish, varying in size from a millet-seed to a large bean. The eruption is invariably symmetrical; the favourite sites are the face, shoulders, wrists, and the extensor surfaces of the upper limbs; but occasionally the scalp, the back, and the lower limbs are attacked. In some cases there is enlargement of the lymphatic glands. The nodules never ulcerate, but after a period, which may be prolonged into years, shrink and disappear, leaving a slight atrophic scar. Both Boeck and Pawlow report good results from the administration of arsenic. 


\section{CHAPTER XIX}

\section{MICROBIC AFFECTIONS (Continued)}

\section{Lupus Vulgaris}

THIS affection is a form of tuberculosis of the skin presenting such marked clinical characteristics as to make it a distinct morbid entity. Though undoubtedly bacillary in its origin, its virulence is comparatively slight.

Symptoms. - The distinctive lesion of lupus vulgaris is a new growth in either the superficial or the deep part of the corium. This neoplastic nodule (called by Leloir lupoma) is soft, brownish-red in colour, and translucent, resembling apple jelly (Hutchinson). The lupous nodule is slow in evolution, and destroys the tissues which it invades, either by ulceration (lupus exedens) or by atrophy (lupus non exedens). The characteristic nodules are at first buried in the skin, on the surface of which after a time they show themselves as papules of the size of a pin's head. These are at first dull-red in colour, and become pale, but do not disappear on pressure. They are discrete and arranged in groups, sometimes in irregular circles. The papules gradually become larger and develop into nodules, the intervening skin meanwhile becoming thickened by cellular infiltration, reddened by inflammatory stasis in the vessels, and somewhat raised so as to form a distinct patch; at this stage the applejelly nodules project slightly above the skin (Plate 37 ). Their translucency varies according to the thickness of 


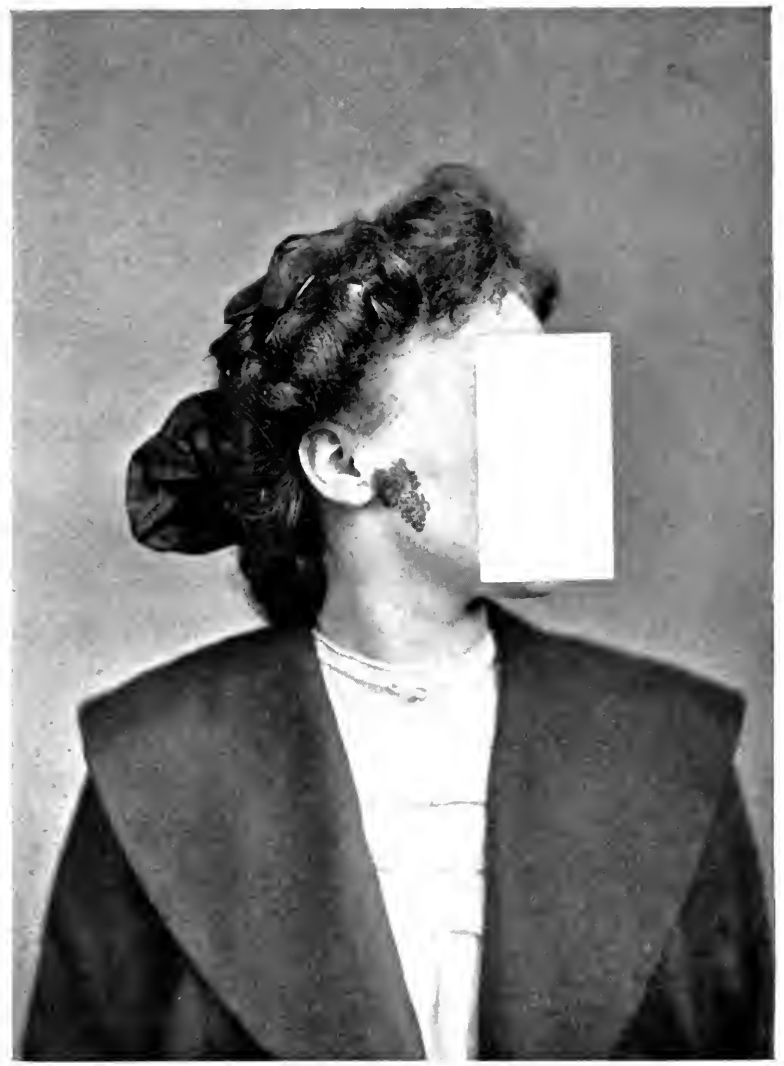

Plate 37.-EARLy LUPUS VUlgaris. 


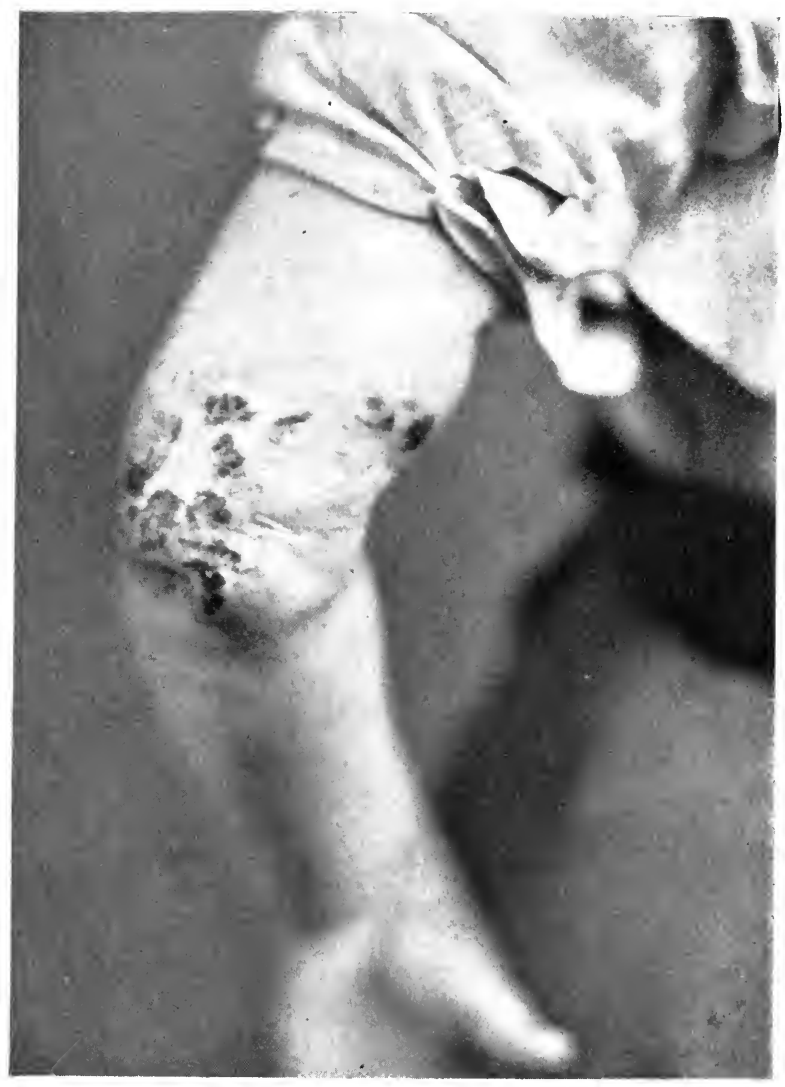

plate 38.- ulcerating lupus vulgaris. 
the epidermis covering them. New nodules spring up around the edge of the patch, which in this way spreads slowly and may gradually invade a large area of skin. The surface of the lesions is covered with fine branny scales, but not so thickly as to hide the red ground of the patch. The lupous tissue tears very easily, in marked contrast to the tough corium. The disease usually starts from a single focus, but others may arise, and, developing separately, or coalescing with neighbouring ones, may involve almost the whole body (lupus disseminatus). The process is, as a rule, extremely slow, and in some cases it may come almost to a standstill for an indefinite time. The patch may slowly undergo involution in the centre, a smooth, firm scar being left resembling that of a burn. This is often bounded by a ridge of bluish-white or reddish tubercles, which continue slowly to invade the surrounding skin.

In the majority of cases, however, ulceration takes place at some time, the lupous tissue breaking down and forming a granular sore covered with greenish-black crusts; dotted around the edge, which is ragged, are apple-jelly nodules in various stages of development (Plate 38). The ulceration may extend through the whole thickness of the skin, and in parts, like the nose, where the integument is thin, it sometimes causes necrosis of cartilage; it rarely, however, erodes bone, though Walter G. Smith ${ }^{1}$ has reported a case in which there was dissolution of bone in the fingers, the process probably being a tuberculous rarefying osteitis. If the inflammatory process reaches any degree of intensity, enlargement of neighbouring lymphatic glands not infrequently follows; this enlargement is considered by Leloir to be due to diffusion of the tuberculous virus by the lymphatics. As a general rule, it may be stated that the ulceration of lupus is extensive rather than

1 Brit. Journ. Derm., 1909, xxi. 69. 
deep. Occasionally, especially after the surface of a lupous patch has been scraped, the process seems to be quickened into considerable activity, the skin becoming hot and hyperæmic, rapid development of fresh nodules taking place, and general febrile symptoms coming on. The phenomena, in fact, recall a mild reaction after the injection of tuberculin, and are probably to be explained by the absorption of bacillary products.

All phases of the lupous process may be present at one and the same time in a given case. Often while one part of a patch is in active ulceration another is cicatrizing, and nodules in all stages of development are to be seen on its surface. In adults sometimes the lesions are infiltrated patches raised more at the edge than in the centre, and with no translucent nodules. The different degrees of infiltration of the skin and of intensity of the inflammatory process, together with the anatomical peculiarities of the part affected, give rise to the greatest diversity in the appearance of the lesion. These variations are expressed by such terms as lupus hypertrophicus, lupus papillomatosus, and lupus serpiginosus, which must be understood as indicating differences of appearance, not of process.

Lupus is seldom symmetrical in distribution. The favourite point of attack is the face, especially the inferior meatus of the nose, whence it may spread backwards to the naso-pharynx and even to the front of the hard palate, or upwards along the nasal duct to the lachrymal sac and the conjunctiva, and the neighbouring part of the cheek. Lupus also occurs on the limbs, and especially the hands and feet, on the trunk, and on the buttocks. No part of the skin is safe from invasion, but, as Hutchinson has pointed out, the warmer a part is, the less likely is it to be attacked by lupus. The disease is rare on the genitals and on the scalp, though it may spread to these parts from 
foci in their neighbourhood. In addition to the mucous membranes affected by extension from the nose, those of the cheeks, gums, palate, and larynx are often the seat of the disease, which may extend to these parts from the skin of the face, or may attack them primarily. The tympanic membrane may be invaded through the external meatus from the ear or through the Eustachian tube from the throat. A patient of mine, a lady past middle age, who for years had been the subject of lupus of the face and other parts of the skin, developed the disease in the vagina and on the os uteri. The appearances in this case bore no resemblance to those described by Matthews Duncan and Thin in a case which they supposed to be an example of vaginal lupus (esthiomène), but which was in all probability syphilitic. In 380 cases collected by Max Bender, lesions of the mucous membrane coexisted with lupus of the skin in 173 cases (about 45 per cent.); in only 6 cases was the disease limited to the mucous membranes. Of 147 of these cases it was ascertained that in 46 (31.2 per cent.) the mucous membrane was the part originally attacked. In many cases the mucous surface in several different regions was attacked. The mucosa most frequently involved was that of the nose; it was implicated in 115 cases, the conjunctiva in 21 , the tear-duct in 24 , the lips in 43 , the palate in 31 , the tongue in 1 , the larynx in 13 , the rectum and vulva in 1. Finsen's statistics of cases treated at the Copenhagen Light Institute show that in from 70 to 80 per cent. the mucous membrane of the nose or mouth was involved.

Clinical course. - The course of lupus is almost always slow, often lasting twenty or thirty years, or longer. The process is more active in ehildhood than in later life, and its activity, as a rule, becomes less with advancing age. The normal sluggishness of the process is diversified by oceasional episodes of unwonted activity, 
during which the disease may make considerable advance. This sometimes occurs under the influence of the physiological changes which take place at puberty, or as the result of an attack of some acute illness, such as measles or scarlet fever, or of external irritation, as by cold. These periods of activity are followed by long intervals of comparative quiescence, the disease seeming almost to die out. Spontaneous cure sometimes takes place, though this is too rare an event to be taken into account in practice. Even when the process does come to a standstill, this often does not occur until it has wrought irreparable destruction on the parts attacked, leaving hideous scars, obliterated passages, and deformed limbs, which would render life all but intolerable for most people. As a rule, lupus is unattended with pain.

The secondary effects of lupus depend on the severity of the process, and also on the situation of the disease. On the face it leaves its mark in destruction of the nose, with scarring of the cheeks, etc., and enlargement of glands, particularly of the parotid. Caseation and breaking down may take place in these, leading to the formation of scrofulous ulcers, and often to profuse suppuration, which undermines the patient's health. Great development of fibrous tissue sometimes takes place in the cicatrices and in the limbs; this leads to contraction and crippling of joints. The skin not uncommonly becomes adherent to the underlying fasciæ and tendons, the whole being glued together into a dense, tough mass, adherent to the bone, which is itself thickened and sclerosed. The ulcerated parts may become the seat of warty vegetations (lupus papillomatosus). There is nothing peculiar to lupus in these secondary changes, which are the results of chronic inflammation in tissues of abnormal vulnerability, complicated by the action of pyogenic cocci which come in to complete 


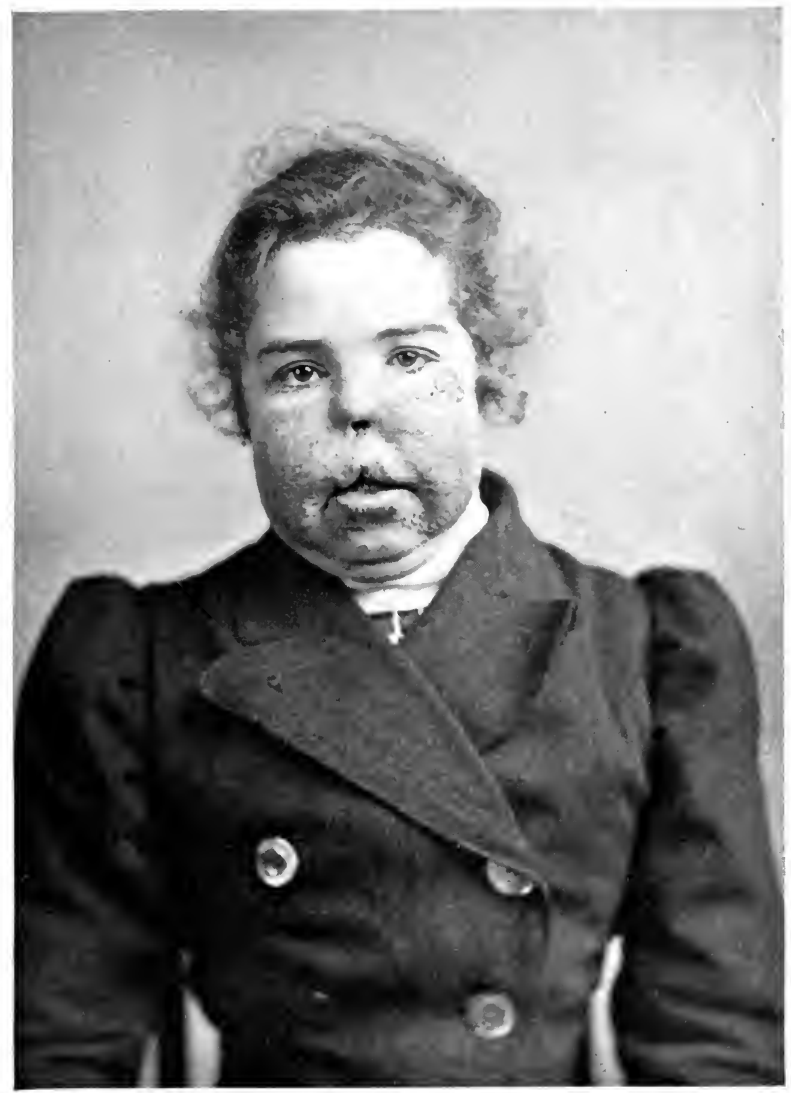

plate 39.- lupus vulgaris With Chronic cedema. 


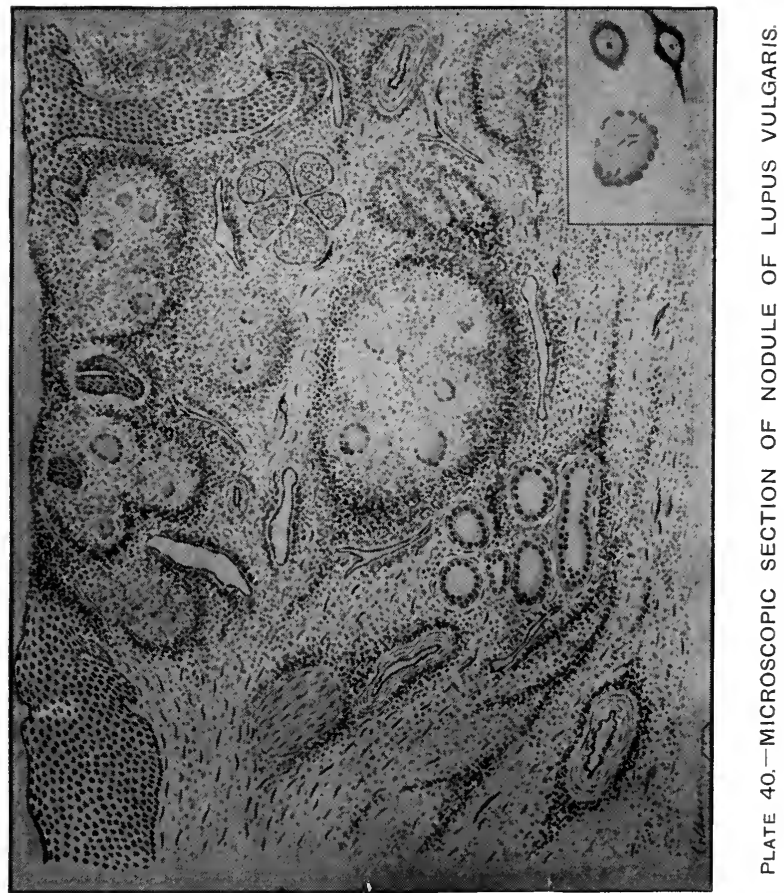


the destructive work of the tubercle bacillus. I have seen pseudo-elephantiasis of the lower limb due to blocking of the lymphatics as a rare result of lupus vulgaris. ${ }^{1}$ A still more formidable complication is the development of epithelioma, which takes place in a certain proportion of cases. This, if I may judge from my own experience, is a not very common occurrence, but Ashibara has collected 122 instances. Lupus is sometimes associated with chronic œedema (Plate 39).

Lupus does not appear to have any effect on the general health, except in rare cases. According to Leloir, however, lupus of the hand may become "a starting-point of tuberculous lymphangitis with production of scrofulo-tuberculous gummata developed along the course of the lymphatics attacked, and finally, under the influence of the absorption of the tuberculous virus by the lymphatics of the upper limb, determine a pulmonary tuberculosis of the corresponding side." Leloir looked upon the enlargement of the glands, which has been described as occasionally taking place in the neighbourhood of lupous patches, as evidence of secondary tuberculous infection, and this fact he claimed to have proved histologically and experimentally. Of 17 patients under his own observation in 1885-86, 10 presented unquestionable evidence of pulmonary tuberculosis. Doutrelepont has reported a case in which a healthy woman, the subject of lupus of the face and limbs, rapidly succumbed to tuberculous meningitis, as proved by post-mortem examination; the lupous lesions were the only discoverable source of infection. Thibierge has recorded the case of a boy aged 15 who suffered from peritoneal and pulmonary tuberculosis, the com-

1 See a report of the case (which was under my care in St. Mary's Hospital), by Leslie Roberts, in the Brit. Journ. Derm. 1888-89, p. 339 . 
mencement of which dated from the cure of a patch of lupus on the cheek. Besnier, from long clinical observation, came to the conclusion that secondary tuberculous infection is a not infrequent result of lupus; sometimes this takes place rapidly (within two or three years), sometimes very slowly (ten, twenty, thirty years, or longer); usually, he says, the subjects of lupus who become phthisical do so in a latent and very slow manner. He gives the proportion of such secondary phthisis in his own practice as 21 per cent. Dubois-Havenith states that among 118 patients under his own care suffering from Jupus, 8 died from pulmonary consumption. Lailler, from observation extending over many years at the St. Louis Hospital, Paris, states that pulmonary tuberculosis is a frequent cause of death among sufferers from lupus. Renouard found that of 137 cases of lupus, fifteen developed pulmonary phthisis. Haslund of Copenhagen puts the proportion of secondary pulmonary infection in the lupus patients in his own clinic at the startling figure of 60 per cent. On the other hand, Nevins Hyde, of Chicago, has never seen a case of such infection; and Brocq's experience has been equally negative.

This divergence of opinion is sufficient to show how difficult it is to obtain clinical evidence on this point which is conclusive one way or the other. My own experience is that the development of secondary tuberculosis in the lungs from a focus of lupus on the skin is decidedly infrequent.

Severe and extensive lupus is sometimes complicated by wasting and anæmia. Many patients, however, have all the appearance of robust health; but as a class sufferers from lupus are not longlived.

Etiology.-The essential etiological factor is local tuberculous infection. The tubercle bacillus of Koch, 
though most difficult to find, is probably always present in some stage of the lesion, and tuberculous infection can be produced by inoculation of cultures made from these lesions. The bacillus gains access through an abrasion or puncture of the skin, as in burns, sores, boils, blisters, infantile eczema, tattooing, vaccination, piercing the ear for earrings, etc., or scratching the lesions of impetigo; the nose may be infected by inoculation from the finger with which it is "picked"; there may be secondary infection of the skin from sinuses, etc., resulting from the breaking-down of tuberculous glands; or the bacillus may be carried to the skin by the blood or lymph after having found its way inside the body through one of the natural passages, or from the softening of some distant focus, as after measles and other acute specific fevers. Wolters reports a case of the nodular form of lupus in which, as the result of microscopical examination, be believes that the bacillus was carried to the skin from the blood-vessels, having reached the veins from a tuberculous gland.

There are, however, a number of secondary causes which play a more or less important part in the production of the disease. Youth is a predisposing influence. The disease usually begins within the first ten years of life, occasionally at puberty, seldom later. In exceptional instances it develops in middle life, or even in old age. Females show considerably greater liability than males. The disease, while sparing no class, numbers more victims among the poor than among the well-to-do. Cold is a predisposing factor of some importance, as evidenced by the greater frequency of lupus on exposed than on covered parts. Even if cold cannot be shown to have any direct influence in the production of the disease, undoubtedly it has a pernicious effect on the process when once established. Measles appears someiimes to be the determining factor 
in the development of lupus. H. G. Adamson ${ }^{1}$ has analysed a series of 28 cases, in nearly all of which multiple cutaneous lupus followed an attack of that disease. The correlation between the two affections was first noticed by $\mathrm{Du}$ Castel. I have myself seen cases of lupus made much worse by the supervention of measles. Discussing cases of multiple cutaneous lupus consecutive to acute exanthemata, F. v. Veress ${ }^{2}$ concludes that the lesions are due to external inoculation, and not to infection conveyed by the blood.

Pre-existing lesions or scars, as I have said, form the starting-points of the disease in a certain proportion of cases. Nasal catarrhs and eczematous eruptions about the nostrils in uncleanly subjects prepare the soil for infection. I have seen cases in which lupus apparently began in the tear-ducts and travelled down into the nose. These facts may account for the marked predilection which the disease manifests for the nose. The affection has been known to begin in the vesicles of herpes (Crocker, Kaposi). Among other conditions which have been found to be starting-points of lupus, besides those enumerated above, are eruptions, suppurating glands, and syphilitic lesions.

Analysing a series of 923 cases of lupus vulgaris treated in the Light Department of the London Hospital, Emlyn Jones ${ }^{3}$ found that $47 \cdot 3$ per cent. arose as a small spot on the face, cheek, or neck (including a few arising round the margin of the eyelids and on the auricle, but not those springing from tuberculous glands, or from scars of gland abscesses or scars left by the removal of old glands); that 28.9 per cent. appeared on the nose or in the nostril; that 11.4 per cent. were secondary to tuberculosis in the glands, bones, or joints

1 Brit. Journ. Derm., Oct., 1904.

${ }^{2}$ Monats. f. prakt. Derm., June 1, 1905, p. 585.

${ }^{3}$ Brit. Journ. Derm., 1907, xix. 305. 
-i.e. surgical tuberculosis; that 1.8 per cent. originated in mucous membranes other than the nasal; and that 1.8 per cent. were secondary to tuberculosis of bone; while 8.5 per cent. arose in miscellaneous ways. Philippson, of Palermo, found that of 135 cases as many as 53 were secondary to surgical tuberculosis, a very much larger proportion than in the London Hospital cases. Twenty-six of the cases were secondary to nasal tuberculosis, 14 were hæmatogenic, and 42 were primary.

The state of the general health has no direct influence on the causation of lupus, and the disease is probably hereditary only in so far as a tuberculous inheritance may create a predisposition thereto. It has been suggested by Baumgarten, however, that the bacillus itself is directly inherited, and in that case the origin of lupus might be explained by the settlement of the microörganism in the skin of the fœtus. Cases in which lupus has been directly inoculated have been reported by Jadassohn and others. In one case a woman was tattooed on the forearm by a man suffering from pulmonary tuberculosis, from which he afterwards died; the operator used his saliva to dilute the ink, and typical lupus nodules appeared on the tattooed parts. Besnier showed a case of lupus in a lad aged 18 , in whom the development of the disease had taken place in a vaccination scar, where it had developed within a few months of the operation. Graham Little has recorded several cases in which lupus developed on vaccination scars; Colcott Fox, however, under whose observation some of them were, does not think they prove anything in regard to the transmission of lupus by vaccination. Dubois-Havenith mentions a case which suggests the possibility of contagion in certain circumstances : two sisters, one of whom had for eight years had a large patch of lupus on the left cheek, shared 
the same bed. For the last two years the other sister had a lupous patch on the lobe of the right ear-that is to say, the ear which was sometimes in contact with her sister's cheek as they lay in bed.

Pathology.-Lupus vulgaris is a local tuberculosis of the skin. The process begins in the deeper layers of the cutis ; the nodules displace the bundles of fibrous tissue, and as they increase in size they grow upwards through the skin, destroying its component elements by pressure, so breaking through the papillary layer and emerging on the surface, where they are covered only by epithelium, more or less translucent, according to its t'ickness. On microscopic examination the nodules are found to be composed of giant cells (Plate 33, Fig. 6, and Plate 40), surrounded by a layer of epithelioid cells, with an outer envelope of ordinary lymphoid or small round cells. The lupous nodule is practically identical in structure with the nodule of tuberculosis, and this fact led Friedländer and Koster to look upon lupus as a local tuberculosis before this was proved bacteriologically by Koch. Tubercle bacilli are present in numbers which probably vary with the acuteness of the case ; even in the growing edge there is often only one in a giant cell. It is not surprising, therefore, that frequently they cannot be discovered on the most careful examination.

In the Final Report (1911) of the Royal Commission on Tuberculosis (Human and Bovine), it is stated that of twenty cases of lupus which were investigated, in nine a bacillus was found presenting the cultural characters of the bovine tubercle bacillus. In only one of the nine, however, did the bacillus obtained from the original material exhibit the high virulence of the bacillus of bovine tuberculosis. In two cases it was found possible to increase the virulence of the culture from the original material by residence in the tissues 
of the calf and rabbit, so as to bring it up to the high virulence of the bovine tubercle bacillus.

When a lupous nodule has reached its highest development, retrogression sets in. This may take one of two directions-namely, either fatty degeneration, followed by the formation of a fibrous cicatrix, or softening and ulceration. Lupus, however extensive or disseminated it may be, shows comparatively little tendency to become generalized.

Diagnosis.-In a well-marked case lupus is easy of recognition. The presence of apple-jelly nodules at once indicates the nature of the process. A typical lupous patch, with its infiltrated raised surface, defined edge studded with apple-jelly nodules, the whole covered with a moderately thick layer of scales, can hardly be mistaken for anything else. The disease, however, may sometimes have to be distinguished from acquired syphilis, scrofulodermia, lupus erythematosus, rodent ulcer, and cancer. The following are the points differentiating it from acquired syphilis: It begins in childhood, whereas syphilis usually begins in adult life; in its rate of progress it is to syphilis as the hour hand to the minute hand of a clock (Payne); the ulcers are ragged instead of sharp-edged; the ulcerative process scarcely ever involves bone; lastly, if the lesions are syphilitic, other traces of the disease are sure to be discoverable. If any doubt should remain, the Wassermann test (p. 495) should be applied. It is seldom that there can be any question as between lupus and hereditary syphilis, for in the latter affection, manifesting itself in adolescence, the unmistakable stigmata of congenital syphilis (p. 486) are almost sure to be present.

In scrofulodermia, also, other evidences of the disease are to be seen, on the neck or elsewhere, in the form of enlarged glands or scars. As lupus and scrofulodermia 
not infrequently co-exist, and as the treatment of both conditions is practically the same, the recognition of what is lupus and what is scrofula is a matter more of academic than of practical importance.

The points of distinction from lupus erythematosus may be summed up as follows: While lupus vulgaris appears before puberty, lupus erythematosus generally shows itself after that period; the soft apple-jelly nodules characteristic of lupus vulgaris are altogether absent in lupus erythematosus; lupus vulgaris usually ulcerates at some time in its course, lupus erythematosus never does so; while lupus vulgaris erodes cartilage, lupus erythematosus scarcely ever extends to the deeper parts ; finally, lupus vulgaris is not symmetrical in its distribution like lupus erythematosus, and it seldom attacks the ears or the scalp. There are cases, however, in which the characteristic lesions of lupus vulgaris are masked by œdematous swelling, and in such circumstances it may be difficult to distinguish it from lupus erythematosus ; even then, however, if the scaly covering of the patch be removed, the prickle-like plugs on the lower surfaces of the crusts will serve to identify the condition as lupus erythematosus. In lupus vulgaris, by stretching the skin at the spreading edge of the disease, small amber-coloured nodules, having the characters of those distinctive of the affection, can generally be seen. Although such patches may not present any trace of ulceration, a tendency to cicatrization is visible at the border; this is never observed in true lupus erythematosus.

In its earliest stage lupus vulgaris may sometimes resemble eczema seborrhoicum, but the appearance of the apple-jelly nodules, the slow course of the process, and the tendency to the formation of scars, will serve to distinguish it from that affection.

Rodent ulcer is essentially a disease of later life. 
The lesion is, as a rule, single; it is much slower in its course than lupus, and when an ulcer is formed it penetrates deeply into the tissues. It differs from a lupous ulcer in having an indurated border and a smooth base.

Epithelioma, also, is a disease of later life. The hard everted edge, the foul base, often roughened with warty formations or sprouting with cauliflower-like excrescences, the implication of neighbouring lymphatic glands, and the secondary deposits in other parts, will serve to identify the disease.

In certain rare cases, where the lesions are numerous and scattered about the body, and where they are exceptionally scaly, lupus may simulate psoriasis; but on careful examination there will almost always be found at least one or two patches presenting the typical characters of lupus.

It should be noted that Neisser uses Oid Tuberculin as a routine method of diagnosis, but with caution if the lungs are affected, and not at all if they are much affected, lest the tuberculous focus should break down and set up general tuberculosis. He asserts that whenever a typical local reaction occurs the lesion is tuberculous, and when absent, non-tuberculous. ${ }^{1}$ The late $\mathbf{M}^{\prime}$ Call Anderson, another strong advocate of the use of the Old Tuberculin in diagnosis, testified that with reasonable care it is both safe and efficient. The methods of its application are described at p. 431.

Prognosis. - The prospect is favourable as regards life, lupus seldom, if ever, directly causing death. The possibility of secondary tuberculous infection, slight as on the whole it may be, must be borne in mind; nor should the possibility of the development of epithelioma be forgotten. Fordyce of New York says that the prognosis

${ }^{1}$ R. Cranston Low, Scot. Med. Journ., May, 1905 (abstr. in Brit. Journ. Derm., Mav, 1906). 
of epithelioma arising on lupus is of greater gravity than that of the ordinary cutaneous form, but this is by no means always the case. As far as recovery from lupus is concerned, the prospects of the patient depend on the severity and extent of the process, and in an almost equal degree on the treatment which is applied. In the most favourable circumstances lupus is an obstinate affection, with a pronounced tendency to recurrence even after the most thorough removal. If the disease be limited in extent, however, and the patient otherwise healthy, persevering treatment will, in a certain proportion of cases, bring about a cure. As already said, the process is most active in childhood, and the older the patient the more hopeful is the prospect of treatment proving successful.

Treatment.-The object to be aimed at is the complete removal or destruction of the diseased tissue. In deciding upon the particular method of local treatment to be pursued, one must not be guided entirely by the destructive energy of a particular agent or procedure; other points, such as the size and situation of the lesions, the tolerance of pain in a given patient, the length of time which the treatment will probably require, and the nature of the scar likely to be left, have to be taken into account, according to the circumstances of the case. Again, the idiosyncrasy of the disease itself must be reckoned with: while in some cases the roughest handling does no harm, in others the disease is of so angry a nature that even the mildest local treatment is resented. In dealing with lupus, as with other affections of the skin, it is necessary to feel one's way, and, while ruthless in warring against the disease, one must never forget that there is a patient behind it.

If lupus be superficial, an attempt should be made to bring about exfoliation of the diseased tissues. The 
best application for this purpose is salicylic acid, which may be used in the form of Unna's salicylic acid and creosote plaster mull, the latter drug being introduced to neutralize the pain caused by the former. The parts should first be softened with an emollient ointment, and then well washed with soft soap, so as to remove the scales. Care must be taken not to continue the use of salicylic acid too long, and to confine its use as nearly as possible to the affected surface, so as not to injure the surrounding skin. Another way of employing salicylic acid is to add it to glycerine in sufficient quantity to form a cream, with a little creosote, and apply it on lint. For either of these applications may be substituted the ointment suggested by Brooke, ${ }^{1}$ which is composed as follows :-

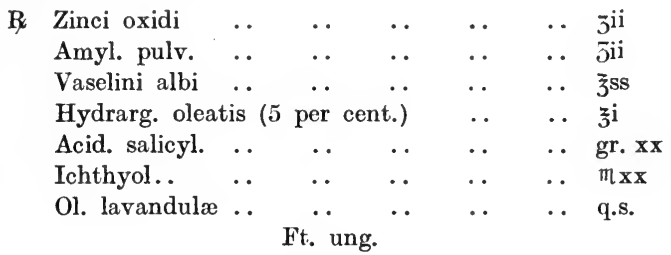

It should be vigorously rubbed in night and morning, the part being then thickly dredged over with potato-starch powder. I have seen excellent results follow the use of this ointment. If the epidermis should break, the surface should be dressed with some simple antiseptic application, such as boric-acid ointment.

Bactericidal applications are sometimes very useful. Mercurial plasters may be applied, or an ointment of gr. i or gr. ii of perchloride of mercury to vaseline ¡ $\mathrm{i}$ may be used. White of Boston says that by this

1 "A Preliminary Treatment of Lupus Vulgaris," Brit. Journ. Derm., May, 1890, p. 145. 
method a cure is effected in a few months. Doutrelepont applies a solution of corrosive sublimate of 1 in 1,000 under guttapercha tissue, and says the method has been very successful in his hands. Dubois-Havenith, on the other hand, who has frequently tried it, has had "variable, but always incomplete, results." Perchloride of mercury has also been injected into lupous patches by Doutrelepont, Tansini, and others, with a benefic al effect.

Chemical caustics are often very useful if applied in a thorough manner. Here the question of anæsthetics naturally presents itself. The injection of cocaine round the patch of lupus to be operated on will often dull the sense of pain sufficiently for the purpose in view. The advisability of a general anæsthetic, and the choice of an agent, if such be thought necessary, must depend on the special circumstances of the case. Among chemical caustics nitrate of silver holds the first place, and is still the favourite remedy for lupus with some very experienced dermatologists. It acts only on the diseased tissue, and may thus be very freely applied. The patch should be deeply grooved with the solid stick in various directions till the whole is destroyed. The procedure is extremely painful both at the time and afterwards. Equally good results can be obtained by milder measures, such as the salicylic-acid 'plaster of Unna. Acid nitrate of mercury, applied on the end of a probe tipped with cotton-wool, is a more efficient caustic than nitrate of silver; but it is also more painful, and gives rise to unsightly scars. Lactic acid is useful for the treatment of ulcerated surfaces ; it causes comparatively little pain, but as it acts impartially on sound and on diseased tissue the neighbouring parts must be protected when it is used. It is most applicable to lupus of mucous membranes. Arsenical paste destroys lupous tissue, but the application causes severe pain, 
and arsenical poisoning is not impossible unless great care be taken. The following is Hebra's formula :-

$\begin{array}{llllll}\text { Arsenious acid .. } & \ldots & \ldots & \ldots & \ldots & \text { gr. } x \\ \text { Artificial cinnabar } & \ldots & \ldots & \ldots & . . & \text { 3ss } \\ \text { Rose ointment } & \ldots & \ldots & \ldots & . . & \text { 弓ss }\end{array}$

This is spread on linen and applied evenly on strips, over which a piece of lint is firmly bandaged. The caustic should be left in situ for twenty-four hours, when the parts are carefully cleansed and the paste reapplied. Chloride of zinc is extremely useful as a caustic agent, especially as a supplement to surgical measures. It may be applied in solution of equal parts of chloride of zinc and alcohol, or as a paste :-

\begin{tabular}{|c|c|c|c|c|}
\hline Chloride of zinc & .. & & .. & \\
\hline Powdered opium & - & • & .. & . \\
\hline Hydrochloric acid & . & - & . & . \\
\hline Boiling water & 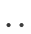 & . & $\ldots$ & $\mathrm{ad}$ \\
\hline
\end{tabular}

Dissolve. To one ounce of the solution add two drachms of wheaten flour. (Middlesex Hosp. Ph.)

Pyrogallic acid is extremely valuable in most cases. It has a selective action on the tissues, and as a rule causes comparatively little pain ; to this rule, however, there are exceptions. It may be applied in the form of a plaster mull or as an ointment (5-10 per cent.). Boeck has found that the pain sometimes caused by pyrogallol is greatly reduced if the drug is made up with a gelanthum or mueilaginous base, as in the following formula :-

\begin{tabular}{|c|c|c|c|c|}
\hline Pyrogalli & $\cdots$ & $\cdots$ & - & \\
\hline Resorcini & . & .. & .. & $\{\bar{a} \bar{a} 7 \cdot 00$ \\
\hline Acid. salicyl. . & . & $\cdots$ & . & \\
\hline Gelanthi & $\cdots$ & .. & . & āā $5 \cdot 00$ \\
\hline Talci pulv. & . & . & $\cdots$ & \\
\hline
\end{tabular}

Any ulcerations in the affected area are anæsthetized with 5 per cent. novocaine, or touched with nitrate of 
silver; the paste is then applied with a spatula all over the patch and a thin layer of cotton-wool spread over it. The paste soon dries, and with the cotton-wool forms a permanent dressing, which is left in situ for a week or longer. Pyrogallic acid is particularly useful in the after-treatment of patches that have been subjected to erasion, scarification, or cauterization. It may be combined with salicylic acid in 10 per cent. in collodion, or in the form of ointment.

The mechanical treatment of lupus includes excision, erasion, scarification, cauterization, simple or electrical, application of the Finsen light and X-rays, and of carbon-dioxide snow.

Excision gives excellent results if the case comes under treatment at an early stage, and the underlying tissue is healthy, and the whole of the disease can be removed without leaving too large a breach of surface. It is also serviceable in dealing with chronic decadent lesions in the adult. The operation is chiefly applicable in the case of limited patches situated on the limbs or trunk. Healing of the wound is greatly aided by transplantation of skin after the method of Thiersch. In this way comparatively large gaps in the tegumentary covering have been filled up. The most thorough removal of the lupous tissue, however, affords no absolute guarantee against recurrence. Excision is, for obvious reasons, seldom, if ever, applicable in lupus of the face.

Erasion, or scraping, is useful when the disease is extensive. The ulcerated surface is scraped out with Volkmann's spoon, just like a tuberculous joint. The scraping must be done with some amount of force; and it will be found that the underlying healthy tissue is much tougher than the diseased structures, which break down readily under the curette. Some powerful antiseptic, such as strong carbolic acid or 
perchloride of mercury ( 1 in 2,000), should be used to wash the raw surface, and the wound should be dressed antiseptically. Erasion, as a rule, requires to be supplemented by chemical agents, such as pyrogallic acid or chloride of zinc, which complete the work of destruction. Veiel supplements crasion by multiple puncture, stabbing the scraped surface in hundreds of points with a narrow-bladed knife.

Scarification consists in ploughing up the diseased patch in close-set parallel furrows, so that all the nodules are broken up. A lupous patch may be scarified in different directions, the lines crossing each other so that no point shall escape the knife. The secret of successful scarification is to use very sharp instruments, and to multiply the incisions so as to cover the whole surface in such a way that the diseased tissue shall be, as it were, thoroughly minced up and the vessels destroyed or occluded. The scarification should be carried below the level of the new formation without going beyond the limit of the corium.

Cauterization with Paquelin's cautery is a severe method, which should hardly ever be used except when it may be of importance to destroy the disease very rapidly. It destroys lupus in a minimum of time, but at the expense of a maximum of cicatrix, with all the subsequent possibilities of deformity and disablement. The method should be reserved for the destruction of small recurrent nodules. The galvano-cautery is more generally applicable, and its effect is much more under the operator's control. It may be used by way of puncture, the affected tissues being, as it were, tattooed with the incandescent point, with which the apple-jelly nodules are individually attacked. Galvano-cauterization can be used as a primary method, the affected surface being, as in the case of scarification, first attacked at the edge. It is also very useful as a supplementary $2 \mathrm{E}$ 
method, after erasion or scarification, for the destruction of recurrent nodules.

The Finsen light, though not the best method for all cases, is unequalled in certain types of the disease, and when combined with other measures it is probably the best all-round treatment for the majority of cases of lupus. In non-ulcerative superficial cases the light alone is sufficient. Where there is much infiltration, or scarring, the process of repair is sometimes hastened by a short course of $X$-rays after the nodules have been partly broken down by the light. If there be deep ulceration it is better to begin with the $\mathrm{X}$-rays, any remaining or outlying nodules being then individually exposed to the light. In more extensive cases, also, it is better to use the X-rays first. The application of pyrogallic acid in a 5 per cent. ointment is a useful adjuvant in reducing thickening of tissues. But in very extensive cases, especially if there be much fibrosis or massive infiltration, or if the disease is rapidly spreading, neither the light nor the $\mathrm{X}$-rays can be recommended. When mucous membranes are the seat of lupus the X-rays are more effectual than the Finsen light. Of both Finsen light and the X-rays it must be admitted that the good results are not always lasting. ${ }^{1} \quad$ Winkler reports excellent results from the use of soft X-ray tubes, followed by application of the static current. Carbon-dioxide snow has also been used with good results, both alone and in conjunction with $\mathrm{X}$-rays.

The fact that the primary focus of lupus is often situated within the nose supplies an explanation of the difficulty of permanently curing the disease. As long as the original source of the trouble remains, re-infection

${ }^{1}$ For reports of cases under the care of the Author, assisted by S. E. Dore, see " Light and X-Ray Treatment of Skin Diseases" (1907). 
of the skin may take place again and again. It is most important to apply effective treatment to lupus in the nasal cavity, so as to prevent the exterior of the nose from being involved.

An important practical point that must be borne in mind in connection with all the severer methods of treatment is to know when to hold one's hand. When inflammation is severe, and the affected tissues are proportionately irritable, soothing applications must be used for a time. For this purpose calamine or lead lotion will be found most useful. Radical treatment should not be proceeded with till the inflammatory condition has been subdued.

With regard to the choice of a method adapted to the situation of the disease, it may be stated in general terms that on the face the Finsen light or X-rays, and on the trunk and limbs erasion, followed by galvanocautery, are the most suitable procedures. In either case the initial advantage obtained by such treatment may need to be followed up by chemical caustics and parasiticidal agents. There is no general formula for the treatment of lupus. Each case must be treated in accordance with its requirements, and each of the methods described has its own special advantages when it is used in the proper circumstances. In the vast majority of cases it will be found that different methods will have to be employed at different stages, while occasionally it will be advantageous to suspend all treatment for a time until the disease has, as it were, lost the tolerance which prolonged medication has produced. The application of chemical substances will cure only milder forms of lupus, where the disease is superficial. On the other hand, there is no mechanical method, however severe, that will infallibly prevent recurrence. The best results will be obtained by a judicious combination of mechanical 
with chemical treatment. The patient should be kept for a considerable time under strict observation, so that any fresh outbreak of the disease may be treated at once.

Constitutional treatment must be carried out on general principles. In a certain proportion of cases the patients present no evidence of ill-health, and therefore require no internal medication. There is no internal remedy, except, perhaps, thyroid extract, that has any specific effect on lupus. If the patient be of scrofulous constitution the treatment appropriate to that condition is indicated. Cod-liver oil in such cases appears to have a decidedly favourable effect. Good food, sea air, and attention to hygiene are powerful adjuvants in the treatment of scrofulous patients. Other unfavourable conditions, such as anæmia or chlorosis, must be dealt with by appropriate measures.

The O'd Tuberculin (T.O.A.), although not the specific which it was at first believed to be, has still, in my opinion, a distinct place in the therapeutics of lupus. The injections sometimes cause an immediate reaction of such violence that it quickens the activity of the process; and in the most favourable circumstances the temporary improvement that follows them speedily disappears. ${ }^{1}$ Notwithstanding this, tuberculin seems to modify the lupous process in such a way that the disease becomes more amenable than before to local treatment. My own experience has been decidedly encouraging, all the more since my earlier expectations were grievously disappointed. Of twelve cases in which I gave the tuberculin treatment a full trial there was not one that did not within a comparatively short time relapse to a condition as bad as before the treatment. Further observation, however, has convinced me that the tuberculin, while

${ }^{1}$ It is worth mentioning that lupus sometimes undergoes considerable temporary improvement under the influence of an attack of erysipelas. 
failing by itself to effect a cure, prevents recurrence when the disease has been destroyed by other means. The patients referred to were, after the failure of the tuberculin, treated by the ordinary chemical and mechanical methods, and may now be looked upon as practically cured. As treatment of the same kind had been tried in all these cases for years previously without permanent success, the apparent abolition of the tendency to recurrence must be placed to the credit of the tuberculin. Neisser has habitually used the Old Tuberculin in treatment, as well as in diagnosis, since its discovery. The late M'Call Anderson, who had a most extensive experience of its use, laid down the following rules for its administration: 1. The initial dose, in the case of an adult, should not generally exceed $\frac{1}{2}$ c.c. of 1 in 1,000, and sometimes it is safer to begin with $\frac{1}{4}$ c.c. 2. Should a dose have little or no effect it is generally safer to give a second of the same strength as the preceding one, the later often acting much more severely than the earlier one. 3. The more pronounced the constitutional reaction the longer should be the interval before the next injection. 4. Much greater care must be exercised in increasing the doses in the earlier than in the later periods of the treatment, when the system has gradually got "acclimatized" to it.

I have tried the New Tuberculin (T.R.) in a series of cases, with results which, though brilliant at first, have since proved disappointing. Others have since reported results which support a rather more favourable view, especially in relation to cases in which parts inaccessible to direct surgical or phototherapeutic measures are involved. Tuberculin treatment by Wright's method is also of value, and may be combined with other measures. 


\section{CHAPTER XX}

\section{MICROBIC AFFECTIONS (Continued)}

Syphilis: Stages, Symptoms, Etiology, Diagnosis, Prognosis

SyPHILIs is a disease caused by the Spirochata pallida (Treponema pallidum) of Schaudinn and Hoffmann. The spirochæte is inoculated-that is, conveyed by direct contact; an abrasion of surface on the part of the recipient facilitates its introduction, but is by no means a necessary condition of infection. The disease is, in the vast majority of cases, transmitted during sexual intercourse, but infection may take place on any part of the body in which the poison is implanted. It may be acquired, or it may be inherited-either from a diseased father (sperm inheritance) or from a diseased mother (germ inheritance). Germ inheritance may take place whether the mother be the subject of syphilis at the time of conception, or whether she contract the disease at any period during gestation; thus, as pointed out by Hutchinson, the child has a much greater chance of being infected by the mother than by the father. Both parents may, of course, be syphilitic, and the offspring will in these circumstances have a double chance of being infected; but there is no evidence to show that the resultant disease is of a severer type than when the poison is drawn from one source only. What is inherited in syphilis is not merely a predisposition to a particular disease, but the actual virus itself, modified, it may be, by its passage through the parent or parents. 
Stages.--Syphilis is really a specific exanthematous fever, "diluted by time" (to use the happy expression of Moxon). It presents a close analogy to smallpox; for instance, if we suppose the eruptive stage to be drawn out into months instead of days, and the sequelæ to come on after years instead of weeks, the following stages can be reeognized in a typical case of acquired syphilis: (1) a latent period, which intervenes between the date of eontagion and the earliest sign of local infection; (2) an incubation period, which includes the formation and development of the chancre and enlargement of the nearest lymphatic glands; (3) a period of invasion, including the specific fever with its associated phenomena up to the appearance of the general eruption; (4) an eruptive period, with early and late development of characteristic lesions on the skin and mucous membranes, and in the glands; a period of quiescence; (6) a period of sequeloe, consisting of late so-ealled "tertiary," and "quaternary" or parasyphilitic lesions. For practical purposes Ricord's division of syphilis into three stages-primary, secondary, and tertiary - is convenient, and corresponds with fair accuracy to natural divisions in the clinical history of the disease. It is necessary, however, that a clear conception should be formed of the exact state of things indicated by these terms. In the early part of the primary stage, syphilis is a local disease, and the spirochæte can be conveyed only by direct contagion from the local sore. The Wassermann test, however (p. 495), may become positive about fourteen days from the appearance of the initial lesion, so that the disease becomes general before the primary stage has passed into the secondary. In the secondary stage-representing the eruptive period of a specific fever-syphilis manifests itself by constitutional symptoms due to the diffusion and multiplication of the spirochæte in the blood: 
in this stage the blood and all the fluid tissues contain the specific virus; and the infection can be transmitted by the secretion from any of the lesions, and by the saliva and other normal fluids.

It must be understood that in many cases it is not only the specific organism of syphilis that is inoculated. The sores become infected by various microörganisms, such as those of soft sore and phagedæna, which cause inflammation and suppuration, and these organisms are often conveyed with the spirochæte. These extraneous infective matters produce lesions of a peculiar kind, which may complicate and in some cases overshadow the specific effect of the syphilitic poison.

Symptoms.-The primary lesion generally appears from three to four weeks after exposure to contagion -hardly ever less than two, or more than six, weeks. The appearance of the lesion varies according to its situation. When situated in a typical position, as on the glans penis or the labium, the first perceptible change is a minute red spot. In a week or ten days this grows into a nodule with definite margin. A marked characteristic of this nodule is its hardness. The induration is seldom very distinct before five weeks have elapsed from the date of inoculation. There is usually more or less itching, though this may be totally absent. Ulceration generally takes place, and the resulting sore presents a minutely granular floor, secreting a small quantity of thin liquid, and bounded by a definite but not raised border. The base of the ulcer is distinctly indurated. Sometimes the lesion is limited to a desquamating papule which does not ulcerate, but may undergo involution so rapidly that the patient, unless he has been on the look out for it, may be unconscious of its presence. On the other hand, it may persist for several months. Simultaneously with the induration of the chancre, the nearest set of lymphatic glands 
becomes enlarged and hard. The primary sore has a natural tendency to heal, the induration gradually disappearing and a scar being left. When unmodified by treatment the primary lesion seldom lasts less than two months. There is usually only one primary sore, but occasionally there may be several, depending on the number of points at which the virus has been inoculated at the time of contagion. I have seen five sores, having the characters of the hard chancre, on a patient's arm at the same time.

Chancres acquired from sexual intercourse are generally situated, in the male, on the frænum and inner surfaces of the prepuce. The glans, the margin of the prepuce (where the chancre is often multiple), the orifice of the meatus, the mucous membranes of the urethra within the meatus, and the skin of the penis, are also common situations. In the female the inner surfaces of the labia majora and the nymphr are the most frequent sites of hard sores; they are also met with on the clitoris and on the os uteri. The vagina seems to be protected from inoculation by the thickness of its epithelium. Chancres are more frequently multiple in women than in men, probably owing to the greater opportunities of auto-inoculation. Extragenital chancres oecur on the fingers (as in midwives and surgeons), on the nipples (in wet-nurses), on the lips, cheeks, tonsils, or tongue, from kissing, smoking infected pipes, drinking out of infected glasses, etc.; they may also develop on vaccination scars or on any part of the body where the poison may be inoculated by a bite or other injury. Dentists' instruments have occasionally been vehicles of the syphilitic poison. The disease has been communicated by tattooing. When the finger is the seat of the chancre, it often first attracts attention as a persistent fissure at the lateral nail-groove. $\mathrm{A}$ hard sore has been known to develop on the penis of an 
infant, after ritual circumcision, when the method-now abandoned in Great Britain-of stopping bleeding by suction was adopted. Hard sores may also be met with in extraordinary situations as the result of unnatural vice; but probably the great majority of extragenital chancres are contracted accidentally.

Wide differences are observed in the appearance of chancres, these being chiefly due to the anatomical peculiarities of the part on which the chancre is situated. Thus, a chancre on the glans is usually definitely circumscribed as well as indurated, whilst a chancre of the cheek presents a diffuse tense œdema in which the edge of the sore is lost. The chancre that affects the bed of the nail is scarcely ever indurated, and often suppurates very freely (Hutchinson). On other parts, and especially on the face, chancres sometimes attain an enormous size, and may lose the ordinary characters of infecting syphilitic sores and simulate malignant disease. Apart, however, from differences in appearance determined by anatomical conditions, and modifications caused by treatment, great variations are observed in chancres. In the incubation period there may be nothing beyond a small dusky spot which lasts for a few days and then disappears, leaving a brown stain. On the other hand, there may be an obstinate ulcer with marked induration, lasting a year or more, and leaving a scar. The induration may recur from time to time (chancre redux), even as long as seven or eight years after its complete disappearance.

The primary sore has a protective influence like that of vaccination, but, like the latter also, the immunity which it confers is neither absolute nor permanent in all cases. Instances of reinfection are not very rare, but the disease is usually much milder in the second than in the first attack.

As already said, there is frequently a double inocula- 
tion, infective matter of inflammatory origin being introduced at the same time as the specific virus of syphilis. In this way soft sores, produced by the streptobacillus of Ducrey, are produced. These sores are hardly ever seen except on the genitals. They are usually multiple, and can reproduce themselves in the patient by secondary inoculation. The typical soft sore has a sharply-cut, punched-out margin, and a grey, unhealthy-looking base with a considerable zone of inflamed skin around it. The lymphatic glands in the neighbourhood become enlarged, and often suppurate, and the several glands of a group become matted together by inflammatory exudation.

It must be clearly understood that although the infective sore is called, in accordance with its most obvious physical character, "hard," and the noninfecting sore by way of distinction "soft," neither of these charaeters is sufficiently constant to be made an absolute criterion of the nature of a given sore. An infecting sore is not always hard; on the lip, for example, there is seldom any marked induration. On the other hand, a sore at first soft may after a few weeks become indurated, and be followed in due course by the develonment of constitutional syphilis.

Herpes, which may occur on the genitals in either sex (herpes genitalis), sometimes follows both infecting and non-infecting sores. A previously existing herpes may conceal a chancre, a fact which should always be borne in mind when the herpes occurs in newly married persons.

The venereal sore may become the seat of phagedana. The ulcerative process assumes a more violent character, and spreads rapidly both in area and in depth; the edge of the ulcer becomes irregular, and sloughing frequently takes place. The ulcer is very painful, and serious hæmorrhage is sometimes produced from erosion 
of the arterioles. Great destruction may be wrought by this process, the penis being sometimes entirely eaten away. The contagion, which probably always originates from venereal sores (Hutchinson), may spread through a hospital, attacking all operation and other wounds.

By the term mixed sore is generally understood a syphilitic chancre contaminated by the presence of septic material, or a sore the result of a double infection. In such sores in recent times the presence both of the streptobacillus of Ducrey and of the Spirochceta pallida has been demonstrated.

Secondary lesions. - If a case of syphilitic infection be left to itself, secondary symptoms may be expected to show themselves in from seven to nine weeks after inoculation. Their onset is, as a rule, marked by little or no constitutional disturbance. In some cases, however, it is ushered in by distinct febrile phenomena. The patient feels unwell, languid, and weak, and complains of loss of appetite, with headache and pains in the joints, muscles, and bones, especially those lying just under the skin-the tibiæ, ulnæ, and clavicles. All these symptoms are usually aggravated at night. The temperature curve often shows a marked evening rise. Occasionally the fever runs extraordinarily high, as in a case reported by Burney $\mathrm{Yeo}$, in which for several weeks it ranged from $100^{\circ} \mathrm{F}$. to $104^{\circ} \mathrm{F}$. The pyrexia may be out of all proportion to the skin eruption; but generally, when the eruption is unusually severe, the fever runs higher than in ordinary cases (Hutchinson). In rare cases the constitutional disturbance is so great, and lasts so long, that the nature of the disease may not be suspected for some time, the symptoms being attributed to some obscure form of toxæmia. The eruption is erythematous in character, and is known as syphilitic roseola. It shows itself as a macular mottling, 


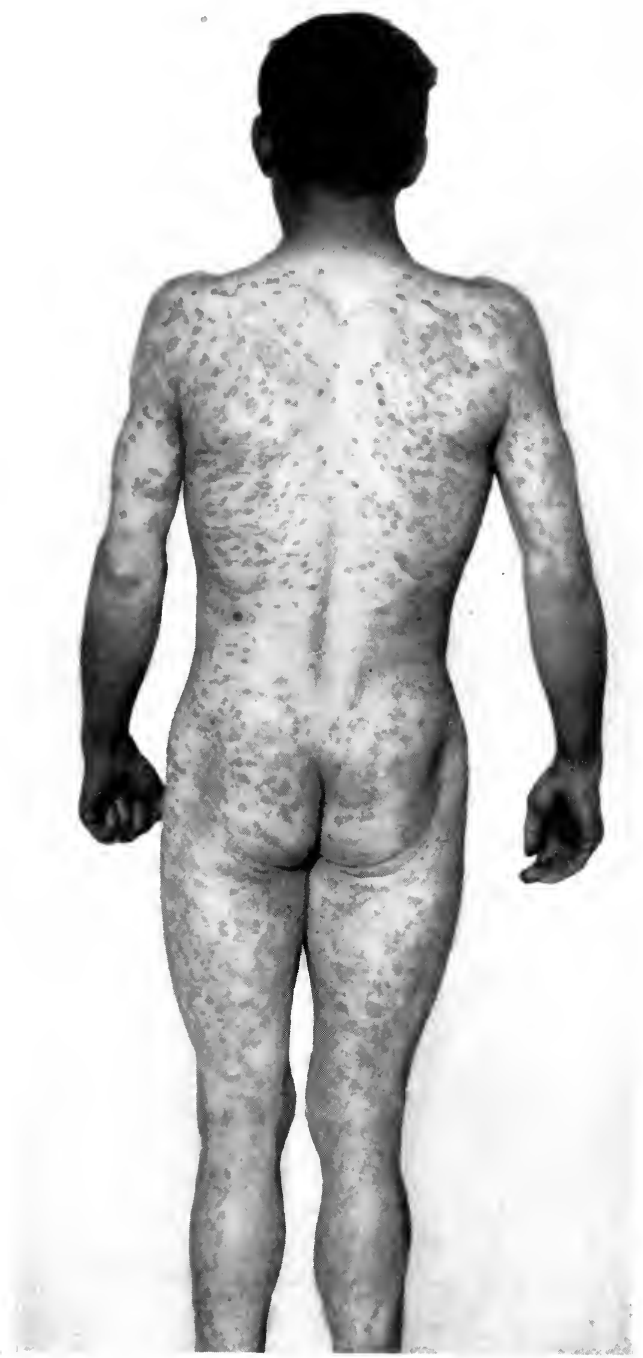

PLATE 41.- PAPULO-MACULO-SQUAMOUS SYPHILIDE.

(Choyce's "System of Surgery.") 


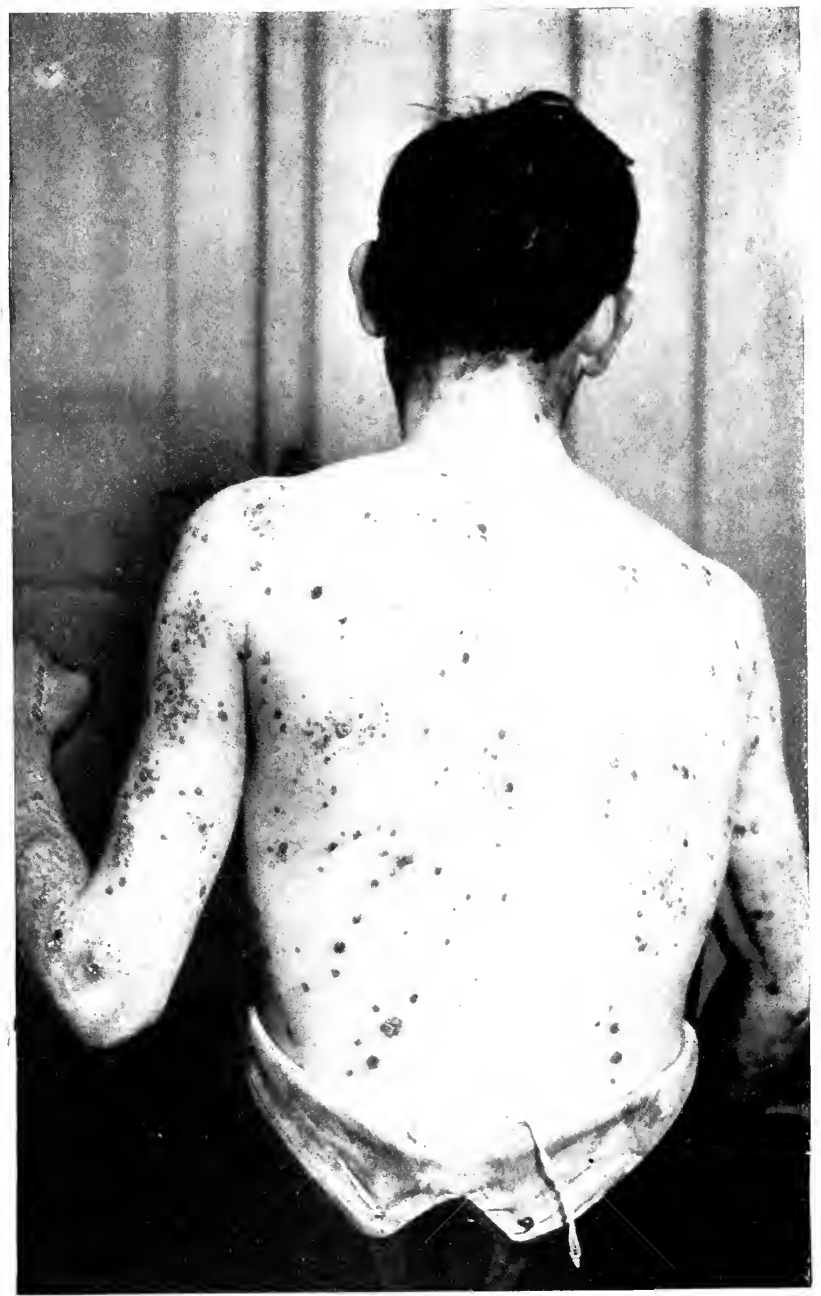

PLATE 42 - CORYMBOSE SYPHILIDE, SEVENTEEN WEEKS AFTER INFECTION.

(IIhitfuld's Cuse. Brit. Journ. Derm., roor.) 
resembling measles, but rather more dusky, scattered more or less thickly over the chest and abdomen. The rash varies in intensity according to the temperature and the amount of clothing worn. It is very evanescent, often disappearing in a few hours, and coming out again as suddenly. Coincidently with the roseola, slight superficial ulcers form on the tonsils; these are often so painless and so transient that the patient may be unaware of their existence. Even if there be no definite ulceration of the throat, the mucous membrane is congested, being, in fact, the seat of an eruption similar to the roseola on the skin. The rash generally begins to fade within a fortnight of its appearance, giving place to a papular or papulo-squamous or papulo-maculosquamous (Plate 41) eruption which comes out on the trunk, limbs, face, and neck. The papules are small, tense, and firm, with smooth or slightly scaly tops. They increase in size by peripheral extension, the older central parts undergoing atrophy or necrosis. Occasionally the eruption assumes a corymbose form (Plate 42). Sometimes, though very rarely, vesicles are formed, or suppuration may supervene and give rise to pustules. The pustules may be either acuminate or flat, and in both the lesion may be either large or small. The small acuminate pustule is known as the miliary lichenoid or follicular syphilide (Plate 44), the large acuminate pustule the acneiform syphilide. The small flat pustule is the impetiginous syphilide, the large flat pustule the ecthymatous syphilide.

In the early stage pustules dry up and form scabs, underneath which no ulceration takes place, and consequently no scar is left. In the later stages the breaking-down of the papule is followed by an ulcerative process with rapid drying of the secretion into crusts; as the ulcer spreads at the edge, each successive layer of crust is necessarily larger than the one immediately 
above it, and a pyramidal structure somewhat resembling a limpet-shell is thus formed, to which, from its dirtybrown colour, the term rupia ( $\dot{v} \pi \operatorname{sis}=$ filth) is applied. Sometimes the crusting process begins in the drying-up of a bulla. Rupial lesions are hardly ever met with till from six months to a year from the appearance of the primary sore, and then usually only in persons who have neglected treatment or whose health has broken down. Rupia always leaves scars, and is symmetrical.

Hyperæmia of the papillæ in particular spots gives rise to patches which may be evanescent (roseola), or may persist as isolated blotches for a longer or shorter time (macular syphilides). These macules, which vary in colour from a delicate rose to a pale violet or duskybluish or even brownish red, have a smooth surface, and, being partly infiltrations, do not disappear completely on pressure. They are seen chiefly on the chest and abdomen, often on the flexor aspects of the extremities, seldom on the face. They cause no subjective symptoms. Scattered among the macules or on them may often be seen papules (maculo-papular syphilides). These syphilides last a variable time, and leave stains the depth of which is proportionate to the length of time the lesions have persisted. A remarkable property in these and other forms of secondary eruption is that they are made more conspicuous by the action of cold on the surface of the skin. In association with the macular syphilides, alopecia, either general or in patches, is often observed. Alopecia areata is in rare cases, however, the earliest sign of secondary syphilis.

Hyperæmia of the papillæ is often followed by infiltration of inflammatory products, and in this way a papule is produced (papular syphilide). Papules, as already said, often arise in connection with the macules; they may also develop independently. Two varieties of these lesions may be distinguished-the small, otherwise 


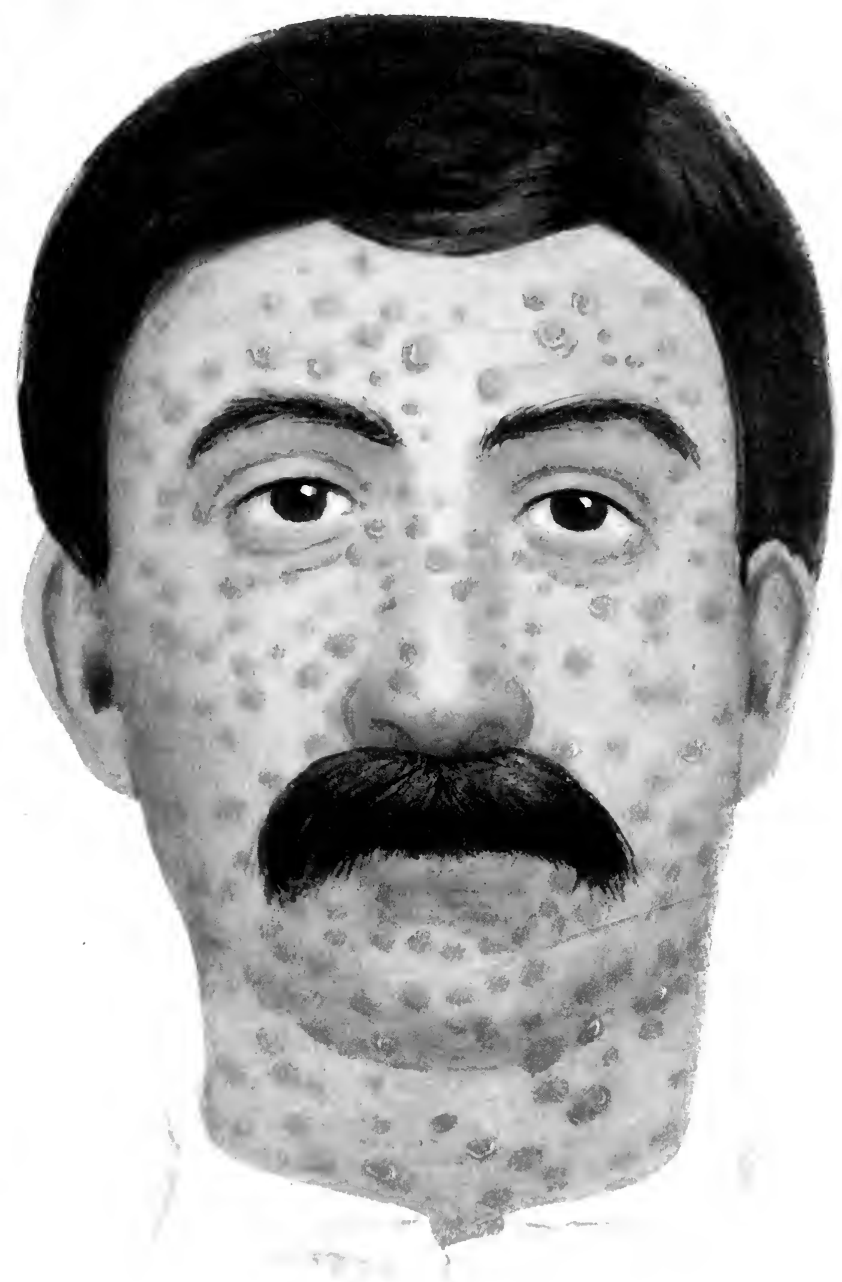

PLATE 43. - LENTICULAR SYPHILIDE,

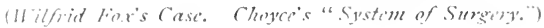


the miliary or follicular papule, and the large, or flat, or lenticular papule (Plate 43). The former vary in size from a pin's head to a linseed; they are at first red, afterwards brownish or of a raw ham or coppery colour, have a shining surface, and feel like small shot. They are thickest over the abdomen, chest, shoulders, and upper limbs, more sparsely scattered over the back and the legs. Involution takes place slowly, and the stain left behind is long in dying away, and is sometimes followed by a shallow depression which may last for years. The small papule is not very common as an early lesion, and is generally looked upon as a sign that the disease is of a severe type. The large papule may develop directly out of the macular syphilide, or may be produced by the gradual enlargement of the small variety. It may be as large as a pea, but is generally flattened on the surface. It affects the whole body pretty impartially, sometimes forming a kind of circlet on the brow round the margin of the hair (corona veneris). Sometimes ringlike patches, the circinate or annular syphilides (Plates $45,46)$, formed by small yellowish-red papules with fine scales, are met with on the chin, around the lips or nostrils, or, it may be, on the vulva. In other cases the flat papules become seborrhœic, and are very obdurate to treatment. When there is pronounced scale formation, these syphilides are styled papulo-squamous; they frequently appear on the palms and on the soles, and may be mixed with maculo-papular and papulo-tubercular lesions; they are often rounded or irregular in shape, have but slight elevation, are at first brownishyellow or brownish-red, but afterwards turn a dirty grey, and on the disappearance of the scales are seen to be of the colour of raw ham. When the scales are unusually horny they form the syphilides cornées of French dermatologists.

The evolution of papules is irregular. Some persist 
as such and increase in size by peripheral extension, undergoing involution meanwhile in the centre. As they shrink they become scaly on the surface; if the formation of scales is at all active, the lesions often come to bear a tolerably close resemblance to patches of psoriasis (Plates 45 and 46). Desquamation frequently persists after complete subsidence of the papule. In other cases, as already said, the papules become transformed into vesicles and pustules. With regard to the vesicular forms, it must be noted that they have no affinity with the eczematous process; the latter is catarrhal, but in the production of syphilitic lesions of the skin the element of catarrh has no place.

A further stage in the development of the papule is reached by the occurrence of overgrowth of the papillæ, giving rise to warty conditions, the favourite seats of which are the tongue and the genitals. If the lesion is situated in a moist part, the hypertrophied papillæ are covered with sodden white epithelium (moist papule, or mucous papule or patch). A more marked degree of hypertrophy transforms the moist papule into a mucous tubercle or condyloma. The difference between warts and condylomas is that while in the former the overgrown papillæ are free, in the latter they are welded into a coherent mass by swelling of the intervening tissue:

At what may be called the height of the eruptive stage of syphilis the lesions may present almost every conceivable variety of type. Not only the simple elementary lesions that have been described may be seen mingled together in every phase of development, but mixed forms, of a complexity that baffles description, may be observed. In this way almost every known skin affection may be more or less closely simulated. Thus one secondary eruption will simulate a copaiba rash, or varicella, or even variola; another, lichen planus : 


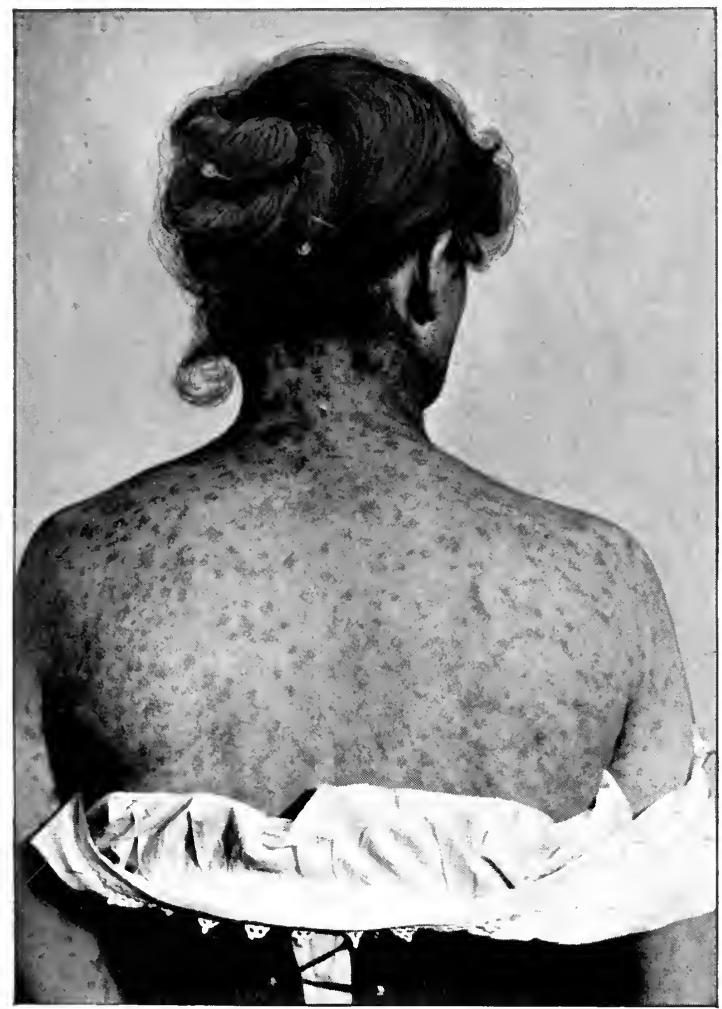

PLATE 44.-FOLLICULAR SYPHILIDE, THIRTEEN WEEKS AFTER INFECTION.

(IITitfield's Case.) 


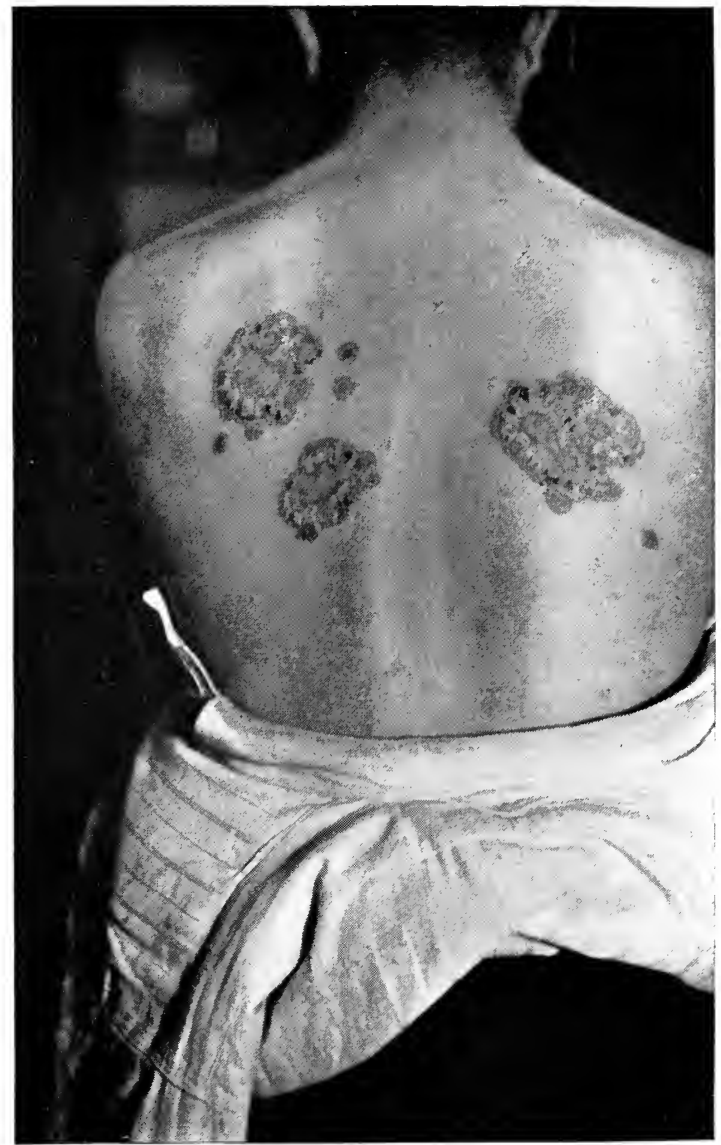

PLATE 45. CIRCINATE SQUAMOUS SYPHILIDE.

(IITitfluld's Case.) 
another, impetigo or acne ; another, alopecia or leucodermia. The leucodermic lesions, almost peculiar to women, take the form of sharply outlined white spots which stud patches of greyish or brownish staining in the neck-hence the term "venereal collar" (Plate 47). Purpura is not infrequently among the manifestations of constitutional syphilis, and pigmentation of the skin may be produced without a pre-existing lesion by transudation of the colouring matter of the blood. At a later period this polymorphous character of the eruption usually gives place to simple ulcerative or squamous lesions. The eruptive period as a whole may last for months. As regards the duration of the individual lesions, little is known. The stains will often last for years. In one case which I have seen the pigmentation was still visible more than twenty years after the secondary lesion of which it was a legacy had disappeared.

There are certain general characters which distinguish secondary eruptions. Though no single one of these is pathognomonic, the combination of two or more of them affords prima-facie evidence of a syphilitic origin, and the combination of several is quite conclusive. In the first place, secondary eruptions are usually symmetrical: This arises from the fact that syphilis in the stage represented by these lesions is a general and not a local disease. Again, secondary eruptions are polymorphous. This is the most distinctive characteristic of the eruption taken as a whole. Not only are the individual lesions multiform, but the grouping of them presents the greatest diversity of appearance. Sometimes the papules are arranged in lines like lichen planus; or the papules, pustules, etc., may be set in isolated patches or irregular clusters ; or the arrangement may be corymbose, several lesions being clustered together, or a large one being surrounded by a circlet of smaller ones, as in $2 \mathrm{~F}$ 
erythema iris. Secondary lesions, both on the skin and on the mucous membrane, have a tendency to assume crescentic outlines. Not only may all the different elementary lesions be present at the same time, but they may be there in all stages of development. Only erythema multiforme and dermatitis herpetiformis in their most variegated aspect can be compared as regards polymorphism with the eruptive stage of syphilis. The lesions in the latter condition, however, have this character distinguishing them sharply from both those affections -namely, the absence of itching. The colour of secondary lesions is remarkable, but not being peculiar to them, can hardly be taken as a trustworthy guide to their nature. The prevailing tone of these lesions is a tint resembling the lean of raw ham, passing into a coppery colour, and leaving a permanent brown pigmentation. The coppery colour of a lesion may be suggestive, but taken by itself it is of comparatively little clinical importance, and a diagnosis of syphilis should never be based on that alone. As regards position, the first rash, as already said, comes out on the abdomen, next on the chest, then on the front of the arms and the back of the legs, next on the palms and soles, the back and sides of the neck, and sometimes on the face. The scaly lesions which simulate psoriasis affect the flexor rather than the extensor surfaces of the limbs, and are seldom seen on the tips of the elbows and knees, the typical situations of true psoriasis. The epigastric and hypochondriac regions, the nape of the neck, and the forehead near the margin of the hair, are situations much affected by syphilitic lesions.

The earliest local manifestations of constitutional syphilis on mucous membranes are, as has been said, small ulcers on the tonsils. These have usually more or less the vutline of a horseshoe, with a yellowish floor and greyish-white borders. They generally pass away 


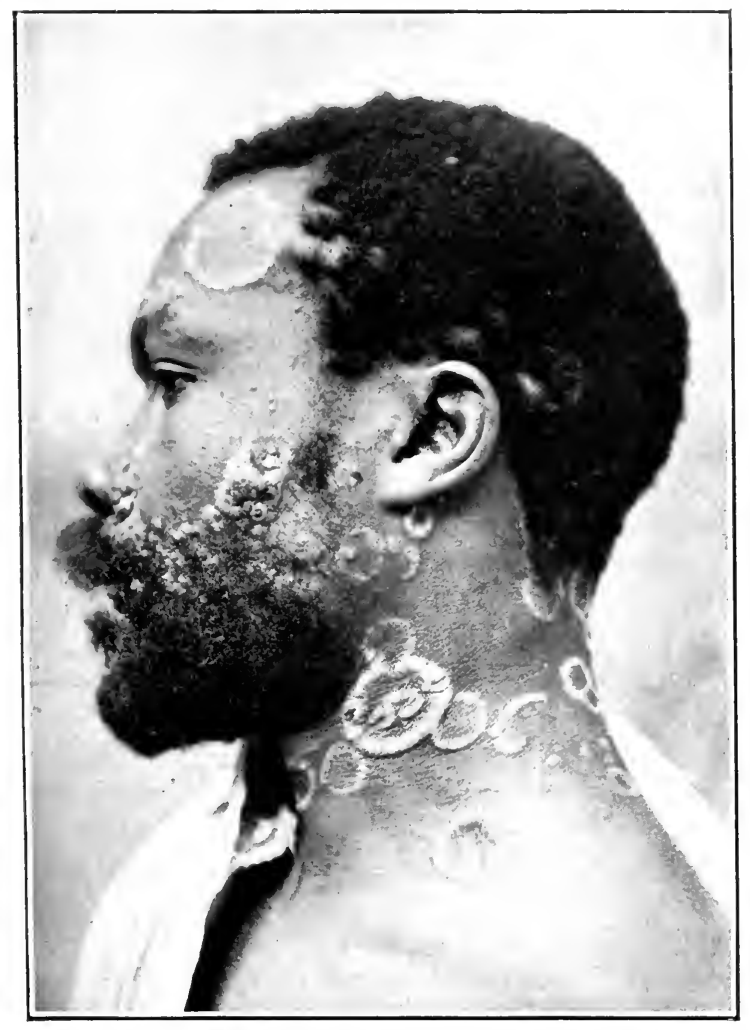

Plate 46.-CIRCINATE SyPhilide in a NEgRo. (Gilchrist.) 

quickly. At a later period mucous patches and mucous tubercles may form on the cheeks, tongue, gums, lips, pharynx, larynx, about the vulva and anus, and under the prepuce; these patches may prove obstinate. From the anus the lesion may spread inwards to the mucous membrane of the rectum.

One of the most important symptoms of constitutional syphilis is the general enlargement of glands. Those most severely affected are the posterior cervical, the inguinal, and the epitrochlear. They are small, separate, free from tenderness, and do not tend to suppurate.

Besides the lesions of the skin and mucous membrane which have been described, all the other tissues of the body-especially the eye, the bones and periosteum, the joints, and the arterial and nervous systems-are liable to become involved. Iritis is of common occurrence from four to seven months after infection; and there may be symmetrical retinitis. The ear may be the seat of otitis media and interna. Periostitis and synovitis, giving rise to tenderness of the bones and joint pains, are common. Localized anæsthesia, due to peripheral neuritis, is said to occur (Fournier). All these symptoms are apt to be symmetrical. Permanent blindness or deafness may, however, result from the inflammation of the retina and internal ear. In describing two unusual forms of syphilitic nails Adamson and McDonagh ${ }^{1}$ give a valuable classification of changes in the nails that may occur in the course of secondary and tertiary syphilis. They divide them into groups-(1) dry onychias (onychia sicca syphilitica), in which the changes appear to be confined to the nails themselves, and (2) paronychias, or perionychias, in which the affection of the nail is a consequence of inflammatory conditions of the parts beneath or around.

In most cases secondary lesions disappear under ${ }^{1}$ Brit. Journ. Derm., [1911, xxiii. 68. 
treatment, and in about six months the patient may seem to be entirely free from the disease. He may, however, remain liable from time to time to "reminders" in the form of lesions intermediate in type between the secondary and the tertiary forms, and partaking to some extent of the characters of both. Among these "intermediate" lesions are sores on the sides of the tongue, and white patches with thickening on its dorsum (the so-called psoriasis lingua or leucoplakia ${ }^{1}$ ), red scaly areas with sinuous outlines on the scrotum, and patches of induration covered with layers of thickened and desquamating epithelium on the palms of the hands (the so-called palmar psoriasis). The character which chiefly differentiates these from tertiary lesions is that they tend to be symmetrical.

The exact duration of the secondary stage is unknown. Almost all the examples of accidental contagion during the secondary period occur within a comparatively short time of its commencement. The cutaneous and other phenomena, as a rule, cease by the end of the first year, but sometimes the later secondary eruptions may continue until the tertiary local lesions make their appearance. This usually occurs in the third year, but it may take place as late as twenty years or even longer after infection. It must be understood that there is no sharp line of demarcation between the secondary and tertiary stages; on the contrary, these occasionally overlap. I have frequently seen in badly nourished patients lesions of tertiary type develop before those of the secondary stage had disappeared.

The course of syphilis as a whole is progressive, with periods of latency of variable length. There are certain circumstances, such as age, sex, personal habits and

1 Leucoplakia is not necessarily syphilitic. Cases are on record in which the subjects of this affection have acquired syphilis. Nor is it alwavs confined to the mouth. (See p.622.) 


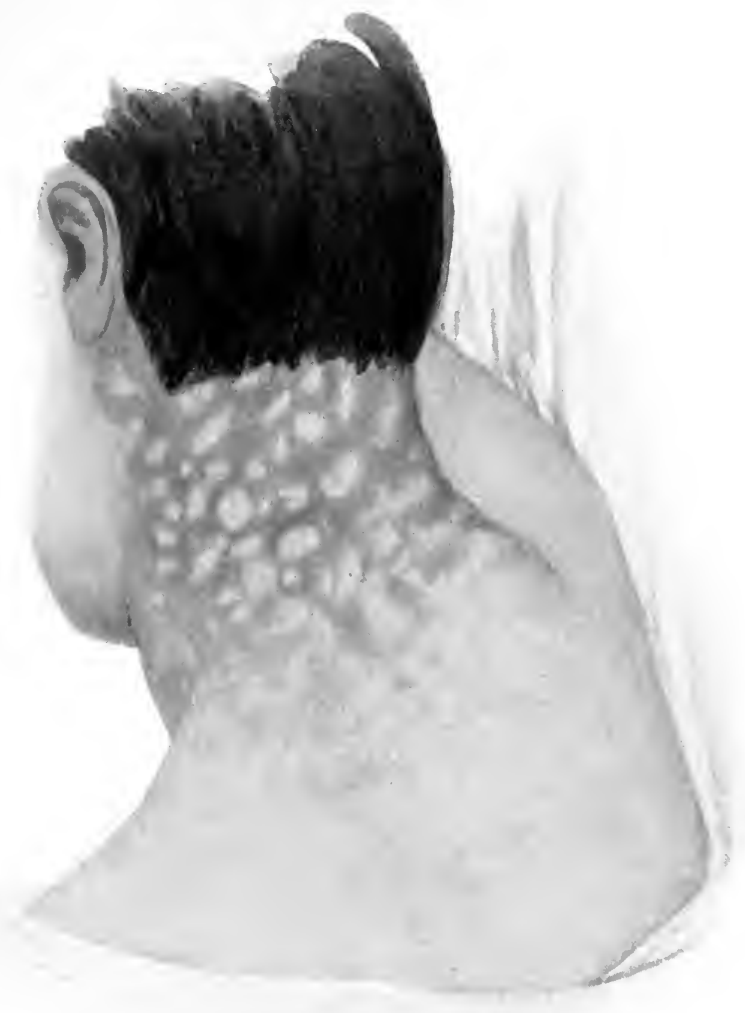

Plate 47.- LEUCODERMIC SYPHILIDE IN NECK. (II'ilfiril Forr's Case.) 

surroundings, the state of the general health, and treatment, which may have a modifying influence on its course and manifestations. As regards age, syphilis is usually mildest in young adults. Females, as a rule, suffer more than males, as the primary sore in them often escapes observation, and treatment is therefore not begun till the disease has become firmly established in the system. The influence of the personal surroundings and habits of the patient is seen in the fact that the disease is generally worst in those who are insufficiently fed and clothed, and who are of uncleanly or dissipated habits. As regards the state of health, scrofula, gout, and rheumatism all seriously aggravate the disease, and the presence of renal mischief is a grave complication. When the disease has ceased to give open proof of its presence, it may be aroused into activity by anything that injuriously affects the health.

Tertiary lesions. - In the tertiary stage the lesions show no tendency to symmetrical arrangement. All tissues may be attacked, the process consisting of slow inflammatory infiltration. The infiltration is at first diffuse, but becomes intensified at certain points, resulting in the formation of nodules (gummata). A gumma is a new growth which begins as a localized infiltration of the connective tissue with small round cells around the blood-vessels. A nodule is thus made in which new vessels appear, and which grows in size by infiltration of the surrounding parts, forming a new growth composed of granulation tissue. After attaining a certain size the tumour undergoes fatty degeneration, subsequently softening and often breaking down into an ulcer when on the surface, which heals when the mass has separated. The effects of the process vary according to the nature of the tissue in which it takes place. When the infiltration is situated near the surface of the integument, the breaking down of gummata gives 
rise to ulcers with a hard raised edge and an indurated base. A characteristic feature of tertiary ulcers is their tendency to become serpiginous. They have sinuous outlines, and show little or no tendency to spontaneous cure. Sometimes, however, they heal and leave dense scars, or they may cicatrize at one part while continuing to spread at another. They are usually few in number.

On the skin the more common position of tertiary lesions is on the forehead at the margin of the scalp (constituting a later form of corona veneris than the papular eruption already described), the upper parts of the legs, the skin of the genitals in both sexes, the nape of the neck, and the back; frequently also the palm or the sole on one side (Plate 48, Fig. 1). Tertiary lesions of the skin are not infrequently lupoid in type, and they may simulate lupus very closely (Plate 48, Fig. 2). The chief point of distinction is that their progress is more rapid than that of lupus vulgaris, and that they occur later in life. On the mucous membranes tertiary lesions have the characters of chronic inflammation with ulceration, followed by the formation of tough cicatricial tissue and thickening. This may lead to great narrowing of natural passages (pharynx, rectum, vulva). Gummata may also form in any of the internal organs; the tongue, the muscles, the bones and the periosteum, the brain and spinal cord and their coverings, the nerves, the testicle, and other viscera, are all liable to attack. Sclerosis of the spinal cord, and of the small blood-vessels and arteries, leading to the formation of aneurysms or amyloid disease, is of occasional occurrence. When the skin is close to the periosteum it is often affected secondarily to the latter. Tertiary lesions nearly always leave enduring marks of their presence in atrophic scars, with thickening. 


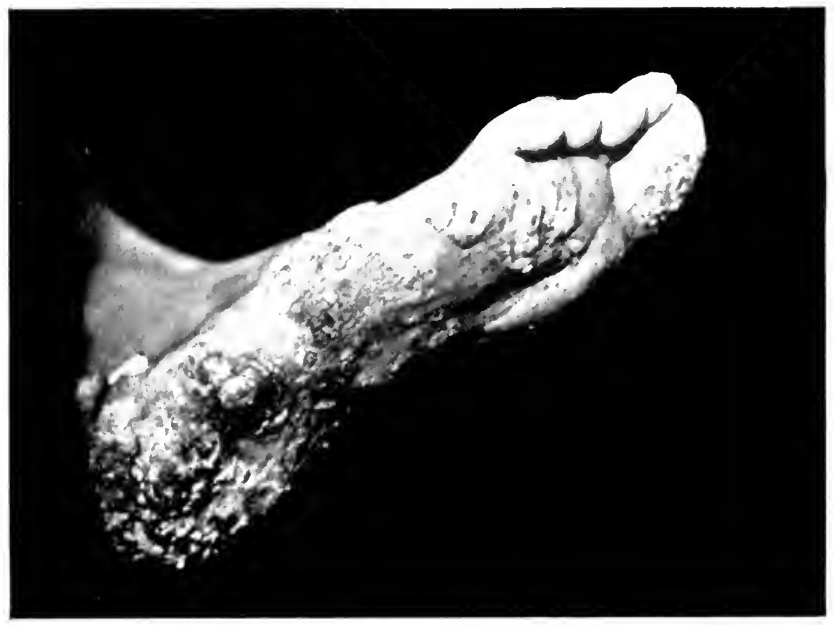

1.- SYPHILIS OF SOLE OF FOOT: CUTANEOUS GUMMATA WITH HYPERKERATOSIS (22 YEARS AFTER INFECTION).

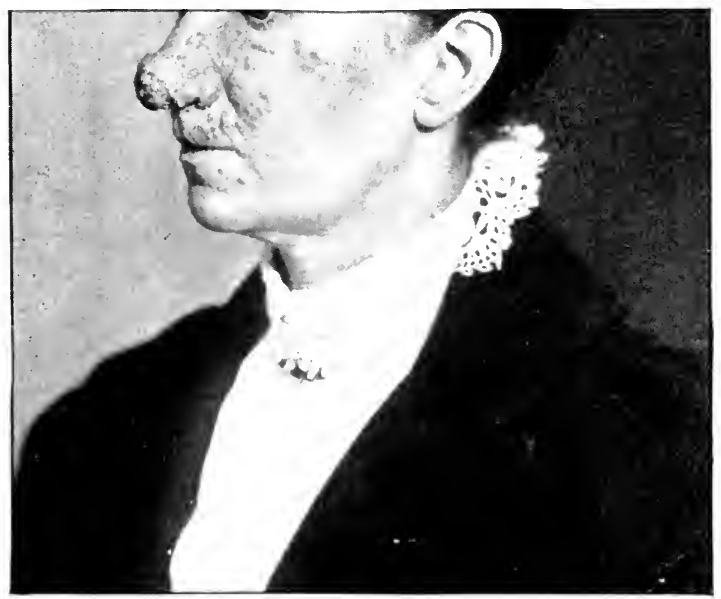

2.-NODULAR LATE SYPHILIS.

(IThitfiele's Cotse.)

Plate 48. 


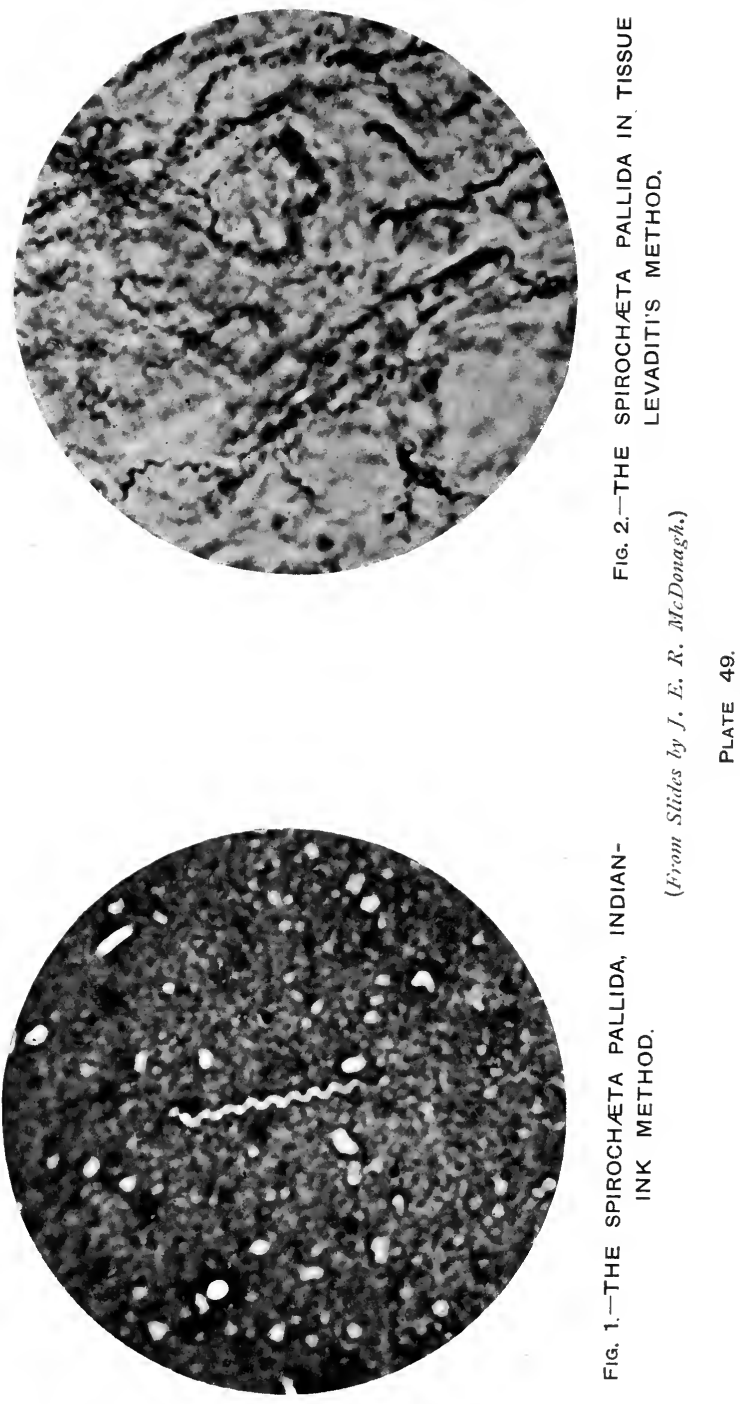


Hereditary syphilis. - The signs of hereditary syphilis do not usually show themselves until three weeks or a month after birth. The child is almost invariably free from any lesion of the skin or other parts when born, but a few cases of undoubtedly congenital syphilis have been reported by those who have had opportunities of seeing large numbers of children immediately after birth in lying-in hospitals. In some cases a form of bullous pemphigoid eruption occurs within a day or two after birth, and may cause death within a week. This attacks any part of the skin, but has a special proclivity for palms and soles. The first symptom, however, is usually a form of chronic coryza (snuffles), sometimes accompanied by laryngitis, causing hoarseness of the cry. This is followed by a skin eruption, which may be papular, scaly, pustular, bullous, or, in rare instances, miliary vesicular. ${ }^{1}$ Condylomas about the buttocks and anus are common. Onychia may occur. In a case described by Heller, that of a child who died at the age of four weeks, several of the nails both of the hands and of the feet were severely affected. In some of the nails the nail-plate had been replaced by a soft mass formed in part of hæmorrhagic crust. Like the secondary eruptions in the adult, the general eruption is symmetrical in distribution and transient in duration. Polymorphism is also a frequent characteristic of infantile syphilis, and the colour approximates to the tint of the lean of raw ham, as is seen in the adult. In fat babies the lesions frequently have the character of intertrigo, and the irritation from the urine and fæces gives rise to sores about the nates, and especially about the anus. Peeling

1 Joseph Grindon (Trans. Amer. Derm. Assoc., 1909, p. 44) has reported two cases of miliary vesicular eruption. One was unmistakably a case of syphilis; in the other the diagnosis seems to me, from the description, open to doubt. 
patches of erythema on the face and neck are common. Sores are also apt to form about the corners of the mouth. The skin is loose, dry, and of a café-aulait tint. The face often presents a peculiarly senile, wrinkled aspect; this, however, is not constant. The eruption is accompanied by wasting, anæmia, debility, and fretfulness. The spleen and sometimes the liver are enlarged, and the testicles may be hard and swollen. Iritis or choroido-retinitis may occur. A little later, bossing of the skull around the anterior fontanelle (Parrot's nodes), or thinning of the skull (craniotabes), may be seen. Alopecia or excessive growth of hair is not uncommon. Epiphysitis, causing pseudo-paralysis and chronic periostitis, especially of the tibiæ, may be early symptoms. These phenomena are generally at their height in the second, third, and fourth months after birth. The affection often ends in death, but if the child survive, the symptoms will, as a rule, have disappeared by the end of the first year of life.

After the first year there comes a period of latency, which may last a variable time. Up to the age of 18 or 20 , inflammatory affections of the eye and ear are frequent; the permanent teeth, as pointed out by Hutchinson, show characteristic changes, particularly affecting the upper central incisors, which are small, widely separated, screw-driver shaped, and notched at their free extremities. The nose is often depressed and saddle-shaped. Gummata may occur in the skin or bones, chronic periostitis, synovitis, and arthritis are not rare, and meningeal and cortical inflammation may lead to mental and paralytic affections. The skin, however, is not usually the seat of any special lesions. There are no scaly or papular eruptions, and only in the rarest cases any ulcerative processes with the serpiginous character which is distinctive of tertiary syphilis in the adult. But ulceration of the palate sometimes occurs. 
Etiology.-The Spirochoeta pallida is a delicate, spiral, slightly refractive organism, $4-15 \mu$ in length and $0.10 .2 \mu$ in breadth (Plate 49). It has 5 to 26 wellmarked regular coils, which are seen when the organism is at rest, as well as when it is in motion. It tapers at both ends, which bear cilia equal in length to 4 to 6 spirals. Schaudinn, who discovered it in the mucous patches in a case of secondary syphilis, not only observed vibratile cilia at each pole, but recognized longitudinal division of the organism in specimens with two cilia at one of the poles. ${ }^{1}$

The spirochrte has been found not only in the primary chancre and secondary lesions of acquired syphilis, but also in the blood and blood-vessels, and in the lymphatics. It has also been demonstrated in the saliva and urine, and in rare cases in the cerebro-spinal fluid. Many observers have also shown its presence in tertiary lesions. Up to the present time it has not been found in parasyphilitic affections.

In hereditary syphilis the spirochæte occurs in abundance in nearly all the organs and fluids of the body as well as in the periosteum and bones. Large numbers of the organism are found in the liver and spleen and in the blood. The bile and urine may also contain them, and they have been shown to exist in the fluid contents and in the walls of the bullæ of syphilitic pemphigus. As yet, the spirochæte has not been found in seminal fluid, and its presence there can only be assumed. Neither is there any evidence of infection of the spermatozoa, but the organism has been demonstrated by Levaditi and Sauvage in the ovary of the child of an infected mother, and numerous observers have found it in the placenta.

Neisser, Baerman, and Halbenstädter in Batavia;

1 Hartmann and Prowazek, quoted by Metchnikoff in "A System of Syphilis," edited by D'Arcy Power and J. Keogh Murphy. 
Finger and Landsteiner, as well as Kraus and Prantschoff, in Vienna, have found the spirochæte in all cases of primary infection in the lower orders of monkeys, as well as in anthropoid apes. Several observers have succeeded in producing syphilitic keratitis in rabbits and dogs by inoculating them with syphilitic products from men and monkeys, and typical spirochætes have been found in these lesions.

Diagnosis.-Usually the diagnosis of syphilis is sufficiently easy. The induration of the primary lesion, together with the enlargement and hardening of the nearest lymphatic glands, is in most cases sufficiently characteristic to enable a positive opinion to be given. It must be remembered, however, that hardness is not a constant feature of infecting sores, especially when seated on the lips or on other parts where the tissue is loose ; nor, on the other hand, can a chancre be at once pronounced to be non-infecting because of the absence of induration. The primary sore is most likely to be overlooked in women, and a very careful examination should therefore be made whenever possible. Primary sores in unusual situations, as on the face, may sometimes present difficulties. One should never allow oneself to be misled by preconceived ideas as to the improbability of contagion, but should judge each case solely on the evidence it offers. The discrimination between a primary sore on the face and malignant disease can often be made by the age of the patient and by the chronicity of the process. In some instances, however, a sure diagnosis can be arrived at only after a certain length of time. Malignant pustule may be excluded by the absence, in chancre, of gangrene and of febrile symptoms, and by the indolence of the sore. For the diagnosis between the primary sore and genital herpes, see p. 171.

In syphilis, more than in any other disease, the 
truth can be determined only by taking a comprehensive view of all the circumstances of the casethe history of the lesions, their characters, course, and termination, and the effect of treatment upon them. The mimicry of syphilis may occasionally perplex the observer. The general distinctive characters of secondary lesions that have been set forth-symmetry, coppery colour, position, polymorphism, and absence of itching, together with enlarged glands, sore throat or tonguewill in most cases suffice to identify the disease, even in the absence of a definite history or mark of a primary sore. It must, however, be repeated that it is not the presence of any one of these characters, nor even the combination of two or three of them, that can be relied upon; only the sum of them can be taken as affording solid ground for the diagnosis of syphilis. When there is any doubt, the whole cutaneous surface should be examined, and in this way a characteristic lesion or mark will usually be discovered which will give the clue that is wanted.

Apart from the general characteristics that have been mentioned, there are certain features whereby the elementary lesions themselves may be distinguished from similar ones not syphilitic in origin. Thus, in the case of macular syphilides, a cool atmosphere will bring them out in vivid colours, even when almost completely faded. From the erythematous drug rashes they are differentiated by the absence of itching or burning, their less livid redness and their longer duration; tinea versicolor and ringworm of the body, both of which are occasionally simulated more or less closely by macular syphilides, can be identified by their respective parasites; measles by the coryza and cough, the crescentic-form of the eruption and the characteristic distribution. Seborrhœa corporis is often very difficult to distinguish from a macular syphilide; indeed, the 
two affections are often associated. The wider distribution of the syphilide and the other evidences of the disease will settle the diagnosis. For the diagnosis of maculo-papular syphilides from pityriasis rosea, see p. 351 .

Squamous syphilides may sometimes be the seat of such an amount of scale formation as to be mistaken for ordinary psoriasis. Again, a papular rash in circles may simulate an annular psoriasis. In either case the syphilitic nature of the lesion can usually be determined by the polymorphism of the eruption and the distribution of the disease, the elbows and knees, which are the favourite situations of psoriasis, being as a rule avoided by the syphilitic eruption. Moreover, while psoriasis prefers the extensor aspects, syphilis prefers the flexor surfaces of the limbs; there is also a difference in the appearance of the scales, those of the syphilitic lesion being thin and dirty white, while those of psoriasis have a characteristic silvery sheen, and are heaped up in layers. The history is of importance in both cases. The subject of true psoriasis will, as a rule, have had several previous attacks, and the disease can often be traced back to early life. In syphilis, on the other hand, a particular lesion is seldom repeated.

The peculiar papular eruption of the palms and soles (see pp. 479, 486) which occurs symmetrically as a secondary, and unilaterally as a tertiary, lesion, and which is sometimes inappropriately called "syphilitic psoriasis," may sometimes be confused with the dry chronic eczema that is met with in the same situation. But in eczema there are heat and itching, and usually, in the case of the palms, the fingers also are involved, and at some point or other there is "weeping." Palmar syphilides may be distinguished from seborrhoic eczema by the presence in the latter condition of lesions in the 
ordinary situations - the scalp and eyebrows, the nasolabial folds, the sternal and interscapular regions; nor do they often assume the crescentic or segmental form. The small papular syphilide may occasionally be difficult to distinguish from a widely diffused lichen planus; in the latter, however, the rash is uniform, the papules are generally arranged in lines, and itching is usually severe.

The large acuminate pustules of syphilis (" acneiform syphilide") have been mistaken for acne vulgaris. From that affection they may be distinguished by their irregular grouping, by the drying-up of the pus into scabs, and by the absence of comedones. In acne, too, the only parts attacked, as a rule, are the face, the back of the neck, the chest, and the back between the shoulders; the eruption is more sluggish and chronic, and there is no cachexia. The small flat pustules ("impetiginous syphilide") have to be distinguished from pustular eczema and impetigo contagiosa. The ulceration which underlies the crusts in the syphilides is not found in either of those conditions. On the other hand, as already remarked, the itching so characteristic of eczema is not present in the syphilides. In impetigo the pustules most frequently affect the face and hands, and the eruption is mild in character. The large flat pustules (" ecthymatous syphilide") are differentiated from severe impetigo contagiosa by the greater number of the pustules, the slow development, the coppery areola and base, the cachexia, and the pigmented scars left when the crusts disappear.

Pustular syphilides may be mistaken for variola, especially when they begin as papules and are preceded by fever and aching pains. But the systemic disturbance in syphilis is less severe, the eruption is more indolent, the lesions appear in successive crops, and the vesicles which are seen on the tops of the papules have an indurated base. Usually, too, the syphilitic eruption 
is either distinctly polymorphic or at any rate more heterogeneous than the variolous.

Subcutaneous gummata may be mistaken for abscess, and on this supposition may be opened, when they give issue not to pus, but to a gummy liquid. The breaking down of a gumma on the leg may give rise to an ulcer resembling the ordinary callous ulcer; the true nature of the sore will be revealed by its proving refractory to ordinary treatment and giving way to antisyphilitic remedies. From lupus, syphilitic lesions can usually be distinguished by the absence of the characteristic applejelly nodules, by the comparative rapidity of the process, and by the age of the patient, lupus usually commencing in early life. Rodent ulcer and epithelioma may sometimes have to be distinguished from tertiary lesions. As a rule, in the cancerous ulcer a process of new growth has preceded the ulceration, and the characteristic hard edge and red, shining, dry floor of the malignant ulcer will generally serve to identify it. The position of rodent ulcer on the upper part of the cheek, near the eyelid, or the side of the nose, or the temple, is another distinguishing feature. Lastly, the age of the patient counts for something-rodent ulcer, or epithelioma of the face, occurring as a rule only in people past middle life. For the diagnosis of lichenoid syphilides from lichen scrofulosorum, see p. 434; for that of the tubercular syphilides from the nodules of lupus vulgaris, see p. 457 ; for that of the nodules of syphilis from erythema nodosum, p. 112 ; for that of rupial crusts from the crusts of nerve leprosy, p. 532. Rupial crusts, it should be added, are distinguishable from the psoriasis rupioides of $\mathrm{M}^{\circ} \mathrm{Call}$ Anderson by the ulceration of the base. The crusts of lupus vulgaris resemble those of rupia in colour, but do not consist of superimposed layers, and the ragged edge is dotted with apple-jelly nodules. 
The diagnosis of inherited syphilis in early infancy is at times easy, but at other times a matter of great difficulty. Snuffles, the wizened old-mannish aspect, the coppery eruption, and the sores about the mouth and anus, make up a sum of clinical phenomena that is characteristic. In certain cases the history of the parents helps to elucidate the difficulty. In the adult who has been the subject of infantile syphilis the signs of the disease are seen in "the square forehead with prominent frontal eminences like budding horns, the sunken nose, the soft, pale, earthy-tinted skin, and the scars about the angles of the mouth," 1 and in the pegged and notched upper incisor and canine teeth. Besides these, signs of interstitial keratitis and choroiditis are often present, and deafness may have been left as a legacy from previous otitis.

The serum diagnosis of syphilis (Wassermann's test) is based on the phenomenon known as "deviation (or fixation) of the complement," described by Bordet and Gengou in 1901. When the blood cells of one animal are introduced into another animal of a different species, the serum of the latter acquires the power of destroying these corpuscles. This hæmolytic property depends on an immune substance, or amboceptor, which resists a temperature of $55^{\circ} \mathrm{C}$., and another substance or "complement" destroyed at that temperature. Heating the serum of the immunized animal to $55^{\circ} \mathrm{C}$. for half an hour destroys its hæmolytic power on account of the destruction of the complement; but this can be restored by adding the serum of a normal animal which contains the natural complement, but which itself has no hæmolytic power, since it contains no immune substance. Bordet and Gengou found that when an antigen, or body capable of giving rise to the formation of antibodies, and the corresponding anti-

1 Hutchinson, "Syphilis," new edition, 1909. 
body are added to the immune substance, the complement which is added afterwards is no longer capable of combining with the immune body, and therefore no hæmolysis occurs. This is known as "deviation of the complement." Wassermann used: (1) red corpuscles of the sheep, washed and suspended in normal saline solution; (2) heated (inactivated) rabbit's serum, immunized against sheep's corpuscles ; (3) fresh guineapig's serum (complement); (4) an extract of virulent syphilitic material, such as congenital syphilitic liver, as the antigen; (5) a known antisyphilitic substance, such as the inactivated serum of immunized monkeys. It was found that if syphilitic antigen and antibody, (4) and (5), were mixed with fresh guinea-pig's serum (3) and incubated at body temperature for three-quarters of an hour, and the corpuscles and hæmolytic serum, (1) and (2), were added, and the mixture incubated for another two hours, no hæmolysis occurred, the complement having been used up by the interaction between the syphilitic antigen and antibody. ${ }^{1}$ In a case of suspected syphilis, the hæmolytic system may consist (a) of 0.4 c.c. of extract of syphilitic liver-antigen; (b) 0.1 c.c. of inactivated serum of the patient-antibody; (c) 0.1 c.c. of normal guinea-pig serum-complement. The mixture is skaken and kept in an incubator at $37^{\circ} \mathrm{C}$. for an hour. At the end of that time 1 c.c. of sheep's corpuscles in inactivated rabbit's serum is added, and the whole mixture is incubated for two hours at $37^{\circ} \mathrm{C}$., removed to an ice-chest and kept there from twelve to eighteen hours. As a control a similar hæmolytic system is arranged in which normal serum is used instead of suspected serum; here there is complete hæmolysis and a "negative" reaction is recorded. If, in the main research, the patient's serum

${ }^{1}$ See Andrewes' description in D'Arcy Power and J. Keogh Murphy's "System of Syphilis," chap. xv., p. 167. 
(b) is syphilitic (that is, if it contain antibody), there will be no hæmolysis, for the complement $(c)$ required for this reaction will have been "fixed" by the antibody to the lipoid of the liver extract-the antigen $(a)$. The test therefore gives a "positive" reaction. If, on the other hand, the patient's serum is non-syphilitic, hæmolysis will take place, for the antibody $(b)$ will be absent and the complement $(c)$ will not have been "fixed," but will be free to react with the antigen $(a)$.

The test is an extremely complicated one, and the technique has to be controlled as well as the hæmolytic system. Boas, of Copenhagen, has elaborated the idea of carrying out the test quantitatively; he always uses five amounts of serum, ranging from 0.2 to 0.01 c.c.

Meier ${ }^{1}$ found that of 21 individuals free from any suspicion of syphilis, every one gave a negative reaction. In 181 persons either admittedly syphilitic or suffering from a parasyphilitic affection, the percentage of positive reactions was 68 in primary syphilis, 93 in secondary syphilis, 100 in both tertiary and malignant syphilis, 75 in congenital syphilis, 83 in latent syphilis, and 75 in syphilis of the central nervous system-an average percentage of $85 \cdot 1$.

In some 8,000 cases of syphilis in which the test had been applied, the percentage of positive results in primary syphilis was 65 , in secondary 90 , in tertiary 83 , and in latent 44 . Boas, by means of the quantitative method referred to above, secured positive reactions in as many as 73 per cent. of primary, 97 per cent. of secondary, 95 per cent. of tertiary, and 37 per cent. of latent cases. In entirely untreated secondary and latent cases, and in tertiary cases which had only been treated, if at all, in the secondary period, he secured 100 per cent. of positive reactions, as well as in cases of tabes

${ }^{1}$ Berl. klin. Woch., 1907, Nos. 50 and 51.

${ }^{2}$ Paul Fildes, Brit. Journ. Derm., 1911, xxiii. 13.

2 G 
in which no specific treatment had been given. The numbers of such cases were-secondary 269, tertiary 63 , latent 15 , tabes $17 .{ }^{1}$

It may be taken as established that while a positive Wassermann reaction is not an absolute proof of syphilis, since the complement is also deviated by the blood in leprosy, and in sleeping sickness, it is conclusive evidence of syphilis wherever those diseases can be ruled out, as can usually be done in this country. Further, while a negative reaction is no proof of the absence of syphilis, or of the cure of syphilis, a series of such reactions at regular intervals, in a patient who has not recently had specific treatment, furnishes trustworthy evidence of the extinction, or suppression, or great attenuation of the virus. The test is least trustworthy in the early stage of syphilis, in which a positive reaction is obtained in only about 70 per cent. of cases. Fortunately, the demonstration of the spirochæte in the primary lesion has now been rendered easy by the dark-field illumination method. The principle of this method consists in shutting off the central rays, and by means of a plano-convex piece of glass reflecting the marginal rays on to the surface of a cover-glass. The suspected material is taken, if possible, from the deeper part of the lesion, care being exercised to prevent it as far as possible from being mixed with blood. A drop of the serum is put on a clean slide of twice the ordinary thickness and covered with a thin cover-slip, which is pressed on the slide as firmly as possible. Some cedar-wood oil is placed between the condenser and the slide, when the Spirochata pallida, if present, will be easily seen. It can be distinguished from other spirochætes by its whiteness and slenderness, the regularity of its spirals, and its less vigorous and less rapid movements. Another means by which the spirochrte is easily demonstrated in

1 Fildes, loc. cit. 
fresh syphilitic material is Burri's indian-ink method (Plate 49, Fig. 1). The material having been emulsified in water, a small quantity is taken up with a fine drawingpen, and dipped into droplets of indian ink on a slide; when the droplets have dried they are examined without a cover-glass with the oil-immersion lens, when the Spirochata pallida, if the case be one of syphilis, will be seen standing out a vivid white on the grey-black background. Yet another method is Levaditi's, in which the spirochæte is stained black with silver nitrate reduced by pyrogallic acid (Plate 49, Fig. 2).

Prognosis.-This depends on the age and general health of the patient, on the severity of the disease, and especially on the treatment. As already said, young adults will, under proper conditions, soon be free from objective symptoms, but patients should not be passed for marriage until there have been a succession of negative reactions, obtained at intervals of six months during a period of at least two years. In persons of unhealthy constitution or of alcoholic habit, or living in insanitary surroundings, the prospect is much less favourable. Syphilis contracted in middle life is very intractable. The mildness of the earlier symptoms affords no guarantee against the appearance of tertiary lesions of great and even fatal severity. The most important element in the prognosis, however, is the treatment. 


\section{CHAPTER XXI \\ MICROBIC AFFECTIONS (Continued)}

\section{Syphilis : Treatment}

Mercury is no longer without a formidable rival in the therapeutics of syphilis, but attention may first be given to that time-honoured specific.

An initial question to be decided is whether it is desirable to administer the drug as soon as the diagnosis is established, or to wait until constitutional symptoms have appeared. Numerous Continental and American authorities are in favour of waiting until the secondary signs appear, while in this country it is held by some that mercury should be given at once.

The next question that arises is as to the method of administration. There are seven ways in which mercury may be given : (1) By the mouth, (2) by intramuscular injection, (3) by subcutaneous injection, (4) by intravenous injection, (5) by inunction, (6) by baths, and (7) per rectum.

1. The oral method is that which many English and American authorities favour, on the grounds that it is clean and convenient and inexpensive, that it can be carried out by the patient himself, and that the therapeutic results are as satisfactory as those yielded by any of the other methods. The disadvantages urged by those who prefer other methods are that there is risk of alimentary disturbance, that in certain patients there appears to be no absorption, and that in others absorption is slow and irregular, and the therapeutic effect tardy. 
2. Intramuscular injection, which is extensively practised in the British Army, is advocated on the grounds that the dosage is definite and the absorption certain, that there is rapid therapeutic action, that the method is clean, and that secrecy is easily maintained. It is specially recommended where the central nervous system is affected, where a rapid therapeutic effect is desired, or where, as in hot climates, there is more than ordinary liability to gastro-intestinal disturbance from the oral administration of the drug. The great objections brought against it are (1) the risk of abscess, and (2) the pain, which, though it varies with the patient, is admittedly in some cases considerable. The favourite sites of injection are the buttocks, the lumbar and scapular regions, and on each side of the vertebral column $4 \mathrm{~cm}$. outside the spinous processes. Either $(a)$ soluble or (b) insoluble salts may be used. Of the former the main drawbacks are the necessity for daily administration and the frequent recurrence of pain; of the latter, that in the rare cases in which mercurial poisoning takes place, it is impossible to get rid of the mercury. Soluble salts are injected daily, the course consisting of 20 to 30 injections; insoluble salts at intervals of seven to fourteen days, from 5 to 10 injections forming a course. Among soluble salts used for injection are perchloride of mercury, biniodide of mercury, neutral mercuric salicylate, and sozoiodolate of mercury. Of insoluble salts, grey oil is considered to be the best for ordinary cases, while calomel is held to be at once the most effective and the most painful. Salicylate, which gives the least pain, appears to be suitable only for cases in which mild treatment is sufficient.

A preparation of mercury for intramuscular injection was introduced by Möller under the name of mercuriol, which can, he claims, be injected with a minimum of local 
irritation. Oil of mercuriol is recommended by Laborie ${ }^{1}$ where energetic treatment or a rapid result is required, where inunction is unsuitable, and large doses of perchloride are not well borne. He gives weekly injections of four to five divisions of a Lüer syringe $(1$ division $=$ $\frac{1}{2}$ c.c.) of a mixture of oil of mercuriol (containing 90 per cent. of mercury) with two parts by volume of dehydrated oil of almonds.

Lambkin's cream consists of pure metallic mercury ろi, palmitin §iv, liquid paraffin (carbol 2 per cent.) ad $\xi x=$ by volume 10 per cent. of mercury. The dose is $m \mathrm{x}-\mathrm{xv}$.

3. Subcutaneous injection is less practised than intramuscular, for the pain is always greater, and the injections are more likely to leave behind them nodules and cause abscess.

4. For intravenous injection it is urged that the dosage admits of accurate regulation, that absorption is certain and the therapeutic action rapid, and that the process is almost painless, nor is there painful induration. The disadvantages are said to be that intravenous injection may cause thrombosis, and that it is not possible where the superficial veins are very small. Moreover, if the vein be missed there is a good deal of swelling and acute pain.

5. The chief advantages claimed for inunction are that the drug is introduced in well-regulated doses, that the process is painless, and that it is followed by no digestive derangement. The disadvantages alleged against it are that in some cases the mercury is not absorbed, that the process is a dirty one, involving the provision of special underclothing, and that it is expensive, requiring a staff of trained rubbers and an adequate supply of hot baths.

6. Baths may be either calomel vapour or mercuric 1 Journ. des Mal. Cut. et Syph., Jan., 1904. 
chloride. The calomel vapour bath is recommended for its speedy therapeutic effect, especially in cases in which there are obstinate ulcers and hard papular syphilides; mercuric baths for cases marked by widespread and ulcerating skin lesions, and for cachectic patients who can ill bear treatment by the oral or other methods.

7. Mercury may be given in the form of suppositories in cases in which other methods are contra-indicated. It is the least effective mode of administering the drug, and is only to be regarded as a dernier ressort.

In cases in which mercury is administered otherwise than by the mouth, there is choice between the intermittent and the continuous methods. On the Continent the intermittent method is usually preferred, courses being given three times a year in the first two years, twice in the third year, and according to circumstances in the fourth year. ${ }^{1}$

Having passed in rapid review the chief methods of treating syphilis by mercury, I proceed to describe my own preferences, the result of long experience of this subject. It is impossible, however, to lay down any rule which shall be equally applicable to all cases. It is of much greater importance to adapt the treatment to the requirements of the individual case than rigidly to conform to any fixed line of treatment. Treatment should vary with the stage at which the case comes under observation, with the severity and locality of the attack, with the general health and habits of the patient, and with the degree of importance which is attached to the maintenance of secrecy. If the disease

${ }^{1}$ For fuller details of the treatment of syphilis by the various methods enumerated in the text, see Captain Pollock's Report to the Sub-committee appointed by the Advisory Board for Army Medical Services to inquire into the Treatment of Venereal Diseases and Scabies in the Army, published in 1904. 
should have reached a comparatively advanced stage before it comes under treatment, or the attack should be marked by unwonted severity, or a structure such as the iris or the choroid should be imminently threatened, it is important to get mercury into the system with the least possible delay, and intramuscular injections should at once be made. Of the superior efficacy of this method as compared with oral administration, the Wassermann test furnishes evidence. In cases where gastric symptoms are present, and there is no imperative need for the maintenance of secrecy, the inunction method may be adopted. In ordinary uncomplicated cases, in which there is no urgency and no occasion for more drastic methods, the drug may be administered orally.

If the primary sore is in a suitable position it should at once be excised. If, however, it has existed some time and the neighbouring glands are enlarged, mechanical removal is useless. The sore should then be treated antiseptically, and specific treatment should be at once begun. I have never been in favour, in ordinary cases, of waiting until the generally accepted signs of secondary syphilis appear, and the Wassermann reaction, which often reveals the presence of antibodies in latent syphilis and furnishes proof of their disappearance under treatment, confirms the view that, once the diagnosis is settled, vigorous treatment should be instituted without delay. The form in which I usually administer mercury by the mouth is blue pill (gr. i to gr. iii t. d.). Grey powder is often used, but in my opinion is more uncertain in its action than blue pill. Plummer's pill is an especially useful form of administering mercury over long periods, as there is little risk of its producing salivation. I usually give gr. iiss night and morning. Or perchloride of mercury may be given (gr: $\frac{1}{32}$ to gr. $\frac{1}{16}$ ).

Inunction, to which resort may be had, as I have 
already said, in cases in which the drug disagrees if given by the mouth, is carried out by rubbing blue ointment vigorously into the patient's skin. It must be rubbed into different places from day to day, otherwise a mercurial eruption is almost sure to be produced. The ointment must not be washed off for some hours; the usual plan is for the patient to wrap himself in flannel and go to bed, taking a warm bath when he gets up on the following morning. The inunction system is very thoroughly carried out by trained rubbers under medical supervision at Aix-laChapelle, Wiesbaden, and other places, and the usual course lasts a month. The method can, however, be carried out with artificial baths at special institutions in this country, or at the patient's own home, though without the advantage belonging to a watering-place "cure," namely, the regimen and general discipline to which patients at such places have to submit.

For intramuscular injections, which are suitable in severe and urgent cases, the insoluble salts are to be preferred, in spite of the serious situation in which the patient might be placed should symptoms of mercurial poisoning appear. They may be administered, for choice, in the form of grey oil, injected into the buttock once a week.

The vapour bath method is especially useful in rupia and ulcerating forms of the disease. Calomel (from one scruple to half a drachm), mixed with water, is vaporized over a small lamp, and the patient sits (from a quarter to half an hour) on a chair over the lamp, enveloped in a cloak. Fumigation has the same advantages as inunction, but both have the drawback of requiring the expenditure of much time and trouble.

The administration of mercury should not at first be pushed to the full physiological limit; it is generally sufficient to produce slight tenderness of the gums. 
Salivation should always be avoided if possible; when it occurs the lesions, indeed, are very rapidly cured, but the suspension of the drug which it necessitates is likely to be followed by troublesome phenomena later.

During a course of mercury the patient should abstain entirely from alcohol, and should be particularly careful to keep his teeth perfectly clean, in order to minimise the risk of stomatitis; and for the same reason it will be prudent for him to refrain from smoking. Septic teeth should be removed or stopped. He must also be careful when he goes out to guard himself against cold, and he must be particular in his diet, so as to avoid disturbance of the bowels. Tonics, such as iron, quinine, etc., are to a certain extent antagonistic to mercury; indeed, the drug has a better chance of producing its fullest beneficial effect when the patient is kept a little below his ordinary standard of health.

Under the influence of mercury administered steadily in small doses - that is, short of purgation and ptyalism -the primary lesion will speedily clear up and the last trace of induration will disappear in about a month. If the administration of the drug be begun before the disease has entered on the constitutional stage, it will often happen that no secondary lesions develop. Nevertheless, the mercurial medication may be continued for six or even nine months; its suspension within that period is apt to be followed by the speedy appearance of secondary manifestations. If these do show themselves they are comparatively trivial. In cases in which secondary lesions have developed it will be wise to persevere with the mercury for at least one year after the disappearance of the eruption. The prolonged exhibition of mercury also makes the subsequent development of tertiary lesions less likely, though it cannot be considered an absolute safeguard. As a general rule of practice it may be laid down that in ordinary 
cases the administration of mercury should be continued, with intermissions of varying length, for twosometimes three and in certain cases even for fouryears. The patient may then be considered tolerably safe from further manifestations of the disease. There is no fear of disordering the health by giving mercury in small doses for several months; on the contrary, patients so treated, as a rule, visibly improve in their general condition.

Mercury may also be used locally with great advantage in the treatment of the more severe secondary syphilides. It may be applied to the skin in the form of an ointment containing $\mathrm{gr} . \mathrm{xv}$ to $\mathrm{xx}$ of the ammoniochloride to the ounce of lard, or oleate of mercury 1 to 2 per cent. The application of calomel to mucous tubercles soon causes their disappearance. In the mouth and throat mercury may be used as a gargle in the form of gr. $\frac{1}{2}$ to gr. i of perchloride of mercury in $\zeta$ viii of distilled water.

In congenital syphilis the best method of administering mercury is the inunction of mercurial ointment. If the skin eruption is very copious, grey powder, gr. i or less thrice daily, should be substituted for the inunction, watch being kept lest the treatment cause diarrhœa. The child should remain under observation at least two years.

In the treatment of tertiary lesions iodide of potassium is the most important drug. It is well to begin with small doses and gradually increase them as required. As this iodide frequently has a very depressing effect on the patient, the iodide of sodium may often advantageously be substituted for it, or the iodides of sodium, potassium, and ammonium may be combined. The addition of ammonia greatly increases the efficacy of the iodides. The tendency of the iodides to cause skin lesions of a peculiar character must not 
be forgotten, and care must be exercised not to push the drug under the mistaken notion that such lesions are syphilitic. When tissue change is slow the iodide may be combined with perchloride of mercury as follows :-

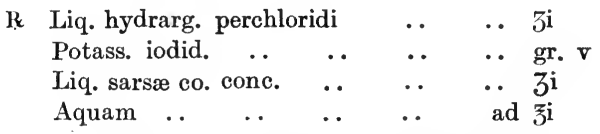

Sometimes, after a prolonged administration of the iodides, they seem to lose their effect. In such circumstances it is well to suspend the drug for a time and give mercury in place of it, returning again at a later period to the iodide, if necessary. The effect of this alternation of treatment is frequently very marked.

Tertiary lesions can often be cured by local treatment alone. For this purpose there is nothing so rapid or so sure in its effects as iodoform, which may be applied either as a powder (dusted on or blown over the affected surface with an insufflator), or in the form of an ointment ( $3 \mathrm{i}$ to $\xi^{\mathrm{i}}$ of vaseline or lard). On account of the disagreeable smell of iodoform, iodol, dermatol, aristol, or xeroform may be substituted for it. Ulcerating patches can frequently be dealt with efficiently by the free application of acid nitrate of mercury, care being taken thoroughly to destroy the lesion. Chromic-acid or silver-nitrate applications should be applied to ulcers on the mucous membrane of the mouth and tongue.

The general principles of the mercurial treatment of syphilis may be summed up as follows: If the patient comes under observation as soon as the primary lesion has appeared, remove it with the knife, if it is in a situation suitable for excision; in either event, save in exceptional cases, begin the internal administration of mercury as soon as the diagnosis is certain. Continue this intermittently for a year, or, if secondary 
symptoms manifest themselves, for two, three, or even four years. If the patient cannot bear the mercury when given by the mouth, try one of the other methods described. If tertiary lesions develop, give iodide of potassium, sodium, or ammonium, or all three, discontinuing the administration if any sign of iodism shows itself. Use antiseptics locally. If at any stage of the disease the general health shows signs of failing, use general tonic treatment, especially cod-liver oil, quinine, and iron, good food, and sea air, as in openair treatment for other chronic infective diseases.

Various serums have been tried, but although some favourable results have been reported, this method has not succeeded in winning a recognized place in the treatment of syphilis.

Arsenic.-Of late years arsenic has been extensively employed in the treatment of syphilis. Its merits were powerfully, but with due qualification, urged by Neisser at the Sheffield meeting of the British Medical Association, in 1908. It was first administered on a considerable scale in the form of atoxyl, an organic compound which had been employed in sleeping sickness and in other affections. As atoxyl came into more extensive use, it was found that its administration was sometimes followed by grave symptoms of intolerance-malaise, nausea, vomiting, gastro-intestinal disturbance, painful micturition, disagreeable sensations in the limbs, and in some cases optic nerve atrophy causing blindness. Other forms of the same arylarsonate salts, such as arsacetin and soamin were then used. That these various compounds exercised a definite influence upon the disease was placed beyond doubt, but they failed to fulfil the desideratum of a substance which should be inimical to the parasite without being injurious to the tissues of the host. There is now ground for hoping that the researches conducted by Ehrlich and Hata, of the 
Frankfort laboratory, in the field of synthetic chemistry have gone far to solve the problem.

Salvarsan, or dioxy-diamido-arseno-benzol, more familiarly " 606 ," is a bichloride in the form of a sulphuryellow powder which is soluble in water ; it has a strongly acid reaction. The formula is $\mathrm{C}_{12} \mathrm{H}_{12} \mathrm{~N}_{2} \mathrm{O}_{2} \mathrm{As}_{2}(\mathrm{HCl})_{2}$; it contains some 34 per cent. of arsenic. A patented preparation is sent out in glass tubes which, having been exhausted of air, are filled with an inert gas. Being highly toxic, it has to be neutralized with an alkali, such as a 15 per cent. solution of sodium hydrate. This is done immediately before use. The preparation is administered either by intramuscular, subcutaneous or intravenous injection. If the method of intramuscular or of subcutaneous injection be employed, the contents of one tube $(0.6 \mathrm{grm}$.) may be rubbed up with 10 drops of the solution of sodium hydrate, sterile water added drop by drop up to about 10 c.c., and the resulting suspension made neutral to litmus by the addition of more of the alkali, or dilute hydrochloric acid may be used. The intramuscular injection is given into the gluteal region, in the same site as mercurial injections (p. 501); for the subcutaneous injection the scapular region is selected. For the intravenous method an average dose is $0.5 \mathrm{grm}$., dissolved in 0.94 c.c. of the sodium hydrate solution; 250 c.c. of 90 per cent. physiological salt solution is added, and solid particles must be removed by solid filtering. Sequeira emphasizes the importance of injecting no solid particles; in one case in which this precaution was omitted, the injection was followed by immediate coma. In Schreiber's method of intravenous injection 10 to 20 e.c. of sterile water is added to the salvarsan, and the mixture is shaken until clear. Normal salt solution is added to make 100 c.c. For each $0.1 \mathrm{grm}$. of the drug, 0.7 e.c. of normal caustic-soda solution is added, and after dissolution of the resulting precipitate 
there is further addition of physiological salt solution to make from 150 to 250 c.c.

The intramuscular and subcutaneous methods frequently cause severe local pain, and are followed by some fever, and there is local infiltration at the site of injection, with the risk, especially in the subcutaneous method, of sloughing of the skin. The intravenous method provokes a much less severe reaction. Sometimes there is immediate nausea, and occasionally vomiting; but ordinarily no symptoms present themselves until some three hours afterwards, when there may be chilliness, and possibly rigors, followed in an hour or so by a rise of temperature, lasting as a rule not more than twenty-four hours. The advantages of this method over the others are obvious; on the other hand, it is more difficult, and must be reserved for those who possess the necessary technical skill. When salvarsan is injected intravenously, it is excreted in the urine much more rapidly than when injected intramuscularly or subcutaneously. Thus, in the intravenous method, Beveridge found no arsenic in the urine after the ninth day, while it has been found on the fourteenth day after subcutaneous, and as late as the thirty-sixth day after intramuscular injection.

With regard to dosage, further experience is required. The relapses which not infrequently occur are attributed by Ehrlich and others to a too cautious use of the remedy. In the opinion of McIntosh and Fildes, ${ }^{1}$ of the London Hospital, where salvarsan has been extensively used since July, $1910,1.0$ to 1.4 grm. may be regarded as an average dose, given in two or three fractions distributed over a period of from three to six days; one or two of the injections must be by the intravenous route. Ehrlich holds that any much less vigorous treatment than this is merely trifling. Major

1 Lancet, 1911, i. 725. 
Gibbard and Capt. Harrison, ${ }^{1}$ of the Rochester Row Hospital, have usually given, in ordinary cases, $0.6 \mathrm{grm}$. by the subcutaneous or intramuscular, and 0.5 to 0.6 grm. by the intravenous, method. Afterwards they gave two intravenous injections of 0.5 to $0.6 \mathrm{grm}$. spaced by a period of two to three weeks. They then began a series of intravenous cases in which an initial dose of 0.5 to $0.6 \mathrm{grm}$. is followed, at intervals of two weeks, by three injections of $0.2 \mathrm{grm}$. each. For children the dose should be calculated at 0.01 grm. per kilogrm. of body weight.

The arsenic in salvarsan is trivalent, and it appears that spirilla succumb to smaller doses of the drug in this form than in that of pentavalent compounds; thus, in salvarsan, the toxic effects of arsenic are avoided. It is necessary, however, to exercise discrimination in the application of this powerful remedy. The cases for which it is unsuitable are those in which there are such grave sequelæ or complications as pronounced cardio-vascular change or a liability to hæmorrhage, marked degeneration of the central nervous system, grave disease of the abdominal viscera, diabetes, or advanced pulmonary tuberculosis. In the many. thousands of cases in which salvarsan has been employed, there is no record of damage to the optic nerve. Its superior activity as compared with mercury can hardly be doubted. Frequently the spirochætes entirely disappear from a lesion in twenty-four to fortyeight hours after the injection. The best response is made in the primary and the tertiary stages, in malignant syphilis, in ordinary recurring active syphilis, and in congenital syphilis. The primary lesion usually heals in about four days. Striking results are reported also in bone syphilis and gummata and in visceral syphilis, while in some cases enlarged glands begin to subside 1 Lancet, 1911, i. 728. 
within a few hours. In more resistant cases belonging to the secondary stage a second injection is sometimes found necessary. The roseolar eruption, as a rule, shows regression in periods varying from one to twenty days; the papular eruption, especially the small variety, is rather more obdurate, and rarely begins to disappear before the fourth day. At Rochester Row, salvarsan was tried in 34 cases, brought together from various military hospitals, in which mercurial treatment had failed to arrest dangerous or troublesome symptoms, or to prevent frequent recrudescences. Gibbard and Harrison ${ }^{1}$ report that the injection of the compound was followed by the complete disappearance of all active signs of the disease in every case but one. In that case, one of ulcerated larynx and hard palate, with neerosis of bone, the ulceration healed, but some hoarseness remained. In parasyphilis, salvarsan has been tried with varying results; in this form of the disease it should be employed very cautiously, and not at all in advanced cases. Whether it can be usefully administered in early stages of parasyphilis, it is too soon to pronounce. Fordyce ${ }^{2}$ reports excellent results in a case of cerebral syphilis, but had little success in nine cases of tabes. Of two cases of optic neuritis, one showed some improvement.

Whether salvarsan has the power of preventing tertiary and parasyphilitic lesions can only be proved by lapse of time. It is too early even to say whether it can permanently prevent lesions of the secondary type. In a minority of cases it fails to check the disease, and the Wassermann reaction remains positive, and, as mentioned above, in cases which do respond to it there is not infrequently recrudescence. Whether inadequacy of dosage is a sufficient explanation of such relapses

1 Loc. cit., p. 729.

2 N.Y. Med. Journ., Nov. 12, 1910. 
remains to be seen. But the remedy has now been tried on so extensive a scale both on the Continent and in this country, as well as in America, and has in so large a majority of cases caused a rapid disappearance of lesions, including some of a most formidable type, followed by negative Wassermann reactions, that there can be little doubt that it, or some modification of it, will hold a prominent and enduring place in the treatment of syphilis. It should, in my opinion, be employed in combination with mercury, rather than as a substitute for the older specific. A second intravenous injection of salvarsan should be given from five to ten days, or longer, after the first, and mercury should then be begun; or the mercurial course may begin after the first injection, the second injection not being given until a month after the first. The mercury should be continued nntil two or three negative Wassermann reactions have been secured, at intervals of a few weeks. Whether salvarsan destroys the parasite, or only temporarily suppresses its activities-in other words, whether the beneficial effects are permanent, and, if not, how they can be made so-it is for further experience and research to determine. There is no evidence that the compound, if administered by skilled hands, is dangerous in any but cases such as those specified above as unsuitable for its exhibition.

In France high claims are made for a derivative of arsenic discovered by M. Mouneyrat-the benzo-sulphonpara-amino-phenyl-arsenate of soda, styled hectine. Hallopeau ${ }^{1}$ states that up to the time of writing, it had been used in 160 cases of primary syphilis, including 25 of his own, without in a single instance failing to abort the disease. In not one of the patients did secondary manifestations present themselves, although the first of them was treated so long ago as November,

1 Bull. Gén. de Thérapeutique, 15 Fév., 1911. 
1908, two in June, 1909, and two in December of the same year, while in nine other cases the interval was eight months or longer. The Wassermann reaction was uniformly negative, usually immediately after the cessation of treatment, in a few cases from two to three months afterwards. In one case, four months after the termination of treatment a patient was reinfected. Hallopeau gives a daily injection of $20 \mathrm{cg}$. of hectine dissolved in 1 c.c. of sterilized water, and keeps up the treatment for thirty days. The site of the injection is the immediate neighbourhood of the chancre, which, if possible, is penetrated on the first two or three occasions. Some pain is felt, which persists for a few hours, but can be mitigated by novocaine, and in any case disappears in twenty-four hours. There is sometimes a diffused swelling of the penis, which ceases with the discontinuance of the treatment; but no unfavourable constitutional symptoms are evoked. Equally emphatic testimony to the value of hectine, not only in aborting syphilis in the primary stage, but in clearing up secondary and tertiary manifestations, is borne by other French dermatologists. Further results must be awaited before forming an estimate of the value of the preparation. 


\section{CHAPTER XXII}

\section{MICROBIC AFFECTIONS (Continued)}

\section{LEPROSY}

Leprosy may be defined as an infective disease endemic in certain parts of the world, manifesting itself primarily by lesions of the skin or of the peripheral nerves, and secondarily attacking most of the other tissues and organs of the body, undermining the constitution, running a slow course, and leading directly or indirectly to death.

Stages and symptoms.-All cases of leprosy have certain features in common, and the disease presents a definite succession of stages. The incubation stage usually extends over two or three years, sometimes much longer. Danielssen and Boeck record a case in which it lasted ten years, and I have seen one in which the evidence pointed to an incubation period of eight years. A prodromal period usually follows, analogous to the febrile stage of syphilis. The patient complains of languor and giddiness, dyspepsia, dryness of the nose, and epistaxis. General sweating is often a prominent symptom, and sometimes local anidrosis may be observed. Constipation or diarrhœea may be present, but according to Leloir this is exceptional. Next comes the period of invasion, usually marked by a rigor and great rise of temperature, as high as $103^{\circ} \mathrm{F}$. or $104^{\circ} \mathrm{F}$.

After a variable period the characteristic leprous macules appear on the face, limbs, or trunk, the most 
common positions being the face, especially the forehead, the nose, the cheeks, and the ears; the extensor surfaces of the limbs and the buttocks are also not infrequently the seat of the eruption. It is now thought that the earliest signs of the disease should be sought on the mucous membrane of the pharynx and upper air-passages; hence the significance of the hoarseness which was looked upon with such suspicion in the Middle Ages. The macules consist of erythematous patches in which not only hyperæmia but a certain amount of infiltration is usually present, and of areas in which the pigment is either increased or diminished. As in smallpox, the fever and other symptoms of invasion subside on the appearance of the eruption. The macules vary according to the natural colour of the skin. In white races they are usually of a light-red colour; in Norway they are generally lenticular crimson patches (Danielssen and Boeck). The colour is brighter at the edge than in the centre, which may become white and atrophic. The size of the spots varies from that of a pin's head to the palm of the hand or larger. They are smooth and shining, with a well-defined outline. On the face they may simulate sunblain, or, by their slightly raised margin and the confluence of two or three of them, they may present the appearance of erythema gyratum. Fresh crops of macules continue to come out at irregular intervals for a considerable time, each outburst being accompanied by some exacerbation of the febrile phenomena. The spots are not at first the seat of altered sensation. They may, however, be hyperæsthetic; but later, as a rule, they become more or less anæsthetic, according to the amount of pressure of the leprous infiltration on the peripheral nerves. The anæsthesia is, however, often not limited to the macules, areas of apparently normal skin being found to have lost their 
sensibility. It is often by an accidental discovery of this kind that the patient is made aware that he is the subject of leprosy.

So far the cutaneous manifestations are common to all cases of leprosy, with the usual variations of intensity in different individuals. As a general rule, the prodromata are more conspicuous and severe in the case of a developing skin leprosy than in the nervous form of the affection. In the latter there may be little or no fever, but rather a persistent feeling of chilliness, and the other symptoms of constitutional disorder may be almost entirely absent.

In its subsequent course leprosy may follow one of two different lines of evolution, according as the disease directs the weight of its attack against the skin or the peripheral nervous system. In a certain proportion of cases both forms may be combined, and thus three distinct types of leprosy are met with-namely, (1) skin, tubercular, or nodular leprosy (Plates 50, 51); (2) nerve, or anæsthetic, leprosy (Plates 52, 53); (3) mixed or complete leprosy. The least common of these varieties is the last. Of the two others the anæsthetic form is more frequent in tropical countries, and the nodular in Europe. Though pathologically the same disease, they present marked clinical differences.

Skin leprosy.-After a period of invasion varying from a few weeks to some months, the macules undergo transformation into nodules by sudden increase of inflammatory infiltration; they also develop independently in the skin and under it. The evolution of the nodules is usually very slow, but in rare cases it may be comparatively rapid, being ushered in by an erythematous blush, simulating erysipelas and accompanied by febrile phenomena. Their size, when fully developed, varies from that of a small shot to that of a filbert or larger. They are round or oval in outline, raised considerably 


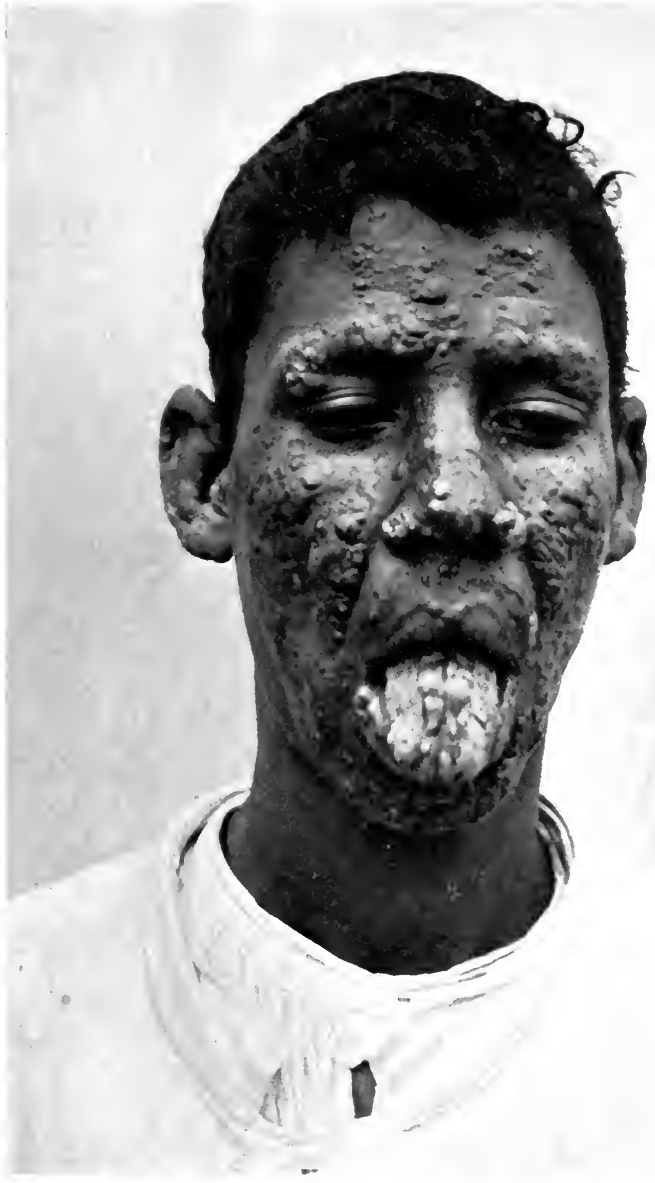

Plate 50.-nodular leprosy With lesions on tongue. (By permission, from the Photographic Album of the School of Hedicine, Cairo.) 


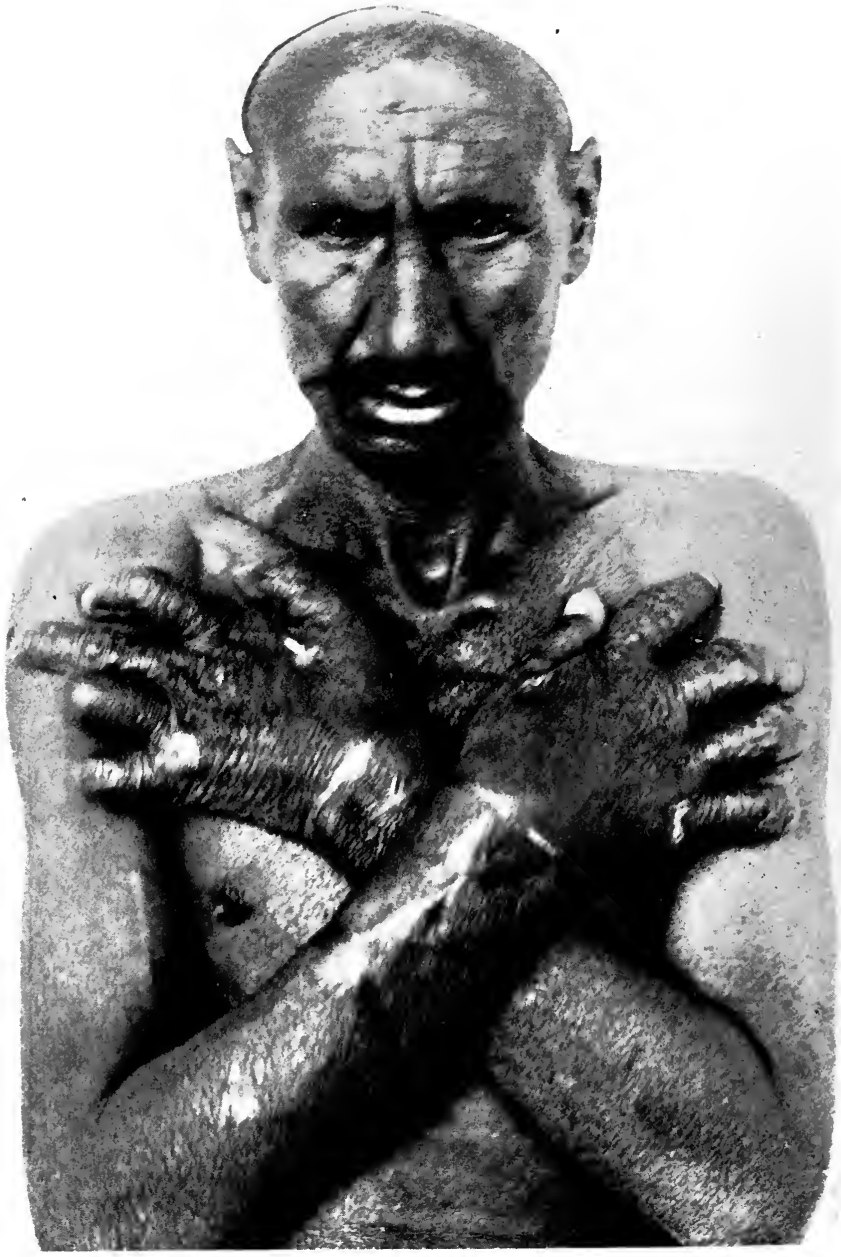

\section{Plate 51.- LEPROSY WITH atROPHIC SKIN LESIONS AND MUTILATED FINGERS.}

(By fermission, from the l'hotegraphic. illum of the School of Hedicine, Cairo. 
above the level of the skin, and sometimes surrounded by a considerable zone of diffuse infiltration. Sometimes they have the normal tint of the skin, but the colour varies greatly according to the degree of inflammatory reaction. When this is slight they may simulate lupous nodules; at other times their brownish-red tint makes them resemble syphilitic papules. When the skin around is congested they may simulate erythema nodosum or rosacea; when suppuration occurs they may resemble sycosis. As in other chronic inflammations of the skin, telangiectasis may be observed on the surface of the nodules. The local temperature is sometimes above the normal. The nodules are elastic to the touch, conveying to the finger an indiarubber-like sensation, resembling that noticed in early gummata. At first they are sometimes hyperæsthetic, later they generally become the seat of temporary or permanent anæsthesia. In some cases sensation is not altered. Extensive areas of skin are frequently involved in the process of inflammatory infiltration, and firm flat plates, as of hard codema, with either a smooth or a nodular surface, can be felt.

This most frequently occurs on the limbs, but is sometimes seen on the face. The colour of these plates is at first red or purple, and afterwards deepens into brown or even black. They are met with chiefly in the most chronic cases. The affected skin, especially in the nodular stage, is often the seat of seborrhoea. This gives the nodules, especially on the face, a characteristic burnished appearance. In negroes the whole skin, even where there are no apparent lesions, is usually greasy, and has a soapy feeling to the touch (Hillis). The hairs in the affected areas fall out. In their distribution the nodules present certain peculiarities distinguishing them more particularly from syphilitic lesions. In the vast majority of cases the face and 
the ears are the first points attacked. The massing of the nodules on the brow, and the consequent deepening of the natural furrows at the root of the nose, give the countenance the characteristic lion-like aspect which was the origin of one of the ancient names for leprosy, leontiasis. The enlargement of the ears also gives a peculiar and characteristic aspect.

The nodules sometimes develop in the first instance on the limbs or the buttocks. They may for a time be confined to the regions in which they first make their appearance, but, as the disease progresses, fresh crops of them come out on the arms, the trunk, and the abdomen. On the upper limbs the usual positions where the nodules are found are the backs of the elbows, the postero-external aspect of the forearms, the wrists, and the postero-lateral aspect of the fingers. The terminal phalanges are the last to be affected. On the lower limbs the corresponding regions are the usual seats of nodules. The nails, especially those of the toes, are often involved, and become deformed. On the chest and belly the nodules are usually small; at the top of the thigh, in Scarpa's triangle, they are larger and more numerous. They are extremely rare on the hairy scalp. Desquamation of the cuticle covering the nodules is of common occurrence; it may be excessive, giving rise to an appearance somewhat resembling ichthyosis.

The mucous membranes are frequently the seat of nodules (Plate 50), the parts usually attacked being the conjunctiva and the mucous lining of the nose, mouth, pharynx, and larynx. In these situations the nodules have a red or grey colour, and may resemble syphilitic lesions. When the tongue is greatly infiltrated the nodules are separated by depressions which may simulate syphilitic fissures. The affected parts are usually anæsthetic in the later stages, though the sense of taste is long retained: The breath has a peculiar sickening 


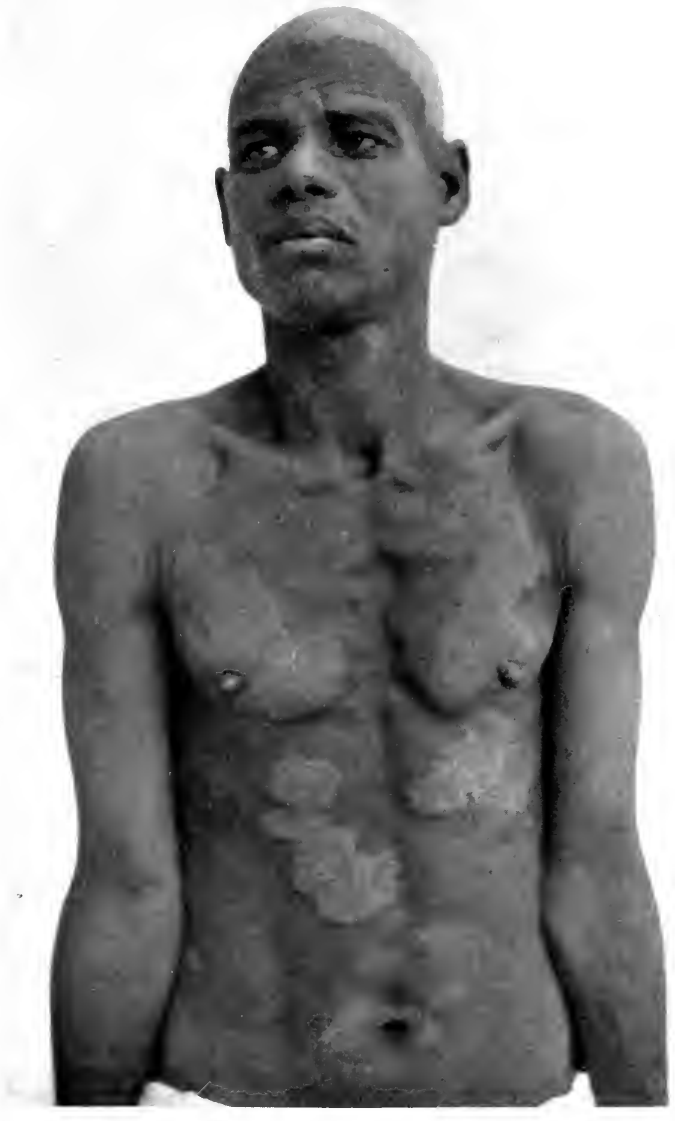

\section{PLATE 52.-NERVE LEPROSY.}

(By permission, from the Photographic Allum of the School of Hedicine, Cairo.) 

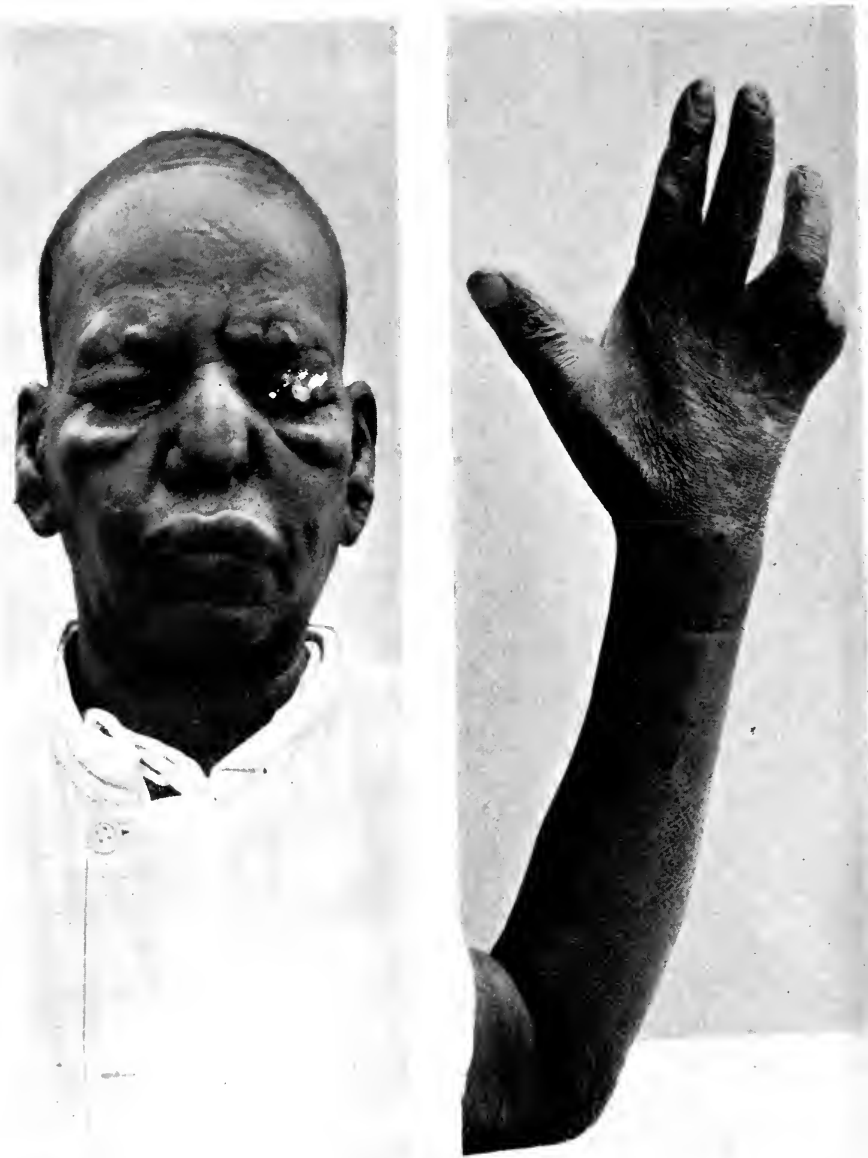

Plate 53.- NeRVE leprosy With muscular atrophy.: (By permission, fiom the Ihotographic Album of the School of Medicine, Cairo.) 
fetor. In the nasal fossæ destructive ulceration of the septum often leads to flattening of the nose, resembling that seen in syphilis. In the eye the leprous inflammation often extends from the cornea to the iris, causing great pain and slow destruction of the globe. The thickening of the laryngeal mucous membrane gives rise to hoarseness, and as the infiltration proceeds and the vocal cords become more and more immobilized, the voice is reduced to a whisper. The gradual narrowing of the glottis that results leads to increasing difficulty in breathing, and the sudden occurrence of ødema of the larynx not infrequently makes trac eotomy necessary.

This stage of leprosy is usually very slowly progressive. The nodules increase in size, and while fresh crops appear from time to time, some of the older nodules undergo softening.

Sooner or later the process enters on a new phase by the supervention of ulceration. Both on the skin and on the mucous membranes nodules have a natural tendency to break down, though in rare instances they may, like the lesions in tubercle and syphilis, undergo cicatricial shrinking without previous ulceration. In a few cases the disintegration is brought about by a suppurative process. The nodules become transformed into pustules which open and discharge their contents, leaving steep-bordered ulcers. These as a rule soon heal, leaving scars.

Often the nodules and plates become red, itchy, or painful, soften and break down, leaving an ulcer with a hard, prominent, sinuous edge and a grey base, which secretes a purulent, sometimes sanious discharge. These ulcers, like those of the corresponding period of syphilis, will usually cicatrize under treatment, but they often leave hideous deformities. When they are neglected a more acute inflammatory process may supervene and cause rapid extension of the ulcer, especially on 
the fingers and toes, and implication of tendons, bones, and joints, which frequently leads to gangrene. These processes may be complicated by the enlargement and suppuration of lymphatic glands, especially those in the inguinal and cervical regions. The liver, spleen, and mesenteric glands may be enlarged. If the patient survive and the ulcers heal, the peripheral nerves may become affected and the phenomena of nerve leprosy supervene.

Nerve leprosy.-As already said, the prodromal symptoms of both varieties of leprosy are essentially the same. There are, however, certain minor differences by which the experienced practitioner can sometimes foretell along which line the evolution of the disease will probably proceed. While the constitutional disturbance and the eruptive phenomena are, as a rule, more marked when the disease is about to make the skin the particular object of its attack, the advent of the anæsthetic form is often foreshadowed by neuralgic pains and cutaneous hyperæsthesia. The patient will experience the sensation of "pins and needles" when slight pressure is made over the track of superficial nerves. Neuralgic pain in the great toe has sometimes been mistaken for gout. Rheumatoid pains, backache, and lumbago are of frequent occurrence. Pigmentary changes in the skin following the macules of the invasion period are much more common in anæsthetic than in nodular leprosy. These changes are of two kinds : in some the affected area is paler than the natural skinsometimes even absolutely white (Plate 52); in others, again, it is deeply pigmented, the staining being brown in light-skinned races, and often of ebony blackness in dark races. The pigmented patches are usually symmetrical, occurring chiefly on the face, limbs, and trunk. They are rare on mucous membranes, and unknown on the scalp. Towards the end of the eruptive period, if not 
before, the pigment often disappears from the macules, and scarring frequently occurs. On these depigmented areas anæsthesia often develops. Anidrosis, which may or may not have been preceded by hyderidrosis, occurs on the affected areas and around them; the secretion of sebum is arrested, the hairs become blanched and fall out. The falling-out of the eyebrows is in some places looked upon by the laity as the first sign of leprosy.

A point of difference between the macules of nodular leprosy and those of the anæsthetic form is that while the former, as already said, become converted into nodules by the formation of inflammatory new tissue, in nerve leprosy only pigmentary changes occur. These may be fugitive; on the other hand, they are sometimes permanent. In some rare cases they may be altogether wanting. The eruptive stage is ushered in by intensification of neuralgic pains, with hyperæsthesia, often intense, of the macular areas and the skin around them, indicating a further development of the neuritic process which is the underlying pathological factor in this form of leprosy. The neuralgic pains increase in severity, and an eruption of bullce takes place; these are independent of the macules, though they may affect the same site (Leloir).

The bullous eruption, like pemphigus, is of extremely rapid development, and its appearance may be preceded by fever and general disturbance. The bullæ usually appear one by one. Their size varies from that of a millet-seed to that of a turkey's egg. They have absolutely the same characters as those of ordinary pemphigus. They increase rapidly, sometimes becoming doubled in size in a few days. They rupture and form a large crust, generally leaving a pale patch bordered by a brown ring, sometimes a brown patch, rarely a scar. On removing the crust a grey surface is exposed, consisting of altered rete, the epidermis being cast off 
by suppuration. Thus thick yellow scabs or crusts may be formed, sometimes resembling rupia. A succession of crusts may form and fall off, leaving at last a granulating surface, which in time gives place to a very white scar. Sometimes the bulla aborts and a parchmentlike scale forms and separates, leaving a hyperæsthetic ulcer. The bullous eruption chiefly affects the hands and feet, the backs of the elbows, and the fronts of the knees, but it may be found on any part of the body. It may continue for years, and after it has disappeared ulcers may remain. During the period occupied by the bullous eruption, nodular thickenings form on the peripheral nerve trunks, and in certain situations, as on the ulnar nerve at the elbow, they can easily be felt.

The eruption at this stage may remain more or less stationary for years, or it may spread all over the body, making the whole skin, or extensive areas of it, atrophied and white. On the face the skin has often a peculiar tense appearance, as though drawn too tightly over the features, giving the countenance a fixed, expressionless look. Meanwhile, the peripheral neuritis becomes more and more general, and as different nerves are involved, a great variety of paralytic and trophoneurotic symptoms are induced. Among these are(1) Hyperasthesia. This may persist for years. It generally begins on the limbs, sometimes on the face, and the trunk is not infrequently affected. Walking, and even the raising of food to the mouth, may be impossible. (2) Neuralgic pains. These are paroxysmal, often coming on at night. They are frequently of extreme intensity. Thickenings can frequently be felt on the affected nerves. (3) Alterations in the sweat secretion. This is a frequent phenomenon. The secretion may be suppressed on the limbs, while at the same time there may be excessive sweating on the trunk. At a later stage, as the neuritis progresses, (4) ancesthesia usually 
takes the place of hyperæsthesia. It begins on the limbs, and very rarely attacks the trunk. On the face it has the same distribution as the previous neuralgia. The loss of sensation is at first confined to the skin, but in time it extends to the subcutaneous tissue and becomes absolute. The mucous membranes of the mouth, soft palate, and back of pharynx may also become utterly insensitive, so that there is difficulty in swallowing, and regurgitation of food often occurs. The nose and the eye may also become anæsthetic. In a case sent to me from the West Indies with very early signs of anæsthetic leprosy there was an ulcer in the left nostril in which numerous acid-fast bacilli, presumably the $B$. leproe, were found. As a result of paralysis, (5) muscular atrophy is observed, especially in the hands. The thenar and hypothenar eminences are the first to waste, then the interossei; wrist-drop occurs, and the second and third phalanges are bent inwards, giving the fingers the aspect of claws. The feet are often similarly affected, so that progressive muscular atrophy is masked by solid œedema.

Among other changes due to the advancing neuritis, purulent conjunctivitis, thickening of the conjunctiva, ulcer of the cornea, and iritis may occur. The septum nasi may undergo absorption, with the result that the nose falls in. The gums may shrink and the teeth fall out. Mutilations are of frequent occurrence. The atrophied, shrivelled skin ulcerates, and, as the ulcers deepen, joints are laid open and phalanges drop off. Such mutilations are confined to the hands and feet; the tarsus and carpus are rarely affected. Mutilation may also result from interstitial absorption of the phalangeal, metacarpal, or metatarsal bones, unaccompanied by ulceration. The stumps are often bulbous. The nails may be greatly deformed, but they do not usually drop off for a long time. In some cases a blue 
soft spot appears on an anæsthetic area after a febrile attack. The skin breaks and the pus finds an exit, leaving an indolent ulcer which gradually excavates the tissues, laying bare muscles anc bones. At this stage the patient may die of pyæmia. In other cases dry gangrene of the fingers and toes supervenes. The hands and feet may become the seat of perforating ulcers, exactly resembling those seen in cases of locomotor ataxy. Gastric "crises" similar to those occurring in that disease are also of not infrequent occurrence in leprosy.

In the last stage of the disease the deformity is horrible. The intelligence is often lost, and death occurs from colliquative diarrhœa, marasmus, tetanic convulsions, intercurrent pneumonia, or pleurisy. In the nodular form of the disease phthisis and nephritis are frequent complications, and one or other of these diseases is in a considerable proportion of cases the direct cause of death.

Mixed leprosy.-In some cases of leprosy both nodular skin lesions and the changes due to leprous neuritis are present. In such circumstances the features of the two forms are combined. Anæsthetic leprosy may supervene on the nodular form, but it is more often the case that the latter shows itself some months after the commencement of the former. Some cases, however, are from the outset of the mixed or "complete" type, and in these the disease runs a more rapid course than in either of the other varieties.

Pathology. - The morbid process is that of inflammation beginning in the skin or in the peripheral nerves, in response to irritation by a specific microörganism. The leprous nodule is composed of granulation tissue together with special "lepra cells" and giant cells. The essential part of the leprous process is the infiltration of the tissues of the affected parts with this modified granulation tissue, and the slowness of the process as 
compared with lupus and syphilis is owing to the slight vascularity of the growth. In the skin the change is usually confined to the corium, and as a rule there is a thin layer immediately beneath the epidermis which is free from the bacilli. They have been found on the surface of the epidermis, but, as MacLeod suggests, ${ }^{1}$ they have probably been deposited there from the nasal secretion or by sneezing and coughing. The gradually increasing pressure of the infiltration material on the vessels, glands, and follicles destroys the normal elements of the integument; these are replaced by the leprous neoplasm, which in turn becomes disintegrated, causing deep ulcers. In nerve leprosy the infiltration takes place around the trunks of the peripheral nerves and penetrates between their fibres, at first irritating them (thus causing hyperæsthesia), then compressing them (causing anæsthesia), and destroying their conductivity (thus giving rise to paralysis). The bacilli occur in clumps within the lepra cells in the lesions of the skin, mucous membranes, and other affected tissues; the blood-vessels going to the part are sometimes seen thickly packed with them. The same bacillus is found in the diseased tissues taken from lepers in every part of the world, but in rare cases no bacilli can be discovered in the skin lesions (Saukrane). The reason of the failure of experimental inoculations may possibly be that passage through an intermediate host is necessary to make the bacillus capable of growing in the animal body. According as the bacilli invade the different internal organs, various complications may be induced. There are many points of resemblance between tuberculosis and leprosy; nothing is yet definitely known, however, as to the connection between them beyond the fact that the affected tissues react to tuberculin, and a considerable proportion of lepers die of phthisis.

${ }^{1}$ Brit. Journ. Derm., 1909, xxi. 309. 
Etiology.-The existence of a specific bacillus in the tissues affected with the disease was proved by Armauer Hansen in 1874, and the evidence that this microörganism is the exciting cause of leprosy is now accepted as conclusive. The Bacillus lepra (Plate 33, Fig. 4), like the tubercle bacillus, is an acid-fast organism; it is straight or very slightly curved, and measures about $5-6 \mu$ in length, by about $0 \cdot 2-0 \cdot 4 \mu$ in breadth. Very little is definitely known as to the actual mode of infection, or as to the conditions necessary for the growth of the bacillus. Among the predisposing causes are prolonged exposure to cold and wet, insufficient and improper food, and generally anything that depresses the health. The wide geographical distribution of leprosy seems to negative the idea that climate is a factor in its causation. It is endemic in certain limited regions in Norway, and to a much less extent in Sweden, in Russia (especially on the shores of the Baltic), in Italy, France, Spain, Portugal, Greece, and Turkey. In Asia it is largely prevalent in China, India, Turkestan, and elsewhere. In Africa, which used to be looked upon as its birthplace, it is also widely diffused. In North America it is found in scattered spots ; in Central America it is relatively common ; and in some parts of South America, especially in Brazil, it may almost be said to be rife. In the West Indies it is not uncommon; it occurs in parts of Australasia, and it rages with almost epidemic virulence in the Sandwich Islands, into which it was first imported within the memory of men not much past middle age. It ceased to be endemic in Great Britain towards the end of the sixteenth century, though what is believed to have been the last case of native origin occurred in the Shetland Isles as late as the beginning of the nineteenth century.

A climatic feature common to most of the favourite 
haunts of leprosy is the proximity of water, "but the exceptions to this rule are sufficiently numerous to forbid its being made the basis of an induction. That some peculiarity of climate, or perhaps rather of soil, has a very decided influence on the development of leprosy is clearly shown by the fact that the children of lepers-who, from living under the same conditions as their parents and in frequent and intimate contact with them, are particularly likely to be attackedhave an excellent chance of escape if they are removed from the infected district at an early age. Even when the disease has actually given signs of its presence, it sometimes seems to be arrested, or at least greatly modified, by transference of the patient to a place free from leprosy (Hutchinson).

The real problem in the causation of leprosy is to determine how the bacillus gains access to the body. From ancient times the food has been regarded as the vehicle of the poison, fish being looked upon with especial suspicion. There is, however, no trustworthy evidence of the disease ever having been conveyed by food of any kind; and, as regards fish in particular, there is abundant proof that persons may contract leprosy who have never had the opportunity of eating fish. From the analogy of kindred diseases like syphilis and tuberculosis, it is not improbable that the virus of leprosy is sometimes transmitted by inoculation, though the prolonged incubation period of the disease makes it very difficult to trace individual cases to definite contagion. But the frequency with which rhinitis, chronic coryza, ozæna, and epistaxis form the earliest symptoms of the disease suggests that in many cases the bacillus gains entrance by way of the nasal mucosa and upper respiratory tract. It is possible that it is inoculated by the bites of fleas and other insects. 
In $a$ few cases leprosy appears to have been communicated by vaccination. ${ }^{1}$

The bacillary origin of leprosy being admitted, it is impossible to escape from the conclusion that the disease is at least potentially contagious ; and what is known as to its mode of spreading, both in ancient times and in our own day, affords strong presumptive evidence that contagion is the principal element in its diffusion. On any other theory it is impossible to explain the development and dissemination of leprosy in a perfectly virgin soil like that of the Sandwich Islands, following the importation of the disease from without. The decrease in the prevalence of the disease which has always followed strict isolation of lepers is a practical proof of its contagious nature. Few people, I imagine, will agree with Hutchinson that the extinction of leprosy throughout Europe in the sixteenth century was a result of the Reformation, and the diminished consumption of fish which was one of the consequences of that movement. The stamping out of the disease is much more likely to have been the result of the terribly drastic methods of "segregation" adopted by our forefathers, combined with a general improvement in the mode of living in all classes.

Confirmatory evidence is afforded by the modern instance of Madagascar, where, since segregation of lepers has been abandoned, the disease, which previously was of very limited distribution, has rapidly increased. It is certain, however, that leprosy is not contagious in the sense in which syphilis is contagious, but only in a limited sense, like tubercle. The bacillus may be implanted by contact, but it can take root only when

1 Two cases in which this occurred have been reported by Daubler, Monats. f. prakt. Derm., Bd. viii., p. 123 . Others have been reported by Arning, Arch. f. Derm. u. Syph., Jan., 1891. 
the soil is particularly favourable to its development. In what this favourable condition of the soil consists is not exactly known, but it is not improbable that the mode of life, the hygienic surroundings, and the constitutional state of the patient have a powerful influence in determining the degree of his susceptibility to the infection.

Heredity has probably only an indirect influence. As the children usually inherit not only their constitution, but their social condition and environment, from their parents, they may no doubt inherit therewith a soil favourable to the growth of the bacillus. Many cases of supposed hereditary transmission of the disease are really examples of contagion, for which the intimacy of family life affords special opportunities. The age at which the disease usually appears-from the nintin to the sixteenth year-is against the notion of its being to any large extent hereditary.

Diagnosis.-In a well-marked case of leprosy, whether of the nodular or the anæsthetic form, the diagnosis presents no difficulty. In the prodromal stage the symptoms may sometimes suggest rheumatism or malaria, but the appearance of the leprous spots or of anæsthetic patches will soon reveal the nature of the disease. In the macular period there may occasionally be some possibility of confusion with erythema simplex or syphilitic roseola. In the former, however, there is no disorder of sensation, and little or no constitutional disturbance, and the lesions are smaller and transitory; the latter can often be excluded by the absence of history of a primary sore and of other characteristic signs of the disease. In the nodular and ulcerative stages the lesions of leprosy sometimes bear a more or less close resemblance to those of lupus and to the tubercular syphilides, but the presence of anæsthesia will generally serve to identify the disease. Moreover, 
the lupous and syphilitic eruptions are both of limited extent, and the syphilides are serpiginous and occur in crescentic groups. It should be remembered, however, that leprosy and syphilis sometimes co-exist. The crust stage of nerve leprosy may offer some resemblances to the scabs of rupia ; the latter, however, are not yellow but greenish or blackish, and instead of being single, consist of several pyramidal layers. In the early stage of nodular leprosy the nodules are occasionally exactly like those of erythema nodosum, and the resemblance may be all the closer from the presence of pains about the joints. The rapid disappearance of the lesions in the former condition will speedily remove all doubt; but if the patient has lived in a leprous district for any time it will be well to reserve judgment for a while as to the nature of the affection. In all cases of doubt, as between leprosy and any other affection, an important test is anæsthesia, which is almost invariably present in leprosy, either in the lesions themselves or in some neighbouring area of the skin. It is most frequently found towards the centre of the macule, in the pale patches that mark the sites of vanished macules, and in the hands and feet. Leprous spots, too, seldom perspire, which constitutes another distinctive feature. In doubtful cases the lepra bacillus should be sought with the microscope in the lesions and the secretions.

Prognosis.-The outlook as regards cure is of the gloomiest. In some very exceptional cases, however, permanent recovery has been known to take place. The prospect is more favourable in the pure anæsthetic than in the nodular form of the disease. The average duration of life in the former is about twenty, and in the latter about ten years. Nodular leprosy sometimes runs a very acute course, however, proving fatal in a year; and, on the other hand, in nerve leprosy life may be prolonged for thirty or forty years. Early 
treatment, and especially removal from an infected district, may do something to improve the patient's condition and increase his chances of recovery.

Treatment,-Attention must be directed mainly to the alleviation of symptoms and to the improvement of the sufferer's general health. There is no antidote for the disease. Tuberculin, which at first seemed to offer a hope that a curative agent had been discovered, only quickens the activity of the process. The serum treatment, which has had a considerable trial, has not up to the present given satisfactory results. Chaulmoogra oil (from Gynocardia odorata) given internally in doses of $m$ iii or more thrice daily after meals, and rubbed in for two or three hours a day in the form of an ointment composed of equal parts of the oil and lard, occasionally does good. Or it may be injected subcutaneously, a method recommended by Tourtoulin, Hallopeau, Du Castel, and others, who report that it yields good results. Arsenic is sometimes of marked use, especially in the skin variety. Gurjun oil (from Dipterocarpus turbinatus) given internally in an emulsion consisting of one part of the oil to three of limewater (亏ss), and applied locally (in the same way as the Chaulmoogra ointment) in a liniment of equal parts of the oil and lime-water, has been well spoken of by those who have tried it in the tropics, but now appears to be little used. The nastin treatment-hypodermic injections of a neutral fat obtained by making extracts from cultures of a streptothrix found in leprotic nodules, and mixed with benzoyl-chloride-has been used with good results in many cases, and in a report of Dr. Otto Peiper ${ }^{1}$ issued by the Imperial German Colonial Office, it is even claimed that of five negro lepers treated with nastin, two were dismissed as cured after an adequate period of observation. The theory of Deycke, who was

1 Arch.f. Schifs- und Tropen-Hygiene, Jan., 1911. 
the first to extract nastin, is that this substance attaches itself to the lepra bacillus, that the benzoyl-chloride is then able to damage the bacillus by removal of its fat, and that its destruction is completed by the normal fluids of the body. Neish, medical officer of the Leper Asylum for Jamaica, reports that all cases are improved by the subcutaneous injection of soluble salts of mercury, the anæsthetic type with more certainty than the tubercular. ${ }^{1}$ In a case of nerve leprosy, Patrick Manson tried thyroidin with apparent success, the patient being free from symptoms at the time of writing. ${ }^{2}$ Sulphur baths are useful, especially in the tropics, where scabies is a frequent complication of leprosy. The ulcers and other lesions must be treated on general surgical principles, the most scrupulous cleanliness and the strictest antisepsis being cardinal principles in the local treatment, for the sake not only of the patient, but of those who have to minister to him. In a case of anæsthetic leprosy under the care of the author and S. E. Dore, the infiltration disappeared after applications of the $X$-rays, the nodules became almost imperceptible, the anæsthesia markedly less, and the elephantiasis distinctly improved. In another case there was marked improvement in the nodules on the face and limbs after $\mathrm{X}$-ray treatment. There is other evidence to show that the local manifestations of leprosy are amenable to radiotherapy. Beurmann reports good results from the application of radium. Nerve-stretching and evacuation of the leprous infiltration lying within the nerve sheath are not infrequently followed by good results to the peripheral portions of the limb supplied by the nerves. When the throat is the seat of disease, tracheotomy may at any time become necessary. 'The consti-

1 "Leprosy in Jamaica," by E. Graham Little, Brit. Journ. Derm., Dec., 1904, p. 447.

2 "Tropical Diseases," 4th edition (1907). 
tutional symptoms may be treated on general principles, quinine being given in full doses when fever is present, and diarrhœa and other complications being dealt with by the usual remedies. Cod-liver oil and a liberal supply of nourishing food, with stimulants accord ing to indications, are most important adjuncts to medical and surgical treatment. The patient should, if possible, be removed at the earliest moment from any place in which the disease is endemic.

Strict isolation is the only trustworthy means of checking the spread of leprosy, as is shown by the experience of Norway. Segregation, if properly carried out, is not only a protection to the community at large, but is greatly to the advantage of the lepers themselves, who thus enjoy far better treatment than they could otherwise, in the majority of cases, command.

\section{Yaws (Frambersia) 1}

This disease, known also as paranghi in Ceylon, as coco in Fiji, as puru of the Malay peninsula, as Amboyna button, etc., is caused by the inoculation of a specific virus, characterized by eruptive and ulcerative lesions of the skin, with involvment of the other tissues in the later stages, and generally by greater or less constitutional disturbance. It is endemic on the West Coast of Africa, in the West Indies, in some parts of North and South America, in Madagascar, Ceylon, and other tropical countries.

Symptoms.-Four distinct stages are recognized in the evolution of the disease. The incubation period is estimated as lasting from three to ten weeks. The primary period corresponds with the life-history of the inoculation sore. This consists of a papule, which may

1 The account of yaws here given is largely founded on the excellent description in Rat's monograph ("Yaws," London, 1891). 
appear on the lip, the breast, the groin, the genitals, or the perineum. In about a week this papule becomes yellow at the apex, and seven days later discharges and dries up into a scab. On removing this scab a small ulcer with raised edge and a floor covered with granulations is discovered. The ulcer heals in a fortnight, but may persist for two months; it leaves an insignificant scar.

The secondary stage begins, about a month after the appearance of the inoculation sore, with febrile phenomena, intermittent in type, and of greater or less intensity; sometimes with graver symptoms of constitutional disorder, such as albuminuria, hæmaturia, or epistaxis. After a variable time from the onset of the fever an eruption of tiny red spots, like those of "prickly heat," appears, the fever generally subsiding as the rash becomes developed. The eruption, which is preceded by itching, appears in the form of small papules on the face and neck, and spreads downwards, the whole body being covered usually by the end of the third day. In a week the papules become yellow on the top, and begin to increase in size, so that by the end of the third week they measure a quarter of an inch in width and an eighth of an inch in height. Meanwhile the yellow heads have become transformed into scabs, beneath which is a heap of granulations grouped together so as to present the appearance of a raspberry (hence frambresia). This is the characteristic lesion of yaws. The granulations secrete a small amount of pus, and the lesions give off a musty odour. After a time the granulations lose their florid aspect and become pale or even white. Sometimes the papules are arranged in rings, especially round the eyes, nose, mouth, and genitals. They are sometimes seen inside the mouth and the vagina, also in the nasal fossæ and the external auditory meatus. 
The ulcerated papules are only slightly sensitive, but itching, as a rule, is very pronounced. In most cases healing takes place beneath the scabs, which separate about the end of the second month from the appearance of the rash. Pale spots are left, which in negroes become darker and in whites lighter than the surrounding skin. The spots are generally permanent, and are most conspicuous about the mouth, the chin, and the lower jaw.

The lesions are always accompanied by a greater or less amount of anæmia; in weakly persons, and in cases where treatment is neglected, healing may be greatly protracted. The papules may remain stationary for many months, or they may extend and by coalescence form large, deep ulcers, which leave considerable deformities or cause death from septicæmia, pyæmia, or exhaustion. In the palms and soles the ulcers usually assume the form of fissures. In children the disease runs an acute course; in the adult the process is more chronic. The description of the eruptive stage which has been given applies to the majority of cases, but variations in the appearance, and especially in the amount, of the eruption are not infrequent. Thus, instead of definite papules, only slightly scaly patches may be visible. Sometimes the eruption is limited to the extensor aspect of the forearm and leg.

In unfavourable cases a tertiary period, characterized by lesions no longer limited to the skin, but involving the deep tissues, may supervene. The most characteristic tertiary lesion is a nodular infiltration of the subcutaneous tissue, generally leading to the formation of superficial ulcers, which spread serpiginously. New nodules frequently appear in the neighbourhood of the older ones, and masses which resemble syphilitic gummata may form and break down into ulcers. The 
favourite position of these late ulcers is the leg below the knee, especially round the ankle. They are also common about the lips, and may be met with in any part of the body. Deep fissures are often present on the hands and feet; the pain on walking caused by them in the latter situation gives rise to a characteristic gait. Among the other lesions of the tertiary period are destructive ulcerations of the pharynx, soft palate, and septum; nodes on the clavicle, sternum, ulna, tibia, and the metacarpal and metatarsal bones, which may give rise to permanent thickening, or break down and cause ulcers; chronic dactylitis ; chronic arthritis, resembling white swelling; and myositis, leading to contractures. If the late affection is severe, grave anæmia may be produced, and may terminate in cachexia and death.

Pathology.-The process is that of a dermatitis limited to the papillary layer, gradually penetrating into the corium, and involving the appendages of the skin. Many observers do not agree with Numa Rat as to the occurrence of a primary sore. The facts that monkeys can be inoculated with the disease and that Castellani has found a spirochæte in association with it point to the existence of a point of inoculation, if not to the presence of an actual sore. The serpiginous ulcerations and gummatous tertiary lesions of Rat are regarded by most of the recent authorities as independent tubercular or syphilitic infections.

It has been contended by some authorities that yaws is a form of syphilis modified by race and climate, but, though it presents points of analogy with syphilis, I agree with Rat and others, who have had extensive opportunities of studying the disease, ${ }^{1}$ that the balance of evidence is against its being syphilis.

${ }^{1}$ See particularly Beaven Rake, "Post-mortem Appearances in Cases of Yaws," Brit. Journ. Derm., 1892, p. 376. 
Yaws is never hereditary or congenital ; yaws and syphilis confer no immunity as against each other, nor does one ever give rise to the other; animals infected with syphilis can be inoculated with yaws; and, as Manson points out, yaws may die out in a community while syphilis persists, or it may be universal in a community while syphilis is unknown. ${ }^{1}$ The clinical and histological differences between the two diseases are set out in the section on Diagnosis.

Etiology.-The specific poison of yaws is conveyed into the system by inoculation, chiefly by direct contact, as by kissing, sexual intercourse, etc.; sometimes apparently indirectly, by insect bites or by flies, which convey the virus from a yaws lesion to an ordinary ulcer. An abrasion of the tegumentary surface appears to be a necessary condition of the implantation of the poison. In 1905, Castellani demonstrated in scrapings of yaws tissues the presence of a very delicate spirochæte, Spirochceta pertenuis or $S$. pallidula, known also as Treponema pertenuis (more correctly pertenue), similar to that of syphilis. His discovery has been confirmed by several observers, amongst them Prowazek, ${ }^{2}$ who believed that he recognized some morphological differences between $S$. pertenue and S. pallida. According to him, in the spirillum of yaws the curves are less steep and more irregular than those of the organism of syphilis; and the former spirillum has flattened curves, which distinguish it from the latter. ${ }^{3}$ Other observers, including Castellani and Schaudinn, have failed to distinguish the two organisms morphologically.

As a result of their experimental researches on the

${ }^{1}$ For a discussion of the distinction between syphilis and yaws, see Daniels, Brit. Journ. Derm., xviii. 426; and Powell, ibid., p. 457.

${ }^{2}$ Arbeiten a. d. k. Gesundheitsamte, 1907, xxvi. 28.

3 "System of Syphilis," p. 100. 
inoculation of yaws into monkeys, Neisser, Baermann, and Halbenstädter ${ }^{1}$ concluded that yaws can be transferred from man to the higher and lower apes, and from ape to ape. These and other observers have successfully inoculated with yaws animals infected with syphilis, and vice versa, which confirms the view of those who have maintained that yaws and syphilis are distinct affections. Ashburn and Craig also successfully inoculated five monkeys, and recovered the Spirochota pertenuis from all the lesions. ${ }^{2}$

An attack usually confers immunity, but in some cases two or more attacks have occurred in the same individual. The lesions are not auto-inoculable. Among the predisposing causes of yaws are-(1) a tropical climate-it is commonest in damp, hilly, isolated regions; (2) tender age-it is most frequent in children under 10, and is hardly ever contracted after 35 ; (3) mode of life-it is commonest in the poor and in those living amidst insanitary surroundings; race-it is most common in Africans : no race, however, is exempt. It is never congenital, and is probably hereditary only in the sense in which leprosy is sothat is, from the inheritance of conditions that favour its production, and from the opportunities of contagion presented by family life.

Diagnosis. - If the tertiary stage of yaws presents resemblances to syphilis, there are striking differences in the primary and secondary stages. In yaws the inoculation lesion is not indurated, there is seldom distinct glandular enlargement, the exanthem, the mucous membrane lesions, the alopecia, the iritis, the affection of the permanent teeth, the bone affections, the polymorphism, the nerve lesions and the gummata of syphilis are absent. The histological differences

1 Münch. med. Woch., July 10, 1906, p. 1337.

${ }^{2}$ Philippine Journ. of Science, Oct., 1907, p. 441. 
between the two affections, as given by MacLeod, are the following: (a) Cellular infiltration: plasma-cells not so definitely arranged in rows or clustered round the blood-vessels as in syphilis; no large multinuclear cells (chorioplaques), or true giant cells, or intracellular hyalin degeneration noted in yaws. (b) Fibrous stroma: rarefaction of the collagen more marked than in syphilis, but no organization or colloid degeneration found, such as occurs in syphilitic gummata. (c) Blood-vessels : no distinct proliferative changes in the vessel-walls or endothelium, as frequently occurs in syphilis. $(d)$ Epidermis: marked proliferation and downgrowth of the epithelium, with great thickening of the horny layer (due to hyperkeratosis or parakeratosis), are characteristic features of yaws, while they are unusual in syphilis. According to the same authority, yaws is distinguished from actinomycosis and rhinoscleroma by the absence of their specific microörganisms; from the lepromata by the absence of Hansen's bacillus; from mycosis fungoides by the absence of "fragmentation" of the infiltrating cells, and of degenerative changes with the formation of products of degeneration in the collagen and elastin, and by the presence of the epidermal changes peculiar to yaws; from tuberculosis, apart from the tubercle bacillus, by the absence of the characteristic architecture with its giant cells, daughter plasma cells, more marked disintegration of the fibrous stroma and complete disappearance of the blood-vessels.

Prognosis.-The disease as a rule tends to spontaneous recovery unless the conditions of life of the patient be of the most unfavourable nature. Death may occur from neglect, but by proper treatment the disease can always be cured, and in most cases its manifestations can be limited to the slin and mucous membranes.

Treatment.-The inoculation sore is best treated 
by simple antiseptic applications. The fever must be dealt with on ordinary principles by quinine or the salicylates. Rat lays a good deal of stress on diaphoresis, and he gives ammonium carbonate with the double object of inducing sweating and promoting the alkalinity of the secretions. In the eruptive stage sulphur baths and calomel fumigations are useful. These should be followed by tonics, especially iron and cod-liver oil. In the tertiary stage mercury and iodide of potassium are the most efficient remedies. Rat is of opinion that in yaws, as in syphilis, the real curative agent is mercury, the iodides helping by promoting the absorption of inflammatory products. Others, however, contend that mercury is useless. In any case it should never be given in the early stage, as it aggravates the disease. Ulcers should be treated locally with black wash, weak solutions of perchloride of mercury, or iodoform. Strong ${ }^{1}$ reports most favourable results from intramuscular injection of the alkaline solution of salvarsan. In all the 25 cases in which he employed it the lesions rapidly disappeared, without the occurrence of unfavourable constitutional symptoms.

I Jour. of Exper, Med., April, 1911. 


\section{CHAPTER XXIII}

\section{MICROBIC AFFECTIONS (Concluded)}

\section{ERysipelas}

Erysipelas may be defined as a streptococcal inflammation of the skin and subcutaneous tissue, usually of the face, attended by constitutional disturbance.

Symptoms.-The disease is usually ushered in by a rigor, followed by a rise of temperature varying between 102 and $105^{\circ} \mathrm{F}$., with malaise, headache, and furred tongue, which later may become dry and brown, while sordes may appear on the lips. There are morning remissions and evening rises of temperature, and an extension of the eruption may be indicated by a further rise. In bad cases vomiting occurs, and the patient becomes delirious. The eruption begins as a raised lesion, shining, swollen, and hot to the touch, with a definite, elevated border, generally scarlet, which usually spreads by peripheral extension, though in erysipelas migrans several parts of the body are successively, and it would seem independently, attacked. In a few days, usually about a week, but sometimes much longer, the eruption ceases to spread; by this time a large part of the face-if this be the seat of the disease-may be involved, and also the neck and the scalp. If the scalp be affected the hair usually falls out, only, however, to grow again. In the centre of the patches small vesicles and bullæ will usually be found, which may become purulent and dry into crusts. As the eruption subsides and is followed by desquamation, the 
temperature gradually declines. Recurrences, however, are frequent. In many cases the symptoms are slighter than here described, and the patient may be able to pursue his ordinary avocations.

Pathology.-There is exudation of serum into the skin and subcutaneous tissues, together with deposit of fibrin and swelling of the connective-tissue fibres, and enlargement of the blood- and lymph-vessels. In severe cases the corium is invaded by the streptococci, extending into the subcutaneous tissues; and occasionally streptococci have been found in various organs.

Etiology.-Erysipelas is due to the Streptococcus pyogenes of Fehleisen (Plate 33, Fig. 8), which gains entrance through a wound, burn, or scald, a variolous or vaccinal lesion, or a microscopic breach of the cutaneous or mucous surface-often in the nasal cavity. Predisposing conditions are alcoholism, Bright's disease, and a low state of general health, however induced. The age-period most liable to attack is that between twenty and forty.

Diagnosis. - The conditions with which erysipelas may possibly be confused are acute eczema of the face and dermatitis from the administration of drugs. From both it is differentiated by the raised, clearly defined border and the presence of marked systemic disturbance. Drug dermatitis may manifest itself simultaneously at several different points, nor does active eczema usually develop from a single point.

Prognosis.-Except in the very old and the very young, and those in low health, or suffering from alcoholism or Bright's disease, the outlook is favourable.

Treatment.-The patient should be kept in bed, the diet should be plain and light but nourishing. The affected parts should be painted with ichthyol-20 to 40 per cent. in lanolin, or, better still, in water (1 in 8). To the nasal cavity, if there are abrasions and sup- 
puration, unguentum hydrargyri nitratis should be applied. Tincture of perchloride of iron and quinine may be given as tonics; strychnine also is sometimes indicated. An autogenous streptococcic vaccine has been injected with advantage in many cases, and favourable results are reported also, in grave cases, from injections of antistreptococcic serum.

\section{Cutaneous Diphtheria}

It has long been known that skin wounds may be infected by the bacillus of diphtheria, and inasmuch as peripheral neuritis may follow such an infection, there is clinical as well as bacteriological evidence of the identity of the affection in the skin and in the throat. It is important, however, that there should be wide recognition of the fact that there may be no membrane or other usual manifestation of the disease, otherwise the affection may be wrongly diagnosed and the causal agent may be left to pursue its ravages in the community unchecked. Cutaneous diphtheria is met with most frequently in children, and usually in the form of an impetiginous eczema, almost invariably associated with severe conjunctivitis, or with otorrhœea or rhinitis. Of ten recent cases described by Dawson ${ }^{1}$ four had a fatal termination. In several of them antitoxin was injected, with excellent results. The ancient view of Trousseau that the bacillus can only effect entrance through a damaged epidermis is probably correct.

In rare cases the vulva or the vagina may be attacked by diphtheria, not only in women during the puerperium, but also in children, in whom it may be either secondary to diphtheria in the throat, or primary. A case of primary diphtheria of the vulva in a child has been

1 "Cutaneous Diphtheria," by G. W. Dawson, F.R.C.S.I., Brit. Med. Journ., 1910, ii. 859. 
reported by Captain L. L. Smith.1 A slight greyish membrane separated from the labia minora and from parts of the labia majora.

\section{Impetigo Contagiosa}

The impetigo contagiosa of Tilbury Fox is a pustular eruption caused by the inoculation of streptococci.

Symptoms.-The appearance of the lesions is occasionally preceded by some amount of febrile disturbance. Soon small erythematous spots appear; on these vesicles are formed, containing a turbid fluid, which rapidly becomes purulent (Plate 54). They soon break, and discharge a fluid that quickly dries up, forming yellowish scabs. In uncleanly persons they are almost always brown, and even black, from dirt. A characteristic feature is that the scabs have no halo of hyperæmia around them, but look as if they were stuck on the skin with gum. Dotted about among them are pustules, which often run together so as to form scabs of considerable size. The scabs are at first loose, but afterwards they adhere so firmly to the skin that their removal requires some force and is followed by a little bleeding. The raw surface thus left secretes a thick purulent discharge, resembling honey in appearance and consistence, which in its turn dries into a fresh scab ("honeycomb scab"). The glands in the vicinity frequently become enlarged and sometimes suppurate. The reddish stain left when the lesions heal completely disappears after a time. The eruption varies greatly in severity, being in some cases limited to a few discrete lesions, and in others extending over nearly the whole body. Sometimes the distribution is annular (impetigo circinata). In a case of impetigo circinata, in a boy of 5 , reported by Adamson, the rings covered the whole trunk, and in addition the extremities, from I N.Y. Med, Journ, Jan. 7, 1901. 


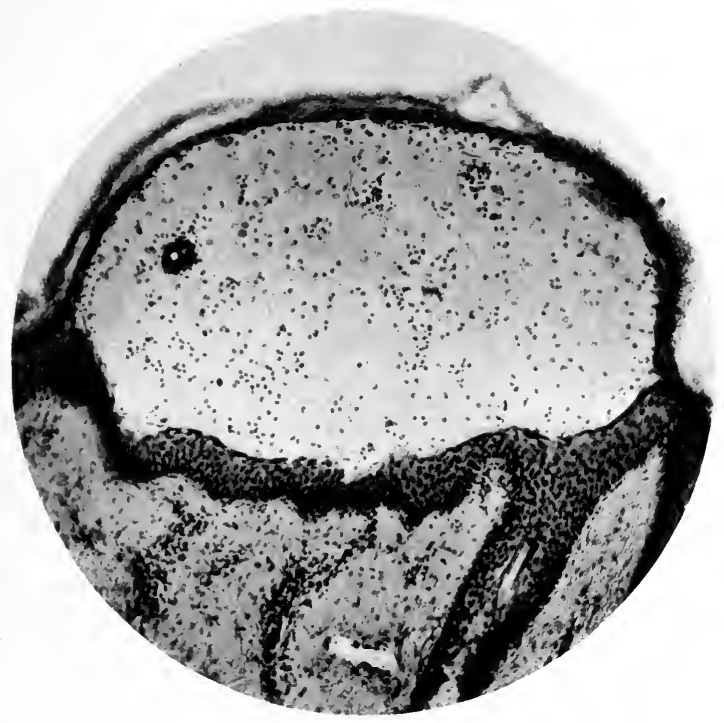

Plate 54.--Section of pustule in impetigo contagiosa. (Gilchrist.) 

the wrists and ankles downwards, were thickly set with macules, vesicles, and bullæ, many of the bullæ being fully three-quarters of an inch in diameter. When the eruption had disappeared the child was brought to hospital again suffering from an affection of the nails. In several of the fingers and toes that part of the nail which overlies the matrix had been destroyed, the area being occupied by a yellowish-brown crust, very tough, and firmly adherent to the matrix beneath. These lesions appeared to be due to infection by the same agent that had caused the skin eruption.

Corlett met with fifteen cases of impetigo bullosa among the troops who had taken part in the HispanoAmerican War. Extensive as was the eruption, there were no grave constitutional symptoms, and recovery took place within a few weeks. In several instances the serous or sero-pustular contents of many of the bullæ were found to be auto-inoculable.

The exposed parts are more likely to be the seat of impetigo than those covered by the clothes. The face is most frequently attacked, the lesions being thickest around the mouth and the nostrils and on the chin; the occipital region is another favourite situation, especially when pediculi are the exciting cause. In all these places the disease is more obstinate than elsewhere. In some cases the confluence of numerous lesions covers the face with a mask of scabs. There may be an accompanying pustular eruption inside the mouth; and Payne has traced a connection between the cutaneous disease and conjunctivitis, otorrhœa, purulent rhinitis, ulcerative stomatitis, and even purulent vaginitis. Conversely he has seen these conditions originate from impetigo on the skin. Next to the face and scalp, the parts most often attacked are, in the order of frequency, the nape of the neck, the neck, the upper extremities, the hands, the lower extremities, the 
abdomen, the back. In the folds of the joints and in the post-auricular sulcus the intertrigo type of impetigo is met with. In these situations, as the result of moisture and of apposition, the vesicles are prematurely ruptured, and red, oozing surfaces are formed. On the limbs, especially in delicate children and debilitated subjects, the pustules, as the result of friction, rupture at an early stage, and a flat irregular scab, not yellow, as in ordinary impetigo, but dirtybrown, and surrounded by a more or less pronounced areola, forms over them. These lesions were formerly believed to constitute a distinct disease, to which the name of ecthyma was applied; the condition is, however, so frequently associated with contagious impetigo as to make it certain that they are modifications of the same process. The streptococcus may be obtained in culture from the membrane that covers the ulcer when the crust has been removed.

Duhring describes a form of impetigo distinct from that here referred to, in that it is not contagious, that it is pustular from the first, and that all the lesions come out at once, not in successive crops. My own experience does not lead me to agree with Duhring that any form of impetigo is non-contagious, and the cases to which his description would apply in other points seem to me to be simply examples of a variety of impetigo contagiosa.

Among the complications of impetigo contagiosa may be mentioned boils and folliculitis. In unhealthy children the eruption is frequently pustular from the first. The disease often occurs epidemically. In such circumstances it runs a definite course, crops of vesicles con. tinuing to come out for about a week, then drying up, the process being completed in about a fortnignt. In the non-epidemic form the affection, if left to itself, may last an indefinite time. 
Etiology.-Contagious impetigo is much more common in children than in adults. The scrofulous diathesis is a powerful modifying factor. The exciting agents were formerly thought to be staphylococcipyogenes aureus and albus (Plate 33, Fig. 7). In Corlett's cases of " impetigo bullosa" (p. 547) the staphylococcus aureus was found in some of the cultures made from the blebs. In 1899, Unna, who called the disease " impetigo vulgaris," thought that it was caused by a special coccus. By the inoculation on himself of pure cultures of these microörganisms, Bockhart produced lesions exactly similar to those of impetigo contagiosa. His results were confirmed by Wickham and others. In addition to the staphyloccoci just mentioned, Leroux, in 1892, found a special micrococcus, which he called the streptococcus of impetigo. In 1901, Sabouraud showed that there are two distinct varieties of impetigo-the impetigo contagiosa of Tilbury Fox, caused by the streptococcus but rapidly becoming infected by the staphylococcus, and the follicular impetigo of Bockhart (p. 556), due to a primary infection of staphylococci. ${ }^{1}$ In the case described by Adamson (p. 546), streptococci as well as staphylococci were found in portions of the crust from beneath the nail-fold of one of the affected fingers. Impetigo contagiosa is not only contagious from one person to another, but is auto-inoculable, the finger-nails being the chief carriers of the infective material. It may be conveyed indirectly-by contaminated garmentsas well as directly; and it is especially prevalent in crowded dwellings and insanitary surroundings. It is a frequent complication of all conditions in which there

1 The history of impetigo contagiosa, and of its separation from the impetigo of Bockhart, will be found in Colcott Fox's article on "Dermatoses of Streptococcic Origin," in vol. ix. of Allbutt and Rolleston's "System of Medicine." 2nd edit., 1911. 
is troublesome itching, notably of scabies and pediculosis. It is often a complication of vaccination.

Diagnosis.-Identification of the disease rests mainly on the scabby appearance and discrete character of the lesions, the absence of hyperæmia around them, and the inoculability of the discharge. The affection with which impetigo contagiosa is most easily confused is pustular eczema, particularly when the impetiginous pustules have coalesced into a patch. The confusion, however, may be avoided by remembering that in eczema the pustules are smaller, that the crusts are surrounded by an inflammatory areola, which is seldom present in impetigo, and that there are severe itching and burning. From the vesicles of eczema those of impetigo contagiosa are distinguishable by being larger and discrete, and by tending to form yellowish crusts without rupturing, or as soon as they have ruptured, as well as by the absence of the areola mentioned above. The points which distinguish impetigo contagiosa from pustular eczema serve also to differentiate it from mild modified smallpox. In impetigo, further, there is no fever, the lesions begin not as papules, but as vesicles or bullæ, they are often limited to the face and extremities, and they dry up into flat, yellowish crusts.

In favourable circumstances contagious impetigo tends to spontaneous cure in a few weeks, but repeated auto-inoculation may cause it to persist indefinitely.

Adamson ${ }^{1}$ regards as possibly a very chronic form of impetigo a benign but most intractable eruption not seldom met with in children, and characterised by sharply circumscribed patches of a superficial dermatitis, distributed more or less symmetrically upon the face, trunk, and limbs. There is no bacteriological proof that the affection is related to impetigo contagiosa, as he frankly avows, and histologically it might

1 Brit. Journ. Derm., 1908, xx. 109. 
be classed with Brocq's group of parakeratosis psoriasiformis, but the clinical evidence, in his opinion, points to a local microbic origin, and possibly a close relationship to impetigo.

Treatment.-The scabs should be removed by soaking in carbolized oil or carbolic acid lotion (1 in 100) or boric starch poultices and the application of a weak mercurial ointment, such as ung. hyd. ammon. or ung. hyd. nitratis dil., sulphur, or other parasiticidal remedy. Plumbi acetatis gr. $\mathrm{x}$, zinci oxidi gr. $\mathrm{xx}$, hydrarg. subchloridi gr. $\mathrm{x}$, ung. hydrarg. nitrat. dil. gr. $\mathrm{xv}$, to $\xi^{\mathrm{i}}$ of lard, or ung. hydrarg. nitratis dil., ung. plumbi subacetatis, ung. zinci, āā ß̌̉, may also be recommended. The whole of the affected parts and the adjacent skin should be washed with a weak antiseptic, such as boric-acid or carbolic lotion, as a measure both of cure and of prevention. Scratching should as far as possible be prevented. Treatment with vaccine has yielded good results, but is not usually required. Weakly and ill-nourished subjects will be benefited, locally as well as generally, by iron and other tonics, nourishing food, change of air, etc.

The pemphigus contagiosus of warm countries is believed by Manson to be a variety of the impetigo contagiosa of temperate climates; it is undoubtedly more nearly related to that affection than to pemphigus. The lesions begin as tiny erythematous specks, which are followed by vesicles and blebs, leading to exfoliation and scaling. In adults they are, for the most part, confined to the crutch and axillæ; in children they are, as a rule, diffuse. Manson has found a diplococcus in the epidermis and fluid of the blister. Others have found the Leishman body, while Castellani and Clayton report the presence of cocci, arranged in pairs, presenting in cultures all the characters of the Staphylococcus pyogenes aureus et albus. Treatment consists in the 
strict observance of cleanliness, the frequent use of a 1 in 1,000 bichloride of mercury lotion, and of a dusting powder of boric acid, starch, and zinc oxide in equal parts.

\section{Bullous Impetigo of Infants}

This affection, often styled pemphigus neonatorum, is met with in new-born infants. Some authorities hold that it is only a sub-variety of impetigo contagiosa, the greater severity of the symptoms being explained by the low resisting power of the skin of new-born infants. It is characterized by the eruption of bullæ on the thighs, buttocks, face, and other parts, accompanied by greater or less constitutional disturbance. The children are free from syphilitic taint, and are often well nourished, but have been exposed to septic infection from insanitary surroundings. The disease is probably due to streptococcal infection, but staphylococci are always found in cultures, and, as Sequeira remarks, ${ }^{1}$ their rapid growth masks the more slowly growing streptococci. In some instances the disease may occur in the form of a limited epidemic, and a particular midwife may occasionally be the means of conveying the disease. Maguire ${ }^{2}$ has described an epidemic of the acute form of the affection at Richmond, Surrey, in 1902, in which there were strong grounds for the belief that the transmitting agent was the midwife to whose practice the cases were confined. The epidenic, he concluded, was due to infection with the Staphylococcus pyogenes aureus, but the source of the contagion, whether the pustular acneiform eruption from which the midwife suffered, or the insanitary surroundings of the first case, was not clear. In another epidemic at Rochester,

1 "Diseases of the Skin." By James H. Sequeira. London, 1911.

${ }^{2}$ Brit. Journ. Derm., Dec., 1903, p. 427. 
U.S.A., in 1907, the disease, in every case but one, began beside the nail of the index finger and in that of the thumb. 1 Though appearing chiefly in the newly born, and fatal only to them, the malady also attacked older children and adults. Adamson, who maintains that pemphigus neonatorum is an infantile form of the impetigo contagiosa of Tilbury Fox, supposes that the Staphylococcus pyogenes aureus is only a secondary infection, and that the Streptococcus pyogenes is the primary cause. Sequeira takes the same view.

Pemphigus neonatorum is not, as a rule, of any gravity, but occasionally it assumes a malignant type, the contents of the bullæ being dark and fetid, and gangrenous ulceration taking place, with symptoms of infection generalized, ending in death in ten or twelve days (Tilbury Fox). Paul Richter ${ }^{2}$ concludes that in some cases the affection is congenital, and that when it so originates the prognosis is unfavourable. The disease which by Ritter and others is styled dermatitis exfoliativa neonatorum (p. 343) is regarded by Richter and Hedinger as a specially malignant variety of pemphigus neonatorum. ${ }^{3}$

\section{Dermatitis Gangrenosa Infantum}

Known also as varicella gangrenosa, this rare affection consists in an eruption of vesico-bullæ, which rapidly undergo necrosis. It occurs in marasmic infants, especially girls, or in those who have suffered from varicella, measles, etc. It is probably a mixed infection, for while pyogenic cocci are always present, the Bacillus pyocyaneus is often found in association with them. The preference sites of the eruption are the lower part of the body and thighs, but sometimes there is wide

1 Trans. Amer. Derm. Assoc., 1908, p. 137.

2 Derm. Zeitschr., Bd. viii., Hft. 5 and 6.

s Arch. f. Derm. u. Syph., July, 1906, p. 349. 
dissemination, and the scalp and neck may be attacked. The general symptoms are high fever, wasting, diarrhœa, convulsions, and grave exhaustion. Death occurs in about one out of two cases. Treatment consists in giving frequent boric-acid baths, or applying boric-acid fomentations to the lesions. A nutritious diet is also indicated.

\section{IMPETIGO HERPETIFORMIS}

Under this name Kaposi ${ }^{1}$ described an affection, -known also as herpes pycemicus-which, while presenting certain affinities with dermatitis herpetiformis, exhibits characteristics sufficiently well marked to entitle it to be classed as an independent disease.

Symptoms.-Impetigo herpetiformis begins with the development of small pustules with opaque contents, which gradually assume a greenish hue. These pustules are arranged in groups on an inflamed base, and lie very close together; they appear first in the groin, on the umbilicus, on the breast, and in the armpit, other parts being attacked at a later stage. They dry up in one or two days, leaving a dirty brown crust. New pustules come out, forming a double and even a triple circle around the first as a centre; these, as they dry, increase the size of the central scab. In this way, starting from a few isolated points, the disease may, by the coalescence of adjacent foci, gradually spread over extensive areas. When the scabs become detached, the skin underneath is found to be red and smooth, sometimes moist, as in eczema, but never ulcerated. In the course of three or four months nearly the whole cutaneous surface may be invaded.

The skin is burning hot, tense, and scabbed all over, the cuirass of crusts being here and there cracked

1 "Maladies de la Peau," French translation by Besnier and Doyon, 2nd edit., i. 799. Paris, 1891. 
and excoriated. The mucous membranes of the tongue, palate, velum, and the back of the pharynx in some cases present circumscribed greyish patches. In one case referred to by Kaposi groups. of pustules were found in the œsophageal folds; in many places, especially near the cardiac orifice, these had ulcerated. The eruption on the skin is accompanied by more or less continuous fever, exaccrbations of which, with rigors and general constitutional disturbance, usher in each fresh crop of pustules. The disease lasts a few weeks, or at most some months, and is almost certain to prove fatal. The cause of death is by no means clear, but in some at least of the cases it was due to marasmus.

Etiology and pathology. - Impetigo herpetiformis is very rare, and has so far hardly been observed anywhere else than in Vienna. Nearly all the patients have been pregnant women, and in one or two there have been uterine complications. Graham Chambers, of Toronto, has, however, reported a case in a man-a farmer who had been a hard drinker, and had smoked to excess, but had enjoyed good health until the eruption appeared. 1 Kaposi appeared to be not disinclined to look upon the disease as infectious, ${ }^{2}$ and the probability is that it is a form of septicæmia or pyæmia. Besnier held that impetigo herpetiformis is not so much a definite pathological entity as a group of closely allied affections. The feature common to them is the formation of vesicles in groups, which quickly become pustules and spread at the circumference while healing in the centre. In this way neighbouring lesions unite and cover large areas. In their evolution the lesions assume at different stages an eczematous, ulcerative, vegetative, or papillomatous aspect. He thought it

${ }^{1}$ Brit. Journ. Derm., 1911, xxiii. 65.

Op. cit., p. 803. 
probable that visceral changes were present in fatal cases. In short, Besnier looked upon impetigo herpetiformis as an expression covering multiple affections of septicæmic type, or reflex lesions leading to trophic changes.

Diagnosis.-Except for the gravity of the affection, there is, as Colcott Fox ${ }^{1}$ points out, little fundamental difference between it and the pustular cases of dermatitis herpetiformis, which, however, may change into another type of eruption. There is little, either, to distinguish it from hydroa gestationis, except the gravity and rarity of the pustules in the latter.

Prognosis.-The outlook is always grave, both for mother and for child. Labour generally comes on prematurely, and even when it occurs at term the infant seldom survives more than a few days.

Treatment.-Impetigo herpetiformis is a very refractory disease. A vaccine, autogenous if possible, should be administered. For the rest, treatment should follow the lines recommended for impetigo contagiosa.

\section{IMpetigo of Bockhart (Follicular Impetigo)}

This condition, now regarded as distinct from the impetigo contagiosa of Tilbury Fox (p. 546), is a staphylococcal infection characterized by pustular lesions about the hair-follicles.

Symptoms.-Each pustule, yellowish in colour, and surrounded by a red halo, has a hair projecting from its centre. The pustules vary in size from a pin's head to a small pea. They dry up into crusts which differ considerably in thickness; the thicker ones, when they fall off, leave a small scar at the mouth of the follicle. They are usually multiple, and may occur at any part of the body. The affection runs a slower course than does streptococcic impetigo.

1 See the article on Pemphigoids in Allbutt and Rolleston's "System of Medicine," 2nd edit., ix. 458. 1911. 
Pathology.-The lesions are primarily small abscesses seated around the summit of the hair-follicle in the epidermis, superficially or deeply. There is also perifollicular infiltration. The cocci enter the horny layer and multiply laterally around the mouth of the follicle. In cultures the white staphylococcus is often found with the yellow.

Etiology.-The affection, like impetigo contagiosa, is most often met with in children, but may occur at any age. The primary lesions may arise spontaneously, but usually follow scratching and other forms of traumatism. Malnutrition of the tissues in such conditions as gastro-intestinal and kidney disorders appears to be a predisposing cause.

Diagnosis.-The affection is differentiated from streptococcic impetigo by the central hair, and the fact that the lesions are pustular from the outset, as well as by its more indolent course.

Treatment.-The disease areas should be cleansed with boric-acid or carbolic fomentations, and a white precipitate ointment then applied. Applications of weak tincture of iodine are serviceable in cases where the scalp is affected. Iron should be given to debilitated subjects, and in refractory cases an autogenous vaccine should be employed.

\section{Sycosis}

Coccogenic sycosis is an inflammatory process caused by staphylococci-usually the Staphylococcus pyogenes aureus-and affecting the hairy parts of the face, and especially the chin, in males. In rare cases, however, the disease may attack the eyebrows, the eyelashes, and the axillary and pubic regions, in both sexes.

Symptoms. - The lesions are papules or nodules, which form round the hairs and develop into pustules, 
each of which is pierced by a hair. They gradually increase in number, and may extend over a large surface. The affection sometimes begins on the upper lip, and may remain limited to that region. As the suppurative process goes on, the hairs are loosened, so that they are easily pulled out, a drop or two of pus generally following them. The pus dries into thin brown or vellow adherent crusts. In severe cases the pustules may be so thickly set together as to form infiltrations which may assume a fungating character. The process never extends beyond the limits of the hairy region. Sycosis does not generally cause baldness, because the papilla is seldom destroyed, the pus lying in a pouch formed by the lining membrane of the follicle and the outer sheath of the hair. The disease may last in varying degrees of severity for an indefinite period. In very chronic cases there is always a good deal of scarring, causing baldness, and occasionally cheloid forms in the scars.

Brocq has described, under the name of sycosis lupoide, a variety of folliculitis which begins at the upper part of the whiskers and travels downwards; there is a narrow erythematous margin, and the process gives rise to marked infiltration, followed by cicatricial atrophy.

Sycosis, of course, in its typical form is peculiar to adult males, but folliculitis of the same character may occur, as has been said before, in women. The disease is often conveyed by the shaving brushes of barbers who are not particular about the cleanliness of their implements. It may begin as a streptococcic infection, the staphylococcic infection being secondary and much more persistent.

Pathology.-The affection is an inflammatory process starting in the hair-follicles, each follicle being, in fact, converted into a small abscess. Sycosis is 
inoculable from one follicle to another by the transference of staphylocncci. Tenderness or excoriation of the skin is probably a necessary condition for the development of sycosis. The sebaceous glands are affected secondarily to the hair-follicles; the sweat-glands are only occasionally involved.

Diagnosis.- The inflammatory nature of the disease, its origin in the follicles, and its limitation to the hairy parts of the face are characteristic. Eczema is not limited to the hairy parts; if the follicles are involved it is only secondarily, and the inflammation is seldom so severe as in sycosis. Itching, on the other hand, is not a feature of sycosis. In cases of widely diffused sycosis, the crusts may have to be removed to clear up the diagnosis; when this is done, the follicular implication will be revealed. Tinea barba is distinguished by its commencing in a circinate scaly patch, by the early breaking of the hair, by the pain caused by extraction of the hair, by the shape of the pustules, which are conical and elevated, by the lumpy masses on the inflamed surfaces, by the fact that it rarely affects the upper lip, and by its special fungus. If doubt should ever arise as between sycosis and acne vulgaris, the presence of acne lesions in non-hairy parts should of itself suffice to decide the question. Tertiary syphilitic ulceration is not limited to the follicles, and is associated with a history of primary infection and marks of previous or coincident specific lesions.

Treatment.-Sycosis is always extremely obstinate ; and recurrence after apparent cure is common. The treatment is first to remove the crusts, then epilate (a process which, owing to the loosening of the hairs by the pus, is not painful), and finally apply soothing and antimicrobic remedies. The removal of the hairs opens the little abscesses, and the mouths of the follicles are thus made patent, so that remedies can penetrate 
to the seat of the disease. In mild cases, oleate of mercury (1 to 2 per cent.) or weak sulphur ointment may be used. When the affection is more severe, strong sulphur or resorcin paste (10 to 20 per cent.) or Unna's carbolicmercury plaster mull should be employed. In ordinary sycosis improvement is brought about by short exposures to $X$-rays, but more radical results are obtained after epilation doses, although these are often followed by relapse necessitating a repetition of the treatment until the hair-follicles are destroyed. The treatment must be applied with great care, the beard region being more sensitive to the rays than the scalp.

This affection is one for which vaccine treatment may be recommended. An autogenous is to be preferred to a stock vaccine, though the latter is useful. Gilchrist, indeed, considers that in infections from staphylococcus aureus a staphylococcus albus gives better results than an autogenous vaccine, but this is probably accounted for by the fact that some of the cases are caused by the staphylococcus albus. It is usual to begin with a dose of 200 millions, gradually increased to 2,000 millions. A dose should be given about every ten days.

\section{Furunculi}

Boils are staphylococcic inflammatory swellings of which the seat is either a follicle or a sebaceous or sweat-gland.

Symptoms.-Boils may form on any part of the skin, but the parts most frequently affected are the face, the neck, the axillæ, and the buttocks. They may be single or multiple, in the latter case being scattered about without any attempt at grouping, and coming out in crops. In such circumstances the process may last a considerable time, constituting a condition known as follicular furunculosis. The lesion begins as a 
minute red papule, which is tender, so that the slightest movement causes pain. Soon induration ean be felt, and the boil shows itself on the skin as a nodule of varying size, presenting the classical characters of inflammation. Resolution may take place within a few days, the boil subsiding without suppuration oceurring. This constitutes the "blind boil." As a rule, however, it "points" more or less distinctly on the third or fourth day, the pustule being seated on an indurated base, surrounded by a raised red area. The inflammatory zone tends to increase, the skin on the surface of the boil becomes purple, tense, and glistening, and finally gives way, about the eighth day, in one or more places. The central part of the swelling is then seen to be occupied by a white pulpy slough or core, which is thrown off in a day or two. Before rupture the boil and the skin around it are exquisitely tender, and the heat, tension, and throbbing may make sleep impossible. Lymphangitis and lymphadenitis are often set up, and there is usually some amount of constitutional disturbance. After separation of the core the symptoms subside, and the resulting eavity heals up by granulation, a scar proportionate to the size of the boil being left.

A special form of boil which becomes developed in the sweat-coils has been described by Verneuil, Dubreuilh, and Pollitzer, the latter of whom styles the affection hydradenitis destruens suppurativa : it is probably a form of acnitis ( $p$. 577). This observer records a case in which the cheeks, chin, parts of the neck, and the upper part of the shoulder were the seat of successive crops of small tumours, which appeared one or two, or by the half-dozen, at a time. The crops came out at intervals of a few days to several weeks, and the process extended over eight months. The lesion began as a nodule deeply seated $2 \mathrm{~K}$ 
in the skin. The nodule was at first neither painful nor tender; it became in a fortnight as large as a pea, and slightly painful; the skin over it was red. If one of these nodules was opened at this stage, a drop of pus exuded; if left untouched, after a few days a little pus was discharged, after which shrinking and cicatrization took place, the whole process occupying about four weeks. Two nodules were excised and examined, when it was found that the tumours were evidently developed in the sweat-coils, the coil being, in the first instance, the seat of infiltration, and its intimate structure being finally lost.

Etiology.-Boils are caused by the action of the Staphylococcus pyogenes aureus and albus, but more often the former. In the case of single boils, local irritation, as by the edge of a stiff collar, or friction, is often the starting-point of the trouble, the slight injury of the tissues thus caused making the part susceptible to the action of the staphylococcus. If the patient is subject to boils, some underlying constitutional state, such as anæmia, lithæmia, or glycosuria, may be present. Furunculosis may also be a sequel of acute specific fevers, particularly smallpox, or it may be an expression of a diabetic or septicæmic condition. Boils may multiply themselves by auto-inoculation, but this does not take place, as a rule, unless the patient is in a bad state of health, or local conditions favourable to the growth of cocci, such as poulticing or scratching, are present. They are secondary in many skir affections, notably scabies, pediculosis, and eczema.

Diagnosis and prognosis.-There can never be any difficulty about the diagnosis, the appearance and course of a boil being absolutely characteristic. The prognosis is always favourable as regards the cure of any given lesion or set of lesions, but the affection is very apt to recur. Single boils are always amen- 
able to treatment, but auto-inoculation of the pus often makes definitive cure somewhat difficult. In furunculosis the prognosis largely depends on the extent to which the underlying constitutional state can be remedied.

Treatment.-The treatment of single boils depends on the stage which the process has reached. When just commencing they may often be aborted by painting the part with glycerine of belladonna, or with tincture of iodine, three or four times a day; by fomenting with a saturated solution of boric acid; by the application of a compress steeped in spirit of camphor for a few minutes several times a day; or by a solution of nitrate of silver or injections of strong carbolic acid. Unna recommends the use of the mercuric-carbolic plaster mull as an abortive in the first stage, and as limiting suppuration to the centre, and causing speedy and painless rupture in the later stages. He says the rupture thus brought about is much smaller than could be made by incision, and soon closes under the plaster. In larger boils that have necrosed the plaster mull accelerates rupture, or, if an incision has already been made, shortens the time of healing and eases pain.

When abortive treatment fails or is inapplicable, the boil should be incised and scraped out, and an antiseptic dressing-iodoform, carbolic acid, or Unna's mercuriccarbolic plaster mull-should be applied. As each boil may be a focus of further infection, it should be destroyed or rendered harmless by thorough antisepsis. For the same reason it is altogether unscientific to promote maturation by the application of poultices and fomentations unless they are made antiseptic. In the treatment of isolated furuncles of long standing I have found radiotherapy efficacious in such a part, for example, as the nape of the neck. Bier's treatment has also been used with success, 
Constitutional treatment may be required for furun. culosis. Insanitary surroundings should be remedied and the health improved by measures appropriate to the special indications of the case, lithæmia, anæmia, glycosuria, etc., being dealt with on ordinary principles. The most useful drugs are iron, quinine, and large roses of diluted sulphuric acid. Duhring finds arsenic, $m$ i-iii of Fowler's solution thrice a day, beneficial. Sulphide of calcium, which is given in doses of gr. $\frac{1}{2}$ in a freshly made pill thrice daily, is a most uncertain remedy.

Sir Almroth Wright's method of treating boils with vaccine consists in injecting dead cultures of staphylococci in measured quantities. Having estimated the patient's opsonic index for staphylococci, 200 millions and upwards are injected about once in ten days, care being taken to allow the negative phase which follows the injection to subside before giving another injection. In this way the opsonic content of the blood is gradually raised, and in successful cases the boils are aborted, and the formation of fresh ones is prevented. Relapses are not uncommon, and it is often necessary to repeat the course of injections. The vaccine should if possible be prepared from the patient's own lesions. By giving small injections and allowing a sufficient interval to elapse between them, this treatment may be carried out without estimating the opsonic index. While conceding that vaccine treatment often yields brilliant results, John T. Bowen (of Boston, U.S.A.) advocates a method of treatment of which the principle is to keep the skin as far as possible sterile by washing the whole body with warm water and soap at morning and at night, bathing the entire surface with a saturated solution of boric acid in water, allowing the skin to dry without wiping, and then dressing the boils with an ointment consisting of boric acid and precipitated sulphur āa $3 \mathrm{i}$, and carbolized petrolatum そi. Every article of clothing 
next the skin is changed daily. Incision is only resorted to after the lesions have become very painful and mature. Bowen finds that these measures often succeed when vaccine treatment has failed. ${ }^{1}$

\section{Carbuncle}

A carbuncle may be defined as a boil affecting several neighbouring follicles. The process is akin to furuncle, but is more severe in its local effects, and accompanied by greater constitutional disturbance.

Symptoms.-The lesion commences as an infiltration in the subcutaneous tissue or the deeper parts of the true skin; it is at first but slightly raised, firm, rounded in outline, and bright red on the surface. In mild cases retrogression may begin at the end of a week, and complete resolution may take place. In most cases, however, the process extends, and in ten days or a fortnight forms a deep-seated, circumscribed swelling as large as the palm or larger, with a brawny base, the skin over it being of a purple colour. Softening takes place in the centre, and the surface becomes dotted with suppurating points, which break, giving exit to blood-stained pus. This cribriform mode of rupture is characteristic of carbuncle. The carbuncle often continues to spread even after the pus has found a vent. The skin between the perforations sloughs, and the necrotic mass or core underneath slowly separatestaking from fourteen days to two months in the process -sometimes as a black, dry eschar, sometimes as a pultaceous mass, more frequently as a yellow, ragged slough, with a most offensive smell. The neighbouring glands are usually swollen. The process is accompanied by rigors, fever, aching in the back and limbs, and general malaise. Death may result, especially in elderly or weakly subjects, from septicæmia or exhaustion, 1 Journ. of Amer. Assoc., 1910, lv. 209. 
especially when the lesion occurs on the face. After separation of the slough a deep, irregular cavity is left, which heals by granulation, a dense, puckered scar, which is not infrequently pigmented, resulting.

Carbuncle is generally single, and occurs especially where the skin is thickest-on the nape of the neck, on the back, the buttocks, shoulders, and forearms. It is sometimes seen on the face.

Pathology.-The process is identical with that of furuncle, but the inflammation is deeper and more destructive, and the abscesses are multiple. The prccess is believed to begin in the pilo-sebaceous follicles and sudoriparous glands.

Etiology.-The exciting cause of carbuncle is, as in furunctulosis, an invasion of staphylococci. Men are more frequently attacked than women. Anything that tends to lower vitality may be a predisposing cause, diabetes in particular being often associated with the disease. It may, however, occur in persons apparently in perfect health.

Diagnosis.-The identification of carbuncle can seldom present difficulty, the multiple yellow points and openings being sufficient to distinguish it from furuncle; and these features, together with its circumscribed outline, differentiate it from diffuse cellulitis.

Prognosis. - In cases of carbuncle a guarded prognosis should be given, especially when the lesion is situated on the face. The size and position of the swelling, and the age and state of health of the patient, are the chief guiding points.

Treatment.-For small carbuncles the treatment is the same as for boils. The free painting of the surface with glycerine of belladonna will ease the pain, reduce the inflammation, and possibly bring about resolution. Unna recommends the application of a mercuric-carbolic plaster mull, the parts being bathed 
with a solution of ammonia or alkali before a new plaster is applied. If the skin is about to break, crucial incisions should be made and the necrotic contents of the swelling cleared out with a sharp spoon. The cavity should be well scraped and all the friable tissue removed, and it should then be syringed out with some strong antiseptic solution such as carbolic acid, and filled with an antiseptic dressing, subsequent treatment being on the accepted lines of antiseptic surgery. Constitutional treatment is usually required. It should be directed to supporting the patient's strength by every available means-liberal diet, careful regulation of the bowels, and the free use of tonics, especially perchloride of iron and quinine. If the pain is very severe morphia should be given, preferably in the form of hypodermic injections. Stimulants should" be withheld till the slough has been cleared out, after which wine, such as port or burgundy, may be given if indicated.

\section{Dissection Wounds}

The inoculation of septic material from a dead body, as when the hands are pricked or scratched in dissecting or post-mortem work, may give rise to pustules or small abscesses at the seat of injury, or to lymphangitis and cellulitis, which may be followed by pyæmia. The skin lesions must be treated antiseptically, and constitutional symptoms, if they arise, be dealt with on general principles. For verruca necrogenica, see p. 439.

\section{ACne Vulgaris}

Acne has been placed in the group of microbic affections, although its title to be looked upon as inoculable in the strict sense is not universally accepted. It is certainly one of the least inoculable of the diseases included in the group under consideration, but its pathological 
affinities with lesions in which microörganisms play a leading part and the bacteriological evidence justify its -provisional inclusion in this category.

Symptoms. - The obstruction may be at the mouth of the sebaceous gland-duct, the plug being visible on the surface as a small black point (comedo), or in the gland itself, when the obstructing material is seen as a tiny whitish mass in the substance of the skin (milium). Gilchrist found the comedo to be formed by a hyperkeratosis of the inner layer of the dilated hairfollicle, a change which was attended by dilatation of surrounding blood-vessels. The primary lesion is a red papule, which may become pustular, the pustule being seated on a raised red base. In the papule, according to Gilchrist, the lower part of the distended follicle is surrounded with polynuclear leucocytes and nuclear detritus. The affection is met with in varying degrees of severity, from a few scattered papules to numerous lesions in all stages of development. The process may be arrested in any stage, some lesions undergoing involution, while others suppurate and in course of time rupture. The individual lesions, as a rule, run an acute course, but the affection as a whole is chronic, fresh crops of papules and pustules coming out as others disappear. The pus may be discharged without any visible scar being left, but where the suppuration has been extensive and deep, considerable scarring and consequent deformity form in the cicatrices. In some cases the inflammatory process extends to the tissues round the sebaceous gland, and a hard, red or purplish nodule is formed, which seldom ruptures, but leaves a livid indurated swelling, that slowly disappears (acne indurata). (Plate 55.)

The favourite situations of the lesions of acne vulgaris are the face, especially on the cheeks, nose, forehead, and chin, the back of the neck, the back between 


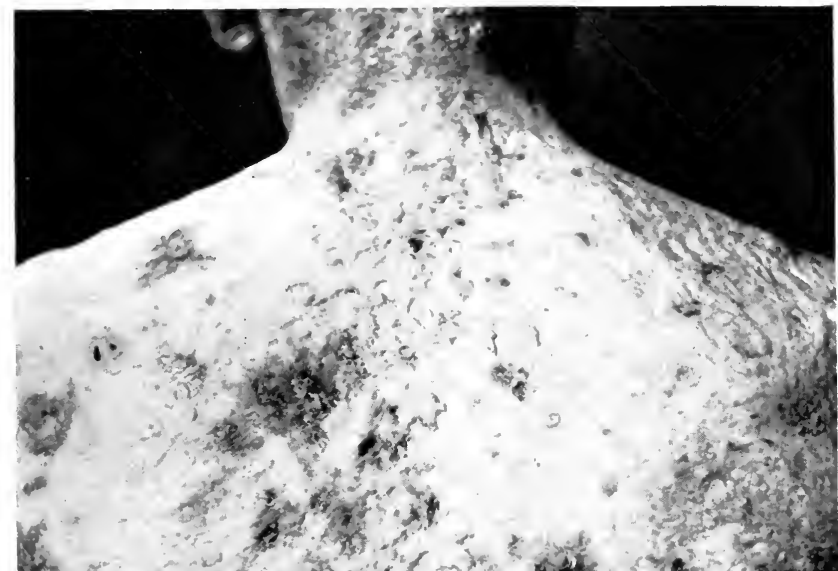

2.

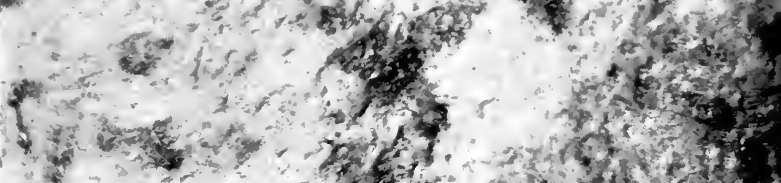

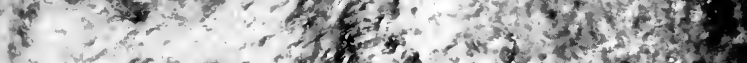

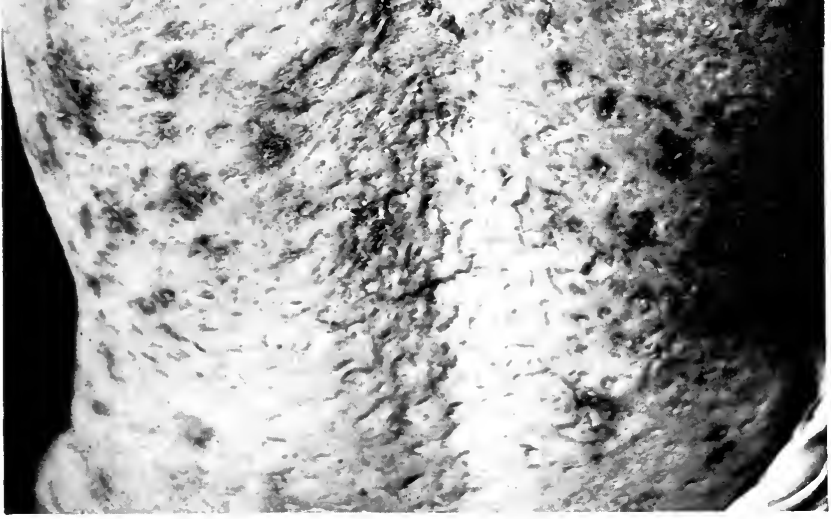

plate 55.-Old acne with severe scarring. 

the shoulders, and the chest. The affection may, however, develop wherever there are sebaceous glands; thus it is sometimes seen on the back of the thigh and arms. The lesions are tender, but do not itch, and beyond the unsightly appearance the affection gives rise to no inconvenience. The skin between the lesions is usually more or less greasy.

Pathology.-Acne is an inflammatory process in and around sebaceous glands, leading to the development of pustules and sometimes to scarring. The inflammation generally supervenes on occlusion of the duct. The plug causing the blockage may be the sebaceous secretion itself, associated with the presence of the bacillus of acne and hyperkeratosis of the mouth of the follicle (acne vulgaris), or some greasy material, e.g. tar, derived from without. The latter and other forms of artificial acne caused by drugs and chemical substances will be found described under the head of "Artificial Eruptions" (Chap. x.). The inflammatory process may also be due to local circulatory disorder, sebaceous obstruction being a secondary occurrence, as in rosacea.

Inflammatory changes are always present in the connective tissue around the follicle. When suppuration occurs, the pus may, if slight in amount, escape by natural drainage through the duct, and the gland may in this way escape destruction; usually, however, both the gland and the follicle are destroyed, and more or less of the perifollicular tissue undergoes necrosis, with consequent scar-formation. In acne indurata, sections of the nodules examined by Gilchrist showed profound changes extending deep into the corium, and in some cases surrounding a highly hypertrophied follicle. There were many giant cells (some containing bacilli), and either plasma cells or lymphoid or connectivetissue cells, besides polymorphonuclear cells, phagocytes 
and pigment cells. In 1908, Gilchrist ${ }^{1}$ exhibited a large number of microphotographs illustrating the histo-pathology of the disease, by way of confirming his conclusion that the acne nodule consists of a giantcell granuloma with large numbers of plasma cells, numerous polynuclear leucocytes, degenerative products, and small and large masses of the acne bacillus. In some of the sections the giant cells were so numerous as to give them a tuberculous appearance.

Etiology.-Gilchrist reported, in 1903, that he had found a definite bacillus, which he styles Bacillus acnes, in all smears taken from 240 typical acne lesions from 86 patients, and that pure cultures were yielded by 62 lesions (mostly acne nodules) from 29 patients. Of the remaining cultures, 82 were sterile from use of an unsuitable medium, and the rest were contaminated by other organisms. The bacillus was found deep down in acne indurata, and was pathogenic in mice and guinea-pigs ; and finally the bacilli were agglutinated by the blood-serum of patients affected with acne. He claims, therefore, to have proved that the Bacillus acnes-probably identical with the microbacillus which had been discovered by Unna and by Sabouraud respectively in 1893 and in 1897-is the primary cause of acne vulgaris, and he suggests that the constitutional and other symptoms often associated with acne vulgaris may be caused by the toxins of that microörganism. In 1908, Gilchrist reported that he had obtained many other pure cultures of Bacillus acnes, and again urged that this organism is the primary cause of acne vulgaris, especially of the nodular type, and that the staphylococcus albus, when present, is only a secondary invader. Fleming ${ }^{2}$ has more recently adduced evidence that the bacillus is the cause of the comedo, and may

1 Trans. Amer. Derm. Assoc., 1908, p. 154.

2 Lancet, 1909, i. 1035. 
also be the cause of the pustules. Like Gilehrist, he found it virtually always present in films made from the pustular lesions (97 per cent.), though in cultures his percentage of positive results was lower. He found, further, that by rubbing a culture of the bacillus into the sterilized skin of a susceptible person a pustular folliculitis was produced from which the microörganism could be recultivated in pure growth. Whitfield's view, for which there is much to be said, is that the seborrhøea is the primary cause of the affection, the acne bacillus being an accidental infection of the excessive secretion in the distended follicle. A. R. Robinson ${ }^{1}$ also is of opinion that the comedo formation is the primary condition, due to changes in the character of the sebaceous secretion, owing to such factors as altered circulation, imprisoned hairs, etc., and Gilchrist ${ }^{2}$ states that he has always found comedones sterile, except for the presence of the staphylococcus.

The predisposing causes of acne comprise (1) an anatomical factor; (2) certain physiological factors; and (3) the bacteriological factor, although the exact measure of its importance is still somewhat obscure. The anatomical factor consists of a structural coarseness of skin, which, from its excessive richness in large sebaceous glands, is naturally greasy and especially liable to retention of secretion. The physiological factors are age and reflex circulatory disorder. Acne vulgaris is essentially a disease of puberty, and, as the time of the great physiological change indicated by that term varies within considerable limits, the age at which acne shows itself ranges from 12 to 25 years. With the advent of puberty certain glands undergo great and rapid development, and in particular there is a growth of new hair in certain parts. These changes in

1 Trans. Amer. Derm. Assoc., 1908, p. 18.

2 Ibid., 1908, p. 27. 
persons whose sebaceous glands are already inclined to over-activity are likely to be followed by plugging of the ducts, with consequent interference with the capillary circulation around the gland and tendency to inflammation. These conditions are increased by reflex circulatory disturbance due to the strain thrown upon the nervous system by the changes taking place at puberty, aggravated in many cases by disorder of the digestive organs, functional disturbance or irritation of the sexual apparatus, anæmia, and in some cases, probably, educational over-pressure. Lastly, the sebaceous matter plugging the duct becomes a suitable soil for microörganisms. Demodex folliculorum (Plate 24, c), which is found in comedones, appears to have no etiological importance. In the suppurative stage staphylococci are present in abundance. Whether they are the cause of suppuration in the comedo, or whether both comedo and pustule are produced by the acne bacillus, is a question as to which bacteriologists are at variance. My own view is that in some cases, at any rate, the bacillus is responsible for the suppuration, while in others the staphylococcus may be the cause of it, just as it is in staphylococcic sycosis.

Diagnosis.-Acne vulgaris can, as a rule, be recognized without any difficulty by the presence of comedones, the discrete character of the eruption and its distribution, and the patient's age. Artificial acne must be excluded by inquiry into the patient's occupation and recent medical history. Rosacea is most common in middle life, chiefly affects the "flush area" of the face, and is markedly congestive in character, dilatation of superficial vessels being a conspicuous feature. Pustular syphilides may attack any part of the cutaneous surface, and are generally grouped, which is never the case with acne pustules, and there is other evidence of the disease. Acne is sometimes mistaken for smallpox, in spite of 
its chronic afebrile character and the absence of subjective symptoms. If the rash is limited to the upper part of the body and a few comedones are found, smallpox may be confidently excluded. For the diagnosis of acne vulgaris from staphylococcic sycosis, see p. 559.

Prognosis.-Acne vulgaris, even if left untreated, tends in the course of years to disappear. The duration of the affection can, however, generally be considerably shortened by treatment.

Treatment. Preventive.-Patients the texture of whose skin predisposes to retention of the sebaceous secretion should wash thoroughly several times a day, with the object of clearing away the coarse epidermis, keeping the mouths of the ducts open, and stimulating the circulation. The face and other parts liable to attack should be vigorously scrubbed with soap and flannel. As a further measure of prevention, some stimulant and parasiticidal ointment should be rubbed in ; for this purpose sulphur ointment (gr. $\mathrm{x}$ to $ろ \mathrm{i}$ ) is very useful. The general health must at the same time be attended to. Alcohol, tea, coffee, and all stimulating food that causes reflex flushing of the skin should be avoided. Smoking and sexual excitement are likely to be injurious for the same reason.

Curative treatment includes local and general measures. If suppuration has not yet occurred, the comedones should be squeezed out by means of an instrument suitable for the purpose; the part should then be washed frequently and energetically with soft soap and coarse flannel. A mixture of spirit and soap, such as the spiritus saponis alkalinus of Hebra, is useful in dissolving and softening the sebaceous matter. The skin should be disinfected by applying sulphur nintment (gr. $\mathrm{xx}$ to $\mathrm{z}_{\mathrm{i}}$ ), resorcin (gr. $\mathrm{xv}$ to $\mathrm{i}$ of $u n g$. paraffini), ichthyol, or carbolic acid in the form of ointment. When suppuration has occurred, the pustules 
should be punctured or incised, and afterwards bathed with hot water so as to encourage bleeding, and then dressed antiseptically. The cavity may with advantage be touched with strong carbolic acid solution. Each pustule must be treated individually; the method requires perseverance, but is effectual. When the inflamed papules are of considerable size, each one should be isolated by covering it with Unna's mercuric-carbolic plaster mull. This should be left on for about twelve hours or more; after removal the part should be cleaned with cotton-wool, soaked in spirit, then washed with corrosive sublimate solution ( 1 in 2,000), and covered with a fresh piece of plaster. In all cases of acne of the body, reinfection from the clothing should be prevented by frequent changes of the garment worn next to the affected part, and washing the adjacent unaffected skin with an antiseptic wash or soap Many observers have testified to the efficacy of treatment by $X$-rays, and though in my experience relapses are not uncommon, even the most severe cases yield to the treatment if it is sufficiently prolonged. Radium also is of service, and Finsen and others have reported favourable results from actinotherapy.

If the skin is tender and sensitive, the local applications should be simple and non-irritating; in such cases weak lead preparations are useful.

Constitutional treatment must be directed to the rectification of any functional disorder that may be a possible source of reflex circulatory disturbance. Par. ticular attention must be paid to the diet on the lines already laid down in speaking of prevention. As chronic constipation is often associated with acne, the judicious combination of mild aperients with tonics is of service. The following mixture, two tablespoonfuls of which should be given after meals twice or three times a day, may be recommended :- 
Rx Mag. sulphatis

Ferri sulphatis

Quininæ sulphatis

Acidi sulphatis dil.

Sp. chloroformi

Infus, quassiæ

$$
\begin{array}{lllll}
\ldots & \ldots & \ldots & \ldots & \text { 5iv } \\
\ldots & \ldots & \ldots & \ldots & \text { gr. viii } \\
\ldots & \ldots & \ldots & \ldots & \text { gr. viii } \\
\ldots & \ldots & \ldots & \ldots & 3 \mathrm{i} \\
\ldots & \ldots & \ldots & \ldots & 3 \mathrm{i} \\
\ldots & \ldots & \ldots & \ldots \text { ad } & \text { 3rii }
\end{array}
$$

A pill of cascara, aloes, and nux vomica may be given at bedtime, instead of the mixture.

Careful regulation of the mode of living is of importance. The patient should be instructed to wear suitable clothing - that is to say, such as keeps the body comfortably warm without causing irritation - to take proper exercise, to bathe frequently (the Turkish bath being especially useful for those whose internal organs are sound), and to live a wholesome life in hygienic surroundings.

Many cases of acne show a marked improvement under vaccine treatment. If the comedo is the outstanding feature of the case, an acne-bacillus vaccine may be given. If the case is one of mixed infection, mixed vaccines of staphylococcus and acne bacillus are indicated. If the case is characterized by deep-seated pustules forming boil-like lesions over a large area with the staphylococcus strongly predominating, a staphylococcus vaccine may be used with advantage, and persisted in for some months. Vaccine treatment should not, however, be regarded as more than an auxiliary of the other measures, both local and general, which have been described.

\section{ACNe Varioliformis}

Acne varioliformis is a somewhat rare form of acne, characterized by red, flat papules.

Symptoms. - The papules become pustules and then dry up, forming scabs. The latter are at first limited to the centre of the lesion, which is depressed below the 
level of the periphery. Later the scab covers the whole surface of the papule, and on separating leaves a small depressed permanent scar resembling a smallpox " pit." This process is regarded by some as a local necrosis, and the affection is sometimes called acne necrotica. A distinctive feature of this affection, as compared with acne vulgaris, is that the lesions are grouped. The forehead is the part most commonly attacked-hence the name acne frontalis; but the scalp and the face may be the seat of the eruption, which has also been seen on the chest and back. The affection causes no inconvenience beyond a little itching and the unsightliness of the lesions when they are on the face.

Etiology and pathology.-Both sexes seem to be equally liable to this form of acne; it is rare under the age of 30 . Some authorities consider it to be connected with syphilis, but with this view I do not agree. According to Touton, the process is inflammatory, and leads to necrosis of the cutis and overlying epidermis. In a case in which he made careful observations he found four species of microörganisms, but he is inclined to look upon their presence as secondary, and probably determined by the antecedent changes in the integument. Sabouraud ascribes the disease to a secondary invasion by the staphylococcus. It has been mentioned among the tuberculides (p. 443), but there is no conclusive evidence of its tuberculous nature.

Diagnosis.-Acne varioliformis can be identified by the absence of comedones, by the grouping of its lesions, the pitting which it leaves, and its preference for the forehead.

Treatment.-This must be directed to the improvement of the general health. Iron and cod-liver oil are particularly useful. The local treatment is that recommended for acne vulgaris. 


\section{Acne Keratosa and Acne Excoriata}

Another rare form of acne was described by Crocker under the name of acne keratosa. It resembles an acne in which the place of the comedo is taken by a horny plug, the presence of which excites inflammation. This plug is apparently formed in the hair-follicle instead of in the sebaceous gland. The eruption is situated about the nose, cheeks, and forehead, on the neck, the extensor aspect of the upper limb, and on the thigh. The lesions, when fully formed, are inflamed, indurated nodules with a flat top, which soften in the centre almost like a carbuncle, the central mass, however, being slow in separating. In all of Crocker's five cases digestive disturbances were present, and in three very prominent, and treatment had no particular effect.

In young girls the persistent form of acne known as acne excoriata (Brocq) is occasionally met with. The "picking" of the spots which causes the excoriation is thought by some observers to be due to a morbid impulse, but Crocker suggested that it is prompted by irritation.

\section{AcNitis}

The condition described under this name by Barthélémy ${ }^{1}$ is probably identical with, or at least closely akin to, the acne agminata of Crocker, the acne telangiectodes of Kaposi, the hydradenitis destruens suppurativa of Pollitzer (p. 561), and the disseminated follicular lupus of Tilbury Fox. J. F. Schamberg ${ }^{2}$ has published an exhaustive study of all the reported cases, with a description of an extensive case of his own.

Symptoms.-The eruption, frequently confined to the face, and always predominant there, usually appears

1 Ann. de Derm. et de Syph., Jan., 1891.

2 Trans. Amer. Derm. Assoc., 1908, p. 97. 
suddenly. The lesions, varying in size from a pin's head to a pea, appear to have their origin in the epidermis, and can be felt like shot beneath the skin, which becomes tumified and red as they grow more prominent. When fully developed they are brownish-red in colour; later a yellowish tint is seen at the centre. If the nodule be incised, a drop of pus usually exudes. They appear in crops, and reach their numerical maximum in from two to three months. In many cases a tendency to grouping is exhibited, especially on the chin, the upper lip, the eyelids, malar regions and temples, linear or oblong patches being thus produced. The smaller lesions have a waxy, glistening appearance quite unlike that of ordinary acne lesions. Barthélémy gives a month as the average duration of each lesion, 10 to 12 days being occupied by the evolution of the nodule, 3 to 4 by the suppuration, and 12 by the drying and falling of the crust. They are, therefore, much more indolent than the lesions of acne. Some of them undergo involution, but most of them end in necrosis and suppuration, with scarring. The disease may persist for a period varying between about six months and four years.

Pathology.-The epidermis remains virtually normal. A large circumscribed cell mass extends from the subpapillary layer throughout the derma, composed chiefly of inflammatory round cells. The infiltration is diffuse, with no particular grouping about the bloodvessels. In the centre of the cell mass is a distinct area of coagulation necrosis. Many giant cells and epithelioid cells are scattered throughout the mass, the latter most abundant in the neighbourhood of the former, which are, for the most part, seen in groups. Typical Langerhans giant cells are also present. There is a noticeable increase in the number of the blood-vessels, with great dilatation of the pre-existing capillaries. 
The lymph spaces and vessels are enormously distended, and there is a general œdema of the tissues. In many of the areas the sweat-glands are extensively involved in the inflammatory process; hence the name hydradenitis destruens suppurativa.

Etiology.-Of the cause of acnitis, nothing is known. The resemblance presented by the disease to folliclis, the tuberculous aspect of the histological sections, and the occasional occurrence of tuberculosis in other members of the families to which the patients belong, have led some French dermatologists and others to regard the lesions as tuberculides. But the tubercle bacillus has never been found in them; the inoculation of guinea-pigs with pus and fragments of nodules has never yielded a positive result; and in Schamberg's case there was no definite reaction to the Calmette test, or to subcutaneous injections of tuberculin. Barthélémy held that intestinal auto-intoxication was one of the factors in the production of the disease ; other authorities have suggested that it is due to a toxic substance eliminated through the sweat-glands. From the character of the lesions, the course of the disease, and the microscopic picture, Schamberg suggests that acnitis may be a parasitic granuloma.

Diagnosis.-The features which distinguish acnitis from ordinary acne have been already mentioned. From folliclis it differs in being more sudden and violent in its onset, in the shorter and more definite course which it runs, in its preference for and its frequent limitation to the head and face, in the deeper origin of the lesions in the skin and their not presenting horny centres, and in the absence of recurrence after the entire disappearance of the eruption.

Treatment.--Cauterization with fuming acid nitrate of mercury or caustic potash solution, the use of the curette and of Berg's comedo extractor, the adminis- 
tration of mercurials and other drugs have been tried, but it is doubtful whether the final disappearance of the lesions is due to the action of any of these remedies. ${ }^{1}$

\section{Glanders}

This affection is due to inoculation with a specific bacillus, which gives rise to lesions of the skin, mucous membranes, and lymphatic glands, and to general constitutional infection, usually ending in death. Glanders may run an acute or subacute or a chronic course, the former as a rule terminating fatally within six weeks, the latter persisting for months or even years, and frequently ending in recovery.

Symptoms.-One of the earliest symptoms is a peculiar discharge from the nostrils, the mucous membrane of which is violently inflamed and ulcerated in the acuter cases; in the chronic form this discharge is often slight or altogether absent. It is not always easy to ascertain the site of inoculation. Generally, however, it is found that the poison has gained admission through a wound or abrasion on the face or the hands. The local inflammatory reaction around this point is usually severe, and ulceration results, the sore having a foul appearance, with irregular edges. The neighbouring lymphatic vessels and glands are generally enlarged and inflamed. The skin lesions appear within three weeks or a month of the date of inoculation as groups of red spots that soon develop into papules. These become vesicles or blebs, which run together and form pustules that give rise to widespread ulceration covered with foul crusts or with black gangrenous shreds. Subcutaneous infiltrations form and break down into large ulcers. The enlargement of the lymphatic glands gives rise to nodules, not

1 For further details of the various aspects of this interesting affection, see Sohamberg, loc. cit. 
only in the neighbourhood of the site of inoculation, but elsewhere ("farcy buds"). These often suppurate and break down into ragged, sloughing ulcers. Nearly the whole surface of the skin may be covered with lesions of these various types. The general symptoms of glanders vary according to the acuteness of the process. They begin in from three or four days to as many weeks of the date of inoculation, and in their general character resemble rheumatic fever. In the acute and subacute cases the symptoms rapidly increase in intensity, and the patient sinks into a typhoid condition, in which he speedily passes away. Death also occurs from pyæmia in a certain proportion of cases. In chronic glanders severe and extensive ulceration may take place.

Etiology.-The causation of glanders has been elucidated by Loeffler, Charrin, and others, who have proved that it is due to a bacillus (B. mallei) somewhat resembling that of tubercle. The affection is almost always conveyed to the human subject from the horse in the discharge from the nostrils or from the ulcers, and is therefore found almost exclusively in persons whose occupation brings them much in contact with that animal. It has, however, been known to be communicated from man to man, and the lamented death of the distinguished Russian scientist Helman not many years ago from glanders contracted in the course of certain laboratory experiments may be taken as an cxample of its direct transmission by inoculation.

Diagnosis.-In a well-marked case the diagnosis can be made from the clinical phenomena alone. In doubtful cases the nature of the disease can be established by the reaction which takes place after the subcutaneous injection of malleïn, a substance discovered by Helman, which it is sufficient to describe here as bearing the same relation to the virus of glanders that tuberculin does 
to that of tuberculosis. Or search may be made for the bacillus with the microscope.

Prognosis.-In the acuter forms death invariably occurs within a few weeks; in the chronic variety recovery takes place in about 50 per cent. of cases.

Treatment.-In acute glanders the injection of bovine or naturally immune serum, or the use of mallein, is indicated. Zieler ${ }^{1}$ has reported successful results from the administration of vaccine. Chronic cases must be treated both constitutionally and locally on general principles. Wright ${ }^{2}$ has in one instance successfully treated chronic glanders with vaccine.

\section{RHiNOSCLEROMA}

This is a chronic microbic affection, commencing in the nostrils and the skin around them.

Symptoms.-The initial lesions are nodules in the cutis and deeper layers of the mucous membrane; these coalesce to form a hard growth with smooth glistening surface, which spreads inwards from the lip and downwards to the pharynx from the posterior nares. According to Kaposi, the larynx may be the primary seat of the disease, and it may extend thence to the trachea. On the mucous membrane the appearance is as if the parts had been infiltrated with glue, which had set to the solidity of stone. When the growth is situated in the skin the epidermis is tense and often cracked, especially about the corners of the nostrils and mouth; from the cracks a glutinous discharge exudes, which dries into yellow scabs. In a singular case described by Schridde, ${ }^{3}$ the nasal lesions, instead of being hard, were soft and ulcerated. Similar cases have been reported by Paltauf and Juffinger. The

1 Mediz. Klin., 1909, No. 18.

2 "Studies on Immunization," p. 406. 1909.

${ }^{3}$ Arch. t. Derm, u. Syph., Jan., 1905, p. 107. 
growth is not painful, but aches on pressure. It causes great deformity, but no symptoms except those due to nasal obstruction; the danger to life is mechanical, from blockage of the larynx. The growth has also been known to perforate the skull and extend into the brain (Kaposi). It does not break down spontaneously, but is generally slowly but surely progressive. The disease may last fifteen or twenty years, or even longer. Spontaneous disappearance after acute fevers has been recorded.

Eticlogy.-Rhinoscleroma is caused by the bacillus of Frisch, which closely resembles Friedländer's pneumococcus. The disease appears to be chiefly prevalent in Austria. Keegan has reported four cases in Hindoos. The sexes are equally liable, and, as far as can be judged from the limited statistics at present available, the disease develops before the age of 40 .

Diagnosis.-Rhinoscleroma may possibly be confused with epithelioma, but that affection usually begins later in life, the upper lip is seldom attacked, the lesions usually have infiltrated edges, and there is a tendency to ulceration. In rhinophyma the growth is not hard, pustules are often present, and there is vascular dilatation.

Treatment.-The growth recurs almost immediately after removal or destruction. All that can be done is to keep the air-passages patent as far as possible, and maintain the patient's strength, if necessary. Salicylic acid injected into the growth and applied to its surface in various ways was found useful by Lang in diminishing the bulk of the tumour in one case.

\section{Anthrax (Malignant Pustule)}

This disease, caused by inoculation with the anthrax bacillus, and corresponding to the splenic fever of animals, gives rise to skin lesions followed by signs of constitutional infection. 
Symptoms. - The most common site of inoculation is an exposed part of the skin, such as the face, the neck, or the hands. The development of the initial lesion is preceded by local itching and burning, and at the spot to which these sensations are referred a livid red papule soon appears. On this a bulla or a pustule quickly forms and soon breaks, drying up into a black gangrenous eschar fringed with tiny vesicles or pustules and surrounded by a wide zone of solid œedematous infiltration, the skin over which is tense and violaceous in colour. The gangrenous process may spread rapidly, the process soon ending in death ; or it may be localized, in which case a slough is thrown off and the resulting sore heals by granulation. The constitutional symptoms are those of septic fever, to which the patient may succumb within a few days. In less severe cases recovery takes place slowly.

Pathology.-The process consists in local inflammatory reaction, followed by gangrene and general septic phenomena due to the introduction of the specific irritant, which grows in the blood and all the tissues. In a case described by Hugo Hermann ${ }^{1}$ the bacilli were lying free in the lymph spaces, and none of them were found in the leucocytes or in any other cells-a fact which the writer notes from its bearing on the theory of phagocytosis.

Etiology.-The exciting cause of the affection is a rod-shaped microörganism, Bacillus unthracis (Plate 33, Fig. 5). Inoculation takes place from handling the hides of diseased animals, and butchers, wool-sorters, etc., are therefore most liable to infection.

Diagnosis.-This rests chiefly on the presence of a gangrenous patch surrounded by infiltraticn in a fatient whose occupation exposes him to infection with the bacillus of anthrax. It is only at the onset that the

${ }^{1}$ Arch. f. Derm. u. Syph., Oct., 1902, p. 203. 
lesion could be mistaken for a carbuncle. The bacillus may be easily detected under the microscope. For the diagnosis from chancre, see p. 490.

Prognosis.-The outlook depends on whether the gangrenous process continues to spread or not. The severity of the constitutional symptoms must also be taken into account.

Treatment.-The initial lesion should at once be thoroughly excised, or freely scraped on the lines indicated for the treatment of carbuncle. In a considerable number of cases Sclavo's serum has been used with excellent results-in this country by Lockwood and Andrewes, Stretton and Mitchell. Sclavo has published statistics of 143 cases so treated, with a mortality of 6 per cent., against the average mortality of 24 per cent.

\section{Gonorrheal Keratodermia}

Under the name of kératodermie blennorragique, Vidal, Jacquet, Jeanselme, Chauffard, and other French writers have described a gonorrhœal affection of the skin, of which there was no recorded case in this country until 1910, when one was shown to the Dermatological Section of the Royal Society of Medicine by Sequeira. ${ }^{1}$ This form of keratosis only occurs in the course of gonorrhœa with swollen and painful joints and severe general manifestations. There is usually serious debility and cachexia, and occasionally visceral complications occur. In the first few weeks of the disease, on the plantar or palmar surfaces, or on both, and rarely on the trunk, coincidently with the affection of the joints there appear nodules of a dark-brown or purple-brown colour, which form irregular horny masses resembling in the aggregate "a mountain range on a relief map." The intervening areas are covered with a parchmentlike thickening of the epidermis, brownish-yellow in

${ }^{1}$ Brit. Journ. Derm., 1910, xxii. 139. 
colour. In the case described by Sequeira the lesions felt like horn; when they were punctured no fluid could be withdrawn. The affected area was surrounded by a narrow hyperæmic border. In this case the palms were unaffected. The upper part of the derma, as well as the epidermis, is affected; there is odema, much infiltration with mononuclear and binuclear plasma cells and a few neutrophile polymorphonuclear leucocytes with scarce eosinophiles; but the deeper strata show no cedema and only insignificant infiltration. As a rule the horny crusts fall off after two or three months, leaving reddish-brown macules.

\section{Ulcus Molle}

Etiology and symptoms.-The "soft sore" has been described in connection with true chancre (p. 475), of which it is frequently a complication. It is due to inoculation-almost always in coitus-with Ducrey's bacillus, which, since it frequently occurs in chains, is often called the streptobacillus. It is demonstrated with difficulty, and in doubtful cases the Spirochata pallida should be sought. It is not often, however, except in cases where soft sore appears in association with-true chancre, that there is much difficulty in distinguishing between the two conditions. The one is seldom single, the other rarely multiple. Soft sore usually appears within two or three days of infection ; and the glands are early involved, and are large and painful, but not shotty. Another distinguishing feature is auto-inoculation, which frequently occurs in ulcus molle.

Treatment.-The most effectual treatment consists in the application of iodoform powder, supplemented by scrupulous cleanliness and hot baths. Instead of iodoform, either iodol, europhen, xeroform, or aristol, as being less offensive, may be used. 


\section{Furunculus Orientalis}

The affection variously known as furunculus orientalis, Delhi boil, Aleppo boil, Biskra button, etc., is a tropical disease the lesion of which is a boil that breaks down, forming a foul ulcer.

Symptoms.-Beginning as a papule, the lesion develops into an ulcer that extends by the erosion of its sharp-cut edge. Around the ulcer other sores may arise, which merge into it. After a period varying from two to many months healing begins, and finally a white or pinkish cicatrix is formed, which by contraction may cause considerable deformity. Occasionally the papule does not proceed to ulceration, but persists as a scaling or scabbing plaque. The process is often unattended with constitutional disturbance.

Etiology.-The disease is caused by the Leishmania tropica, named by $\mathrm{H}$. Wright, an American observer who, in 1903, found the organism in great profusion in the granulation-cells of one of the lesions. Cunningham had previously described these "Leishman bodies," and had been inclined to regard them as parasites, and the observations of Manson and others have established the fact that L. tropica, or an organism morphologically identical with it, is the cause of the affection. Manson's view is that there are two probable methods of infection : in one the organism is directly inoculated, without undergoing developmental changes; in the other it is first ingested by some insect, and, after undergoing evolutionary changes, is conveyed to the new host. The incubation period varies greatly in different cases; it may last only a few days, or may extend over several weeks.

Treatment.-This should proceed on the same lines as the treatment of ordinary boils. In some cases the $X$-rays have been tried with encouraging results. 


\section{Elephantiasis Arabum}

This disease of tropical and subtropical countries, only very rarely seen in Europe, is marked by chronic hypertrophy of the skin and subcutaneous tissue, giving rise to enormous enlargement of a particular part of the body-generally one (Plate 56, Fig. 1), and in rare cases both (Plate 56, Fig. 2), of the lower limbs; sometimes it is the scrotum (Plate 56, Fig. 2), one of the labia, or the mamma (Plate 57). The face is occasionally the seat of the disease.

Symptoms. - The disease is often ushered in by febrile disturbance ("elephantoid fever"). The part attacked becomes rapidly swollen, owing to inflammation of the lymphatics, the skin being tense and red as in erysipelas. There is great infiltration of the areolar tissue, and vesicles and bullæ often form and discharge a serous or chyle-like fluid. When fully developed the limb is often three or four times its natural size (Plate 56, Fig. 2), the swelling being hard and solid for the most part, though pitting moderately under strong pressure. The surface is often roughened by a network of dilated lymphatic vessels; varicose ulcers also frequently form. Exacerbations may take place at irregular intervals; their occurrence is always heralded by febrile disturbance. Except at these times there is generally little pain, but the patient is greatly inconvenienced by the bulk of the affected part. After some years the attacks of fever cease and the part remains permanently swollen. The scrotum sometimes forms a tumour reaching quite to the ground, and weighing over a hundred pounds. Cutaneous lesions of an eczematous type, which give rise to much itching, are frequent complications. The tension is often so great that the integument gives way and 


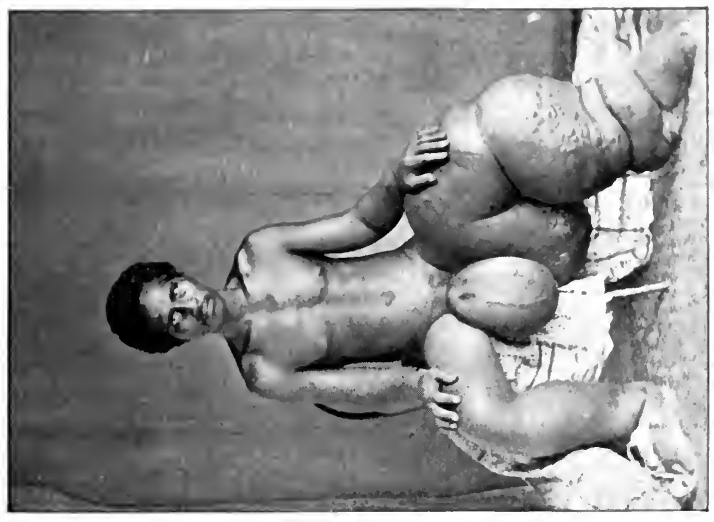

○

比

○岂

《虫 :

$\infty$

O

山う

แ

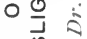

ब

$\leq \sum_{\infty}$

占这

I

U $\frac{I}{0}$

$\frac{1}{\omega} \approx$

ن $\ddot{\Sigma}$

N

ن

ㄴ.

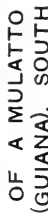

○

인

u.

○

$\frac{\omega}{\omega} \underline{3}$

$\leq$

云 z

I

믐

岁它

Ш

- $\sum_{0} \frac{\pi}{w}$

i $3<$ 는 

milky fluid escapes. The patient is much weakened by the loss of this fluid.

Pathology.-The principal change is in the subcutaneous tissue, which is greatly hypertrophied; the corium and epidermis are also considerably thickened, and papillary growths are not uncommon. Both bloodand lymph-vessels, muscles, fasciæ, nerves, and bones are also greatly enlarged.

Etiology.-The condition is the result of plugging of the lymph channels of the affected part. This has been shown by Manson and others to be due, in tropical countries, to the Filaria sanguinis hominis, now known also as the Filaria Bancrofti (Plate 24, H, and Plate 33, Fig. 10), which takes up its abode in the lymphatic trunks and discharges its ova into the lymph-stream; obstruction of the lymphatic circulation is brought about by the embryos, either mechanically or by setting up inflammation. Lymphatic obstruction may also be the result of violent or repeated inflammation, as in erysipelas, phlegmasia dolens, long-continued eczema, etc.; in fact, anything that interferes with the lymphatic circulation may cause elephantiasis. It is occasionally found in association with syphilis, as in a case shown by Adamson; ${ }^{1}$ the relation between the two diseases is not understood. Elephantiasis spares neither age nor sex, but is more common in men; it is sometimes congenital. A malarial clima'e and poor living are predisposing factors. Where it is endemic, its geographical distribution appears to coincide with that of certain species of mosquito, which serve as the intermediate host of the filaria (Manson).

Treatment. - The disease can sometimes be checked by removal from a district where it is endemic. The symptoms can generally be mitigated by improvement of the health, and by soothing applications to the ${ }^{1}$ Brit. Journ. Derm., 1910, xxii. 161. 
affected part. In confirmed cases of elephantiasis of the leg or scrotum there is no cure but amputation, though sometimes in the former case benefit is derived from excision of redundant masses of skin. The $X$-rays have often given good results, and the same may be said of electricity. A galvanic current should be applied from five to ten minutes, with the positive pole on or near the sound part, and the negative at different spots in the affected region.

\section{Granuloma Pyogenicum}

In this condition, styled also botryomycosis hominis, ${ }^{1}$ fungating granulomas occur as a sequel to wounds, the result of infection with cocci, probably the Staphylococcus pyogenes aureus. They vary in size from a pea to a small cherry, are of slow growth, of florid colour, and usually pedunculated. Care must be exercised not to mistake them for malignant tumours. Treatment consists in removing them.

\section{Ulcerating Granuloma of the Pudenda}

This affection, known also as sclerosing granuloma of the pudenda and as serpiginous ulceration of the genitalia, is met with in India, West Africa, British Guiana, and the West Indies. A similar, not improbably the same, disease is reported from Northern Australia and Polynesia.

Symptoms and pathology.-The growth is a chronic vascular granuloma, which is specially liable to cause a deep formation of dense, fibrous, very contractile tissue, but betrays no tendency to caseation or suppuration. The disease most frequently begins on the penis or the labia minora, or in the groin, as a nodular thickening or elevation of the skin. Excoriation, which readily occurs, exposes a surface prone to bleed and

1 Bodin, Ann. de Dermatol., 1902, iii. 298. 


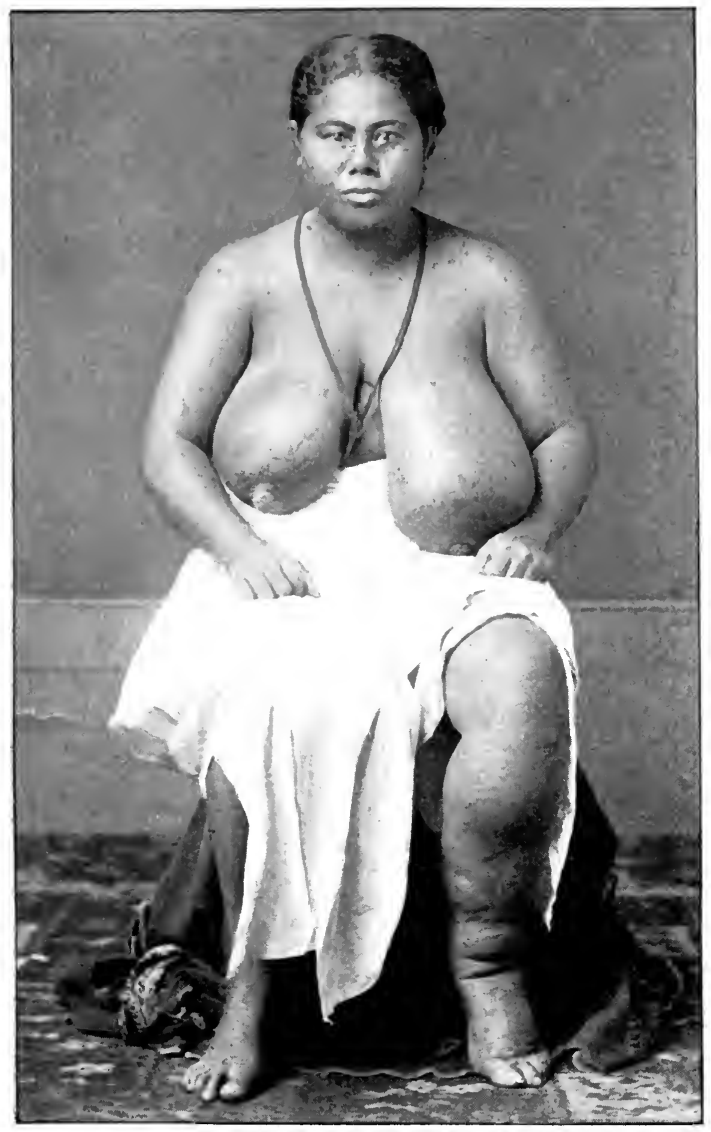

Plate 57.- elephantiasis of mamme; left leg and FOOT ALSO AFFECTED.

(From flotograph by D). Daziles, Samoa.) 

break down. On the glans, or the mucous surface of the labia, the growth extends rapidly; on the skin, much more slowly. It may remain limited to the glans or the labia for years. It may spread by autoinfection of an opposing surface, or by continuous excentric peripheral extension; it runs a chronic course, and the general health is only affected indirectly. In rare instances the granuloma is completely converted into fibrous tissue, and so spontaneous cure occurs, but usually there is cicatrization in parts and slow extension elsewhere. In the male the growth tends to spread over the penis, scrotum, and upper part of the thighs; in the female, over the labia, into the ragina, and along the flexures of the thighs. In both sexes it may ultimately involve the urethra, the pubes, the perineum, and the rectum.

Etiology.-The affection is generally, though not invariably, a venereal disease. Wise has found in the ulcerations spirochætes closely resembling S. pallida and $S$. refringens, but their precise relation to the malady has yet to be determined.

Diagnosis.-From malignant disease and from tertiary syphilides this condition can be distinguished by microscopical examination, and from the latter also by its irresponsiveness to specific remedies. From lupus vulgaris, which superficially it most resembles, especially when it occurs in the groin or on the perineum, it differs histologically as well as in being virtually limited to the pudendal region, in affecting mucous as well as cutaneous surfaces, and in tending to spread by way of the folds of the skin.

Treatment.-Radiotherapy is the only effective treatment, or, in the early stage, before large areas or important passages are affected, excision. Scraping is temporarily beneficial, but as a rule there is recurrence from portions of growth remaining in the fibrous tissue. 


\section{CHAPTER XXIV}

\section{AFFECTIONS OF THE SEBACEOUS AND SWEAT- GLANDS}

The sebaceous and sudoriparous glands may be disordered in their functions by excess, diminution, or alteration in the character of their natural secretion; and they may become inflamed, either primarily, or secondarily to the functional disorder, or as the result of invasion by micro organisms, with the result that structural changes are often brought about in the integument and its appendages.

\section{AFFECTIONS OF THE SEBACEOUS GLANDS}

\section{SEBorRhGa}

Seborrhœea is a condition of over-activity of the sebaceous glands, leading to increase and alteration of their secretion. It occurs in two principal forms: (1) a dry form, in which the solid fatty constituents of the sebum are in excess, and the excreted material appears in the shape of dense, scaly masses, generally more or less greasy both to sight and touch (seborrhoea sicca, otherwise pityriasis capitis); (2) an oily form; in which the fluid part of the secretion predominates, the discharge often being visible as oily drops at the mouth of the ducts, and making the skin look and feel as if it had been anointed with oil (seborrhoo oleosa).

Symptoms.-Both forms of seborrhœa are most common on the scalp. The condition most frequently observed is a slight greasy scurfiness, the thickness and 
colour of which vary greatly, according to the trouble that is taken to keep the parts clean. A peculiar salmon tint which is often seen in seborrhœic lesions is probably, as suggested by Brooke, due to the superposition of the yellow colour of the greasy secretion and masses of cornified epithelium upon the red of the subjacent hyperæmia. On the heads of infants seborrhœa may give rise to large dirty-yellowish greasy masses, generally thickest about the anterior fontanelle; the surface underneath these accumulations is usually pale, but sometimes it is inflamed. In adults such concretions are rare, but desquamation may be very abundant, the scales being sometimes distinctly greasy, sometimes dry and powdery. The condition is often accompanied by more or less itching, but not infrequently the only sign that reveals its presence to the patient is the shower of scales (dandruff) which falls from his head when he brushes his hair or which accumulates on the collar of his coat during the day. Dry seborrhœa is generally accompanied by loss of hair, which loses its gloss from the want of its natural lubricant, and withers from lack of nourishment. The eyebrows, moustache, and beard are sometimes, though much less frequently than the scalp, the seat of dry seborrhœa. According to Sabouraud, pityriasis, seborrhœa, and so-called seborrhœic eczema constitute a group of disorders which includes half the dermatoses of the scalp occurring between the ages of 15 and 20 in both sexes. Before puberty the heads of certain children are covered with dry, white scurf, which falls easily. This "pityriasis alba" (seborrhœa sicca), the first stage of seborrhœa capitis, is caused by a cryptogamic parasite-the spore of Malassez or bottle-bacillus of Unna, which lives in the superficial layers of the epidermis and causes it to desquamate. The scurf is, therefore, not dry seborrhøea, but epidermal scales. The second stage occurs about 
puberty. The scurf becomes more greasy, adherent, and yellowish-brown in colour (pityriasis steatoides). The hair now shows a tendency to fall out. This change is due to a secondary infection by the Staphylococcus epidermidis albus, which yields a greyish-white culture. It is therefore styled the coccus grise, whilst Unna, from the mulberry-like masses which it forms as it grows, calls it the "morococcus." The third stage, or that of oily seborrhœea, is due to an infection of the fatty cylinder contained in the sebaceous duct by the microbacillus of acne, Bacillus acnes. The bacillus leads to increased secretion of the gland, and excretes a toxin which causes loss of hair. The above course of events holds good for men only; in women, save in exceptional cases of a masculine type, the process usually remains at the second stage.

On the face the oily form is the more common. The discharge dries and gives rise to yellowish or reddishbrown cakes of greasy scales that often have a hyperæmic base and a fringe of papules about the edge. Reddish blotches frequently remain for a considerable time after the seborrhœic process has come to an end. The affection shows a marked preference for the middle third of the face, especially the alæ of the nose and the naso-labial furrow, and it is often limited to that region. In elderly persons the condition in this situation sometimes appears to be connected with the development of epithelioma (Jamieson). The corners of the mouth and the ears are also frequent seats of oily seborrhœa. The dry form is chiefly seen in parts away from the middle line. It is met with in the form of small scaly patches that are sometimes slightly hyperæmic.

Both forms of seborrhœa occur on the trunk and limbs. The lesions have the same general characters as those on the face. On the genitals and perineum, 
and in the genito-crural fold, seborrhøa of the oily variety is common, but the distinctive characters of the lesions are in these regions often lost in the secondary erythematous and eczematoid conditions that are apt to develop there. On the labia the irritation of the rancid, greasy masses not infrequently gives rise to excoriation, which might possibly be mistaken for soft chancre.

Seborrhoor almost invariably begins on the scalp, and in the large majority of cases it is confined to that part. From the scalp it spreads downwards to the face, the body, and the limbs ; and it may be taken as a rule, to which the exceptions are fewer in proportion to the care used in investigation, that when seborrhœic lesions are found on any part of the body, clear evidence of seborrhœa, present or past, will be found on the scalp (Unna).

Pathology.-The process is "apparently a dermatitis caused by the presence of one or possibly several microörganisms, and leading to a specific irritation of the fat-forming functions of the skin" (Brooke). Unna's view, that the seat of the process is the sudoriparous and not the sebaceous apparatus, has not found general acceptance among dermatologists, but it is not improbable that among the processes comprised under the name of seborrhœa there may be some in which the sweat-glands are concerned as well as the sebaceous glands. Consistently with the opinion just referred to, Unna regards all the conditions that have been described in the present section not as seborrhœa proper, but as seborrhœic eczema. That seborrhœa prepares the soil for other diseases, and notably for eczema, has already been stated, and it may be admitted that it is often difficult to draw the line accurately between the two conditions, so as to be able to say just where seborrhœa ends and seborrhœic eczema begins. 
But to call every case of scurfiness of the scalp eczema (which is essentially a catarrhal process) seems to me either a pathological misconception or an abuse of terms.

Etiology.-Reference has already been made to the exciting causes of seborrhœea. Among predisposing causes are all conditions that give rise to constitutional weakness, notably syphilis and acute fevers. Jacquet ${ }^{1}$ maintains that the starting-point of the affection is always some form of gastro-intestinal disturbance, constipation being that most frequently met with. His theory is that, the chemical processes of digestion being disordered, toxins are produced which affect the sebaceous glands, either through the medium of the sympathetic or during their elimination through the skin. I agree with Brooke, ${ }^{2}$ however, that the majority of persons who are the subjects of seborrhœa are in robust health. Indeed, the affection is so common that if constitutional weakness or derangement were a necessary condition of its production, the general standard of health in civilized countries must be assumed to be much lower than medical experience shows it to be. Sabouraud's theory is that the hypertrophy of the sebaceous in common with other glands which takes place in some subjects at puberty offers a suitable soil for the growth of the microbacillus of seborrhœa, and that its toxin, irritating the glands to further secretion, is thus carried out into the hair-follicles. He suggests that there is some connection between large and active sebaceous glands and over-activity of the testicle, and that this explains the frequent occurrence of the affection in virile and hairy men and the comparative immunity

1 “ De l'Etat séborréique de la Peau et de ses Rapports avec les Dermatoses." Paris, 1892.

${ }^{2}$ See his careful and suggestive paper, "The Relation of the Seborrhœic Processes to some other Affections of the Skin," Brit. Journ. Derm., vol. i., 1888-89, p. 253. 
enjoyed by women, who, when they are attacked by it, are of the masculine type.

That the severer forms of the disease are often associated with some disorder of the health proves nothing more than that, like other pathological processes, it flourishes best in a congenial soil. The determining factor in the production of seborrhoea is no doubt the irritation set up by a microbic agent. It must be admitted that the reasons that can be given for this belief are at present mostly of an a priori character, but they are nevertheless of considerable weight. It is difficult to explain the occurrence of the affection in persons of all ages, classes, and modes of life, and in the most diverse circumstances of health and skin texture, without postulating an external cause working independently of such conditions. That the amplest opportunities for invasion by microörganisms exist has been shown by Taenzer, who isolated about eighty varieties of bacteria and fungi from the scales and secretion of eczema seborrhoicum. The fact, established by clinical observation, that seborrhœa almost always spreads downwards from the head is probably to be accounted for by direct infection by the patient's fingers, and possibly also by falling scales.

Diagnosis.-Of typical seborrhœa of the oily variety the diagnosis can hardly ever present any difficulty. The characteristic greasiness of the lesions, the marked preference of the eruption for and its frequent limitation to the scalp, and its downward spread, make up a clinical picture that in most cases is readily recognized. The dry form is often by no means easy to distinguish from psoriasis. The character of the scales differs considerably in well-marked cases, those of psoriasis being bright and silvery and heaped up, while those of seborrhœa are less glistening, softer and greasier, and more diffuse. This alone, however, is not a safe guide. 
In cases otherwise doubtful the starting-point is the distinctive feature-seborrhœa beginning, as already said, on the scalp and tending to spread downwards; while psoriasis almost invariably commences on the elbows and knees and spreads upwards. When this mark fails us a diagnosis may be almost impossible. It is important to bear in mind that the two affections may co-exist. The presence of the bottle bacillus in the scales serves in doubtful cases to rule out pityriasis rosea.

Treatment.-As seborrhœa is a local disease, it can be cured by local measures, internal medication being necessary only when the general health is not satisfactory. The scaly masses must be removed by washing with soap and water, and the surface underneath soothed with emollient applications if inflamed. Parasiticides should next be applied, the strength being carefully adapted to the tolerance of the skin. Of these I trust most to sulphur in the ordinary run of cases. It may be applied as a lotion composed of $3 \mathrm{ss}$ to $3 \mathrm{i}$ of precipitated sulphur in ऊviii of distilled water. It should be rubbed gently in (after being thoroughly shaken) with a small brush, care being taken to touch the hair as little as possible. The best time for the application is at bedtime, on account of the smell of the sulphur; in severe cases it should be made twice a day. When the mixture of the lotion and the products of secretion have formed a crust, this should be removed and the sulphur reapplied. The sulphur may also be applied in the form of a powder mixed with oxide of zinc, powdered talc, etc. Brocq speaks well of the following combination :-

$\begin{array}{lllllll}\text { B Salicylic acid } & \ldots & \ldots & \ldots & \ldots & \ldots & \text { gr. } \mathbf{x x x}\end{array}$

Powdered hydrochlorate of pilocarpin $\quad . \quad$ gr. $x \mathbf{x}$

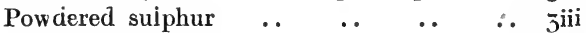

$\begin{array}{llllll}\text { Borate of soda.. } \quad . . & \ldots & \ldots & \text {. } & \text { gr. } \operatorname{lx} \times \mathrm{v}\end{array}$

Starch powder.. $\quad \ldots \quad \ldots \quad \ldots \quad \ldots \quad \ldots$ Jiiss

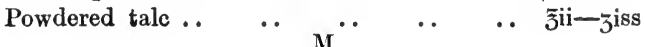


The amount of sulphur may be inereased to $3 \mathrm{v}$, that of borate of soda to 3iiss, the powder forming the vehicle being proportionately inereased; or the latter may be replaced by finely powdered calcined magnesia, oxide of zinc, subnitrate of bismuth, and talc. After cleansing the head, if necessary, a layer of this powder is carefully applied to the scalp (not to the hair) every night. When the scalp is dry, sulphur is best applied in the form of an ointment consisting of gr. $\mathrm{x}-\mathrm{lx}$ of precipitated sulphur to adeps lance $3 \mathrm{i}$, or $3 \mathrm{i}$ of the sulphur to $\bar{\jmath} \mathrm{i}$ of pure ung. paraffini with the addition of a little salicylic acid. Precipitated sulphur in eold eream in the proportion of 1 in 10 makes a good application. The following formula, proposed by Vidal, is useful :-

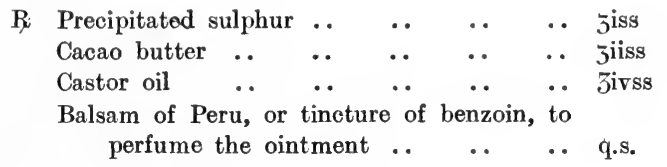

If sulphur irritates the skin, resorcin is an efficient substitute. It ean be used in the form of an ointment: Resorcin gr. x, ung. paraffini zi. Euresol (resorcin monoacetate), while not less effectual than resorcin, is much less irritating, and has the advantage of but slightly discolouring grey hair. It may be combined with corrosive sublimate, formic acid and alcohol as a head wash for the cure of dandruff. Another recent remedy for seborrhœa which has yielded good results is captol, an impure mixture of chloral hydrate and tannic acid; it may be combined with chloral hydrate. tartaric acid, and alcohol. Yet another remedy of recent introduction is anthrasol, a colourless tar. Mercurial applications may be used, either in the form of a lotion containing corrosive sublimate gr. $\mathrm{i}-\mathrm{v}$, in alcohol $\left(90^{\circ}\right.$ C.) 3 iii-vi, with distilled water or rose-water to 
make up to two pints; or of an ointment composed of yellow precipitate gr. viiss- $\mathrm{xv}$, pure vaseline or lanolin $\mathrm{z}^{\mathrm{i}}$; or calomel gr. $\mathrm{xv}$, tannin gr. $\mathrm{xxx}-\mathrm{xlv}$, vaseline or lanolin 3 i. $\beta$-Naphthol is often of great service. The scalp should first be thoroughly cleansed with naphtholated oil (1 per cent.) and naphthol soap, and afterwards washed for a week with an alcoholic solution of naphthol (1 to 2 per cent.). The same substance may be used in the form of an ointment in the strength of $\mathrm{gr}$. $\mathrm{xx}$ to $\xi \mathrm{i}$. Jamieson uses an ointment composed of tannic acid $3 \mathrm{i}$, pure glycerine q.s., vaseline $3 \mathrm{i}$, unguentum aq. rosa $\mathrm{z}_{\mathrm{i}}$. The use of this should be combined with daily washing with spiritus saponis alkalinus and warm water, the washing becoming less frequent as the seborrhoea improves. When only one washing a week is required, the spiritus saponis alkalinus may with advantage be replaced by infusion of quillaia bark applied hot. The restoration of the hair may be promoted by the methods for the treatment of baldness which are described at p. 635 .

\section{Seborrhoea Corporis (Pityriasis Circinata)}

Under the former of these names Duhring and others have described what they consider to be a special form of seborrhoea.

Symptoms.-The eruption, which is of microbic origin, is most frequently seen on the front of the chest over the sternum, and on the back between the shoulderblades. The lesions appear first in the form of small red papules (hence the affection is sometimes called seborrhoe papulosa), which speedily coalesce into patches. These clear up in the centre while continuing to spread at the edge; circinate lesions are thus formed, the ring, however, being seldom complete. The rings, by coalescence, form patches to which Unna has applied the term "petaloid." When one circle meets another, the 
parts touching each other, as usual, fade away, the remaining segments often forming wavy lines. The lesions are slightly raised, covered with greasy scales, and usually of a pinkish-salmon tint; if the scales are rubbed off an actively growing patch, the underlying surface is seen to be bright red. The affection sometimes spreads extensively over the trunk by rapid advance of the edge of already existing lesions, and by the development of new foci among them. The only symptom is slight itching. The disease is almost invariably associated with seborrhœe of the scalp, and the lesions are sometimes actually continuous with those on the head. There can be little doubt that it is identical with seborrhoea of the scalp, though possibly, as suggested by Brooke, the more highly developed type of lesions may be the result of the action of some more deeply penetrating and aggressive microorganism than is present in the ordinary forms of seborrhœea.

In the psoriasis type of seborrhœa the distribution is the same as that described above, but it consists of disks or of plaques of varying sizes, and covered with scales which, though usually greasy, sometimes resemble those of psoriasis.

Pathology.-There has been a great deal of discussion as to the true nature of the affection, which has been variously regarded as a form of lichen ( $L$. circumscriptus, annulatus, gyratus, etc.) and an eczema, as well as a seborrhœe. Sabouraud regards the condition as a superseborrhœic pityriasis, the pathology being the same as that of steatoid pityriasis of the scalp. Payne, while admitting that the starting-point of each so-called papule is a sebaceous gland, held that there is something more than over-secretion. In his opinion, the bright-red colour of the papules and margins of the patches indicates not only hyperæmia but dilatation 
and elongation of the capillary vessels. My own view is that the process is originally a seborrhøa, the hyperæmia being the response to irritation caused by the sweat and by the friction of the underclothing. As a matter of fact, the affection is chiefly seen in persons who perspire freely, and especially in those who wear thick, coarse underclothing; hence it has been termed "flannel rash." If neglected, it passes generally into eczema, and in many cases it is doubtless a seborrhœic eczema from the first.

Diagnosis.-The affection may sometimes be mistaken for tinea versicolor, but the absence of the fungus peculiar to the latter is decisive. For the diagnosis from pityriasis rosea, see p. 351. In the psoriasis type of seborrhœa the scales are less profuse than those of psoriasis, they are not silvery, nor will they be found on the extensor surfaces, especially of the knees and the elbows; the distribution, further, is less extensive than in psoriasis. When seated in the folds they may become eczematous, or they may be infected with staphylococci.

Treatment.-This should be on the same general lines as that of seborrhœic eczema. The underclothing must be of unirritating texture, and should be frequently changed. A simple parasiticidal application, after the parts have been thoroughly cleansed, may be relied upon to effect a cure.

J. F. Payne, though holding that no internal treatment is necessary, considered that two points had to be borne in mind: Gastric dyspepsia, though it cannot produce seborrhœea, may aggravate it when present, chiefly by causing cutaneous irritation and scratching; the same is true of constipation. Hence it is well to correct these conditions. Again, the general nutrition of the skin may be bad, and it may be advantageous to give a short course of arsenic. 


\section{MiLium}

A milium is a small, white, pearly mass, generally of the size of a millet-seed (hence the name), situated just under the epidermis, chiefly in situations where the skin is thin and there is little or no subcutaneous fat.

Symptoms.-Milia are seen most frequently on the face, especially on the cheeks, temples, eyelids, and forehead; sometimes on the penis and scrotum, and on the inner surface of the labia minora. They vary in number, and are generally scattered about without any sign of grouping, except occasionally, when they may be seen massed about the inner canthus. In the early stage of their development they are sometimes translucent, and after growing to a certain size they may remain stationary. They are hard and freely movable in the skin. On the eyelids and scrotum, however, they usually run together, forming flat masses which are sometimes so hard as to deserve the name of "cutaneous calculi." Crocker has described a special congenital form in which finely granular patches occur on the head and face, of a pale reddish-yellow, and slightly raised upon the surface. He suggested that it may be due to adherence of the amnion at an early stage of fotal life, or to a deep-seated intra-uterine inflammation.

Milia are not infrequently seen in children at the breast, but most often in young adults. They sometimes follow acute forms of inflammation of the skin, as pemphigus and erysipelas; they occasionally form in the scars left by the lesions of syphilis and lupus.

Pathology.-Milia are often considered to be plugs of sebaceous material, differing from comedones in being deeply seated in the acini of the glands and in having no opening towards the exterior. Robinson, however, holds, with Unna, Balzer, and other authorities, 
that they have no connection with the secretory portion of the sebaceous gland, and that whenever sebaceous material is present the case is not one of milium. According to Unna, milium is due to a hyperkeratosis of the middle portion of the root-sheath of a short lanugo hair, a hyperkeratosis of the epithelium of the hair-follicles causing an outward expansion of the follicle sheath at one point; this outgrowth presently extends into the perifollicular tissue, and is ultimately separated from the follicle. Milia are often associated with acne.

Treatment.-The little tumours can be turned out through a small incision. A little iodine or strong carbolic may be applied to the sac to prevent recurrence.

\section{Comedones}

Comedones (Plate 58) are small bodies consisting of concentric layers of horny cells due to hyperkeratosis at the pilo-sebaceous orifices and containing fatty material and colonies of microbacilli plugging the ducts of sebaceous glands.

Symptoms.-Comedones are most common in adolescents, but are sometimes seen in children. They show on the surface of the skin as pointed papules with a black top. The black colour is due to excessive cornification and pigmentation of the epidermic cells. They are most frequently seen on the face, especially about the nose, the cheeks, and the forehead, and on the back and chest. When numerous they produce an appearance like grains of gunpowder embedded in the skin, and when squeezed out they look not unlike small maggots. A parasite, the Demodex (or Acarus) folliculorum, can sometimes be found in comedones, but does not seem to have any causal relation therewith. By themselves comedones are harmless, except for the disfigurement which they cause; but persons in whom they are numerous 


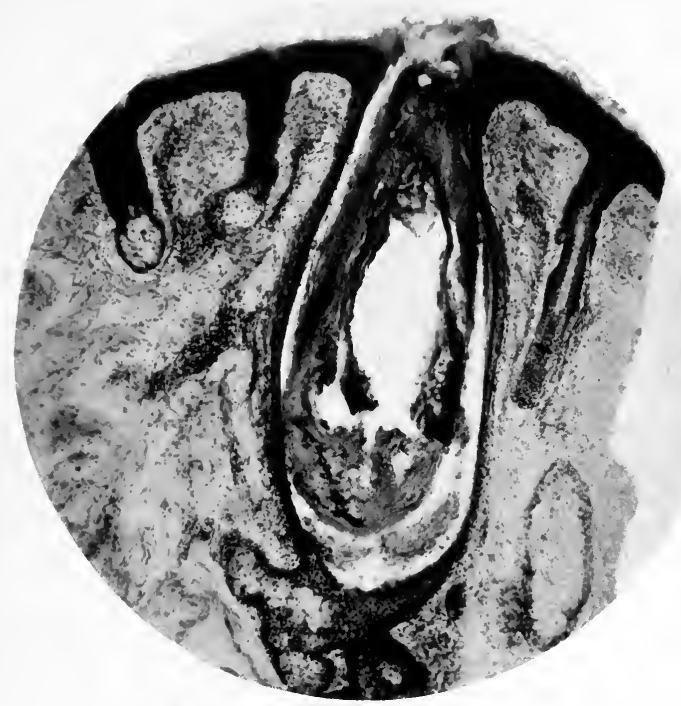

Plate 58.-microscopic section of a comedo. (Gilchrist.) 

are generally the subjects of oily seborrhœa, and the inflammation of the plugs very frequently gives rise to acne (p. 567).

Treatment.-The comedones should be squeezed out, either with the finger-nails or with a special instrument. This little operation should be done gently, as they are apt to become inflamed if roughly handled. Extrusion of the plugs should be followed by washing with soft soap and hot water and vigorous friction, and the application of a paste composed of kanlin $\zeta \mathrm{ss}$, glycerin 3iii, and vinegar 3ii, or a weak sulphur or resorcin ointment. Internal treatment directed to the regulation of the hepatic, digestive, and menstrual functions, according to indication, is often of service.

Grouped comedones have been described by Thin and others which appear to be etiologically connected with dyspepsia and to have no relation to acne. Their favourite situation is the "flush area" of the face, and they form symmetrical groups of black points smaller than ordinary comedones. Similar lesions have been seen on the trunk, but without grouping, on the forehead and cheeks of young children, and also in the aged.

\section{DISEASES OF THE SWEAT-GLANDS}

The sweat-glands may be the seat of functional disorder, the secretion being increased in amount, suppressed, or altered in character; or they may be obstructed, with or without inflammation.

\section{HYPERIDRosis}

In this condition the secretion of sweat is excessive either over the whole skin or in some particular region. With the so-called "critical sweating" of certain febrile conditions we have nothing to do here. Universal hyperidrosis may occur as a result of excessive heat, as in the sudarium of a Turkish bath; or it may be 
due to unwonted muscular exertion in a person "out of training"; or to violent mental emotion of a depressing kind ("cold sweat") ; or it may be a form of rapid tissue-waste in phthisis, leprosy, or other wasting diseases, or a symptom associated with general paralysis or Graves's disease. In a case described by Amenita $^{1}$ it appeared to be due to excitation of the sweat-centre in the cord by the toxins of syphilis or by a syphilitic inflammatory lesion. When localized, hyperidrosis may be unilateral, or may be confined to particular regions, such as the palms and soles, and especially hot covered parts, such as the axillæ and genital regions, where the glands are larger. In the latter situations the secretion may not only be excessive, but it may have an offensive smell (bromidrosis). In such situations intertrigo and eczematoid eruptions are often induced by the irritation caused by the decomposed secretion. Occasionally hyperidrosis may be limited to the area of distribution of a particular nerve-e.g. the fifth. The symptom may be continuous, or it may be excited by mental emotion or by movement, as in mastication, etc. When the palms and soles are the seat of the affection it is often symmetrical, and may be so severe as to lead to a thickened, sodden, macerated condition of the skin, making the use of the hand or foot painful and difficult. In some cases Jamieson ${ }^{2}$ has noticed a peculiar delicate pink tint of the inner side of the palm and the ball of the little finger and thumb. Hyperidrosis may be persistent, or it may disappear with the temporary disorder of health on which it is dependent.

Etiology,-The affection is probably due to disordered innervation; it is sometimes congenital, and it may be hereditary. Physiological experiments have

1 Gaz. d. Ospedali e d. Cliniche, June 29, 1903.

8 "Diseases of the Skin," p. 75. 
shown that sweating may follow paralysis of the sympathetic and stimulation of sensory nerves. Localized sweating is also sometimes associated with central nervous disease, or with injury to nerve cords. It is occasionally of hysterical origin. The fluid itself presents no abnormality.

Prognosis.-This depends on the nature of the cause producing the condition, and often it cannot be recognized

Treatment.-The indication is the improvement of the general health by tonics, etc. Belladonna is sometimes useful; it may be given in the form of full doses of the tincture, or of hypodermic injections of atropia, gr. $\frac{1}{150}$, increased up to gr. $\frac{1}{80}$. Ergot may also be of service. Crocker speaks well of sulphur, a level teaspoonful of the precipitated sulphur being given in milk twice a day. Diuretics, as suggested by Besnier, may be useful by diverting the excess of fluid into another channel. Locally the inunction of belladonna ointment or liniment is often bencficial, and faradization may do good. When the soles of the feet are thickened and tender a useful plan is to powder the stockings and boots with very fine boric acid every day, the boots being fitted with cork socks, which should be washed in boric acid lotion daily (Thin). Excessive sweating in the axilla or elsewhere may be checked for a time by pressing a very hot sponge to the part for a few minutes ; boric-acid powder or salicylicacid powder (3 per cent.) may then be dusted on. In a severe case treated by the author and S. E. Dore the condition yielded to the $X$-rays given in pastille dos $\mathrm{s}$, and the patient has remained well for four years.

\section{Granulosis Rubra Nasi}

Associated with hyperidrosis is a rare affection of the skin of the nose in delicate children, for which 
Jadassohn, ${ }^{1}$ who observed seven cases, has proposed the name granulosis rubra nasi.

Symptoms and pathology.-The skin of the front of the nose becomes intensely red. In the cases described the reddened area was dotted over with minute deep-red specks and papules, irregular in distribution, and showing no tendency to coalesce. The papules gradually developed into pustules and quickly dried up. There was infiltration of cells around the sweatducts, and foci of similar cells, consisting of mononuclear leucocytes and cells with vesicular nuclei, with a few plasma cells, were found in the neighbourhood of the hair-follicles and scattered throughout the corium. Both the ducts and the sweat-coils were dilated, as were also the blood-vessels and lymphatic spaces of the corium. The epidermis was normal, save for a slight parakeratosis around the sweat-pores.

Diagnosis.-Pick distinguishes the affection from lupus erythematosus by the absence of scales, from lupus vulgaris by the histology, and from rosacea by the age of the patients and the absence of telangiectases and of changes in the sebaceous glands. MacLeod ${ }^{2}$ describes a case, and further differentiates the affection from eczema by the local chronicity, the absence of vesiculation and weeping, and of infiltration, and by its irresponsiveness to local treatment. The disease, he points out, is not always limited to the nose, but may involve the upper lip, or the cheeks, or the eyebrows.

Treatment.-The cases described by the writers named, and by Hermann, were intractable, but two later cases of $\mathrm{H}$. Malherbe's ${ }^{3}$ yielded to quadrillated

${ }^{1}$ Arch. f. Derm. u. Syph., Oct.-Nov., 1901, p. 145 (abstr. by MacLeod in Brit. Journ. Derm.).

${ }^{2}$ Brit. Journ. Derm., June, 1903, p. 197.

${ }^{3}$ Journ. des Mal. Cut. et Syph., Feb., 1905, p. 97. 
linear scarification. In a case described by W. Pick, the use of ichthyol liniment and salicylic plaster was followed by improvement, but the hyperidrosis continued, and the affection of the nose returned. It disappears spontaneously at puberty.

\section{Bromidrosis}

Foul-smelling sweat sometimes occurs in general conditions, such as rheumatic fever, uræmia, and scurvy. It is only, however, as an idiopathic condition that it concerns us here.

Symptoms.-Bromidrosis may or may not be associated with excessive secretion. Though occasionally general, it is most commonly localized, the parts most frequently affected being the feet, where the decomposition of the sweat gives rise to a rank and sickening stench. The soles of the feet become sodden and macerated, and so tender that walking is sometimes impossible; in severe cases inflammation and exfoliation of the skin often occur. In other parts of the body, such as the axillæ and perineum, the smell is less rancid and more fusty in character. The sweat has no smell when first secreted, and the fetor is mainly due to the presence of a special microörganism-the Bacillus foetidus (Thin). Other bacteria of various kinds which are fornd in the decomposing sweat may assist in the process. ${ }^{1}$

Etiology.-Bromidrosis of the feet is usually observed in young persons whose occupation involves a great deal of standing, especially domestic servants and soldiers. It is often associated with flat-foot, and is not infrequent in those who wear waterproof coverings

1 See "Diseases of the Sweat Apparatus," by J. J. Pringle and H. G. Adamson, in Allbutt and Rolleston's "System of Medicine," ix. 641. 2nd edit., 1911.

$2 \mathrm{~N}$ 
for the feet, especially when this practice is combined with deficient cleanliness.

Treatment.-The most scrupulous cleanliness must be observed, the feet being frequently washed, and the stockings being changed before the sweat with which they are soaked has had time to decompose. The method of disinfection with boric acid, described on $\mathrm{p}$. 607, should also be employed. The plan adopted in the German army of rubbing the feet with mutton suet mixed with 2 per cent. of salicylic acid is very useful both in correcting fetor and in preventing tenderness. The occasional application of a 5 to 10 per cent. solution of chromic acid is also beneficial.

\section{Chromidrosis}

Under certain very rare conditions both the sweat and the sebaceous secretion may be coloured, the tint generally being some shade of blue; but red, green, yellow, violet, and even black sweating has been observed.

Symptoms.-The phenomenon is generally localized and occurs symmetrically. A favourite situation is the eyelids ; the cheeks, forehead, and side of the nose coming next in frequency. In rare cases the whole of the face, the chest, the abdomen, the backs of the hands and bends of the limbs, and especially the axillæ and groins, are the seat of the affection. The amount of pigmentation varies at different times in the same case, being generally worst in women just before a menstrual period. Constipation or some other disorder of the health is generally associated with the condition.

Etiology.--In the large majority of cases the patients are women-mostly young and unmarried. The neurotic temperament is a predisposing cause, the determining factor of the attack often seeming to be mental shock or emotion. The disease is in all 
probability primarily a neurosis. It has been suggested that the coloration is due to the presence of indican, which becomes oxidized by exposure to the air or by some ferment into indigo. Arthur Hall has shown that blue aniline dye, such as is used in cheap stockings, is dissolved by the acid sweat, and stains the epidermis to a considerable depth.

Diagnosis.-Our first care in such cases must be to exclude fraud. So suggestive of imposture, indeed, is the whole thing that some have expressed their disbelief in the genuineness of the phenomenon. Renewal of the pigmentation has, however, sometimes been observed in circumstances that appear to preclude the possibility of deception.

Prognosis and treatment.-Prognosis is always good as regards the ultimate disappearance of the coloration, but the condition may persist for years. Treatment must be directed to the improvement of the general health; local medication is useless.

Coloured sweating has also been observed as the result of the ingestion of copper (green sweat) or iron (blue sweat), or associated with the presence of eertain bacteria, as in the red sweat not infrequently seen in the axillæ and genital regions, and sometimes in yellow and blue sweat. In such cases of red sweat the bacteria attach themselves to the hair-shaft and worm themselves into its substance. The affection is not strictly a form of chromidrosis, the sweat itself not being coloured at the time of its excretion, but acting as a solvent for the colouring matter in the masses attached to the hair. The fungus has been cultivated by Kneas. Examination of pure cultures showed the cocci frequently arranged in pairs and tetrads, like the Micro. coccus tetragenus.

Bloody sweat may result from the extravasation 
of blood into the coils and ducts of the sweatglands. This condition may in very rare cases follow great mental emotion in persons of excitable temperament, or it may be a form of vicarious menstruation. It is sometimes also seen in new-born babes, and in such a case has been known to prove fatal. Treatment can only be directed to the removal of the cause, which in adults is almost invariably the hysterical temperament.

Phosphorescent sweating has been seen in certain rare cases after eating phosphorescent fish, or even as an idiopathic phenomenon. A case in which the body-linen became luminous after extraordinary exertion on record. The phosphorescence is believed to be due to bacilli.

\section{Uridrosis AND ANIDrosis}

Uridrosis is due to the presence of urea and other urinary constituents in the sweat. Urea is normally present in minute quantities in that secretion, but under certain conditions, as in cholera, uræmia, etc., the amount may be so much increased that the skin may be coated with white crystals, as if it were covered with hoar-frost. The sweat has a urinous smell.

Anidrosis, or diminution of the sweat secretion, may be associated with certain general conditions, such as diabetes and fever; or it may be due to a congemital anomaly in the structure of the skin, as in ichthyosis, or to a diseased condition of the skin, as in psoriasis, eczema, or sclerodermia; or it may be the result of disordered innervation, as in anæsthetic leprosy, or of malnutrition. It may also be dependent on a purely mechanical cause, such as obstruction of the sweat-ducts by epithelial débris, owing to imperfect washing. The secretion may be merely diminished or may be totally suppressed, and the whole skin or only 
some particular area may be affected. Anidrosis rarely occurs as an independent affection. In cases due to congenital anomaly, treatment is useless; in other cases, general invigorating treatment, stimulation of the skin by massage, hot baths, and the constant and faradic currents, and also the ingestion of hot drinks, may be useful. Jaborandi may be given by the mouth, or nitrate of pilocarpin may be injected hypodermically in doses of $\frac{1}{6} \mathrm{gr}$.

\section{SUDAMINA}

Sudamina, or miliaria, are small vesicles, looking like drops of dew on the skin (Jamieson). They are caused by obstruction of the sweat-ducts, with or without inflammation. The fluid contained in the vesicles is simply the imprisoned sweat, which, being prevented from issuing by way of the natural orifice, is effused under the horny layer. The obstruction is generally caused by an epithelial plug, formed while the functional activity of the sweat-gland is suspended, as in fevers. Sometimes sudamina occur on a dry and hot skin, where perspiration, so far from being excessive, has been deficient.

Symptoms.-The parts chiefly affected are the chest and abdomen, but the vesioles may form wherever there are sweat-ducts to be blocked up. They are, as a rule, set close together, but are not often confluent. They undergo no change, and disappear completely in a few days. Sometimes they come out in successive crops. In certain cases an inflammatory process, which may be primary or secondary, develops in and about the glands. The lesions in these cases are bright-red papules (miliaria rubra), the size of a pin's point, and sometimes vesicular or pustular (miliaria alba) on the top. The lesions are discrete, though thickly aggregated, and the fluid contained in the vesicles is serous, being the 
result of inflammatory exudation. The vesicles and pustules do not burst spontaneously, but dry up in a few days, forming small scales, which soon separate. The individual lesions are very short-lived, but the affection may be kept up for some time by successive crops of eruption. More or less itching is generally complained of.

Diagnosis.-The appearance of the lesions is so characteristic that there can hardly ever be any doubt as to the diagnosis. Miliaria rubra may sometimes resemble the vesicular stage of eczema, but there is no formation of patches and no "weeping"; the affection, moreover, is very transitory. A sweat rash in a child may suggest the exanthem of an acute specific fever, but the absence of constitutional disturbance will generally prevent such a mistake. It is important, however, to remember that sudamina may be associated with a scarlatinal or other febrile rash; they are especially common in typhoid.

Treatment.-Miliaria almost always yield readily to treatment, but relapse is common. It is only when the retention of the secretion is complicated by inflammation that treatment is required. Dusting with a little protective powder and the application of a cooling ointment are all that is necessary.

The so-called "red gum," or "lichen infantum," is a form of miliaria due to too warm clothing. The remedy is obvious.

\section{Miliaria Papulosa}

"Prickly heat" is a form of miliaria rubra, sometimes called "lichen tropicus" on account of the papular lesions by which it is characterized. The special pathological feature of prickly heat is that the inflammation in the sweat-gland is primary, and is the cause of the obstruction of the duct. 
Symptoms.-The lesions are tiny acuminate papules, bright red in colour and thickly clustered together, but not confluent, with a few vesicles and pustules scattered about between them. The eruption is preceded by profuse sweating. The lesions come out suddenly, and give rise to pricking and tingling of extreme intensity. The affection shows a preference for covered parts (trunk, limbs, upper part of forehead), and it usually extends over large areas. Prickly heat is most common in the tropics, but it is not unknown in England, especially in persons who have had it before. Fat people, and those who perspire freely, are most liable to it, and the irritation of clothing, especially flannel, sometimes appears to be a determining factor. One attack predisposes to another. Prickly heat in some degree resembles papular eczema, but the circumstances of its occurrence and its sudden disappearance will serve to distinguish it.

Treatment.-Saline diuretics, such as the acetate and nitrate of potash, are very useful. Locally a soothing or evaporating lotion or a cooling ointment will give relief. Alkaline or bran baths are also beneficial. The diet should be non-stimulating, and alcohol should be taken only in the greatest moderation. Any cause of irritation in the clothing should be removed. Care should be taken to prevent chill, and for this reason woollen underclothing should be worn.

A peculiar form of miliary eruption on the face has been described by G. T. Jackson and Rosenthal under the name of hidrocystoma, and by Crocker as dysidrosis of the face. The lesions consisted of small vesicles resembling sudamina, but grouped so as to form patches, which persisted without any apparent tendency to spontaneous recovery. The lesions gave rise to itching, but there was no sign of inflammation. The condition appeared in Crocker's case to be associated with dyspepsia, the lesions being more prominent after meals. 


\section{CHAPTER XXV}

\section{AFFECTIONS OF MUCOUS MEMBRANES OF THE LIPS, TONGUE, MOUTH, ETC.}

IN the chapter on the Principles of Diagnosis it has been pointed out that while the mucous membranes and epithelial surfaces adjoining the integument are not infrequently involved in cutaneous affections, they are in rare instances the seat of primary lesions, the skin being only affected, if at all, secondarily and subordinately. In this chapter such affections will be briefly considered.

\section{Perlitche}

Under this name Justin Lemaistre described for the first time, in 1885, a variety of chronic stomatitis affecting the lips, chiefly at the commissures. The disease is almost peculiar to childhood.

Symptoms.-The lesions, which are generally symmetrical, consist of an exuberance of the epithelium, whitish in hue, which looks as if it were macerated, and is easily detached. The lesion sometimes extends along the epithelium some way across the lip, involving the surrounding skin and the mucosa of the inside of the lips. The derma is not exposed, the affection being limited to the epithelium. The characteristic feature is a kind of whitish pellicle, projecting and wrinkled about the corners of the lips, which have thus a fissured appearance. There is often only a single patch, divided by the fissure into two equal parts so as to present the appearance of the two pages of an open book (P. Raymond). 
In other cases the patch is multiple, and may extend to the inside of the lip at the commissure. There is little or no pain, but a feeling of discomfort and heat, which makes the child constantly lick its lips (pour lécher; hence the name perlèche). When the fissures extend deeply, however, they sometimes reach the derma, and pain is felt on opening the mouth. Other lesions are often associated with perlèche-diphtheroid or impetiginous stomatitis, crusts, vesicular erythema, etc. It has been suggested that these are all manifesta. tions of one disease.

Etiology.-Perlèche is said by Lemaistre to be caused by the Streptococcus plicatilis, which he found in all the cases he examined, and which he successfully cultivated. According to Sabouraud, it may be due to a streptococcic salivary infection, or accompany streptococcic impetigo of the face. Others have attributed the affection to a variety of the staphylococcus albus, to the staphylococcus aureus, and to any of the microbes present in the mouth. It is admitted by all that the disease is contagious and may be epidemic. Hence care should be taken to prevent the common use of pencils, drinking-mugs, towels, etc., in schools.

Diagnosis and prognosis.-The diagnosis is easy, the only difficulty being to distinguish perlèche from syphilitic lesions. It is well to regard all children presenting the lesions described as affected with syphilis till it is clearly established that there is no syphilis. Perlèche is distinguishable from herpes by its bilaterality and its not beginning as a vesicular eruption. The duration of perleche is usually from a fortnight to a month; sometimes it lasts longer--possibly, as suggested by Lemaistre, as the result of successive inoculation. It tends to spontaneous recovery, but leaves behind a smooth, mother-of-pearl tinted surface, which may persist for months. Relapse is common. 
Treatment.-This consists in cauterization with sulphate of copper or nitrate of silver, followed by the application of a protective ointment of vaseline and oxide of zinc. ${ }^{1}$

\section{Cheilitis Exfoliativa}

This rare condition, a persistent and repeated exfoliation of the vermilion of the lips, is associated with a slight seborrhœea of the scalp. Stelwagon 2 has reported two cases. In a similar though not identical case of mine, that of a lady from the United States, the lips as a whole, and not merely the red part, were covered with a thick and disfiguring accumulation of scales. When the scales were removed the vermilion was seen to be blue. The patient complained of some burning pain, but chiefly of a sensation of "deadness" in the lips. The condition appeared to have been set up by a habit of gnawing the lips and "picking" the skin. The subjects of cheilitis exfoliativa are usually neurotic, ${ }^{3}$ and in this case there were some indications of that temperament, but there was no associated seborrhœa.

Treatment.-In the case just described there was marked improvement under the application of radium; in an earlier one, that of a man from Johannesburg, an apparent cure was effected by the same treatment.

\section{Cheilitis Glandularis}

Cheilitis glandularis is a chronic inflammation of the lower lip, with swelling of the mucous glands.

${ }^{1}$ For a fuller account of perlèche (by Jacquet) see Brocq's “Pratique Dermatologique," t. iii., p. 839 et seq. Paris, 1902.

2 "A Report of two Cases of Persistent Exfoliation of the Lips," Journ. Cut. Dis., June, 1900.

${ }^{3}$ In a case exhibited by Graham Little there was no neurotic histoiy or symptoms, and the patient denied licking the lips. Brit. Journ. Derm., 1909, xxi. 252. 
Symptoms,-It is chiefly the vermilion that is affected, but the inflammation spreads to the inside of the lip. When the skin is affected, it presents an erythematous aspect. The mucous glands swell to the size of a hemp-seed or larger, the follicular openings are greatly dilated, and fistulous tracks may be formed. A viscid secretion oozes out, and the lips may be found glued together in the morning.

Etiology. - Of the cause of this rare affection ${ }^{1}$ nothing is known, but it appears to originate in catarrh of the mouth and pharynx. In a case of Galloway's the cheilitis was associated with seborrhœea of the face and scalp, but this association is rare, nor are the patients neurotic, as is usually the case in cheilitis exfoliativa (p. 618).

Treatment.-The measures that appear to have been most successful are mild cauterization with silver nitrate, the use of a potassium chlorate gargle, and the internal administration of iodide of potassium.

\section{Fordyce's Disease}

This affection, first described by Fordyce in 1896, specially attacks the red of the lips and the oral mucous membrane.

Symptoms.-The lesions consist of small whitish or yellowish milium-like bodies, which may be scanty or profuse, discrete or agminated. Inside the mouth the milium-like bodies are whiter than those on the lip, and more projecting. As a rule there are no subjective symptoms, but if present they take the form of slight burning or itching in the affected parts, with a feeling of stiffness. The condition, according to Stelwagon, ${ }^{2}$ is not so rare as might be supposed from the infrequency

${ }^{1}$ The tenth case was reported by R. L. Sutton in 1909. Journ. Cut. Dis., April, 1909.

2 "Diseases of the Skin;" by H. W. Stelwagon. 
with which advice for it is sought. A patient of Fordyce's, who was a physician, detected it in all of the members of his family, as well as in one out of every two negroes whom he examined.

Pathology and etiology.-The nature and causes of the formations are not understood. Fordyce's mature view is that they are sebaceous glands, but C. J. White ${ }^{1}$ holds that, as Fordyce first thought, they are due to a granular change in the mucous membrane. Among those who accept the former theory there is difference of opinion as to whether the glands are a late development of embryonic elements or are of postnatal origin.

Diagnosis.-The signs are too distinctive to be confounded with those of any other affection. When the lesions are very profuse they may mimic a solid patch, but by stretching the tissues the milium-like bodies can be distinguished.

Treatment.-By scraping away the supericial epithelium Fordyce was able to squeeze out some of the bodies and so diminish the discoloration. But the results of treatment are not encouraging, and the condition tends to become permanent.

Ichthyosis Glossa (Schwimmer) or LeUcokeratosis (ButLIN)

This is a condition of the buccal mucous membranes which closely resembles tylosis (p. 726). Rosenheim ${ }^{2}$ describes two cases, in one of which a whitish patch on the mucous membrane of the cheek appeared to be consequent upon the habit of holding a quid of tobacco against the cheek when not chewing. In the other no determining cause was suggested. In neither case was there any admission of syphilis. Rosenheim maintains

1 Journ. Cut: Dis., March, 1905, p. 97.

${ }^{2}$ Bull. Johns Hopkins Hosp., Feb., 1904, p. 47. 
that the essential changes in leucokeratosis and in tylosis are much alike.

\section{LeUCOPLAKIA OF the ToNgue}

Leucoplakia of the tongue is most frequently a manifestation of syphilis, but it sometimes appears as an idiopathic affection. It is known variously as psoriasis of the tongue, ichthyosis linguce, tylosis lingua, leucoma, leucoplasia, and "smoker's patch."

Symptoms.-The white, pearly patches, consisting of thickened epidermis and hypertrophied papillæ, form slowly, are clearly circumscribed, and raised above the normal surface of the tongue. The warty cap of the growth may be shed, leaving a red, tender surface, which again becomes covered with cornified epithelium. The plaques may be furrowed, and in bad cases fissured, and sometimes the fissures ulcerate and become the seat of epithelioma. The mucosa of the cheeks and other parts of the oral cavity may be involved.

Etiology.-Leucoplakia of the tongue is no doubt due to the action of some such direct irritant as hot tobacco-smoke, badly fitting dentures, or ragged stumps of teeth. It is more frequent in men than in women. Gout or so-called rheumatism, or chronic dyspepsia, may be a predisposing factor.

Diagnosis.- The condition may be distinguished from lichen planus patches on the buccal mucous membrane by the irregular streaks and discrete papules characteristic of the latter condition. The mucous patch of early syphilis forms rapidly, and there is usually no hardening of the epithelium. As the condition is so frequently syphilitic, it is a safe rule to apply the Wassermann test in all cases.

Treatment.-Unless remedial measures are applied early, and the irritants to which the affection is due are avoided, leucoplakia tends to become chronic. Piquant 
flavours, hot food or liquids, etc., must be given up; mildly antiseptic mouth-washes are sometimes serviceable. Strong iodide of potassium solution (1 in 5) may be applied three times a day. If other remedies fail, as in many cases they will, the galvano-cautery should be used, or radium, or the patches may be excised with the knife.

\section{Leucoplakia of the Penis, Vulva, and Vagina}

In rare cases the penis, in the subjects of marked phimosis, is the seat of leucoplakia. Less infrequently the vulva or the vagina is affected by this condition. In the opinion of Pflanz ${ }^{1}$ the pathological process is identical, whether the condition appears in the penis or in the vagina or vulva. In the penis it is frequently due to balanitis. In the vulva it begins with redness and swelling of the parts; in the next stage the labia minora decrease in size, and the surface involved becomes of an opaque white; in the third stage, cracks and ulcers are found, sometimes with carcinomatous degeneration; finally, the whole vulval orifice becomes white and smooth, the labia minora and the clitoris contract and atrophy, and subjective symptoms-itching and soreness-disappear. The urinary orifice and the vestibule are obliterated. The thighs and the perineum may be involved. According to Berkeley and Bonney, ${ }^{2}$ the beginning of the pathological process is swelling of the epithelium and desquamation, with vascularity of the subepithelial tissue and lymphocytic infiltration. Subsequently plasma and connective-tissue cells accumulate and lymph-nodes form, and finally, with hypertrophy of the epithelium and disappearance of the elastic fibres

1 Derm. Zeitschr., Nov. 1909, xxi. 710 (abstr. Brï. Journ. Derm., 1910, xxii. 205).

2 Trans. Roy. Soc. of Med., Obstet. Section, 1909, p. 29; and "Gynæcological Surgery." 
of the connective tissue, there is complete sclerosis. These observers have shown that leucoplakic vulvitis is distinct from kraurosis vulvæ, of which it has usually been regarded as a less advanced stage.

Treatment.-This consists in application of the $X$-rays or of radium. Should such measures fail, a wide excision is recommended.

\section{Kraurosis Vulve}

Atrophy of the vulva with stenosis of the orifice is sometimes met with in sterile young women, or after the menopause, or as a sequela of oöphorectomy.

Symptoms and pathology.-The parts affected are the labia minora, the vestibule, the meatus urinarius, and the vagina, but there is no extension to the thighs and perineum as in leucoplakic vulvitis. The mucocutaneous surface becomes red and shiny, and dotted with bright-red spots; at the urinary orifice a caruncle will usually be found. Afterwards the surface becomes pale yellow and glistening, atrophy of the labia minora and clitoris supervenes, and the mons veneris wastes. The subjective symptoms are soreness, pain on micturition, and dyspareunia. The epithelium becomes thinned, and the papillæ atrophy. There is subepithelial infiltration of plasma cells, lymphocytes, and polymorphonuclear leucocytes.

Treatment.-Berkeley and Bonney advise removal of the affected areas and enlargement of the vaginal orifice.

\section{Grooved Tongue}

Grooved tongue, known also as furrowed, wrinkled, fluted, ribbed, scrotal, or sulcated tongue, is often congenital and familial. The dorsum of the tongue is lobulated and grooved; the furrows may be few in number, parallel with the exaggerated central depressions, or so numerous as to present the appearance of a network. 
No symptoms appear, except that in the deeper grooves tiny particles of food may accumulate, and the only treatment necessary is to keep the tongue clean, if necessary, with mild antiseptic solutions. The condition is a permanent one.

\section{Glossitis Areata Exfoliativa}

This affection, though rare, bears a number of synonyms, such as transitory benign plaques of the tongue and erythema migrans.

Symptoms.-On the dorsum of the tongue, usually near the tip, appear small greyish, well-defined, slightly elevated spots, which, as they spread peripherally, undergo slight exfoliation or desquamation in the centre. They also become red, the colour being brightest near the border, but the border itself is greyish, or yellowish, or bluish, or even quite white. The patches may form circles or festoons, and when they run into each other they may present an aspect which has suggested the expression langue géographique. Exceptionally the under-surface of the tongue may be affected. The patches are constantly undergoing modification, old spots fading and new ones appearing. The mucous membrane is not, as a rule, indurated, and there are seldom subjective symptoms, though itching is sometimes complained of. The condition is most frequent in young children, and is often a family peculiarity. A furrowed tongue is a predisposing factor. There is no evidence that the affection is syphilitic or fungous.

Prognosis and treatment.-The affection is indolent, and usually persists during some years, or indefinitely, in spite of treatment. Mildly astringent and antiseptic mouth-washes are to be recommended, with attention to the digestion and regular action of the bowels. 


\section{Black Tongue (Hairy Tongue)}

The affection thus designated is a nyperkeratosis, in which, usually in front of the circumvallate papillæ, dark-brown or black patches appear, studded with filiform projections which sometimes simulate true hairy growths. The lesions start on the middle line, and extend forwards and laterally. The filiform projections are most pronounced in male adults. When the patch is well developed the patient may complain of an unpleasant taste, and in rare cases slight pain is present, but as a rule there are no objective symptoms. Some observers have suspected the action of a fungus or a microörganism, but nothing is definitely known as to causation. The affection may persist for a few weeks, or for years, but sooner or later it disappears. It makes no response to treatment, and nothing but mildly antiseptic mouth-washes should be prescribed. 


\section{CHAPTER XXVI}

\section{AFFECTIONS OF THE HAIR AND OF THE NAILS}

\section{AFFECTIONS OF THE HAIR}

Diseases of the hair depend on pathological changes in the follicle. These consist of inflammation in and around the hair-sac, and trophic changes leading on the one hand to overgrowth and on the other to deficiency of pigment, atrophy, and total destruction of the hair. Concretions of various kinds may also form on the hairshaft. Besides these conditions there are the parasitic diseases, such as ringworm and favus; these have already been considered (Chapters xvi., xviI.).

\section{Folliculitis Decalvans}

This special form of chronic folliculitis of the scalp, which leads to cicatricial baldness, has been described by Quinquaud and others.

Symptoms.-The affection is at first sight somewhat like alopecia areata (p. 636), but at the edge of the bare patches a small red papule or patch of erythema can be seen surrounding each hair-follicle. I have had several well-marked cases under my care. The microscopic appearances are those of perifolliculitis. Quinquaud found microörganisms which he thinks peculiar to this condition.

Treatment.-In this extremely chronic process treatment has little effect. The indications are to check the spread of the disease and promote the growth of the hair. For this purpose parasiticides, followed by 
stimulating applications, should be tried. Pringle has found epilation successful.

\section{Pseudopelade}

This is a rare condition first described by Brocq in 1884, in which rounded or irregular-shaped islands of baldness occur on the scalp. The patches gradually spread and coalesce to form large, smooth, shiny areas. Unlike those in alopecia areata, they are cicatricial; the follicles are destroyed, so that the hair is never restored; the note-of-exclamation stumps, too (p. 637), are absent. The disease usually occurs in adults with dark, coarse, wiry hair. Nothing is known of the etiology, cultures from the hairs usually giving negative results, and treatment is of little or no avail.

\section{Dermatitis Papillaris Capilliti}

Another inflammatory process, affecting the scalp and ending in atrophy of the hair-follicles, is desoribed by Kaposi under this name. It commences at the edge of the scalp on the back of the neck, and spreads upwards towards the crown of the head. The initial lesions are small papules, which soon coalesce into large raspberrylike vegetations in the occipital region. They bleed easily, and an offensive discharge oozes out between the papillæ, while abscesses form beneath and undermine them. These masses are composed of granulation tissue. After a time they shrink and become converted into connective tissue. The process causes baldness in some places from atrophy of the hair-follicles, while in others a kind of cheloid, with tufts of hair projecting through the hypertrophied scar tissue, is produced (acne cheloid). The affection is known in France as sycosis papillomateuse and folliculite dépilante ; in Germany as Nackenkeloid (Unna), and in Vienna as Sycosis framboesiformis (Neumann). The adjective "frambœsiformis" indicates 
the raspberry-like (frambosia) growth which is the characteristic lesion of the disease. ${ }^{1}$

\section{Overgrowth of Hair (Hirsuties)}

This condition may occur either as an exaggeration of the natural growth in hairy parts or as an abnormal growth in hairless regions, as on the upper lip or the chin in women; or it may be universal. Some anomaly of dentition is often associated with general hirsuties. Dark-complexioned persons are more liable than fair persons. The condition is not infrequently hereditary; it may be congenital, or may become developed at any period of life, being most common at and after the climacteric. Hirsuties is a frequent accompaniment of insanity in women, and it is sometimes associated with disorder of the menstrual function, and with barrenness. Sometimes the condition follows a severe illness. Overgrowth of hair may also be the result of local irritation, as by blistering or stimulating applications.

Prognosis.-The condition is, as a rule, persistent, unless it can be got rid of by treatment. It is only in the slighter cases, however, that this offers any chance of success.

Treatment.-The only satisfactory procedure is electrolysis, and this is applicable in but a small proportion of cases. Electrolysis should be used only when the superfluous hairs are thick, dark, and well defined; the method is unsuitable in cases where there is a large undergrowth of finer hair which cannot be dealt with. Each hair-bulb should be destroyed separately with a needle connected with the negative pole of a galvanic battery passed down to the bottom of the follicle, in

1 For a careful description of this disease-which is very rare in this country-with a full account of the histological examination of a raspberry-like growth, see Fritz Porges, Arch. f. Derm.

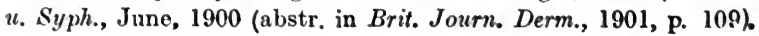


a direction parallel to the hair-shaft. The eircuit is completed by the patient's grasping the positive pole. When bubbles of froth are seen the needle is withdrawn and the hair is extracted with forceps; if it is not per. fectly loose the needle must be reintroduced. 'Two or three dozen hairs may thus be destroyed at a sitting. The operation is not very painful, and the patient is usually so anxious to be rid of the deformity that she will bear the discomfort without flinching. The operation leaves a small red papule, which in time gives place to a macule so small as to be invisible except on elose inspection. If the procedure is followed by any discomfort, the part should be bathed with warm water and a soothing lotion applied. 'The operation sometimes requires to be repeated, in consequence either of the follicle having been imperfectly destroyed in the first instance or of the fine downy hairs becoming coarser when the others have been got rid of. The great source of failure is the uncertainty of the direction taken by the hair within the follicle, and the consequent diffculty of reaching the bulb with the electrode. To meet this difficulty Stern 1 suggests that the hair first be pulled out with forceps, and the needle then at once passed into the follicle while it is still wide open. The method undoubtedly requires skill on the part of the operator and perseverance on that of the patient, but when properly used it gives satisfactory results in a limited number of cases. The $\boldsymbol{X}$-rays have also been used with satisfactory results; but I do not recommend this method on account of the risk involved. When the growth is too abundant for electrolysis to be practicable, shaving is the only alternative. Pulling out the hair with tweezers only makes it grow more vigorously. Depilatories hardly ever do permanent good, and often do harm. As some uterine affection

1 Therapeut. Monats., Aug., 1892. 
or other derangement of the health is sometimes associated with the condition, the local procedure should, when necessary, be complemented by appropriate treatment of the visceral disorder.

\section{Atrophic Changes in the Hair}

These changes may be the result of senile decay, or of some constitutional affection, such as an acute fever, phthisis, or diabetes. They may also occur, independently of any systemic cause, as the result of local processes. The hairs become dry, lose their natural glossiness, and split or break. When the hair is long it often splits at the end; in some cases the splitting appears to take place from the root, so that at first sight there would appear to be several hairs emerging from one follicle. Associated with this condition, pustular folliculitis is sometimes observed, but it is not clear whether it is a cause or a consequence of the affection of the hair.

\section{T'RICHORRHEXIS NoDosa}

Symptoms.-The nodular condition of the hair which bears the above designation, and was first described by Erasmus Wilson and afterwards more fully by Beigel, occurs chiefly in men. The beard, the whiskers, and the moustache are more liable to attack than the hair of the head, but the hair of any part of the body may be affected. Little bead-like swellings of a whitish appearance, like "nits," are seen at regular intervals along the hair-shaft, and at these spots the cortex gives way under the slightest strain, the medulla remaining unbroken. Between the nodes the hair is normal.

Etiology.-The condition is considered by $P$. Raymond ${ }^{1}$ to be of microbic origin, the cortex being eroded by a diplococcus somewhat larger than Staphylo${ }^{1}$ Ann. de Derm. et de Syph., tome ii., 1891. 
coccus pyogenes. Raymond believes the affection to be communicable, a fact which may account for its apparent hereditary transmission in certain cases. Sabouraud maintains that it is due simply to injuries to the hair ; and Lassueur, who takes the same view, has shown that it can be produced experimentally in the hair of the moustache by the free use of soap. ${ }^{1}$

Treatment.-This consists in strengthening the hair by frequent shaving. In view of the possible microbic origin of the affection, epilation of the diseased hairs, followed by the application of an antiseptic lotion, would appear to offer the best chance of success. The general health must also be improved by appropriate. measures.

\section{Monilethrix}

In this curious condition of the hair, known also as beaded hair, and first described by Walter Smith, ${ }^{2}$ of Dublin, the hair-shaft all along its length presents spindle-shaped enlargements at intervals, connected by constricted portions; the latter are almost devoid of colour, the pigment seeming to be massed in the nodes. The hairs break at the narrow parts. The condition affects the hair all over the body.

Etiology and pathology.-The affection generally begins soon after birth, and is occasionally hereditary; it has also been known to come on after nervous shock (Unna). It appears to me to be due to a succession of atrophic changes at periodic intervals, the apparently swollen parts of the hair representing the normal shaft, and the constrictions the atrophied portions. Some consider the affection to be of tropho-neurotic origin. F. Bering ${ }^{3}$ holds that the changes in the hair are due to the pressure exerted by a cornified mass which he

1 Ann. de Derm. et de Syph., Nov., 1906, p. 911.

${ }^{2}$ Brit. Med. Journ., 1879, ii. 291.

${ }^{3}$ Arch. f. Derm. u. Syph., May, 1905, p. 11. 
found plugging the funnel of the follicle. A recent case shown by S. E. Dore ${ }^{1}$ appeared to confirm this view that the affection may be the result of keratosis follicularis, the affected area having been the site of keratosis of the follicles for three years before the monilethrix appeared. In this case the patient was an eight months' child. Later, however, a full-term child of the same parents developed the disease; in this case there was but a slight degree of keratosis of the hair-follicles, which was concurrent with and not precedent to the growth of moniliform hairs. ${ }^{2}$ Dore suggests that the condition may be due to an error of cornification affecting both hair and follicle.

A condition somewhat resembling monilethrix is that known as "bayonet hair." In heads which are beginning to grow bald in the occipital region hairs are seen with a spindle-shaped swelling near the tip, gradually thinning off until it becomes continuous with the normal hair. The thin end may have a double joint, which gives it some resemblance to a bayonet set on the end of a weapon, or it may be bent round. The spindle is strongly pigmented. This peculiar condition is sometimes found among the hairs of the body where the skin is somewhat ichthyotic. Pinkus, ${ }^{3}$ who was the first to describe it, attributes the peculiar form of the hair to its having taken more than the normal time to force its way to the surface. Clubbed and tapering hairs are sometimes seen as the result of $\mathrm{X}$-ray exposures.

\section{Canities}

Greyness of the hair is generally a senile change, but may occur quite early in life as a result of disease,

1 Brit. Journ. Derm., 1910, xxii. 164.

2 Ibid., 1911, xxiii. 111.

${ }^{3}$ Derm. Zeitschr., 1910, Bd. xvii., p. 253 (abstr. in Brit. Journ. Derm., 1910, xxii. 365). 
nervous shock, or long-continued nervous exhaustion. It is sometimes congenital, and occasionally hereditary. There is a family in the South of France both the male and female members of which have had for three hundred years a natural badge in the shape of a lock of white hair, generally situated over the forehead. The hair has been known to become grey or even white suddenly under the influence of terror or grief. In neurotic sub. jects greyness may be temporary, coming on with an attack of neuralgia and disappearing when the pain subsides. The hair generally, however, remains discoloured, though occasionally the pigment may be restored if the cause that produced the greyness is removed.

Treatment.-It is only when the condition has followed some exceptional nervous strain in a person not beyond middle age that treatment can be of use. Even then the prospect of a cure is doubtful. Nervetonics may be of service, and jaborandi given internally in the form of tincture $\left(\mathrm{m}_{\mathrm{xv}}\right)$, or hypodermically as hydrochlorate of pilocarpin (gr. $\frac{1}{10}$ to $\frac{1}{5}$ ), may be useful.

Some cases are on record of the natural colour of the hair changing from fair, for instance, to black, under the influence of pilocarpin injections used for some other purpose. The hair has also been known to change colour after a severe illness. Artificial discoloration may be produced without the application of a dye. Thus, workmen who have to handle aniline dyes have often deep red-brown hair; the hair of copper-smelters often turns green, and that of workers in cobalt mines blue.

\section{Alopecia}

Etiology.-Baldness is usually a senile change, but may occur quite early in life, or may be congenital, and the congenital types may be met with in several 
members of the same family (alopecia congenita familiaris). Alopecia is often said to be comparatively rare in women, but C. J. White, ${ }^{1}$ analysing 794 cases of loss of hair, from various causes, in his own practice, finds that women are more prone to the affection than men, though men are attacked earlier than women. The tendency to baldness is often hereditary. It is a frequent symptom of secondary syphilis, and it sometimes occurs in the later stages of that disease as the result of ulcerative processes. Early baldness may also be a consequence of any fever or other general disease that interferes with nutrition. Apart from any such cause, however, it may be produced by a complex set of factors, such as (1) the shape of the skull, the sides being so prominent that the temporal arteries are easily compressed by the hat; (2) venous stagnation owing to the same cause; (3) profuse perspiration with decomposition of the secretion, and afterwards invasion by bacteria, leading to rotting of the hair; (4) chronic seborrhoea of the scalp. These factors may be combined in varying degrees. The great cause of premature baldness is, however, the one last named, its effect being no doubt largely aided by the wearing of hard, unventilated hats. White's statistics point to dandruff as being the factor in 79 per cent. of cases, heredity in 30 , systemic depression in 20 , fever in 11 , and maltreatment of the scalp in 50. The microbic theory is considered in connection with seborrhœea capitis (p. 596). Parker $^{2}$ has propounded an hypothesis that alopecia is due to auto-intoxication with some substance derived from the lungs owing to decomposition of organic material normally present in respired air when this air is retained in the air vesicles. The apices are the places of retention, owing to diaphragmatic breathing.

1 Journ. Amer. Med. Assoc., 1910, Iv. 1074.

${ }^{2}$ N.Y. Med. Record, July 13, 1901. 
Treatment.- In confirmed baldness no treatment is very satisfactory. If the falling out of the hair has followed an acute illness or is a symptom of syphilis, the hair will generally grow again as the patient recovers his health. Local stimulation will often hasten the process. In elderly people no treatment will restore hair lost through natural decay, but in younger persons the development of commencing baldness may sometimes be checked and the growth of new hair promoted by local treatment directed to the prevention of dryness, the cure of seborrhœa, and the improvement of the nutrition of the hair-roots. For the former purpose the application of fatty or oily matter, and especially of lanolin diluted with vaseline, and with some weak antiseptic-such as sulphur-added, is very useful. For seborrhœa the treatment recommended for that condition should be adopted (p. 598). The nutrition of the hair-bulbs may be improved by stimulating lotions which redden the scalp and bring a larger amount of blood to the affected parts. For this purpose the following formula is of use :-

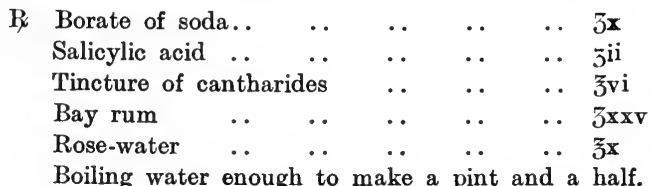

The borax and salicylic acid should first be dissolved in the boiling water; the bay rum, etc., should then be added to the solution, which should be filtered before being used.

Hebra's formula, tr. macidis grm. v, ol. oliva grm. l, may in some cases be useful. I have sometimes had good results from the following:-

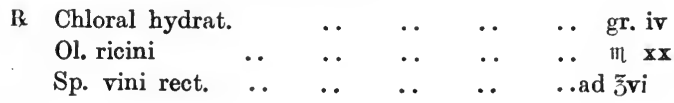


This lotion should be sprayed on the scalp night and morning. C. J. White mentions chloral hydrate as one of the best remedies, the others being uresol, perchloride of mercury, and tannic acid. Besnier recommended the application of equal parts of acetic acid and chloroform; it must, however, be used with caution, or it may cause irritation. The following ointment is also sometimes of service :-

$$
\begin{aligned}
& \begin{array}{lllllll}
\text { B } & \text { Acid. salicyl. } & \ldots & \ldots & \ldots & \ldots & \text { gr. } \mathbf{v}
\end{array} \\
& \begin{array}{lllll}
\text { Sulph. præcipitat. } & \ldots & \ldots & \ldots & \text { gr. } \mathbf{x v}
\end{array} \\
& \begin{array}{llllll}
\beta \text {-Naphthol } & \ldots & \ldots & \ldots & \ldots & \text { gr. } x
\end{array}
\end{aligned}
$$

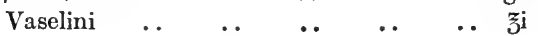

Restoration of the hair has been observed to follow the administration of thyroid extract in myxœdema.

\section{Alopecia Areata}

In this condition patches of baldness appear upon the scalp or other hairy parts of the body.

Symptoms.-The characteristic patches of baldness may gradually spread over a considerable area. The affection is marked by suddenness of onset, and in severe cases by the rapidity of its extension. It generally begins on the scalp, and is often limited to that region: it may, however, be universal, the hair falling out all over the body, and leaving the patient not only with an absolutely bald head, but without eyebrows, eyelashes, whiskers, beard, moustache, axillary or pubic hairs. In such cases the nails both of the fingers (Plate 59) and toes occasionally atrophy coincidently with the falling-out of the hair.

The usual course of events is somewhat as follows: One or more small patches suddenly make their appearance on a scalp otherwise perfectly healthy. These initial patches are most commonly situated on one side or other of the occiput, over the ridge marking the point of insertion of the trapezius muscle; on one side 

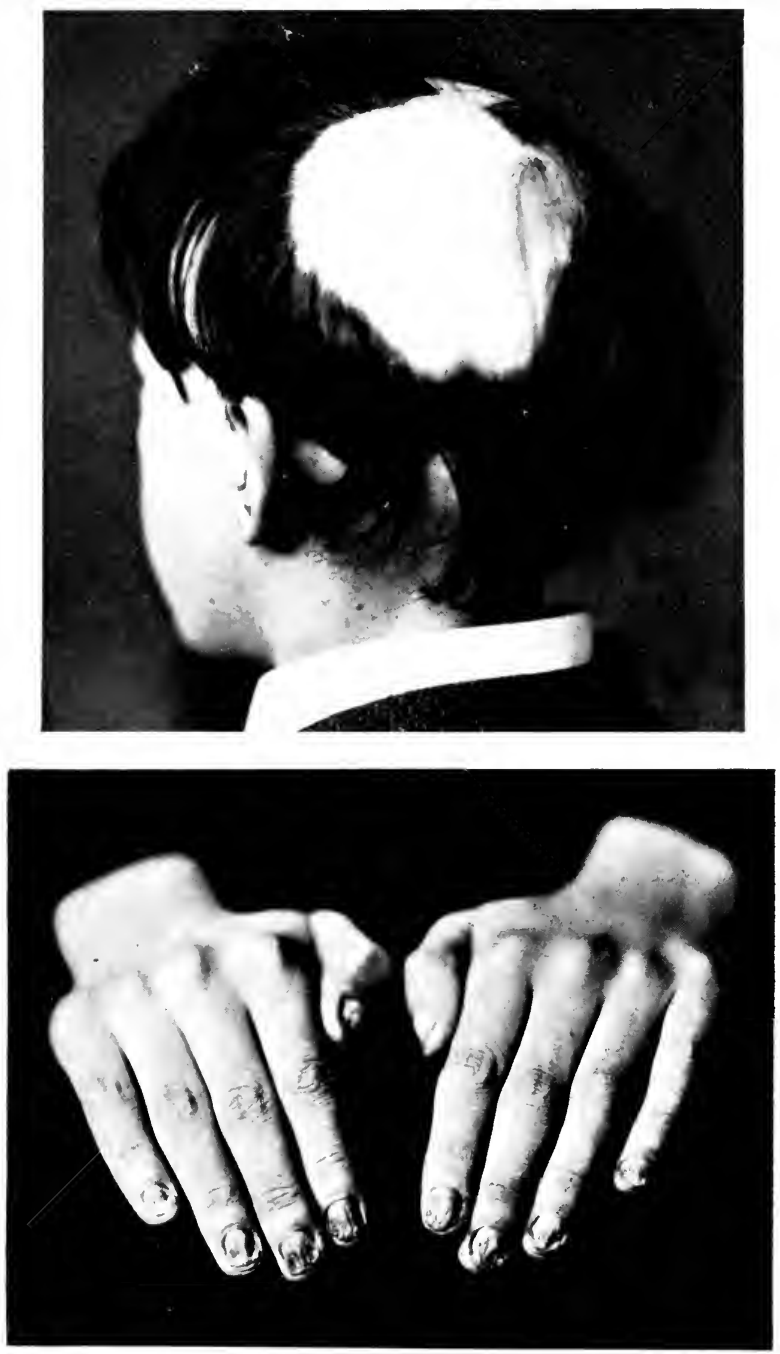

Plate 59.-ALOPECIA AREATA WITH ATROPHIED NAILS. 

or other of the vertex; and above and behind the ears. In the early stage the skin of the patch is somewhat red, but later it acquires the whiteness and smoothness of a billiard ball. Sensation is unaffected, but the skin on the patch reacts decidedly less to stimulant substances than the rest of the scalp. The smooth bald patches are sharply defined from the neighbouring healthy parts, but the hairs at the edge are looser than normal, and, on careful search, in many cases short hairs can be found that show distinct signs of atrophy close to the root, giving them somewhat the shape of a note of exclamation ( 1 ). Sometimes the patches are small, round, and distinctly depressed below the level of the surrounding skin. Generally they continue spreading for a time, and may coalesce with others, forming denuded areas of irregular outline. When the affection has lasted some time the skin of the patches is thinned and adherent to the underlying tissues, so that it cannot be pinched up or moved upon them.

Etiology.-There are two theories as to the production of this disease, some considering it to be a neurosis, others inclining to the belief that it is due to microorganisms. That it is at least sometimes neurotic in origin appears to me to be proved by its not infrequent occurrence as an immediate sequel of mental shock, such as fright; it also often seems to be directly connected with prolonged mental distress or worry. I have known total alopecia occur in a lady within forty-eight hours of receiving news of the death of her son. Stepp has recorded a case in which complete loss of the scalp hair followed the shock of a railway accident. Jacquet regards dental irritation as one of the causes of the affection, having frequently observed the coexistence with it of dental lesions. It is generally stated to be more common in the male than in the female sex: Lassar gives the proportion as seven males to three females; Crocker, on 
the ground of his own experience, denies that there is any marked difference between the sexes, though even the figures which he gives show a preponderance of males. It is most common in young persons, and is rare after 40 . The largest body of statistics collected is that which formed the basis of Norman Walker's introductory remarks at the International Congress of Dermatology held in Paris in 1900. A statistical examination of the records of 4,000 cases of skin diseases at the Edinburgh Royal Infirmary showed that alopecia areata formed, roughly, 5 per cent. of the whole; that there was a greater susceptibility in males than in females, and that the most common age of incidence was between 10 and 20 .

Alopecia areata occasionally follows the track of a particular nerve, and it has been known to be consecutive to injury to the sympathetic nerve. Leloir examined histologically cutaneous nerves from the affected surface, and in one case they presented all the signs of atrophic neuritis. Cases illustrating the marked influence of nerve lesions in producing alopecia areata have been published by Schutz. A fact in some degree confirmatory of the neurotic origin of alopecia areata is its occasional association with leucodermia. On the other hand, in only one of the 4,000 cases investigated by Norman Walker and Marion Marshall-Rockwell was a possible nerve influence suggested, and these writers conclude that the nervous element in the disease has been greatly overrated.

The microbic theory at present rests more on clinical than on pathological evidence. Some years ago Kazanli ${ }^{1}$ reported the discovery of a microbe which he believed to be specific, and micrococci have been found by Robinson and others in the root-sheaths of the hair around the affected areas, and also in the lymph-spaces

${ }^{1}$ Brit. Journ. Derm., 1888-9, p. 132. 
of the corium and subpapillary layer; but the few investigators who have seen these microörganisms are not agreed as to their characters; and even if their existence be admitted, there is no clear proof of their causal relation to the process. Sabouraud, ${ }^{1}$ who has made extensive researches on the subject, concludes that alopecia areata is of microbic origin, the follicles being occupied by innumerable colonies in the early stage of the disease ; later, when the area patch has been definitely constituted, no microörganism can be found. He holds that seborrhœa oleosa and alopecia areata are essentially identical processes, due to the same microbacillus. Norman Walker and Miss Marshall-Rockwell found that affected hairs inoculated on Sabouraud's medium produced a glistening white growth due to the Staphylococcus epidermidis albus (Welch). Welch himself, however, states that this organism is constantly found on the skin even after sterilization of the surface with antiseptics. In only one of Norman Walker's cases did a brick-red growth develop such as is produced by Sabouraud's bacillus. In two cases pieces of skin were excised, and organisms were found in the follieles and slight inflammatory changes in the epidermis. In 70 per cent. of the cases seborrhœa was present. It is possible that in some cases alopecia areata is a manifestation of late syphilis.

There is some reason to believe that alopecia areata may in certain circumstances be transmitted from one patient to another, and in France epidemics of pelade are said to be not infrequent in schools and in regiments ; but Sabouraud declares, after long experience, that he has never met with an epidemic of pelade, the diagnosis in every alleged instance investigated by him being at fault. In 18 of the 4,000 cases investigated

${ }^{1}$ Ann. de Derm. et de Syph., 1896, vii. 253, 460, 677, 824; and Ann. de l'Inst. Pasteur, 1897, xi. 134. 
by Norman Walker there was a history of possible contagion. Decisive proof of contagion is still wanting, however; and it is certain that, even if the affection be contagious, transmission is altogether exceptional.

A condition similar to alopecia areata is occasionally produced by exposure to the Röntgen rays.

Diagnosis.-Alopecia areata is distinguishable from lupus erythematosus of the scalp in that the bald areas are not cicatricial, there is no destruction of the follicles, nor are the borders of the patches inflamed. Folliculitis decalvans also is cicatricial, and at the edge of the bare patches a small red papule or patch of erythema will be seen around each follicle. For the diagnosis between alopecia areata and pseudopelade, see p. 627.

Prognosis.-Restoration of the hair takes place, sooner or later, in most cases, but the process is as a rule a protracted one, and several successive crops of downy hair may grow and wither away again before the patches are definitely covered over. Even after complete restoration, relapse is not at all uncommon. In total cases the baldness usually is permanent, but it is difficult to give a definite prognosis on this point, as complete restoration of the hair has been known to take place after ten and, in one case, sixteen years. ${ }^{1}$ As long as there is no great thinning or loss of mobility in the affected skin there is a fair prospect that the hair will be restored within a year. The chances of early recovery diminish in proportion to the shrinkage of the skin and the age of the patient, the prognosis being bad after the age of 45 .

Treatment.-Attention should be directed to improvement of the general health, if there be any need for this, by tonics (especially iron), by sea-bathing, and other invigorating measures, such as massage and elec-

${ }^{1}$ Michelson, quoted by Jamieson, "Diseases of the Skin," 1888, p. 406. Edinburgh. 
tricity. The subcutaneous injection of hydrochlorate of pilocarpin ( $\frac{1}{30}$ gr.) has proved successful in my hands in a limited number of cases. Locally, strong stimulation is indicated; for this purpose chrysarobin ointment should be rubbed into the patches night and morning with proper precaution. The most usually accepted treatment is blistering, for which purpose acetum cantharidis may be used; it should be applied to the patches and the scalp around them. The same effect may be produced by croton oil, or oil of mustard in the following formula :-

\begin{tabular}{|c|c|c|c|c|}
\hline Ol. sinapis & .. & .. & . & \\
\hline Ol. ricini & .. & .. & .. & .. \\
\hline Sp. rosmarini & .. & M. & & 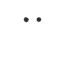 \\
\hline
\end{tabular}

This should be painted on, not rubbed in, once or twice a day. A good substitute for croton oil in obstinate cases is trikresol, which contains the ortho-, meta-, and parakresols. Iodine in the form of strong liniment applied daily is often of service. Jamieson speaks highly of the following formula of Erasmus Wilson's :-

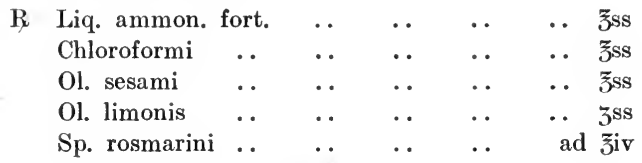

M.

This is to be rubbed gently into the bald part, at first once, afterwards twice a day; in the later stages faradism is sometimes useful. All these various remedies act in the same way - that is to say, by increasing the flow of blood to the part and thereby improving the nutrition of the hair-follicles. Sabouraud recommends the use of sulphur in a vehicle of some fatty substance which will mix readily with the fats of the skin.

The routine treatment adopted by Lassar is anti2 P 
septic in character. The head is washed daily for a few minutes with a strong tar soap, which is then sluiced off, after which the head is dried. The scalp is then treated successively with 2 per cent. sublimate solution, absolute alcohol with the addition of $\frac{1}{2}$ to 1 per cent. naphthol, and finally with 2 per cent. salicylic acid in oil. By this treatment Lassar affirms that in all fairly recent cases the disease is brought to a standstill at once.

It must be remembered that, whatever remedy may be employed, spontaneous cure often takes place, especially in young people, so that too much credit must not be given to drugs.

In some cases the Finsen light, high-frequency current, or $X$-rays may be used with advantage.

\section{LEPOTHRIX}

Certain concretions are sometimes seen on the hairs. The most common of these is lepothrix, which is confined to the hairs of the axillæ and the scrotum. To the naked eye the hairs are dull and lustreless, with ragged borders; they are so brittle that they break on the least traction. On microscopic examination the affected hair is seen to be surrounded more or less completely with irregular masses of concretion, in which some of the fibres of the cortex are embedded. In the axilla the concretion is often red in colour, owing to the presence of the micrococcus which produces red sweat in that situation; as this red colour is not seen in the scrotal hairs the association in the axillæ is probably accidental. Glasgow Patteson discovered a short bacillus which penetrates under the cortical scales and is constantly present in lepothrix. The condition, which is tolerably common, gives rise to no symptoms. The application of parasiticidal agents would probably be the most hopeful line of treatment. 


\section{Trichomycosis Capilliti}

Under this name Winternitz ${ }^{1}$ describes an affection of the hair closely resembling but not identical with lepothrix, and due, he believes, to a different organism, a straight or slightly bent bacillus, frequently with spores at each end, and measuring 1.8 to $3.6 \mu$ in length, and 0.6 to $0.9 \mu$ in breadth. The affected hairs were thickened, owing to the presence of a concretion which was dark brown in colour, and irregularly margined or lobed.

\section{Piedra}

This affection is seen almost exclusively among the natives, especially the women, in the district of Canca, in Colombia (South America). It has also been seen in Europe. In men the beard sometimes suffers. The concretions are small, black, gritty particles, which cling to the shaft of the hair. They are so hard that they rattle when the hair is combed: hence piedra, a stone. They consist of closely aggregated pigmented spore-like bodies, due to a fungus, whioh, according to Castellani, is the Trichosporum giganteum (Behrend, 1890); they are as large again as trichophyton spores, and very refringent. The affected hair has an acid smell, and the condition is locally believed to be connected with the use of a peculiar oily substance for lubricating purposes. ${ }^{2}$ The treatment should evidently be antiparasitic; but it may be necessary to shave the head.

1 Arch. f. Derm. u. Syph., July, 1903, p. 81.

2 See a paper by the author in Path. Trans., vol. xxx., 1879, p. 441, and Juhel-Rénoy (Ann. de Derm. et de Syph., vol. ix., 1888, p. 777, and vol. i., 1890, p. 776). Cases have also been recorded by Unna (Lewin's Festschrift, Berlin, 1896) and Behrend. See the report of a microscopical examination of these cases by Tracheler (Monats. f. Derm., xxi.). 


\section{Tinea Nodosa ${ }^{1}$}

This name denotes a nodular concretion, also consisting of fungus spores, sometimes affecting the hair of the whiskers, beard, or moustache. It weakens the hair, which splits and breaks. Clipping the hairs short and the use of antiparasitic remedies are the methods of treatment indicated.

\section{AFFECTIONS OF THE NAILS}

The nails are often involved in processes-such as infection by cocci, eczema, psoriasis, lichen planus, favus, ringworm-which affect the integument generally. The lesions of these epidermic appendages in such cases have been described with the diseases in question. The nails may also be the seat of trophic changes which may be due to senile atrophy or to acute illness, or may occur without any apparent cause. Sometimes the longitudinal striæ are exaggerated, and the nails may be "fluted" or "reeded" (onychorrhexis) ; sometimes transverse furrows (Beau's lines) remain as records of a fever or other severe illness; sometimes white spots are developed, and sometimes a large part or the whole of the nail may become white (leuconychia), due, according to Unna and Joseph, to the presence of air in and between the nail-cells; according to Giovanni, to abnormal cornification; according to Heidingsfeld, to parakeratosis without infiltration of air. Shedding of the nails (onychoschizia) may occur, as said elsewhere, as a part of the process of alopecia areata, or in association with diabetes, syphilis, locomotor ataxy, and other nervous disorders. In koilonychia, or "spoonnail," the nail becomes concave instead of convex, owing to inversion of the sides and the free end. This condition

1 The affection was first described and named by Cheadle and the Author (Lancet, vol. i., 1879, p. 190). 
is sometimes associated with leuconychia totalis, with Raynaud's disease, with lichen planus, or with acanthosis nigricans. Pigmentary and degenerative changes may also occur in the nails as the result of occupation, as in dyers, washerwomen, jewellers, and others.

\section{Onychia and Paronychia}

Apart from the various causes enumerated above, the matrix of the nails may be the seat of pathological processes similar to those affecting other tissues. Inflammation (onychia) may occur; this may be idiopathic or may follow injury, or may be a manifestation of syphilis or the result of direct tubercular infection (onychia maligna). In the latter case the condition is frequently associated with scrofulous lesions in the eyelid and elsewhere. If the process is acute there are great pain and redness; suppuration takes place beneath the nail, which is discoloured and thickened, and is finally pushed out of its bed and thrown off, leaving an unhealthy sore. This may heal, or the inflammation may involve the lymphatics, and give rise to paronychia or whitlow.

Treatment.-The treatment for onychia is to remove the nail, if it has not already been thrown off, and apply antiseptic dressings. The general health may also require attention.

\section{Ingrowing ToE-NaIL}

A special variety of paronychia is caused by ingrowing toe-nail, a condition that generally occurs as the result of pressure by tight boots, or of irritation by the edge of a badly-cut nail. Ulceration takes place on one side of the nail (generally that of the big toe), which becomes embedded in inflammatory tissue, so that walking is rendered impossible.

Treatment.-In severe cases the nail should be divided with scissors, and the two halves removed 
separately. As this operation is extremely painful, an anæsthetic will be necessary. The bare surface must then be dressed antiseptically. In less severe cases the granulations may be destroyed with acid nitrate of mercury, the nail scraped thin in the middle, and an antiseptic dressing applied. In cutting toe-nails they should not be rounded at the edges.

\section{Hypertrophy OF THE NAILS}

Onychauxis, or hypertrophy of the nails, sometimes occurs, the whole nail becoming thickened, and the free end growing out to a great length, and sometimes becoming twisted like a ram's horn (onychogryphosis). Onychogryphosis is frequently associated with congenital ichthyosis, and Müller records cases in which it appeared in association with congenital hypertrichosis and with a chronic psoriasis.

Treatment.-The superfluous part of the nail should be removed after soaking in hot water.

\section{EgG-Shell NaIL}

Under this designation, Dr. Nevins Hyde, of Chicago, describes a form of modified nutrition of the nails intimately associated with hyperidrosis. The patients were all young women below the standard of sound health, who exhibited an unusual translucency of the nails of both fingers and toes, an enfeeblement of their connection with the distal portion of the nail-bed, and a tendency to growth in an upward rather than in a forward direction. The colour of the nail in wellmarked cases is precisely that of the inner face of the shell of a hen's egg-a delicate combination of white and purple: hence the name. He suggests that constant maceration of the distal portion of the nail-bed, the result of the hyperidrosis, interferes with the normal cornification of the nail-plate. 


\section{CHAPTER XXVII}

\section{NEW GROWTHS}

As our knowledge of the etiology of disease extends so will the group of new growths diminish, and already it is a moot question whether some of the supposed new growths may not be of microbic origin. Until this question is finally settled there must always be a group of affections of doubtful causation which, from the presence of more or less circumscribed tumours, may be classed as new growths. The term must, however, be taken strictly in its anatomical sense, and not as meaning something sui generis. Neoplasms may be provisionally classified into growths affeeting connective and other tissues of mesoblastic origin, and growths affeeting epithelial tissues either alone or in addition to the connective tissues. But since a seientific and final classification of new growths is at present impossible, it has been thought best here to adopt the familiar clinical division into tumours of benign and tumours of malignant nature, which has at least the advantage of being practically convenient.

\section{BENIGN NEOPLASMS}

Under this head are placed all new growths which are strictly local in their development, and, though sometimes attaining great dimensions, remain localized throughout their course, and which when completely removed do not recur. As a rule, benign tumours are homologous in structure-that is to say, they are over- 
growths of tissue normally present in the region from which they spring. Thus the group embraces cystic tumours, arising from the distension of pre-existing spaces (sebaceous and atheromatous cysts), and local overgrowth of gland structure (adenoma sebaceum), of connective tissue (cheloid and fibroma), of muscular tissue (myoma), of nerve (neuroma), of blood-vessels (telangiectasis, nævus), and of the lymphatic system. In addition to these are certain growths associated with degenerative changes in the skin, and of doubtful pathological nature, though known clinically to be benign-such as colloid milium of the skin, xanthoma, and molluscum contagiosum.

\section{Cysts}

Sebaceous cysts are most commonly seen on the scalp, the face, and the back, but they may develop in any part of the skin supplied with sebaceous glands. They occur more frequently in women than in men. There may be one or several cysts. They are rounded in shape, often somewhat flattened on the top, and may be as large as an orange. They grow slowly, and cause no pain unless they become inflamed. To the touch they feel like lumps of dough. The duct may be patent, so that some of the contents can be pressed out, or it may be closed; the latter is the more common condition when they are situated on the scalp. The skin over them is generally normal, though somewhat redder than the surrounding parts. When the cysts are inflamed the skin becomes bright red and the tumour itself feels softer and sometimes breaks down into a fungating ulcer.

There is some doubt as to the pathology of these growths. Paget regarded them as new growths, but most observers believe them to be simply retention cysts, the accumulation of epidermic débris and sebaceous 
matter in the follicle causing expansion of its cavity, with secondary hypertrophy of their walls. Sebaceous cysts are distinguished from fatty tumours by the absence of lobulation and the fact that the contents can be squeezed out when there is an opening.

Treatment.-This consists in incising the cysts and carefully dissecting or scraping out the cyst wall.

Dermoid cysts occasionally occur on the skin. They are often very numerous, and resemble fibromas, but on cutting into them a sebaceous-looking material escapes. They should be excised, unless their number makes interference undesirable.

The cystic tumours of the skin caused by $C y s t i$ cercus cellulosa, echinococeus, etc., are referred to elscwhere.

\section{Adenoma Sebaceum}

Clinical features.-Adenoma sebaceum occurs chiefly on the face. The lesions are small, firm, papules - or rather, tiny solid tumours-firmly embedded in the skin at different depths or projecting from it, and varying in size from a pin's point to a pea. They may be whitish or yellowish (Balzer), or pink (Pringle), or the surface may be warty (Hallopeau and Leredde). Sometimes they are red, owing to dilatation of the capillary vessels on their surface, and intermingled with them are numerous telangiectases. The lesions are usually symmetrical in distribution, and, though thickly crowded in places, do not run together to form patches. The tumours present no opening, but, when they are pricked, inspissated sebum can be squeezed out of them. They cause no inconvenience as a rule, though occasionally they are painful in cold weather. The condition is congenital, but further crops of lesions appear after birth, especially at puberty. Adamson ${ }^{1}$ has shown two cases of adenoma sebaceum in mother and child. In the

1 Brit. Journ. Derm., 1911, xxiii. 109. 
mother the lesions dated from birth; in the child, a boy of 5 , the lesions first appeared at the age of 4 . In 1893 three cases in one family were reported. ${ }^{1}$ The lesions undergo little change, though some of them may undergo spontaneous involution. Rosacea is sometimes associated with the condition. Other textural defects in the skin-warts, nævi, keratosis pilaris, etc.-often coexist with adenoma sebaceum; and the patients are generally of a low grade of mental development, often imbeciles or epileptics.

Pathology.-According to Pringle, to whom we owe an excellent account of this disease, ${ }^{2}$ the essential lesions consist of an increase in number and complexity of the sebaceous glands, recalling at first sight the general appearance of sections of the hypertrophic masses that are sometimes seen in advanced rosacea. The condition is not improbably due to excessive development of gland structures from superfluous embryonic remains in the skin.

Diagnosis.-The appearance of the little firm tumours, thickly grouped about the sides of the nose, intermingled with telangiectases, with the history of congenital origin and the association of other anomalous conditions of the skin and mental deficiency, will suffice in most cases to identify the disease.

Treatment.-No internal medication has any effect on the condition. Pringle found that attempts to scoop, gouge, or bore out the little tumours with instru. ments used for such purposes in cases of lupus were painful and unsatisfactory, owing to the depth at which they were situated and the firmuess with which they were embedded. Superficial scarification was also unsuccessful. Electrolysis was used by Radcliffe-Crccker

1 Brit. Journ. Derm., 1893, v. 360.

2 Ibid., vol. ii., 1890, p. 1 et seq. (with a good coloured illustration). 
with success in a case in which the nodules were not large.

When adenoma sebaceum is not congenital the lesions appear as pink, sometimes lobulated, growths on the scalp, back, and face, varying in size from a pea to a nut. The subjects are usually elderly persons. The growths may be removed by electrolysis, by the galvano-autery, or by carbon-dioxide snow.

Adenoma may arise not only from the sebaceous glands, but also from the sweat-glands. The latter growths (hidradenomas) are met with $(a)$ on the lower eyelids of adult women, seldom in men, as small tumours varying little from the colour of the skin; or (b) as pink lesions on the neck, and sometimes on the trunk, between the ages of 10 and 20 .

\section{Cheloid}

The normal process of healing by second intention is a transformation of vascular embryonic (granulation) tissue into fibrous tissue. Sometimes the transformation is tardily effected; the granulations continue to form, and are converted into imperfect but excessive scar tissue-hypertrophied cicatrix. A still further departure from the normal results in the formation of distinct fibrous growths-scar cheloid. In some cases growths of fibrous tissue resembling scar cheloid arise without any previous wound having been noticed; these cases have been classed together as spontaneous or true cheloid. A remarkable example of this has been recorded by Walter Smith. ${ }^{1}$ The apparently spontaneous cheloid is most frequently observed on the trunk, especially over the sternum, and on the face; and when it is remembered how frequently acne pustules or slight injuries and the resulting scars are overlooked in these parts the use of the term

${ }^{1}$ Brit. Journ. Derm., 1888-9, p. 157. 
"spontaneous" is probably unjustifiable. This view is confirmed by a histological investigation by Krzysztalowicz, ${ }^{1}$ who concludes that a hard-and-fast distinction between spontaneous and scar cheloid is untenable. The term "cheloid" will therefore be used here to denote both forms. The term " hypertrophied cicatrix" should be confined to cases in which the growth does not extend beyond the limits of the wound, "cheloid" being used to denote the condition when it has so extended.

Clinical features. - The primary lesion is a white or pinkish swelling, which may project above the level of the skin or may lie within the corium. Sometimes dilated vessels are visible on the surface. The shape of the swelling differs according to its origin. Usually it tends to assume a rounded contour, but it may be depressed in the centre and it may extend laterally by claw-like processes-whence the name "cheloid" (from $\chi \eta \lambda \eta$, a claw). A less accurate derivation is from $k \eta \lambda i s$, a scar. Occasionally the growth has a warty aspect, constituting the verrucose cicatricial tumour or warty scar. Cheloid is a result of active growth; it is at least possible that the process may be infective.

Whilst cheloid may appear on any part of the body, it is commonest, as I have said, over the sternum and the rest of the trunk, and on the face and head. Extensive formation of cheloid tumours has been observed after smallpox. The tumours form in a few weeks, and usually continue to enlarge for a long time. Sometimes they undergo involution. In a case of Goodhart's, large cheloid tumours, which formed all over the body after smallpox, had disappeared at the end of a few months. Hutchinson thinks that this tendency to involution is most marked in young subjects. As a rule, during many years the tumours either remain stationary

1 Monats. f. prakt. Derm., Nov. 1, 1909, p. 381. 
or, at any rate, enlarge very slowly. Cases are recorded in which cheloid has undergone sarcomatous change.

The tumours are usually tender, and may be the seat of itching, pain, and burning. Sometimes, however, they give rise to no subjective symptoms. The tumour occurs at all ages, but chiefly between 15 and 50 . It is more common in negroes than in whites.

Pathology and diagnosis. - The bulk of the growth consists of fibrous tissue, more cellular and vascular than normal scar tissue. The tumours are covered by epidermis, which may be considerably thinned, so that the papillæ may be absent. The diagnosis presents no difficulty, the scar-like appear. ance and claw-like processes of the tumours being characteristic.

Treatment.-Removal or destruction of cheloid is never successful. Pressure with an elastic bandage, massage, and deep gashing of the tumour in different directions, so as to divide as many vessels as possible, have given good results in some cases. The application of unguentum hydrargyri and other preparations of mercury is often followed by good results. Electrolysis answers well when the growth is small. Even in the case of growths of moderate size I have seen complete cure effected by electrolysis applied once a week for some time, followed by daily massage. Radium is one of the most effective methods of treatment. I have found that application of the $X$-rays has led to marked diminution of the growth, and that pain has been greatly eased. For small growths the light treatment is also satisfactory, either used alone or in combination with X-rays. When cheloid is attended with pain, cocaine should be injected in and around the tumour, or belladonna or opium may be applied locally. 


\section{Fibroma}

Under this head are included soft fibrous growths (fibroma molluscum), firm fibromas, neuro-fibromas, and diffuse fibroma, which is one form of dermatolysis.

\section{Fibroma Molluscum}

Clinical features.-Fibroma molluscum is a pearshaped or rounded fibrous tumour, covered as a rule by smooth skin, and varying in size from a pin's head to an orange. This tumour is not uncommon, and is almost always multiple. Usually the growths are pedunculated, but sometimes they form flat masses embedded in the corium. Occasionally they occur in immense numbers, and then the sebaceous glands in the skin covering them may be dilated, and in uncleanly persons the excessive secretion of sebum by decomposition may give rise to offensive odours. Wickham ${ }^{1}$ has called attention to the occurrence of brownish pigmentary stains and violet-coloured prominences and blotches in association with these growths. They are commonest on the trunk (Plate 60), then on the head and face, and after that on the limbs; they are rare on the palms and on the soles. They have been met with on the tongue and the buccal mucous membrane (Crocker). The tumours tend to increase in size and number, but they may remain stationary for a number of years. Occasionally they slough and ulcerate. They cause no pain, except when they are inflamed as the result of accidental injury. A case in which fibroma molluscum was associated with multiple benign cystic epithelioma has been reported (p. 680).

Pathology and etiology.-The growths consist chiefly of lax fibrous tissue sparingly supplied with

1 Brit. Journ. Derm., 1890, p. 151. 


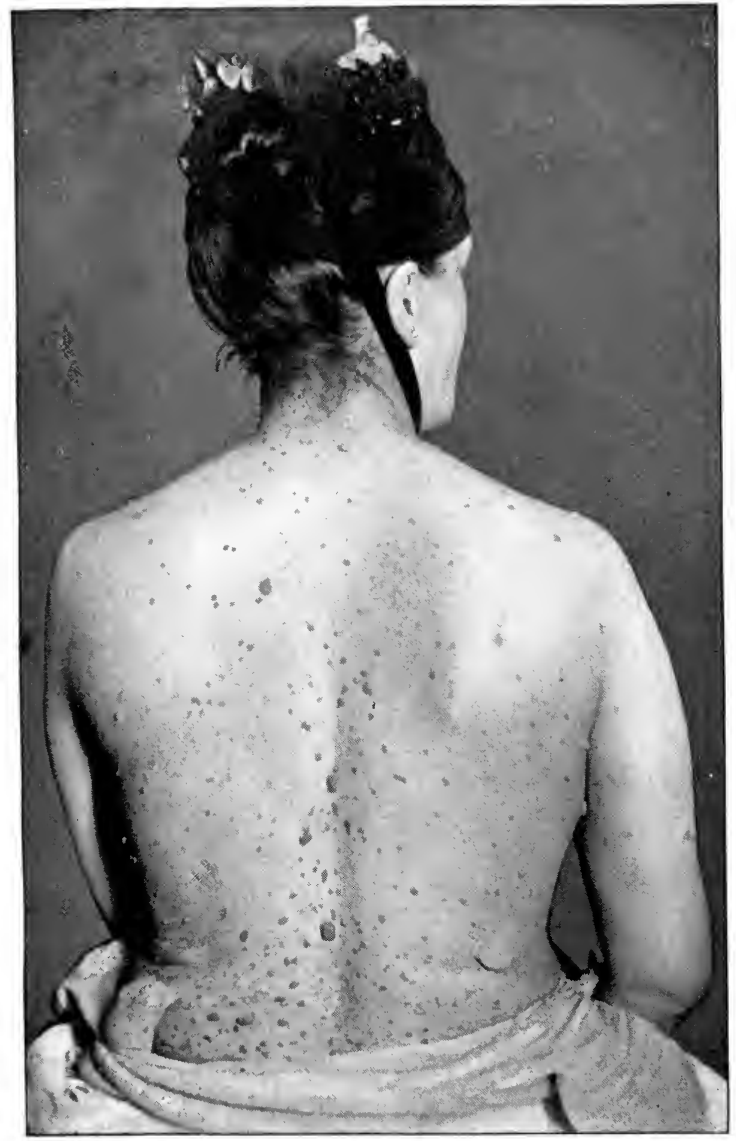

Plate 60.-Fibroma molluscum. 


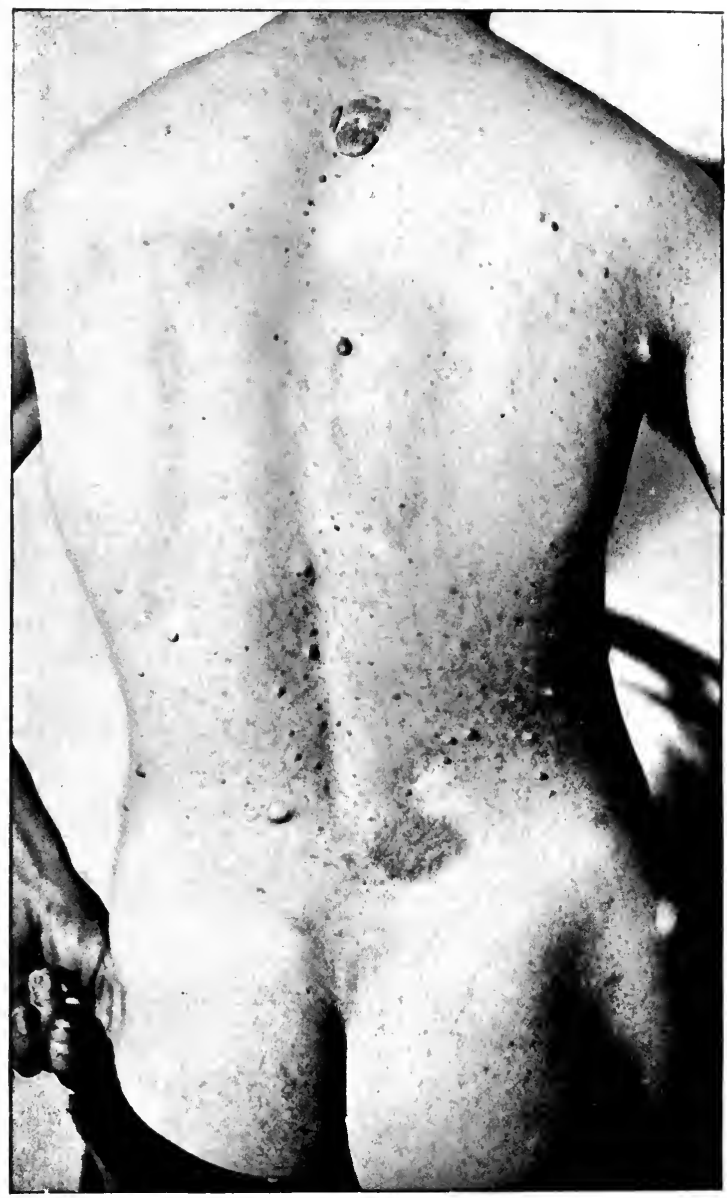

Plate 61.-VON RECKLINGHAUSEN'S DISEASE.

(I'hitfield's Case. We'st London Aled.-Chir. Journ.) 
blood-vessels and containing a few nerves. The origin of the growths has been variously traced to the corium and the subcutaneous tissue (Virchow). They may appear in early childhood. Nothing is known as to the etiology of the condition.

Diagnosis.-The tumours are distinguished from fatty tumours by the fact that they are pedunculated and present no trace of lobulation, and from sebaceous cysts by their solid structure.

Treatment.-The growths may be removed by ligature, galvano-cautery, or the knife, special precautions being taken against hæmorrhage, which may be formidable. They may, however, be so numerous as to render treatment inadvisable.

\section{Diffuse Fibroma}

This is a variety of fibroma molluseum in which the tumours are large and attached by broad bases. As they are usually multiple they overlap each other, forming large folds of loose skin with dilated sebaceous orifices. The condition must be distinguished from elastic skin, which is an anatomical peculiarity.

\section{Von Recklinghausen's Disease (Plate 61)}

A special type of multiple fibroma of the skin, described by von Recklinghausen and known by his name, is characterized by coffee-coloured pigmentation on and around the nodular tumours, which are irregularly distributed, though in exceptional cases they follow the course of one or more individual nerves. In one of my own cases there were only nine tumours altogether, irregularly scattered over the trunk and limbs in relation to different cutaneous nerves. In association with the growths there is perceptible thickening of the nerves of the arms. The distinctive feature of fibroma of the von Recklinghausen type is that whereas ordinary 
fibroma is composed of fibrous tissue, this is made up of fibrous and nervous tissue. ${ }^{1}$ A case has been recorded by Preble and Hektoen in which multiple neurofibromas of the skin were associated with arthritis deformans. In an atypical case under the author and Wilfrid Fox there were no large pendulous tumours on the skin, only very fine fibrils in the skin were affected, the pigmentation was mottled, and there were fits -due, not, as might have been supposed, to intracranial neurofibromas, but to hysteria. ${ }^{2}$ In some cases decided pigmentation develops long before neurofibromas of nerve-trunks or molluscous tumours of the skin present themselves. In other cases the neurofibromas or the molluscous tumours, with or without cutaneous pigmentation, are complicated by the presence of papillomatous growth. Parkes Weber ${ }^{3}$ has classified these anomalous or incomplete forms of the disease as follows: (1) Cases of plexiform neuroma unaccompanied by multiple molluscous tumours of the skin, with or without cutaneous pigmentation; (2) cases of multiple molluscous tumours of the skin unaccompanied by obvious neuro-fibromatosis of nerve-trunks, with or without decided cutaneous pigmentation; (3) cases of pigmentation of the skin not (or not yet) accompanied by obvious neuro-fibromatosis of nervetrunks or molluscous tumours of the skin; (4) anomalous cases of neuro-fibromatosis complicated by the coexistence of bony or papillomatous changes.

\section{Hard Fibromas and Neuro-Fibromas}

Clinical features.-These forms of fibroma vary in size from a pin's head to very large dimensions. They

${ }^{1}$ For an exhaustive study of this affection, see work by Alexis Thomson.

${ }^{2}$ Brit. Journ. Derm., A pril, 1907, p. 109.

s Ibid., 1909, p. 52. 
usually arise in the corium, but may start in the tendonsheaths or the sheaths of the nerve fibres. In the latter case they are called neuro-fibromas: these lie as a rule in the subcutaneous tissue, but in rare cases have been found in the skin. Several tumours may coalesce into a single lobulated mass (Schwimmer). They occur on the trunk and extremities, and are usually isolated. Neuro-fibromas are usually multiple, and are movable in the subcutaneous tissue. The tumours tend to enlarge slowly, but sometimes calcification or fatty degeneration takes place. Thus blood-vessels may become dilated into blood cysts (telangiectatic form). The neuro-fibromas, owing to the nervefibres stretched over or included in them, are often extremely sensitive to pressure. The other forms are not sensitive. On section, hard fibromas resemble tendon tissue.

Etiology and diagnosis.-Nothing is known as to the etiology of these growths. Like the soft fibromas, they may develop very early in life. The diagnosis is usually easy. Neuro-fibromas may possibly be mistaken for rheumatic nodules. The latter, however, occur chiefly in the region of the elbows and about tle scalp, and there is a history of rheumatism.

Treatment. - This is the same as that of soft fibromas. Neuro-fibromas may be successfully dealt with by excising a portion of the nerve cords supplying the tumours.

\section{Myoma Cutis}

Occurs either as a superficial growth or as a tumour originating from the subcutaneous muscular structures. Of the former kind Crocker ${ }^{1}$ collected ten cases recorded in medical literature, and added one of his own. Later, Leslie Roberts ${ }^{2}$ collected five further cases, and added

${ }^{1}$ Brit. Journ. Derm., Feb., 1897.

2 Ibid., April, 1900. 
one of his own. Another case was published by Marschalko. ${ }^{1}$ The author also had two cases under observation, the patients being father and daughter.

Clinical features.-In one of my cases the father, aged 54, a nervous subject, was troubled with " rheumatic" pains about the ankle-joints at the age of 20 , when the tumours were first noticed. These were situated on the left side of the chest, and the man's attention was first called to them by pain described as being "'like the cutting of a knife," and greatly aggravated by cold. The daughter, who suffered from neuralgia, had similar tumours in various parts of the body. They began as hard red pimples on the leg (Plate 62), which caused "cramp," aggravated by cold. Similar growths appeared later in the right hypochondriac region and on the arm: as a rule several appeared at once and at some distance from each other; occasionally they coalesced. They were not painful for four or five years after their first appearance, but as they grew larger they became tender, and were the seats of frequent attacks of neuralgic pain.

Superficial myoma generally occurs in the form of nodular tumours on the arms, back, chest, and cheek. The deeper kind occurs as a solitary tumour, chiefly on the breasts and the genitals. The superficial growths are soft, elastic, and often very painful. They are sometimes sessile, sometimes pedunculated; they do not, as a rule, attain a very large size. The back is the commonest site, but they may occur on the scrotum, the nipple, and in other parts. They develop very slowly, and often start in an ecchymotic spot: The skin over them is generally red, but may be natural in colour. Sometimes they undergo involution, but as a rule they slowly increase in size and also in number,

${ }^{1}$ Monats. 1. prakt. Derm., Oct. 1, 1900. 


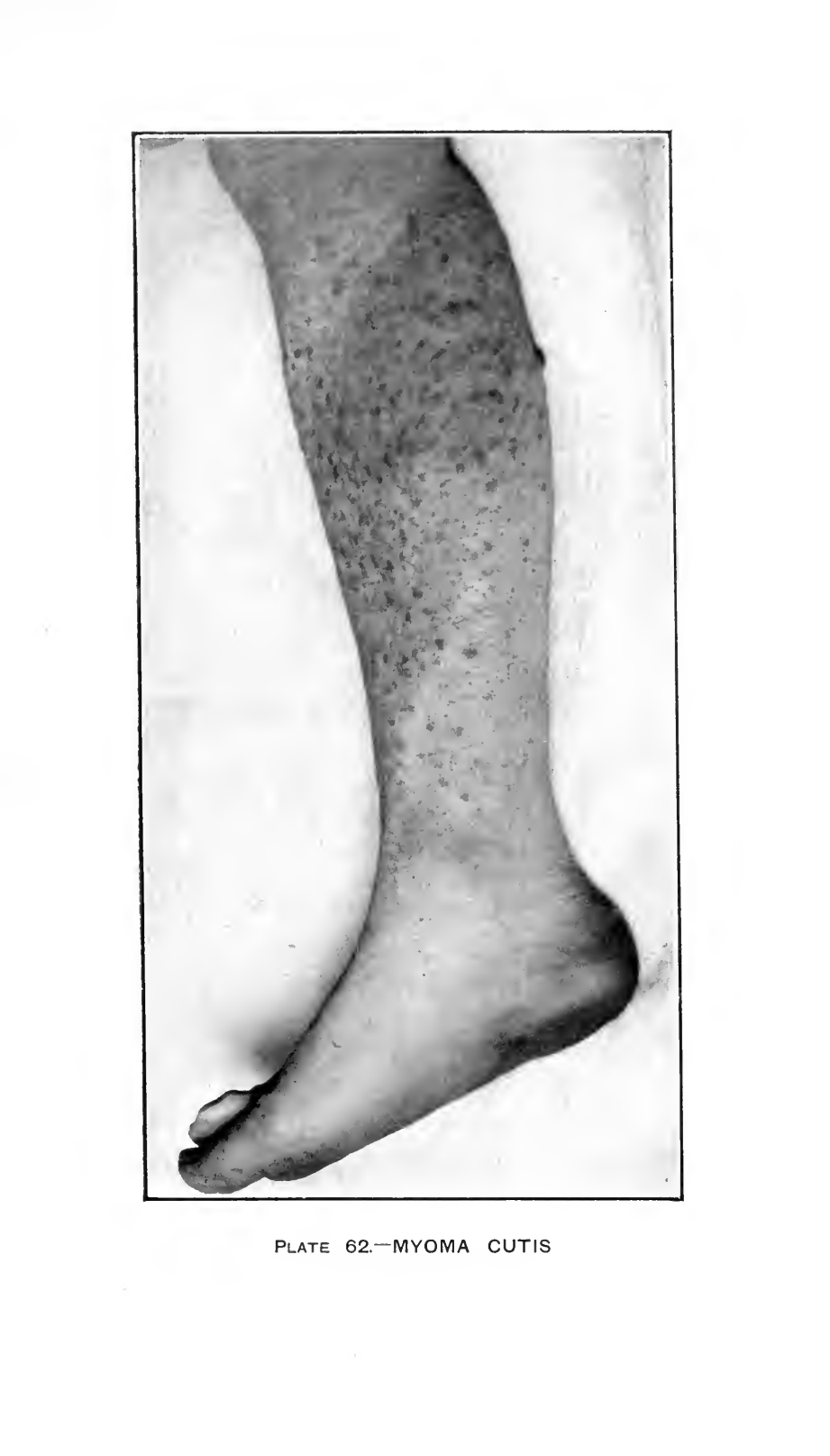



often coming out in crops. As they develop they become more painful.

Diagnosis and pathology.-The distinctive clinical feature of myoma cutis is that it contracts under the influence of cold. But the diagnosis can be made only by exclusion. ${ }^{1}$ The growth is principally made up of unstriped muscular fibre, with which may be mixed a greater or lesser amount of fibrous tissue, constituting fibro-myoma; or the structure may be largely erectile (angio-myoma); or the lymphatics may be involved (lymphangio-myoma). These growths may arise from (1) the vessel walls, (2) the arrectores pilorum, (3) the deep layer of unstriped muscle in the nipple, scrotum, etc.

Treatment.-The only treatment for myomas, of whatever kind, is to remove them by surgical methods when they become inconvenient from their size or are seriously painful.

Neuroma, so far as it affects the skin, has been described under the head of Neuro-Fibroma.

\section{MYхома}

Myxoma, when it arises in the skin, usually forms rounded pedunculated translucent tumours. It is commonest in the loose slin of the scrotum and the labia, but it may occur in any part. The growths are usually multiple. They tend to enlarge slowly. The gelatinous appearance of the tumours is characteristic. The absence of a central depression distinguishes them from molluscum contagiosum. They are made up of lax tissue, chiefly fibrous, with wide interstices filled with

1"A Case of Myoma Multiplex of the Skin" (illustrated). Crocker, Brit. Journ. Derm., vol. ix., p. 1, 1887. In a paper contributed to the same journal (Jan., 1907) Wallace Beatty described what he believed to be the twenty-seventh recorded case. 
mucilaginous matter. The treatment of myxoma consists in removing the growths by ordinary surgical methods.

Myxœdema may be regarded as diffuse myxoma. The condition belongs more to the province of general medicine than to that of dermatology.

\section{Telangrectasis}

Clinical features.-Telangiectasis is an acquired condition in which the capillaries are considerably dilated over a larger or smaller area of skin. The lesions are often stellate in shape, a number of vessels radiating from a raised central dot which is the enlarged loop of an arteriole.

These lesions are generally seen in persons with a delicate skin; occasionally they follow injury, and in a person who has been struck by lightning the vessels of the skin can sometimes be' seen clearly marked out as if they had been injected. Telangiectasis also occurs in the upper part of the body, and on the face and neck, particularly in elderly people, in the form of small spots constituted by small tufts of dilated capillaries. Clinically, telangiectasis resembles the slighter forms of vascular nævus. The condition is most common on the face, especially in persons much exposed to the weather.

Etiology.-Telangiectasis is often associated with inflammatory and other morbid processes, such as rosacea, adenoma sebaceum, etc. (Plate 63). Telangiectasis of the skin occurring in the subjects of Graves' disease have been reported by Létienne and Arnal $^{1}$ and by Nevins Hyde. ${ }^{2}$ The author and Dr. Dore showed at the Dermatological Section of the Royal Society of Medicine, in May, 1908, a case of acquired bilateral

${ }^{1}$ Arch. Gén. de Méd., 1897, s. vii., pp. 513-23.

${ }^{2}$ Brit. Journ. Derm., Feb., 1908, p. 33. . 

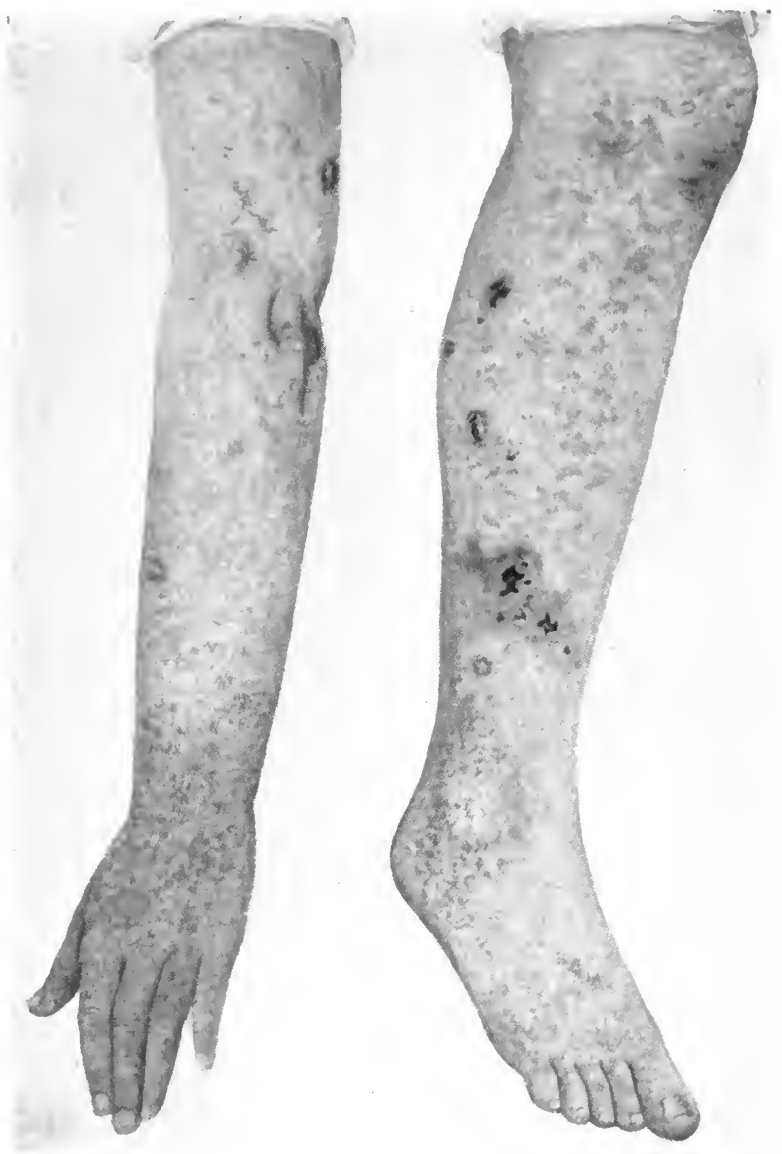

Plate 63.- telangiectasis with ulceration. 

telangiectases of legs and feet caused by pressure and exposure to cold. Colcott Fox reports a case of bilateral telangiectases of the trunk with a history of marked epistaxis in childhood and recent rectal hæmorrhage, and gives an account of other cases. ${ }^{1}$

Treatment.-If treatment is required, the dilated vessels should be obliterated by electrolysis or galvanocautery.

\section{Nevus Vascularis}

Cutaneous angioma is a congenital condition charac. terized by the over-development of the vascular tissue in the skin. Frequently at first cutaneous nævi resemble flea-bites; by the formation of new capillary vessels they cover a wider area, and constitute either the "strawberry mark" or, if somewhat larger, the "port-wine mark." If they remain smaller than these varieties, with dilated vessels at the outer part, the spider-like novus, novus araneus, or stellate novvus, is formed. With or without a growth of new capillaries in the corium there may be a new formation of veins in the subcutaneous tissue, thus constituting the capillary-venous or the venous novvus, soft, compressible, slightly lobulated tumours of greater or less extent, and either bright red or purple in colour according as the corium is or is not affected. Sometimes a venous nævus contains a considerable amount of fat, constituting the ncevus lipomatodes, which has the combined characters of a venous nævus and a lipoma.

Clinical features.-Capillary nævi occur most frequently on the face, head, and neck, but they are met with in other parts. Venous nævi are found on any part of the body, not infrequently on the lips and tongue. Nævi may be fully formed at birth and remain stationary throughout life. Frequently,

${ }^{1}$ Brit. Journ. Derm., May, 1908, p. 145. 
however, they are small at birth and extend slowly for some years, after which they remain stationary. Very many nævi which are present at birth disappear within a few months. Slight injuries to venous nævi frequently eause hæmorrhage or result in inflammation which may lead to extensive ulceration or to spontaneous eure by thrombosis or sloughing. Cysts may form from obliteration of vessels.

Pathology and etiology.-The microscope shows the growth to consist almost entirely of blood-vessels. In the case of the nævus lipomatosus, there is also a good deal of new-formed fat. Of the causation of the condition nothing is known. The newly-formed vessels arise from pre-existing vessels in the corium or subcutaneous tissue.

Treatment.-Vascular nævi occasionally disappear spontaneously, but more frequently they either remain stationary or become larger as the child grows, or else they grow rapidly. In very slight cases there is always a chance that the condition may disappear spontaneously; compression of the dilated vessels, as by the application of collodion, may help to bring this about. Various methods of treatment have been found successful. In suitable cases good results have been obtained from applications of radium. To Louis Wickham, of Paris, the credit is due for the pioneer work with this potent remedy. Cases of ordinary raised nævus may be treated successfully with carbon-dioxide snow, which is at once simpler and more rapid and less costly than treatment by radium. Treatment with liquid air is less effectual on account of the absence of pressure, and much more costly. Inflammation induced in the nævus will sometimes effect a cure. The X-rays should not be used for this purpose, but vaccination or the injection of irritants and astringents, such as tincture of iodine, perchloride of iron, or tannin, may be found of service. 
Electrolysis gives good results in suitable cases. The needle must be passed entirely through the tumour in several directions. Multiple puncture with the galvanocautery has also proved satisfactory in some cases, especially for spider nævi. Caustics, such as fuming nitric acid and acid nitrate of mercury, have their advocates. In choosing a method it is necessary to have regard to the size and structure of the nævus, and the nature of the scar that is likely to be left. For large projecting nævi the best treatment is $e x-$ cision.

\section{Nevus Anfemicus}

This is a rare condition, first described by Vörner in 1906, in which areas paler than the normal skin occur. It is frequently associated with telangiectasis. Vörner considers it to be due to an error of development, the place of arteries and veins in the affected areas being taken by capillaries. Fischer's view is that it is the result of some nervous disturbance of vessel innervation.

\section{Nevus Pigmentosus (Mole)}

Pigmentary nævus has no pathological or clinical relation with vascular nævus, though the two are frequently present in the same person.

Clinical features.-The lesions consist of pigmentary macules, or slightly raised pigmented patches, with or without an excessive growth of hair. The ordinary smooth, pigmented mole is known as noevus spilus. The distribution of pigmented nævi is usually irregular, but sometimes they form bands, as a rule unilateral; they are then termed linear navi. The pigmentation, which Fabry ${ }^{1}$ holds to be hæmatogenous, may be slight or absent (white moles-Hutchinson).

1 Arch. f. Derm. u. Syph., Feb., 1902. 
Sometimes moles are more distinctly raised, and contain a quantity of fat. Another variety consists of pigmented papillomas, which may have a wide extent. These moles, which are not hairy, are called verrucose navi.

Moles are commonest on the face, neck, and trunk, but they are also met with on the limbs. Usually small, in some cases they occupy extensive surfaces. They are often single, but frequently occur in great numbers. They have been observed to be distributed in the region supplied by a particular cutaneous nerve. Warty moles are usually unilateral. Congenital in origin, or developing very soon after birth, they remain stationary or grow with the growth of the individual. Not infrequently they become the starting-point of carcinoma or of sareoma, usually of the melanotic variety. Some have maintained that all melanotic growths of the skin have their origin in moles, but, after a careful examination of the subject, Wilfrid Fox ${ }^{1}$ concludes that, while in the vast majority of cases such growths originate in the skin, both melanotic carcinoma and sarcoma may arise entirely apart from moles. This observer believes that the process by which a mole is formed in an infant is identical with that which is seen when a mole becomes malignant, and suggests that every mole in an infant would be a melanotic carcinoma but for the power possessed by the young corium of cutting off the cells, a power which may be lost after middle age or severe injury. He also concludes that the pigment appears to be intimately connected with the prime eause by virtue of which moles become malignant, whatever that cause may be.

Treatment.-If moles cause great disfigurement or threaten to become malignant they should be destroyed by means of carbon-dioxide snow. If this treatment is

1 Brit. Journ. Derm, Jan., Feb., March, 1906. 
not suitable they may be freely removed with the knife. They should never be irritated with caustics, lest they become malignant. This caution applies especially to melanotic moles, which should either be removed by a wide excision or left alone.

\section{Angio-Keratoma}

This rare affection is characterized by the development of capillary telangiectases, with small warty growths, on the hands and feet, chiefly on their dorsal aspects. It has been known, however, to occur on the scrotum.

Clinical features.-The initial lesions are tiny red or violet spots, at first discrete, afterwards becoming clustered into irregular groups and forming small patches distinctly raised, rough on the surface, hard, and sometimes covered with thickened epidermis so as to resemble warts. They become paler on pressure, but the blood cannot be completely forced out of them, a small bright red or black spot, clearly representing a capillary loop, always remaining in the centre. On pricking them blood escapes. No retrogressive changes occur in the lesions, but fresh telangiectases develop from time to time. The condition gives rise to no subjective symptoms.

Etiology.-Angio-keratoma commences, as a rule, in early adult life, and is always associated with a marked tendency to chilblains. Most of the patients in the cases so far recorded have been young women. There appears to be in some cases an hereditary element in the disease. I have had under my care a father and two children, the condition in the latter being congenital. Though all of them suffered from chilblains, no telangiectases developed on the parts affected by chilblains. Pautrier holds angio-keratoma to be a tuberculous affection, but in summarizing his paper MacLeod ${ }^{1}$ justly remarks that

\section{${ }^{1}$ Brit. Journ. Derm., Aug., 1904.}


if the slight histological resemblances and the clinical associations described by this author are sufficient ground for including this affection among the tuberculides, many other forms of dermatitis might be brought within the group. ${ }^{1}$ S. E. Dore reports a case of angiokeratoma with chilblain circulation, erythema pernio, and Bazin's disease, which appears to bear out the view that the inefficiency of the circulation is a predisposing factor rather than a result of tuberculous infection.

Pathology.-The essential feature of the process is telangiectases resulting from repeated temporary dilatation of the small blood-vessels. The hyperkeratinization which gives the fully-developed lesions their warty appearance is a secondary change. The lesions show no tendency to spontaneous involution, and fresh ones usually come into existence every winter. Angiokeratoma can hardly be mistaken for anything else.

Treatment.-Electrolysis, which has been successfully employed by Pringle, is the best treatment. A fine steel needle connected with the negative pole is inserted into the telangiectasis, the positive pole being held in the other hand, and a current of two to three milliamperes allowed to pass for thirty seconds. The procedure causes some pain, but is followed by only slight scarring. The general measures for the improvement of the circulation in persons subject to chilblains are also indicated.

\section{Infective Angioma}

Under this name Hutchinson has described an affection characterized by minute red points "like grains of cayenne pepper" embedded in the skin. The lesions are arranged in groups which spread out peripherally while clearing up in the centre, thus forming rings. Outside these, fresh points or "infective satellites" arise, and by the meeting of adjacent rings large

${ }^{1}$ Brit. Journ. Derm., Sept., 1903, p. 323. 
areas of skin become affected, the lesions having the gyrate serpiginous outline common in such circumstances. Most of the little points can be obliterated by pressure, but some, larger than the others, cannot. The limbs are generally the seat of the affection, which has also been seen on the face and trunk. The disease spreads slowly, with intervals of remission. It begins in early life, vascular nævus appearing sometimes to be a predisposing factor ; indeed, Jamieson thinks the condition itself is simply a superficial nrevus, and with this view I agree. Hutchinson, on the other hand, looks upon it as a kind of lupus, and allied to lymphangiectodes. The fact of the development after birth and the serpiginous character of the telangiectases will suffice to identify the affection.

Treatment.-The only procedure that seems likely to be successful is electrolysis.

\section{Angio-Fibroma Contagiosum Tropicum}

Unna and Bassowitz have described, as occurring in Southern Brazil, an affection consisting in an eruption of red papules which quickly develop into nodules varying in size from a large pea to an almond. Any part of the cutaneous surface may be affected, and the mucous membrane may also be attacked. In some cases, as the result of secondary infections, the nodules ulcerate ; in others, they disappear spontaneously. Of the etiology of the affection, nothing is known.

Treatment.-Bassowitz's treatment is to inject into the base of the nodules a few drops of formalin, or to apply the galvano-cautery, iron and arsenic being administered internally.

\section{LYMPHANGIOMA}

Lymphangioma circumscriptum cutis (Plate 64) is an affection characterized by an overgrowth and 
dilatation of lymph-vessels and the formation of new ones in circumscribed areas of the skin. Patches of greater or less extent are formed, covered with clusters of small vesicles. These are deep-seated and have thick walls, and sometimes have a superficial resemblance to warts. They have been met with on the limbs, the face, the neck, and the shoulders. They are pale or straw-coloured, sometimes marked with red striæ, and contain clear alkaline fluid in which a few lymph corpuscles are found. The condition is very chronic, spreading slowly at the circumference, where fresh vesicles develop. In a case recorded by the author, ${ }^{1}$ the patient was a delicate, fair-complexioned little girl aged 7. The disease made its first appearance when she was a few months old, as a group of vesicles in the left scapular region; the affection spread slowly and caused but little inconvenience. There were no attacks of lymphangitis.

The affection is probably congenital, though generally first noticed in early childhood, but the conditions which lead to the development of the vesicles are involved in obscurity.. Pollitzer, as the result of histological examination, inclines to the view that a deep obstruction, either lymphatic or partly venous and partly lymphatic, is the first link in the chain of events that leads to the formation of lymphangioma. The sexes appear to be equally liable to the affection. Sometimes the patches are partly fibro-cavernous in structure, and the occasional association of the lesions with venous nævi suggests that the blood-vessels are, at least in some cases, concerned in the process. Bernard held, as does Brocq, that the disease is primarily one of the lymphatics, and that the appearance of blood is due either to the rupture of capillaries into the lymphatic dilatation, in which case 1 "Internat. Atlas of Rare Skin Diseases," fasc. i., pt. i. 


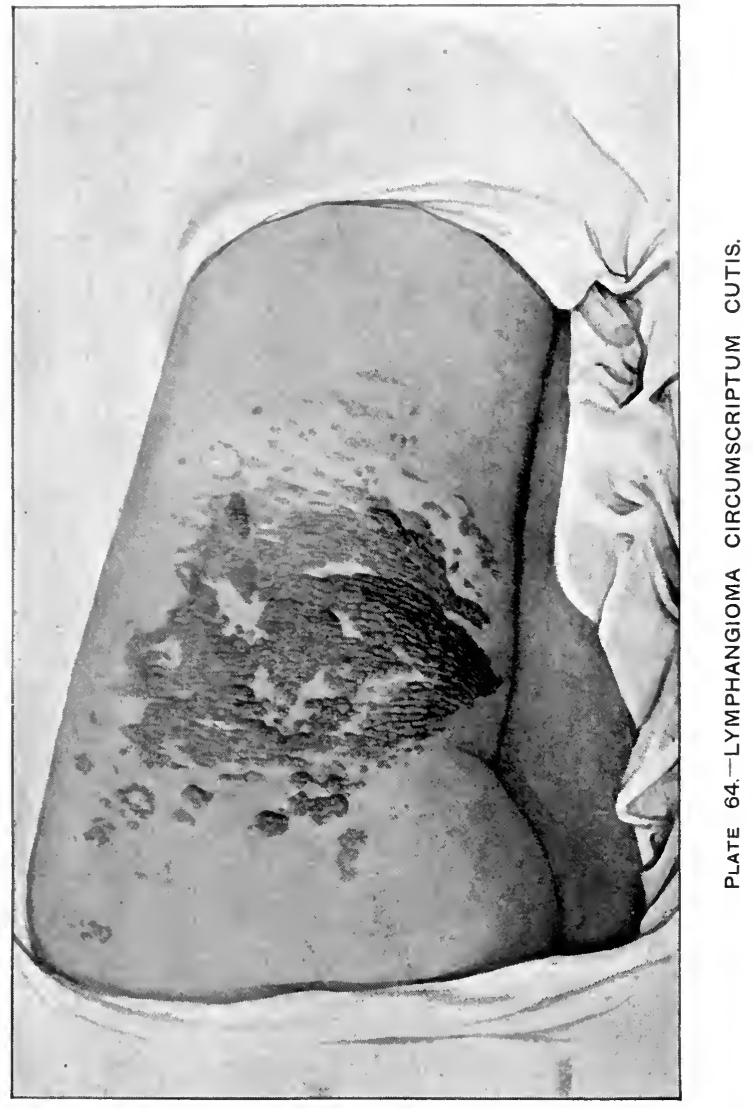



the fluid of the vesicle is pinkish, or to the rupture of capillaries into the floor of the vesicles but not into the cavity, this form giving rise to the appearance of a dark tuft in the centre of the vesicle.

In a case recorded by Hoggan, that of a boy aged 9 , dilatation of the lymphatics occurred after two attacks of inflammatory character in two successive years. The dilated vessels filled and became tense when the patient stood up, and collapsed, leaving only indefinite traces, when he lay down. Microscopic examination showed plexiform dilatation of lymphatics, the walls of which, as well as the surrounding tissues, were normal. For this condition A. G. Francis 1 has proposed the term "lymphoma simplex."

Under the name of lymphangioma tuberosum multiplex, Kaposi described a condition met with on the trunk and neck of a woman aged 32 ; it had been noticed during childhood, but had extended in the few years previous to her coming under observation. The lesions consisted of close-set vesicles, the size of lentils and smaller. Microscopic examination showed small lymphatic dilatations throughout the corium. Besnier and Doyon consider that such cases may be examples of cystic adenoma developed in the sweatglands. After reviewing all the cases reported, Francis concludes that they are examples of lymphangioma, and advocates the adoption of the term first proposed by Török, lymphangioma cavernosum. The etiology is very obscure, and many different names have been suggested for the affection, among them eruptiva

${ }^{1}$ See "Lymphangioma Circumscriptum Cutis," Brit. Journ. Derm., Feb. and March, 1893, where a comprehensive account of the whole subject is given ; also "Five Cases of Lymphangioma," Leslie Roberts, Brit. Journ. Derm., vol. viii., p. 309; “ Lymph angioma Circumscriptum s. Cystoides Cutis," Max Freidweiler Arch. f. Derm. u. Syph., Bd. xli., Hft. 3, p. 323. 
hydradenoma, cystic benign epithelioma, and epithelial cystadenoma.

Under the head of hæmato-lymphangioma, Francis has classed several groups of cases. One group eontains the modification of ordinary angiomas (nævi) of the skin and mucous membranes, termed "warty degeneration," the best known example of which is the "sago tongue." The white wart-like prominences contain cystic spaces filled with clear fluid. Most observers in England regard them as dilated lymphatics; others-for example, Besnier and Doyonconsider them to be the result of the occlusion of blood-vessels, and hold that the condition is allied to angio-keratoma.

In another group the primary condition is a wellmarked angioma, upon which a condition of lymphangioma afterwards develops. The first case was described by Tilbury and Colcott Fox. A man aged 21, born in Mauritius of English parents, had two large nævi on the left thigh, which had remained unehanged. At the age of 6 months the veins of the left calf began to enlarge. At the age of 2 years a number of little "warty" growths appeared on the skin of the left buttock, the flexor surface of the left knee, and the left half of the perineal region, on areas quite distinct from those occupied by the nævi. At the same time the patient had an attack of fever, which left him very prostrate for six months. On each occasion the skin affection became worse, the "warts" enlarging and becoming more vesicular in appearance.

Treatment.-In all these forms of lymphangioma treatment consists in destruction of the growth by electrolysis, by cautery, by X-rays, or by excision. Whatever the method employed, the operation must be thorough, or recurrence is almost certain to take place. 


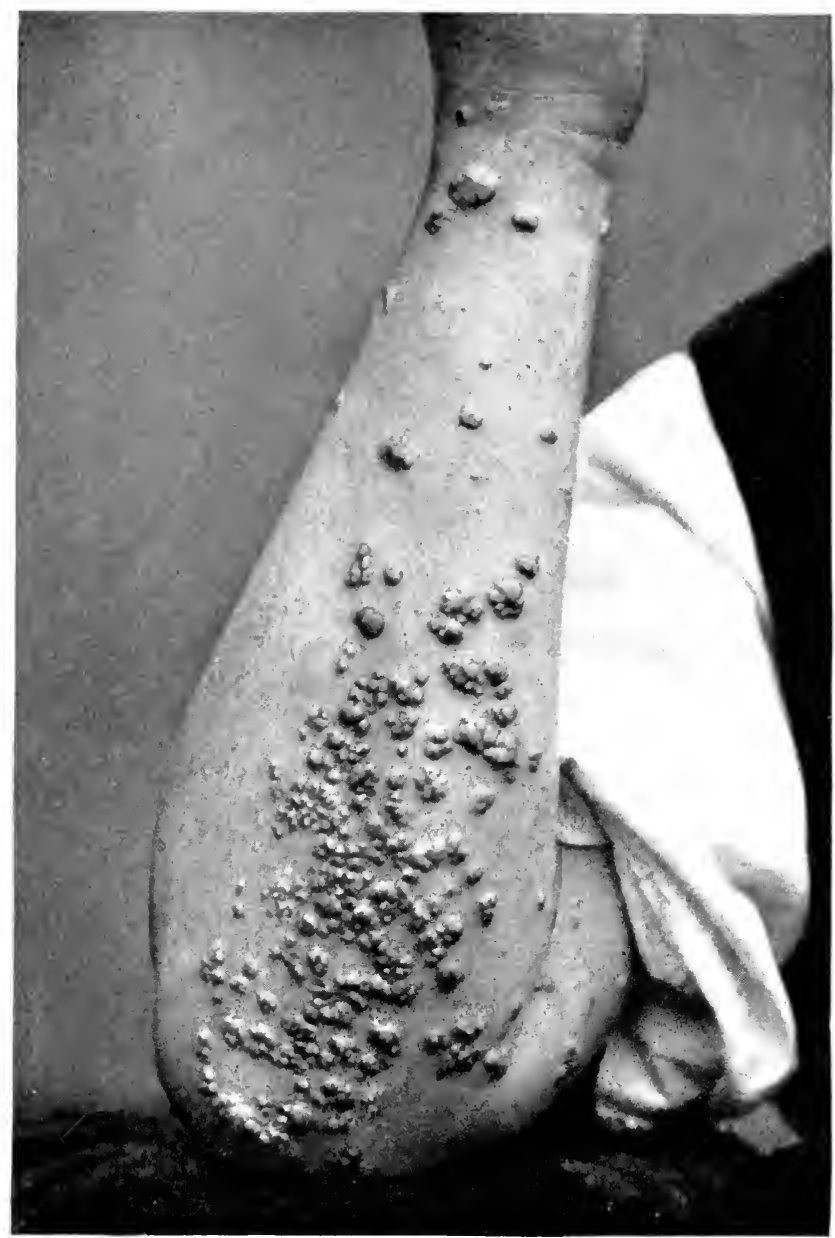

PLATE 65.-XANTHOMA DIABETICORUM.

(linnis (irsi') 



\section{Xanthoma}

This term is applied to a somewhat rare disease, first described by Addison and Gull, marked by the formation of plates or nodosities or larger growths of a yellow or yellowish-white colour embedded in the corium. If the lesions appear as plates the disease is styled xanthoma planum; if as nodules, xanthoma tuberosum; if as large growths, xanthoma en tumeurs (Besnier). In one form they are associated with glycosuria-xanthoma diabeticorum (Plate 65). There are two main types of distribution. In one, only the eyelids are affected (xanthoma palpebrarum); in the other the cutaneous surface generally, and also the mucous and serous membranes (xanthoma multiplex).

Clinical features.-Xanthoma planum is nearly always met with in the form of plates, very rarely of nodules. Occasionally cystic spaces form within the lesions. Commencing, as a rule, in the upper eyelid near the inner canthus on one side, it soon makes its appearance on the other side, and extends. In many cases the lower lids are affected as well as the upper, and sometimes a zone of xanthoma is formed, looking like a circle of wash-leather let into the lids. Xanthoma planum has also been met with on the ears, the nose, the mucous membrane of the mouth, the tongue, the palate, and other mucous membranes. The affection usually begins after the age of 40 ; when it appears in childhood it is generally as part of a xanthoma multiplex. In xanthoma multiplex the lesions are nearly always of the nodular form, but the plane variety is occasionally met with. The colour varies greatly; a mixture of blackish pigment with the yellow has been noticed. Linear grouping of lesions is often observed, especially along the lines of flexion. No part of the skin is exempt. The eruption is usually widespread, but it may be 
limited to one part. Although it generally starts on the eyelids, many cases are recorded in which these were spared. The condition has been noted in the mouth, pharynx, and esophagus, the respiratory passage, the aorta, the bile-duet, peritoneum, etc. The hands and the penis are often affeeted, and around the anus and in the gluteal folds the lesions may be present in great numbers, and by their aggregation constitute small tumours. Some cases are congenital, others begin within the first few years of life. In some of these early cases an hereditary disposition seems to have existed. The distinctive feature of the lesions is that they are embedded in the corium.

Xanthoma diabeticorum.-The special features of xanthoma diabeticorum consist in its rapid evolution, its swift and complete involution, and its association with diabetes mellitus or glycosuria. 1 drew attention to these peculiarities in $1883,{ }^{1}$ in eonnection with the fourth ease then on record. Since that time several other cases have been brought forward, and the affection is now everywhere recognized, though it is extremely rare. In a recent severe case of mine there was no sugar in the urine, nor did there ever appear to have been any; but the typical character of the lesions, and their disappearance about a week after the patient had been put on antidiabetie diet, left no doubt as to the nature of the case. The lesions are distinguished from those of other forms of xanthoma by the presence of a raised red area around the yellow spots. The spots appear first on the extensor surfaces of the limbs, next on the lower part of the back and abdomen, on the buttocks, and on the penis. They have also been met with on the palms in several cases. T'he eyelid is very rarely affeeted. The affection is commonest in young adults, especially in those inelined to obesity.

${ }^{1}$ Path. Soc. Trans., London. 
Pathology.-The process consists in the accumulation of large, often multinuclear cells, of connectivetissue type, filled with fat-drops. In addition to this there is a formation of new and a destruction of preexisting fibrous tissue. The epidermis, when not normal, is simply pigmented. The view of Chambard is that now generally received, namely that the affection is essentially of inflammatory nature, and that the xanthoma cells (which are practically the same as the cells met with in atheroma of arteries) are developed partly from leucocytes, partly from connective-tissue corpuscles. Krzysztalowicz, from a histological study of a case, specially insists on the hypertrophied connective-tissue cells with which the collagen and the fat lying in and between them build up the papule. The so-called xanthoma cells are, he sars, merely the greatly enlarged ordinary connective-tissue cells filled with fat. Pollitzer, as a result of the study of thirteen cases, concludes that xanthoma palpebrarum differs essentially in structure from the multiplex variety. The former, he holds to be a degeneration of embryonically misplaced muscle-tissue rather than a new growth, while xanthoma multiplex is an irritative hyperplastic growth of connective tissue of which the cells either produce fibrous tissue, or undergo degeneration. Pick's view is that the xanthomatous elements are not inflammatory products infiltrated with fat, or undergoing fatty degeneration, but are formed of matter of which the significance is not understood. James C. Johnston $^{1}$ believes the nodules of xanthoma multiplex to be neoplastic, and not inflammatory as in xanthoma diabeticorum. My own observations on xanthoma diabeticorum, made in conjunction with G. C. Henderson and Jackson Clarke, point distinctly to the process of that form of xanthoma being of inflammatory

\& Brit. Journ. Derm., June, 1905. 
nature. The elastic fibres remain unaffected. In rare instances xanthomatous plates have been met with in mucous membrane, in the peritoneum, and in the walls of the arteries. Malassez reports a case in which he found them on an ovarian cyst. ${ }^{1}$

Etiology.-Of the causation of xanthoma little is known, except that it is sometimes hereditary and congenital, and is often associated with glycosuria and with hepatic disorders. Galloway ${ }^{2}$ suggests that in most cases xanthoma multiplex is accompanied by irregularities of metabolism, associated especially with inadequacy of the functions of the liver. Cases of this form of xanthoma have been recorded by Köbner and others in which the lesions developed in capillary nævi and had a reddish hue. Xanthoma multiplex has also been found associated with thickening of tendons (Hutchinson) and with lesions both in tendon-sheaths and in the heart, and possibly in the liver (Cranston Low). ${ }^{3}$ MacLeod ${ }^{4}$ has shown a case of xanthoma tuberosum multiplex in which there were changes in the joints that progressed simultaneously with the cutaneous lesions. Xanthoma planum is commoner in women than in men, in the proportion of about three to one.

Diagnosis.-An ordinary case of xanthoma palpebrarum is easily recognizable from the distinotive appearance of the yellow plates, embedded in the corium, and almost imperceptible to the touch. Xanthoma

${ }^{1}$ A summary of all the cases recorded up to that date, and a complete account of a second one observed by myself, with the "esults of a histological examination by Jackson Clarke and a discussion of the whole subject, will be found in the Brit. Journ. Dirm., Aug., 1892.

2 Brit. Med. Journ., March 21, 1908.

3 Brit. Journ. Derm., 1910, xxii., loq. The case here described presented features of all three types of xanthoma.

4 Ibid., 1910, xxii. 267. 
multiplex may be mistaken for acne vulgaris until the lesions are punctured and found to be solid. In the early stages the lesions may also mimic those of lichen planus, but the resemblance soon ceases. From urticaria pigmentosa this form of xanthoma is distinguished by the absence of wheals and of itching, and the impossibility of inducing factitious lesions. In the early stage xanthoma diabeticorum may simulate lichen planus or acne, but the resemblance soon disappears.

Prognosis.-When it has reached its height, xanthoma palpebrarum persists through life. Xanthoma multiplex usually progresses for a time and then remains stationary. In xanthoma diabeticorum the prognosis is good so far as the skin lesions are concerned. They generally disappear in a few weeks, involution sometimes being preceded by an increase of itching in the growths. New lesions may, however, continue to come out for some time.

Treatment.-Under the careful application of the galvano-cautery or the thermo-cautery the lesions may disappear; good results have also been claimed for radium and the $X$-rays. The lesions may be removed with the knife in cases in which other methods are not suitable. In xanthoma diabeticorum the treatment must be antiglycosuric.

\section{Xanthoma of Balzer}

This extremely rare affection is characterized by hypertrophy and deformity of the elastic tissue in limited areas of the skin. The lesions have a general similarity to those of ordinary xanthoma. They consist of slightly-raised lenticular pinkish-yellow areas, soft to the touch, and having no inflammatory zone around them. In the only case of this affection that has come under my notice the patient was a young lady aged 21 . The lesions were situated on the left side of the lower 
part of the neck and the shoulder of the same side. They had appeared about puberty, and very slowly increased in size and numbers. On microscopic examination by Jackson Clarke the elastic fibres were found greatly thickened, fibrillated, and knobbed. There were neither fatty cells nor inflammatory exudation. In Balzer's case a slight inflammatory infiltration was present. The diagnosis can be made with certainty only by the aid of the microscope. The prognosis is bad, the affection, after progressing slowly, persisting indefinitely. No treatment has yet been found successful.

\section{Molluscum Contagiosum}

Molluscum contagiosum is characterized by the formation of small growths like tiny mother-of-pearl shirt-buttons (Hutchinson).

Clinical features.-The growths are roundish in shape and generally flattened on the top, where there is usually a depression, in which there is a small aperture leading into the interior of the tumour. Through this hole a whitish material, or sometimes a milky fluid, can be squeezed out, which under the microscope is seen to consist of oval bodies formed of degenerated epithelial cells. The little growths are firm in consistence. At first they are sessile, but as they develop they not infrequently acquire a pedicle. They are most commonly seen on the face, the eyelids being a favourite situation. They are also met with on the neck, the breast, the limbs, the genitals, and about the anus. They are never seen on the palms or soles. They are generally multiple, sometimes very numerous, and widely distributed. After attaining a certain size they may remain stationary for an indefinite time. They often undergo involution or drop off owing to strangulation of the pedicle. Sometimes they become inflamed and are destroyed by suppuration. 


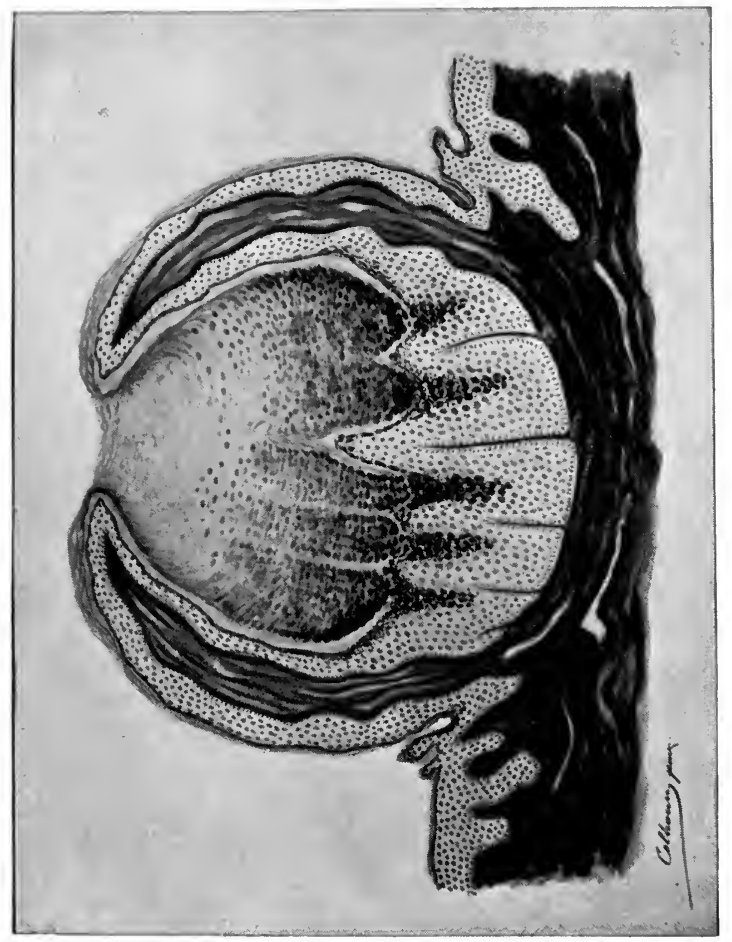

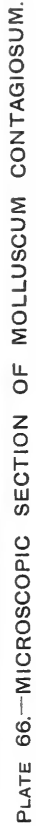



Etiology and pathology.-Molluscum contagiosum is most common in the young, and poverty seems to be a predisposing condition. The disease is generally believed in England to be contagious, and many cases are on record in which several members of the same family suffered from it at the same time. The infection may be conveyed from the mother's breast to the infant's face during lactation. Successful experimental inoculations have been made by Vidal and by Pick. ${ }^{1}$ It is clear, however, that the contagion is operative only under certain exceptional conditions, about which nothing is definitely known. The disease affects fowls and other birds, and is usually contracted from them; but it has been known to develop after the use of Turkish baths, probably as the result of infection. Some years ago at least half a dozen cases came under my care within a very short time of each other, in all of which the disease appeared to have followed a visit to the same Turkish bath. At Damascus it is known by an Arabic designation which means "itch of the bath." 2 Pick concluded from his experiments that there is a period of incubation lasting two months, and that the inoculated disease requires from three to four months for its complete evolution. In structure (Plate 66) molluscum contagiosum resembles a racemose gland, and many authorities believe it to be merely an enlarged and altered sebaceous gland. This view finds some support in the fact that the growth never occurs in the palms and soles, where no sebaceous glands exist. Virchow, however, believed that the hair-follicle is the starting-point of the process. His observations were confirmed by Thin, Crocker, and others; but the process is now known to consist in an overgrowth of the cells of the rete. Psorosperms, developing in the epithelial

1 Brit. Journ. Derm., 1892, p. 234.

${ }^{2}$ See paper by Norman Walker, Brit. Journ. Derm., 1910. xxii. 286 . 
cells, were considered by Neisser and Mansuroff to be the real etiological factors in the process, and a micrococcus has been found by Shaw. ${ }^{1}$ The researches of C. J. White and Wm. H. Robey, jun., under the direction of the Cancer Commission, have led them to the conclusion that the lesion in molluscum contagiosum is not due to the action of a protozoon, and is not analogous to cancer. $^{2}$

Diagnosis.-The appearance of the little pearly growths, with the central depression and the hole leading into the substance of the tumour, is characteristic. Sometimes when they are very small they resemble vesicles, and might suggest varicella. The microscopic examination of the contents will show the nature of the affection. A small tumour on the genitals has been mistaken for a chancre, but the presence of similar growths elsewhere and the other clinical aspects of the case will generally prevent such an error. For the diagnosis from keratosis follicularis, see p. 684 .

Treatment.-Molluscum contagiosum sometimes disappears spontaneously. Touching with pure liquid carbolic acid, followed by painting with flexible collodion, will often get rid of the tumours. But the best treatment is to split them from below upwards with the knife and squeeze them out; or they may be cut off.

\section{Colloid Milium}

This rare condition is characterized by small yellow, translucent, cyst-like formations in the skin, chiefly on the upper part of the face. They do not contain fluid, but a gelatinous material. Sometimes they become depressed in the centre and are slowly absorbed; sometimes they become inflamed and afterwards dry up. The affection occurs in both sexes, and does not generally

${ }^{1}$ Amer. Journ. of Cut. and Gen.-Urin. Dis., vol. x., 1892.

${ }^{2}$ Journ. Med. Research, April, 1902 
begin before puberty. The condition appears to be one of colloid degeneration of the skin. Spontaneous recovery has been known to occur, but the condition is refractory to treatment. Erasion with the sharp spoon, or electrolysis, might be tried. ${ }^{1}$

\section{Epithelioma Adenoides Cysticum}

The growth which bears this designation was first reported by Jacquet and Darier in 1887, under the name of hydradénome éruptif, and since then it has been deseribed by Török, Perry, Quinquaud, Philippson, Besnier, Fordyce, W. Pick, and others, each writer seeming to make it a point of honour to invent a new name for the tumour. The simplest of these names is benign cystic epithelioma, employed by Dr. Hartzell. The one used here was proposed by Brooke, ${ }^{2}$ who made a most painstaking investigation of the disease. The following brief description is mainly condensed from Brooke's.

Clinical features.-The lesions are small tumours, at first of the same colour as the surrounding skin, which afterwards, as they increase in size, become shining and translucent, but hardly sufficiently so to suggest that they contain fluid. Nearly all contain one or more minute white, brightly refracting, milium-like bodies. The little growths are firm, but not hard, and can be felt to be embedded in the skin. In the four cases observed by Brooke the most common sites of the growths were the space between the eyebrows, the root of the nose, the nostrils, the cheeks, the upper lip, and to a

${ }^{1}$ The affection was first described by Wagner, Arch. d. Heilk., Bd. vii., 1866. The pathology of the condition has been discussed by L. Philippson, Brit. Journ. Derm., vol. iii., 1891 ; and by Besnier, French translation of Kaposi, vol. ii., p. 370. See also a paper by C. J. White in the Journ. Cut. and Gen.-Urin. Dis., vol. xx., Feb., 1902.

${ }^{2}$ Brit. Journ. Derm., 1892, p. 269. 
less extent the chin. In these situations they were so thickly clustered together as to form disfiguring lumpy patches. The growths may occur on any part of the body from the occiput to the pelvis, and on the arms and legs. The lesions never attain any great size. They are painless, and the only symptom to which they give rise is slight prickling or itching. As a rule they show no tendency to ulceration, but occasionally they undergo malignant transformation. The course of the affection is very slow, subject, however, to sudden acceleration, even after it has lasted many years. It begins, as a rule, between the tenth and fourteenth years. E. Wood Ruggles ${ }^{1}$ has reported a case-the first of its kind-in which multiple benign cystic epithelioma and fibroma molluscum occurred consentaneously in the same patient, a girl of 12 , in whom the tumours first appeared at the age of 6 or 7 .

Pathology.-Clinically the growths seem to be absolutely benign, but histologically they are epitheliomas in the wider sense of that term, denoting only a tumour composed of epithelial elements and not necessarily malignant. Microscopically the growths, according to Brooke, consist of finger-like prolongations of epithelium coiled on themselves so as to form masses, in which are cysts filled either with purely colloid material or with concentric layers of flattened horny cells round a colloid centre. Some believe that the growth has its starting-point in the sweatglands, but Brooke holds that it originates directly from the epidermis and from the epithelium of the hair-sacs. In a case described by W. Pick there was marked proliferation of the epithelium at the periphery of the sebaceous acini. The new cells are probably of embryonic origin.

Treatment.-The usual treatment is removal by 1 Trans. Amer. Derm. Assoc., 1909, p. 231. 
excision or destruction with the cautery. Healing takes place readily. Fordyce succeeded in removing most of the larger tumours by means of the dermal curette, some of the smaller ones being expressed with a comedo extractor. In Wood Ruggles's remarkable case, after the high-frequency current and electrolysis had been tried with little benefit, striking improvement resulted from the application of carbon-dioxide snow. The cosmetic effect was admirable.

\section{Keratosis Follicularis}

Keratosis follicularis is a rare disease, which was formerly termed by E. Wilson ichthyosis sebacea cornea. The subject has been carefully studied by Darier, ${ }^{1}$ from whom the following account is chiefly derived. It was independently described simultaneously by J. C. White of Boston, and Darier applied the name "vegetating follicular psorospermosis" (psorospermose folliculaire végétante) to the process.

Clinical features.-The affection usually begins in early life, and is more often met with in males than in females. The lesions first appear as small brown or yellow crusts, which are removable after maceration, but readily form again. The crusts project sometimes as much as 3 to $4 \mathrm{~mm}$. above the surface. They are hard, dry, and adhere firmly to the underlying tissue. When detached, as they may be by squeezing with the fingers, they are found to present on their under surface a softish prolongation which dips into a follicle. This soft part can be squeezed out by pressing with the finger-nails. The . lips of the depression are slightly everted, and are redder and firmer than normal. The lesions are at first discrete, but may become confluent by extension, and the thickening of the affected parts increases so that nodular masses are formed, from which oozes an offensive discharge.

${ }^{1}$ Ann. de Derm. et de Syph., No. 7, July 25, 1890. 
The eruption in Darier's first case (a woman aged 30) began on the epigastrium and on the flanks, and rapidly spread to the sternal region, the face, and the scalp. Finally the whole of the trunk was affected, and also, to a slight extent, the limbs. In certain parts -e.g. the scalp, temples, naso-labial furrows, axillæ, groins, and anal cleft-the lesions were confluent three years after the commencement of the disease. In a second case recorded by the same author the eruption began over the sternum when the patient (a man) was 38. Seven years later the scalp was covered with yellowish-brown crusts, covering pits from which tufts of hair projected. The face, except the eyelids and the circumference of the orbits, was covered with papules, which were confluent at the roots of the hair of the eyebrows and around the mouth. The shoulders and the neck were moderately affected. The middle of the back was covered with a mass of lesions resembling large comedones. A similar condition existed on the sternum and epigastrium. On the hypogastrium, about the pubes, and in the groins the lesions constituted large hemispherical bosses, with a central pit, from which stinking puriform matter escaped. There were many lesions on the outer and posterior aspects of the forearms, and some in front of the anus. The inner surfaces of the thighs and legs were slightly affected. The palms and soles were studded with yellow dots from thickening of the horny layer.

In all the cases hitherto recorded the affection has been slowly progressive. Fresh areas may become rapidly covered with papules. In ten out of twelve cases the patients were males. The affection does not seem to react to any marked extent on the general health:

Pathology and etiology. - The lesions sometimes, but not invariably, implicate the hair-follicle, 
the outer part of which is dilated. The granular layer of the epidermis is slightly, the mucous layer greatly, thickened, and its interpapillary processes are enlarged. Some of the cells of these layers contain "round bodies" about as large as the epithelial cells themselves, and presenting a granular protoplasm and a nucleus with a doubly contoured nuclear membrane. The sebum-like plug which fills the depression contains numerous round or oval highly refracting "grains," in which a trace of a nucleus can sometimes be made out. Darier regarded the "grains" as psorosperms, which he believed to be the cause of the disease, and this view received the support of Malassez, Balbiani, and some subsequent observers, but Darier afterwards recognized that the corps ronds were simply due to cornification of an anomalous type. Török and Tommasoli, on account of the resistance of these bcdies to mineral acids and alkalis, had regarded them as products of degeneration, and Unna believes them to be largely the result of hyalin degeneration. Kreibich suggests that possibly the affection is angio-neurotic in origin. Audrey and Dalous hold the disease to be an expression of dystrophy of the whole epidermis, due to a congenital force which remains latent until liberated by conditions as yet unknown. In a case reported by Ormerod and MacLeod, ${ }^{1}$ and in others, the initial lesion occurred independently of the follicles, contrary to Darier's original view that the primary seat of the initial lesions was the funnel or upper third of the pilo-sebaceous follicle.

Bowen $^{2}$ is inclined to adhere to the view originally enunciated by J. C. White, that the process is essentially a keratosis of the mouths of the follicles. MacLeod regards the eruption as "a type of dyskeratosis asso-

${ }^{1}$ Brit. Journ. Derm., Sept., 1904, p. 321.

${ }^{2}$ Journ. of Cut. and Gen.-Urin. Dis., June, 1896. 
ciated with a peculiar cellular degeneration, which may affect any portion of the epidermis, and is frequently located at the upper third of the pilo-sebaceous follicle or the openings of the sweat-ducts." The name given to the affection by Darier is, as MacLeod points out, a most unfortunate one, since it is not essentially follicular, and is only vegetating in severe cases and in a late stage. "Keratosis follicularis" is not more appropriate, since the disease is not an ordinary keratosis and may not be follicular.

Diagnosis.-In the early stage keratosis follicularis may have to be distinguished from keratosis pilaris, but the distribution is different (see p. 722). When there is prolongation into a follicle there may be some resemblance to molluscum contagiosum; but the distribution is less limited than in that affection, the aperture in the individual lesions is larger, and the growths have not the characteristic pearly appearance of the molluscum bodies.

Treatment.-The only procedure which offers any chance of success is that proposed by Schwimmer, viz. to destroy the lesions as they appear with the thermocautery. A case described by Bukovsky showed some improvement under arsenic, but recovery was incomplete. Palliative measures are the application of salicylic and sulphur ointments, and the thorough cleansing of the offensive discharge by mild antiseptic lotions, dusting powders, or baths.

\section{Acanthosis Nigricans}

This extremely rare disease was described in $\mathbf{1 8 9 0}$ by Pollitzer and Janowski, and named by Unna. It had previously been described by Darier under the name dystrophie papillaire et pigmentaire. It consists in a roughening of the skin, with warty growths and a peculiar pigmentation (Plates 67, 68). 

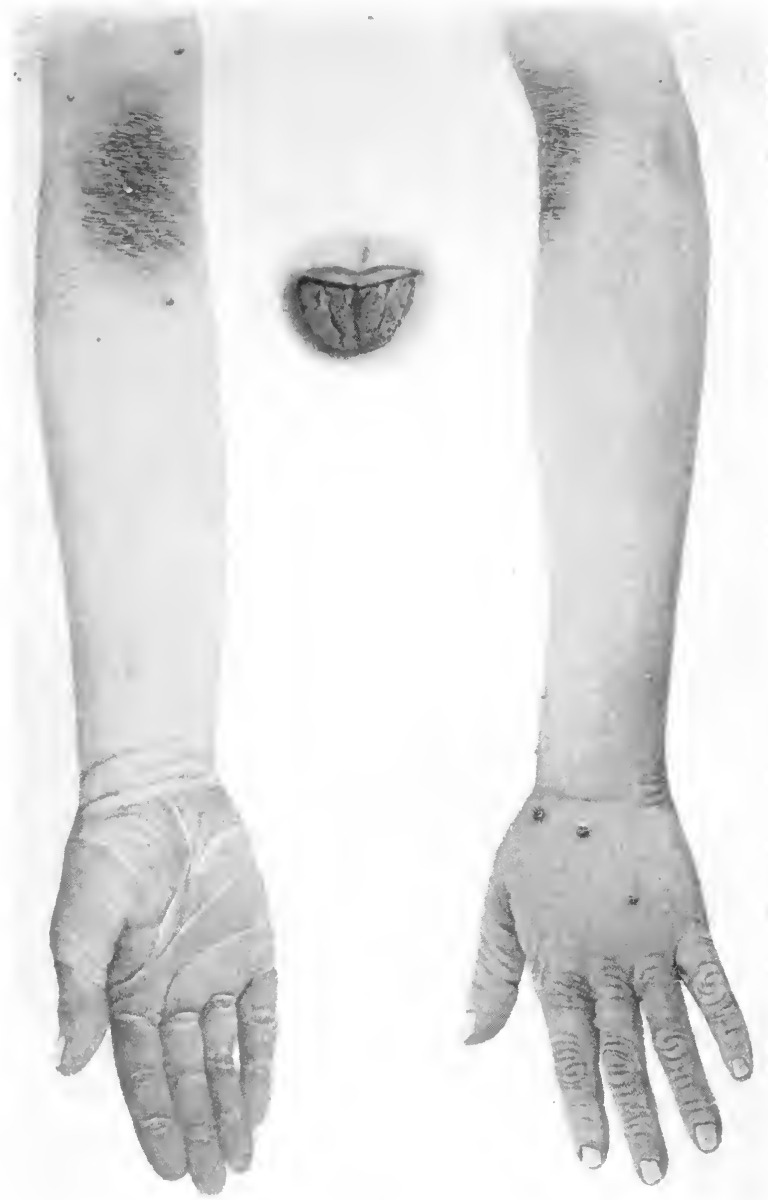

PLATE 67.-ACANTHOSIS NIGRICANS. 

Clinical features.-A harshness and bronzing of the skin about the neck and the front of the body generally are first noticed, followed by patches of deeper discoloration. Masses of fissured, wart-like vegetation, for the most part blackish in colour, appear in the axilla and umbilicus, on and about the breast and around the anus and genitals. From the fissures there is sometimes a viscid exudation. The natural lines of cleavage are deepened, and the thickened skin is closely dotted with warts, both sessile and pedunculated. The fingerends are usually deeply cracked, the nails thickened, dull, striated and brittle, the hair dry and wiry, and it may fall out, until the whole body is denuded. The mucous membrane of the mouth becomes dry and wrinkled, and warts appear; the tongue is swollen and fissured. In some cases the mucosa of the vagina is covered with warts, but none of the mucous membranes are pigmented. The distribution of the lesions is roughly symmetrical, and the pigmentation shows a disposition to follow the lines of cleavage and flexure. The patient is usually cachectic when the cutaneous affection first manifests itself, but there is seldom any pain associated with the condition of the skin. With the progress of the affection the warty growths become more luxuriant, and appear in fresh places, and the hypertrophy of the skin becomes more pronounced.

Pathology.-In structure the wart-like growths present the characters of papilloma. In a case of Darier's in which a post-mortem examination was made the suprarenal capsules appeared to be normal, but the nerves going to the capsule on each side were in contact, close to the gland, with cancerous growth. In another necropsy (Kuznitzky's case) the suprarenals were rather large, smooth and red; in the right one was a cavity, the medullary substance was red, and there was a thin, greyish-yellow cortex. In a case of my own the patient, 
though somewhat neurotic, had no symptom pointing definitely to abdominal disease, but for a year before coming under my care she had had a vaginal discharge, and I have reason to believe that after a period of apparent improvement she died of an "internal tumour." Microscopic examinations of scrapings of the pigmented skin were made by Jackson Clarke, and in them were found (1) epidermal scales, yellowish in colour, with hyphæ of fungi, spores, etc.; (2) a number of black, opaque, angular and irregular particles; (3) a few brown translucent granules resembling amorphous hæmatoidin. He concluded that the brown masses probably consisted of hæmatoidin from minute intra-epidermal hæmorrhages and that the black masses were adventitious. The pathology of the affection is still shrouded in obscurity.

Etiology.-In the majority of cases-twenty-five out of thirty, according to Darier ${ }^{1}$ - the affection is associated with cancer of the abdominal cavity, either primary or secondary, and Darier suggests that in such cases it arises from the action of the new growth on the abdominal sympathetic centres. It occurs twice as often in women as in men, and usually between the ages of 35 and 45 . In the very few cases which have been reported in young children there has been no associated cancer.

Diagnosis.- The only conditions from which acanthosis nigricans can require to be differentiated are Addison's disease, keratosis follicularis (Darier's disease), and arsenical pigmentation. In Addison's disease the bronzed skin is never rough or warty, the discoloration has not the same distribution, and the mucous membranes are pigmented. Keratosis follicularis is not associated with abdominal cancer, the lesions consist of closely agminated, crust-covered papules, and under 1 "Précis de Dermatologie." Paris, 1909. 


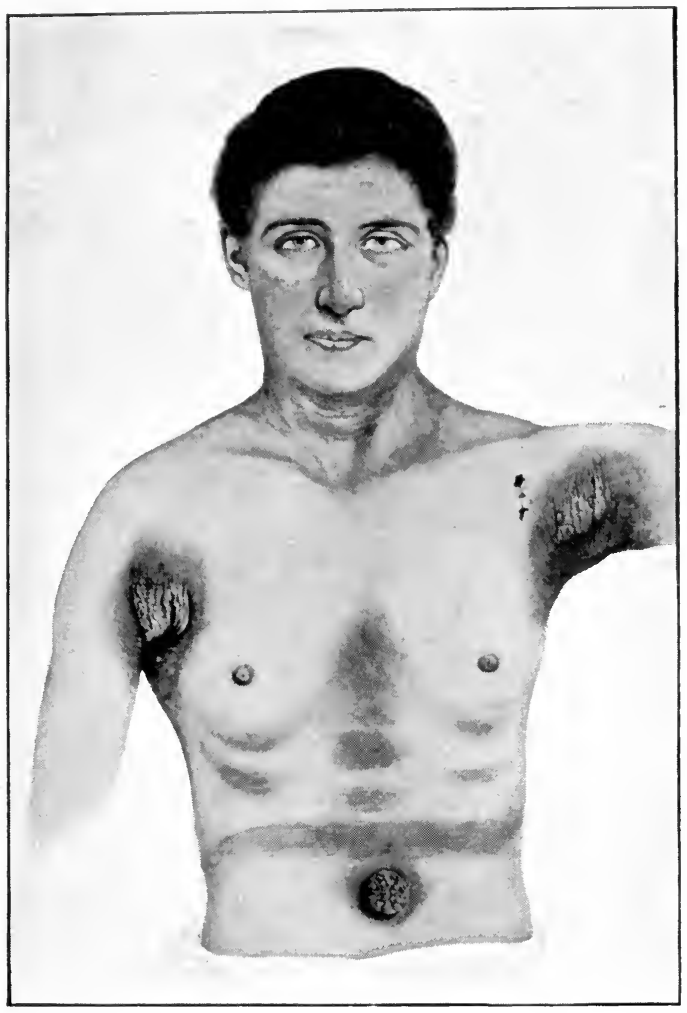

Plate 68.-ACANTHOSIS NIGRICANS. 


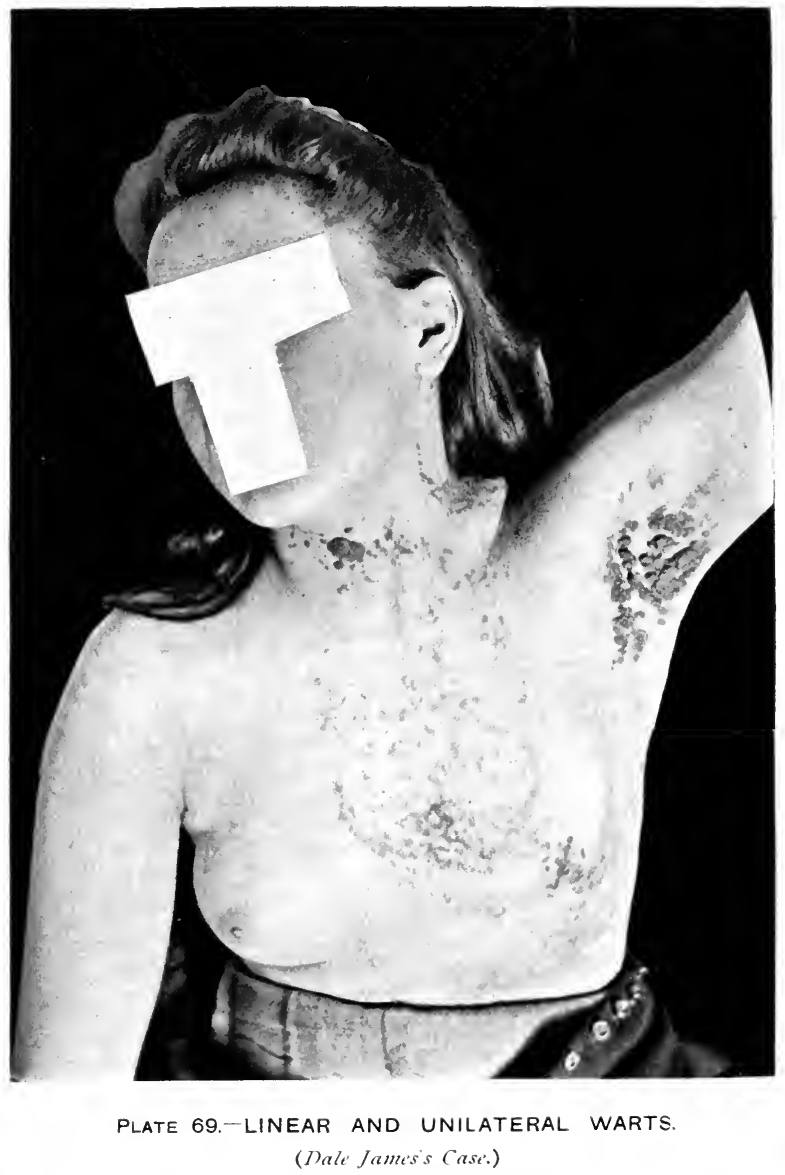


the microscope the corps ronds and granules peculiar to the affection can be seen. The pigmentation that is due to arsenic is more diffuse than the staining of acanthosis.

Prognosis.-When the affection is associated with malignant growth the prognosis is that of cancer. Hodara ${ }^{1}$ has reported a case in which acanthosis followed cancer of the breast, appearing two months after the beginning of the cancerous growth. After the removal of the breasts the pigmentation almost disappeared, but there was recrudescence with considerable extension six months later.

Treatment.-Should the warty growths become so large as to occasion inconvenience they may be excised, or removed by the application of caustics.

\section{Lentigo}

Clinical features and pathology.-The colour of the small pigmented spots popularly known as "freckles" is usually yellow or yellowish-brown, occasionally sepia. Their commonest situations are the face, especially about the nose and cheeks, and the backs of the hands. Sometimes they are seen on covered parts, such as the arms, the back, the buttocks, etc. Generally the number of them is moderate, and they are small and light in tint; occasionally the face is so thickly covered with them, and they are so large and dark, as to constitute a disfigurement. Pathologically, freckles are patches formed by the localized deposit of pigment in the basal layer of the epidermis.

Etiology,-Freckles are sometimes congenital, but generally first appear in childhood about the age of 10 . A fair, delicate skin is a predisposing condition. The exciting cause is sunlight; hence they are always most marked in summer, and fade more or less in winter. The

1 Monats. f. prakt. Derm., June 15, 1905, p. 629. 
affection tends to disappear as age advances. In rare cases freckles develop in adult life and in old age, particularly on covered parts; in such circumstances the condition is probably connected with impairment of nutrition or senile atrophy. A freckle-like pigmentation is sometimes associated with osteo-arthritis.

Treatment.-If treatment be considered necessary, the indication is to remove the patches by inducing localized blistering or desquamation. The best remedy is a solution of perchloride of mercury, gr. ii or iii to the ounce of water, applied several times a day. Pure carbolic acid applied with a match to each spot separately, and salicylic acid used in the form of Unna's plaster mull, are also useful. In most cases, however, the removal is merely temporary.

\section{Papilloma}

Papilloma of the skin includes various conditions characterized by the formation of papillary growths. These may be of syphilitic, tuberculous, cancerous, or inflammatory nature, and as such they are treated of in connection with the process of which they are the result. In this section only innocent papillary growths arising independently of any general process are considered. Such growths are often, in Continental dermatological literature, designated nævi, a term which is used in this volume in a much more limited sense. ${ }^{1}$ Sometimes the distribution of the growths is linear and unilateral, as in the case of Dale James's figured in Plate 69.

Warts are of several kinds, the differences being in their shape, general appearance, and situation; structurally they are all essentially the same. The common wart (verruca vulgaris) is generally seen on the hands, but also on other parts, as a small sessile growth with

${ }^{1}$ See ante, p. 661. 
a surface at first smooth, afterwards roughened with enlarged papillæ, which can sometimes be seen projecting like coarse bristles; occasionally the little mass is fissured here and there to its base. The colour is at first that of the skin, but after a time, owing to changes in the keratin, and in uncleanly persons to griming with dirt, it becomes brown or even black. Warts may be single or multiple; sometimes they are seen clustered together on the fingers. The condition is most common in childhood, and tends to disappear with the advent of puberty. It may, however, persist much longer, and may even be developed in adult life. Warts give rise to no symptoms, unless they are of such a size or in such a situation as to interfere with the holding of a pen, etc.

Flat wart (verruca plana) may occur in youth, but is generally seen in old age. In young persons such warts are most common on the face (particularly the forehead) and on the backs of the hands; in the elderly the back and the arms are the ordinary situations. The warts are, as the distinguishing epithet implies, flat; they are smoother, as a rule, than the common wart, and often square in outline, resembling the papules of lichen planus, but distinguishable from them by being smaller, by being lighter in colour and more uniform in shape, by the absence of itching, and of any tendency for the growths to coalesce into scaly, infiltrated patches. The changes found on examination are hypertrophy of all the layers of the epidermis, with elongation of the papillæ. In old people warts are often large and prominent, dark in colour, and associated with other senile changes in the skin. They are generally situated on the back, the forearms, and the face. They often itch intensely. Such a wart may form the starting-point of a malignant growth.

Another form of wart is characterized by raggedness $2 \mathrm{~s}$ 
of surface, the overgrown papillæ being separated and forming finger-like processes; hence this kind of wart is known as verruca digitata. Such warts are most common on the scalp, especially in women. They sometimes cause inconvenience in dressing the hair.

A long thread-like wart (verruca filiformis) is sometimes seen on the neck and the eyelids.

Seborrhœic wart (verruca seborrhoica or verruca plana senilis) occurs sometimes in young persons, but generally in old age. It is sometimes referred to under the name of senile wart, an obviously inappropriate designation, for not only is it not confined to the aged, but the growth is a condition of acanthosis rather than a true wart. L. Waelsch, therefore, suggests that it be called acanthosis verrucosa seborrhoica. The lesions consist of multiple patches of pseudo-warty growth on the back, arms, neck, sternum, and abdomen; the face is sometimes, though rarely, attacked. The lesions are generally more or less grouped in lines following the natural lines of cleavage in the skin. The patches are generally rounded in outline, and usually present varying degrees of pigmentation, from brown to black, associated in some cases with abnormal cornification. In cleanly persons the patches are of a pale fawn tint, and the surface, which is soft and greasy to the touch, has a reticulated appearance. In those who wash seldom and imperfectly the warts are covered with a dark crust of dirt which can be scraped off with a knife; a pinkish-yellow reticulated surface will then come into view. The only symptom to which the warts give rise is itching, which, especially in elderly persons, may be very troublesome. According to Pollitzer, the histological characters of the growth are slight thickening of the horny layer, with considerable hypertrophy of the Malpighian layer, while in the papillary and subpapillary layers there are epithelioid 
cells in groups and lines separated by bundles of connective tissue, with a peculiar infiltration of fat, affecting the coil-gland epithelium, the middle and papillary layers of the cutis, and the epithelium of the rete, and perhaps some atrophy of the sebaceous glands and hair-follicles. He looks upon these warts as benign growths developed out of "misplaced" embryonic cells, resembling in respect of the presence and peculiar arrangement of the epithelioid eells the growth called by von Recklinghausen "lymphangio-fibroma." The fatty infiltration in the skin may perhaps be regarded as evidence of a seborrhœic process, if Unna's view that the coil-gland glomerulus not only secretes sweat, but is the chief source of fat for the skin, be accepted. Poór ${ }^{1}$ holds that senile warts are due simply to failure of the sebaceous glands to extrude their secretion while the openings are blocked by the abnormal cornification typical of senility, cystic degeneration thus being set up.

Venereal warts (verruca acuminatce) are papillary excrescences usually seen about the genitals in both sexes, and sometimes in the axillæ and other moist, warm parts. They are generally reddish in colour, pointed, tufted, or cauliflower in shape, bathed in decomposed sweat and purulent discharge; sometimes, especially about the vulva, they grow with an unrestrained luxuriance. These warts are nut syphilitic, but they are most frequently gonorrhœal in origin, proliferation of the papillæ being due to the irritation of the discharge. Somewhat similar warts are sometimes seen in pregnant women who have not suffered from gonorrhœa. In such cases the warts quickly disappear after delivery; but the gonorrhœal warts show little tendency to do so, and may continue to grow for years. The latter are highly contagious.

${ }^{1}$ Derm. Zeitsch., Bd. x., p. 462. 
Etiology.-Except as regards the form last mentioned, the causation of warts is obscure. The flat wart, as has been said, is sometimes a result of senile degeneration of the skin; and Jamieson says he has in some cases traced the origin of the digitate wart to the use of rancid hair-oil or pomade. ${ }^{1}$ The popular notion that the common wart is auto-inoculable is no doubt well founded; and it is not unusual to see a large wart surrounded by several smaller ones derived from it. Cocci and bacilli have been found in the little growths by Cornil, Kühnemann, and others, but the significance of these microörganisms is doubtful. Arthur Hall ${ }^{2}$ regards the sudden disappearance, under the administration of drugs so different as magnesium sulphate, nitro-hydrochloric acid, liquor arsenicalis, thyroid extract, and tuberculin, of persistent warts as pointing to their being of parasitic origin, and due to a microörganism of low vitality. In a later paper ${ }^{3}$ he reports a case in which warts of several years' standing disappeared when the patient's costiveness was corrected and free action of the bowels set up. Chalmers Watson, too, had a case, that of a boy of 13 , in which verrucæ planæ on the face and hands disappeared and verruca vulgaris of the hands improved on the administration of large doses of castor oil-a result which suggested to him that in some cases a chronic infection from the alimentary tract is an important etiological factor. $^{4}$

Treatment.-Ordinary warts may be destroyed by the galvano-cautery, or by an application of carbondioxide snow, or by cataphoresis with magnesium sulphate. Exfoliation may be caused by means of salicylic acid

1 “Diseases of the Skin," 1888, p. 375.

2 Brit. Journ. Derm., July, 1904, p. 177.

s Ibid., March, 1906, p. 107.

4 Ibid., May, 1903, p. 178. 
in the form of a plaster, or dissolved in collodion $(3 \mathrm{j}$ ad $\jmath_{j}$ ), and then applying chromic acid to the base of the growth. If this fail, a strong caustic, such as acid nitrate of mercury, should be used, with precautions to limit the range of its destructive action. A good method is to moisten the wart with strong acetic acid, and when damp to apply the solid stick of nitrate of silver. Radium also is effectual in many cases. I have seen warts completely disappear under $X$-ray treatment, but in some cases there has been recurrence. As the dose required has to be a large one and to be repeated, there is some risk of telangiectasis, and on the whole the other methods mentioned are to be preferred. Seborrhœic warts may be treated in the same way as ordinary warts.

Digitate warts should be removed with the elastic ligature or the knife, the galvano-cautery, or carbondioxide snow. Gonorrhœal warts, if very luxuriant, should be snipped off with scissors or destroyed with the galvano-cautery; if they are small they may be got rid of by applying chromic or glacial acetic acid. An important element in the treatment of these moist warts is to keep them dry and clean, and the surrounding parts protected from infection. The conditions (irritating discharge, etc.) keeping up the papillary hypertrophy must also be dealt with.

\section{Keratosis Senilis (Senile Keratoma)}

In this affection, often met with in elderly persons who have lived in the tropies, dry yellow or brownish spots, or warty growths like senile warts, or red telangiectatic spots with an irregular margin, appear, generally on the face. After a while the lesions are covered with a rough-surfaced, pigmented layer, which sends conical processes into the skin, so that its removal may cause a little hæmorrhage. The growths sometimes 
take on an epitheliomatous character. Their distribution, and the age of the patient, usually suffice to distinguish them from syphilides, from the lesions of lupus erythematosus, and from acne rosacea.

Treatment.-At an early stage resorcin and salicylic acid will cause the growths to disappear. Later, they should be removed with the knife; or the $X$-rays, carbon-dioxide snow, or radium may be applied.

\section{Verruca Peruviana}

In certain elevated valleys of Peru there occurs an affection known as verruca Peruviana, and sometimes as verrucome de Carrion, after the student who lost his life in an experiment that proved the acute febrile and the eruptive chronic types to be identical. In some cases the disease ends, either fatally or by recovery, with the febrile stage; in others the fever is followed by the appearance of numerous small elevations, usually on the extensor surfaces of the limbs. The verruca may be of a miliary kind-when the eruption resembles small ripe cherries-or larger, and both kinds may occur in the same case, and simultaneously. When the eruption appears the fever may diminish; if it persist, the prognosis is unfavourable. Escomel ${ }^{1}$ believes the disease to be caused by long, thin, straight bacilli, which he found in sections examined by him. The treatment is the same as for yaws, ${ }^{2}$ to which the affection offers much clinical resemblance.

\section{Clavus}

Corns are circumscribed thickenings of the epidermis, in the centre of which a horny peg or nail (hence the name clavus) projects downwards among the papillæ

${ }^{1}$ Ann. de Derm. et de Syph., Nov., 1902, p. 961 (abstr. in Brit. Journ. Derm.).

2 See ante, p. 541. 
so that its point rests on the sensitive cutis, causing sharp pain when driven inwards by pressure. Corns also "shoot" spontaneously, especially under the influence of barometric depression. The most common situations for corns are the outer surfaces of the little toes, the upper surfaces of the other toes, and the sole, especially the part where the weight of the body falls in walking. A softer but not less painful kind of corn often forms between the toes. Pressure and friction are the causes chiefly responsible for corns, but some persons show a much greater proclivity than others to their production. They may be congenital, or at any rate may develop in early childhood on feet that have never been imprisoned in tight or ill-fitting boots. Anatomically, the condition is hyperplasia of the horny layers. Corns sometimes become inflamed, and suppurate, and break down into deep ulcers.

Treatment.-The corn may be removed by the application of salicylic acid in a plaster or ointment, in the following form :-

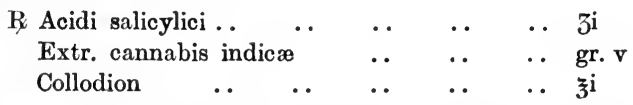

This should be painted on with a camel-hair brush or a glass rod after the corn has been soaked in hot water and the top shaved off. After a day or two the thickened epidermis can easily be picked off. A method which I have found most successful is to soak the corn with acetic acid and then rub it thoroughly with nitrate of silver. After treatment the part should be protected from pressure by perforated pads of felt plaster, and boots adapted to the shape of the foot should be worn. Good powders for soldiers and others whose feet are tender are:-Acid. salicyl. 3i, French chalk ad ろiv; and Acid. salicyl. ऊiv, pulv. acid. boric. ऊii, talc ad ऊiv. 


\section{Caluus}

Callosities differ from corns chiefly in the absence of the "peg." The thickening of the epidermis may be congenital, but is usually acquired. It occurs on parts exposed to pressure, as on the hands of labouring men, the fingers of harpists, etc. Callosities may also develop on the feet from the pressure of boots or from going barefoot. The condition seldom calls for treatment, but if any is required the hypertrophied horny layer can be got rid of by means of salicylic-acid plaster.

\section{Cornu Cutaneum}

Horny excrescences resembling the horns of animals have in rare cases been observed in human beings. The horny excrescences sprout generally from the scalp, the forehead, the temples, sometimes from the face, the extremities, the genitals, and the trunk. They are not painful, except when injured; occasionally they are the starting-point of malignant disease. They are rare under middle age, but have been observed in children. They are essentially overgrown warts (Crocker). In most cases cornu cutaneum originates in sebaceous cysts; sometimes in warts or cutaneous injuries, or scars, or in a broken-down molluscous tumour of the eyelids (Jamieson). Whitfield has reported a case in which a horn developed on the site of a cut on the left knee in a patient affected with atrophic lichen planus. ${ }^{1}$ The horn should be removed and the base thoroughly cauterized.

1 Brit. Journ. Derm., 1910, xxii. 360. 


\section{CHAPTER XXVIII}

\section{NEW GROWTHS}

\section{MALIGNANT NEOPLASMS}

THe essential feature of a malignant growth is that in its extension it does not thrust aside the structures in which it grows, but destroys them and takes their place. A tumour may be locally malignant-that is, it may spread indefinitely from a given centre and recur after removal, not, however, becoming generalized in the system-or it may be malignant in the full sense of the word, not only invading the surrounding parts, but giving rise to secondary formations in distant regions. Thus sarcoma is often only locally malignant, while carcinoma is typically malignant. The group of malignant growths affecting the skin includes Paget's disease, carcinoma, sarcoma (of various types), epithelioma, mycosis fungoides, and xerodermia pigmentosum.

\section{Paget's Disease}

This affection, the individuality of which was established by Paget in a paper based on the study of fifteen cases, ${ }^{1}$ has since that time been the subject of many memoirs, one of the most important being that of Wickham. ${ }^{2}$ It occurs chiefly in women after the age of 40 .

1 St. Bartholomew's Hosp. Repts., 1874, p. 83 et seq.

2 "Contribution à l'Étude des Psorospermes cutanées et de certaines Formes de Cancer." Paris, 1890. 
Clinical features.-The first visible lesion is reddening of a patch of skin on or around the nipple, which has the appearance of an inflammatory hyperæmia, followed by branny desquamation. The infiltration soon deepens, producing a bright-red granular, distinctly indurated surface, from which there usually oozes a sticky yellowish discharge. This may form crusts and obscure the nature of the lesions, save at the border, which is characteristic, being sharply defined, indurated, and sometimes distinctly raised. In the later stages of the disease itching and burning are the chief subjective symptoms. The process usually commences in the nipple and areola, but undoubted instances have been recorded of its attacking the skin of the breast away from the nipple, the scrotum, the scrotum and thigh, the penis, the glans penis, the anus and perineum, the abdominal wall, the axilla, the umbilicus, and other parts. Dubreuilh has recorded a case in which the vulva was the seat of the disease. After a period, which is usually about two, but has been known to be. extended to thirty, years, deep-seated parts may become affected by the cancerous process. On the breast this shows itself by retraction and induration of the nipple, and the formation of a tumour in the substance of the gland.

Pathology and etiology.-The histological changes consist in great proliferation of the deeper layers of the epidermis and inflammatory infiltration of the corium. In the thickened epidermis the bodies described as psorosperms by Darier, Wickham, and Jonathan Hutchinson, jun., abound. Most English writers have looked upon the cancerous disease in which the affection terminates as having no closer connection with the original malady than as being the effect of prolonged irritation; Thin, however, regarded the affection as cancerous throughout, and suggested the name " malignant papillary der- 
matitis." Wickham attributes both the affection of the skin and the cancer in which it terminates to psorospermal infection, but the bodies are now generally regarded as a peculiar form of cell degeneration. Fabry and Trautmann ${ }^{1}$ isolated from the skin, in one of their patients, a vegetable fungus of the yeast variety, which they held to be the cause of the disease and not a secondary infection. It readily grew on cultivation media and was pathogenic to white mice. But, as MacLeod remarks in his abstract of the paper, a fungus so readily isolated could scarcely have been overlooked in other cases had it been present. Whitfield's view, with which I agree, is that the change is definitely carcinomatous from the start, and is allied to that met with in malignant disease originating in moles. ${ }^{2}$

Diagnosis and prognosis.-The age of the patient, the bright-red granular surface exposed after removal of crusts, the induration, especially marked at the welldefined edge, the retraction of the nipple, with the obdurate nature of the affection, distinguish it from chronic eczema, which it most closely resembles. The diagnosis is made certain by the microscopic examination of scrapings in iodized serum (Darier) or liquor potassæ (Hutchinson, jun.). The bright oval nucleated bodies appear, some still contained within the host-cells, others surrounded by distinct capsules. The non-symmetrical character of the affection is another distinctive feature. The course of the disease is steadily progressive, and if left untreated it terminates in death.

Treatment.-Complete amputation of the whole breast or part affected should be carried out as soon as the diagnosis is established. Cures by radiotherapy have been reported, but the results on the whole have not

${ }^{1}$ Arch. f. Derm. u. Syph., March, 1904, p 37.

2 See Whitfield's article on "Tumours of the Skin," in Allbutt and Rolleston's “System of Medicine," 2nd edit., ix. 601. 1911. 
been such as to justify the neglect or postponement of amputation. A case of Cheatle's in which, although clinically there was only a small patch of about the size of a shilling, carcinomatous changes were found in ducts at the deepest part of the breast, points to the inadequacy of any less drastic method of 1 reatment. Where, however, amputation is refused, radium may be employed; I have used it in such cases with complete removal of the growth. In inoperable cases the rays may be used for palliative purposes.

CANCER OF THE SKIN

$$
\text { Cancer "en Cuirasse" }
$$

Cancer en cuirasse may occur primarily in the skin, without previous mammary cancer. I have myself seen several such cases. In one of them it commenced in the skin over the breast, and in two others at some distance from that part (Plate 70). The first visible lesion is a thickening of the skin somewhat resembling sclerodermia. After a time nodules develop, and by causing pressure on the lymphatics give rise to bead-like chains over the breast. The blocking of the lymphatics causes œedema of the arm; this in one case was the first symptom observed. The disease spread rapidly, and death occurred within four months in all the cases by forming a sort of breastplate, which compressed the ribs and caused a very painful form of death by gradually increased interference with the breathing.

The treatment for this condition is the careful use of $X$-rays, radium, and various other measures for the relief of pain.

\section{Melanotic Cancer}

According to Unna, Gilchrist, Whitfield, and others, nearly all the cases of sarcoma derived from pigmented 


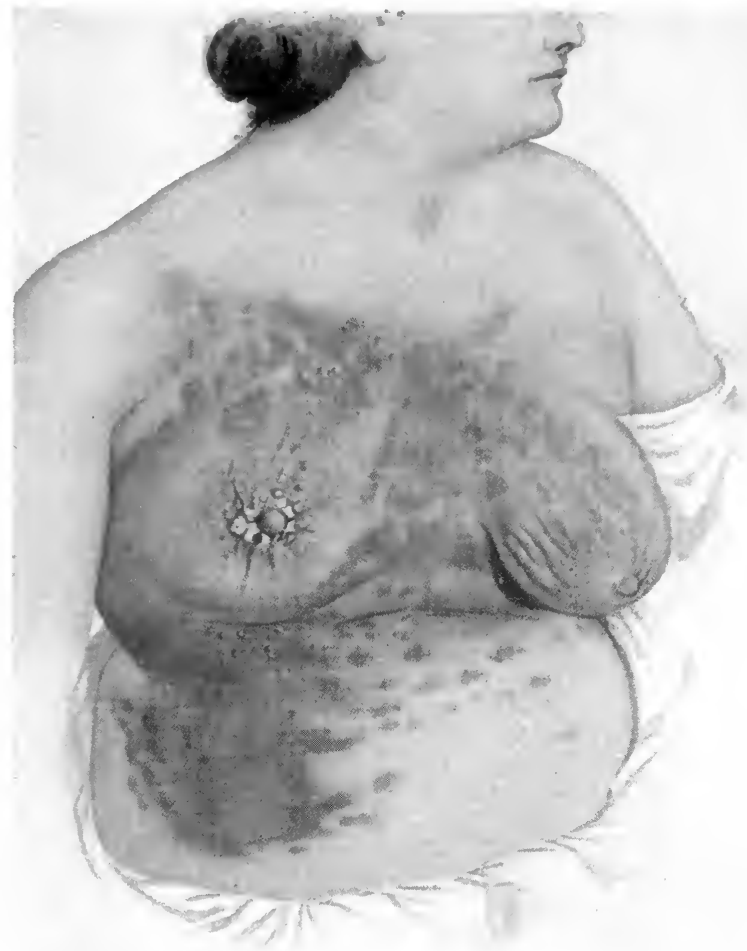

plate 70.-cancer "en cuirasse." 

moles described as melanotic sareoma of the skin have been instances of melanotic carcinoma. Cases of undoubted melanotic cancer occurring elsewhere have been marked by the development of dark areas rapidly changing to tumour along the course of the lymphatics, with early implication of glands.

\section{Epithelioma}

Clinical features and pathology (Plate 71).Cancerous tumours are chiefly characterized by overgrowth of epithelium at the expense of surrounding tissues. Thus on the skin cancerous growths usually begin as slight papillary elevations, but if the process begins in a gland a nodule forms the starting-point. To take the more usual case, the papule becomes firmer and extends laterally, involving the skin immediately around it, the infiltration being evidenced by the characteristic firm raised border. Extension in depth is also effected by continual growth of the deeper layers of the rete mucosum. The rapid growth of the epidermis at the sides and the base of the growth causes the central and superficial part to perish for want of nutrition, so that ulceration occurs in the middle while extension is going on in the depths and at the sides of the growth. If this be of moderate degree the surface remains covered by a certain thickness of epithelium, and there is no bleeding from denudation of vessels - in fact, no true ulceration, although there is a moist discharge which dries and forms crusts. If the necrotic process extend to the vascular tissues there is more or less hæmorrhage. When the lateral growth predominates, the so-called discoid epithelioma is the result. This is typically seen in sweep's cancer of the scrotum; the surface is raised, with a steep border, and bright red, with firm granular surface. If there be luxuriant formation of new tissue at the margin and deep ulceration in the centre, the 
crateriform ulcer of Hutchinson is the result. If the granulations are of large size the cancer is said to be of the papillary form. This phase may be so marked as to deserve the name "cauliflower growth," such as is met with on the external genitals and the os uteri. Frequently the appearance of the lesions is modified by some pre-existent morbid condition. Thus epithelioma may arise in a chronic ulcer, simple or syphilitic, or from lupus ; in a wart or mole, etc.

All the forms of epithelioma have the following common characters: peripheral extension, infiltration and destruction of neighbouring parts, central ulceration; and in all cases (with the exception of rodent ulcer) there is a tendency to form secondary growths in lymphatic glands, in the viscera, and elsewhere. Just as a cancer of the tongue which has its starting-point at the bottom of a deep fissure may widely infiltrate the organ before there is any appreciable induration or ulceration of the surface, so an epithelioma of the skin beginning in the deepest part of the glands may widely infiltrate the corium and subcutaneous tissues before the surface is ulcerated. These deep-seated epitheliomas are the more dangerous by reason of their anatomical connections. The amount of pain caused by cutaneous cancer varies according to the structure involved. Cancer of the skin has a predilection for certain sites, such as the natural orifices-mouth; anus, vulva, and eyelids; moist parts, as the glans penis; exposed regions, as the face and hands; parts exposed to slight injuries, such as the feet from friction of the boots-but it may occur in any part. As already said, a wart, a mole, or an ulcer may be the starting-point: Epithelioma occasionally appears as a complication of long-standing lupus vulgaris lesions, and cases have been recorded in which it has developed on a cicatrix caused by the X-rays applied to lupus lesions. Sequeira 


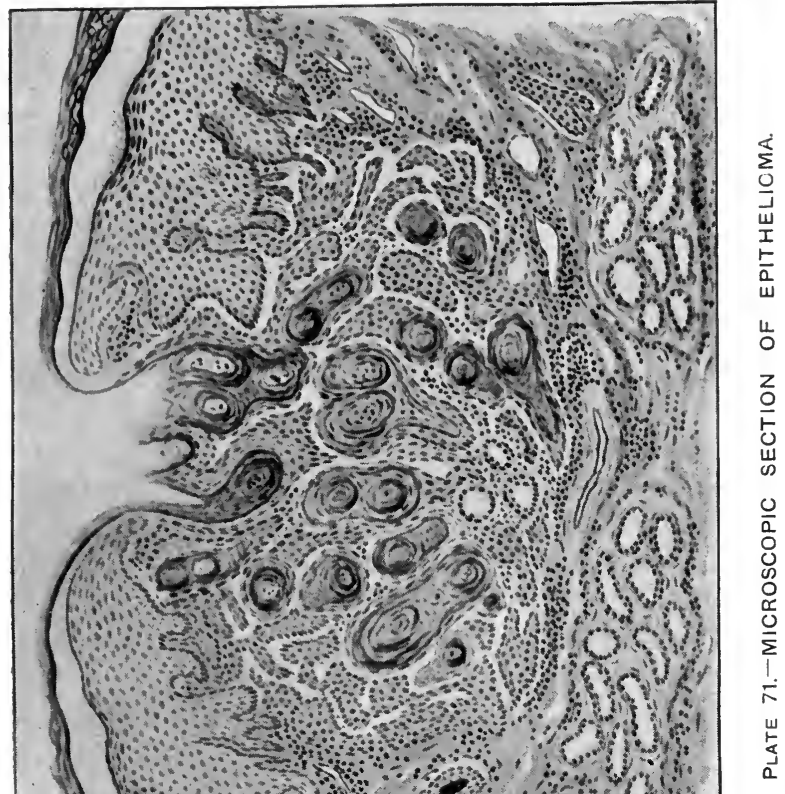



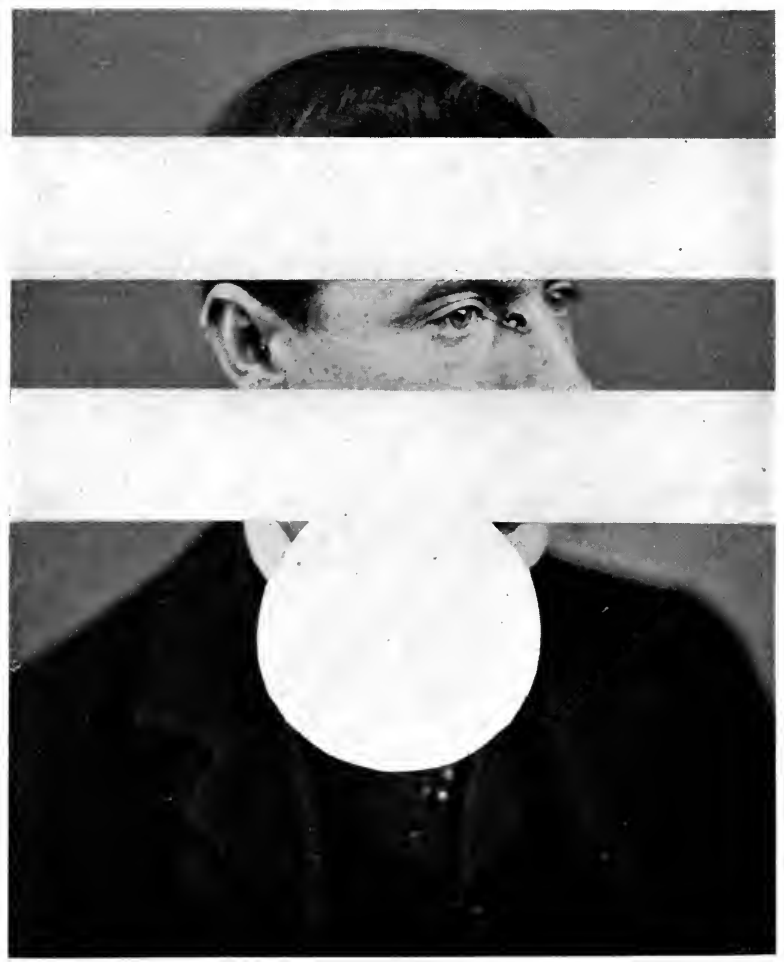

PLATE 72.--EARLy RODENT UlCER. 
believes that there is considerable risk of the development of what he calls lupous carcinoma when frequent exposures to the rays are carried out over long periods. An ulcerated and everted sebaceous cyst simulates in appearance a malignant growth, and also tends to terminate in veritable epithelioma. The group of "trade cancers" includes those met with in workers in peat-tar, coal-tar, paraffin, and lamp-black, in addition to the scrotal cancers of sweeps, mentioned above. The malignant infiltration varies in rapidity in different cases, but, as with cancer generally, it may be said that, unless speedily and thoroughly removed, sooner or later the disease causes the death of the patient by gencralization and exhaustion.

Cancers vary in structure according to the particular epithelium in which they arise. On the skin they are usually of the stratified squamous type, with wellmarked cell-nests in the central parts of the older lobules. These nests are due to the older cornified cells becoming flattened and arranged in concentric layers as the result of pressure. When the process starts in a tubular gland the glandular-tubular type of epithelioma is the result. Not only is there overgrowth of epithelium, but the connective tissue is altered by inflammatory exudation and by formation of new vessels.

I. C. Pfeiffer and other observers have described parasitic sporozoa in cutaneous as in other epitheliomas. Malassez and some others believe that there may be a causal relationship between these parasites and the growth. The question is still unsettled.

Diagnosis.-In the diagnosis of epithelioma attention must be paid to the fact of its being usually a single growth, to the starting-point, the site, the slight discharge, the border, as described above, and the secondary growths. To distinguish epithelioma from a wart or a mole, microscopic examination or protracted 
observation may be necessary; if signs of crustation or ulceration should appear, epithelioma should be suspected. For the diagnosis from rhinoscleroma, see p. 583 ; from lupus vulgaris, p. 459.

Treatment.-Early and free removal with the knife is the safest treatment of cancer of the skin: When this is impossible the sharp spoon, followed by the actual cautery or chloride of zinc, gives some hope of cure. For inoperable cancer, C. W. Allen recommended arsenious acid, equal parts by weight of acid. arsenios. and orthoform, or one part of white arsenic to two or three parts of orthoform mixed with enough water to form a butterlike paste. Orthoform is said largely to mitigate the pain of epithelioma. Van Harlingen ${ }^{1}$ treated fifty-five cases of epithelioma-almost all of them facial-with caustic potash, and found that in the majority the results were satisfactory. The cases suitable, in his opinion, for this form of treatment are such as display small, well-defined pearly lesions. Larger lesions, he holds, are best treated by the $X$-rays, but here caustic potash may be used to dissolve the horny epithelium. Ravogli and Stelwagon also speak well of caustic potash in epithelioma, and Hartzell reports that some of his best results have been obtained by the use of this substance in conjunction with pyrogallol. ${ }^{2}$

On the whole, the results of treatment of cancer by Finsen light and $X$-rays have been disappointing, though one or other of these methods may with advantage be used as a palliative. In cases of superficial epithelioma in which operation is undesirable, or is refused by the patient, radium may be applied. In such cases I have seen the tumour disappear under its influence, though it is too soon to say that there will be no recurrence.

1 Trans. Amer. Derm. Assoc., 1905, p. 57.

${ }^{2}$ Ibid., p. 63. 


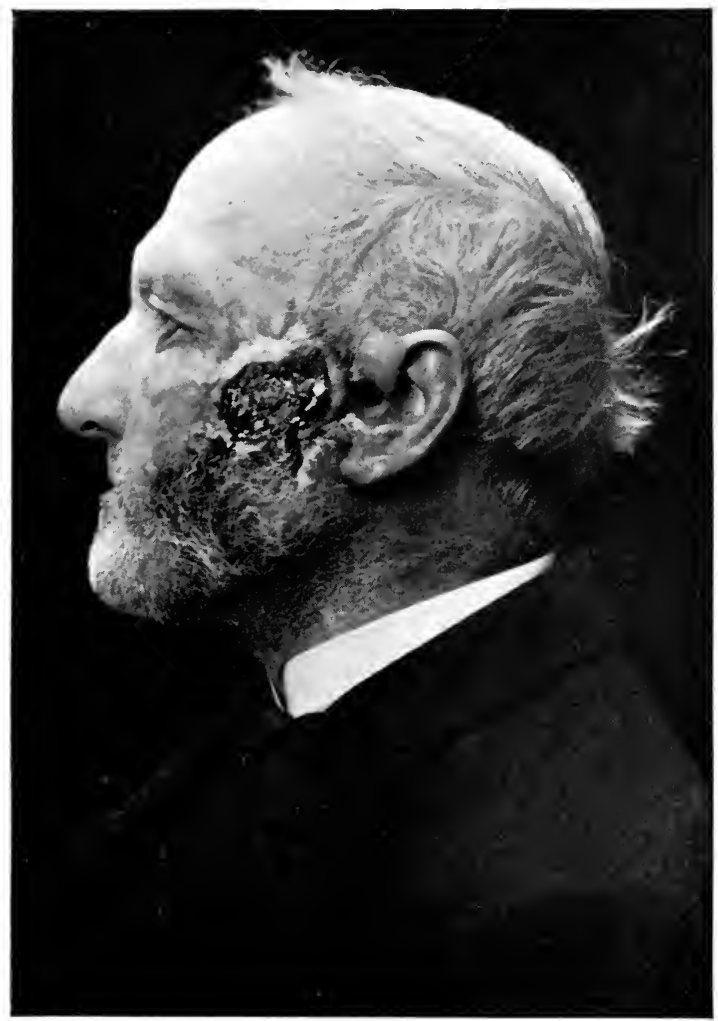

Plate 73.-Rodent ulCER-MORE advanced stage. 


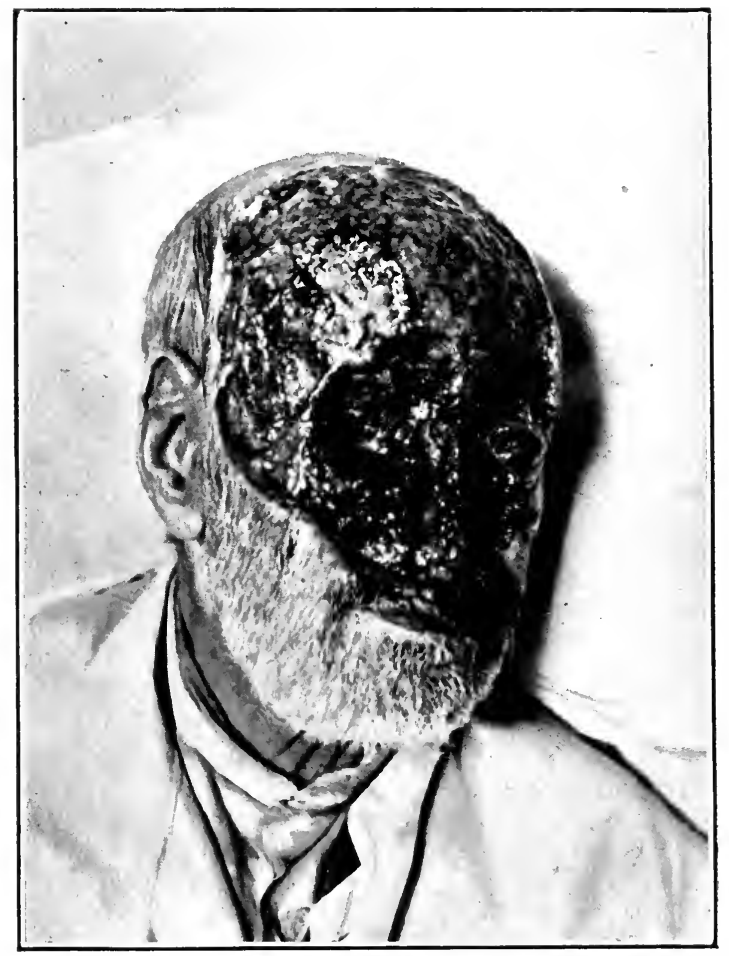

Plate 74.-rodent ulcer-late stage. 
Cylindroma of the skin is a very rare but wellmarked variety of cutaneous epithelioma. The growth, according to Nicolau, ${ }^{1}$ is composed of cylinders of cells (hence the name, which originated with Billroth), most of them anastomosing to form trabeculæ. Its most characteristic feature is the mucoid degeneration of the connective tissue imprisoned in the network formed by the cylindrical growths.

\section{RODENT UlCER}

The individuality of rodent ulcer is well explained in the words of A. Jacob, ${ }^{2}$ by whom its claim to be considered a distinct clinical entity was first established : "The characteristic features of this disease are the extraordinary slowness of its progress, the peculiar condition of the edges and surface of the ulcer, the comparatively inconsiderable suffering produced by it, its incurable nature, unless by extirpation, and its not contaminating the neighbouring lymphatic glands."

Clinical features.-The affection is a form of epithelioma. In the first stage the lesion is a small circumscribed nodule in the skin, flat and depressed in the centre, with unbroken cuticle, firm to the touch, and of a dull brownish-red colour. It often remains for some years without undergoing any perceptible change, but at length it enters upon the second stage, the cuticle covering it is broken, and an ulcer with depressed granular centre and infiltrated border is formed (Plate 72). The ulcer becomes slowly larger and deeper; it infiltrates and destroys the subjacent tissues, attacking and replacing bones as well as soft tissues (Plates 73, 74)-the third stage. Sometimes it spreads superficially, with cicatrization of the centres,

${ }^{1}$ Arch. de Med. Exper. et Anat. Pathol., Nov., 1903 (abstr. Brit. Journ. Derm., June, 1904).

2 Dublin Hosp. Repts., 1827, p. 232. 
but usually the destruction of the parts beneath it is more marked in the centre, so that a crater-like form results. In some cases true fluid-containing cysts are present in the growth. It is remarkable that almost every case of rodent ulcer has its seat within an area bounded by a line drawn from the uppermost part of the pinna to the root of the nose, and another drawn from the lobule of the ear to the columella of the nose. Cases are, however, recorded in which it has been met with in other parts-e.g. the thigh, ${ }^{1}$ and the back of the hand. In 1888, Colcott Fox showed a case of multiple rodent ulcers (three)-that of a man who died ten years later after great extension of the disease.

Pathology.-The structure is that of an epithelioma, the cells being smaller than those of the ordinary epithelioma, and only exceptionally being arranged in cell nests (Plate 75). Most histologists are agreed that it begins in the deepest layers of the rete mucosum, but others have traced it variously to the sweat-glands, the sebaceous glands, and the hair-follicles. In a case studied by Carle it started in the arrector muscles of the hairs. Norman Walker maintains that there is no connection between rodent ulcer and squamous epithelioma, except the fact that both are largely composed of epithelial cells. He argues that its type and the arrangement of its cells correspond to that described as glandular or tubular carcinoma. MacCormac has, however, shown that rodent ulcer and squamous epithelioma are identical, the apparent difference being explained by difference in the rate of cell-growth.

Etiology.-The causation of rodent ulcer, like that of cancer in general, is not yet definitely settled. Dubreuilh and Wickham have described psorosperms in

1 See case reported by Herbert E. Friend and T. Crisp English, Lancet, May 4, 1911, p. 579. 


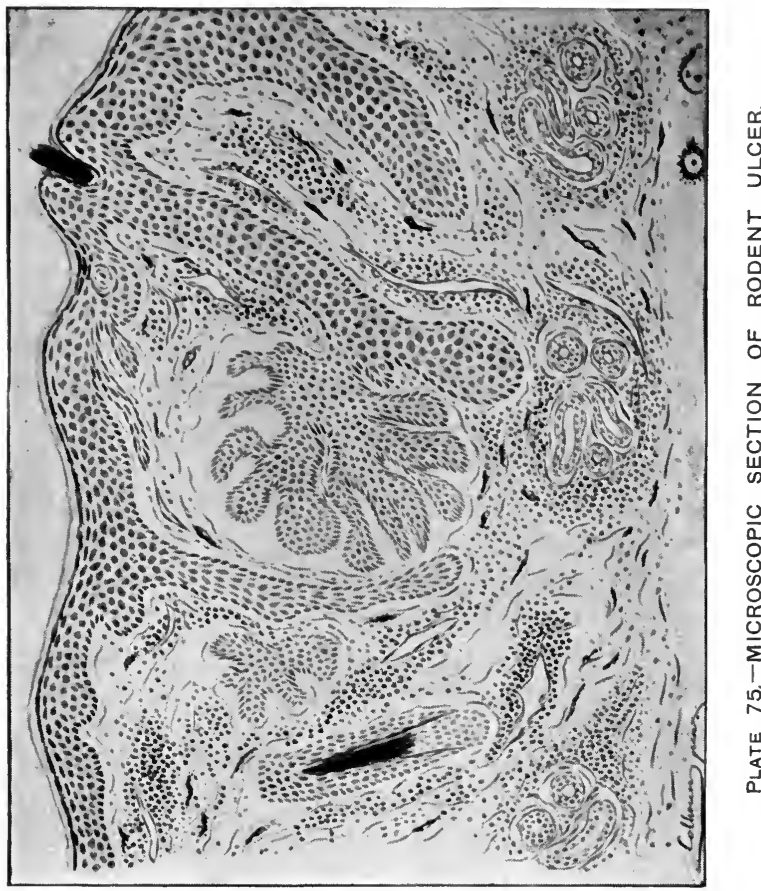

品 

association with the process. Like cancer in general, rodent ulcer is a disease of old age, but it is not rare about 30 , and its occurrence has been recorded in patients under 20-in a case of Sequeira's as early as the twelfth year. Norman Walker's statistics give the average age for the commencement of the disease as 40. The affection occurs with about equal frequency in the two sexes.

Diagnosis.-Rodent ulcer differs from epithelioma not only in the size and arrangement of the cells, as mentioned above, but also in having a hard and everted edge, in not infecting the glands, in not having a foul base roughened with granulations, in not being attended by severe pain except in late stages, and in the much slower course which it runs. The absence of gland infection and the slow course, with the limited amount of new growth, also distinguish it from other cancers. From lupus vulgaris it is differentiated by the absence of apple-jelly nodules, by the age of the patient, and by the mode in which it begins. From tertiary syphilitic ulcers it is distinguished by the granular base, the usually solitary character of the ulcer, and the resistance to treatment. One must be careful not to be misled by the temporary improvement sometimes observed.

Prognosis.-The outlook is favourable in the first stage. It is gloomy in proportion to the implication of the deeper tissues, especially periosteum and bone. Hideous deformity may be produced by the extension of the ulcer; and if nerves are affected the pain may be so great as to render life almost unendurable.

Treatment.-In appropriate parts, where disfigurement can be disregarded, free excision is to be recommended. In parts where this is impossible, such as between the nose and eye, radium, applied with proper precautions, is the best remedy. I have used it with the most satisfactory results in an inveterate case which 
had been treated by electrolysis without advantage. In deep ulcerative cases, also, with invasion of bone or periosteum or mucous membrane, in which, though there was healing of the ulceration under the X-rays, small burrowing foci remained and continued to spread, I have found radium useful. This treatment is more suitable in cases where there is no cartilage or mucous membrane to be implicated than when the growth arises on the eyelids or in one of the canthi, on the nose, or on the lip. In cases of the latter group I have had both successes and failures with radium. Treatment with carbon-dioxide snow has also yielded good results in the pre-ulcerative stage, both alone and in conjunction with X-rays. The actual cautery followed by caustics may sometimes effect a cure. Caustics, such as chromic acid, may be used alone in appropriate cases, but there is a risk of their stimulating the growth if they do not destroy it completely. Carle recommends, after curetting, an application of arsenious acid-acid. arsenios. 1 part, alcohol and water equal parts to a dilution of 1 in 150. Finsen light treatment has been successful in several cases in my hands, but in my experience it is inferior to the $X$-rays. Some early cases are much benefited and even cured by zinc ions, as recommended by Lewis Jones and others.

\section{Sarcoma of the Skin}

The skin, according to Babes, is the most frequent source of sarcoma; but, as Kaposi points out, in the majority of cases the process in the skin is secondary to growths commencing in the lymphatic glands or the deeper structures.

Clinical features.-The clinical forms of sarcoma of the skin are so various that it is impossible to describe them all. The following is only a general outline of the disease in its commoner varieties. Sar- 
comas vary greatly in consistence, the spindle-celled tumours being fairly firm, the small-celled ones soft, with all intervening grades of density. The description would apply also to mycosis fungoides in its later stages, for that affection is sometimes regarded as a form of sarcoma (Kaposi). When a sarcoma arises in a congenital papilloma its surface is frequently warty, and the tumour is then usually melanotic. In colour sarcomatous tumours vary greatly: the pigmented varieties are bluish-black or brown; the non-pigmented, reddish in hue. Sarcoma may arise in any part of the body; but moles, warts, and pre-existing ulcers all predispose to sarcoma. It has already been stated that many tumours arising in this way are in reality carcinomas. Hutchinson has drawn attention to a form of melanotic sarcoma which begins at the side of the nail, and for a time shows nothing more than a blue mark, which afterwards becomes a tumour of extremely malignant character. Very frequently cutaneous sarcomas are exceedingly numerous, and may form rapidly over the whole body. Sarcoma is commonest at and after middle age, but may occur in childhood. Ulcerated sebaceous cysts often present the appearance of malignant growths, and indeed, if long neglected, may form starting-points of such tumours.

Sarcoma may be taken as a type of malignant growth. Its chief characters are unlimited local extension, with infiltration and destruction of neighbouring tissues and (when situated on skin and mucous membranes) rapid ulceration. Secondary deposits in sarcoma are usually due to the growth invading and projecting into veins, in which detached particles are carried to the heart, lungs, etc. Extension along lymphatics is also met with, and the glands may be enlarged throughout the body. Subsidence and scarring are very rare. Congenital sarcoma, sarcoma appearing early in life, and 
sarcomatous tumours of the melanotic variety are usually highly malignant.

Pathology.-A round-celled sarcoma differs but little in structure from a mass of granulation tissue. The blood-vessels have thin walls, and active budding takes place from the cells which constitute the walls. All sarcomas are remarkably vascular, and, the walls of their vessels being thin, hæmorrhages are frequent. The central parts of sarcoma tend to degenerate, like those of gummata and tuberculous masses. Melanotic sarcomas owe their special features to granules of a pigment, known as melanin, being deposited in some of the sarcoma cells. When there is a formation of spindle cells parallel to the long axis of the vessels of the tumour, while round cells collect in the spaces so formed, the "alveolar sarcoma" is constituted. In one variety (lipomatous) the cells become loaded with fat. Until Virchow established the differential characters of sarcomas as compared with epitheliomas, these two groups were included together as cancers.

Diagnosis. - This usually depends upon histological examination. But a tumour which appears in previously normal skin, or at the site of an injury, or in a wart or mole, which is soft and reddish from vascularity, or bluish from pigment, and which, after growing slowly, undergoes rapid enlargement, projects above the surface, and readily bleeds and ulcerates, is probably a sarcoma.

Prognosis.-The outlook is extremely bad, recurrence being the rule, however freely removal be carried out.

Treatment.-Complete removal at the earliest possible moment should be enjoined, whenever this is practicable. The results of radiotherapy and other physico-therapeutic methods have been disappointing, except where they are used simply as palliatives. Lassar, 
Köbner, and Shattuck have reported good results from subcutaneous injections of Fowler's solution, diluted with two parts of distilled water, beginning for an adult with miv, increased after a time to mvi. Pospelow has given arsenic internally in the form of Asiatic pill with good results. I have also used this method with some success. Coley's fluid, obtained from cultures of virulent strains of the Streptococcus erysipelatis to which an emulsion of Bacillus prodigiosus is added, with glycerine and thymol, has given the best results in spindle-celled sarcoma, and it may be tried in inoperable cases. Melanotic sarcomas seem to make no response to the injections.

\section{Idiopathic Multiple Pigmented Hamorrhagic SARCOMA $^{1}$}

This disease is very rare in these islands; two cases have been described by Hutchinson and one by Stephen Mackenzie. The latter's patient was previously under the care of Pringle. Sequeira has reported three cases, and Weber and Daser also had a case. In Italy the disease appears to be relatively common. De Amicis had 50 cases in Naples. In other parts of Europe and in America it is occasionally met with. Kaposi, who first described the disease, had 30 cases; in Russia, Stoukovenkoff of Kief and Semonoff had 10 each. Wigglesworth, Lustgarten, Fordyce, Brayton, Sherwell,

1 J. H. Sequeira and Bulloch, Brit. Journ. Derm., June, 1901, p. 201 (gives summary). Radcliffe-Crocker, "Diseases of the Skin," 3rd edition, 1903, p. 963. Stephen Mackenzie, Brit. Journ. Derm., June 4th, 1890. Jonathan Hutchinson, Arch. of Surg., v. 237 and vi. 132; "Smaller Atlas of Clinical Surgery," Plate 61; Brit. Journ. Derm., Jan. 7, 1905. Weber and Daser, Brit. Journ. Derm., April, 1905, p. 135. Weber, Proc. Roy. Soc. Med., Clin. Section, 1908, p. 235. J. M. H. MacLeod, Brit. Journ. Derm., May, 1905, p. 173. J. H. Sequeira, Proc. Roy. Soc. Med., Derm. Section, 1909, p. 15 ; 1910-11, p. 8. 
Corlett and others have reported cases in America. The disease is much commoner in males than females; all Kaposi's cases were males, and in 73 cases from the literature, Sequeira found only five females. It usually attacks middle-aged men, but a few cases have occurred under 20 years of age and several between 20 and 30 . Corlett had three cases in one family, the youngest being 2 years of age, and de Amicis observed the disease in a child of 5. A majority of the cases have occurred in Polish and Galician Jews.

Clinical features.-The disease begins with the formation of bluish-red infiltrated areas or nodules on the palms, soles, or dorsal surfaces of the hands and feet, and subsequently attacks the legs and arms, and spreads to the trunk and face in the course of two or three years. The nodules are about the size of a pea, and may be discrete or in groups. They are tender and painful. Some may have a central depression, and complete involution may occur, leaving depressed pigmented scars. They rarely ulcerate or suppurate. Dilated vessels and hæmorrhages may occur in and around the nodules, and the pigmentation is due to this hæmorrhage. As the disease progresses, fresh growths and infiltrations appear. Edema and thickening of the legs give rise to a condition of elephantiasis, which causes much deformity. The mucous membranes are attacked and later the visceral organs. Death may occur in the first, second, or third year, but the average duration of the disease is about four years. A few cases of recovery are on record. The patient under Pringle and Stephen Mackenzie lived for twenty-five years after he was first seen in this country.

Etiology and pathology.-The causation of the disease is obscure; exposure to cold, rheumatism, and valvular disease of the heart are said to have been predisposing causes in some of the cases. Hutchinson 


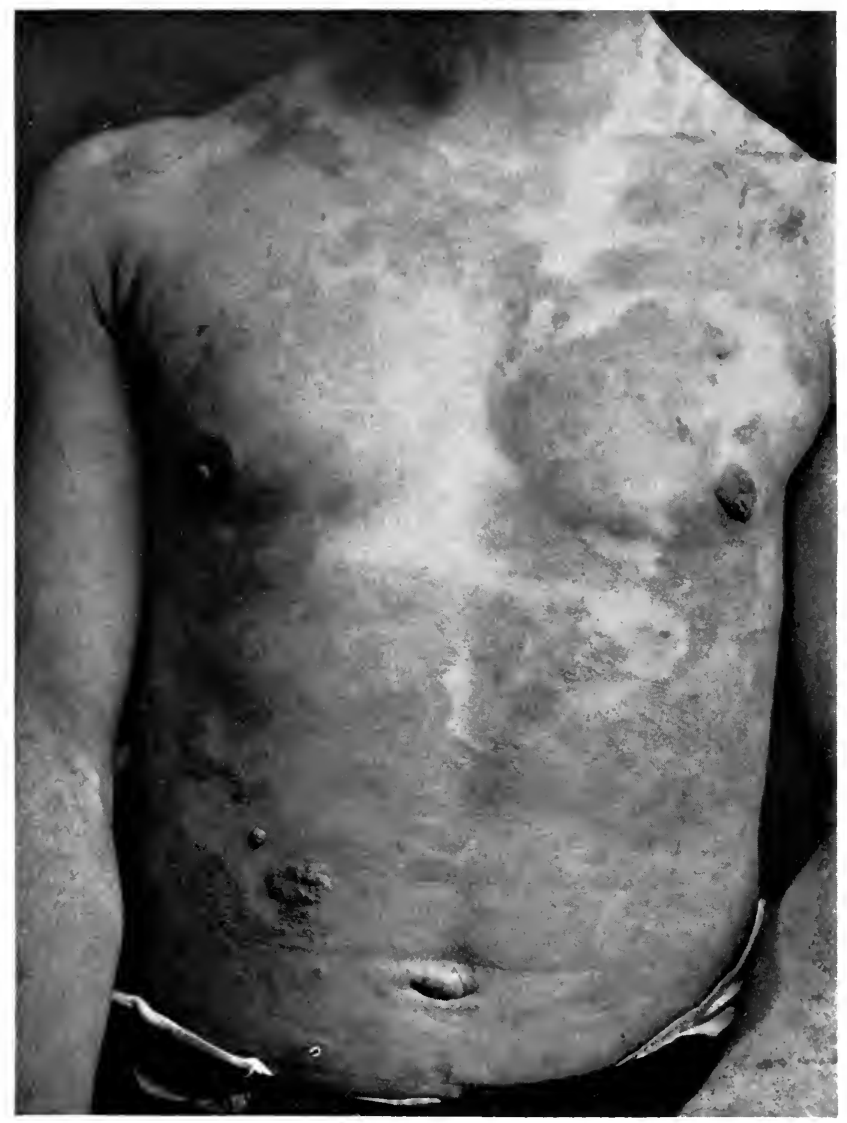

Plate 76.- mycosis fungoides.

(Stoze'r's' Cast.) 

has pointed out an association with gout in several cases. Of Sequeira's cases, Bulloch regarded the tumours as inflammatory in the first; in the second, Turnbull concluded that the process was inflammatory and not sarcomatous; and MacLeod came to the same conclusion with regard to the case of Weber and Daser. He described the condition as a growth of organizing connective-tissue cells associated with marked vascular dilatation and œedema and deposit of blood pigment. Sequeira thinks that the same name has been given to two different classes of case, one benign and the other malignant. He regarded his first case as of the same type as those described by Hutchinson under the name of symmetrical purple congestion of the skin.

Treatment.-Some instances of cure under the administration of arsenic have been recorded. Radiotherapy might be tried; in a case reported by Lustgarten it effected a marked improvement.

\section{Mycosis Fungoides}

To this now well-recognized disease various names have been given, but the one used here was applied to it by Alibert, as indicative of the naked-eye appearance, and not as a pathological description.

Clinical features.-The lesions in the early stage (Plate 76) are dull-red or livid patches, varying in size from the finger-nail to the palm of the hand, with borders sometimes well marked, sometimes fading off, occasionally flat, more often raised or thickened. The patches are smooth and dry at first; later they become scaly, and at last they may be moist or covered with crusts. From the appearance of the lesions, Erasmus Wilson termed the affection "eczema tuberculatum," and to this first stage of the disease French dermatologists have given the name eczéma prémycosiforme. Nevins Hyde 
and F. H. Montgomery, who have made a special study of this stage, ${ }^{1}$ consider that it is just as much a manifestation of the disease as is the stage of tumour formation, and that the process is in operation as soon as the early pruriginous symptoms are manifested. They sum up as follows: "The facts point to a systemic origin for mycosis fungoides as definitely and as unmistakably as a glycosuric xanthoma points to a condition which by no possibility could have been explained by any examination merely of its cutaneous lesions." In the later stage the moist eczematous surface becomes more and more infiltrated, so that tumours project above the level of the skin (Plate 76). They may be as small as a pea, or as large as an apple. They are firm and lobulated, broader at the free end than at their attachment (somewhat resembling tomatoes, whence the term "fungoides"). Their surface may be smooth and moist, or else excoriated and covered with crusts.

The lesions are not arranged according to any plan. Any part of the body may be affected. They have been observed on the mucous membranes of the mouth, the uvula, and the soft palate. In an atypical case exhibited by Sequeira ${ }^{2}$ the greater part of the cutaneous surface was affected; in another, shown on the same occasion, the tumour was single. The premycotic stage may last for many months or even years before tumour formation begins. This is not always regularly progressive, for many of the patches may disappear whilst new ones are forming around them. As a rule, however, progressive thickening occurs, a papillated condition (lichenoid plaques) often being observed before fungation. Fresh tumours may develop on the site of pre-

${ }_{1}$ Journ. Cut. and Gen.-Urin. Dis., June, 1899. See also a case reported by Allan Jamieson and Miss Huie, Brit. Journ. Derm., April, 1904, p. 126.

2 Brit. Journ. Derm., 1910, xxii. 23. 
vious tumours that have disappeared. On the whole, the disease steadily progresses and wears out the patient, who becomes emaciated and dies of septicæmia, pneumonia, diarrhœa, or some other complication. In one case, however (Bazin), recovery is recorded to have taken place after an attack of erysipelas. The duration of the disease is usually from six months to as many years, or even longer. In the early stage the subjective symptoms are intense itching, and sometimes burning pain, causing loss of sleep and impairment of health. As a rule, however, the general health remains fairly good until many tumours have formed. The growths are devoid of sensibility. The lymphatic glands may be enlarged throughout the body, as in lymphadenoma.

Pathology.-The tumours are composed of small round cells, supported by scanty fibrous tissue, thus resembling lymphadenoma. Indeed, some authors regard the affection as a lymphadenoma of the skin; others look upon the new growth as granulation-tissue tumour due to the action of micrococci. Professor Zeit, in an autopsy, found lymphatic leukæmia with hyperplasia of the spleen, cervical, axillary, inguinal, mediastinal, bronchial, cœliac, mesenteric, and retroperitoneal lymphatic glands and leukæmic lymphomas in the lungs, skin, liver, spleen, and intestines. There were general jaundice, brown atrophy of the breast, and acute parenchymatous degeneration of the kidneys, chronic cholecystitis, and a submucous fibroid of the uterus. These findings suggest a true lymphatic leukæmia, although the clinical picture was that of mycosis fungoides. Zeit and Pardoe $^{1}$ therefore conclude that mycosis fungoides is a chronic lymphatic leukæmia.

Etiology.-The direct cause of the affection is unknown. The extremes of age are 20 to 60 years, but it is commonest between 40 and 50. Males are

${ }^{1}$ Journ. Cut. Dis., xxix. 7. 
more frequently attacked than females: of 28 cases collected by Stowers, 22 were males and 6 females, and the age limits were 25 on the one side and 72 on the other. The general aspect of the affection, like sarcoma and cancer, suggests an infective origin, but this as yet has not been proved. Payne showed that the micrococci described by various authors are probably accidental. Posada, working under Wernicke, has described coccidia in the lesions. ${ }^{1}$ In a case of Riecke's the growth followed an injury to the back of the head from a fall, the consequent swelling becoming livid red and gradually spreading. The duration has been as short as nine weeks, and as long as thirty years, as in a case recorded by Dubreuilh.

Diagnosis.-In the premycotic or eczematous stage it may be difficult and sometimes impossible to distinguish between mycosis fungoides and an eczematous or urticario-eczematous condition. In mycosis fungoides, however, the red of the patches may be tinged with yellow; they are more persistent and more infiltrated than those of eczema, and as a rule the edges are more raised, and they will be refractory to the therapeutic measures appropriate to eczema. In the mycotic stage, mycosis fungoides resembles no other malignant condition but sarcoma. The resemblance is seldom sufficiently close to mislead.

Treatment.-The only measure that has any influence on the process is radiotherapy. I have seen some cases much benefited by this treatment; Allan Jamieson has reported cases in which the lesions completely disappeared under the rays, and favourable results have also been recorded by E. Stainer, Radcliffe-

1 For an exhaustive study of the histology and bacteriology of mycosis fungoides, see a paper by J. Galloway and J. M. H. MacLeod, Brit. Journ. Derm., May and June, 1900. The results of the bacteriological examination were negative. 
Crocker and Pernet, and others. White and Burns, of Harvard, have reported a case in which the patient, under the use of only moderate doses of $\mathrm{X}$-rays, "succumbed to the effects of a too rapid relief from his malignant disease," being, in fact, poisoned by the products set free by the rays. Arsenic is sometimes of use, and Coley's fuid should be tried, and various vaccines. In a case under my care the use of resorcin ointment (gr. $\mathrm{xx}$ to $\mathrm{z}^{\mathrm{i}}$ ) was followed by considerable local improvement. In the premycotic stage, antipruritic remedies should be used. When the tumours have broken down, antiseptic dressings are necessary.

\section{Leuk zmia Cutis}

The cutaneous lesions which may appear in association with leukæmia present resemblances in some cases to mycosis fungoides. They may take the form of diffuse œdema-like infiltration, usually attacking the face ; of large subcutaneous tumours ; of smaller nodules in the skin widely dispersed and more or less symmetrically arranged. Cases have also been reported in which papules, both of the prurigo and of the lichen type, eczematous lesions, and eruptions resembling pityriasis rubra or general exfoliative dermatitis, all of them associated with severe pruritus, have been interpreted as manifestations of leukæmia; but it has been suggested by Rolleston and Wilfrid Fox, in their report of a case of atypical myeloid leukæmia, ${ }^{1}$ that this group of cases belongs to lymphadenoma rather than to leukæmia. In the case just referred to the nodules were of a grey or plum colour-a feature peculiar to this case-oval in shape, firm to the touch, and quite superficial.

Prognosis and treatment.-The prognosis of 1 Brit. Journ. Derm., 1909, xxi. 377. 
leukæmia cutis is very bad. In some cases the cutaneous symptoms have been mitigated by radiotherapy, and in others by arsenic, but leukæmia is almost always a fatal disease.

\section{Kaposi's Disease (Xerodermia Pigmentosum)}

This extremely rare affection, also known as melanosis lenticularis progressiva (Pick), was first described by Kaposi. It is characterized by the formation of numerous tumours, which, though apparently benign in the early stage of their development, run a malignant course and, after extensive destruction of tissues, cause the death of the patient by exhaustion.

Clinical features.-The initial lesions are small pigmented spots, resembling freckles, but rather darker, which appear chiefly on the face, neck, arms, and legs, the area of distribution corresponding pretty exactly with the parts of the skin often left uncovered in infancy. Erythematous patches or papules, like those of measles, sometimes precede the "freckles." The latter usually come and go for a time, disappearing in the winter, to return in the summer; after a time they become permanent, and the colour tends to deepen till they are often quite black. They are irregular in outline, vary in size from a pin's head to a pea, and are generally thickly crowded together, especially on the face. For some time the condition suggests nothing more than excessive freckling, but by-and-by further lesions appear among the "freckles" in the form of white glazed atrophic spots, which often run together, forming scar-like areas; telangiectases, stellate and striate; and superficial ulcers discharging pus that is auto-inoculable, and that dries into yellow crusts under which healing takes place, followed by a good deal of cicatricial contraction. Lastly, after some years, small warty-looking growths develop on the "freckles." 
This event marks the entrance of the disease on a more formidable phase of its evolution. Tumours form and ulcerate, producing fungous masses, the process extending both widely and deeply, and destroying every tissue that comes in its way. In a case recorded by $M^{\prime}$ Call Anderson ${ }^{1}$ the whole of the face and part of the neck were eaten away, the ulcerating process, starting from three foci (nose, lip, and cheek), having resulted in the excavation of a huge pit, the greatest depth of which in the face was about $1 \frac{1}{2}$ inches, in the neck about $\frac{3}{4}$ of an inch. The destruction involved the external auditory canal and the lower portion of the temporal bone, the zygoma, which had entirely disappeared, the posterior half of the lower jaw, including the condyles, the palatal and the superior maxillary bones. There was no lesion in any of the other organs. Generalization rarely if ever occurs. This accounts for the relatively slight effect which the disease has on the health until near the end, when emaciation (from difficulty experienced in taking food) and exhaustion supervene, and a slight hæmorrhage may close the scene.

Pathology.-The morbid anatomy of the disease is very obscure. Kaposi believes that the change conmences in the papillary body and epidermis, extending thence to the true skin. The primary pigmentation is due to atrophy. The tumours are epitheliomatous in structure, a fact which suggests that the process is analogous to the cancerous degeneration that not infrequently takes place in pigmented moles in elderly persons.

Etiology.-Nothing is known as to the causation of this affection, beyond the fact that exposure to the sun may be an exciting influence, but it is clear that some special predisposition must exist. The disease

${ }^{1}$ Brit. Journ. Derm., Dec., 1892. The paper is illustrated. 
generally attacks two or more members of the same family, often selecting its victims exclusively from one or other sex. The sexes are equally liable. It generally begins within the first two years of life, but it has been known to commence in middle life. Several authors have drawn attention to the comparative frequency of the affection in Jews, among whom consanguineous marriages are not uncommon. According to von Halle, writing in 1901, consanguinity was present in 10 per cent. of the cases, while Bayard in 1903 found that the percentage was 12.5 . The parents, however, never suffer from the same affection.

Prognosis.-When the disease is fully established it tends steadily to a fatal issue. The commencement of tumour formation, which may be called the patient's death-warrant, has, however, been known to be delayed for many years, but this is altogether exceptional.

Treatment.-Only palliative measures can be attempted. Auto inoculation of pus from the early ulcers should as far as possible be prevented, and the tumours should be excised as soon as they are noticed. The early and thorough application of this method offers the only chance of checking the disease. The work of Bowles and others on the action of light, especially reflected light, on the skin suggests that possibly something might be done in the early stages of the disease by the application of reddish and brown pigments (salve sticks), and exclusion of the sun's rays. 


\section{CHAPTER XXIX}

\section{MALFORMATIONS}

THERE remain to be considered certain conditions which, though clearly not belonging to any of the categories in which the diseases already described have been provisionally grouped, it is difficult to classify on the basis of any distinctive feature common to them all. Inasmuch as, though not always, strictly speaking, congenital, they depend on an error of development of some kind, I have ventured to bring them together under the head of "malformations." This term must not, however, be understood as implying a definition; it is used merely as a designation, neutral and temporary in character, for conditions which await the dawn of a fuller knowledge of their pathogenesis before they can be finally classified. These conditions include ichthyosis, with its degrees and varieties, tylosis, sclerema neonatorum, œdema neonatorum, and albinism.

\section{IChthyosis (Congenital Dyskeratosis)}

Ichthyosis is characterized by dryness of the skin, which becomes scaly (hence the name, from i $\chi \theta \dot{u}$ s, a fish) and rough, and often warty. There are many varieties of the affection, and Lenglet ${ }^{1}$ recognizes seven chief groups, related to one another by transitional forms : (1) the lamellar desquamation of the new-born of Grass and Török, (2) fœtal ichthyosis properly so-

1 Ann. de Derm. et de Syph., vol. iv., May, 1903.

$2 \mathrm{U}$ 
called, (3) the congenital ichthyosiform erythrodermia of Brocq (p. 208), (4) keratodermia of palms and soles, (5) atrophic lesions circumscribed and generalized, (6) types of bullous lesions complicated by one of the preceding morbid types, and (7) ordinary ichthyosis. But ichthyosis may be said to occur in four principal forms, distinguished as xerodermia, ichthyosis follicularis, ichthyosis simplex, and ichthyosis hystrix. The first three, though clinically distinct, are pathologically identical, being the results of a process that manifests itself in varying degrees of intensity, of which they may be taken as the extremes. The fourth, though belonging to the same nosological genus, is a distinct species. All four are, as a rule, congenital; in exceptional cases the condition is acquired.

Keratosis pilaris, or xerodermia, which is the commonest form of ichthyosis, is often nothing more than a dry scaly condition of the skin ; little or no sweat is secreted, and the hair-follicles, especially on the extensor aspects, project on the surface of the skin, giving to the hand, when passed over it, the feeling of a nutmeg grater (hence the name keratosis pilaris). In the more marked cases the epidermis is distinctly thickened, and the natural lines are better defined than in normal skin: Chalmers Watson ${ }^{1}$ describes the case of a boy of 6 in which the keratosis pilaris was associated with absence of hair from the skin, baldness, an abnormally slow pulse, and constipation with fetid stools. Histological examination disclosed a sclerosis or thickening of the corium with cellular infiltration in its papillary layer, marked thickening of the bloodvessels with cellular infiltration around them, imperfect development of hair, and changes in the sebaceous and sweat-glands, especially the former. The affection was virtually cured by the inunction of myelocene, with ${ }^{1}$ Brit. Journ. Derm., Jan., 1904, p. 1. 
the administration first of castor oil, afterwards of sulphate of magnesia, and an occasional enema of plain water. Chalmers Watson regards the condition as a chronic irritation and defective nutrition of the skin, the changes in the derma being primary and the epidermic changes secondary, the irritant acting primarily on the cutaneous vessels, and being derived, in all probability, from the alimentary tract. ${ }^{1}$ It is distinguished from goose-flesh by the roughness and scaliness of the lesions, and by their being less evanescent; from keratosis follicularis by the different distribution. For the diagnosis from lichen scrofulosorum, see p. 435.

Ichthyosis follicularis.-Three unusual cases of follicular disease associated with baldness, in one family, have been described by J. M. H. MacLeod. ${ }^{2}$ In all three patients, whose ages were 19,13 , and 10 , there was not only more or less complete baldness of the scalp, with absence of eyebrows and eyelashes, but also trachoma and conjunctivitis. In certain situations the hairs were replaced by pin-head papules, the colour of the horny skin, with no inflammatory halo ; the papules were surmounted by horny spines. Occurring more or less all over the skin, except the palms and soles, they were most marked on the extensor aspects of the upper arms, the back and sides of the neck, and the back; in distribution they were roughly symmetrical. The skin was dry and harsh from the presence of fine scales. The affection in each case developed within the first two years. This affection obviously presents much resemblance to keratosis follicularis, but it is non-inflammatory in origin, and MacLeod proposes for it the name ichthyosis follicularis.

Ichthyosis simplex is marked by extreme scali-

${ }^{1}$ For the results of a histological examination of 25 cases by S. Giovanni, see Arch. f. Derm. u. Syph., Dec., 1902.

2.Brit. Journ. Derm., 1909, xxi. 165. 
ness of the skin, which sometimes appears to be covered with a dense horny cuirass, like the hide of a crocodile. The colour of the scales varies, according to their age and position, from white to dark green and black. The whole skin is affected, but in widely different degrees of severity, the extensor surfaces, especially the elbows and knees, nearly always suffering most; occasionally warty growths develop in these situations. On the other hand, the flexures and the palms and soles are comparatively little affected, and the face also is more or less spared. The hair participates in the general dryness, and becomes dull and brittle; the nails break easily. The sebaceous as well as the sweat secretion is deficient, though neither is entirely suppressed; and patients are usually better in summer, when the glands act more freely.

The most marked subjective symptom is an exaggerated sensitiveness to cold, but there is also a good deal of itching. The skin "chaps" readily and deeply, and is particularly prone to become the seat of eczema, which adds greatly to the sufferings of the patient.

Acquired ichthyosis is seldom general, and has usually been seen in association with neuritis or some central nerve disease.

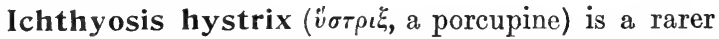
affection than those just described. It is never universal, but is occasionally seen in association with xerodermia. Its distribution often appears to correspond to that of the cutaneous nerves, the lesions being arranged longitudinally on the limbs and transversely on the trunk. Unna, however, thinks that it follows the embryonic lines of fissure. The lesions are small papillary growths with horny tops, which stud the skin as with tiny nailheads; these may develop into large warty masses or concretions like limpet-shells, rising sometimes to a 
height of half an inch or more above the level of the surrounding skin. An unusually well-marked case was exhibited by Stowers in 1907. The affection may be very widespread, and in situations where the warty projections are liable to injury may cause a good deal of inconvenience, but otherwise it gives rise to no symptoms. When localized in the track of a particular nerve it is sometimes described under the name of papilloma neuroticum.

Etiology and pathology of ichthyosis.-All varieties of ichthyosis are, as a rule, congenital, though there is usually no very obvious abnormality in the skin till some little time after birth. In some cases, however, the skin is seen to be peculiarly smooth and glazed as soon as the vernix caseosa is removed; and in others a remarkable condition, styled by some writers hyperkeratosis congenita, has been observed in the skin of the fœtus, which is covered with thick epidermic plates, separated by vertical and horizontal fissures into square patches, like the parti-coloured garment of Harlequin ("harlequin fœtus "). ${ }^{1}$ Unna ard most other writers regard hyperkeratosis congenita as a distinct affection from ichthyosis, but on both clinical and histological grounds F. Bering ${ }^{2}$ leans to the view that no definite line of demarcation can be drawn between the two conditions. Of the cause of hyperkeratosis congenita as little is known as when Lebert first described it more than forty years ago.

Both sexes are equally liable to ichthyosis. Beyond the fact that the condition clearly depends on an error in development, nothing is known as to its pathogenesis. The process appears to consist in increased formation of epithelial cells, which undergo rapid keratinization.

1 See a case recorded by Bland-Sutton (Trans. Med.-Chir. Soc, vol. Ixix., 1886), with coloured illustration and bibliography.

${ }^{2}$ Arch. f. Derm. u. Syph., Sept., 1905, p. 379. 
Tommasoli found lesions in the cutis as well as the epidermis, as indicated by the presence of round or fusiform cells, dilated vessels, and large numbers of oval or flattened nuclei. He is therefore inclined to regard ichthyosis as the expression of a catarrhal condition of the skin. Hutchinson thinks ichthyosis "an intensified form of psoriasis, beginning at a very early period, and deriving peculiarities accordingly." With that opinion I cannot agree. The disease, whatever its nature, can hardly be mistaken.

Treatment.-There is little prospect of a cure being effected, but the condition may be greatly alleviated. Thyroid extract has been administered with temporary benefit. The scales should be removed and the skin kept soft and flexible. This is best done by the free use of soft soap with warm baths, alkaline or bran, and vigorous friction. Inunction with lanoline or other fatty material should follow the cleansing process. The treatment must be regularly persevered with, otherwise any advantage gained will speedily be lost. Salicylic acid will suffice for smaller growths of the hystrix variety, but the large ones must be excised or scraped away. Weak sulphur ointment may be recommended, and benefit may be obtained from a course of sulphur baths at certain spas.

\section{Tylosis (Keratosis of Palms and Soles):}

Tylosis is a condition, affecting the palms and soles, which consists in the thickening of the epidermis into a horny plate, generally dry and smooth on the surface, sometimes worm-eaten. In the foot, only the part that comes in contact with the ground in walking is affected. The condition is as a rule congenital, but may be the result of the long-continued administration of arsenic, or of either hyperidrosis or dysidrosis. When due to arsenic the affection begins with the formation 


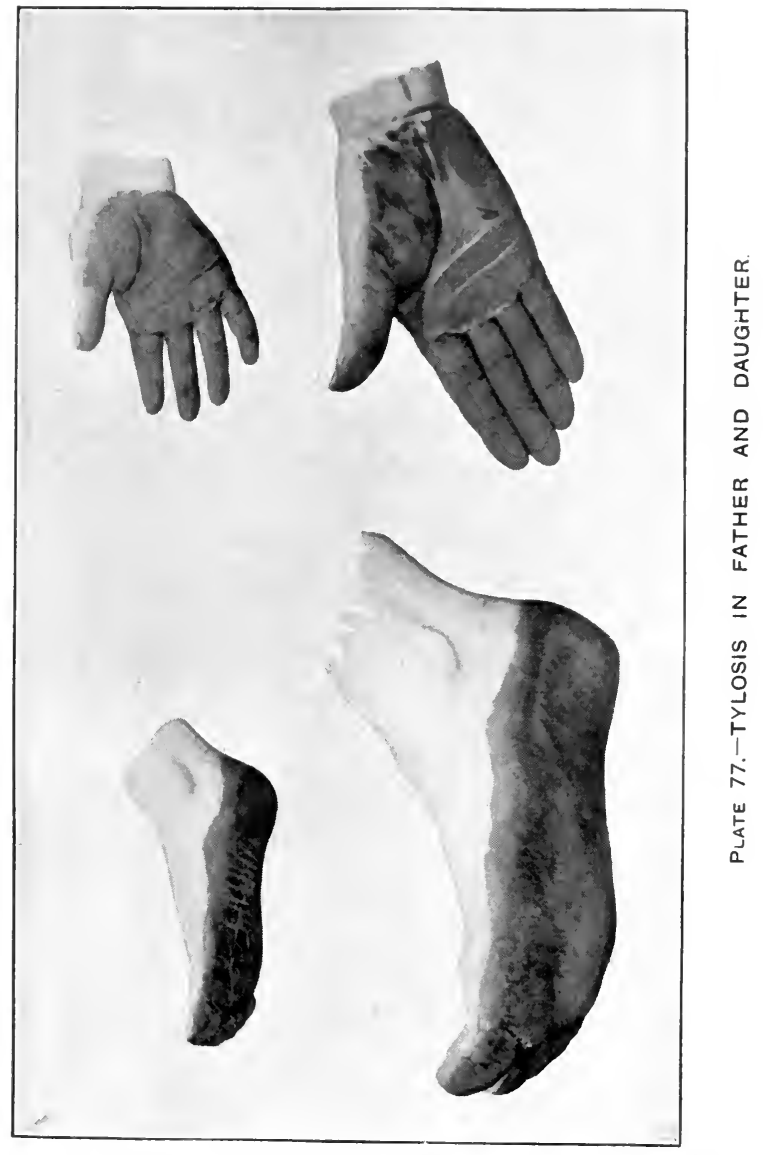



of papules, which develop into nodules and by-and-by into a uniform callosity; when caused by hyperidrosis the thickening commences round the sweat-follicles, and the affected epidermis is sodden as well as thickened. Both sexes are equally liable, and the condition is sometimes hereditary (Plate 77 ).

Horny thickening of the palms and soles may be a secondary condition, due to inflammatory processes, such as eczema, psoriasis, syphilis, gonorrhœa, etc. Such forms of tylosis have been referred to in connection with the several diseases of which they are the result.

Treatment.-In congenital cases little good can, as a rule, be looked for from treatment, but the persevering use of salicylic acid in ethereal solution (10 per cent.) or in a plaster mull has been successful in Unna's hands. The acquired condition may be dealt with in the same way. Ichthyol combined with salicylic acid in an ointment has been found satisfactory by Brooke. Mayer recommends the rubbing in of rheumasan, a salve-soap containing 10 per cent. of free salicylic acid, the drug to be discontinued for a few days on the first appearance of redness.

\section{Sclerema Neonatorum and CEdema Neonatorum}

Sclerema neonatorum is a peculiar induration of the skin which is generally congenital, but occasionally becomes developed within a few days after birth. The skin has a waxy appearance, is hard, tense, and cold, the baby lying motionless as if its face and limbs were fixed in death. The body can be lifted with one hand as if it were frozen. The temperature is subnormal, the breathing very slow and feeble. The child cannot open its mouth to suck, so that what little flicker of life there may be is speedily extinguished. The condition may be acquired consecutively to acute wasting illness (diarrhœa, pneumonia, etc.), or may be the result 
of malnutrition. According to Parrot, the anatomical changes are desiccation of the skin, with thickening of the layers and diminution of the fat, but no true sclerosis:

A somewhat similar condition is odema neonatorum, which is almost unknown in England. It is said to commence on the third day after birth. The œdema begins in the lower limbs and spreads upwards: The skin has a doughy feel, and pits with difficulty: The child is drowsy from the first, and, as a rule, quickly dies of collapse, diarrhœa, convulsions, or other complications. Constitutional feebleness, bad feeding, and exposure to cold are considered to be the causes of the condition.

Treatment.-The indications in both affections are to raise the temperature to the normal standard and to improve nutrition. The child should be wrapped in cotton-wool or kept in an incubator, such as Tarnier's couveuse, and fed artificially. The circulation should be stimulated by friction.

\section{Albinism}

Congenital absence of pigment in the skin and other tissues may be general or partial. The skin for the most part is perfectly white, but where it is thin enough for the vessels to show through it is pinkish. Owing to the same cause the iris looks pink, and there being no screen of colouring matter in front of the retina, photophobia exists. Albinism is often, but by no means invariably, associated with delicacy of body and some mental inferiority. When the condition is partial, irregular patches of white skin are seen here and there, sometimes arranged in correspondence with the distribution of a particular set of nerves, but seldom symmetrical. The hairs on the unpigmented spots are white. Albinism is generally hereditary, and is more common in coloured than in white races; in some tropical regions the condition is endemic. 


\section{N D E X}

Acanthia lectularia, 352, 366

Acanthosis, 4

nigricans, 684

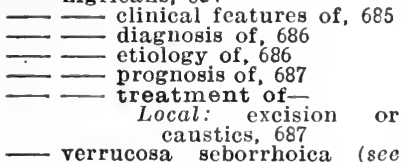
Wart, seborrhœic)

Acaro-dermatitis urticarioides (see Grain itch)

Acarus folliculorum hominis, 352, 604

scabiei hominis, 352, 355

Achorion gallina, 411

gypseum, 411

Quinckeanum, 411

Schönleinii, 411

Acne agminata, 577

bromide, 236

cachecticorum, 445

treatment of, 445

cheloid, 627

confluent, 236

excoriata, 577

frontalis, 576

indurata, 568

keratosa, 577

necrotica, 576

rosacea (see Rosacea)

scrofulosorum, 444 treatment of, 445

telangiectodes, 443,577

varioliformis, 443,575

diagnosis of , 576

etiology of, 576

pathology of, 576

symptoms of, 575

treatment of, 576

vulgaris, 567

diagnosis of, 493, 559, 572,573

distribution of lesions of, 568

etiology of, 570

pathology of, 569

predisposing causes of, 571

prognosis of 573

symptoms of, 568
Acno vulgaris, treatment of-

Constitutional : diet. 574; mild aperients. with tonies, 574; vaccine, 575 ; nonirritating clothing, 575; exercise, 575: Turkish bath, 575

Local : if no suppuration, squeeze out comedones, 573 ; spiritus saponis alka. linus, 573; sulphur, resorcin, ichthyol, or carbolic-acid ointment, 573; if suppuration has occurred, puncture or incise pustule, 574; Unna's mercuric: carbolic plaster mull, 574; corrosive sublimate solution. 574; X-rays, 574; radium, 574; Finsen light, 574

Preventive : frequent ablutions, 573; sulphur ointment, 573 ; avoid stimulating foods and drinks, smoking, and sexual excitement, 573

Acneiform syphilide, 493

tuberculide (see Acne scrofulosorum)

Acnitis, 443, 577

- diagnosis of, 579

- etiology of, 579

pathology of. 578

symptoms of, 577

treatment of, 579

Aconite eruptions, 250

Acrodermatitis chronica atrophi. cans, 224

Acrodynia, 137

etiology of, 138

Acrosclerodermia (see Sclerodactylia)

Actinomycosis, 418

diagnosis of, 419

etiology of 419

prognosis of. 419 
Actinomycosis, treatment of Constitutional : iodide of potassium, 419

Local: removal or destruction of diseased tissues, 420; X-rays, with sulphate of copper internally, 420

Actinotherapy (see Finsen light)

Acuminate cultures, 376

Acute bed-sore. 219

- circumscribed œdema of Quincke, 81

Addison's disease, bronzing of skin in, 211, 365, 686

Adenoma sebaceum. 651 congenital, 649 clinical features of, 649 diagnosis of, 650 pathology of, 650 treatment of-Local: electro.

Ainhum, 186 ly sis, 650

Albinism, 728

Aleppo boil (see Furunculus orientalis)

Alibert's classification, 20

- dartres, 21

- teignes, 20,21

Allergy (see Anaphylaxis)

Alopecia, 633

etiology of, 633

treatment of -

Constitutional: thyroid ex. tract, 636

Local: lanolin and sulphur, 635; stimulating lotions. 635; Hebra's lotion, 635; chloral hydrate, 636; euresol, 636; bichloride of mercury, 636; tannic acid, 636; acetic acid and chloroform. 636; salicylic acid, sulphur, $\beta$-naphthol, and vaseline, 636

areata, 636

clinical course of, 636

contagiousness of, 639

diagnosis of, 385,640

etiology of, 637

parasitic theory of, 638

prognosis of. 640

symptoms of, 636

syphilis and, 639

treatment of-

Constitutional: tonics, 640 ; iron, 640

Hygienic sea-bathing, 640

Local: massage and electricity, 640; hydrochlorate of pilocarpin, 641: chrysarobin, 641 ; blistering with acetum can- tharidis, croton oil, oil of mustard, trikresol, 641; in later stages, faradism, 641; sulphur, 641; wash head with strong tar soap. then treat with sub. limate solution, absolute alcohol and naphthol, and salicylic acid in oil, 642 ; Finsen light, 642; high-frequency current. 642 ; X-rays, 642 Alopecia congenita familiaris, 634 Amboyna button (see Yaws)

Anæsthesia, 63

- in leprosy, 522,524

Anaphylaxis in eczema, 291

- in ringworm, 380

Anetodermia erythematosa, 222

Angio-fibroma contagiosum tropicum, 667

Angio-keratoma, clinical features of, 665

etiology of, 665

pathology of. 666

- treatment ofLocal: electrolysis, 666

Angioma, cutaneous (see Nævu vascularis)

_ infective, 667 treatment of

Local: electrolysis, 667

Angio-myoma, 659

Angio-neuroses, 60

Angio-neurotic œdema, 86

- etiology of, 87

— p pognosis of, 87

- - symptoms of, 86

- treatment of, 87

Anidrosis, 612

Animal irritants, 226

- parasites, diseases caused by. 39. $352-69$

Anomalies of pigmentation, 209

- of secretion. 2

Annular lichen planus, 188

syphilide, 479

Anthrax (see Malignant pustule)

Antifebrin eruptions, 250

Antimony eruptions, 250

Antipruritic remedies, 67

Antipyrin eruptions, 250

Antitoxin eruptions, 258

Appendages, epidermic, affections of, $626-46$

Apple-iel'y nodules, 445

Argenti nitras eruptions, 214, 251

Argyria, 214, 251

Arnica eruptions, 251

Arsenic, epithelioma caused by protracted use of, 243

eruptions, 242

_- keratosis caused by, 243

- peripheral neuritis caused by. 244 
Arsenical pigmentation, diagnosis of, 687

Artificial eruptions (see Eruptions, artificial)

Asiatic pill in lichen planus, 194

in psoriasis, 330

- in sarcoma, 711

Aspergillus and mycetoma, 418

Asthma and eczema, 294

- and urticaria, 79

Asymmetrical distribution of lesions, significance of, 26

Atrophic changes in hair, 630

Atrophodermia, idiopathic, 222. 224

linear, 222

macular. 223

maculatum, 222

primary, 222

senile, 224

- striatum, 222, 223

Atrophy of skin (see Atrophodermia)

Atropine eruptions, 246

Auspitz's classification, 22

Autographism. 80

Bacillus acnes, 570, 594

anthracis, 584

foetidus, 609

lepræ. 525, 528

mallei. 581

pyocyaneus, 553

tuberculosis, 429

- infective power of, 430

Bakers, eruptions in, 228

Bald ringworm (see Tinea decalvans)

Baldness (see Alopecia)

Balsam of Peru cruptions, 251

Balsamic erythema, 245

Balzer, xanthoma of, 6,675

Barber's itch (see Sycosis, coccogenic)

Barley itch, 360

Bateman's classification, 21

"Bayonet hair," 632

Bazin's classification. 22

Bazin's disease (see Erythema induratum scrofulosorum)

Beaded hair (see Monilethrix)

Beau's lines on nails, 644

Bed-bug, 352

Bed-sore, acute, 219

Charcot's. 219

Belladonna eruptions, 246

Benign cystic enithelioma (see Epithelioma adenoides cysticum)

Bichromate of potash eruptions, 228

Bilateral hernes in hereditary syphilis. 176

Bilharzia hæmatobia, 369

Bird-mite, 352
Biskra button (see Furuuculus orientalis)

Blastomycetic dermatitis, 423

- diagnosis of, 425

- prognosis of, 426

symptoms of, 424

treatment of -

Constitutional: iodide of potassium, 426

Black tongue, 625

"Black-dot" ringworm, 382

Bladder-worm, 352

Blastomycosis, cutaneous (8ee Blastomycetic dermatitis)

systemic, 425

Bleb, definition and characters of, 14

“Blind boil," 561

Bloody sweat. 612

Blue sweat, 611

Bockhart's impetigo (see Impetigo of Bockhart)

Boil. "blind," 561

Boils (see Furunculosis and Furunculosis orientalis)

Borate of sodium eruptions, 251

Boric-acid eruptions, 251

Botfly, 369

Botryomycosis hominis, 590

Bottle bacillus in eczema seborrhoicum, 279

Brain, influence of, in production of skin affections, 55

Bromide acne, 236

eruptions, 235

- compared with iodide eruptions, 241

- diagnosis of, 238

— - symptoms of, 236

treatment of-

Constitutional : arsenic, 259; salol. 259

Local: lead lotion, 259

Bromidrosis, 609

etiology of, 609

symptoms of, 609

treatment of-

Local: frequent washing, 610; disinfection with boric acid, 610; rub feet with mutton suet and salicylic acid, 610; chromic acid, 610

Bronson's classification, 22

Bronzing of skin in Addison's disease, 211

Brooke's ointment for lupus, 461

Bug, bed, 352

harvest, 352

Bug-bites, 366

treatment of-

Local: eau de Cologne, toilet vinegar, lead lotion. strong ammonia or "eau de luce," 367; solu- 
tions of soda or boric acid, 367

Bulla, definition and characters of, 14

Bullous impetigo of infants, 552

Burri's indian-ink method, 499

Cade oil eruptions, 251

Calculi, cutaneous, 603

Callosities, 696

Callous ulcer, diagnosis of, 494

Callus, 696

Calmette's ophthalmic reaction, 432

Cancer en cuirasse, 700

\section{treatment of -}

Local: X-rays, radium and other analgesic melanotic, 700 measures, 700

of the skin, 700

originating in nævus pigmentosus, 664

Canities, 632

treatment of -

Constitutional: nerve tonics, 633; jaborandi, 633

Cannabis indica eruptions, 251

Cantharides eruptions, 251

feigned eruptions caused by, 230

Carate (see Pinta)

Carbolic-acid eruptions, 251

feigned eruptions caused by, 230

Carbon-dioxide snow in adenoma sebaceum, 651

in epithelioma adenoides cysticum, 681

in keratosis senilis, 694

in lupus erythematosus, 132

in lupus vulgaris, 465

in næevus pigmentosus, 664

in nævus vascularis, 662

in rhinophyma, 135

Carbuncle. 565

in warts, 692, 693

diagnosis of, 392,566

etiology of, 566

pathology of, 566

prognosis of. 566

symptoms of, 565

treatment of -

Constitutional: nourishing diet, 567; regulation of bowels, 567; perchloride of iron and quinine, 567; if pain is severe, mor. phia, 567; no stimulants, 567

Local : paint with glycerine of belladonna, 566; Unna's mercuric-carbolic plaster mull, 566; bathe with ammonia or alkali,
567; crucial incision it skin is about to break, 567; scrape and syringe cavity with carbolic acid. and fill with antiseptic dressing, 567

Carcinoma (see Cancer)

"Cauliflower growth," 702

Cautery in epithelioma adenoides cysticum, 681

— in lichen planus, 195

- (see also Galvano-cautery and Paquelin's cautery)

Cestodes, 352

Chancre, hard, 472

- diagnosis of, 678

— extragenital, 473

$\longrightarrow$ extragenital

- - herpes and, 475

- in females, 473

$\longrightarrow$ in males, 473

phagedæna in, 475

protective influenco of, 474

regional peculiarities of. 474

soft (see Ulcus molle)

Charcot's bed-sore, 219

Cheilitis exfoliativa, 618

treatment of, 618

glandularis. symptoms of, 619 etiology of, 619

Cheiropompholyx (see Pompholyx)

Cheloid, 19, 651

clinical features of, 652

diagnosis of. 653

forms of, 651

in scars of sycosis, 558

pathology of, $€ 53$

treatment of -

Local: pressure, massage, and deep gashing, 653; mercurial ointment, 653 ; electrolysis. 653: radium, 653; X-rays, 653; Finsen light for small growths, 653 ; cocaine, belladonna. opium, for pain, 653

Chemiotaxis, 3 ,

Chemists, eruptions in, 228

Chickenpox eruption, 34, 678

Chigoe, 367

Chilblain (see Erythema pernio)

Chin, ringworm of (see Tinea sycosis)

Chloasma, 210

- diagnosis of, 210

- idiopathic, 210

- symptomatic, 210

uterinum, 210

Chloral eruptions, 244

Chlorine eruptions, 246

Chromatophores, 3

Chromidrosis, 610 diagnosis of, 611

etiology of. 610 
Chromidrosis, prognosis of, 611 symptoms of, 610 treatment of, 611

Chrysarobin eruptions, 252

Chrysophanic-acid eruptions, 252

Cicatrices, 19, 651

Cinchona eruptions, 249

Circinate syphilide, 479

- diagnosis of, 392, 395

Classification of animal parasites, 352

of dermatophytes, 377

of skin affections, Alibert's, 20

Auspitz's, 22

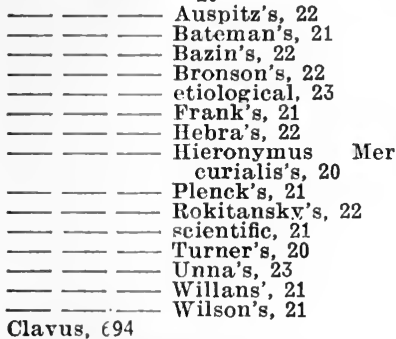

Clavus, $€ 94$

Bateman's, 21

Bronson's, 22

etiological, 23 curialis's, 20

Plenck's, 21

Rokitanskv's, 22

ientific, 21

Unna's, 23

Willans', 21

treatment of -

Local: salicylic acid after shaving off top of corn, 695; acetic acid and nitrate of silver; powders for tender feet, 695

Cleavage lines, relation of, to distribution of lesions, 26

Clinical tests for tuberculosis, 431

Coccogenic sycosis (see Sycosis, coccogenic)

Coccus grise. 594

Co-co (see Yaws)

"Cold sweat," 606

Coley's fluid in mycosis fungoides, 717

in sarcoma of skin, 711

Colloid milium, 678

\section{treatment of -}

Local: erasion or electrolysis. 679

Coloured sweat (see Chromidrosis)

Comedones, 568. 570, 604

- grouped, 605

treatment of -

Constitutional: regulation of hepatic, digestive, and menstrual functions, if necessary, 605

Local: squeeze out plugs, 605: wash with soft soap and hot water, and apply paste of kaolin. glycerine, and vinegar, or weak sulphur or resorcin ointment, 605
Concretions on hair, 642

Condyloma in acquired syphilis, 480

in hereditary syphilis, 487

Confluent acne, 236

Congelation (see Carbon-dioxide snow)

Congenital dyskeratosis (see Ich. thyosis)

ichthyosiform erythrodermia, 208

keratodermia of palms and soles, 722,726

syphilis (see Syphilis, hereditary)

Conjunctiva. " essential shrinking " of, in pemphigus, 156

Contagious impetigo (see Impetigo contagiosa)

pemphigus (see Pemphigus contagiosus)

Copaiba eruptions, 245

Cord. spinal, and cutaneous erup. tions. 56

Cordylobia anthropophaga, 367

Corium, pathological processes in, 8

Corns (see Clavus)

Cornu cutaneum, 696

Corona seborrhoica, 278

- veneris, 479,486

Corps ronds, 683

Crab-louse, 352,364

Craniotabes in hereditary syphilis, 488

Crateriform cultures, 376

ulcer of Hutchinson, 702

Craw-eraw. 368

Creams, 306

Croton-oil eruptions, 228, 230, 252

Crust. definition and characters of, 17

Cubebs eruptions. 245

Culture media. for ringworm parasites, 377

Cultures, ringworm, 376

Cutaneous angioma (see Nærus vascularis)

blastomycosis (see Blastomycetic dermatitis) calculi. 603

diphtheria. 545

— in children, 545

- - of vulva, 545

test for tuberculosis, 432

Cvlindroma of skin, 705

Cysticercus cellulosæ, 352, 369

Cysts. dermoid. 649

- sebaceous, 648

Dandruff, 593

Darier's disease (see Keratosis follicularis)

Dark-field illumination method, 498

Dartres, Alibert's, 21 
“Dead fingers," 214

Degeneration of skin, 6

Delhi boil (see Furunculus orientalis)

Demodex follieulorum, 352, 572, 604

Derma, pathological processes in, 8

Dermanyssus avium, 352

Dermatitis, artificial, 226

blastomycetic (see Blastomycetic dermatitis)

caused by external irritants, 4,226

drug. diagnosis of, 544

exfoliativa infantum, 343

- neonatorum, 343, 553

- - (see also Pityriasis

rubra) rubra)

gangrenosa infantum, 553 symptoms of, 554 treatment of-

Boric-acid baths or boric-acid fomentations, 554 : nutritious diet. 554

gestationis (see Herpes gestationis)

herpetiformis, 139

diagnosis of, 146, 147, 556

etiology of, 144

pathology of, 144

symptoms of, 139

treatment of-

Constitutional : mor. phine, 147; opium. 147; arsenic 147; an. timony, 148; quinine, 147; iron. 148; phosphorus, 148; nerve tonics. 148; phenacetin, 148; antipyrin, 148

Hygienic : regulated diet, 148: no alcohol or coffee, 148; avoidance of disturbing emotions, 148

Local : weak sulphur ointment. 147; almond oil, 147; carbolic oil, 147; olive oil with lime water, 147; salicylic acid, 147: thiol. 147; weak ichthyol ointment, 147; warm bathing, 148

malignant papillary, 698

nodular, 368

nodularis necrotica, 444

papillaris capillitii, 627

repens, 216

seborrhœic, of infancy, 279

toxic, 226
Dermatitis variegata, 201

- vegetans, 143

- venenata, 226

X-ray, 228

Dermatobia cyaniventris, 367

Dermato-neuroses (see Neurodermatoses)

Dermatophiliasis, 367

Dermatophilus penetrans, 367

Dermatophytes, mycology of, 377

Dermic papules, 10

Dermo-epidermic papules, 11

Dermoid cysts. 649

Desquamation, process of, 5, 16

Desquamative scarlatiniform erythema, 340

Devergie's disease (see Pityriasis rubra pilaris)

Dhobie's itch, 400

Diabetic gangrene, 217

Danthoma (see Xanthoma)

Diagnosis by exclusion, 29

positive, 40

of generalized eruptions, 40

of local and regional erup. tions, 41

principles of, 24

Diet in acne vulgaris, 573, 574

- in angio-neurotic œdema, 87 in carbuncle. 567

in dermatitis gangrenosa infantum, 554

in dermatitis herpetiformis, 148

in eczema. 312

- in erysipelas, 544

- in herpes zoster, 179

- in impetigo contagiosa, 551

in leprosy, 535

in leucoplakia of tongue, idiopathic, 622

in lupus erythematosus, 130

— in lupus vulgaris, 468 in miliaria papulosa, 615

in pemphigus, 167

in pompholyx, 152

In pruritus. 68

in psoriasis, 332

in purpura rheumatica, 120

simplex, 118

in Raynaud's disease, 216

in rosacea, 135

in strumous ulcers. 437

in syphilis, 506

in urticaria, 85

Diphtheria. cutaneous (see Cutaneous diphtheria)

Discomyces and mycetoma, 418:

Dissection wounds, 567

Disseminated ringworm, 383

Distribution of lesions, signiffcance of, 26

Dracunculus medinensis, 368

Drug dermatitis (see Drug eruptions)

- eruptions, 231 diagnosis of, 234. 544

$\longrightarrow$ pathology of, 232 
Drug eruptions, treatment ofConstitutional : discontinue drug. 259; diuretics, 259; iodido of potassium, 259: salino purge, 259; arsenic, 259; salol, 259

Ducrey's bacillus, 476, 586

Duhring's disease (see Dermatitis herpetiformis)

Dyers, eruptions in, 228

Dysidrosis (see Pompholyx) of face. 615

Dyskeratosis, congenital Ichthyosis)

(see

Dystrophie papillaire et pigmentaire (see Acanthosis nigricans)

Ecchymoses, definition of, 10

Echinococcus hydatid, 352, 369

Ecthyma, 548

Ecthymatous syphilide, 477, 493

Eczema, 261

- age and, 273

analogy of process with in-

flammation of joint, 314

anaphylaxis in, 291

artificial dermatitis and, 285

asthma and, 294

at climacteric, 276

at puberty, 275

auto-inoculability of, 296

catarrhal nature of, 262

chill and, 292

complexion and, 285

complications of, local, 284

- internal, 284

constitutional disturbance in, 266

contagiousness of, 297

definition of, 261

dentition and, 292

diagnosis of, 297-300, 326, 346 , $386,395,396,413,492,544$, $559,608,609$

disease of brain or spinal cord and, 294

distinguished from artificial dermatitis, 262

distribution of lesions in, 269

erythematous, 265

etiology of. 285

- microbic theory of, 287 summary of, 297

folliculorum, 281

functional neurosis and, 293 "gouty," 295

in children, 273

in elderly persons, 276

in infants, 274, 292

in insane, 294

in middle life, 275

influence of climate in, 268
Eczema, influences modifying, 268 initial lesion of, 263

intertriginous, 265

intestinal toxiemia and, 292

madidans," 269

marginatum, 371, 397 anomalous cases of, 398 diagnosis of, 298, 399. 400,408 etiology of, 399 symptoms of, 398 simulating eczema, 298 treatment of-

Local : chrysarobin, 399; naphthol, 399; sulphurous acid, 400; tincture of iodine, 400

microbic theory of, 287

motoring and, 292

"nervous," 281

nervous shock and, 291

of anus, 272

of arms, 269

of chin, 271

of conjunctiva, 271

of ear, 270

of face, 271 in infants, 274, 292

of feet, 271

Unna's three types of, 274

- - of genitals, 271

- of legs, 269

of lips, 270

of nails, 271

of nipple, 271

of nostrils, 270

of scalp, 270

of umbilicus, 272

of wrists, 271

papular, 265

diagnosis of. 434, 493, 615

pathology of, 284

polymorphous character of, 263

prognosis of, 300

pruriginosum, 139

pustular, 550

reflex irritation and. 293

regional peculiarities of 269 relation of, to functional neurosis. 294, 296

rickets and, 296

rimosum, 271

rubrum, 269

scrofula and, 296

seborrhœa and, 286

seborrhoicum, 275,277

bottle bacillus in, 279

corona in, 278

diagnosis of $395,399,404$, $408,458,492$

in infants, 279

microbacillus of Sabouraud in, 279

staphylococcus epidermidis. albus in, 279 
Eczema, sex and, 272

special forms of, 277

stage of, crustaceous, 267

desquamative, 267

dry, 267

ery thematous, 267

exudative, 267

staphylococcal influence in, 288

sweat. 280

symptoms of, 264

subjective, summary of, 282

thin, dry, anæmic skin and, 289

treatment of-

Constitutional : no drug a specific, 302 ; vinum antimoniale, 302; iron and arsenic contra-indicated in acute inflammatory conditions, 303; opium. 303 ; morphia, 303 ; veronal, trional, chloral, sulphonal, or phenacetin, 303; quinine, 304 ; arsenic, strychnine, phosphorus, or ergot in chronic neurotic conditions, 304 ; in climacteric women, musk or valerian, 304; when discharge is profuse, ouinine with belladonna, 304 ; vaccine, 304 ; thyroid extract, 304; ichthyol at menopause, 311; opium when irritation is severe in aged, 312

Hygienic : no stimulants, 303; simple diet, 303; light clothing 303,313 ; rest. 303; "lowering", diet contra-indicated, 312; hydrotheranv, 313: sulphur waters, 313; “indifferent" waters, 313; bromo-iodide waters, 313; other waters, 313; tar baths, 313; sea-bathing. 313; climate, 313

Local: when disease is rebellious, counter-irritation over vaso-motor centres of part, 304 ; conditions of success, 305; removal of crusts and scales, 305; wash with boric acid, 306; “creams," 307; pastes, 307 ; sticks, 307 ; salve muslins, 307 ; plaster muslins, 308; glycerine jelly or varnish, 308; starch jelly, 308; sulphur, 308; resorcin, 309; ichthyol. 309; tar lotion, 310; yel- low oxide of mercury, 310; tar ointment, 310; salicylic acid, 310: chrysarobin for chronio patches, 310; modification of remedies according to situation, 310 ; adaptation to age, 311; radiotherapy, 312; danger of over. treatmont, 314

Eczema tuberculatum, 714

vesicle-formation in, 264

"visceral manifestations" and, 275

xerodermia and, 286

Eczéma érysipélatoinde récidivant des arthritiques, 296

- prémycosiforme, 714

Eczematous folliculitis, diagnosis of, 392

Egg-shell nail, 646

Ehrlich-Hata's "606" (see Sal. varsan)

Electrolysis in adenoma sebaceum, 651

in angio-keratoma, 666

in cheloid, 653

in colloid milium, 679

in hirsuties, 628

in infective angioma. 667

in lymphangioma, 670

in morphœa, 185

in nævus vascularis, 663

in rosacea, 135

in telangiectasis, 661

Electro-plate workers, eruptions in, 228

Elephantiasis arabum, etiology of, 589

pathology of, 589

symptoms of, 588

treatment of -

Local : amputation, 599; X-rays, 590; galvanism, 590

Elephantoid fever, 588

Endodermophyton Castellani, 407 concentricum, 402 indicum, 402

Endo - ectothrix trichophytons, 374,375

Endothrix trichophytons, 374. 375

Enteric fever rash, 32

Epidermic papules, 11

Epidermis, pathological processes in, 7 thickening of, 6

Epidermolysis bullosa, 153

Epidermophyton inguinale, 377, 398, 399. 400

Perneti, 400

rubrum, 400

Epithelioma. 44, 701

adenoides cysticum, 679 
Epithelioma adenoides cysticum, clinical features of, 679

pathology of, 680

treatment of -

Local: excision or cautery, 681 ; dermal curette, 681; carbon-dicutaneous, 701

oxide snow, 681

clinical features of, 701 diagnosis of, 459, 494, 583,703

pathology of, 701

treatment of-

Local: surgical re. moval by preference, 704: sharp spoon with actual cautery or chloride of zinc, 704; arsenious acid and orthoform, 704; caustic potash. 704; caustic potash and pyrogallol, 704; Finsen light and $\mathrm{X}$-rays as palliatives, 704 ; radium in superficial epithelioma in which operation

Ergot eruptions, 252 is undesirable, 704

Eruptions, antitoxin, 258

arsenic, 242

artificial, 226

caused by animal irritants, 226

by external agents, 226

by inorganic substances, 226

by internal agents, 231

by vegetable irritants, 226,227

belladonna, 246

bromide (see Bromide eruptions)

characters of, in diagnosis, 25

chloral, 244, 246

cinchona, 249

copaiba, 245

cubebs, 245

drug, 231

feigned, 36,230

formalin. 246

general diagnosis of 40

iodide (see Iodide eruptions)

iodoform, 242

local, diagnosis of, 41

mercury, 247

of infectious fevers, 30

of infective granulomas, 37

on vermilion of lips, diagnosis of. 46

on face, diagnosis of, 43 $2 \nabla$
Fruptions on feet, diagnosis of 49

on fingers, diagnosis of, 48

on genitals, diagnosis of, 50

on hands, diagnosis of, 46

on mucous membranes (sce

Mueous-membrane lesions')

on nails, diagnosis of, 49

on scalp diagnosis of, 41

opium, 248

orth of orm, 242

quinine, 249

salicylic, 250

serum, 258

trade. 228

tuberculin, 257

vaccinatioli. 254

Erysipelas, 543

diagnosis of, 36, 298, 544

etiology of, 544

in vaccination, 256

migrans, 543

pathology of, 544

prognosis of, 544

symptoms of, 543

treatment of-

Constitutional: perchloride of iron, 545; strychnine, 545 ; vaccine, 545 ; serum, 545

Local : ichthyol, 544; unguentum hydrargyri nitratis, 545

Erysipeloid (see Erythema serpens)

Erythema, 92

- ab igne, 95

annulare, 104, 106

atrophicans, 120

elevatum diutinum, 110, 197

etiology of, 93

fugax, 95

gyratum, 106

hyperæmic, 92, 94

induratum ${ }_{440}$ scrofulosorum, clinical features of, 440

diagnosis of, 442

etiology of, 441

treatment of-

Cod-liver oil, 442 ; rest, 442: compression by bandaging, 442

infectiosum, 100

infiammatory, 93, 96

intertrigo, 96

\section{treatment of -}

Local : separate the surfaces by pads or powder bags, 113; strictest cleanliness, 113; wash with solution of boric acid, 113

iris, 106

keratodes, 103 
Erythema keratodes, treatment of-

Constitutional : ich. thyol, 115; iodide of potassium, 115

Local : ointment of ichthyol and salicylic acid, 115; diachylon ointment with salicylic acid, 115

marginatum, 106

migrans (see Glossitis areata exfoliativa)

multiforme, 104

diagnosis of, 109, 110 . 178,297

etiology of, 109

forms of, 104

pathology of, 108

prognosis of, 110

symptoms of, 105

treatment of-

Constitutional : in in. testinal cases, antiseptics such as salol, and vaccine, 115; arsenic, 116; antimony. 116; adrenalin, 116; sedatives in severe cases, 116

Local : calamine lotion, 116

nodosum, 111

diagnosis of, 112, 113, 532

etiology of, 112

pathology of, 112

prognosis of, 113

treatment of-

Rest, with soothing or cooling lotions, 116; salicylate of soda, 116; iron, 116

of Jacquet, infantile (see Infantile)

papulatum, 104, 105

paratrimma, 98

treatment of-

Local: neutralize pressure, 114; local cleanliness, 114 ; stimulating applications, 114 : treat sore on surgical principles, 114

pernio, 102

\section{treatment of -}

Constitutional: ferru. ginous tonies in anzmic cases, 115; digitalis. if heart is weak. 115; calcium chloride or lactate, 115: adrenalin, 115

Local: stimulate circulation, 114; iodine. 114; Friar's balsam and camphorated spirit, 114: ichthyol, 114; warm covering for hands and feet, 115; toilet vinegar for hardening skin, 115

Erythema perstans, 110

etiology of, 111

treatment of-

Local : chrysarobin ointment, 116

rubeoloid, 101

scarlatiniforme, 98

diagnosis of, 100

etiology of, 99

symptoms of, 98

treatment of -

Constitutional : remove cause, 114 ; quinine in large doses, 114; sodium salicylate, 114

Local: boric-acid ointment, 114 ; calamine liniment, 114; dusting powders, 114

serpens, 216

\section{treatment of-}

Local : salicylic-acid or ichthyol ointsimplex, 94 ment, 217

\section{- simplex, 94}

\section{diagnosis of, 531}

treatment of -

Local: remove source of irritation, 113; antipruritic remedies, 113

solare, 101

tuberculatum, 105

uræmicum, 101

Erythematous drug rashes, diagnosis of, 491

Erythrasma, 407

- diagnosis of, 408

— etiology of, 407

- prognosis of, 408

— symptoms of, 407

_- treatment of -

Local: paint with solution of permanganate of potash, or apply sulphur, cade, or naphthol ointment, 408

Erythrodermia, congenital ichthyosiform, 208

desquamativa, 209

Erythrodermias, resistant maculo-papular scaly. 2.02

Erythrodermie pityriasiane en plaques disséminées, 337

Ervthromelalgia, 63

Fsthiomène, 449

Evolution of lesions, significance of, 27

Fxamination of patient, 24

Exanthem (see Rash) 
Exclusion, diagnosis by, 29

Excoriation, process of, 5,17

Excrescences, horny, 696

Exfoliative dermatitis, 339

Face, eczema of (see Eczema of face)

lesions on, diagnosis of, 43 nodular, 45

tumours on, 45

ulcers on, 44

Faradism in alopecia areata, 641

in hyperidrosis, 607

" Farcy buds." 581

Fasciola hepatica, 352, 369

Faviform cultures of trichophytons, 376

Favus, 409

diagnosis of, 39, 396, 413

distribution of lesions of, 409

etiology of, 410

geographical distribution of, 409

mouse, 411

of animal origin, 411

of scalp, diagnosis of, 299

parasites, mycology of, 411

pathology of, 412

prognosis of, 413

scutula of, 409,412

symptoms of, 409

transmission of, 411, 412

treatment of -

Local: remove crusts by soaking with carbolized oil and wash with soft soap. 414; epilation by X-rays, 414 ; parasiticides as for ringworm, 414; avulsion of nails, 414; Leiter's tubes, 414; watch for fresh disks, 414

Feet, lesions on, diagnosis of, 49

ringworm of, 398

Feigned eruntions, 36, 230

Fever, enteric, rash in. 32

- scarlet. rash in, 30

tvnhoid, rash in. 3?

t.yphus. rash in, 32

Fibroma. diffuse, 655

hard, 656

molluscum. 654

clinical features of, 654

diagnosis of, 655

etiolog $y$ of. 655

nathology of, 655

treatment of-

Local : ligature, galvano-cautery, or the knife, 655

Fibro-mvoma, 659

Filaria Bancrofti. 589

medinensis. 352.368

- sanguinis hominis, 352. 589

Finpnrs, lesions on, diagnosis of, 48
Finsen light in acne vulgaris, 574

- in alopecia areata, 642

—. in cheloid, 653

— in cutaneous epithelioma, 704

in lupus erythematosus, 132

vulgáris, 466

in rodent ulcer, 708

Fissure, definition and characters of, 18

"Flannel rash." 602

Flea-bites, 32,366

"Flush area," 43

Flushing, 132

Fluted tongue, 623

Foetal ichthyosis, 721

Folliclis, 443

Follicular eczema, 281

- furunculosis, 560

- impetigo (see Impetigo of Bockhart)

- papule, 479

vegetating psorospermosis (see Keratosis follicularis)

Folliculite dépilante, 627

Folliculitis decalvans, 626

$\longrightarrow$ diagnosis of, 640

- - symptoms of, 626

- treatment of, 626

exulcerans, 443

scrofulosorum, 443

Foot, perforating ulcer of, 219 (see also Feet)

Fordyce's disease, 619

- diagnosis of, 620

- - etiology of, 620

- patholog. $V$ of, 620

- - symptoms of, 619

Formali treatment of, 62

Foxia Mansoni, 406

Frambœsia (see Yaws)

Frank's classification, 21

Freckles (see Lentigo)

Freezing (see Carbon-dioxide snow)

Frost-bite. 103

treatment of -

$\mathrm{Rub}$ affected part with nitrate of silver, carternally and locally, 115: massage and galvanism, 115

Frotte, 1a, 357

Fungi. diseases caused by, $370-427$

Fungus foot of India (see Mycetoma)

Furrowed tongue, 623

Furunculosis, 560

diagnosis of, 562,566

etiology of. 562

follicular. 560

- prognosis of. 561

_- symptoms of. 560

treatment of-

Constitutional : iron. 564: quinine, 564; diluted sul- 
phuric acid, 564; arsenic, 564; sulphide of calcium, 564: vaccine, 562

Hygienic : remedy insanitary surroundings, 564; improve health by appropriate measures, 564

Local : try to abort with glycerine of belladonna, tincture of iodine, boric acid, spirit of camphor, nitrate of silver, carbolic acid, or Unna's mercuric-carbolic plaster mull. 563; if abortion inapplicable, scrape out and dress with iodoform, carbolic acid or Unna's mercuric - carbolic plaster mull, 563; no poultices or fomentations unless made aseptic, 563; X-rays for isolated boils of long standing, 563; Bier's treatment, 563; wash with soap and water, bathe with boric-acid solution. dress with boric acid, precipitated sulphur, and carbolized petrolatum, 564 ; incision. 565

Furunculus orientalis, 587

etiology of, 587

symptoms of, 587

treatment of-

As for ordinary boils; X-rays, 587

Gadfly, 369

Galvanism in diffuse sclerodermia, 183

in elephantiasis Arabum, 590 in frost-bite, 115

in herpes zoster. 179

in Raynaud's disease, 215

in sclerodermia, 183

Galvano-cautery in adenoma sebaceum, 651

in angio-fibroma contagiosum tropicum, 667

in fibroma molluscum, 655

in idiopathic leucoplakia of tongue, 622

in lupus vulgaris, 465

in nævus vascularis. 663

in telangiectasis. 661

in tuberculous ulcers, 439 . 440

in verruca necrogenica, 440

in warts, 692, 693

in xanthoma, 675

Gangrene, diabetic. 217

" hysterical," 218

in vaceination, 256

"spontaneous," 218
Gangrene of extremities, symmetrical (see Raynaud's disease)

Gangrenous urticaria, 60

Genital region, lesions in; 50 ulcers in, 50

German measles, 131

Gibert's pityriasis rosea (see Pityriasis rosea)

Glanders, 580

diagnosis of, 581

etiology of, 581

prognosis of, 582

symptoms of, 580

treatment of -

Constitutional: bovine or naturally immune serum, 582; malleïn, 582; vaccine, 582

Glands, sebaceous, affections of, 592

- sweat, affections of, 605

Glandular disturbances. skin affections dependent on, 55

Glossitis areata exfoliativa, 624

Glossy skin, 221

Gnat-bites, 367

Gonorrhœal keratodermia, 585

Grain itch. 360

etiology of, 360

- symptoms of. 360

treatment of, 360

Granuloma annulare, 198

of pudenda (see Ulcerating granuloma of pudenda)

pyogenicum, 590

Granulosis rubra nasi, 607

- — diagnosis of. 608

- - pathology of, 608

- - - symptoms of, 608

Graves's disease, 211

Green sweat, 611

Greyness of hair (see Canities)

"Grog-blossoms," 133

Grooved tongue, 623

Growths (see New growths)

Guinea-worm, 368

Gum rash, 80

Gummata, 485

- diagnosis of, 442

- in hereditary syphilis, 488 subcutaneous diagnosis of, 494

Gunshot wound, skin lesions fol. lowing. 58

Gypseum cultures, 376

Hæmato-lymphangioma, 670

Hæmochromatosis. 213

Hair, affections of, 626-44

atrophic changes in, 630 "bavonet," 632

beaded (see Monilethrix)

- greyness of (see Canities) overgrowth of (see Hirsuties)

Hands, lesions on, diagnosis of, 46 
Harlequin fotus, 725

Harvest-bug, 352

bites, 367

\section{treatment of -}

Local : naphthol or weak mercurial ointment, 367

Uebra's classification. 22

- prurigo (see Prurigo of Hebra)

Hectine, 514

Henoch's purpura, 119

Hereditary syphilis (see Syphilis, hereditary)

Herpes, 167

bilateral, in hereditary syphilis. 176

circinatus bullosus, 139

diagnosis of, 299

facialis, 168

febrilis (see Herpes, irrita tive)

frontalis, etiology of, 58

genitalis, 168, 169

and syphilis, 475

gestationis, 148

symptoms of, 148

- treatment of, 149

iris, 107

irritative, 168

diagnosis of, 170

symptoms of, 168

treatment of-

Local : soothing and anti-pruritic lotions or ointments, 179 ; protective powder or muslin bags, 179 : boric-acid or calamine lotion, 179; black wash for genitals, 179

labialis, 168

phases in evolution of lesions of, 168

progenitalis, 168, 169

prognosis of, 178

pyæmicus (see Impetigo herpetiformis)

symptomatic (see Herpes, irritative)

zoster, 168, 171

diagnosis of, 177, 299

distribution of lesions in, 172

etiology of, 57, 175

pathology of, 175

symptoms of, 171

treatment of -

Constitutional : su bcutaneous

injections of morphine, 179 ; iodide of potassium, 179; antipyrin. 179; quinine. 179 ; iron, 179 ; strychnine. 179; codliver oil, 179
Herpes zoster, treatment of

Local : menthol, 179; protective powders, 179; collodion, 179; ethyl chloride, 179; continuous current, 179 ; division, stretching, or resection of supraorbital nerve, 179

Hidradenoma, 651

Hidrocystoma, 615

Hieronymus Mercurialis's class1fication, 20

High-frequency current in alopecia areata, 642

in lupus erythemato sus, 132

in pruritus, 67

in psoriasis, 335

in Raynaud's disease. 215

Hirsuties, 628

prognosis of, 628

treatment of -

Local : electrolysis, 628 ; X-rays, 629; depilatories not advisable, 629

"Honeycomb scab," 546

Horny excrescences, 696

House-flies, 352

Hydatid echinococcus, 369

Hydradenitis destruens suppura tiva, 443, 561

Hydradénome éruptif (see Epitheliom a adenoides cysticum) Hydroa restivale, 143

- gestationis, 148

gravidarum, 148

herpetiforme, 139

puerorum, 143

vacciniforme, 139, 143

Hydrosis oleosa in eczema, 278

Hydrotherapy in alopecia areata, 640

in eczema, 313

- in ichthyosis, 726

- in miliaria papulosa, 615

- in pityriasis rosea, 351

- in prurigo of Hebra, 75

- in pruritus, 68

— in psoriasis. 329, 335

- in syphilis, 502

in ulcus molle, 586

Hyperamic ery thema, 92, 94

Hyperæesthesia, 62

in leprosy, 524

Hyperidrosis, 605

etiology of, 606

prognosis of, 607

treatment of-

Constitutional: tonics, 607 ; belladonna, 607; atropia, 607; ergot, 607; sulphur, 607: diuretics, 607

Local: inunction of belladonna ointment or lini- 
ment, 607; faradization, 607; powder stockings and boots with boric acid, 607; dust on boricacid or salicylic-acid powder after application of wet sponge, 607 ; X-rays, 607

Hyperkeratosis congenita, 725

Hyperplasia, 3

Hyperirophic scar, 19

Hypertrophy of nails, 646

Hypoderm, pathological processes in, 8

Hypodermic sarcoids, 442

Hysteria and skin affections, 60

“Hysterical gangrene," 218

“- hemiplegia," 60

“- œdema," 60, 87

- treatment of, 88

Ichthyosiform erythrodermia, congenital, 208

Ichthyosis, 721

diagnosis of, 402

etiology of, 725

forms of, 721

follicularis, 723

glossæ, 620

hystrix, 724

linguæ (see Leucoplakia of tongue. idiopathic)

ordinary (xerodermia), 722

pathology of, 725

rubra, 209

sebacea cornea (see Keratosis follicularis)

simplex, 724

treatment of-

Constitutional: thyroid extract, 726

Local : alkaline or bran baths and inunction with lanolin, 726; salicylic acid for small hystrix growths, excision or scraping for larger ones, 726; weak sulphur ointment and sulphur baths, 726

Idiopathic multiple pigmented hæmorrhagic sarcoma, 711

Impetiginous syphilide, 477, 493

Impetigo bullosa, 547, 549

circinata, 546

contagiosa, 546

complications of, 548

diagnosis of, 39, 493, 550

etiology of, 549

symptoms of, 546

treatment of-

Constitutional: iron, 551 ; n o u ris hing food, 551; vaccine, 551
Impetigo contagiosa, treatment of (contrnued) -

Local: removal of scabs by soaking in carbolized oil or carbolic-acid lotion, 551; boric starch poultices, 551 ; weak mercurial ointment, 551 ; wash with boric-acid or carbolic lotion, 551

diagnosis of. 385

due to raying, 388

follicular (see Impetigo of Bockhart)

herpetiformis, 554

diagnosis of, 556

- etiology of, 555

- - prognosis of, 556

prognosis of, 556
symptoms of, 554

treatment of-

As for impetigo contagiosa (q.v.); autogenous vaccine, 556

intertrigo, type of, 548

of Bockhart, 556

diagnosis of, 557

etiology of, 557

pathology of, 557

symptoms of, 556

treatment of-

Constitutional : iron, 557; a u tog en ou s vaccine, $65 \%$

Local : cleanse with boric-acid or carbolic fomentations, and apply white precipitate o int $\mathrm{ment}, 557$; we a k tincture of iodine where scalp is atfected, 557

varioliformis, 443

vulgaris (see Impetigo contagiosa)

Indian hemp eruptions, 251

Indiella genus. 418

Infantile ery thema of Jacquet, 96 diagnosis of, from congenital syphilis, 97

treatment of -

As for erythema intertrigo (q.v.)

Infective angioma, 667

granulomata, 37

Inflammation of skin, 2

- pigmentation as sequela of, 3

Inflammatory erythema. 93, 96

Ingrowing toe-nail, 645

Injuries to nerves, skin diseases following, 58

Inoculable díseases, diagnosis, 39 Inoculation rashes. 254 
Intertrigo (see Erythema intertrigo)

Iodide eruptions, 239

- compared with bromide eruptions, 241

diagnosis of 241

symptoms of, 239

Iodine, felgned eruptions caused by, 230

Iodoform eruptions, 242

lonization in lupus erythematosus, 132

- in rodent ulcer, 708

Iron eruptions, $\angle 5 \%$

Irritants, animal, 226

- external, dermatitis caused by, 4

— inorganic, 228

Itch (see Scabies)

Itch-mite, 352

Itching, 2, 64

(see also Pruritus)

Jacob's ulcer (see Rodent ulcer)

Jigger, 367

Joint affections associated with psoriasis, 320

Kaposi's disease, 718

clinical leatures of, 718

etiology of, 719

pathology of, 719

prognosis of, 720

ointment, 358

Keloid (see Cheloid)

Keratodermia erythematosa symmetrica, 104

gonorrhœal, 585

of palms and soles, congenital, 722,726

Kératodermie blennorragique, 585

Keratolysis neonatorum, 343

Keratoma, senile (see Keratosis senilis)

Keratosis follicularis, 681

- clinical features of, 681

- diagnosis of, $684,686,723$

- etiology of, 682

— pathology of, 682

- spinulosa, 198

L o c a $l$ : thermo-cautery, 684; palliative measures, 684

— pilaris, 722 diagnosis of, 435,684 senilis, 693

treatment of -

Local.: resorcin and salicylio acid in early stage, 694; later, exeision, X-rays, radium, or carbon-dioxide snow, 694
Kerion, 376, 383

Kienböck-Adamson method of eplation in favus, 414

Koilonychia, 644 in ringworm, 388

Koplik's spots, 31

Kraurosis vulva, 623

Labiomycosis, 426

Lacquer poisoning, 228

Lamellar desquamation of newborn, 721

"Lancet" Special Commission on Vaccination, 260

Land scurvy, 118

Langue géographique, 624

Larvæ, eruptions caused by, 369

Lassar's paste, 307

Lead (acetate and carbonate) eruptions, 252

Leishman body, 551

Leishmania tropica, 587

Lenticular papule, 479

Lentigo, clinical features of, 687

— etiology of, 687

- pathology of, 687

Leontiasis, 520

Lepocolla repens, 325

Lepothrix, 642

"Lepra" psoriasis, 317

Lèpre vitilige, 180

Leprosy, 516

- anæsthetic, 518

bacillus of, 525,528

climatic features of, 528

complete, 518

contagiousness of, 530

diagnosis of, 38, 404, 531, 532

etiology of, 528

fish-eating theory of. 529

heredity and. 531

incubation period of, 516

invasion period of, 516

macular, 517

- diagnosis of, 416

mixed, 518, 526

nerve, 518, 522

altered sebum secretion in, 523

sweat secretion in, 523

anæsthesia in, 523, 524

bullous eruption in, 523

claw-like fingers in, 525

hyperæsthesia in, 522, 524

muscular atrophy in, 524

neuralgic pains in. 522 . 523,524

peripheral neuritis in. $522,523,524,525$

pigmentary changes in, 522 
Leprosy, nerve, stages of, 524 symptoms of, 522 ulcers in, 524, 526

nodular, 518

pathology of, 526

prodromal period of, 516

prognosis of, 532

skin, 518 leontiasis in, 520

- nodules of, 518

pustules in, 521

ulcerative stage of, 521

stages of, 516

starting-point of, supposed, 517, 529

symptoms of, 516

treatment of -

Constitutional: tuberculin, 533; serum, 533; chaul. moogra oil, 533 ; arsenic, 533; gurjun oil, 533; nastin, 533; mercury, 534 ; thyroidin, 534; quinine, 535; cod-liver oil, 535

Local : Chaulmoogra ointment, 533; gurjun oil, 533; X-rays, 534; radium, 534; n erve-stretching, 534 ; evacuation of leprous infiltration, 534

tubercular, 518

Leptus autumnalis, 352, 367

Lesions, asymmetrical, 26

distribution of, 26

elementary. 9

evolution of, 27

on face, diagnosis of, 43

on feet, diagnosis of, 49

on fingers, diagnosis of, 48

on genitals, diagnosis of, 49 , 50

on hands, diagnosis of, 46

on mucous membranes, diagnosis of, 51

on nails, diagnosis of, 49

on scalp. diagnosis of, 41

on vermilion of lips, diagnosis of. 46

primary, 9

secondary, 16

symmetrical, 26

Leucodermia, 211

diagnosis of. 213, 416

etiology of, 212

prognosis of 213

symptoms of, 211

treatment of -

Local : corrosive sublimate. 213 ; peroxide of $\mathrm{hy}$ drogen, 213

Leucodermic lesions on neck in secondary synhilis, 481

Leucokeratosis, 620

Leucoma (see Leucoplakia of tongue. idiopathic)

Leuconychia,. 644

Leucoplakia of penis, 622
Leucoplakia of tongue, idiopathic, 621

- - diagnosis of, 621

etiology of, 621

symptoms of, 621

treatment of-

local: avoid irri-

tants, 621; io-

dide of potas-

sium solution,

622; galvano-

cautery, 622 ; ra-

dium, 622; ex-

of vagina, 622 cision, 622

— of valva, 622

- syphilitic, 484

Leucoplasia (see Leucoplakia)

Leukæmia cutis, 717

treatment of -

X-rays or arsenic as a palliative, 718

Levaditi's method, 499

Lice (see Pediculus)

Lichen, 187

- acuminatus, 204

agrius, 187

annularis, 196

annulatus, 601

- atrophique, 189, 191

circumscriptus, 601

gyratus, 601

hypertrophicus, 191

infantum, 614

neuroticus, 205

nitidus, 196

pilaris, 198

planus, 187

acute, 191, 192

atrophicus, 191

chronic, 191

diagnosis of, 193, 207 . $299,327,346,493,621$ distribution of lesions in, 188

etiology of, 193

of mucous membranes, 190

pathology of, 192

sclerosus, 191

symptoms of, 188

treatment of-

Constitutional : a r senic, 194 ; antipyrin. 195; biniodide of mercury in generalized lichen planus. 195; salicyl-arsenate of mercury. 195

Local : corrosive sublimate and carbolicacid ointment. 195; pyrogallic acid, 195; mercurial plasters, 195; cautery, 195; ruber, 187 X-rays, 196

$\longrightarrow$ acuminatus, 187 
Lichen ruber moniliformis, 189 $\underset{\text { planus) }}{\text { planus (ichen }}$

scrofulosorum, 187, 433 clinical features of, 433 diagnosis of, 434 etiology of, 433 pathology of, 434 treatment of -

Local: calamine lotion or boric-acid ointment, 435

scrofulosus (see Lichen scrofulosorum)

simplex, 187

- chronieus, 76

spinulosus, 198

diagnosis of, 435

strophulosus, 187

strophulus, 80

tropicus (see Miliaria papulosa)

urticatus, 80,187

variegatus, 201

verrucosus, 192

Lichenification in eczema, 77

- in lichen planus, 77

in prurigo 76

- in psoriasis, 77

Lichenization (see Lichenification)

Lichenoid parapsoriasis, 337

syphilides, diagnosis of, 434

Light treatment (see Finsen light)

"Lily rash," 228

Lime, handling of, eruptions caused by, 228

Linear atrophodermia, 222

Lips, lesions on vermilion of, diagnosis of, 46

mucous membranes of, affections of, 616

Liquid-air treatment. 745

Livedo reticularis, 95

Liver-fluke, 352

Iouse, body. 352, 364

— crab. 352.364

— head, 352,364

pubic. 352,364

Lupoma, 446

Lupus disseminatus, 447

- erythematosus, 120

aggregatus, 121

course of, 124

diagnosis of, 128, 129 , $328,458,608$

discoides, 121

disseminatus, 121

etiology of, 126

multiple epithelioma following, 125

nodular, 122

of mucous membranes, 122

of scalp, diagnosis, 640

patholog $v$ of, 125

prognosis of, 124
Lupus erythematosus, relation of, to tuberculosis, 128

symptoms of, 120

telangiectatic, 121

"transformation" of , into lupus vulgaris, 130 treatment of -

Constitutional : vaccine, 130; salol, 130; ichthyol, 130, 131; adrenalin, 130; quinine, 131

Hygienic: avoidance of a l cohol, tea, and coffee, 130 ; easily digested food, 130; care of teeth and gums, 130 : fresh air. 131; exercise, 131 ; sea air contraindicated, 131

Local : evaporating lotions, 131; calamine lotion, 131; lotio picis carbonis, 131 ; solution of subacetate of lead, 131; ichthyol, 131; Hebra's spiritus saponis alkalinus, 131 ; iodine, 131 ; resorcin. 131 ; salicylic acid, 131; pyrogallic acid, 131; lin. iodi fortis. 131; linear scarification, 131; carbon-dioxide snow, 132: thermo-cautery, 132: X-r a y s, 132 ; high-frequency current, 132; Fin sen light, 132 ; ionization, 132

— exedens, 446 hypertrophicus, 448

non-exedens, 446

papillomatosus. 448,450

serpiginosus, 448

verrucosus, 440

vulgaire érythématöide, 129

vulgaris, 446 age and, 453

bovine tubercle bacillus and, 456

clinical course of. 449

complications of, 450,451

diagnosis of, 437, 457 . $494,531,608$

distinctive lesion of, 446

distribution of lesions of. 448

enithelioma and, 451

etiology of, 452

general health and, 451, 455

measles and, 453

mucous - membrane lesions in, 448,449 
Lupis vulgaris, nasal catarrh and, 454

pathology of, 456

prognosis of, 459

pseudo - elephantiasis caused by, 451

puimonary phthisis and, 452

secondary causes of, 453

- effects of, 450

sex and, 453

spontaneous cure of, 450 starting-points of, 454

symptoms of, 446

transmissibility of, 455 - by vaccination, 256

treatment of-

Constitutional : no internal $r$ emed $y$ is a specific except perhaps thyroid extract, 468; cod-liver oil, 468; Old Tuberculin, 468; New Tuberculin, 469

Hygienic : good food, 408; sea air, 468

Local : appıy salicylic acid, 461; rub in Brooke's ointment, dredge with potatostarch powder, 461; if skin breaks, dress with boric-acid ointment, 461; bactericidal applications, such as mercurial plasters, corrosive sublimate solution, or perchloride of mercury injections, $461-2 ;$ c hom i c a l caustics : nitrate of silver, 462; salicylic plaster of Unna, 462; acid nitrate of mercury, 462; lactic acid, 462; arsenical paste, 462 ; chloride of zinc, 463; pyrogallic acid, 463 ; novocaine. 463; mechanical treatment: excision, 464; era sion, 464; multiple puncture, 465 ; scarification, 465; Paquelin's cautery, 405; galvano - cautery, 465; Finsen light in non - u l cerative cases, 466; X-rays for deep ulceration and when mucous membranes are affected, 466; static current, 466; carbondioxide snow, 466; when inflammation is severe, calamine or lead lotion, 467; choice of method, 467

Lupus vulgaris, warty vegetations in, $450^{\circ}$

Lymphangioma circumscriptum cutss, 667

treatment of-

Electrolysis, cautery, X-rays, or excision, 670

tuberosum multiplex, 669

Lymphangioma-myoma, 659 Lymphoma simplex, 669

Maculæ cœruleæ, 363

Macular atrophodermia, 223

- leprosy (see Leprosy, macu. lar) syphilide, 478

Macule, definition and characters of, 9

Maculo-papular syphilide, 478

- diagnosis of, 350

Maculo-papulo-squamous syphilide, 479

Madura foot (see Mycetoma)

Madurella genus, 418

Malassezia tropica, 405

Malformations, 721-8

Malignant disease (see New growths, malignant)

pustule, 583 diagnosis of, 490, 584, 585

etiology of, 584

pathology of, 584

prognosis of, 585

symptoms of, 584

treatment of-

Excision or free scraping, 585; Sclavo's

Malleīn, 582 serum. 585

Massage in alopecia areata, 640

in cheloid, 653

__ in diffuse sclerodermia, 183

in frost-bite, 115

- in morphœea, 185

— in psoriasis, 335

— in Ravnaud's disease, 215

- in sclerodermia, 183

Measles, diagnosis of, 491

German, rash of, 31

Medicinal rashes (see Drug eruptions)

Megalery thema epidemicum, 101

Mégaspore trichophytons, 374,376

Melanin, 3

Melanoblasts, 3

Melanosis lenticularis progressiva (see Kaposi's disease)

Melanotic cancer, 700

Meralgia paræsthetica, 63

Mercury eruptions, 247 
Mibelli's porokeratosis, 199 Microbacillus of sabouraud, 570 Microbic aflections, 428-591 Micrococcus tetragenus, 612 - vesicans, 369

Microtilaria perstans, 368

Microïde trichophytons, 374, 376

Microörganisms, diseases caused by, sy, 428-591

Microsporon anomœen, 350

Audouini, 372

canis, 372

felineum, 372

furfur, 400, 404

lanosum, 393

Mansoni, 406

minutissimum, 400,40 '

tardum, 372, 373

Microsporons and trichophytons, differences between, 373

of ringworm, 372

- cultures of, 376

Microsporosis, 373, 382, 384, 385 (see also Microsporons)

Miliaria (see Sudamina)

alba, 614

- papulosa, 187, 614

symptoms of, 615

treatment of-

Constitutional : a c etate and nitrate of potash, 615; avoid stimulating foods and drinks, 615

Local: soothing or evaporating lotions, 615; alkaline or bran baths, 615 ; removal of irritation, 615

rubra, 614

Miliary benign lupoid (see Multiple benign sarcoid)

Tiliupule, 478

Milium, 568, 603

$$
\text { colloid (see Colloid milium) }
$$

pathology of, 603

symptoms of, 603

treatment of -

Expel milia through small incision, 604; iodine or strong carbolic, 604

Mixed sore in syphilis, 476

Mole (see Nævus pigmentosus)

Molluscum contagiosum, 676

—— clinical features of, 676

- diagnosis of, 678,684

etiology of, 677

pathology of, 678

treatment of -

Local: touching with

liquid carbolic and painting with collodion, 678; surgery, Monilethrix, 631

etiology of, 631

pathology of, 631
Morbid anatomy of skin, 9

Morbus maculosus, 118

Morococcus, eczema and, 287, 294

Moro's test for tuberculosis, 432

Morphoea, 183

- diagnosis of, 185

etiology of, 184

guttata, 185

pathology of, 184

prognosis of, 185

symptoms of, 183

treatment of-

Local: gentle massage, 185; electrolysis, 185

Morvan's disease, 220

Mosquito-bites, 307

Motor disturbances, 55

Mouse favus, 411

Mucous-membrane lesions, diagnosis of, 51

in acanthosis nigricans. 685

in arsenic eruptions, 243

in chloral eruptions, 245

in dermatitis herpeti. formis, 140

in eczema, 270, 271

in eczema marginatum, 398

in epidermolysis bullosa, 153

in erythema multiforme, 105

scarlatiniforme, 98

in glanders, 580

in herpes, irritative, 168 , 169

zoster, 174

in impetigo contagiosa, 547

herpetiformis, 555

in leprosy, 520

in lichen planus, 190

in lupus erythematosus, 122

vulgaris, 448, 449

in pemphigus foliaceus, 158

vegetans, 158

vulgaris, 156

in psoriasis, 318

in rhinoscleroma, 582

in sclerodermia, 180

in syphilis, secondary. 482

tertiary, 486

in tinea circínata, 394

in ulcerating granuloma of pudenda, 590

- in urticaria, 79

Mucous membranes of lips, tongue, and mouth, affections of, $616-25$

patch, 480

— tubercles, 483, 507

Multiple benign sarcoid, 445

Multiple benign sarcoid, 445 
Multiple pigmented sarcoma (see Sarcoma)

Musca cadaverina, 352

domestica, 352

- vomitaria, 352

Muscular atrophy in leprosy, 525

Mustard, feig ned eruptions caused by, 227, 230

Mycetoma, 417

\section{- etiolog $\mathrm{v}$ of, 417,418}

melanoid, 417

ochroid, 417

pathology of, 417, 418

symptoms of, 417

treatment of-

Local : potassium iodide, 418 ; amputation, 418

Mycology of dermatophytes, 377

Mycosis fungoides, 713

clinical features of, 713

diagnosis of , 541,716

etiology of, 715

pathology of, 715

\section{Treatment of-}

X-rays, 716; arsenic, 717; Coley's fluid, 717 ; resorcin ointment, 717

Myoma cutis, 657

clinical features of, 658

diagnosis of, 659

pathology of, 659

treatment of-

Local: excision, 659

Myxœedema, 660

Myxoma, 659

Nackenkeloid, 627

Nævus anæmicus, 663

araneus, 661

capillary-venous, 661

linear, 653

lipomatodes, 661

pigmentosus, 663

clinical features of, 663

malignant transformation of, 664

treatment of-

Carbon-dioxide snow, 664; excision, 665; caustics contra. spider-like, 661 indicated, 665

spilus, 663

stellate, 661

vascularis, 661

clinical features of, 661

etiology of, 662

pathology of, 662

treatment of -

L o c a $l$ compression, 662 ; radium, 662 ; carbon-dioxide snow, 662 ; liquid air, 662 ; injection of irritants or astringents,
Nævus, venous, 661

662; electrolysis, 663 ; galva n o - a utery, 663 ; caustics, 663

Nails, affections of, 644-6

Niagnosis of, 49

hypertrophy of, 646

ringworm of (see Onychomy cosis)

Nematodes, 352

Neo-endothrix trichophytons, 375

Nerve disorder, skin affections caused by, 6, 39

Nerves, injuries to, skin diseases following. 58

Nervous diseases of skin (see Neuro-dermatoses)

“- eczema," 281

Nettle-rash (see Urticaria)

Neuralgic pains in leprosy. 524

Neurasthenia and skin affections, 60

Neuro-dermatoses, $54-225$ classification of, 54

- general characters of, 39 hysterical, 60

- sensory, 62

Neuro-fibroma, 656

Neuroma, 659

Neuroses of skin (see Neurodermatoses)

New growths, 39, 647-728

- benign, 647-96

$\longrightarrow$ malignant, 697-728

New Tuberculin (see Tuberculin)

Nikolsky's sign, 141, 157, 158

Nitric acid, feigned éruptions caused by, 230

Niveum cultures, 376

Nodular lesions on face, 45

Nodule, definition and characters of, 11

Nodules, rheumatic, diagnosis of, 657

Norwegian itch, 359

Nux vomica eruptions, 252

Ochronosis, 211

Edema, acute circumscribed, 81

- angio-neurotic (see Angioneurotic odema)

hysterical, 87

- nysterical, 87,727

Edème bleu des hystériques. 88 Estrus, 369

Onychauxis, 646

Onychia, 645

dry, in secondary syphilis, 483

maligna. 645

—_ sicca syphilitica, 483

Onychogryphosis. 646

Onychomyosis, 371, 396

diagnosis of, 396

prognosis of, 396 
Onychomyosis, symptoms of, 396 treatment of-

Local: serape nails and apply parasiticides, 397; chrysarobin, 397; remove affected parts and apply creosote, acetic acid, benzine, corrosive sublimate, mercurial plaster, resorein, or tincture of iodine, 397; soak nails with Fehling's solution, remove them, and dress with copper sulphate, 397

Onychorrhexis, 644

Onychoschizia. 644

Oospora canina, 411

genus, 418

Ophthalmic reaction of Calmette, 432

Opium eruptions, 248

itching caused by, 248

Opsonins, 3

Orthoform eruptions, 242

Osteo-arthritic pains in psoriasis, 320

Overgrowth of hair (see Hirsuties)

Oxyuris vermicularis, 352

Paget's disease, 697

clinical features of. 698

diagnosis of, 300, 699

etiology of, 698

pathology of 698

prognosis of, 698

treatment of-

Local: amputation by preference, 699; radium. 700 ; X-rays in inoperable cases, $7(11$

Palm, dry scaly eruptions on, 47

Paludal urticaria, 82

Paperhangers, eruptions in, 228

Papilloma (see Wart)

Papular syphilide, 478

Papule, definition and characters of, 10

formation of, 4

Papulo-squamous syphilide, 479

Paquelin's cautery in keratosis follicularis. 684

in lupus erythematosus, 132 vulgaris, 465

- - in pruritus, 67

—_ in rosacea, 135

- in xanthoma, 675

Paraffin eruptions, 228

Parakeratosis, 4

- variegata, 200, 337

- - symptoms of, 200

- treatment of, 203

Paranghi (sec Yaws)

Paransoriasis, 336

diagnosis of, 329, 338
Parapsoriasis en goutte, 336

en plaques, 337

etiology of, 338

guttata, 336

lichenoid, 337

pathology of, 337

sy mptoms of, 336

treatment of-

Constitutional : arsenic 338; mercury, 338; deal with symptoms, 338

Local: weak resorcin or salicylic-acid ointment, 338

Parasites, animal. 352

- accidental, 352 diseases caused by, 39

miscellaneous, 366

occasional. 352

stationary, 352

vegetable, 370

Parasitic affections, $7,352-427$

Parasyphilis, 471, 489, 497, 498, 513

Paronychia, 645

in secondary syphilis, 483

Parrot's nodes, 488

Pars papillaris, pathological processes in, 8

Pastes, 307

Pathological processes in derma, 8 in epidermis, 7 - seats of, 7

Pathology of skin, 1

Pediculoides ventricosus, 360

Pediculosis, 361

diagnosis of, 365

— etiology of, 363

of body, 362 symptoms of, 362

treatment of -

Local: bake clothes, 366; alkaline or hot baths, with antiseptic soap, 366

of head, 362

symptoms of, 362

treatment of-

Local: white precipitate ointment, 365 ; acetic acid, 365 : ether and oleate of mercury, 366; petroleum, turpentine, or oil of sassafras, 366 ; remove crusts with carbolized oil or carbolic-acid lotion and treat impetigo with mercurial or boricacid lotions, 366

of pubes, 363

symptoms of, 363

\section{treatment of -}

Local : as for pediculosis of head, but hair not to be cut, 
Pediculus capitis, 364

366; white precipitate ointment, 366; oleate of mercury and æther. sulph. 366; application of soothing lotion, 366

- corporis. 364

- pubis, 364

Pelade (see Alopecia areata)

Peliosis rheumatica (see Purpura rheumatica)

Pellagra, 135

diagnosis of, 137

etiology of, 136

pathology of, 137

prognosis of. 137

symptoms of, 135

treatment of-

Symptomatic : opium, 137; quinine, 137; calomel, 137; arsenic, 137

Pemphigus, 152

acute, 157

contagiosus, 551

treatment of-

Cleanliness, 552: bichloride of mercury lotion, 552; dust with boric-acid, starch, and zinc oxide powder, 552

etiology of 162

foliaceus, $153,158,346$

diagnosis of, 165

prognosis of. 165

symptoms of, 158

treatment of-

Local : bland ointments, 167

Constitutional : treat fever and other symptoms on general principles

Hygienic : nourishing diet, 167

neonatorum, 552

of mucous membranes, 153, 156. 158. 159

pathology of, 161

nruriginosus, 139

treatment of-

Constitutional : vaccine, 166; arsenic, 166; opium, 166: nhosnhorus. 166 ; ichthvol, 166; belladonna, 166

Hyirienic: nourishing food, 167

Local: prick large bulla with sterilized needle. 166: dress with boric-acid ointment, carron oil. or weak lead lotions. 166; cooling nintments, 166

vegetans. 153

diagnosis of, 165
Pemphigus vegetans, prognosis of, 166 symptoms of, 159

- vulgaris, 153 diagnosis of, 164

- prognosis of, 165

- symptoms of, 155

Percutaneous test for tubercu. losis, 432

Perforating ulcer of foot, 219

Perionychia in secondary syphilis, 483

Perlèche, 616

diagnosis of. 617

etiology of, 617

prognosis of, 617

symptoms of, 616

treatment of, 618

Pernio (see Erythema pernio)

Perspiration (see Sweat)

Peruol, 358

Petaloid patches of seborrhœa, 600

Petechia, definition of, 10

Petroleum eruptions, 228

Phagocytosis, 3

Phenacetin eruptions, 252

Phosphorescent sweat, 612

Piedra, 643

Pigmentation, 17

- anomalies of, 209

- as result of inflammation, 3 cause of. 3

- general, 17

- in syphilitic lesions, 6

- local. 17

Pinta. 414

diagnosis of, 416

diatribution of lesions in, 414, 415

etiology of, 416

forms of 414

geographical distribution of, 414

prognosis of, 417

prophylaxis of. 417

symptoms of, 414

treatment of -

Local: in early cases, tincture of iodine, 417; in more advanced, chrysarobin, 417; for facial lesions, resorcin, resorcin-sulphur, or salicylicacid ointment, 417 ; mercury nitrate ointment. 417

Pirquet's (von) test for tuberculosis, 432

Pityriasis, 339

alba, 593

- capitis. 592

circinata (see Seborrhœa corporis)

of scaln. diagnosis of, 385 rosea. 339.348 circinate lesions in, 349 diagnosis of, 350,351 , 404,408 
Pityriasis rosea, etiology of, 350 maculate lesions in, 349 prognosis of, 351 symptoms of, 348

treatment of -

Local : anti - pruritic applications such as liquor picis carbonis, 351; tepid bran or alkaline baths, 351; vaseline with salicylic acid after bath, 351

rubra, 339, 340

contagious form of, 343

diagnosis of, 100, 328, 345,346

etiology of, 344

following other affections, 341

in new-born infants, 343

pathology of, 344

primary. 341

secondary, 341

prognosis of, 346

symptoms of, 340

treatment of-

Constitutional : a $\mathbf{n}$ timony in acute cases, 347; arsenic in chronic cases, 347 ; opium for sleeplessness, 347

Hygienic : no stimulants, 347 ; protection against cold, 347; nutritious food and cod-liver oil, 347

Local: tepid bran or alkaline baths, 347; tarry preparations, 347; application of liquor picis carbonis, 347: creoline, 347 ; oil of cade ointment, 347: carbolized oil, 347 ; swathing in subacetate of lead lotion, 347,348 ; mercurial prepara tions contra-indicated. 348; dust with starch and oxide of zinc powder, then cover with cottonwool or bandages steeped in calamine liniment, 348: in infants, smear skin with fatty substances, then cover with cotton-wool, 348

pilaris. 188, 204, 339

diagnosis of, 207

distribution of lesions in, 206

pathology of, 207

symptoms of, 205
Pityriasis rubra pilaris, treatment of -

Constitutional:

arsenic contraindicated, 208; a $r$ sen i t 0 of sod a, 208; $\beta$-naphthol, 208; guaiacol c a rbonate. 208 ; carbolic a c id, 208; hypodermic injection of a r sen ic, 208; a t o x y 1, 208 ; cacodylic acid, 208; pilocarpin, 208; jaborandi, 208

Hygienic: attention to diet, 208; violent exercise. 208

Local: tar. 208; oil of cade, 208 ; nyrogallic acid, 208; soothing applications.

simplex. 339 208

steatoides, 594

- versicolor (see Tinea versicolor)

Pix liquida eruptions, 252

Plasma cells, 4

Plenck's classification, 21

Plica nolonica, 362

Podophrllin eruptions, 253

Pompholyx. 149

diagnosis of, 151, 152, 300

etiology of, 150

pathology of, 150

prognosis of, 152

simulated bv ringworm, 152

symptoms of, 149

treatment of -

Constitutional : iron. 152; arsenic, 152: ouinine, 152; strychnine. 152

Hyrienic: no violent exercise, 152; no alcohol, 152; regulated diet. 152; change of scene, 152

Local: as for nruritus

Porokeratosis (Mibelli), 199

"Port-wine mark," 661

Positive diagnosis, 40

Post-mortem wart (see Verruca necrogenica.)

" Prickly heat," 187 (sre also Miliaria papulosa)

Primary sore (see Chancre)

Primula nconica eruptions, 22.8

Proof medium, Sabouraud's, 377

Prurigo. 73

ptiologr of, 73

ferox, 74 
Prurigo, lichenification in, 76 1 mitis, 74

-... of Hebra, 73

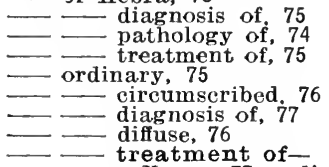

X-rays, 77 ; radium, 77 ; static electricity, 77 ; adhesive dressing, 77; sedatives, 77 ; di et, 77

simple, 80

Pruritus, 64

$$
\text { ani, } 66
$$

bath, 66

diagnosis of, 66

generalized, 65

etiolog of, 65

hiemalis, 65

local, 66

etiology of, 65

of anus, 66

of nares, 66

of palms of hands, 66

of scrotum, 66

of soles of feet, 66

of vulva, 66

opii. 248

palmaris et plantarum, 66

senilis, 66

treatment of -

External : menthol, 67; cocaine, 67; Paquelin's thermo-cautery, 67; Xrays, 67 ; high-frequency current, 67; radium, 67; Turkish baths. 69; emollient and alkaline baths, 69: hot water, 69; evaporating lotions, 70 ; liquor plumbi subacetatis, 70 ; carbolic acid, 70; mercury, 71; naphthol. 71; cocaine, 71 ; menthol, 71 ; chloroform. 71; chloral, 71 ; salicylic acid, 72; tar, 72 ; nitrate of silver in solution, 72 ; benzoin, 72 : ichthyol, 68,72

Hygienic: cloth ing. 67; diet, 68

Internal : calomel, guaia. cum, and sulphurated antimony for gouty patients. 68; sulphur w a te $\mathrm{r}$, 68 ; indifferent waters in senile cases, 68: carbolic acid, 68; cannabis indica 68; ichthyol, 69; aspirin, 69; antipyrin, 69

universalis, 65

"Pseudo-lupus vulgaris," 423
Pseudopelade, 627

Psoriasis, 315

abrasion and, 324

after vaccination, 257

auto-intoxication and, 325

clinical course of, 320 types of, 321

diagnosis of, 207, 299, 326-9, $346,350,393,396,459,492$, 597

distribution of lesions in, 317

erysipelas and, 324

etiology of, 323

gout and. 323

guttata, 316

- inoculability of, 325

- inveterata, 316

joint affections in, 320

"lepra," 317

linguæ, 484

- nervous influences and, 324

nummularis, 316

of hairy skin, diagnosis of, 385

of scalp, diagnosis of, 413

of tongue, 621

palmar, 484

pathology of, 321

prognosis of, 329

punctata. 316

rupioides, 316

season and, 324

symptoms of, objective, 315 subjective, 319

syphilitic, 492

treatment of -

Constitutional : Fowler's solution. 330; Asiatic pill, 330; antimony in acute cases, 320 ; phosphorus. 332; salicylate of sodium 331 , bromide of potassium, bromide of sodium, or hydrobromate of quinine, 331; salicin, 331 ; iodide of potassium, 331; biniodide of mercury, 331; naphthalan, 331; thyroid extract, 331; vaccine, 332; in cases associated with osteoarthritis, a r s e n i c, 332 ; meat diet and hot water, 332

Hygienic : clothing, 329 ; avoidance of chill. 329: climate, 329; sea-bathing, 329

Local: removal of scales, 332; salicylic acid in spirit. 332 ; antimicrobic remedies, 332; alkaline baths, 333: mercurial ointments, 333; tar, 333 ; anthrasol, 333; resorcin, 333; chrysarobin. 333; 
traumaticin, 334; pyrogallic acid, 334 ; eugallol, 334; pyraloxin, 334; caustics, 335 ; massage of skin, 335; radiotherapy, 335; high - frequency current, 335 ; radium, 335 ; sulphur baths, 335 ; carbolic oil or weak lead preparations if tendency to development of pityriasis rubra is present, 336 iasis, types of, 321

vaccination and, 324

ospermose folliculaire végéante (see Keratosis follicuaris)

rosperms," 683

$x$ irritans, 352, 366

ura, 116

etiology of, 117

hæmorrhagica, 118

lesions of, 117

rheumatica, 118

diagnosis of, 120

etiology of, 119

pathology of, 119

prognosis of, 120

symptoms of, 118

treatment of -

Rest in horizontal position, quinine and iron, 120 ; liberal diet, 120

sinplex. 118

treatment of -

Rest in bed, with generous diet, 118; iron (or arsenic) and chlorate of potassium, 118; adrenalin, 118

urticans, 82

(see Yaws)

ular syphilide, 477

ule, definition and characters of, 15

formation of, 5

nia in vaccination, 257

ermite végétante, 144

cke, acute circumscribed edema of, 31

ine eruptions. 249

um dermatitis, 230

in acne vulgaris, 574

in cancer en cuirasse. 700

in cheilitis exfoliativa, 618

in cheloid, 653

in cutaneous epithelioma, 754

in genital leucoplakia. 623

in idiopathic leucoplakia of tongue, 622

in keratosis senilis, 694

$2 \mathrm{~W}$
Radium in leprosy, 534

- in næevus vascularis, 662

- in Paget's disease, 700

- in prurigo, 77

in pruritus, 67

in psoriasis, 385

in rhinophyma, 135

in rodent ulcer, 707

in rosacea, 135

in warts, 693

in $\mathrm{x}$ anthoma, 675

Rash of chickenpox, 34 of enteric fever, 32

of erysipelas, 36

of measles, 31

of rötheln, 31

of scarlatina, 30

of smallpox, 33

of typhus, 32

(see also Eruptions)

“Raspberry excrescence," 255

Ray fungus, 419

Raynand's disease, 214

etiology of. 215

prognosis of 215

symptoms of, 214

treatment of-

Constitutional : i $\mathrm{e} \mathrm{h}$ thyol, 216; arsenic, 216; quinine, 216; diet rich in hydrocarbons, 216

Local: galvanism, 215; high-frequency current, 215; massage, 215

Recklinghausen's (von) disease, 655

Red gum, 614

sweat, 610, 611

"Reminders" in syphilis. 484

Rete, lower, pathological processes in, 8

upper, pathological processes in, 8

Rhagades, 18

Rheumatic nodules, diagnosis of, 657

Rhinophyma, 133

- diagnosis of, 583

treatment of-

Local: carbon - dioxide snow, 135 ; radium, 135 (sec also under Rosacea)

Rhinoscleroma, 582

diagnosis of, 583

etiology of 583

symptoms of, 582

treatment of. 583

Rhus eruptions, 226

vernix eruptions, 228

Ribbed tongue, 623

Ringworm, 370

anaphylaxis in, 380

animal origin of some cases of, 378

bald (see Tinea decalvans)

"black-dot," 382 
Ringworm, diagnosis, 39, 129, 413

- (see also Tinea)

etiology of, 371

- epitome of, 378

infection in, 378

microsporon cultures in, 376

microsporons of, 372

and trichophytons of, differences between, 373 mycology of, 377

of beard (see Tinea sycosis)

of body (see Tinea circinata)

of chin (see Tinea sycosis)

of eyebrow (see Tinea palpebralis)

of feet, 398

of genital region (see Eczema marginatum)

of hairless skin (see Tinea circinata)

of hairy parts (see Tinea tonsurans and Tinea sycosis)

of mucous membranes, 371, 394

of nails (see Onychomyosis)

of scalp (see Tinea tonsurans)

parasites, culture media for, 377

pathology of. 379

prophylaxis of, 391. 393

Tokelau (see Tinea imbricata)

trichophyton cultures in, 376

trichophytons of, 373 and microsporons of, differences between, 373

endo-ectothrix, 374, 375

endothrix. 374, 375

Ritter's disease, 343

Rodent ulcer, 44, 705

clinical features of. 705

—- diagnosis of, 459, 494, 707

—_ etiology of, 706

pathology of, 706

prognosis of, 707

stages of, 705

treatment of-

Local : free excision in appropriate parts, 707; radium, 707 ; c ar bon-dioxide snow in pre-ulcerative stage, 708; cauterv and caustics, 708; caustics al on e, 708; curetting and arsenious acid, 708 : Finsen light, 708; X-rays, 708: zinc ions in early stages, 708

Rokitanskv's classification, 22

Röntgen-ray dermatitis. 229

- treatment (see $\mathrm{X}$-rays)
Rosacea, 133

diagnosis of, 129,134, 608

pathology of, 134

prognosis of, 134

symptoms of, 133

treatment of-

Constitutional : vaccine, 134: intestinal antiseptics, 134; arsenic, 135; ichthyol, salol, or $\beta$-naphthol, 135

Hygienic : regulation of diet, 135; no alcohol, 135;

Local: as for acne vulgaris, 135; scarification, 135; Paquelin's cautery, 135 ; electrolysis, 135 ; $\mathbf{X}$ rays, 135 ; radium, 135

Roseola, 95

syphilitic, 476

Rötheln, ragnosis of, 531

Royal Commission on Tuberculosis, Human and Bovine, final report of, 456

Rubella, rash of, 31

Rubeoloid erythema, 101

Rupia, 478

diagnosis of, 494, 532

Sabouraud's microbacillus, 570

proof medium, 377

Sacculated tongue, 623

"Sago tongue," 670

Salicylate of soda eruptions, 250

Salicylic-acid eruptions, 250

Salt, handling of, eruptions caused by, 228

Salvarsan in syphilis, 510

in yaws, 542

Salve muslins, 307

Sand-flea, 367

Sarcoma, alveolar, 710

cutaneous, 708

- clinical features of, 708

- - diagnosis of, 710

— - pathology of, 710

—- prognosis of, 710

treatment of-

Excision, 710 ; arsenic, 711; Colev's fluid in inoperable cases, 711; physicotherapeutic measures as palliatives, 711

idiopathic multiple pigmented hæmorrhagic, 711 melanotic, 709

originating in nævus pigmentosus, 664

Sarcoptes scabici hominis, 352, 355

Scab, definition and characters of, 17

Scabies, 353 
Scabies, diagnosis of, 39, 67, 298, $365,356,365$

etiology of, 353

of animal origin, 359

pathology of, 355

prognosis of, 356

secondary lesions in, 354

symptoms of, 353

subjective. 353

treatment of-

Local : sulphur ointment. 357: "la frotte," 357; St. Louis Hospital and other ointments, 357; Kaposi's ointment, 358; Hebra's modification of Wilkinson's ointment, 358 ; Vleming kx's lotion. 358 ; sulphur precipitate soap, 358; balsam of Peru, 358; peruol, 358; for delicate skin, stavesacre. 358: applications should be vigorous but not violent. 358; if secondary lesions are inflamed. first subdue inflammation, 359

Scale, definition and characters of, 16

Scalp, lesions on, diagnosis of, 41

ringworm of (see Tinea tonsurans)

Scar. definition and characters of, 19

hypertrophic, 19

Scarlatina rash, 30

Scarlatiniform erythema (see Erythema scarlatiniforme)

Schistosomum hæmatobium, 369

Schönlein's disease (see Purpura rheumatica)

Sclavo's serum in malignant pustule. 585

Sclerema neonatorum, 727

treatment of, 728

Sclerodactylia, 185

prognosis of, 186

symptoms of. 185

treatment of, 186

Sclerodermia, 180

circumscribed (see Morphœa) diffuse. 180

atrophic, 181

etiolog. 5 of, 182

infiltrated, 181

pathology of, 182

prognosis of. 183

symptoms of, 180

trantment of-

Constitutional : cod-

liver oil. 183: arsenic, 183; thy roid feeding. 183

Local : massage, 183 ; galvanism, 183

progressive (see Sclerodacty. lia)
Sclerosing granuloma of pudenda (see Ulcerating granuloma of pudenda)

Scrofula, 428

relation of, to tubercle, 428

Scrofulodermia, 433

diagnosis of, 457

Scrofulous abscess, sub-cutaneous, 436

Scrotal tongue, 623

Seaman's skin, 225

Sebaceous adenoma (see Adenoma sebaceum)

cysts, 648

glands, affections of, 592-605

Seborrhoea, 592

capitis, 592

diagnosis of, 386, 392, 413, 597

distribution of lesions of. 594

etiology of, 596

- in acne vulgaris, 571

oleosa, 592

- papulosa, 600

- pathology of, 595

- psoriasis type of, 601

sicca, 592. 593

starting-point of, 595

symptoms of, 592

treatment of -

Local : wash with soap and water, 598; apply parasiticides, 598 ; sulphur, 598; resorcin, 599; euresol, 599; captol, 599; anthrasol, 599; mercurial applications, 599 ; $\beta$-naphthol, 600; Jamieson's ointment, 600 ; wash with spiritus saponis alkalinus and warm water. 600 ; infusion of quillaia bark, 600

corporis, 600 diagnosis of, 351, 491, 602 pathology of, 601 symptoms of, 600 treatment of-

As for seborrhœic eczema: course of arsenic, if general nutrition of skin is bad, 602

Seborrhœic dermatitis of infancy, 51,279

eczema (see Eczema seborrhoicum)

of infancy. 51,279

Secondary lesions. 16

Secretion, anomalies of. 2

Senile atrophodermia, 224

keratoma (see Keratosis senilis)

neuroses of skin. 54,55, 62

Serpiginous ulceration of geni. talia (see Ulcerating granuloma of pudenda)

Serum diagnosis of syphilis, 495 
Serum eruptions, 258

- in erysipelas, 545

- in glanders, 582

in leprosy, 533

in malignant pustule, 585

Silver, working with, eruptions caused by, 228

Skin, atrophy of (see Atrophodermia)

glossy, 221

- pathology of, 1

Smallpox, diagnosis of, 356, 493, 550

distribution of lesions, diagnostic import of, 34

eruption, 33

mild modified, diagnosis of, 550

"Smoker's patch" (see Leucaplakia of tongue idiopathic)

"Snuffles" in hereditary syphilis, 487

Sodium salicylate eruptions, 250

Soft sore (see Ulcus molle)

Sore, primary (see Chancre)

Spinal cord and cutaneous affections, 56

Spiradenitis disseminata suppurativa, 443

Spirochæta pallida, 470,489

Burri's indian-ink method and, 499

dark-field illumination method and, 498

Levaditi's method and, 499

pallidula, 539

pertenuis, 539,540

"Spontaneous" gangrene, 218

“Spoon-nail," 644

Spore of Malassez, 593

Sporotrichium Beurmanni, 422

indicum, 422

Schenki, 422

Sporotrichosis, 421

diagnosis of, 423

etiology of . 422

forms of, 422

symptoms of, 422

treatment of-

Iodides in large doses, 423; puncture nodules, inject iodine solution, dress with iodine lotions, 423

"Spotted sickness" (see Pinta)

Squame, definition and characters of, 16

Staphylococcus epidermidis albus. $279,594,639$

pyogenes, 630

albus, 288, 551, 560, 562, 570

aureus. 288. 369. 551. 552. $553,557.562,590$

Static current in lupus vulgaris. 466

in prurigo, 77
Sticks for antiseptic remedies, 307

Stramonium eruptions, 253

Stratum corneum, pathological processes in, 718

germinativum, pathological processes in. 8

granulosum, pathological processes in, 8

lucidum, pathological processes in, 8

“Strawberry tongue," 31

Streptococcus plicatilis, 617

- pyogenes, 553

- of Fehleisen, 544

Streptothrix infection, 420

Stria, linear, 222

Strophulus, 80

Strumous ulcers, 435

- diagnosis of. 436

origins of, 435

- svmptoms of, 435

treatment of-

Constitutional: plenty of good food. 437; cod - liver oil, 437; i ron and ot her to n i s, 437; N e w Tuberculin, 437

Local: open abscesses and scrape their walls, 437; remove caseous glands, 437; cleanse ulcers, 437; a p p 1 y antiseptic dressing, 437; X-rays, 437

Strychnia eruptions, 252

Subcutaneous scrofulous abscess. 436

- injection test for tuberculosis, 431

Sudamina, 613

- diagnosis of. 614

_- symptoms of. 613

- treatment of, 614

Sugar, handling of, eruptions caused by. 228

Sulphonal eruptions. 253

Sulnhur eruptions, 228, 253

Sunburn, 101

Sweat. bloody, 612

blue. 611

coloured, 611

eczema, 280

foul-smelling, 609 (see also Bromidrosis)

green. 611

phosphorescent, 612

red, 610,611

secretion of, altered in leprosy. 524 vellow. 611

Sweat-glands, affections of, 605-15 Sycosis, coccogenic, 557 diagnosis of, 298, 392, 559

pathology of, 558 
Sycosis, coccogenic, symptoms of, 557

treatment of-

Constitutional : vac. cille, 560

Local: removal of crusts, 559; epilate, 559 ; in mild cases, oleate of mercury or weak sulphur ointment, 560; in more severe cases, strong sulphur or resorcin paste, or Unna's carbolic mer. cury plaster mull, $560 ; \mathrm{X}$-rays, 560

frambœsiformis, 627

lupoïde, 558

papillomateuse, 627

Symmetrical distribution of lesions, significance of, 26

gangrene (see Raynaud's disease)

Syphilide, acneiform, diagnosis of. 493

annular, 479

circinate, 479

cornée, 479

ecthymatous, 477

diagnosis of, 493

impetiginous, 477

- diagnosis of, 493

large acuminate pustular, 477

flat pustular, 477

lenticular, 479

macular, 478

diagnosis of, 491

maculo-papular, 478

maculo - papulo - squamous, 479

miliary papular, 478

papular, 478

of palms and soles, diag. nosis of, 492

papulo-squamous, 479

pustular, 477

diagnosis of, 493

small acuminate pustular, 477

flat pustular, 477

squamous, diagnosis of. 492

tubercular, diagnosis of, 531

Syphilis, 470

course of, 484

diagnosis of, 37, 328, 474, $491-99,540$

serum, 495

eruptive period in, 471

etiology of, 489

germ inheritance of, 470

hereditary, 487

bilateral herpes in, 176

condylomata in. 487

diagnosis of, 495

gummata in, 488

intertrigo in, 487
Syphilis, hereditary, later symp. toms, of, 488

onychia in, 487

period of latency in, 488

permanent teeth in, 488

skull in, 488

"snuflles" iv. 487

symptoms of, 487

ulceration of palate in, 488

herpes genitalis and, 475

incubation period in, 471

intluence of age in, 485

- of health in, 485

latent period in, 471

period of sequelæ in, 471

primary, 471,472 (see also

Chancre)

proguosis of, 499

"reminders", in, 484

secondary, 471,476

colour of lesions in, 481

condylomata in, 480

constitutional disturb. ance in. 476

diagnosis of, 297

duration of, 484

enlargement of glands in, 483

leucodermic lesions on neck in, 481

moist papule in, 480

_ - mucous papule in, 480

mucous-membrane lesions in, 482

nails in, 483

polymorphous stage of, 480

symmetrical character of lesions in, 481

ulcers on tonsils in. 482

warty conditions in, 480

sperm inheritance of, 470

stages of, 471

sýmptoms of, 472

tertiary, 471,485

bone lesions in, 486

brain lesions in, 486

gummata in, 485

diagnosis of, 494

lesions of blood-vessels in, 486

of internal organs in, 486

mucous-membrane lesions in, 486

nerve lesions in, 486

periosteum lesions in, 486

spinal cord lesions in, 486

ulceration in, diagnosis of, 559

transmission of, by vaccination, 256

treatment of -

Mercury: general principles, 500 ; by mouth, 500 ; 
by intramuscular injection, 501; mercuriol, 501; Lambkin's cream, 502 ; by subcutaneous injection, 502; by intravenous injection, 502 ; by inunction, 502; bv baths, 502; by suppositories, 503 . Author's preferences, 503: in urgent cases, intramuscular injection; in ordinary cases, oral administration; excision of primary sore, 504; blue pill, 504; grey powder, 504; Plummer's pill, 504 ; perchloride of mercury, 504; inunction if oral administration disagrees, 504 ; intramuscular injection, 505; vapour bath. 505 ; calomel, 505 ; special precautions, 505 ; length of administration, 506; local administration, 507: ammonio-chloride and lard, or oleate of mercury. 507; calomel for mucous tubercles, 507; perchloride gargle for mouth and throat, 507; in congenital syphilis. inunction, 507; grey powder if skin eruption very severe, 507 : for tertiary lesions, iodide of potassium, 507; iodide of sodium, 507; addition of ammonia, 507; iodoform, iodol. dermatol, aristol. or xeroform, 508

Arsenic: atoxyl, 509; arsacetin, 509; soamin, 509 . Salvarsan : b y int ramuscular injection, 510 ; by subcutaneoûs injection, 510 ; bv intravenous injection, 510 ; $\mathrm{s} u$ p e $\mathrm{r}$ iorit $v$ of intravenous method. 511: dosage. 511; unsuitable cases, 512 ; results. 512 : in parasyphilis. 513: combination of salvarsan and mercury, 514. Hectine, 514

Syphilis, Wassermann's test for. 495

Syphilitic lesions, vigmentation as a result of. 6

“- psoriasis," 492

u? cer. diagnosis of, 437

Svringomvelia, 220

Systemic blastomycosis, 425

T.O.A. (see Tuberculin. Old) T.R. (see Tuberculin, New)
Tabanidæ, 352

Tambu-fly disease, 367

Tanners, eruptions in, 228

Tar eruptions, 228, 252

Teignes, Alibert's, 20, 21

Telangiectasis, clinical features of, 660

etiology of, 660

treatment of -

Local: electrolysis or galvano-cautery. 661

Terebene eruptions, 253

Thapsia, feigned e ruptions caused by, 230

Thermo-cautery (see Paquelin's cautery)

Thickening of epidermis, 6

Thyroid extract in eczema, 304

—— in ichthyosis, 726

—_ in lupus vulgaris, 468

- in psoriasis, 331

Tilbury Fox, impetigo contagiosa of (see Impetigo contagiosa)

Tinea alba, 406

albigena, 406

barbæ (see Tinea sycosis)

circinata, 371, 393 diagnosis of, 298, 351, $395,402,491$

etiology of, 393

prognosis of, 395

simulating eczema, 394

symptoms of, 393

treatment of-

Local : rem oval of superficial layers of epidermis with iodine or liquor epispasticus, 395; chrysarobin. 395 ; salicylic and benzoic acid, 395 ; oleate of copper or oleate of mercury, 396: milder applications for young children, 396

cruris (see Eczema marginatum)

decalvans, 382

flava. 405

imbricata, 401

- diagnosis of, 402

- etiology of, 402 .

prognosis of 402

symptoms of, 401

trastment of -

Hygienic: disinfection of elothes. 403

Local : linimentum iodi, 402: resorcin dissolved in tinct,ure of benzoin. 402: chrvsarobin. 403; Cassia alats. leaves, 403

intersecta, 407

large-spored. 382

marginata, 371

nigra, 406 
Tinea nigro-circinata. 407

- nodosa, 644

- palpebralis, 370

Sabouraudi, 406

small-spored, 373

sycosis, 370,391

diagnosis of, 392, 559

infection in, 392

pathology of, 392

prognosis of, 393

symptoms of, 391

treatment of-

Local : epilation by X-rays. 393; with forceps, 393 ; para siticides such as chrysarobin, sulphur, or oleate of copper, 393

tonsurans, 370

diagnosis of, 298,385

itching in, 384, 385

mierosporic, 381, 382, 384, 385

diagnosis of, 385

symptoms of, 381

prognosis of, 386

symptoms of, 380

trichophytic, 382, 383, 385

symptoms of, 382

treatment of-

Local: epilation by X-rays, 387; epilation with forceps, 388 ; paint with aniline pencil, 388; tincture of iodine, oarbolized glycerine or vaseline, 388 ; clear away débris with spirit and ether lotion, 389; antiseptic wa shes, 389; salicylic acid dissolved in chloroform or ether, 389 ; in chronic cases, chrysarobin, 389; Hodara's solution. 389; sulphur for voung children, 390; mercury, 390; Coster's paste, 390: strong sulphur and mercurial ointments, 390 . oleate of copper. 390 ; carbolic acid. 390; croton oil, 390: needling with croton oil for refractorv stumps, 390 . Vidal's treatment. 391

versicolor. 339, 403

diagnosis of, 39, 404, 405, 416. 491

etiology of. 404

prognosis of, 405
Tinea versicolor, symptoms of, 403

treatment of -

Local : washing with soft soap and warm water, and friction with flesh brush, 405; i o d in e, 405; hydrosulphate of soda, 405; sulphurous acid, 405; naphthol, sulphur, or salicylicacid ointment, 405

Toe, trophic ulcer of, 220

Toe-nail. ingrowing, 645

Tokelau ringworm (see Tinea imbricata)

Tongue, black, 625

- groove. 623

- leucoplakia of (see Leucoplakia of tongue)

- scrotal, 623

- (see also Glossitis areata exfoliativa)

Toxins, intestinal, and skin affections, 59

Trade eruptions, 228

- treatment of-

Scrupulous cleanliness, 259; avoidance of contact if possible, 259

Transitory benign plaques of tongue, 624

Trematodes, 352

Treponema pallidum, 470

pertenuis (pertenue), 539, 540

Trichomycosis capillitii, 643

'Trichophytic infection, anomalous cases of, 398

Trichonhyton acuminatum, 373

albiscicans, 406

- Blanchardi, 406

- Ceylonese, 407

crateriforme, 373

Macfadyeni, 406

Mansoni, 402

- Sabotion, 380

- sulfureum, 373 violaceum, 373

Trichophytons and microsporons, differences between. 373

cultures of, 376

endo-ectothrix, 374, 375

endothrix, '374, 375'

mégaspore. 374. 376

microĩde. 374,376

neo-endothrix, 375

true endothrix, 375

Trichophytosis, 382, 383, 385, 391, 393,396

Trichorrhexis nodosa, etiology of, 630

symptoms of, 630

- treatment of.' 631

Trichosporum giganteum, 643 
Trophic disturbances, 55 ulcers, 219

Tropical skin, 225

Tubercle, anatomical, 12

- bacillus, 429,430

relation of, to scrofula, 428

pathological, 428

Tuberculides, 442

Tuberculin eruption, 257

in treatment of leprosy, 533

- (New) in treatment of strumous ulcers, 437

of lupus vulgaris, 469

(Old) in diagnosis of lupus vulgaris, 459 of tuberculosis, 431

Original Alt (see Tuberculin, old)

Rügkstand (see Tuberculin, New)

Tuberculosis, 428

- bacillus of, 429

clinical tests for, 431

cutaneous, diagnosis of, 37

cutaneous test for, 432

cutis orificialis (see Tuberculous ulcers)

diagnosis of, 541

Human and Bovine, final report of Royal Commission on, 456

ophthalmic test for, 432

percutaneous test for, 432

predisposing causes of, 430

subcutaneous test for, 431

transmissibility of, by vaccination, 256

verrucosa cutis, 440

Tuberculous lesions, 37

ulcers, 437

- - diagnosis of, 439

- - etiology of, 438

—- symptoms of, 437

- - treatment of, 439

Tumour, definition and characters of, 13

Tumours, small, on face, 45

Turner's classification, 20

Turpentine eruptions, 230, 253

Tylosis, 726

- linguæ (see Leucoplakia of tongue, idiopathic)

treatment of-

Salicylic acid, with or without ichthyol, 727; rheumasan, 727

Typhoid rash, diagnosis of, 32

Typhus rash, diagnosis of, 32

Ulcer, callous, 494

- crateriform, of Hutchinson, 702

definition and characters, 18

of foot, perforating. 219

rodent (see Rodent ulcer)

trophic, of toe, 220
Ulcerating granuloma of pudenda, 590

diagnosis of, 591

etiology of, 591

pathology of, 590

symptoms of, 590

treatment of-

Local: X-r a ys, 591; in early stage, excision, 591; scraping,

Ulceration, 5 591

Ulcers of genital region, 50

on face, 44

strumous (see Strumous ulcers)

trophic, 219

tuberculous (see Tuberculous ulcers)

Ulcus molle, 475,586

- etiology of, 586

treatment of -

Hygienic : scrupulous cleanliness and hot baths, 586

Local: iodoform powder, or iodol, europhen, xeroform, or aristol, 586

Ulerythema centrifugum, 120

Umbilication, 5

Unna's classification, 23

Uridrosis, 612

Urine, feigned eruptions caused by, 230

Urticaria, 78

— asthma and, 79

- bullosa, 82

- diagnosis of, 84

—_ etiology of, 82,83

factitia, 80

fugax, 78

"gangrenous," 60

gigas, 81

hæmorrhagica, 82

cdematosa, 81

of mucous membranes, 79

"paludal," 82

papulosa, 80 .

diagnosis of, 84

pathology of, 83

perstans, 79

prognosis of, 85

symptoms of, 78

treatment of-

Constitutional : removal of cause, 85; emetic, 85 : purge, 85 ; quinine. if of malarial origin, 85; calcium of chloride in gouty cases, 85; regulation of bowels in chronic cases, 86; vaccine and salol in bacillary cases, 86; sulphate of atropia, 86 ; adrenalin, 86; ichthyol, 86 
Urticaria, treatment of (continued)-

Hygienic : prevent chill, 85 ; rest, 86 ; sea voyage, 86

Local : anti-pruritic applications, 85; starch powder for body linen and bedclothes, 85 ; light clothing, 86

pigmentosa, 88

diagnosis of, 91.675

etiology of, 90

pathology of, 90

symptoms of, 88

treatment of -

Belladonna and atropine (?), 91; antipruritic measures, 91; improve general health, 91

Vaccination, complications of, 260

eruptions, 254

"Lancet" Special Commis. sion on, 260

Vaccine in acne vulgaris, 575

in coccogenic sycosis, 560

in eczema, 304

in erysipelas, 545

in ery thema multiforme. 115 , 116

in furunculosis, 564

in glanders, 582

in impetigo contagiosa, 551 herpetiformis, 556

of Bockhart. 557

in lupus erythematosus, 130

in pemphigus, 166

in psoriasis, 332

in rosacea, 134

in urticaria, 86

Vacciniform ecthyma of infants, 257

“Vagabond's skin," 362, 363

Varicella, 34, 678

- gangrenosa (sce Dermatitis gangrenosa infantum)

Variola (see Smallpox)

Vaso-motor disturbances, pure, 55

Vegetable irritants, 227

- parasites, 370

- diseases caused by, 39

Vegetating follicular psorospermosis (see Keratosis follicularis)

Veld sore, 369

\section{etiology of, 369}

treatment of -

Remove superficial epidermis, rub ulcersurface with lint soaked in perchloride of mercury, 369

Velvety eultures of trichophytons, 376
"Venereal collar," 481

Venereal sores (see Chancre and Ulcus molle)

warts, 691

Ver du cayor, 368

macaque, 367

Vermilion of lips, lesions on, 46, 616-20

Verruca acuminata, 691

digitata, 690

filiformis, 690

necrogenica, 439

symptoms of, 439

treatment of -

Local: salicylic acid applied by Unna's plaster mull or Brooke's ointment, 440 ; if lesions are spreading rapidly, destroy them with caustics, galvanocautery, or X-rays. 440

Peruviana, 694

plana (see Wart, flat)

senilis, 690,693

seborrhoica, 690,693

vulgaris (see Wart, common)

Vesicle, definition and characters of, 13

formation of, 4

umbilication of, 5

Vibices, definition of, 10

Vitiligo (see Leucodermia)

Vleming $\mathrm{kx}^{\prime} \mathrm{s}$ lotion, 358

Von Pirquet's test, 380. 432

Von Recklinghausen's disease, 655

Vulnerability of skin, 5

Wart, 688

common, 688

etiology of, 692

treatment of -

Galvano-cautery, carbon-dioxide snow, or cataphoresis, 692; salicylic and chro$\mathrm{m}$ i c a cid. 693; strong caustics. 693; radium, 693; X-rays, flat, 689 693

- etiology of. 692

treatment of (see Wart. common)

seborrhœic. 690, 693

venereal, 691

treatment of -

Snip off with scissors or destroy with galvano-cautery, 693 ; chromic or glacial acetic acid for small ones; keep dry and clean, 693 
Wassermann's test for syphilis, 471,495

Werlhof's purpura, 118

Wheals, 12

"White-spot disease" (see Morphoa)

Willan's classification, 21

Wilson's classification, 21

Wood-tick, 369

Wrinkled tongue, 623

Xantho-erythrodermia perstans, 203. 337

Xanthoma, 671

clinical features of, 671

diabeticorum, 671, 672, 674, 675

diagnosis of, 674

en tumeurs, 671

etiology of, 674

multiplex, 671, 673, 674, 675

of Balzer. 6, 675

palpebrarum, 671, 674, 675

patholog $y$ of, 673

planum, 671, 674

prognosis of, 675

treatment of-

Galvano-cautery or thermo-cautery, 675: radium, 675; X-rays, 675; excision, 6\%5: anti-glycosuric, in xanthoma diabeticorum, 675

tuberosum, 671, 674

pigmentosum (see Kaposi's disease)

X-ray dermatitis, acute, 229

X- chronic, 229

$\mathrm{X}$-rays in acne vulgaris, 574

in actinomycosis, 420

in alopecia areata, 642

in cancer en cuirasse, 700

in cheloid, 653

in coccogenic sycosis, 560

- in cutaneous epithelioma, 704

- - sarcoma, 711

in eczema. 312

in elephantiasis Arabum, 590

in favus, 414

in furunculosis, 563

in genital leucoplakia, 623

in hirsuties, 629

in hyperidrosis, 607

in idiopathic multiple pig. mented hæmorrhagic sarcoma, 713
X-rays in keratosis senilis, 694

- in leprosy, 534

- in leukæmia cutis, 718

in lichen planus, 196

- in lupus erythematosus, 132 vulgaris, 466

- in lymphangioma, 670

- in mycosis fungoides, 717

— in Paget's disease, 699, 700

in prurigo, 77

- in pruritus, 67

in psoriasis, 335

in rosacea, 135

in sarcoma, 711

in strumous ulcers, 437

in tinea tonsurans, 387

in tubcosis, 393

n tuberculous ulcers, 439.

in ulcerating granuloma of pudenda, 591

in verruca necrogenica, 440

in warts, 693

in xanthoma, 675

Yaws, 535

diagnosis of, 540,541

distribution of lesions of, 535

etiologv of, 539

incubation period of, 535

pathology of, 538

primary period of, 535

prognosis of, 541

secondary period of, 536

stages of, 535

symptoms of, 535

tertiary period of, 537

treatment of-

Constitutional : for fever, quinine or salicylates. 542; ammonium carbon: ate, 542; iron and codliver oil, 542; in tertiary stage, mercury, 542; iodide of potassium, 542; salvarsan, 542

Local: sulphur baths, 542 ; c a $10 \mathrm{mel}$ fumigations, 542; for ulcers, black w a sh, perchloride of mercury, or iodoform,

Yellow sweat, 611

Zona (see Herpes zoster)

Printed by Cassell and Company, limiten, La Belle Sacvage, London, E.C. 


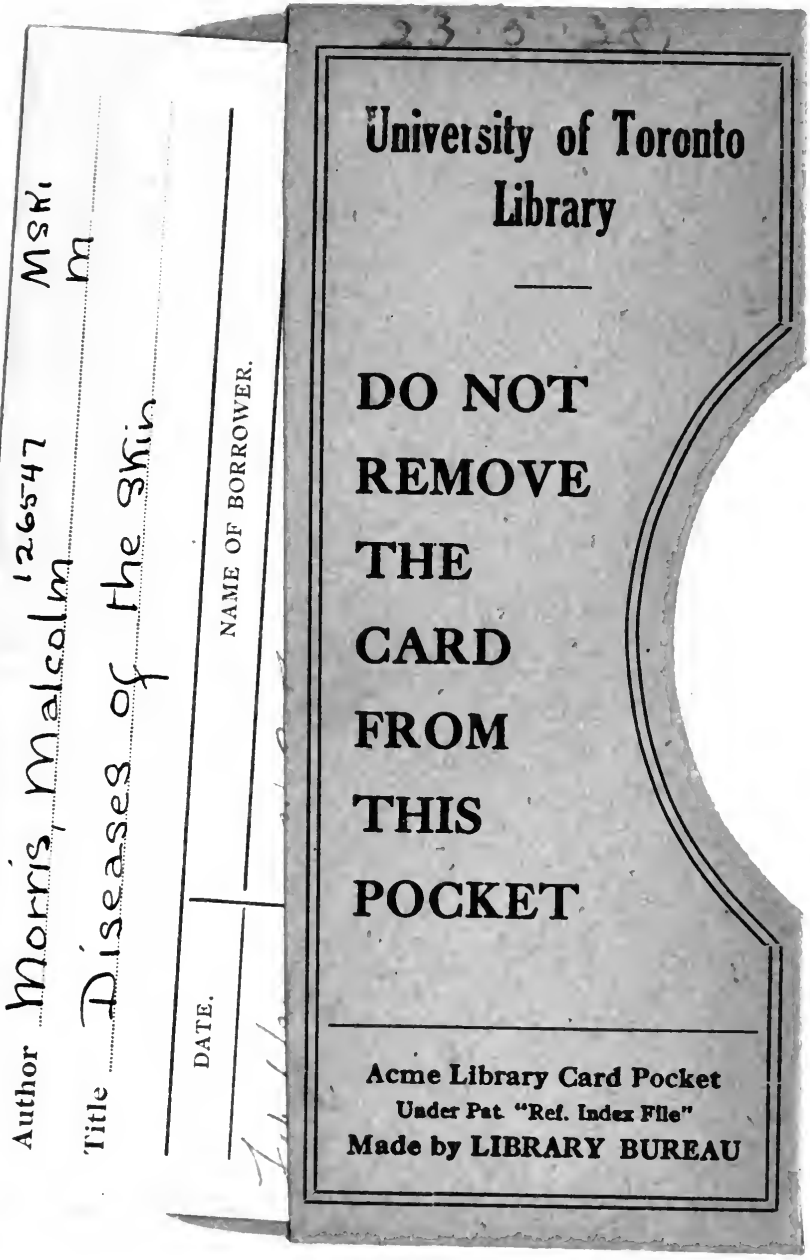


